\title{
Modelización multivariante de los procesos de enseñanza - aprendizaje basados en competencias en educación superior
}

Andrea Conchado Peiró 
UNIVERSIDAD POLITÉCNICA DE VALENCIA

Departamento de Estadística e Investigación Operativa Aplicadas y Calidad

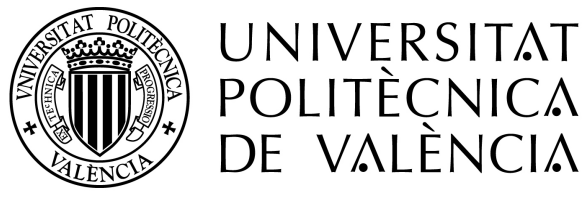

\title{
Modelización multivariante de los Procesos de Enseñanza - Aprendizaje basados en Competencias en Educación Superior
}

\author{
TESIS DOCTORAL \\ Presentada por: \\ Andrea Conchado Peiró
}

Dirigida por:

José Miguel Carot Sierra

José Jabaloyes Vivas 


\section{Esta editorial es miembro de la UNE, lo que garantiza la difusión y comercialización de sus publicaciones a nivel

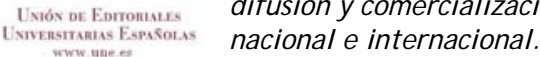

\section{(c) Andrea Conchado Peiró, 2011}

Primera edición, 2011

(c) de la presente edición:

Editorial Universitat Politècnica de València

www.editorial.upv.es

ISBN: 978-84-695-1039-1

Ref. editorial: 5512

Queda prohibida la reproducción, distribución, comercialización, transformación, y en general, cualquier otra forma de explotación, por cualquier procedimiento, de todo o parte de los contenidos de esta obra sin autorización expresa y por escrito de sus autores. 


\section{Agradecimientos}

La elaboración de esta tesis doctoral no hubiera sido posible sin la ayuda de otras muchas personas a las que quisiera agradecer su apoyo durante la realización de este trabajo.

En primer lugar a mis directores de Tesis, José Miguel Carot y José Jabaloyes, quienes me introdujeron en los fundamentos de la estadística, la metodología de encuestas y el análisis multivariante, no sólo por el esfuerzo y dedicación que les ha supuesto la dirección de este trabajo, sino también por su confianza y valiosas orientaciones, que me han sido de gran ayuda en mi crecimiento a nivel profesional.

Asimismo quisiera agradecer a los profesores José Ginés Mora y Andrés Carrión sus aportaciones y valoraciones en el desarrollo de la investigación, que directa o indirectamente, sin duda han contribuido a la orientación del trabajo hacia el enfoque más adecuado en cada momento, así como a mis compañeros del Centro de Gestión de la Calidad y del Cambio, por su apoyo incondicional día tras día.

Me gustaría igualmente agradecer a mis padres y mi hermana Beatriz la ayuda recibida en tantas ocasiones, gracias a los que he podido conseguir muchos de mis objetivos personales y profesionales. Y por último a Guillermo por apoyarme siempre, y haber soportado con infinita paciencia los momentos más difíciles en la realización de este trabajo. 
To err is human, to forgive divine, but to include errors in your design is statistical

Leslie Kish, 1978 


\section{Resumen}

La importancia de las competencias en el ámbito universitario se ha incrementado de forma muy significativa en los últimos años a consecuencia del proceso de adaptación de las universidades al Espacio Europeo de Educación Superior. Los nuevos títulos de Grado y Máster enfatizan en mayor medida en los resultados del aprendizaje que el estudiante obtendrá al finalizar los estudios, en contraposición a las antiguas titulaciones diseñadas en torno a la adquisición de conocimientos. Este cambio de enfoque implica la necesidad de una transformación metodológica, no sólo en cuanto a la definición y planificación de las materias sino en lo que se refiere a la selección de nuevas metodologías docentes. En este contexto algunos profesores universitarios ya se están planteando si las metodologías docentes tradicionales resultan adecuadas para afrontar estas nuevas circunstancias y progresivamente están introduciendo en sus clases innovaciones metodológicas, basadas en los denominados contextos activos de aprendizaje.

En los próximos años cabe esperar que sean las universidades las que asuman la planificación y gestión del cambio a nivel institucional hacia este nuevo enfoque metodológico . Sin embargo, la responsabilidad de la ejecución en la práctica de dichos planes recae en última instancia sobre el profesorado universitario. Esta situación supone para muchos de ellos no sólo una dificultad añadida a las ya inherentes a su trabajo, sino que adicionalmente experimentan ciertas carencias en su formación a la hora de desarrollar sus tareas diarias con este nuevo enfoque. Por todo ello resulta absolutamente necesaria la investigación orientada al estudio de los procesos de enseñanza aprendizaje de competencias en el ámbito universitario, con el fin de facilitar la asimilación de las innovaciones metodológicas y evitar que la adaptación de los planes de estudio se convierta en un mero ajuste estético y formal de las metodologías docentes clásicas.

El objetivo de este trabajo es modelizar estos procesos de enseñanza aprendizaje de competencias en Educación Superior, considerando los distintos factores del entorno académico y personal de los estudiantes que potencialmente pueden influir en los resultados del aprendizaje obtenidos. Para ello se realiza en primer lugar una extensa revisión de los conceptos de competencia y enfoque del aprendizaje, así como una descripción de los contextos académicos universitarios y los modelos de enseñanza - aprendizaje en la Universidad más relevantes.

Seguidamente se evalúan cada una de las relaciones que se establecen 
individualmente entre los factores relativos al contexto de los estudiantes y las competencias que adquirieron en la Universidad según su percepción. Del mismo modo se estudia cómo la presencia de determinadas variables modificadoras del efecto, como el área de estudio, influyen en las relaciones entre variables mediante la introducción de relaciones de interacción y confusión.

Una vez examinadas las relaciones entre los factores del contexto de los estudiantes, se validan los instrumentos de medida diseñados en el cuestionario REFLEX para cuantificar los Métodos de enseñanza - aprendizaje empleados y las Competencias adquiridas en la Universidad, mediante técnicas de Análisis Factorial Confirmatorio e Invarianza Métrica, cuyo ajuste se considera excelente o muy bueno en todos los casos. A partir de ambos modelos de medida se propone en base a la teoría y análisis exploratorios previos, un modelo causal de relaciones estructurales. La validación de este modelo resulta aplicable a distintas áreas de estudio y robusto a posibles diferencias institucionales y su ajuste se evalúa como bueno tomando en consideración el incremento de complejidad respecto a los modelos de medida anteriores. Concretamente para el área de Ciencias se plantean ciertas modificaciones a este modelo fundamental desde una perspectiva exploratoria, cuya interpretación y significado resultan coherentes con el contexto de la educación universitaria en esta área de estudio. 


\section{Resum}

La importància de les competències en l'àmbit universitari s'ha incrementat de forma molt significativa en els últims anys a conseqüència del procés d'adaptació de les universitats a l'Espai Europeu d'Educació Superior. Els nous títols de Grau i Màster emfatitzen més que abans en els resultats de l'aprenentatge que l'estudiant obtindrà al finalitzar els estudis, en contraposició a les antigues titulacions dissenyades entorn de l'adquisició de coneixements. Aquest canvi d'enfocament implica la necessitat d'una transformació metodològica, no sols quant a la definició i planificació de les matèries sinó pel que fa a la selecció de noves metodologies docents. En aquest context alguns professors universitaris ja s'estan plantejant si les metodologies docents tradicionals resulten adequades per a afrontar aquestes noves circumstàncies i progressivament estan introduint en les seues classes innovacions metodològiques, basades en els denominats contextos actius d' aprenentatge.

En els pròxims anys cal esperar que siguen les universitats les que assumisquen la planificació i gestió del canvi a nivell institucional cap aquest nou enfocament metodològic. No obstant això, la responsabilitat de l'execució en la pràctica d'aquests plans recau en última instància sobre el professorat universitari. Esta situació suposa per a molts d'ells no sols una dificultat afegida a les ja inherents al seu treball, sinó que addicionalment experimenten certes carències en la seua formació a l'hora de desenvolupar les seues tasques diàries amb aquesta nova perspectiva. Per tot això resulta absolutament necessària la investigació orientada a l'estudi dels processos d'ensenyança - aprenentatge de competències en l'àmbit universitari, amb l' objectiu de facilitar l'assimilació de les innovacions metodològiques i evitar que l'adaptació dels plans d'estudi es convertisca en un simple ajust estètic i formal de les metodologies docents clàsiques.

L'objectiu d'aquest treball és modelizar estos processos d'ensenyança aprenentatge de competències en Educació Superior, considerant els distints factors de l'entorn acadèmic i personal dels estudiants que potencialment poden influir en els resultats de l'aprenentatge obtinguts. Amb aquest objectiu es realitza en primer lloc una extensa revisió dels conceptes de competència i enfocament de l'aprenentatge, a més de una descripció dels contextos acadèmics universitaris i els models d'ensenyança - aprenentatge en la Universitat més rellevants.

A continuació s'avaluen cada una de les relacions que establides indi- 
vidualment entre els factors relatius al context dels estudiants i les competències que van adquirir en la Universitat segons la seua percepció. De la mateixa manera s'estudia com la presència de determinades variables modificadores de l'efecte, com l'àrea d'estudi, influïxen en les relacions entre variables per mitjà de la introducció de relacions d'interacció i confusió.

Una vegada examinades les relacions entre els factors del context dels estudiants, es validen els instruments de mesura dissenyats en el qüestionari RÈFLEX per a quantificar els Mètodes d'ensenyança - aprenentatge empleats i les Competències adquirides en la Universitat, per mitjà de tècniques d'Anàlisi Factorial Confirmatori i Invarianza Mètrica, l'ajust del qual es considera excel-lent o molt adequat en tots els casos. A partir d'ambdós models de mesura es proposa basant-se en la teoria i anàlisis exploradores prèvies, un model causal de relacions estructurals. La validació d'aquest model resulta aplicable a distintes àrees d'estudi i robust a possibles diferències institucionals i el seu ajust s'avalua com adequat prenent en consideració l'increment de complexitat respecte als models de mesura anteriors. Concretament per a l'àrea de Ciències es plantegen amb caràcter explorador certes modificacions a aquest model fonamental, la interpretació i significat del qual resulten coherents amb el context de l'educació universitària en aquesta àrea d'estudi. 


\section{Abstract}

The importance of Competence-based Higher Education has increased in recent years as a result of the process of adaptation of universities to the European Higher Education Area. The new study programmes of Grado and Master emphasize on learning outcomes that students obtain after their studies, as opposed to the previous degrees, mainly focused on the acquisition of knowledge. This change of approach implies a need for a methodological change, not only regarding the definition and planning of the academic resources but also related to the selection of new teaching methods. In this context, some university professors are already wondering whether the traditional modes of teaching and learning are appropriate to meet these new circumstances and consequently they are gradually introducing methodological innovations, based on the so-called active learning contexts.

In the next years universities are expected to plan and manage this change towards this new methodological approach from the institutional level. However, the responsibility for implementation in practice of such plans eventually rests on professors. Sometimes this situation involves an additional difficulty to their work, as they feel some lack of training to develope their daily tasks with this new approach. Therefore, research about teaching - learning skills in university seems to be absolutely necessary in order to facilitate the assimilation of the methodological innovations and prevent the adaptation of old study programmes becomes a mere aesthetic and formal adjustment of the traditional modes of teaching and learning.

The aim of this work is to model these processes of teaching and learning for the acquisition of competencies in Higher Education, taking into consideration how different factors related to the academic and personal environment of students could influence the final achievement of learning outcomes. With this aim, it is exposed an extensive review of the concepts of competence and learning approach, and a description of the university academic contexts and models of teaching and learning in Higher Education.

Secondly, each of the relationships between competencies acquired during Higher Education and the different factors belonging to the academic and personal environment of student is analyzed. Additionally the effect of effect modifiers, such as the field of study, is studied. This effect is mainly focused on the introduction of relations of interaction and confusion between variables. 
Finally, after examining the relationships between contextual factors of students, the measuring instruments used in the REFLEX Project have been validated. This instruments are based on different modes of teaching and learning used by professors and competencies acquired at University. In this stage Confirmatory Factor Analysis and Measurement Invariance techniques have been applied. In both cases the fit of these measurement models has been considered excellent or very high. From here on, a structural model has been proposed, based on previous researches and exploratory analysis. This model has been successfully applied to different fields of study and the differences between universities have been valued as no significant. The fit of this model has been considered good, considering the increase of complexity regarding to the previous models. Specifically for the Science field some changes to the basic model have been suggested from an exploratory point of view. These modifications is consistent with the learning environment in this field of study. 


\section{Índice general}

3. Los procesos de enseñanza - aprendizaje en la Universidad 41

3.1. Las competencias . . . . . . . . . . . . . . . 41

3.1.1. El capital humano . . . . . . . . . . . . . . . 41

3.1.2. Concepción tradicional de las competencias . . . . . . 44

3.1.3. Carácter práctico de las competencias . . . . . . . . 45

3.1.4. Carácter integrador de las competencias . . . . . . . 46

3.1.5. Medición de competencias: La autoevaluación . . . . . 46

3.1.6. Clasificaciones de competencias . . . . . . . . . . 48

3.1.7. Las competencias en el Espacio Europeo de Educación

Superior: Los resultados del aprendizaje . . . . . . 5 50

3.2. Los enfoques del aprendizaje . . . . . . . . . . 53

3.2.1. Motivaciones y estrategias . . . . . . . . . . . 54

3.2.2. Categorización de los enfoques del aprendizaje . . . . 55

3.2.3. Instrumentos para la medición de los enfoques del aprendizaje . . . . . . . . . . 5 56

3.2.4. La gestión del tiempo y las características personales . 58

3.3. El contexto académico universitario . . . . . . . . . . 61

3.3.1. El profesional flexible en la nueva sociedad del conocimiento . . . . . . . . . . . . . 61

3.3.2. El cambio metodológico en la Universidad hacia los contextos activos de aprendizaje . . . . . . . . . 62

3.3.3. El cambio paradigmático en las metodologías docentes y la formación del profesorado universitario . . . . . . 68

3.4. Los modelos de enseñanza - aprendizaje en el contexto universitario . . . . . . . . . . . . . . 69

3.5. Proyectos de investigación internacionales sobre adquisición de competencias en la Universidad . . . . . . . . . . . 76

3.5.1. El Proyecto "Tuning Educational Structures in Europe" (TUNING) . . . . . . . . . . . . 76 
3.5.2. El Proyecto "Careers After Higher Education - An European Research Study" (CHEERS) . . . . . . . . . 80

3.5.3. El Proyecto "El Profesional Flexible en la Sociedad del Conocimiento: Nuevas Exigencias en la Educación Superior en Europa" (REFLEX) . . . . . . . . . . 82

4. El contexto académico y personal de los estudiantes de Educación Superior

4.1. Introducción . . . . . . . . . . . . . . . . 85

4.2. Material y métodos . . . . . . . . . . . . 86

4.2.1. Análisis Factorial Exploratorio . . . . . . . . . . . 86

4.2.2. Análisis de Componentes Principales . . . . . . . . . . 89

4.2.3. Análisis de Datos Cualitatitivos . . . . . . . . . . . . . 104

4.3. Resultados y discusión . . . . . . . . . . . . . . . . 163

4.3.1. Análisis descriptivo . . . . . . . . . . . . . . 163

4.3.2. Metodologías docentes empleadas en la Universidad y adquisición de competencias . . . . . . . . . . . . 170

4.3.3. El contexto académico y personal de los estudiantes . 190

4.3.4. Relaciones entre el contexto de los estudiantes y la adquisición de competencias en la Universidad . . . . 216

4.4. Resumen y conclusiones . . . . . . . . . . . . . . . 236

5. Modelización de los procesos de enseñanza - aprendizaje de competencias

5.1. Introducción . . . . . . . . . . . . . . . . . . 239

5.2. Material y métodos . . . . . . . . . . . . . . 240

5.2.1. Consideraciones generales . . . . . . . . . . . 240

5.2.2. Formalización matemática . . . . . . . . . . . . . . 244

5.2.3. Supuestos estadísticos y análisis previos . . . . . . . 250

5.2.4. Procedimiento para el análisis . . . . . . . . . . . 255

5.2.5. Modelos sobre múltiples muestras . . . . . . . . . . . 291

5.2.6. Análisis parcial de invarianza . . . . . . . . . . . 295

5.2.7. Modelos multinivel . . . . . . . . . . . . . . . 296

5.2.8. Programas informáticos . . . . . . . . . . . 298

5.3. Resultados y discusión . . . . . . . . . . . . . . 299

5.3.1. Supuestos estadísticos y análisis previos . . . . . . . 299

5.3.2. Métodos de Enseñanza - Aprendizaje . . . . . . . . . . 302

5.3.3. Competencias adquiridas en la Universidad . . . . . . 329

5.3.4. Modelización causal de los Procesos de enseñanza aprendizaje de competencias en la Universidad . . . . 349

5.3.5. Modelización multinivel de los Procesos de enseñanza - aprendizaje de competencias en la Universidad . . . 386

5.4. Resumen y conclusiones . . . . . . . . . . . . . . 387 
6. Conclusiones y líneas futuras de investigación 389

\section{Referencias 393}

8. Apéndices 423

8.1. Anexo A: Preguntas seleccionadas del cuestionario REFLEX 423

8.2. Anexo B: Agrupación de titulaciones universitarias en áreas de estudio . . . . . . . . . . . . . . . . . 4 427

8.3. Anexo C: Medidas de asociación . . . . . . . . . . 430

8.4. Anexo D: Relaciones entre variables contextuales y factores competenciales. Figuras . . . . . . . . . . . . 4 435

8.5. Anexo E: Abreviaturas y siglas . . . . . . . . . . . . 445 


\section{Indice de tablas}

3.1. Modelo Motivo/Estrategia 3x3 formulado por Biggs. Dimensiones . . . . . . . . . . . . . . . . 54

3.2. Modelo Motivo/Estrategia 3x3 formulado por Biggs. Enfoques del aprendizaje . . . . . . . . . . . 56

3.3. Modalidades organizativas y Métodos de enseñanza - aprendizaje ....................... 63

3.4. Correspondencia entre Modalidades organizativas y Métodos de enseñanza - aprendizaje . . . . . . . . . . . 63

3.5. Correspondencia entre Competencias y Modalidades organizativas . . . . . . . . . . . . 76

4.1. Elementos ilustrativos, por categoría y grupo . . . . . . . . 102

4.2. Elementos de la tabla de contingencia estándar . . . . . . . 105

4.3. Variables contextuales del entorno académico y personal de los encuestados. Abreviaturas . . . . . . . . . . . . 165

4.4. Descripción de los ítems incluidos en la matriz de correlaciones. Competencias adquiridas en la Universidad . . . . . . . . 166

4.5. Análisis descriptivo. Características de los estudios y Métodos de enseñanza - aprendizaje . . . . . . . . . . . 167

4.6. Análisis descriptivo. Gestión del tiempo . . . . . . . . . . 167

4.7. Análisis descriptivo. Características personales . . . . . . 168

4.8. Análisis descriptivo. Área y Nivel de estudios . . . . . . . . . 169

4.9. Análisis descriptivo. Competencias adquiridas en la Universidad170

4.10. Contraste de esfericidad de Barlett. Características de los estudios y Métodos de enseñanza - aprendizaje . . . . . . . . 170

4.11. Contraste de esfericidad de Barlett. Competencias adquiridas en la Universidad . . . . . . . . . . . . . . . . . . . . . . 170

4.12. Matriz de correlaciones observadas. Características de los estudios y Métodos de enseñanza - aprendizaje . . . . . . . . 171

4.13. Matriz de correlaciones observadas. Competencias adquiridas en la Universidad . . . . . . . . . . . . . . . . . . . . . . . 171

4.14. $\alpha$ Cronbach con exclusión de cada uno de los ítems. Características de los estudios y Métodos de enseñanza - aprendizaje 173 
4.15. $\alpha$ Cronbach con exclusión de cada uno de los ítems. Competencias adquiridas en la Universidad . . . . . . . . . . . . . 173

4.16. Comunalidades de los ítems excluidos del análisis. Características de los estudios y Métodos de enseñanza - aprendizaje 174

4.17. Comunalidades de los ítems incluidos en el análisis. Características de los estudios y Métodos de enseñanza - aprendizaje 174

4.18. Comunalidades de los ítems incluidos en el análisis. Competencias adquiridas en la Universidad . . . . . . . . . . . . 175

4.19. Autovalores y porcentaje de varianza acumulada. Caracteristicas de los estudios y Métodos de enseñanza - aprendizaje . . 176

4.20. Autovalores y porcentaje de varianza acumulada con y sin eliminación del factor tamaño. Competencias adquiridas en la Universidad . . . . . . . . . . . . . . . . . . . . . 178

4.21. Matriz de componentes sin eliminación de factor tamaño. Competencias adquiridas en la Universidad . . . . . . . . . 180

4.22. Matriz de componentes y rotación Varimax. Características de los estudios y Métodos de enseñanza - aprendizaje . . . . . 180

4.23. Interpretación de las componentes principales. Características de los estudios y Métodos de enseñanza - aprendizaje. . . . . 182

4.24. Matriz de componentes con eliminación de factor tamaño y rotación Varimax. Competencias adquiridas en la Universidad 182

4.25. Interpretación de las componentes principales con eliminación del factor tamaño. Competencias adquiridas en la Universidad 183

4.26. p-valor del Contraste $\chi^{2}$ de independencia. Características de la carrera y Métodos de enseñanza - aprendizaje . . . . . . . 192

4.27. p-valor del Contraste $\chi^{2}$ de independencia. Características de la carrera y Métodos de enseñanza - aprendizaje frente a Gestión del tiempo y Características personales . . . . . . . 193

4.28. p-valor del Contraste $\chi^{2}$ de independencia. Gestión del tiempo y Características personales . . . . . . . . . . . . . 193

4.29. Cociente de ventajas. Características de la carrera y Métodos de enseñanza - aprendizaje . . . . . . . . . . . . 195

4.30. Coeficiente de correlación de Pearson. Características de la carrera y Métodos de enseñanza - aprendizaje . . . . . . . . . 196

4.31. Medida $\Phi^{2}$ de Pearson. Características de la carrera y Métodos de enseñanza - aprendizaje . . . . . . . . . . . . 197

4.32. Medida $\Phi^{2}$ de Pearson. Características de la carrera y Métodos de enseñanza - aprendizaje frente a Gestión del tiempo, Características personales y Área y Nivel de estudios . . . . . 197

4.33. Medida $\Phi^{2}$ de Pearson. Gestión del tiempo, Características personales y Área y Nivel de estudios . . . . . . . . . . . . . 198

4.34. Valor del estadístico $\chi^{2}$ para el Contraste de independencia global. Características de la carrera y Métodos de enseñanza - aprendizaje . . . . . . . . . . . . . 200 
4.35. Valor del estadístico $\chi^{2}$ para el Contraste de independencia parcial. Características de la carrera y Métodos de enseñanza - aprendizaje . . . . . . . . . . . . . . . 201

4.36. Valor del estadístico $\chi^{2}$ para el Contraste de independencia condicional. Características de la carrera y Métodos de enseñanza-aprendizaje . . . . . . . . . . . 202

4.37. Valor del estadístico $\chi^{2}$ para el Contraste de asociación parcial no condicional. Características de la carrera y Métodos de enseñanza - aprendizaje . . . . . . . . . . . . 203

4.38. Riesgo ponderado para el Contraste de asociación parcial no condicional. Características de la carrera y Métodos de enseñanza-aprendizaje . . . . . . . . . . . . . . 204

4.39. Valor del estadístico $\chi^{2}$ para el Contraste de asociación condicional. Características de la carrera y Métodos de enseñanza - aprendizaje . . . . . . . . . . . . . . . 205

4.40. Interacción y confusión entre variables. Características de los estudios y Métodos de enseñanza - aprendizaje . . . . . . . . 206

4.41. Cociente de ventajas para el Contraste de asociación parcial no condicional, con y sin segmentación. Características de la carrera y Métodos de enseñanza - aprendizaje . . . . . . . . . 208

4.42. Diferencias entre los cocientes de ventajas para el Contraste de asociación parcial no condicional, con y sin segmentación. Características de la carrera y Métodos de enseñanza - aprendizaje . . . . . . . . . . . . . . . . 209

4.43. Máximas diferencias de los cocientes de ventajas para el Contraste de asociación parcial no condicional, con y sin segmentación, por área de estudio. Características de la carrera y Métodos de enseñanza - aprendizaje . . . . . . . . . . . 209

4.44. Análisis de correspondencias múltiple. Valores propios y porcentajes de inercia en los dos primeros factores . . . . . . . 210

4.45. Coordenadas factoriales, contribuciones absolutas y contribuciones relativas. Gestión del tiempo y Características personales 212

4.46. Valores - test. Gestión del tiempo y Características personales 213

4.47. Coordenadas factoriales y valores - test de la proyección en suplementario. Area y Nivel de estudios . . . . . . . . . . . . 214

4.48. Coordenadas de los antiguos ejes rotados. Competencias adquiridas en la Universidad . . . . . . . . . . . . . . . . . . . 217

4.49. Coordenadas sobre las componentes principales de Competencias adquiridas en la Universidad. Características de la carrera y Métodos de enseñanza - aprendizaje . . . . . . . 218

4.50. Valores-test sobre las componentes principales de Competencias adquiridas en la Universidad. Características de la carrera y Métodos de enseñanza - aprendizaje . . . . . . . . . . . 219 
4.51. Valores-test sobre las componentes principales de Competencias adquiridas en la Universidad. Gestión del tiempo y $\mathrm{Ca}$ racterísticas personales . . . . . . . . . . . . . . . . 221

4.52. Valores-test sobre las componentes principales de Competencias adquiridas en la Universidad. Área y Nivel de estudios . 223

4.53. Proyección de las componentes de Características de la carrera y Métodos de enseñanza - aprendizaje sobre las componentes de Competencias adquiridas en la Universidad . . . . 226

4.54. Valores-test de las proyecciones de Características de la carrera y Métodos de enseñanza - aprendizaje sobre las componentes de Competencias adquiridas en la Universidad . . . . 226

4.55. Proyección de componentes del Competencias adquiridas en la Universidad sobre las componentes de Características de la carrera y Métodos de enseñanza - aprendizaje. . . . . . . . 22

4.56. Valores-test de la proyección de las componentes de Competencias adquiridas en la Universidad sobre las componentes de Características de la carrera y Métodos de enseñanza -

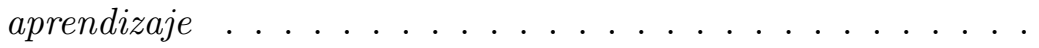

4.57. Coordenadas sobre las componentes principales de Competencias adquiridas en la Universidad. Asociación de En tu carrera había que trabajar mucho para aprobar y Tu carrera era una carrera con prestigio académico. . . . . . . . . . . 231

4.58. Coordenadas sobre las componentes principales de Competencias adquiridas en la Universidad. Asociación de El profesor era la principal fuente de información y Proyectos de inves-

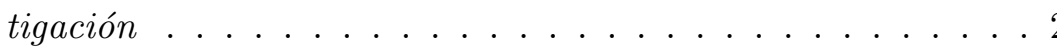

4.59. Valores-test sobre las componentes principales de Competencias adquiridas en la Universidad. Asociación de En tu carrera había que trabajar mucho para aprobar y Tu carrera era una carrera con prestigio académico . . . . . . . . . . . . .

4.60. Valores-test sobre las componentes principales de Competencias adquiridas en la Universidad. Asociación de El profesor era la principal fuente de información y Proyectos de investigación . . . . . . . . . . . . . . . 232

5.1. Notación LISREL . . . . . . . . . . . . . . . . . . . . . 245

5.2. Análisis descriptivo. Métodos de enseñanza - aprendizaje . . . 300

5.3. Análisis descriptivo. Competencias adquiridas en la Universidad300

5.4. Prueba de Kolmogorov-Smirnov. Métodos de enseñanza - apren-

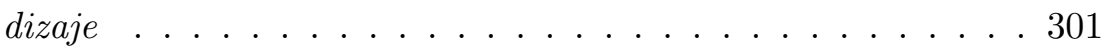

5.5. Prueba de Kolmogorov-Smirnov. Competencias adquiridas en la Universidad . . . . . . . . . . . . . . . . . . . 302 
5.6. Fiabilidad compuesta y varianza extraída. Modelo de medida inicial para Métodos de enseñanza - aprendizaje con 4 factores latentes . . . . . . . . . . . . . . 306

5.7. Fiabilidad compuesta y varianza extraída. Modelo de medida modificado para Métodos de enseñanza - aprendizaje con 4 factores latentes . . . . . . . . . . . . . . 307

5.8. Modelo de medida final para Métodos de enseñanza - aprendizaje con 4 factores latentes. Fiabilidad compuesta y varianza extraída . . . . . . . . . . . . . . . . . . . 309

5.9. Ecuaciones no estandarizadas. Modelo de medida final para Métodos de enseñanza - aprendizaje con 4 factores latentes . 311

5.10. Matriz de varianzas - covarianzas. Modelo de medida final para Métodos de enseñanza - aprendizaje con 4 factores latentes311

5.11. Matriz de correlaciones. Modelo de medida final para Métodos de enseñanza - aprendizaje con 4 factores latentes . . . . . . 311

5.12. Ecuaciones estandarizadas. Modelo de medida final para Métodos de enseñanza - aprendizaje con 4 factores latentes . . . . 312

5.13. Fiabilidad compuesta y varianza extraída. Modelo de medida inicial para Métodos de enseñanza - aprendizaje con 3 factores latentes . . . . . . . . . . . . . . . 315

5.14. Fiabilidad compuesta y varianza extraída. Modelo de medida final para Métodos de enseñanza - aprendizaje con 3 factores latentes . . . . . . . . . . . . . . 316

5.15. Ecuaciones no estandarizadas. Modelo de medida final para Métodos de enseñanza - aprendizaje con 3 factores latentes . 318

5.16. Matriz de varianzas - covarianzas. Modelo de medida final para Métodos de enseñanza - aprendizaje con 3 factores latentes318

5.17. Matriz de correlaciones. Modelo de medida final para Métodos de enseñanza - aprendizaje con 3 factores latentes . . . . . 318

5.18. Ecuaciones estandarizadas. Modelo de medida final para Métodos de enseñanza - aprendizaje con 3 factores latentes . . . . 319

5.19. Índices de ajuste y contraste $\chi^{2}$ por área de estudio. Invarianza métrica del modelo para Métodos de enseñanza - aprendizaje con 4 factores latentes . . . . . . . . . . . . . 320

5.20. Promedios de las matrices residuales de varianzas-covarianzas por área de estudio. Invarianza métrica del modelo para Métodos de enseñanza - aprendizaje con 4 factores latentes . . . . 321

5.21. Índices de fiabilidad por área de estudio. Invarianza métrica del modelo para Métodos de enseñanza - aprendizaje con 4 factores latentes . . . . . . . . . . . . . . . . . . . 322

5.22. Ecuaciones no estandarizadas. Invarianza métrica del modelo para Métodos de enseñanza - aprendizaje con 4 factores latentes322 
5.23. Errores de medida por área de estudio. Invarianza métrica del modelo para Métodos de enseñanza - aprendizaje con 4 factores latentes . . . . . . . . . . . . . . . . . . . . 323

5.24. Varianzas de los factores latentes por área de estudio. Invarianza métrica del modelo para Métodos de enseñanza aprendizaje con 4 factores latentes . . . . . . . . . . . . 323

5.25. Covarianzas de los factores latentes por área de estudio. Invarianza métrica del modelo para Métodos de enseñanza aprendizaje con 4 factores latentes . . . . . . . . . . . . . 324

5.26. Correlaciones de los factores latentes por área de estudio. Invarianza métrica del modelo para Métodos de enseñanza aprendizaje con 4 factores latentes . . . . . . . . . . . 324

5.27. Índices de ajuste y contraste $\chi^{2}$ por área de estudio. Invarianza métrica del modelo para Métodos de enseñanza - aprendizaje con 3 factores latentes . . . . . . . . . . . . 325

5.28. Promedios de las matrices residuales de varianzas-covarianzas por área de estudio. Invarianza métrica del modelo para Métodos de enseñanza - aprendizaje con 3 factores latentes . . . . 325

5.29. Índices de fiabilidad por área de estudio. Invarianza métrica del modelo para Métodos de enseñanza - aprendizaje con 3 factores latentes . . . . . . . . . . . . . . . . 326

5.30. Ecuaciones no estandarizadas. Invarianza métrica del modelo para Métodos de enseñanza - aprendizaje con 3 factores latentes327

5.31. Errores de medida por área de estudio. Invarianza métrica del modelo para Métodos de enseñanza - aprendizaje con 3 factores latentes . . . . . . . . . . . . . . . . . 328

5.32. Varianzas de los factores latentes por área de estudio. Invarianza métrica del modelo para Métodos de enseñanza aprendizaje con 4 factores latentes . . . . . . . . . . 328

5.33. Covarianzas de los factores latentes por área de estudio. Invarianza métrica del modelo para Métodos de enseñanza aprendizaje con 4 factores latentes . . . . . . . . . . . 328

5.34. Correlaciones de los factores latentes por área de estudio. Invarianza métrica del modelo para Métodos de enseñanza aprendizaje con 4 factores latentes . . . . . . . . . . . . 329

5.35. Contraste $\chi^{2}$ para los 4 modelos planteados inicialmente. Competencias adquiridas en la Universidad . . . . . . . . . . . 335

5.36. Índices de ajuste para los cuatro modelos planteados inicialmente. Competencias adquiridas en la Universidad . . . . . . 336

5.37. Fiabilidad compuesta y varianza extraída. Modelo de medida inicial para Competencias adquiridas en la Universidad con 6 factores latentes . . . . . . . . . . . . . 338 
5.38. Fiabilidad compuesta y varianza extraída. Modelo de medida final para Competencias adquiridas en la Universidad con 6 factores latentes . . . . . . . . . . . . . . . . . . . 339

5.39. Ecuaciones no estandarizadas. Modelo de medida final para Competencias adquiridas en la Universidad con 6 factores latentes . . . . . . . . . . . . . . . 340

5.40. Matriz de varianzas-covarianzas. Modelo de medida final para Competencias adquiridas en la Universidad con 6 factores latentes . . . . . . . . . . . . . . . . . . . 341

5.41. Matriz de correlaciones. Modelo de medida final para Competencias adquiridas en la Universidad con 6 factores latentes 341

5.42. Ecuaciones estandarizadas. Modelo de medida final para Competencias adquiridas en la Universidad con 6 factores latentes 342

5.43. Índices de ajuste y contraste $\chi^{2}$ por área de estudio. Invarianza métrica del modelo para Competencias adquiridas en la Universidad . . . . . . . . . . . . . . . . . . . . . 344

5.44. Promedios de las matrices residuales de varianzas-covarianzas por área de estudio. Invarianza métrica del modelo para Competencias adquiridas en la Universidad . . . . . . . . . . . . . 344

5.45. Indices de fiabilidad por área de estudio. Invarianza métrica del modelo para Competencias adquiridas en la Universidad . 345

5.46. Ecuaciones no estandarizadas. Invarianza métrica del modelo para Competencias adquiridas en la Universidad . . . . . . . 346

5.47. Errores de medida por área de estudio. Invarianza métrica del modelo para Competencias adquiridas en la Universidad . . . 347

5.48. Varianza de los factores latentes por área de estudio. Invarianza métrica del modelo para Competencias adquiridas en la Universidad . . . . . . . . . . . . . . . . . . 347

5.49. Covarianzas entre factores latentes por área de estudio. Invarianza métrica del modelo para Competencias adquiridas en la Universidad . . . . . . . . . . . . . . . . . . . . . 348

5.50. Correlaciones entre factores latentes por área de estudio. Invarianza métrica del modelo para Competencias adquiridas en la Universidad . . . . . . . . . . . . . . . . . . . . . . . . . 348

5.51. Matriz de varianzas-covarianzas entre factores latentes. Modelo estructural inicial entre Métodos de enseñanza - aprendizaje y Competencias adquiridas en la Universidad con problemas de infraidentificación empírica . . . . . . . . . . 351

5.52. Matriz de correlaciones entre factores latentes. Modelo estructural inicial entre Métodos de enseñanza - aprendizaje y Competencias adquiridas en la Universidad con problemas de infraidentificación empírica . . . . . . . . . . . . 351 
5.53. Autovalores y autovectores de la matriz de varianzas - covarianzas para la identificación de problemas de falta de identificación empírica . . . . . . . . . . . . . . . . . . . 353

5.54. Fiabilidad compuesta y varianza extraída. Modelo estructural final entre Métodos de enseñanza - aprendizaje y Competencias adquiridas en la Universidad . . . . . . . . . . . . . . . 355

5.55. Ecuaciones no estandarizadas del modelo de medida. Modelo estructural final entre Métodos de enseñanza - aprendizaje y Competencias adquiridas en la Universidad . . . . . . . . . . 357

5.56. Ecuaciones no estandarizadas del modelo estructural. Modelo estructural final entre Métodos de enseñanza - aprendizaje y Competencias adquiridas en la Universidad . . . . . . . . . . 358

5.57. Matriz de varianzas-covarianzas. Modelo estructural final entre Métodos de enseñanza - aprendizaje y Competencias adquiridas en la Universidad . . . . . . . . . . . . . . . . . . . 358

5.58. Matriz de correlaciones. Modelo estructural final entre Métodos de enseñanza - aprendizaje y Competencias adquiridas en la Universidad . . . . . . . . . . . . . . . . . . . . . . 358

5.59. Ecuaciones estandarizadas del modelo de medida. Modelo estructural final entre Métodos de enseñanza - aprendizaje y Competencias adquiridas en la Universidad . . . . . . . . . . 359

5.60. Ecuaciones no estandarizadas del modelo de medida. Modelo estructural final entre Métodos de enseñanza - aprendizaje y Competencias adquiridas en la Universidad . . . . . . . . . . 360

5.61. Contraste $\chi^{2}$ e índices de ajuste por área de estudio. Invarianza estructural del modelo entre Métodos de enseñanza aprendizaje y Competencias adquiridas en la Universidad . . 361

5.62. Contraste $\chi^{2}$. Invarianza estructural del modelo entre Métodos de enseñanza - aprendizaje y Competencias adquiridas en la Universidad . . . . . . . . . . . . . . . . . 362

5.63. Indices de ajuste obtenidos sobre la totalidad de las áreas de estudio, y el subconjunto de 7 áreas excluyendo Ciencias. Invarianza métrica y estructural del modelo entre Métodos de enseñanza - aprendizaje y Competencias adquiridas en la Universidad . . . . . . . . . . . . . . . . 363

5.64. Imposición de restricciones de invarianza parcial. Invarianza métrica y estructural del modelo entre Métodos de enseñanza - aprendizaje y Competencias adquiridas en la Universidad

5.65. Promedios de las matrices residuales de varianzas-covarianzas por área de estudio. Invarianza métrica y estructural del modelo entre Métodos de enseñanza - aprendizaje y Competencias adquiridas en la Universidad . . . . . . . . . . . . . . . 364 
5.66. Indices de fiabilidad por área de estudio. Invarianza métrica y estructural del modelo entre Métodos de enseñanza - aprendizaje y Competencias adquiridas en la Universidad . . . . . 364

5.67. Ecuaciones no estandarizadas del modelo de medida. Invarianza métrica y estructural del modelo entre Métodos de enseñanza - aprendizaje y Competencias adquiridas en la Uni-

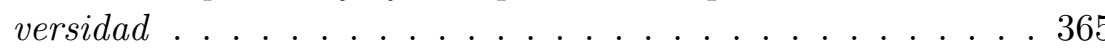

5.68. Ecuaciones no estandarizadas del modelo estructural. Invarianza métrica y estructural del modelo entre Métodos de enseñanza - aprendizaje y Competencias adquiridas en la Universidad . . . . . . . . . . . . 366

5.69. Matriz de varianzas-covarianzas. Invarianza métrica y estructural del modelo entre Métodos de enseñanza - aprendizaje y Competencias adquiridas en la Universidad . . . . . . . . . 366

5.70. Matriz de correlaciones. Invarianza métrica y estructural del modelo entre Métodos de enseñanza - aprendizaje y Competencias adquiridas en la Universidad . . . . . . . . . . . 366

5.71. Errores de medida por área de estudio. Invarianza métrica y estructural del modelo entre Métodos de enseñanza - aprendizaje y Competencias adquiridas en la Universidad . . . . . 367

5.72. Perturbaciones por área de estudio. Invarianza métrica y estructural del modelo entre Métodos de enseñanza - aprendizaje y Competencias adquiridas en la Universidad . . . . . . 368

5.73. Covarianzas y correlaciones entre errores de medida por área de estudio. Invarianza métrica y estructural del modelo entre Métodos de enseñanza - aprendizaje y Competencias adquiridas en la Universidad . . . . . . . . . . . . . . . . . . . . 368

5.74. Ecuaciones estandarizadas del modelo de medida en Educación. Invarianza métrica y estructural del modelo entre Métodos de enseñanza - aprendizaje y Competencias adquiridas en la Universidad . . . . . . . . . . . . . . . . . . . . 369

5.75. Ecuaciones estandarizadas del modelo de medida en Humanidades. Invarianza métrica y estructural del modelo entre Métodos de enseñanza - aprendizaje y Competencias adquiridas en la Universidad . . . . . . . . . . . . . . . . . . . . 370

5.76. Ecuaciones estandarizadas del modelo de medida en Ciencias Sociales. Invarianza métrica y estructural del modelo entre Métodos de enseñanza - aprendizaje y Competencias adquiridas en la Universidad . . . . . . . . . . . . . . . . . . . . . . 371

5.77. Ecuaciones estandarizadas del modelo de medida en Economía y empresa. Invarianza métrica y estructural del modelo entre Métodos de enseñanza - aprendizaje y Competencias adquiridas en la Universidad . . . . . . . . . . . . . . . . 372 
5.78. Ecuaciones estandarizadas del modelo de medida en Derecho. Invarianza métrica y estructural del modelo entre Métodos de enseñanza - aprendizaje y Competencias adquiridas en la Universidad . . . . . . . . . . . . . . . . . 373

5.79. Ecuaciones estandarizadas del modelo de medida en Técnicas. Invarianza métrica y estructural del modelo entre Métodos de enseñanza - aprendizaje y Competencias adquiridas en la Universidad . . . . . . . . . . . . . . . . . . 374

5.80. Ecuaciones estandarizadas del modelo de medida en Salud. Invarianza métrica y estructural del modelo entre Métodos de enseñanza - aprendizaje y Competencias adquiridas en la Universidad . . . . . . . . . . . . . . . . . . . 375

5.81. Ecuaciones estandarizadas del modelo estructural en Educación. Invarianza métrica y estructural del modelo entre Métodos de enseñanza - aprendizaje y Competencias adquiridas en la Universidad . . . . . . . . . . . . . . . . . . . 376

5.82. Ecuaciones estandarizadas del modelo estructural en Humanidades. Invarianza métrica y estructural del modelo entre Métodos de enseñanza - aprendizaje y Competencias adquiridas en la Universidad . . . . . . . . . . . . . . . . . . . . . . 376

5.83. Ecuaciones estandarizadas del modelo estructural en Ciencias Sociales. Invarianza métrica y estructural del modelo entre Métodos de enseñanza - aprendizaje y Competencias adquiridas en la Universidad . . . . . . . . . . . . . . . . . . . . . . 376

5.84. Ecuaciones estandarizadas del modelo estructural en Economía y empresa. Invarianza métrica y estructural del modelo entre Métodos de enseñanza - aprendizaje y Competencias adquiridas en la Universidad . . . . . . . . . . . . . . . . 377

5.85. Ecuaciones estandarizadas del modelo estructural en Derecho. Invarianza métrica y estructural del modelo entre Métodos de enseñanza - aprendizaje y Competencias adquiridas en la Universidad . . . . . . . . . . . . . . . . . . . 377

5.86. Ecuaciones estandarizadas del modelo estructural en Técnicas. Invarianza métrica y estructural del modelo entre Métodos de enseñanza - aprendizaje y Competencias adquiridas en la Universidad . . . . . . . . . . . . . . . . . . . . . 377

5.87. Ecuaciones estandarizadas del modelo estructural en Salud. Invarianza métrica y estructural del modelo entre Métodos de enseñanza - aprendizaje y Competencias adquiridas en la Universidad . . . . . . . . . . . . . . . . . 378

5.88. Contraste $\chi^{2}$ para los 4 modelos planteados inicialmente. Modelos estructurales entre Métodos de enseñanza - aprendizaje y Competencias adquiridas en la Universidad para Ciencias . 379 
5.89. Indices de ajuste para los 4 modelos planteados inicialmente. Modelos estructurales entre Métodos de enseñanza - aprendizaje y Competencias adquiridas en la Universidad para Ciencias . . . . . . . . . . . . . . . . 380

5.90. Fiabilidad compuesta y varianza extraída. Modelo estructural entre Métodos de enseñanza - aprendizaje y Competencias adquiridas en la Universidad para Ciencias . . . . . . . . . . 382

5.91. Ecuaciones no estandarizadas del modelo de medida. Modelo estructural entre Métodos de enseñanza - aprendizaje y Competencias adquiridas en la Universidad para Ciencias . . . . . 383

5.92. Ecuaciones no estandarizadas del modelo estructural. Modelo estructural entre Métodos de enseñanza - aprendizaje y Competencias adquiridas en la Universidad para Ciencias . . . . . 384

5.93. Matriz de varianzas-covarianzas entre factores latentes. Modelo estructural entre Métodos de enseñanza - aprendizaje y Competencias adquiridas en la Universidad para Ciencias . . 384

5.94. Matriz de correlaciones entre factores latentes. Modelo estructural entre Métodos de enseñanza - aprendizaje y Competencias adquiridas en la Universidad para Ciencias . . . . . . . 384

5.95. Ecuaciones estandarizadas del modelo de medida. Modelo estructural entre Métodos de enseñanza - aprendizaje y Competencias adquiridas en la Universidad para Ciencias . . . . . 385

5.96. Ecuaciones estandarizadas del modelo estructural. Modelo estructural entre Métodos de enseñanza - aprendizaje y Competencias adquiridas en la Universidad para Ciencias . . . . . 386

5.97. Correlaciones intraclases. Modelo estructural entre Métodos de enseñanza - aprendizaje y Competencias adquiridas en la Universidad . . . . . . . . . . . . . . . . . 387

8.1. Coeficiente de correlación Rho de Spearman. Características de la carrera y Métodos de enseñanza - aprendizaje . . . . . 430

8.2. Coeficiente de contingencia. Características de la carrera y Métodos de enseñanza - aprendizaje . . . . . . . . . . . 430

8.3. Coeficiente de contingencia. Características de la carrera y Métodos de enseñanza - aprendizaje frente a Gestión del tiempo y Características personales . . . . . . . . . . . . . 431

8.4. Coeficiente de contingencia. Gestión del tiempo y Características personales del individuo . . . . . . . . . . . . . . . 441

8.5. V de Cramer. Características de la carrera y Métodos de enseñanza-aprendizaje . . . . . . . . . . . 431

8.6. V de Cramer. Características de la carrera y Métodos de enseñanza - aprendizaje frente a Gestión del tiempo y Caracteristicas personales . . . . . . . . . . . . . . 432

8.7. V de Cramer. Gestión del tiempo y Características personales 432 
8.8. Medida Lambda de Goodman y Kruskal simétrica. Características de la carrera y Métodos de enseñanza - aprendizaje 432

8.9. Medida Lambda de Goodman y Kruskal simétrica. Características de la carrera y Métodos de enseñanza - aprendizaje frente a Gestión del tiempo y Características personales . . . 433

8.10. Medida Lambda de Goodman y Kruskal simétrica. Gestión del tiempo y Características personales . . . . . . . . . . . . 433

8.11. Coeficiente de incertidumbre simétrico. Características de la carrera y Métodos de enseñanza - aprendizaje . . . . . . . . 433

8.12. Coeficiente de incertidumbre simétrico. Características de la carrera y Métodos de enseñanza - aprendizaje frente a Gestión del tiempo y Características personales . . . . . . . . . . 434

8.13. Coeficiente de incertidumbre simétrico. Gestión del tiempo y Características personales . . . . . . . . . . . . 444

8.14. Variables contextuales del entorno académico y personal de los encuestados y Competencias adquiridas en la Universidad.

Abreviaturas . . . . . . . . . . . . . . . . . 445 


\section{Índice de figuras}

3.1. Modelo Conductista del Aprendizaje . . . . . . . . . . . . 69

3.2. Modelo de Diferencias del Aprendizaje . . . . . . . . . . 70

3.3. Modelo ATI de Cronbach . . . . . . . . . . . . . 70

3.4. Modelo de Aprendizaje en Contexto . . . . . . . . . . . 71

3.5. Modelo Heurístico del Proceso de Enseñanza - Aprendizaje . 72

3.6. Modelo de Factores . . . . . . . . . . . . . . . . . . . 72

3.7. Esquema del modelo mediacional propuesto por Biggs . . . . 73

3.8. Modelo 3P de Enseñanza - Aprendizaje propuesto por Biggs . 74

4.1. Subespacio engendrado por los dos primeros vectores de la matriz simétrica $X^{\prime} X$ en el espacio $R^{p} \ldots \ldots \ldots 2$

4.2. Subespacio engendrado por los dos primeros vectores de la matriz simétrica $X^{\prime} X$ en el espacio $R^{n} \ldots \ldots$. . . . . 94

4.3. Distribución de variables e individuos activos y suplementarios en la base de datos original . . . . . . . . . . . . . 96

4.4. Nube de individuos en $R^{p}$. Representación de variables nominales ilustrativas . . . . . . . . . . . . . . . . 98

4.5. Nube de individuos en $R^{n}$. Representación de variables continuas ilustrativas . . . . . . . . . . . . . . . 9 98

4.6. Relaciones establecidas por las variables de confusión . . . . . 119

4.7. Autovalores. Características de los estudios y Métodos de enseñanza-aprendizaje . . . . . . . . . . 176

4.8. Porcentaje de varianza acumulada. Características de los estudios y Métodos de enseñanza - aprendizaje . . . . . . . . 177

4.9. Autovalores con y sin eliminación del factor tamaño. Competencias adquiridas en la Universidad . . . . . . . . . . . 178

4.10. Porcentaje de varianza acumulada con y sin eliminación del factor tamaño. Competencias adquiridas en la Universidad . 179

4.11. Círculo de variables activas en las componentes 1 y 2. Características de los estudios y Métodos de enseñanza - aprendizaje 185

4.12. Círculo de variables activas en las componentes 3 y 4 . Características de los estudios y Métodos de enseñanza - aprendizaje 186 
4.13. Círculo de variables activas en las componentes 5 y 6 . Características de los estudios y Métodos de enseñanza - aprendizaje 187

4.14. Círculo de variables activas en las componentes 2 y 6 . Competencias adquiridas en la Universidad . . . . . . . . . . . 188

4.15. Círculo de variables activas en las componentes 4 y 3. Competencias adquiridas en la Universidad . . . . . . . . . . . . . 189

4.16. Círculo de variables activas en las componentes 1 y 5 . Competencias adquiridas en la Universidad . . . . . . . . . . . . . 190

4.17. Proyección del Área y nivel de estudios sobre los ejes definidos sobre Gestión del tiempo y Características personales. Factores 1 y $2 \ldots \ldots \ldots \ldots$. . . . . . . . . . 215

4.18. Representación simultánea sobre las componentes principales 1 y 2 de Competencias adquiridas en la Universidad. Características de los estudios y Métodos de enseñanza - aprendizaje 220

4.19. Representación simultánea sobre las componentes principales 1 y 2 de Competencias adquiridas en la Universidad. Gestión del tiempo y Características personales . . . . . . . . . . . . . 222

4.20. Representación simultánea sobre las componentes principales 1 y 2 de Competencias adquiridas en la Universidad. Área y Nivel de estudios . . . . . . . . . . . . . . . . . . . . . . 224

4.21. Representación simultánea de las componentes Características de la carrera y Métodos de enseñanza - aprendizaje sobre las componentes 1 y 2 de Competencias adquiridas en la Universidad . . . . . . . . . . . . . . . . . . . 227

4.22. Representación simultánea de las componentes de Competencias adquiridas en la Universidad sobre las componentes 1 y 2 de Características de la carrera y Métodos de enseñanza -

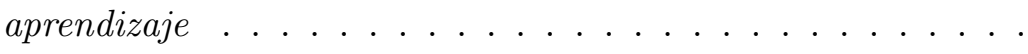

4.23. Representación simultánea sobre las componentes 1 y 2 de Competencias adquiridas en la Universidad. Asociación de En tu carrera había que trabajar mucho para aprobar y Tu carrera era una carrera con prestigio académico . . . . . . . . . . . 233

4.24. Representación simultánea sobre las componentes 1 y 2 de Competencias adquiridas en la Universidad. Asociación de El profesor era la principal fuente de información y Proyectos de investigación . . . . . . . . . . . . . . .

4.25. Representación simultánea sobre las componentes 5 y 6 de Competencias adquiridas en la Universidad. Asociación de En tu carrera había que trabajar mucho para aprobar y Tu carrera era una carrera con prestigio académico . . . . . . . . . . .

5.1. Modelo de medida inicial con 4 factores latentes. Métodos de enseñanza-aprendizaje . . . . . . . . . . . 303 
5.2. Modelo de medida final con 4 factores latentes. Métodos de enseñanza - aprendizaje . . . . . . . . . . . . . 310

5.3. Modelo de medida inicial con 3 factores latentes. Métodos de enseñanza - aprendizaje . . . . . . . . . . . . 313

5.4. Modelo de medida final con 3 factores latentes. Métodos de enseñanza - aprendizaje . . . . . . . . . . . . 317

5.5. Modelo de medida 1, con 5 factores latentes y exclusión del ítem Hablar y escribir en idiomas extranjeros. Competencias adquiridas en la Universidad . . . . . . . . . . . . . . . . . 331

5.6. Modelo de medida 2, con 6 factores latentes y exclusión del ítem Hablar y escribir en idiomas extranjeros. Competencias adquiridas en la Universidad . . . . . . . . . . . . . . . . 332

5.7. Modelo de medida 3, con 5 factores latentes e inclusión del ítem Hablar y escribir en idiomas extranjeros. Competencias adquiridas en la Universidad . . . . . . . . . . . . . . . . 333

5.8. Modelo de medida 4, con 6 factores latentes e inclusión del ítem Hablar y escribir en idiomas extranjeros. Competencias adquiridas en la Universidad . . . . . . . . . . . . . . . . . 334

5.9. Modelo de medida final para Competencias adquiridas en la Universidad con 6 factores latentes . . . . . . . . . . . . . 343

5.10. Modelo estructural inicial entre Métodos de enseñanza - aprendizaje y Competencias adquiridas en la Universidad con problemas de infraidentificación empírica . . . . . . . . . . 350

5.11. Modelo estructural inicial entre Métodos de enseñanza - aprendizaje y Competencias adquiridas en la Universidad . . . . . 354

5.12. Adquisición de competencias mediante métodos de enseñanza - aprendizaje. Modelo estructural para el área de Ciencias . . 381

8.1. Representación simultánea sobre las componentes principales 3 y 4 de Competencias adquiridas en la Universidad. Características de los estudios y Métodos de enseñanza - aprendizaje 435

8.2. Representación simultánea sobre las componentes principales 5 y 6 de Competencias adquiridas en la Universidad. Características de los estudios y Métodos de enseñanza - aprendizaje 436

8.3. Representación simultánea sobre las componentes principales 3 y 4 de Competencias adquiridas en la Universidad. Gestión del tiempo y Características personales . . . . . . . . . . . 437

8.4. Representación simultánea sobre las componentes principales 5 y 6 de Competencias adquiridas en la Universidad. Gestión del tiempo y Características personales . . . . . . . . . . 438

8.5. Representación simultánea sobre las componentes principales 3 y 4 de Competencias adquiridas en la Universidad. Área y nivel de estudios . . . . . . . . . . . . . . . . . 439 
8.6. Representación simultánea sobre las componentes principales 5 y 6 de Competencias adquiridas en la Universidad. Área y nivel de estudios . . . . . . . . . . . . . . . . . . . . 440

8.7. Representación simultánea sobre las componentes principales 3 y 4 de Competencias adquiridas en la Universidad. Características de la carrera y Métodos de enseñanza - aprendizaje

8.8. Representación simultánea sobre las componentes principales 5 y 6 de Competencias adquiridas en la Universidad. Características de la carrera y Métodos de enseñanza - aprendizaje

8.9. Representación simultánea sobre las componentes principales 3 y 4 de Competencias adquiridas en la Universidad. Características de la carrera y Métodos de enseñanza - aprendizaje

8.10. Representación simultánea sobre las componentes principales 5 y 6 de Competencias adquiridas en la Universidad. Características de la carrera y Métodos de enseñanza - aprendizaje 


\section{Capítulo 1}

\section{Introducción}

Resulta evidente la importancia que en los últimos años han alcanzado las competencias como elemento clave de la formación universitaria. El principal responsable de la introducción de este nuevo enfoque en la educación universitaria es el proceso de adaptación de las universidades al Espacio Europeo de Educación Superior, bien conocido ya por la mayoría de los profesionales que trabajan en este sector. Sin embargo, no es la única razón. Los recientes desarrollos en sistemas de gestión de la calidad universitaria, han propiciado igualmente que las competencias se conviertan en la piedra angular de la evaluación interna en la gran mayoría de las universidades españolas. En ambos casos, la principal aportación de la orientación por competencias es que la simple definición de los conocimientos vinculados a cada materia ya no resulta suficiente. Además de estos conocimientos, es preciso concretar las habilidades y actitudes que el estudiante deberá haber adquirido como consecuencia del proceso de aprendizaje.

En el ámbito laboral, las competencias constituyen el punto de referencia para el estudio de los requisitos formativos de los titulados universitarios en su transición al mercado laboral y concretamente en la denominada sociedad del conocimiento, basada fundamentalmente en el uso intensivo de las tecnologías de la comunicación y la información, se han incrementado de forma muy significativa las expectativas puestas en las Instituciones de Educación Superior. Ya no es suficiente que los titulados universitarios posean los conocimientos necesarios para desempeñar las tareas laborales en un campo específico de estudio. Ahora se espera de las universidades que formen a sus estudiantes para que se desenvuelvan como profesionales flexibles, capaces de enfrentarse a muy diversas situaciones laborales. Es decir, que ya no son suficientes las competencias específicas del área de estudio sino que debe proporcionarse a los estudiantes las competencias genéricas necesarias para facilitar el aprendizaje posterior de las competencias que les sean requeridas en el futuro. 
En este contexto se está replanteando si las metodologías docentes tradicionales, basadas en lecciones magistrales aportadas por profesores expertos a grupos de estudiantes que actúan pasivamente como receptores de la información, resultan adecuadas para afrontar estas nuevas circunstancias. Por ello, los profesores universitarios están introduciendo progresivamente nuevas metodologías docentes, basadas en los denominados contextos activos de aprendizaje, con el fin de desarrollar nuevos modelos educacionales basados en la formación en competencias y los resultados del aprendizaje. Estas transformaciones apuntan a un cambio paradigmático metodológico más allá de las pequeñas innovaciones introducidas por cada profesor, a partir del cual las instituciones de Educación Superior ya no se considerarán instituciones donde simplemente se proporciona enseñanza sino que adicionalmente se produce aprendizaje. Sin embargo, la introducción de estos cambios hacia el desarrollo de nuevos contextos activos de aprendizaje aporta diversas ventajas, como el incremento de la implicación de los estudiantes en el proceso de aprendizaje, o simplemente el hecho de que los estudiantes aprenden a través de la aplicación de los conocimientos teóricos a problemas reales.

Independientemente de la planificación y gestión del cambio hacia este nuevo enfoque metodológico realizada por las universidades a nivel institucional, no cabe duda de que finalmente recae sobre el profesorado universitario la responsabilidad de la ejecución en la práctica de dichos planes. Para muchos de ellos este nuevo enfoque de su trabajo no supone sino una dificultad añadida a las anteriores. La principal carencia que percibe este colectivo como consecuencia del cambio metodológico es la falta de formación para el desempeño de sus tareas docentes. Con frecuencia los profesores universitarios se ven a sí mismos como profesionales o investigadores reconocidos en su campo, pero faltos de interés por la docencia. De hecho, es común tropezar con ciertas actitudes escépticas hacia el papel de la Universidad en la formación de los estudiantes y la implantación de nuevos enfoques destinados a mejorar la calidad de la enseñanza.

Contrariamente a esta actitud negativa, que afortunadamente no es habitual, la gran mayoría del profesorado universitario se encuentra concienciado de la gran importancia de transmitir a sus alumnos las competencias que posteriormente necesitarán en sus trabajos, y no simplemente los conocimientos de la forma tradicional. No obstante, la impartición de docencia en este contexto supone un reto. Este requerimiento adicional resulta especialmente complejo en el sentido de que implica un cambio radical en el enfoque tradicionalmente aplicado a las metodologías docentes, tan arraigado en determinados ámbitos universitarios. Específicamente, frente al enfoque tradicional que centra la enseñanza en el trabajo del profesor, este cambio metodológico sostiene que sólo es posible un aprendizaje eficaz cuando es 
el propio estudiante el que asume la responsabilidad de su aprendizaje, lo cual constituye el pilar fundamental de la llamada formación basada en el estudiante. En este contexto de transición resulta primordial proporcionar al profesorado universitario cierta información sobre cómo se producen los procesos de enseñanza - aprendizaje de competencias en la Universidad. De esta forma no sólo se facilita la asimilación de las innovaciones metodológicas sino que se evita que la adaptación de los planes de estudio se convierta en un mero ajuste estético y formal de las metodologías docentes clásicas.

\section{Motivación}

La investigación sobre Enfoques del aprendizaje comienza con los trabajos de Marton y Säljö (1976a, 1976b) en la Universidad de Gotemburgo (Suecia) y constituye el precedente directo de posteriores estudios sobre modelos de enseñanza - aprendizaje. El término Enfoque del aprendizaje aparece por primera vez en 1984 y se define como los procesos de aprendizaje que emergen de la percepción que el estudiante tiene de la tarea académica influida por sus propias características personales (Marton, Hounsell y Entwistle, 1984). Estos enfoques del aprendizaje están influidos tanto por las características personales del estudiante y el contexto de enseñanza, como por la interacción entre ambos elementos. Concretamente, el aprendizaje académico se define como la integración de tres dimensiones: la conciencia que el estudiante tiene de su propio aprendizaje, el contenido de la materia y el contexto de enseñanza - aprendizaje (Marton y Svensson, 1979; Säljö, 1979).

La gran mayoría de las contribuciones sobre esta cuestión coinciden en que existen dos componentes fundamentales en su definición: las motivaciones que revelan las intenciones que mueven al estudiante a aprender y unas estrategias coherentes con dichas intenciones (Hernández, García, Martínez y Maquillón, 2002). Atendiendo a ambos elementos, es posible identificar hasta tres enfoques diferentes de aprendizaje, siendo formas transculturales o universales de aproximarse al aprendizaje, independientemente del sistema educativo nacional (Abalde et al., 2001). En primer lugar el enfoque superficial se basa en una motivación extrínseca. En este caso el aprendizaje en el contexto académico se considera un medio para lograr un objetivo concreto, como conseguir trabajo o aprobar un examen y las estrategias para lograrlo se basan en minimizar el esfuerzo y reproducir lo aprendido a través de un aprendizaje memorístico. A continuación el enfoque profundo se basa en una motivación propia del estudiante por las materias. Las estrategias surgen de esa motivación y se utilizan para maximizar la comprensión, de forma que la curiosidad del individuo sea satisfecha. Por último el enfoque de alto rendimiento se basa en la valoración que el individuo hace de sí mismo a través de la obtención de un alto rendimiento académico. Esta motivación constituye una forma particular de motivación extrínseca. En estas circunstancias las 
estrategias se relacionan con la organización del tiempo, la organización del espacio y el dominio de las materias de la forma más eficiente posible (Ruiz, Hernández y Ureña, 2008).

No obstante estas tipologías de enfoques del aprendizaje sólo tienen sentido si se contextualizan en el contexto de enseñanza - aprendizaje (Biggs, 1993). En este aspecto se ha planteado que el análisis del proceso de aprendizaje sólo es posible si se consideran tanto las características personales del estudiante como la relación con su contexto particular de aprendizaje. De esta forma resulta determinante la percepción que el estudiante tiene de este contexto a partir de sus características personales (Laurillard, 1979).

El primer modelo de enseñanza - aprendizaje que se plantea es el Modelo de Aprendizaje en Contexto. Este modelo se fundamente en tres aspectos primordiales: la enseñanza, la forma de evaluación y el currículum, referido al contenido y estructura de lo que se aprende (Ramsden, 1988, 2003). Inmediatamente posterior es el Modelo Heurístico del Proceso de Enseñanza Aprendizaje. Este modelo pretende reflejar la compleja naturaleza del proceso de enseñanza - aprendizaje a través de las distintas conexiones que lo componen: estudiante, enseñanza y departamento (Entwistle, 1987). Posteriormente Pérez propone el Modelo de Factores en el que afirma que la clave de los procesos de enseñanza - aprendizaje reside en la interacción de tres elementos: la actividad educativa del profesor, los contenidos de la materia y los factores cognitivos y metacognitivos de los alumnos (conocimientos previos y capacidad y motivación para el aprendizaje) (Pérez, 2001). No obstante en esta cuestión resulta ineludible el análisis de los modelos propuestos por Biggs, siempre estructurados de forma que los aspectos relativos al proceso de aprendizaje median las relaciones entre las características personales del estudiante, el contexto de aprendizaje y el consecuente rendimiento académico. Estos modelos se plantean como una variante del paradigma Presagio Proceso - Producto desarrollado por Dunkin y Biddle (1974) y desde su planteamiento inicial han experimentado sucesivas modificaciones y mejoras hasta alcanzar su configuración definitiva (Biggs, 1978, 1984, 1987, 1993). El modelo definitivo se formula en 2001 y recibe el nombre de Modelo 3P de Enseñanza - Aprendizaje por estructurarse en tres factores (Biggs, Kember y Leung, 2001). En primer lugar el factor de presagio, constituido por variables independientes, que existen previamente al proceso de aprendizaje. Incluye las características personales del estudiante, las particularidades del contexto de enseñanza así como la interacción entre ambos elementos. A continuación el factor de proceso, compuesto por variables mediadoras, está integrado por los enfoques del aprendizaje. Finalmente el factor de producto, compuesto por variables dependientes, representa los resultados del aprendizaje en todas sus posibles formas, desde el rendimiento académico hasta la adquisición de competencias o el grado de satisfacción con los estudios. A partir de este 
modelo, De Miguel et al. (2005) plantean el análisis de las relaciones entre los cuatro elementos fundamentales del modelo: las competencias a alcanzar por los alumnos (resultados del aprendizaje), las modalidades u escenarios organizativos a tener en cuenta para realizar los procesos, los métodos concretos a utilizar a la hora de desarrollar las actividades de enseñanza-aprendizaje y las estrategias de evaluación que se estiman oportunas para verificar si los alumnos alcanzan los aprendizajes previstos. En su investigación se somete este modelo a un contraste empírico de expertos y a consulta externa de profesores implicados en procesos de renovación metodológica con el fin de detectar las interacciones más comunes que se establecen entre los diversos elementos del modelo. Esta investigación constituye uno de los pocos estudios sobre los procesos de enseñanza - aprendizaje realizados en base a una metodología cuantitativa, y no exclusivamente teórica. La práctica totalidad de los modelos mencionados anteriormente, e igualmente las investigaciones previas sobre enfoques del aprendizaje, se fundamentan en investigaciones teóricas o planteamientos cualitativos.

Por tanto, las últimas décadas han supuesto un avance muy significativo de los conocimientos teóricos sobre enfoques del aprendizaje y modelos de los procesos de enseñanza - aprendizaje. Sin embargo, en muy pocas ocasiones se han validado empíricamente estos modelos, en la mayoría de los casos propuestos exclusivamente en base a la teoría. Esta situación de falta de soporte empírico se ve notablemente acentuada por el hecho de que los actuales estudios en el campo de la educación tienden a utilizar de forma generalizada muy diversas técnicas estadísticas multivariantes con el fin de respaldar las hipótesis planteadas y las conclusiones obtenidas durante el proceso de investigación. Por otro lado, la adaptación de los estudios universitarios al Espacio Europeo de Educación Superior ha supuesto un importante cambio paradigmático en las metodologías docentes del profesorado universitario. En consecuencia, este colectivo precisa ser dotado de la información necesaria, fundamentada en la teoría y validada empíricamente, para abordar con éxito la transición y la asimilación de las innovaciones metodológicas. 


\section{Capítulo 2}

\section{Objetivos}

El objetivo principal de esta Tesis Doctoral es modelizar los procesos de enseñanza - aprendizaje de competencias en la universidades españolas mediante la aplicación de técnicas estadísticas multivariantes. Con este objetivo, se ha tratado de describir en detalle y comprender las relaciones establecidas entre distintos factores pertenecientes a los contextos de aprendizaje de los estudiantes universitarios en toda su complejidad, tanto a nivel académico como personal, así como profundizar en el concepto de competencia como resultado final de los procesos de enseñanza - aprendizaje. Para ello se ha estructurado el trabajo en tres secciones, cada una de las cuales posee unos objetivos y metodología específicos.

En primer lugar se realiza una extensa revisión bibliográfica sobre diversos conceptos relativos al aprendizaje en el ámbito universitario, desde un enfoque pedagógico con el fin de obtener una perspectiva global de las investigaciones precedentes en este campo de investigación sobre la que fundamentar los resultados obtenidos y sustentar los modelos causales propuestos en secciones posteriores del trabajo.

A continuación se realiza un estudio detallado desde un enfoque exploratorio del contexto académico y personal de los estudiantes universitarios, considerando en el primer caso las características de los estudios y los métodos de enseñanza - aprendizaje empleados con mayor frecuencia por el profesorado universitario, y la gestión del tiempo y las características personales de los estudiantes como parte del segundo. El objetivo de esta etapa es comprender las relaciones que se establecen entre los factores pertenecientes a estos contextos e identificar posibles variables mediadoras que introduzcan modificaciones del efecto significativas en las relaciones entre variables.

En la última fase se plantea como objetivo la validación de un modelo sobre los procesos de enseñanza - aprendizaje de competencias en la Uni- 
versidad que tenga en consideración no sólo las contribuciones precedentes en este campo de investigación, sino también el conocimiento adquirido en la fase anterior sobre el contexto de aprendizaje de los estudiantes y su influencia en los procesos de adquisición de competencias. El enfoque con que se han abordado los análisis en esta etapa tiene un carácter estrictamente confirmatorio, en contraposición al apartado anterior.

El objetivo general del trabajo puede concretarse en los objetivos específicos siguientes:

1. Identificar tanto las competencias que los estudiantes universitarios adquieren fundamentalmente gracias a sus estudios de Educación Superior como los métodos de enseñanza - aprendizaje empleados con mayor frecuencia por el profesorado universitario, a partir de las valoraciones de los titulados universitarios

2. Comprender las relaciones establecidas entre distintos factores del contexto académico y personal de los estudiantes e identificar posibles variables modificadoras del efecto, responsables de la introducción de relaciones de interacción y confusión

3. Estudiar las relaciones de dependencia e interacción establecidas entre competencias adquiridas en la Universidad y los factores potencialmente influyentes en estos procesos pertenecientes al contexto académico y personal de los estudiantes

4. Contrastar empíricamente la fiabilidad y validez de los ítems planteados en el modelo de medida como indicadores de los constructos teóricos subyacentes definidos en investigaciones teóricas precedentes sobre métodos de enseñanza - aprendizaje y adquisición de competencias en Educación Superior

5. Diseñar modelos multivariantes, en base a una estrategia de modelización confirmatoria, que permitan validar las teorías sobre modelos de enseñanza - aprendizaje en el ámbito de la Educación Superior, estimando los coeficientes de regresión correspondientes a los modelos de medida y estructural así como los errores de medida y las perturbaciones vinculadas a variables manifiestas y latentes, respectivamente

\section{Estructura del trabajo}

La Tesis Doctoral se ha estructurado en siete capítulos y cuatro anexos como se describe a continuación.

En el capítulo 3 se hace una primera aproximación a la cuestión sobre el aprendizaje de competencias en el ámbito universitario. Para ello se 
profundiza en los conceptos de capital humano y competencias así como su equivalencia con los denominados resultados del aprendizaje. Asimismo se resume el estado del arte sobre los enfoques del aprendizaje adoptados por los estudiantes universitarios y la gestión de su tiempo durante los estudios. Desde una perspectiva metodológica se describen las modalidades de enseñanza - aprendizaje empleadas tradicionalmente por el profesorado universitario y se justifica la necesidad de realizar una transformación hacia el desarrollo de nuevos contextos activos de aprendizaje. Finalmente se exponen los principales modelos de enseñanza - aprendizaje empleados en la Universidad y se enumeran los proyectos de investigación a nivel internacional más relevantes en relación con el tema.

En el capítulo 4 se describen detalladamente las relaciones entre variables del contexto de aprendizaje de los estudiantes y se profundiza en las competencias que adquieren gracias a sus estudios universitarios y los métodos de enseñanza - aprendizaje empleados con mayor frecuencia por sus profesores. De este modo se identifica el área de estudio como principal variable mediadora a tener en consideración en los modelos de enseñanza - aprendizaje de competencias y se analiza la modificación del efecto introducida por esta variable en las relaciones restantes.

En el capítulo 5 se valida el modelo propuesto por Biggs en 2001 sobre los procesos de enseñanza - aprendizaje en el contexto universitario basado en el paradigma Presagio - Proceso - Producto. Para ello se validan previamente los instrumentos de medida empleados para la medición de los métodos de enseñanza - aprendizaje y las competencias adquiridas en la Universidad mediante un conjunto de indicadores medidos en escala Likert en base a las percepciones de los encuestados de su experiencia en la Universidad. De este modo se obtiene un modelo generalizable a la práctica totalidad de las áreas de estudio consideradas en este trabajo, con excepción del área de ciencias que constituye un caso aparte cuyo estudio se ha enfocado desde una perspectiva exploratoria.

En el capítulo 6 se exponen las conclusiones alcanzadas como resultado del trabajo de investigación desarrollado en la presente Tesis Doctoral así como las líneas futuras a desarrollar a resultas de este estudio y por último en el capítulo 7 se muestra la bibliografía consultada para la redacción de la memoria.

Finalmente en los anexos A y B se adjunta información sobre las preguntas del cuestionario REFLEX seleccionadas para la investigación y la agrupación de titulaciones universitarias en áreas de estudio. Asimismo los anexos $\mathrm{C}$ y D contienen las tablas relativas a medidas de asociación y figuras sobre relaciones entre variables contextuales y factores competenciales. 


\section{Capítulo 3}

\section{Los procesos de enseñanza - aprendizaje en la Universidad}

\subsection{Las competencias}

El concepto de competencia ha evolucionado notablemente en los últimos años. Desde las primeras investigaciones sobre la importancia del capital humano en las organizaciones hasta el momento actual en que las competencias han tomado la condición de elemento clave e indispensable para la adaptación de las universidades al Espacio Europeo de Educación Superior este término ha tenido diversas acepciones que deben ser consideradas y analizadas como punto de partida y referencia en el estudio de cuestiones más avanzadas.

\subsubsection{El capital humano}

Vinculado a las competencias también el capital humano ha experimentado un incremento en el reconocimiento a su relevancia mediante los compromisos adquiridos en el Consejo Europeo de Lisboa, celebrado en esta misma ciudad en el año 2000, en el cual se enfatizó el hecho de que "todo ciudadano debe poseer los conocimientos necesarios para vivir y trabajar en la nueva sociedad de la información" y en que "un marco europeo debería definir las nuevas cualificaciones básicas que deben proporcionarse a través de la formación continua: cualificaciones en materia de tecnologías de la información, idiomas extranjeros, cultura tecnológica, espíritu empresarial y habilidades para la socialización" (De la Fuente y Ciccone, 2002).

Asimismo el Consejo Europeo de Lisboa señala la necesidad de "moder- 
nizar el modelo social europeo mediante la inversión en capital humano y la lucha contra la exclusión social". Como es bien sabido en la sociedad del conocimiento es fácil que parte de los conocimientos adquiridos durante los periodos formativos queden rápidamente obsoletos. Con el fin de garantizar la validez y actualización de los conocimientos adquiridos los individuos experimentan continuamente la necesidad de seguir aprendiendo a lo largo de su vida. Sin embargo para aquellos individuos que no poseen una formación básica la dificultad para continuar aprendiendo es mayor. Estas diferencias entre capacidades de los individuos tienden a acentuarse con el tiempo. Por ello, existe la posibilidad de que con el paso del tiempo los individuos con poca formación difícilmente puedan acceder a los puestos de trabajo de los ya formados e incluso algunos de ellos ni siquiera puedan acceder a la formación que les permita mejorar sus condiciones de trabajo por falta de formación básica. A largo plazo esta situación puede generar tensiones sociales debido a las desigualdades laborales. Por esta razón, la inversión en capital humano se contempla desde la perspectiva global como una herramienta de prevención contra la exclusión social.

No existe una definición única sobre el concepto de capital humano. Keeley lo define como el conjunto de conocimientos, habilidades, competencias y atributos característicos de los individuos que facilitan la creación de bienestar personal, social y económico, en cuyo caso enfatiza en la relevancia del capital humano para el desarrollo de las regiones (Keeley, 2007). En términos generales el concepto de capital humano se refiere al valor de las destrezas productivas de las personas, y se basa en el hecho de que algunos individuos deciden invertir parte de sus recursos en la mejora de sus propias capacidades para obtener mayores rendimientos futuros (Vila, 2005). Sin embargo, Hartog (2000) señala que en algunas ocasiones este concepto se restringe a las capacidades y habilidades que los individuos adquieren como resultado de una inversión en formación excluyendo las capacidades innatas. El concepto de capital humano también debería incluir este conjunto de competencias propias que no necesariamente están dirigidas a obtener un beneficio laboral inmediato. De esta forma la inversión en capital humano podría valorarse en términos generales como el coste de todas las acciones emprendidas con el objetivo de mejorar el bienestar de las personas.

Los beneficios de la educación pueden ser monetarios o no monetarios. Los beneficios monetarios hacen referencia a la contribución de la educación al incremento de los ingresos de las personas con estudios respecto a los de las personas sin estudios. Este incremento puede deberse no sólo a las diferencias en los salarios sino también a ingresos adicionales relacionados con el contexto socioeconómico del individuo. En cambio los beneficios no monetarios de la educación son aquellos no directamente relacionados con un incremento de la riqueza. Estos beneficios que pueden ser tanto públi- 
cos como privados. Ejemplos de beneficios no monetarios públicos son la estabilidad de las estructuras sociales o la disminución de desigualdades en la distribución de riqueza de la región. Sin duda ambos están relacionados puesto que en la mayoría de los casos las desigualdades sociales implican una ruptura del equilibrio social. En el ámbito privado la educación está ligada a beneficios relacionados con mejoras en la salud, el control efectivo de la natalidad, la educación de los hijos, la gestión doméstica o incluso incrementos de la efectividad en los procesos de búsqueda de trabajo (Vila, 2000).

Así como se ha estudiado la relación entre educación y productividad también se ha analizado en profundidad la relación entre educación e ingresos. Según Hartog (1988) existe una relación directa entre la complejidad de los puestos de trabajo y el nivel de ingresos demandado por el individuo en compensación por la necesaria inversión en formación para satisfacer los requerimientos del trabajo. Sin embargo las distintas capacidades de los individuos no son valoradas de igual forma. Concretamente se identifican tres dimensiones del capital humano con influencia en el nivel de ingresos: intelectual, social y manual. Entre estas tres la capacidad intelectual es la mejor remunerada con mucha diferencia respecto a las otras dos. La capacidad social se valora en cierta medida pero en cualquier caso la capacidad que obtiene los ingresos más bajos es la manual (Hartog, 1980).

Este hecho está muy relacionado con el reciente desarrollo de la nueva sociedad del conocimiento que se inició en la década de los noventa. En este contexto recursos intangibles como la información y el conocimiento han logrado desplazar a los recursos tradicionales relacionados con el capital físico y humano. Este entorno inestable basado en el uso intensivo de las tecnologías de la información y las comunicaciones es la razón de que los graduados universitarios hayan de adaptarse rápida y fácilmente a continuos cambios en sus puestos de trabajo.

De hecho en determinadas ocasiones es habitual encontrar desajustes entre el nivel de educación alcanzado por determinados individuos y requerido en sus puestos de trabajo (Dekker, De Grip y Heijke, 1980). Esta situación recibe el nombre de Desajuste Educativo y está directamente relacionada con el Desajuste Competencial. En este caso es posible que, aunque formalmente el nivel de estudios sea el requerido en el puesto de trabajo, las competencias necesarias para el adecuado desempeño de las tareas asociadas sean distintas a las que se adquirieron durante los estudios (Badillo-Amador, García-Sánchez y Vila, 2005). Ambos desajustes están claramente relacionados y en el caso de los graduados universitarios influyen por igual en la disminución de la satisfacción con su trabajo (Allen y De Weert, 2007).

Por esta razón se ha criticado la opinión de muchos expertos sobre la 
relación directa y exclusiva entre los estudios superiores y los requerimientos de los puestos de trabajo. Sin embargo, con el fin de compensar estos desajustes entre la educación recibida y las exigencias del trabajo los individuos pueden optar por emprender actividades de formación encaminadas a satisfacer las necesidades de sus trabajos (Ishikawa y Ryan, 2002).

En el caso de que el individuo opte por una formación generalista se ampliará el rango de oportunidades de empleo que le ofrece el mercado laboral ya que este tipo de formación contribuye a incrementar las capacidades de aprendizaje y adaptación a nuevos entornos de trabajo. Sin embargo si opta por formación específica el incremento de productividad en su puesto de trabajo será claramente significativo pero esto no garantizará la posibilidad de acceder a mejores puestos de trabajo en otros entornos. Por esta razón se ha propuesto que sean los individuos los encargados de financiar su formación en competencias genéricas mientras que las organizaciones deberían ser las responsables de la inversión en formación específica del puesto de trabajo (Acemoglu y Pischke, 1998).

En cualquier caso es obvio que para los graduados universitarios la Universidad juega un papel fundamental en el sentido de que los años inmediatamente posteriores a la graduación suelen ser cruciales en su incorporación al mercado laboral y el nivel de competencias que poseen en ese momento depende en gran medida de su paso por las instituciones de educación superior, y concretamente del área y nivel de estudios (Mora et al., 2007b).

Tradicionalmente se han planteado dos cuestiones fundamentales respecto a esta situación: ¿cuáles son las competencias fundamentales para el desempeño de los trabajos de los graduados? (Busato, Prins, Elshout y Hamaker, 2000; Heijke, Meng y Ris, 2003; Leckey y McGuigan, 1997) y ¿en qué medida contribuye la universidad al desarrollo de dichas competencias? (Belfield, Bullock y Fielding, 1999; Meng y Heijke, 2005; Van der Velden y Wolbers, 2004). Esta última cuestión constituye el eje central de la investigación desarrollada en este trabajo.

\subsubsection{Concepción tradicional de las competencias}

Los inicios de la formación en competencias se encuentran en los Estados Unidos e Inglaterra durante las décadas de los años sesenta y setenta. El objetivo de este tipo de formación estaba muy condicionado a cubrir las necesidades del sector industrial que en aquel momento se encontraba en pleno desarrollo y expansión. Debido a esta situación particular el diseño de esta nueva metodología formativa se realizó desde un enfoque más económico que educativo. Esta concepción de la formación basada en competencias se 
corresponde claramente con las definiciones aportadas por determinadas instituciones en las cuales se incide en el desarrollo de las capacidades manuales o instrumentales para el adecuado desempeño de las tareas correspondientes al puesto de trabajo.

Frente a esta concepción tradicional de las competencias, como desarrollo de habilidades físicas y manuales cabe resaltar las diferencias con otros significados relacionados con el mismo concepto. Entre los términos que aluden al desarrollo de capacidades manuales e instrumentales se encuentra el vocablo destrezas. Las destrezas se refieren al desarrollo de determinadas aptitudes innatas desde un punto de vista motor e intelectual sin que medie la educación. El diccionario de la Real Academia de la Lengua define la destreza como la "habilidad, arte, primor o propiedad con que se hace algo". La existencia de destrezas justifica que determinadas personas sean capaces de realizar ciertas actividades físicas con mayor precisión que otros. Del mismo modo se pueden encontrar en estas categorías los términos Habilidad, Aptitud o Capacidad. Tanto las habilidades como las aptitudes y capacidades implican la formación en ciertas tareas o actividades para llevarlas a cabo con perfección aunque las aptitudes o capacidades se refieren en mayor medida a potencialidades innatas que los seres humanos poseen y que necesitan ser desarrolladas mediante la educación.

\subsubsection{Carácter práctico de las competencias}

En la actualidad se considera que una persona competente es aquella que no solo sabe aplicar sus conocimientos en la práctica, sino que es capaz adaptarse al entorno modificando su modo de actuación en función de las circunstancias. Al mismo tiempo debe ser capaz de resolver los problemas que se presenten en cada momento recurriendo a las estrategias de las que dispone para solventar los imprevistos e incertidumbres. Asimismo, y con el fin de evaluar el desarrollo de la competencia, deben existir criterios previos claros sobre los objetivos a alcanzar en cada momento (Tejada et al., 2006). Esta misma idea ya la expresó Bunk al afirmar que posee competencia profesional quien dispone de los conocimientos, destrezas y aptitudes necesarios para ejercer una profesión, puede resolver los problemas profesionales de forma autónoma y flexible, y está capacitado para colaborar en su entorno profesional y en la organización del trabajo (Bunk, 1994) al igual que lo hizo la Comisión Europea al definir las competencias como la combinación de conocimientos, capacidades y actitudes adecuados para afrontar una determinada situación (Organización para la Cooperación y el Desarrollo Económico, 2005). Asimismo en el contexto del proyecto TUNING, posteriormente comentado con mayor detalle en este trabajo, el término Competencia implica no sólo que una persona pone en práctica determina- 
da capacidad o habilidad para desempeñar una labor sino que puede hacerlo de un modo que permita evaluar su nivel de consecución. Este concepto de competencia supone que es posible tanto desarrollar como valorar el grado de adquisición de las competencias y por tanto es posible desarrollarlas continuamente mediante el ejercicio y la educación (González y Wagenaar, 2005).

\subsubsection{Carácter integrador de las competencias}

Afortunadamente se han incorporado progresivamente al concepto de competencia nuevos enfoques enriquecedores de forma que ha sido posible superar esta simple concepción manual o práctica para aportar nuevas dimensiones que reflejen su actual complejidad y multidisciplinariedad. Concretamente, Hartog (1992) define las competencias como las aptitudes, destrezas y capacidades de los graduados en Educación Superior que contribuyen a aumentar su productividad con un enfoque multidimensional.

Cabe resaltar la importación del carácter multidimensional como elemento novedoso en el concepto de competencia. Esta misma idea es desarrollada por Tejada et al. (2006) al definir las cuatro dimensiones principales de las competencias (cognoscitiva, social, comunicativa y ética) e igualmente por el Proyecto TUNING al definir las competencias como una combinación dinámica de las capacidades cognitivas y metacognitivas, de conocimiento y entendimiento, interpersonales, intelectuales y prácticas así como de los valores éticos (González y Wagenaar, 2005).

\subsubsection{Medición de competencias: La autoevaluación}

El estudio de los nuevos procesos de enseñanza - aprendizaje implica la selección de los indicadores más adecuados para medir las competencias de los titulados. Si bien no se ha alcanzado un consenso sobre el mejor método para la medición de las competencias, sí existen ciertas directrices sobre cómo no debería llevarse a cabo. Una de las posibles opciones es recurrir directamente al empleo de indicadores, de carácter interno o externo a la institución, que aporten una aproximación de la formación en competencias del titulado. Posibles indicadores internos podrían ser las calificaciones obtenidas en la titulación, el nivel de educación alcanzado o los años dedicados a la educación, mientras que los referentes como al nivel salarial, el cargo ocupado en la organización e incluso la satisfacción laboral, podrían ser empleados como indicadores externos. La utilización exclusiva de ambos tipos de indicadores no resulta completamente satisfactoria en este ámbito ya que pueden estar condicionados a elementos no controlables por las instituciones. En el caso de los indicadores internos, sus resultados pueden verse afectados 
por la estructura del sistema educativo, o incluso de la propia institución, mientras que los indicadores externos no cabe duda de que oscilarán inevitablemente en consonancia con las fluctuaciones del entorno laboral.

Del mismo modo se ha planteado la posibilidad de realizar una evaluación, diseñada por expertos, del nivel de competencias de los titulados. Aunque éste parece un buen sistema para obtener una panorámica de su perfil competencial, únicamente tendría sentido su aplicación en el momento exacto de la finalización de los estudios, ya que no sería un buen instrumento para evaluar las competencias adquiridas en el entorno laboral de modo informal. La solución para esta dificultad implicaría entrevistar a los supervisores de los titulados. Sin embargo, esto supondría una grandísima inversión de recursos que probablemente no resultaría factible, tanto por la dificultad de diseñar una muestra representativa de este colectivo, como por la de contactar con ellos y lograr tasas de respuesta viables.

Por todo ello un buen método para la obtención de información sobre el nivel de competencias de los titulados universitarios parece ser su propia autoevaluación. Esta opción resulta más factible que las anteriores ya que puede integrarse en las encuestas dirigidas a titulados que la mayoría de las universidades ya han desarrollado como parte de sus sistemas de evaluación interna, permitiendo al mismo tiempo el análisis longitudinal de los resultados.

No obstante, la aplicación de este tipo de estudios requiere el desarrollo de un instrumento de medida válido y fiable que permita controlar el error de medida, ya que al basarse en la autopercepción de los encuestados es difícil que aporte una perspectiva precisa de la realidad (Bou - Llusar, EscrigTena, Roca-Puig y Beltrán-Martín, 2009). El diseño de este instrumento constaría de una lista estándar de competencias, formuladas adecuadamente de forma objetiva y fundamentada en diversas dimensiones teóricas. Estas dimensiones harían referencia al nivel de competencias de los titulados, independientemente de su área de estudios, factor que únicamente cobraría significado en la importancia asignada a cada una de las dimensiones.

Con el fin de diseñar convenientemente el instrumento de medición de competencias es necesario conocer las clasificaciones de competencias propuestas en investigaciones precedentes. La mayoría coinciden en que es posible realizar una clasificación básica en competencias generales y específicas, en función del nivel de vinculación a un contexto de trabajo específico. A partir de esta premisa se han planteado otras muchas agrupaciones y en la actualidad no se ha alcanzado un consenso sobre si la utilización generalizada de alguna de ellas resulta más adecuada. Como contrapartida, en la actualidad existe la tendencia de trabajar con clasificaciones de competen- 
cias diseñadas en función de los objetivos específicos de cada proyecto de investigación, sin pérdida de coherencia con los fundamentos teóricos.

\subsubsection{Clasificaciones de competencias}

Tal y como propuso Becker en 1964, e investigaciones posteriores han confirmado, el capital humano de los graduados universitarios constituye una combinación de competencias genéricas y específicas (Becker, 1964; Heijke, Meng y Ris, 2003; Kellerman, 2001; Semejin, Van der Velden, Heijke, Van der Vleuten y Boshuizen, 2005). Las competencias genéricas son las consideradas esenciales para el adecuado desarrollo del graduado en la sociedad actual. Estas competencias no están asociadas a un contexto de trabajo determinado aunque contribuyen al desarrollo de la capacidad de aprendizaje necesaria para la adquisición de nuevas competencias requeridas en el puesto de trabajo. Asimismo contribuyen a desarrollar las capacidades de comunicación y trabajo en equipo de los estudiantes. En los últimos años su relevancia en los estudios universitarios se ha incrementado progresivamente puesto que permiten a los graduados adaptarse a los continuos cambios en los requerimientos de sus puestos de trabajo e incluso del mercado laboral. El proyecto TUNING diferencia tres tipos de competencias genéricas en función de su naturaleza: Instrumentales, interpersonales y sistémicas. Las competencias instrumentales se refieren a la adquisición de conocimientos y tecnologías e incluso al aprendizaje de idiomas mientras que las competencias interpersonales incluyen no sólo el ámbito de la interacción social y personal sino también la autocrítica y la capacidad de expresar los propios sentimientos. Por último las competencias sistémicas requieren el desarrollo previo de las competencias instrumentales puesto que conciernen a la comprensión de los sistemas como totalidad (González y Wagenaar, 2005).

Por otro lado el ámbito de las competencias específicas se limita a los conocimientos y destrezas requeridas en el puesto de trabajo. Su desarrollo no contribuirá a incrementar las posibilidades del graduado en el mercado laboral de igual forma que lo hacen las competencias genéricas pero sí influye considerablemente en la posibilidad de que los graduados encuentren trabajos relacionados con su área de estudio en la Universidad (Boshuizen, 2004). Por esta razón su adquisición se considera indispensable en el diseño de estudios universitarios con cierto grado de especialización. Bishop aboga por el desarrollo de este tipo de competencias frente a las genéricas ya que considera estas últimas como una simple herramienta para el posterior desarrollo de competencias específicas (Bishop, 1995). Sin embargo, otros investigadores como Bowen inciden en la importancia de las competencias genéricas frente a las específicas (Bowen, 1977). En este aspecto Heijke considera que los titulados en estudios con enfoque especializado tienen mayores probabilidades de ocupar puestos de trabajo directamente relacionados con su área de es- 
tudio, mientras que los titulados en estudios generalistas tienden a situarse en empleos que demandan este tipo de competencias en mayor medida. En cualquier caso Heijke y Meng (2006) alertan del riesgo que corren los titulados en estudios que pretenden aportar a sus estudiantes una combinación mixta de ambos tipos de competencias. En estos casos es probable que estos titulados en este tipo de estudios terminen aceptando trabajos mal remunerados en que los no que se les valora ni por sus competencias específicas ni por las genéricas. De hecho, considerando el cambio que están realizando los mercados laborales hacia una mayor demanda de competencias genéricas, los responsables de los estudios con estas características deberían replantearse la necesidad de realizar ciertos ajustes en el enfoque de los estudios.

Nordhaug distingue entre competencias específicas de las empresas, de las tareas y de los sectores económicos (Nordaug, 1993). No obstante otras clasificaciones como las de Bunk y Kellermann no consideran la diferenciación principal en competencias genéricas y específicas. Bunk agrupa las competencias en cuatro tipos: especializadas, metodológicas, participativas y socio-individuales (Bunk, 1994) mientras que Kellermann las clasifica en cinco grupos. En primer lugar las Competencias genéricas y académicas, referidas a los conocimientos generales y las Competencias científicas y operativas, relativas a la precisión y atención al detalle. Seguidamente se consideran las Competencias personales y profesionales, basadas en los conocimientos y métodos específicos, así como las Competencias sociales y reflexivas que fomentan el liderazgo y el trabajo en equipo. Finalmente las Competencias físicas y manuales, referentes a las destrezas y aptitudes manuales (Kellerman, 2007).

Al mismo tiempo Heiijke clasifica las competencias en tres grupos en función del contexto donde se fomenta en mayor medida su aprendizaje. En primer lugar, las competencias específicas de la disciplina y que pueden adquirirse a través de la educación básica. Seguidamente las competencias genéricas que pueden adquirirse mediante la educación inicial pero también en la formación adicional posterior. Por último las competencias de gestión desarrolladas en la mayoría de los casos en el contexto de trabajo del graduado (Heijke, Meng y Ramaekers, 2003).

Otras clasificaciones como las de Vila y García-Aracil diferencian con mayor precisión las distintas competencias en función de su naturaleza. En la clasificación de Vila las competencias se agrupan en ocho categorías: Organizativa, Ejecutiva, Instrumental, Conocimiento Especializado, Disciplina, Físico-manual, Conocimiento Genérico y Cooperación (Vila, 2005) mientras que García-Aracil y Van der Velden (2008) resumen esta clasificación en seis categorías: Organizativa, Métodológica, Participativa, Conocimiento Especializado, Conocimiento Genérico y Socio-Emocionales. Sin embargo, a 
pesar del esfuerzo de los investigadores por definir una clasificación estándar, en la mayoría de ocasiones se emplean las clasificaciones ad-hoc diseñadas en función de los objetivos específicos de cada proyecto (Allen y Van der Velden, 2001).

\subsubsection{Las competencias en el Espacio Europeo de Educación Superior: Los resultados del aprendizaje}

A pesar del gran número de referencias a la importancia de los resultados del aprendizaje en el proceso de Bolonia existe cierta confusión sobre el significado concreto de este concepto, posiblemente relacionada con la falta de implementación práctica en las universidades europeas ya implicadas en el proceso de adaptación al nuevo Espacio Europeo de Educación Superior. Un número sustancial de las instituciones que participaron en el informe TRENDS IV afirmaba conocer o estar familiarizada con este concepto. Sin embargo, sólo los profesores que tenían conocimiento del proyecto TUNING declararon ser conscientes de la dimensión europea del concepto (Reichter y Tauch, 2003).

No existe una definición única de lo que se entiende como resultado del aprendizaje. Sin embargo todas las definiciones encierran matices similares. En líneas generales, tal como se define en el marco de trabajo del Proyecto TUNING, los resultados del aprendizaje pueden definirse como aquello que se espera que el estudiante sepa, entienda y/o sea capaz de demostrar al finalizar un periodo de aprendizaje. Este periodo puede referirse tanto a una unidad de un módulo de aprendizaje, un curso o una cualificación (González y Wagenaar, 2005).

La relación entre resultados del aprendizaje y competencias es una cuestión compleja y confusa. Fundamentalmente se ha establecido claramente la diferencia entre ambos términos. De hecho, algunos documentos evitan explícitamente utilizar ambos términos para evitar mayor confusión (Kennedy, Hyland y Ryan, 2006). Por otro lado en la guía para los usuarios de créditos ECTS se define el término competencia como una combinación dinámica de atributos, habilidades y actitudes que pueden ser genéricas o específicas, y enfatizan en que su desarrollo debe ser el objetivo principal de los planes de estudio. Este desarrollo debe estructurarse en varios módulos o cursos y evaluarse al concluir diferentes etapas. Según este mismo documento, los resultados del aprendizaje son conjuntos de competencias que expresan lo que se espera que el estudiante sepa, comprenda o sea capaz de hacer al finalizar el proceso de aprendizaje. Así, los resultados de aprendizaje se expresan habitualmente en términos de competencias. Atendiendo a las definiciones anteriores los resultados del aprendizaje se considerarán en 
este trabajo como conjuntos de competencias adquiridos por los estudiantes como resultado del proceso de aprendizaje.

Los resultados del aprendizaje constituyen un concepto ampliamente referenciado en numerosos documentos relacionados con el proceso de Bolonia. Sin embargo, no fueron mencionados en la Declaración de Bolonia de 1999 ni tampoco en el Comunicado de Praga (2001). El primer documento que alude claramente a la importancia de los resultados del aprendizaje es el Comunicado de Berlín:

"Los Ministros animan a los estados miembros a que elaboren para sus Sistemas de Educación Superior un marco de titulos comparables y compatibles, con el fin de describir estos en términos de cantidad de trabajo, nivel, resultados del aprendizaje, competencias y perfil. Se comprometen a elaborar un marco global de títulos para el Espacio Europeo de Educación Superior. (...) Los títulos de primer ciclo deben dar acceso, en el sentido de la Convención sobre Reconocimientos de Lisboa, a programas de segundo ciclo. Los títulos de segundo ciclo deben dar acceso a los estudios de doctorado. (...)"

En el Comunicado de Bergen (2005), los Ministros de Educación de los países participantes incidieron en la necesidad de contar un marco de cualificaciones:

"Adoptamos el marco general de cualificaciones en el EEES, que comprende tres ciclos (incluyendo, dentro de cada contexto nacional, la posibilidad de cualificaciones intermedias), los descriptores genéricos basados en resultados del aprendizaje y competencias para cada ciclo y los intervalos de créditos en el primer y segundo ciclo."

"Nos comprometemos a elaborar marcos de cualificaciones nacionales, compatibles con el marco general de cualificaciones en el EEES para el 2010 y a presentar avances sobre el mismo en 200\%. Solicitamos que el Grupo de Seguimiento informe sobre la puesta en marcha y los posteriores desarrollos del marco general."

"Subrayamos la importancia de asegurar que el marco general de cualificaciones del EEES y el marco más amplio de cualificaciones para el aprendizaje a lo largo de la vida sean complementarios, incluyendo la educación general y la formación profesional, tal y como se está desarrollando dentro la Unión Europea, asi como entre los países participantes. Solicitamos que la Comisión Europea consulte a todos los grupos participantes en el Proceso de Bolonia conforme progrese el trabajo."

Por otra parte, la Comisión Europea propuso crear un Marco Europeo de 
Cualificaciones para el aprendizaje a lo largo de la vida enfocado principalmente a la implantación a nivel europeo y nacional. El propio Comunicado de Bergen señalaba la necesidad de compatibilizar ambos marcos:

"We underline the importance of ensuring complementarity between the overarching framework for the EHEA and the proposed broader framework for qualifications for lifelong learning encompassing general education as well as vocational education and training as now being developed within the European Union as well as among participating countries. We ask the European Commission fully to consult all parties to the Bologna Process as work progresses."

En el Comunicado de Londres (2007) se enfatizan las múltiples aplicaciones de los resultados del aprendizaje. En primer lugar, como punto de referencia en la definición del Sistema Europeo de Transferencia y Acumulación de Créditos (ECTS) y ayuda e innovación a la reforma curricular:

"We underline the importance of curricula reform leading to qualifications better suited both to the needs of the labour market and to further study. Efforts should concentrate in future on removing barriers to access and progression between cycles and on proper implementation of ECTS based on learning outcomes and student workload."

"We urge institutions to further develop partnerships and cooperation with employers in the ongoing process of curriculum innovation based on learning outcomes."

Asimismo, se aplican igualmente en la definición de nuevos planes de estudio basados en los Marcos Nacionales de Cualificaciones:

"Qualifications frameworks are important instruments in achieving comparability and transparency within the EHEA and facilitating the movement of learners within, as well as between, higher education systems. They should also help HEIs to develop modules and study programmes based on learning outcomes and credits, and improve the recognition of qualifications as well as all forms of prior learning."

Finalmente pueden aplicarse con el objetivo de promocionar el aprendizaje centrado en el estudiante:

"With a view to the development of more student-centred, outcome-based learning, the next exercise should also address in an integrated way national qualifications frameworks, learning outcomes and credits, lifelong learning, and the recognition of prior learning." 
Desde su primera aparición en el Comunicado de Berlín de 2003 se ha aludido a los resultados del aprendizaje en un gran número de documentos y comunicados oficiales. En la actualidad un gran número de universidades han iniciado ya las tareas correspondientes para su implementación. En esta fase concreta del proceso resulta esencial ser consciente del riesgo de hacer un uso exclusivamente teórico y retórico de los resultados del aprendizaje como solución irreal para todas las dificultades inherentes al proceso de Bolonia. Más que como un concepto teórico los resultados del aprendizaje deberían considerarse como parte de un conjunto de herramientas que, adecuadamente aplicados, pueden facilitar la implantación de los objetivos del proceso de Bolonia (Adam, 2008).

De este modo, los resultados del aprendizaje pueden contribuir a la adaptación de diferentes niveles y dimensiones de la educación. En términos generales existen tres posibles niveles de aplicación para los resultados del aprendizaje. A nivel institucional pueden aplicarse a todas las tareas de enseñanza, aprendizaje y evaluación de la institución. No sólo son útiles para los estudiantes al definir claramente las competencias, habilidades y destrezas que adquirirán al finalizar el curso sino que facilitan al profesor la definición de objetivos didácticos e indican el modo de enseñanza más adecuado en cada caso. Asimismo su principal aplicación a nivel nacional es la aportación de descriptores de nivel explícitos para la creación de los Marcos Nacionales de Cualificaciones. Por otro lado la aplicación de los resultados del aprendizaje a nivel internacional es más amplia e imprecisa comparada con los dos niveles anteriores. Los genéricos Descriptores de Dublín cumplen esta función en el EHEA. El objetivo de estos descriptores es proporcionar un contexto de trabajo en el cual los Ministerios de Educación de cada país puedan desarrollar sus propios descriptores de nivel con mayor detalle. Estos descriptores se han definido en función de los tres ciclos de cualificaciones correspondientes a los niveles de Bachelor, Máster y Doctorado.

\subsection{Los enfoques del aprendizaje}

La investigación sobre Enfoques del aprendizaje (también denominada Modelo SAL o Student Approaches to Learning) es una área de investigación que se inició con los trabajos de Marton y Säljö (1976a, 1976b) en la Universidad de Gotemburgo (Suecia) y constituye el precedente directo de posteriores estudios sobre modelos de enseñanza - aprendizaje. El término Enfoque del aprendizaje aparece por primera vez en 1984 y se define como los procesos de aprendizaje que emergen de la percepción que el estudiante tiene de la tarea académica influida por sus propias características personales (Marton et al., 1984). Estos enfoques del aprendizaje están influidos 
tanto por las características personales del estudiante y el contexto de enseñanza, como por la interacción entre ambos elementos. Concretamente, el aprendizaje académico se define como la integración de tres dimensiones: la conciencia que el estudiante tiene de su propio aprendizaje, el contenido de la materia y el contexto de aprendizaje (Marton y Svensson, 1979; Säljö, 1979). Esta conciencia del estudiante sobre el acto del aprendizaje es una de las claves para el éxito académico y se relaciona con el desarrollo de competencias metacognitivas. Por otro lado, estas estrategias están condicionadas por el contenido de la materia, segunda dimensión del concepto de aprendizaje académico. Al mismo tiempo, el contexto de aprendizaje abarca tanto las experiencias de aprendizaje previas, como las propias expectativas y valores del estudiante, el estilo de enseñanza del profesorado e incluso la institución, la metodología docente y los recursos y materiales docentes empleados en el aula así como el sistema de evaluación seleccionado por el profesor.

Los enfoques de aprendizaje no son características psicológicas estables, sino que dependen de la naturaleza de la tarea, el sistema de evaluación, el método de enseñanza, la percepción que el estudiante, la relevancia del curso, el interés del estudiante o el estilo de aprendizaje (Laurillard, 1979).

\subsubsection{Motivaciones y estrategias}

La gran mayoría de los investigadores en Enfoques del aprendizaje coinciden en que existen dos componentes fundamentales en su definición: las motivaciones que revelan las intenciones que mueven al estudiante a aprender y unas estrategias coherentes con dichas intenciones (Hernández et al., 2002). El modelo Motivo/Estrategia 3x3 formulado por Biggs (1984) refleja esta estructura:

\begin{tabular}{|l|l|l|}
\hline Dimensiones & Motivos & Estrategias \\
\hline D1. Utilización & M1. Instrumental / Extrínseco & E1. Reproducción \\
\hline D2. Internalización & M2. Intrínseco & E3. Organización \\
\hline D3. Logro & M3. Logro & E1. Reproducción \\
\hline
\end{tabular}

Tabla 3.1: Modelo Motivo/Estrategia 3x3 formulado por Biggs. Dimensiones

Según Beltrán las estrategias de aprendizaje hacen referencia a operaciones o actividades mentales que facilitan y desarrollan los diversos procesos de aprendizaje (Beltrán, 1993). La combinación de las componentes motivacional y estratégica ha sido una constante desde la formulación del modelo y sigue vigente en la actualidad, aunque se han propuesto ciertas modificaciones sobre la definición y clasificación de cado uno de los términos. 
No obstante, se considera que el conjunto formado por ambos componentes refleja adecuadamente las dimensiones que definen el complejo proceso de estudio desarrollado por los estudiantes universitarios.

\subsubsection{Categorización de los enfoques del aprendizaje}

Atendiendo a ambos elementos, es posible identificar hasta tres enfoques diferentes de aprendizaje, de modo que todas constituyen formas transculturales o universales de aproximarse al aprendizaje, independientemente del sistema educativo nacional (Abalde et al., 2001). En primer lugar el Enfoque Superficial se basa en una motivación extrínseca. En este caso el aprendizaje en el contexto académico se considera un medio para lograr un objetivo concreto, como conseguir trabajo o aprobar un examen, y las estrategias para lograrlo se basan en minimizar el esfuerzo y reproducir lo aprendido a través de un aprendizaje memorístico. Este enfoque superficial es adoptado como consecuencia de una percepción insatisfactoria del estudiante en cuanto al ambiente de aprendizaje, de forma que considera que las tareas y procesos de aprendizaje son algo impuesto externamente. Por ello, el estudiante se limita a completar los requisitos mínimos exigido en cada tarea y memorizar el contenido de la materia con el fin de superar la evaluación sin relacionar los contenidos nuevos con los adquiridos previamente ni reflexionar sobre su posible transferencia a otros ámbitos. Según Valle, en este caso el esfuerzo de los estudiantes no está guiado por ninguna de las metas relacionadas con la motivación (aprendizaje y rendimiento), sino por la meta de evitación del trabajo (Valle et al., 2000). El Enfoque Profundo se basa en una motivación propia del estudiante por las materias, de forma que el estudiante se interesa por el trabajo académico y tiene la intención de comprender e interaccionar con los contenidos previos. Las estrategias surgen de esa motivación y se utilizan para maximizar la comprensión, de forma que la curiosidad del individuo sea satisfecha. Por último, el Enfoque de Alto Rendimiento o Enfoque de Logro se basa en la valoración que el individuo hace de sí mismo a través de la obtención de un alto rendimiento académico. Esta motivación constituye una forma particular de motivación extrínseca características de estudiantes competitivos, auto-disciplinados, esmerados y sistemáticos y cumplen todos los requisitos que demanda la educación superior. En estas circunstancias las estrategias se relacionan con la organización del tiempo, la organización del espacio y el dominio de las materias de la forma más eficiente posible (Ruiz et al., 2008). En uno de los escasos estudios sobre la cuantificación del porcentaje de estudiantes con mayor tendencia a adoptar uno u otro enfoque del aprendizaje Hernández estima que, en una muestra de 360 estudiantes universitarios, aproximadamente la mitad de los encuestados adopta un enfoque profundo $(47,5 \%)$ o un enfoque superficial $(42,2 \%)$ mientras que los estudiantes que adoptan el enfoque de alto rendi- 
miento constituyen una minoría (10,2\%) (Hernández y Hervás, 2005).

La Tabla 3.2 refleja estos tres enfoques del aprendizaje según la formulación de Biggs junto con sus componentes motivacional y estratégica correspondientes (Biggs, 1989):

\begin{tabular}{|l|l|l|}
\hline Enfoques & Motivos & Estrategias \\
\hline (ES) Superficial & (MS) Instrumental / Extrínseco & (EsS) Reproducción \\
\hline (EP) Profundo & (MP) Intrínseco & (EsP) Significado \\
\hline (EL) Logro & (ML) Logro & (EsL) Organización \\
\hline
\end{tabular}

Tabla 3.2: Modelo Motivo/Estrategia 3x3 formulado por Biggs. Enfoques del aprendizaje

A pesar de que Biggs considera excluyentes los enfoques profundo y superficial (Biggs, 1988) sí pueden darse simultáneamente otras combinaciones de enfoques, concretamente los que contienen el enfoque de logro o de alto rendimiento. Así, es posible encontrar situaciones en las que se observe la presencia simultánea de este enfoque y el superficial, al igual que puede darse el caso de que este enfoque de logro coexista con el profundo.

No obstante, estas tipologías de enfoques del aprendizaje sólo tienen sentido si se contextualizan en el contexto de enseñanza - aprendizaje (Biggs, 1993). En este aspecto se ha planteado que el análisis del proceso de aprendizaje sólo es posible si se consideran tanto las características personales del estudiante como la relación con su contexto particular de aprendizaje. De esta forma resulta determinante la percepción que el estudiante tiene de este contexto a partir de sus características personales (Laurillard, 1979).

\subsubsection{Instrumentos para la medición de los enfoques del aprendizaje}

Con el fin de medir los enfoques del aprendizaje adoptados por los estudiantes universitarios se han desarrollado diversos instrumentos de medida en forma de cuestionarios, que adicionalmente plantean la relación entre dichos enfoques y otros posibles factores implicados en el proceso de aprendizaje. El primer instrumento definido en esta línea de investigación fue el cuestionario Inventario de Enfoques de Estudio (ASI: Approaches to Studying Inventory) propuesto por Entwistle y Ramsden (Entwistle, Hanley y Hounsell, 1979; Entwistle y Ramsden, 1982). Este cuestionario consta de 64 ítems que conforman 16 subescalas: Enfoque Profundo, Interrelación de Ideas, Uso de la Evidencia, Motivación Intrínseca, Enfoque Superficial, Se- 
guimiento de Normas, Miedo al Fracaso, Motivación Extrínseca, Enfoque Estratégico, Métodos de Estudio Desorganizados, Actitudes Negativas hacia el Estudio, Motivación de Logro, Aprendizaje por Comprensión, Injustificación, Aprendizaje por Operación e Imprevisión. Este cuestionario fue revisado posteriormente por Entwistle y Tait (1990) y se renombró como Revised Approaches to Studying Inventory (RASI). La revisión de este cuestionario se compone de seis subescalas, tres para cada uno de los enfoques.

A pesar de que éste fue el primer instrumento propuesto, su uso no se encuentra tan generalizado como el elaborado por Biggs, aplicable al ámbito universitario (Biggs, 1987). El cuestionario recibe el nombre de Cuestionario de Procesos de Estudio (CPE) (SPQ: Study Process Questionnaire) y está compuesto por cuarenta y dos ítems con tres escalas, correspondientes a los tres enfoques de aprendizaje profundo, superficial y logro y seis subescalas que contienen ítems referentes a los componentes motivacionales y estratégicos. Este cuestionario fue revisado posteriormente por Biggs et al. (2001) y se renombró como Revised two factor Study Process Questionnaire (R-SPQ-2F). Esta revisión se realizó con el fin de adaptar la versión original a las características propias de los estudiantes universitarios actuales. Esta nueva versión del cuestionario está compuesta por veinte ítems y presenta dos escalas, correspondientes a los enfoques profundo y superficial, y cuatro subescalas en función de la componente, motivacional o estratégica. El Cuestionario de Procesos de Aprendizaje ha sido posteriormente adaptado y aplicado a dos muestras españolas con resultados muy similares a los obtenidos por otros estudios, tanto en lo que respecta a su fiabilidad como a la validez de constructo (Hernández et al., 2002; Hernández, García y Maquillón, 2005).

Existen otros cuestionarios equivalentes desarrollados como instrumentos para la medición de los enfoques del aprendizaje, entre los que se encuentra el cuestionario Approaches and Study Skills Inventory for Students desarrollado por Tait, Entwistle y McCune (1998) y que pone de manifiesto el grado de correspondencia existente entre el ASI y el SPQ. Igualmente el cuestionario Inventory of Learning Styles (ILS) propuesto por Vermunt está compuesto por 120 ítems agrupados en tres escalas: concepción del aprendizaje, estrategias de regulación y estrategias de procesamiento (Vermunt, 1996). Todos estos instrumentos han sido convenientemente traducidos y adaptados a contexto cultural español, alcanzando índices de fiabilidad y validez aceptables. 


\title{
3.2.4. La gestión del tiempo y las características personales
}

\author{
La influencia de los factores personales en los enfoques del apren- \\ dizaje
}

Respecto a la relación entre la gestión del tiempo y las características personales y los enfoques del aprendizaje se han planteado numerosos estudios descriptivos desde muy diversas perspectivas, aunque es posible encontrar elementos comunes entre ellos. En muchos de ellos se plantea el análisis de los enfoques de aprendizaje de los estudiantes en función de su género, concluyendo que en la gran mayoría de ellos no existen diferencias significativas (Biggs, 1984; Buendía y Olmedo, 2002; Clarke, 1986; Hernández, 1999; Hernández et al., 2002; Richardson y King, 1991; Zeegers, 2001), aunque si existe alguna diferencia es que las mujeres muestran mayor tendencia a adoptar un enfoque profundo que los hombres (Biggs, 1982). En este aspecto Cano remarca que alumnos y alumnas utilizan distintas estrategias de aprendizaje, pero la razón para esta diferencia no se debe al género sino a la incidencia del contexto de aprendizaje que incide significativamente, en interacción con el género, sobre las estrategias desplegadas por los alumnos (Cano, 2000).

Otros factores relevantes considerados en diversos estudios son la edad, la etapa educativa y el curso, factores que parecen fomentar la adopción de un enfoque más profundo a medida que se incrementan en el tiempo (Clarke, 1986; Watkins y Hattie, 1981; Zeegers, 2001). Por el contrario, la procedencia geográfica y académica no parecen ser determinantes en el enfoque de aprendizaje adoptado por el alumno (Biggs, 1982; Hernández y Maquillón, 2000; Kember, 2000; Porto, 1994; Rosario, 1997). Sobre la relación entre el área de estudio y el enfoque de aprendizaje adoptado se han realizado muy diversos estudios pero no se han alcanzado un consenso en base a conclusiones coherentes debido a las limitaciones de recursos en la fase de recogida de datos así como por la heterogeneidad en los planteamientos de investigación (Biggs, 1982; Hernández y Maquillón, 2000; Kember, 2000). Uno de los estudios más particulares realizados en esta línea de investigación es el desarrollado por Salas sobre una muestra de estudiantes chilenos en el que confirma la inexistencia de relación entre la dominancia del cerebro y la propensión a adoptar un enfoque profundo en los estudios, definiendo la dominancia cerebral como la tendencia observada en los seres humanos a utilizar más las funciones de un hemisferio que las del otro para interactuar con su medio en acciones como percibir, pensar, conocer, resolver problemas o actuar (Salas, Santos y Parra, 2004). 


\section{La gestión del tiempo de los estudiantes universitarios}

En este aspecto cobra mucha mayor importancia el uso del tiempo que hacen los estudiantes universitarios en cuanto al rendimiento que obtienen de sus estudios. Según Heijke, Meng y Ramaekers (2010) el tiempo de los estudiantes universitarios se distribuye fundamentalmente entre las siguientes actividades: asistencia a clases, estudio individual o en grupo, estudio de otra posible disciplina o materia, actividades formativas fuera del ámbito de los estudios y trabajo remunerado (opcional). Brennan, Patel y Tang (2009) apuntan que los graduados españoles encuestados en el Proyecto REFLEX (cohorte de titulados universitarios en 2005/06) perciben una dedicación semanal promedio a sus estudios de 38 horas. Este número de horas resulta inferior al que reportaron anteriormente los graduados españoles encuestados en el Proyecto CHEERS (cohorte de titulados universitarios en 1999/2000), cuya percepción asciende a 42 horas. Asimismo, en ambos casos estos resultados son inferiores a los promedios señalados por los graduados europeos en conjunto, con un promedio de 35 horas semanales para los encuestados en el Proyecto REFLEX y 32 para los encuestados en el Proyecto CHEERS.

Heijke et al. (2010) señalan que desempeñar un trabajo remunerado de forma paralela a los estudios parece tener cierta utilidad en la adquisición de competencias específicas, al igual que ocurre con el tiempo de estudio individual o en grupo, aunque en este último caso esta actividad muestra adicionalmente un efecto positivo en la adquisición de competencias genéricas. La dedicación de tiempo a estudiar una disciplina adicional y las actividades formativas fuera del ámbito de los estudios contribuyen al desarrollo de competencias genéricas. En cuanto al trabajo remunerado, resulta determinante el hecho de que las tareas se encuentren relacionadas con el área de estudio del estudiante. En los casos en que no ocurre así, únicamente contribuye a la adquisición de competencias de comunicación, mientras que cuando está relacionado con los estudios tiene un efecto significativamente positivo sobre la adquisición de competencias, tanto genéricas como específicas.

En principio no es posible conocer la proporción de estudiantes que se encuentran en esta última situación, debido a la temporalidad e inestabilidad de los empleos que desempeñan los estudiantes durante su estancia en la Universidad. Sin embargo, sí es posible obtener una aproximación mediante el análisis de los indicadores sobre la realización de estudios a tiempo parcial. El informe de la encuesta desarrollada por Eurostat, revela que España se encuentra en una posición promedio en este aspecto respecto a otros países de la Unión Europea (Eurostat, 2009). Así, el número de estudiantes que manifiestan dedicar más de 20 horas semanales a sus estudios alcanza el $78 \%$, resultado superior al mínimo obtenido en Estonia (56\%) e inferior al máximo obtenido en Portugal (95\%). En este informe se alude a otros 
factores pertenecientes al contexto personal de los estudiantes y que igualmente pueden resultar determinantes en el rendimiento de los estudios. Así, el nivel de estudios de los padres es uno de los condicionantes tradicionalmente considerados en estos estudios. En términos generales, el porcentaje de estudiantes cuyos padres tienen estudios secundarios y que logran finalizar sus estudios universitarios apenas alcanza el $32 \%$, mientras este mismo resultado se incrementa hasta el $63 \%$ para aquellos estudiantes cuyos padres tienen estudios universitarios. Curiosamente este factor mantiene cierta relación de interacción con el género del estudiante, puesto que en la gran mayoría de los países, las mujeres cuyos padres tienen un nivel de estudios elevado tienen más posibilidades de finalizar sus estudios universitarios que los hijos varones, con excepción de Italia y Holanda donde el patrón de comportamiento es precisamente el contrario.

Todos estos factores en conjunto tienen un efecto directo o indirecto sobre el desempeño académico de los estudiantes. No obstante Salanova destaca la importancia del llamado Circulo Vicioso o Virtuoso que propone que dos situaciones contrapuestas. En el caso del circulo virtuoso, "a mayor rendimiento en el pasado, mayor bienestar psicológico en el futuro y por tanto influirá en un mayor rendimiento posterior". Por el contrario el círculo vicioso propone que, "a peor rendimiento pasado, peor bienestar psicosocial presente y por tanto peor rendimiento futuro" (Salanova, Martínez, Bresó, Llorens y Grau, 2005). De este modo, el autoconcepto del estudiante en cuanto a su eficacia académica pasa a ser una variable mediadora entre las posibilidades de éxito académico del estudiante, su nivel de compromiso con los estudios y futuro desempeño.

Evidentemente esta descripción de la distribución del tiempo de los estudiantes universitarios no ha tenido en consideración la dedicación del tiempo a actividades de ocio y tiempo libre. En principio no se ha planteado el estudio del potencial efecto que estas actividades podrían tener sobre la adquisición informal de competencias, pero resulta apropiado conocerlas con el fin de comprender mejor el entorno personal de los estudiantes universitarios. Así, según Rodríguez las actividades de ocio desarrolladas con más frecuencia por los estudiantes universitarios tienen un claro componente social como son salir con los amigos o ir a bares o pubs. Al mismo tiempo dedican una parte importante de su tiempo al seguimiento de los medios de comunicación, ya sea la televisión, la radio o la lectura de periódicos. Respecto a las actividades artísticas y culturales muestran mayor inquietud que el resto de los jóvenes y la mayor parte de ellos realiza algún deporte. Asimismo la mayoría de ellos se consideran creen ser realistas, responsables y maduros, quizá algo conformistas y faltos de interés pero liberales, pacíficos y tolerantes (Rodríguez y Agulló, 1999). Por último, los valores de los graduados universitarios relativos al ámbito laboral se desarrollan entorno 
al desarrollo personal y profesional, el estatus profesional y económico, el ocio y la familia, la utilidad para la sociedad y la estabilidad y autonomía laboral (Conchado, Cortés, Mora y Carot, 2009; Mora et al., 2008).

\subsection{El contexto académico universitario}

\subsubsection{El profesional flexible en la nueva sociedad del cono- cimiento}

La denominada sociedad del conocimiento, basada fundamentalmente en el uso intensivo de las tecnologías de la comunicación y la información, ha supuesto un incremento significativo de las expectativas en las Instituciones de Educación Superior. Ya no es suficiente que los titulados universitarios posean los conocimientos necesarios para desempeñar las tareas laborales en un campo específico de estudio. Ahora se espera de las universidades que formen a sus estudiantes para que se desenvuelvan como profesionales flexibles, capaces de enfrentarse a muy diversas situaciones laborales. Es decir, que ya no son suficientes las competencias específicas del área de estudio sino que debe proporcionarse a los estudiantes las competencias genéricas necesarias para facilitar el aprendizaje posterior de las competencias que les sean requeridas en el futuro. Evidentemente, la formación en las competencias específicas relativas a los contenidos y procedimientos del campo de estudio concreto de la titulación continúa siendo fundamental para el posterior desempeño de las tareas, sobre todo en los contextos laborales en que se requieren conocimientos muy especializados en la materia (Heijke et al., 2010). No obstante, según Stenberg las Instituciones de Educación Superior tienen actualmente mayor demanda de formación en competencias genéricas y señala que en los próximos años será necesaria una nueva generación de expertos, cuya formación y experiencia sea mucho más amplia que sus conocimientos técnicos (Stenberg, 2003). Por todo ello ha aumentado notablemente el énfasis en la formación continua, factor que ya es considerado en determinados sectores como el elemento más determinante en el crecimiento económico de un país o una región. En estos términos, todos estos cambios ya se están reflejando en el mercado laboral al que acceden los titulados universitarios, Tal como señala Schmid es posible introducir un nuevo concepto en esta línea, denominado Mercado Laboral en Transición para hacer referencia a la movilidad de los titulados y la flexibilidad de los perfiles demandados por las organización, en estos momentos características de los mercados laborales actuales (Schmid, 2001).

En este contexto se está replanteando si las metodologías docentes tradicionales, basadas en lecciones magistrales aportadas por profesores expertos a grupos de estudiantes que actúan pasivamente como receptores de la infor- 
mación, resultan adecuadas para afrontar estas nuevas circunstancias. Por ello los profesores universitarios están introduciendo progresivamente nuevas metodologías docentes con el fin de desarrollar nuevos modelos educacionales basados en la formación en competencias y los resultados del aprendizaje. Estas transformaciones apuntan a un cambio paradigmático metodológico más allá de las pequeñas innovaciones introducidas por cada profesor, a partir del cual las instituciones de Educación Superior ya no se considerarán instituciones donde simplemente se proporciona enseñanza sino que adicionalmente se produce aprendizaje.

\subsubsection{El cambio metodológico en la Universidad hacia los contextos activos de aprendizaje}

\section{Los contextos de aprendizaje}

En el contexto actual de los mercados laborales en transición, las competencias específicas presentan mayor probabilidad de quedar obsoletas con el tiempo mientras que las competencias genéricas aportan beneficios a largo plazo. La cuestión de qué tipo de competencias obtienen los estudiantes al finalizar sus estudios universitarios radica en gran medida de cómo se hayan transmitido, en términos de contextos de aprendizaje y métodos docentes. Concretamente, cabe esperar que los contextos de aprendizaje que no sólo se basan en la enseñanza de las competencias específicas sino que adicionalmente incorporan competencias genéricas, aporten una formación más completa y versátil (Heijke et al., 2010; Vaatstra y De Vries, 2007). Idealmente, las instituciones deberían proporcionar a sus estudiantes formación en una combinación adecuada de ambos tipos de competencias, de modo que la interacción entre genéricas y específicas generase la capacidad de afrontar situaciones laborales complejas, poco habituales y no rutinarias (Stasz, 1998).

\section{Modalidades y métodos de enseñanza - aprendizaje}

De Miguel define los métodos de enseñanza - aprendizaje como la "forma de proceder que tienen los profesores para desarrollar su actividad docente" (De Miguel, 2006). Sin embargo, tal y como señala Zabalza la formulación de este concepto puede resultar demasiado laxa y engloba muchos aspectos de la docencia universitaria como para resultar efectivo en la indentificación de indicadores de calidad (Zabalza, 2010). Por ello, más que hablar de métodos concretos suele ser preferible hacer referencia a orientaciones metodológicas, o como denomina De Miguel, Modalidades de Enseñanza Aprendizaje. En este sentido las modalidades constituyen "las distintas maneras de organizar y llevar a cabo los procesos de enseñanza - aprendizaje". 
En este aspecto en docencia universitaria es posible plantear muy distintas formas de organizar las enseñanzas dependiendo de los objetivos de aprendizaje planteados por el profesor así como los escenarios y recursos de que dispone.

\begin{tabular}{|l|l|}
\hline Modalidades organizativas & Métodos de enseñanza - aprendizaje \\
\hline Clases teóricas o Expositivas & Lección magistral \\
Seminarios o Talleres & Estudio de casos \\
Clases prácticas & Resolución de problemas \\
Prácticas externas & Aprendizaje basado en problemas \\
Tutorías & Aprendizaje orientado a proyectos \\
Estudio y trabajo en grupo & Aprendizaje cooperativo \\
Estudio y trabajo individual o autónomo & Contrato de aprendizaje \\
\hline
\end{tabular}

Tabla 3.3: Modalidades organizativas y Métodos de enseñanza - aprendizaje

Aunque modalidades y método de enseñanza - aprendizaje se han presentado como elementos distintos, resulta evidente la existencia de vinculación entre ambos, ya que algunas modalidades facilitan el empleo preferente de unos métodos sobre otros. Para evaluar las relaciones entre ambos elementos De Miguel realizó una investigación en la que se analizaban las valoraciones de un grupo de expertos sobre esta cuestión. Los resultados de la investigación mostraron que "las clases teóricas se relacionan casi en exclusiva con el método expositivo o lección; los seminarios, con el estudio de casos y la resolución de problemas; las clases prácticas, con la resolución de problemas y con el aprendizaje basado en problemas; las prácticas externas, con el aprendizaje basado en problemas; las tutorías, con el aprendizaje orientado a proyectos y con los contratos de aprendizaje; el trabajo en grupo, con el aprendizaje cooperativo y con el aprendizaje basado en problemas, y el estudio individual, con los contratos de aprendizaje y con el aprendizaje orientado a proyectos".

\begin{tabular}{|l|c|c|c|c|c|c|c|}
\hline & $\begin{array}{c}\text { Lección } \\
\text { magistral }\end{array}$ & $\begin{array}{c}\text { Estudio } \\
\text { de casos }\end{array}$ & $\begin{array}{c}\text { Resolución } \\
\text { de problemas }\end{array}$ & $\begin{array}{c}\text { Aprendizaje } \\
\text { basado en } \\
\text { problemas }\end{array}$ & $\begin{array}{c}\text { Aprendizaje } \\
\text { orientado } \\
\text { a proyectos }\end{array}$ & $\begin{array}{c}\text { Aprendizaje } \\
\text { cooperativo }\end{array}$ & $\begin{array}{c}\text { Contrato de } \\
\text { aprendizaje }\end{array}$ \\
\hline Clases teóricas & 3 & 1 & 1 & 0 & 0 & 1 & 1 \\
Seminarios & 0 & 3 & 3 & 2 & 1 & 2 & 1 \\
Clases prácticas & 0 & 2 & 3 & 3 & 0 & 1 & 1 \\
Prácticas externas & 0 & 1 & 2 & 3 & 2 & 1 & 1 \\
Tutorias & 1 & 1 & 1 & 2 & 3 & 2 & 3 \\
Estudio grupo & 1 & 2 & 2 & 3 & 2 & 3 & 1 \\
Estudio individual & 2 & 2 & 1 & 1 & 3 & 1 & 3 \\
\hline
\end{tabular}

Tabla 3.4: Correspondencia entre Modalidades organizativas y Métodos de enseñanza - aprendizaje 


\section{Los contextos activos de aprendizaje}

\section{Críticas a los contextos de aprendizaje tradicionales}

Tradicionalmente la enseñanza proporcionada por las Instituciones de Educación Superior se ha fundamentado en que los estudiantes estudien los contenidos, asistan a clase para comprender mejor dichos conceptos y finalmente demuestren sus conocimientos en los exámenes finales. Así, estas clases teóricas se estructuran fundamentalmente en torno a la presentación de los contenidos de la materia de forma sistemática por parte del profesor.

Sin embargo, en este contexto de aprendizaje se corre el riesgo de que los estudiantes adquieran conocimientos sin ningún significado para ellos, con la consiguiente probabilidad de ser olvidados rápidamente después de la evaluación (Gerritsen, 1999). Asimismo, este contexto de aprendizaje apenas fomenta la implicación de los estudiantes, lo cual es habitual que se manifieste en una motivación muy baja en el aprendizaje de la materia. Por último, se ha señalado que la subdivisión de la materia de estudio en diversas asignaturas y temarios resulta un tanto artificial y alejada de la realidad laboral, generalmente organizada en torno a problemas, cuya resolución habitualmente requiere la aplicación de conocimientos procedentes de diversas materias. En otras palabras, se considera que la estructuración de contenidos realizada en la lección magistral no refleja la interdisciplinariedad de las situaciones que posteriormente deberán afrontar los titulados universitarios. $\mathrm{Al}$ mismo tiempo presenta algunos inconvenientes que la convierten en una opción poco recomendable para la formación en determinadas competencias. Por su propia naturaleza su aporte en la adquisición de las capacidades de liderazgo, innovación y cooperación resulta prácticamente nulo. Asimismo, en otras competencias como organización personal y comunicación, se considera que tiene un efecto parcial, ya que aunque existe comunicación, ésta se produce en un único sentido, desde el profesor hacia el estudiante. Del mismo modo la capacidad de organización la adquiere posteriormente el alumno, fundamentalmente gracias a su trabajo autónomo y no a consecuencia del discurso recibido en el aula.

Debido a estas críticas a los contextos tradicionales de aprendizaje, y concretamente al empleo de la lección magistral como método docente, se ha fomentado la introducción de nuevos contextos de aprendizaje que demandan una participación más activa por parte del alumnado. En estos contextos de aprendizaje activos es habitual aplicar determinados métodos de enseñanza - aprendizaje como trabajos en grupo, prácticas en empresa, trabajos escritos o aprendizaje basado en problemas u orientado a proyectos. De este modo las instituciones tratan de introducir cambios en las metodologías docentes con el fin de garantizar que los titulados universitarios posean 
las competencias genéricas que posteriormente les permitirán desenvolverse con soltura en el ámbito laboral. Concretamente tanto profesores universitarios como empleadores coinciden en que determinadas competencias son especialmente relevantes en el entorno laboral, como son la resolución de problemas, la comunicación y el trabajo en equipo (Stasz, 1998).

\section{Ventajas de los contextos activos de aprendizaje frente a las modalidades tradicionales}

Una de las principales aportaciones de los contextos activos de aprendizaje es el incremento de la implicación de los estudiantes en el proceso de aprendizaje. Aunque la investigación en este campo se desarrolló fundamentalmente en la década de los 70 y su aplicación es relativamente reciente, sus inicios se remontan a principios del siglo XX, con los trabajos de Dewey (1916). La hipótesis que plantean tanto Dewey como otros estudios posteriores es que, puesto que al finalizar el proceso de enseñanza - aprendizaje los estudiantes han adquirido experiencia en la aplicación de los conocimientos teóricos a la resolución de problemas, son más capaces de aplicar estos conocimientos en la práctica. Asimismo, estos estudiantes adquieren otras competencias genéricas de forma adicional, como trabajo en equipo, búsqueda de información o capacidad de trabajar de forma autónoma (Biggs, 2003; Everwijn, Bomers y Knubben, 1993; Ramsden, 2003).

Otra característica de los contextos de aprendizaje activos es que los estudiantes aprenden a través de la aplicación de los conocimientos teóricos a problemas reales. Esta experiencia obtenida mediante ejemplos prácticos y/o reales o incluso mediante simulación de situaciones prácticas, resulta una condición muy relevante para una adecuada preparación profesional, y adicionalmente facilita la adquisición de experiencia profesional. Schön incide en que los profesores envíen a los estudiantes a que tomen contacto con la realidad de su entorno laboral desde el inicio de los estudios, de modo que el aprendizaje se lleve a cabo mediante la ejecución de tareas (Schön, 1987). De hecho, las estancias en entornos laborales a través de prácticas en empresa son una metodología muy valorada por los empleadores, y en los últimos años también por las Instituciones de Educación Superior así como por los titulados universitarios, como un método muy eficiente para la adquisición de las competencias demandadas en el puesto de trabajo (Falconer y Pettigrew, 2000).

\section{Aprendizaje basado en problemas}

El aprendizaje basado en problemas parte de una serie de problemas diseñados por el profesor que el estudiante ha de resolver para desarrollar determinadas competencias previamente definidas. Habitualmente se soli- 
cita a los estudiantes que desarrollen las soluciones adecuadas o correctas mediante la ejercitación de rutinas, la aplicación de fórmulas o algoritmos, la aplicación de procedimientos de transformación de la información disponible y la interpretación de los resultados y se suele utilizar como complemento de la lección magistral. Así, se considera que el estudiante aprende de un modo más adecuado cuando tiene la posibilidad de experimentar, ensayar o, sencillamente, indagar sobre la naturaleza de fenómenos y actividades cotidianas (De Miguel et al., 2005). Este aprendizaje se considera más motivador cuando se plantean preguntas que requieren del esfuerzo intelectual del estudiante y no de la mera repetición de una rutina de trabajo aprendida. Asimismo resulta todavía estimulante si se plantea en un contexto cuya aplicación tenga significado práctico para los estudiantes (Gerritsen, 1999). Este método de enseñanza se relaciona con las modalidades de Clases prácticas y Prácticas externas, a su vez asociadas en gran medida con las competencias de innovación y organización personal. Asimismo, aunque es evidente que contribuye a desarrollar la capacidad de resolución de problemas, también fomenta la toma de decisiones, comunicación y determinadas actitudes como la meticulosidad, la precisión, la revisión, la tolerancia y el contraste de los resultados obtenidos, y por supuesto fomenta el trabajo grupal e interprofesional si el profesor permite que los estudiantes trabajen en grupo. En este caso la colaboración facilita el aprendizaje no sólo porque requiere del estudiante que exponga y argumente sus puntos de vista o soluciones sino que adicionalmente debe debatirlas con otros. Se trata de un método de trabajo activo, centrado en el estudiante, en el que el profesor es sobre todo un facilitador de la información, y no un mero transmisor.

La finalidad de estas modalidades de enseñanza - aprendizaje es mostrar a los estudiantes cómo deben actuar en un contexto controlado y aproximadamente similar al laboral, pero con un margen de error más amplio. Se ofrece así al estudiante la posibilidad de establecer una conexión entre lo aprendido y la realidad. Asimismo sitúan al estudiante ante situaciones cercanas al desarrollo de la profesión, que exigen de su capacidad de innovar, integrar y aplicar conocimientos y habilidades asociados a la titulación o incluso o a otros campos del saber; y, por supuesto, le exige que aprenda a debatir y argumentar ante personas que tienen una formación similar a la suya. Quizá la única limitación que presentan es que requieren mayor preparación por parte del profesor, así como una buena organización de los recursos disponibles si se realizan en el entorno académico.

\section{Aprendizaje orientado a proyectos}

La base de este método de enseñanza - aprendizaje es que los estudiantes llevan a cabo la realización de un proyecto en un tiempo determinado para resolver un problema o abordar una tarea mediante la planificación, 
diseño y realización de una serie de actividades, y todo ello a partir del desarrollo y aplicación de aprendizajes adquiridos y del uso efectivo de recursos (De Miguel et al., 2005). En este caso los estudiantes trabajan como grupo autónomo sobre un problema real, aplicando sus conocimientos y habilidades para la resolución del problema. Se considera que esta metodología contribuye a desarrollar un amplio abanico de competencias tanto relacionadas con los conocimentos (análisis, síntesis y conceptualización, desarrollo y profundización de conocimientos, investigación e innovación en las soluciones técnicas) así como diversas habilidades y destrezas (pensamiento sistémico y crítico, comunicación oral y escrita, trabajo en equipo y competencias sociales y planificación y organización del trabajo) y actitudes (iniciativa y responsabilidad individual y grupal).

Existen muchas similitudes entre el aprendizaje basado en problemas y el basado en proyectos. En ambas metodologías los estudiantes obtienen experiencia específica mediante el aprendizaje autónomo, trabajan en grupo y aprenden procedimientos sistemáticos para abordar problemas relacionados con su área de estudio. La principal diferencia entre ambos es que se espera de los estudiantes que trabajan mediante aprendizaje basado en proyectos que generen un informe final. Por el contrario, el resultado final del aprendizaje basado en problemas es la interacción discursiva entre los estudiantes que resulta una aportación fundamental de cara al posterior estudio individual de los distintos miembros del grupo.

\section{Estudio y trabajo en grupo y Aprendizaje cooperativo}

Se define como un enfoque interactivo de organización del trabajo en el aula en el cual los alumnos son responsables de su aprendizaje y del de sus compañeros en una estrategia de corresponsabilidad para alcanzar metas e incentivos grupales. En definitiva, más que una metodología de enseñanza aprendizaje se considera un enfoque global de la enseñanza o una filosofía. Esta metodología se basa en la organización de actividades por parte del profesor a través del trabajo en equipo. Durante las primeras fases del trabajo el profesor deberá supervisar el funcionamiento del equipo y les indicará algunas pautas para desarrollar el trabajo. Esta tarea supone un mayor esfuerzo del profesor al inicio, quien deberá confiar en la capacidad de aprendizaje autónomo de sus estudiantes en fases más avanzadas del trabajo. En cualquier caso, esta forma de trabajo resulta mucho más motivadora para los estudiantes puesto que les aporta la riqueza de las interacciones grupales y les permite asumir un papel activo en la realización de las tareas. En contraposición al aprendizaje cooperativo se encuentra el aprendizaje competitivo, en el que se fomenta que los estudiantes compitan con sus compañeros por un objetivo o una calificación que sólo algunos de ellos podrán alcanzar. En este caso la referencia para los estudiantes no es la adquisición de competen- 
cias sino el trabajo realizado por sus compañeros de modo que no se produce interacción entre ellos, y por supuesto no se dan situaciones de colaboración. Según D. W. Johnson, R. Johnson y K. Smith (1991), el trabajo cooperativo constituye una alternativa mucho más recomendable que el aprendizaje competitivo. Concretamente cuando se aplica sobre grupos pequeños puede llegar a ser un contexto de aprendizaje muy motivador, en el sentido de que materias que inicialmente pueden resultar aburridas para algún estudiante en concreto, pueden surgir aspectos interesantes al escuchar a sus compañeros hablar sobre él, sin mencionar el interés que inmediatamente despiertan si en algún momento alguno de sus compañeros ha tenido alguna experiencia personal o profesional relacionada con el tema. Adicionalmente, puede ser el medio de evitar el abandono de la materia en caso de dificultades académicas gracias al apoyo de los compañeros.

Respecto a la adquisición de conocimientos se considera que es una metodología adecuada para aprender a buscar y seleccionar información, obtener una comprensión profunda de los conocimientos así como aprender a aplicarlos en la práctica. Es evidente que fomenta la resolución creativa de problemas así como la expresión oral y el desempeño de roles grupales. Entre las actitudes desarrolladas mediante esta metodología se encuentran la expresión de los sentimientos y opiniones así como la práctica de la escucha activa (De Miguel et al., 2005).

\subsubsection{El cambio paradigmático en las metodologías docentes y la formación del profesorado universitario}

Indudablemente los docentes universitarios tienen a su alcance otras metodologías igualmente efectivas y útiles para el desarrollo de estas competencias, como las tutorías o los seminarios y talleres. Estas metodologías cuyo estudio escapa a los objetivos de este trabajo, conllevan el aprendizaje de otras competencias. Asimismo su empleo en el aula debe ser reforzado mediante el trabajo autónomo del estudiante, al igual que ocurre con las anteriores. Sin embargo, la cuestión en este aspecto no debería ser la identificación de la metodología óptima mediante la cual es posible adquirir mayor número de competencias, sino la selección de la metodología más adecuada en el contexto de aprendizaje del alumno. A pesar del énfasis puesto por las instituciones de Educación Superior en la necesidad de realizar este cambio metodológico, recae sobre el profesorado universitario la responsabilidad de su ejecución en la práctica. Para muchos de ellos este nuevo enfoque de su trabajo no supone sino una dificultad añadida a las anteriores (masificación de las aulas, deterioro de la relación profesor - alumno, desfase generacional, etc.) La principal carencia que percibe este colectivo como consecuencia del cambio metodológico es la falta de formación para el desempeño de sus 
tareas docentes en este nuevo contexto. Por otro lado con frecuencia los profesores universitarios se ven a sí mismos como profesionales o investigadores reconocidos en su campo, pero faltos de interés por la docencia. De hecho, es común tropezar con ciertas actitudes escépticas hacia el papel de la Universidad en la formación de los estudiantes y la implantación de nuevos enfoques destinados a mejorar la calidad de la enseñanza.

Contrariamente a esta actitud negativa, que afortunadamente no es habitual, la mayoría del profesorado universitario se encuentra concienciado de la gran importancia de transmitir a sus alumnos las competencias que posteriormente necesitarán en sus trabajos, y no simplemente los conocimientos según los procedimientos tradicionales. No obstante, la impartición de docencia en este contexto supone un reto ya que exige a los profesores que formen a sus estudiantes para que se desenvuelvan como profesionales flexibles, capaces de enfrentarse a muy diversas situaciones laborales. Este requerimiento adicional resulta especialmente complejo en el sentido de que implica un cambio radical en el enfoque tradicionalmente aplicado a las metodologías docentes, tan arraigado en determinados ámbitos universitarios.

\subsection{Los modelos de enseñanza - aprendizaje en el contexto universitario}

El Modelo Conductista de Aprendizaje es el más simple de todos los propuestos, y parte de la idea de que el medio más eficaz para fomentar el aprendizaje es manipular el contexto de aprendizaje, obviando así las características personales o diferencias individuales estudiante y la gestión del tiempo durante los estudios universitarios. Este modelo, que igualmente obvia las diferencias en cuanto a la aptitud y competencias previas de los alumnos, habitualmente a través de la comparación de resultados entre grupos experimentales y grupos de control.

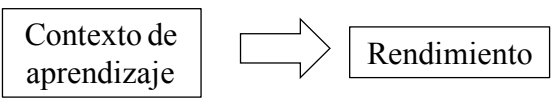

Figura 3.1: Modelo Conductista del Aprendizaje

Con el fin de solventar las limitaciones del modelo anterior se plantea el Modelo de Diferencias Individuales que, sin obviar los efectos de la manipulación del contexto de aprendizaje, considera las diferencias entre los 
estudiantes como un factor igualmente determinante de los resultados obtenidos, analizando para ello los niveles de correlación entre niveles de aptitud y rendimiento.

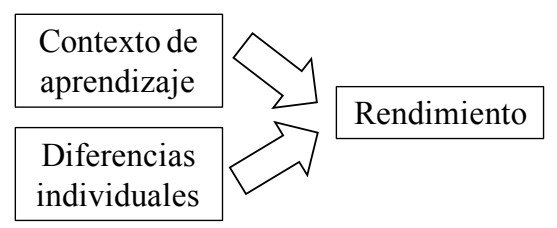

Figura 3.2: Modelo de Diferencias del Aprendizaje

Puesto que ambos modelos presentan ciertas limitaciones en su formulación, Cronbach propone el Modelo ATI de Cronbach, en el cual se considera que el rendimiento es un resultado de la interacción entre el contexto de aprendizaje y las diferencias individuales de los estudiantes y puntualiza que ambos resultan determinantes sobre los resultados del aprendizaje (Cronbach, 1967). Sin embargo este modelo presenta dos limitaciones que deben ser tenidas en consideración. En primer lugar los resultados de su aplicación mostraron que las interacciones entre ambos factores apenas resultaron significativas. Asimismo la relación entre aptitud, rendimiento y contexto fue posteriormente contrastada empíricamente por Biggs y resultó ser mucho más débil de lo que en principio se había hipotetizado (Biggs, 1976).

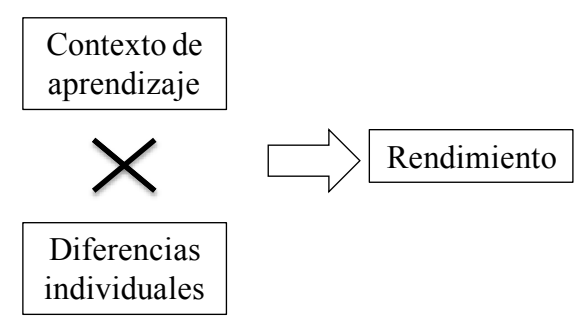

Figura 3.3: Modelo ATI de Cronbach

Un modelo de enseñanza - aprendizaje relativamente más complejo que los anteriores es el Modelo de Aprendizaje en Contexto (Ramsden, 1988, 2003). Este modelo se fundamente en tres aspectos primordiales del contexto de enseñanza - aprendizaje: la enseñanza, la forma de evaluación y el currículum, referido al contenido y estructura de lo que se aprende. Adi- 
cionalmente, las experiencias educativas previas resultan determinantes en la determinación de la percepción del alumno en el aprendizaje, puesto que influyen inevitablemente en su enfoque del aprendizaje. Específicamente, el hecho de cambiar el contexto de aprendizaje al ingresar en una institución de Educación Superior, conlleva que el bagaje del alumno a la hora de afrontar los procesos de aprendizaje se modifique por la percepción de nuevos requisitos y tareas.

En este modelo la importancia de la percepción del alumno del contexto de aprendizaje puede llegar a sustituir otros factores primordiales en otros modelos, como por ejemplo las diferencias individuales entre estudiantes.

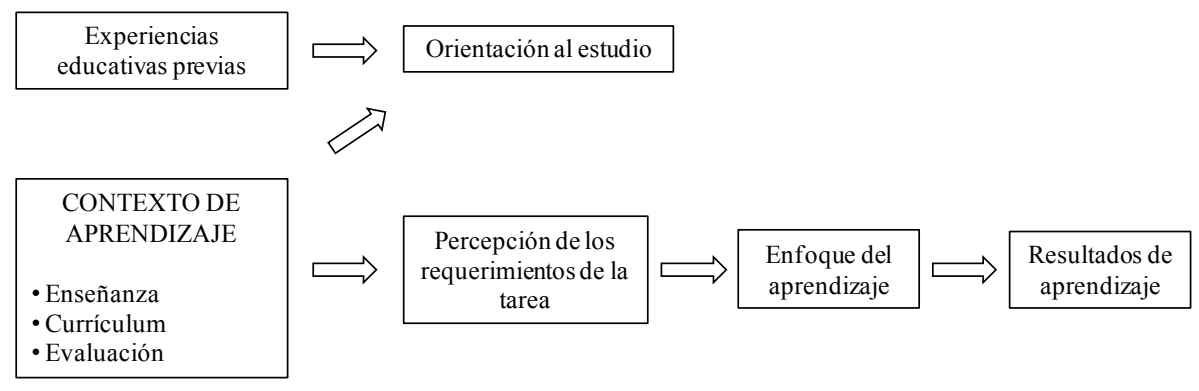

Figura 3.4: Modelo de Aprendizaje en Contexto

Inmediatamente posterior es el Modelo Heurístico del Proceso de Enseñanza - Aprendizaje (Entwistle, 1987). Este modelo pretende reflejar la compleja naturaleza del proceso de enseñanza - aprendizaje a través de las distintas conexiones que lo componen: estudiante, enseñanza y departamento. Con este modelo se pretende aportar la importancia adecuada a todos y cada uno de los elementos potencialmente influyentes en el contexto de aprendizaje académico.

Como principales características del modelo, Entwistle destaca que el modelo ofrezca un marco de referencia con el fin de fomentar la reflexión del profesorado universitario sobre el contexto de aprendizaje. Para ello es necesario que dicho modelo describa la realidad de este proceso considerándolo en su toda complejidad al tiempo que reconozca la responsabilidad e individualidad del alumno.

Posteriormente Pérez propone el Modelo de Factores en el que afirma que la clave de los procesos de enseñanza - aprendizaje reside en la interacción de tres elementos: la actividad educativa del profesor, los contenidos de la materia y los factores cognitivos y metacognitivos de los alumnos (conoci- 


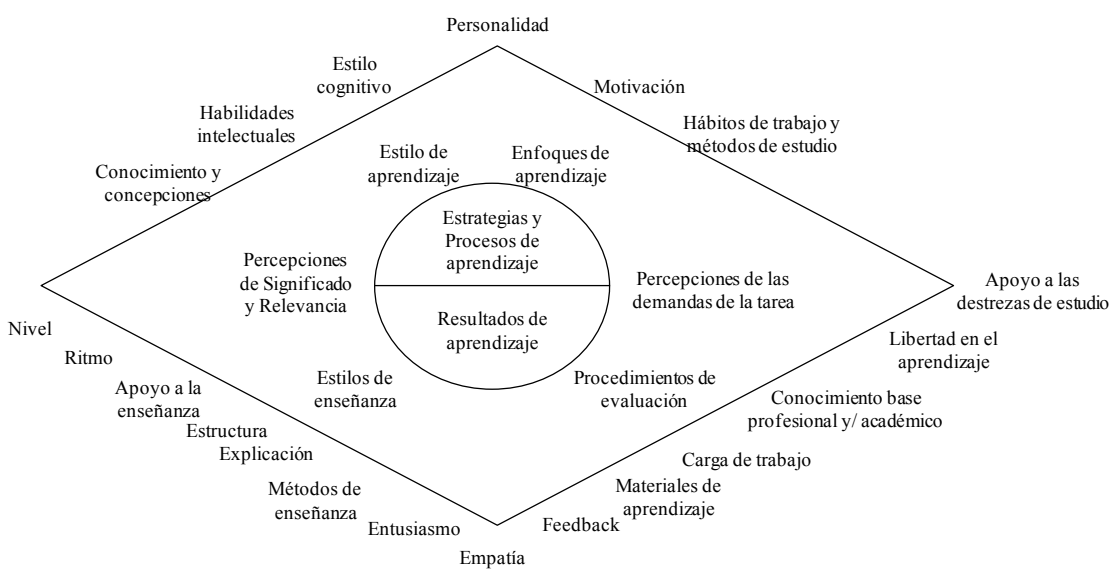

Figura 3.5: Modelo Heurístico del Proceso de Enseñanza - Aprendizaje

mientos previos y capacidad y motivación para el aprendizaje) (Pérez, 2001). El elemento sobre la actividad educativa del profesor hace referencia al modo de planificar el trabajo en el aula mientras que con el término contenidos de la materia alude a su selección en cantidad y organización. Asimismo el elemento relativo a los factores cognitivos y metacognitivos no son más que las concepciones del aprendizaje de los alumnos, y que inevitablemente se relacionan con la adopción de uno u otro enfoque del aprendizaje.

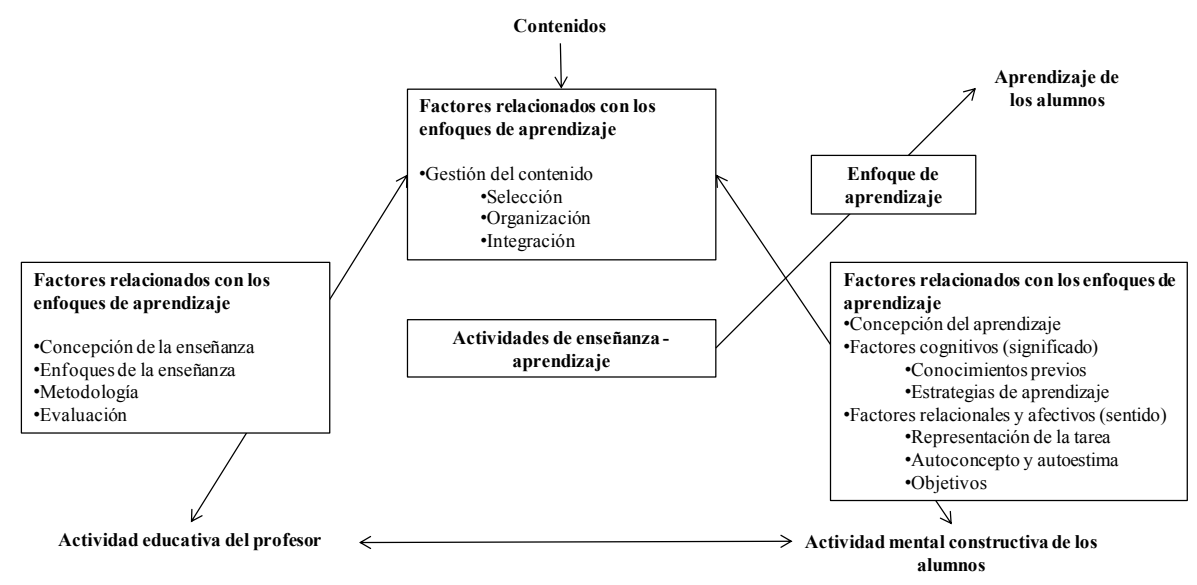

Figura 3.6: Modelo de Factores

No obstante, en esta cuestión resulta ineludible el análisis de los mode- 
los mediacionales propuestos por Biggs, siempre estructurados de forma que los aspectos relativos al proceso de aprendizaje median las relaciones entre las características personales del estudiante, el contexto de aprendizaje y el consecuente rendimiento académico. En este sentido las investigaciones realizadas por Biggs han demostrado la existencia de diferencias en el rendimiento en función de la personalidad, las actitudes de los estudiantes y las actitudes de los mismos frente a diversas situaciones de aprendizaje, justificando así la formulación del cuestionario SPQ.

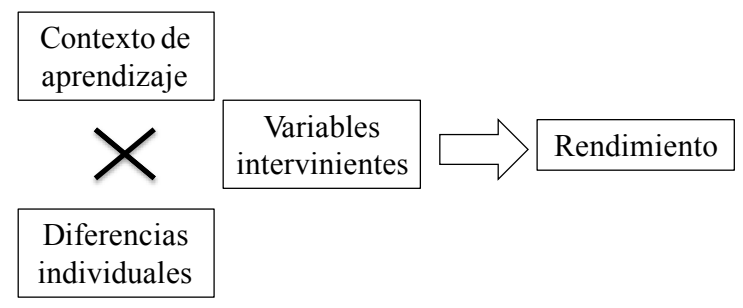

Figura 3.7: Esquema del modelo mediacional propuesto por Biggs

Estos modelos se plantean como una variante del paradigma Presagio - Proceso - Productodesarrollado por Dunkin y Biddle (1974) y desde su planteamiento inicial han experimentado sucesivas modificaciones y mejoras hasta alcanzar su configuración definitiva (Biggs, 1978; Biggs, 1984; Biggs, 1987; Biggs, 1993; Biggs et al., 2001).

El Factor de Presagio está compuesto principalmente por variables independientes que ya existían de forma previa al proceso de aprendizaje. Hay dos tipos de variables que pueden ser clasificadas en esta categoría. En primer lugar las variables relativas al estudiante, o simplemente las características personales, como son las capacidades innatas, expectativas y valores, los estilos cognitivos o los rasgos de la personalidad. Asimismo pertenecen a esta categoría las variables relativas al contexto de enseñanza, o variables situacionales o contextuales como son los métodos de enseñanza aprendizaje empleados por el profesor, el clima de aprendizaje en el aula, la disponibilidad de recursos institucionales y sobre todo el sistema de evaluación.

El Factor de Proceso está compuesto para variables de proceso o variables intervinientes, y tratan de referenciar a lo que Biggs denomina el "Complejo Proceso de Aprendizaje" (Biggs, 1984). Este concepto hace referencia a las intenciones de los estudiantes, previas al desarrollo de sus estrategias de aprendizaje, y que forman parte de la personalidad del estudiante. Estas 
variables actúan como mediadoras en el proceso y proceden de la percepción que el alumno tiene del contexto de aprendizaje. En esencia constituyen los motivos y estrategias que componen cada uno de los posibles enfoques del aprendizaje.

El Factor de Producto, planteado en el modelo como variable dependiente, considera el rendimiento académico o de forma más general los resultados del aprendizaje. Puede tratarse tanto de las calificaciones obtenidas por el alumno como por las competencias adquiridas gracias al proceso de aprendizaje o simplemente una valoración del grado de satisfacción con el proceso de aprendizaje.

El modelo definitivo recibe el nombre de Modelo $3 P$ de Enseñanza Aprendizaje (Biggs et al., 2001) por estructurarse en los tres factores anteriores. Este modelo plantea diversas relaciones bidimensionales entre todos sus componentes, tanto los factores personales y contexto de aprendizaje, ambos pertenecientes al factor de presagio, como los enfoques del aprendizaje representantes del factor de proceso y los resultados del aprendizaje como factor de producto. Una de las aportaciones más novedosas de este modelo es la idea de que el enfoque de aprendizaje contextual resulta un indicador tan apropiado de la calidad de la enseñanza en el aula que debe ser incluido como resultado del aprendizaje.

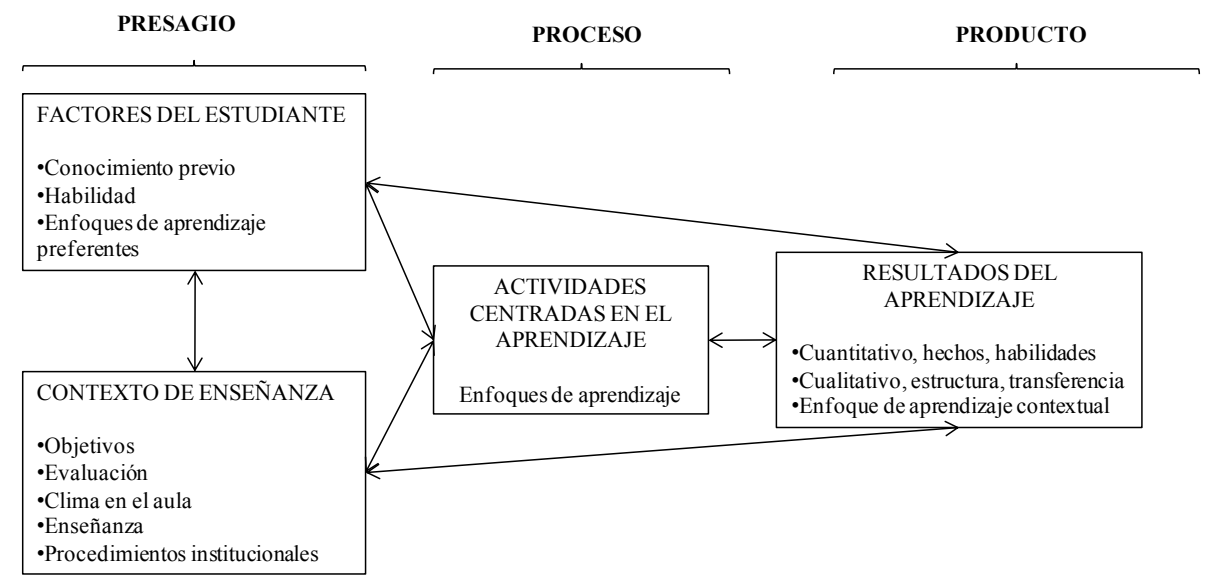

Figura 3.8: Modelo 3P de Enseñanza - Aprendizaje propuesto por Biggs

La práctica totalidad de los modelos mencionados anteriormente, e igualmente las investigaciones previas sobre enfoques del aprendizaje, se fundamentan en investigaciones teóricas o planteamientos cualitativos. Asimismo, 
apenas se han realizado validaciones empíricas de los modelos planteados anteriormente. Esta situación evidencia cierta falta de coherencia en los planteamientos metodológicos en esta área considerando las actuales tendencias en el área de la Educación hacia la aplicación de técnicas estadísticas multivariantes. No obstante, De Miguel et al. plantean el análisis de las relaciones entre los cuatro elementos fundamentales del modelo de Biggs: las competencias a alcanzar por los alumnos, las modalidades u escenarios organizativos a tener en cuenta para realizar los procesos, los métodos concretos a utilizar a la hora de desarrollar las actividades de enseñanza-aprendizaje y las estrategias de evaluación que se estiman oportunas para verificar si los alumnos alcanzan los aprendizajes previstos. En su investigación se somete este modelo a un contraste empírico de expertos, así como a la consulta externa de profesores implicados en procesos de renovación metodológica con el fin de detectar las interacciones más comunes que se establecen entre los diversos elementos del modelo. Esta investigación constituye uno de los pocos estudios sobre los procesos de enseñanza - aprendizaje realizados en base a una metodología cuantitativa, y no exclusivamente teórica.

La Tabla 3.5 muestra uno de los principales resultados obtenidos en esta investigación, en forma de matriz de relaciones entre distintas modalidades de enseñanza y competencias. Cada una de las celdas de esta tabla muestra la moda de las valoraciones de los expertos a las distintas relaciones. En ella se observa que tanto el Estudio y trabajo en grupo como los Seminarios y talleres son las modalidades con mayor peso (20 y 19 puntos, respectivamente) mientras que las Clases Teóricas constituye la modalidad con peso mínimo (10 puntos).

Sobre las valoraciones de los expertos a las relaciones entre modalidades de enseñanza y competencias se ha señalado que corresponden a estudios genéricos, que en cualquier caso deberían matizarse en función del área de estudio concreta de la titulación considerada. 


\begin{tabular}{|c|c|c|c|c|c|c|c|c|}
\hline & & \begin{tabular}{|c|} 
Clases \\
teóricas
\end{tabular} & Seminarios & \begin{tabular}{|c|} 
Clases \\
prácticas
\end{tabular} & \begin{tabular}{|c|} 
Prácticas \\
externas
\end{tabular} & Tutorías & $\begin{array}{l}\text { Estudio } \\
\text { en Grupo }\end{array}$ & \begin{tabular}{|c|} 
Estudio \\
Individual
\end{tabular} \\
\hline \multirow{3}{*}{ Conocimientos } & Generales & \begin{tabular}{|l|}
2 \\
\end{tabular} & 2 & \begin{tabular}{|l|}
2 \\
\end{tabular} & 1 & 1 & 2 & 3 \\
\hline & Académicos & 3 & 2 & 2 & 1 & 2 & 2 & 3 \\
\hline & Profesionales & 1 & 2 & 3 & 3 & 2 & 2 & 1 \\
\hline \multirow{4}{*}{ Habilidades } & Intelectuales & 2 & 2 & 2 & 1 & 1 & 2 & 3 \\
\hline & Comunicación & 0 & 3 & 2 & 2 & 2 & 3 & 1 \\
\hline & Interpersonales & 0 & 2 & 2 & 2 & 2 & 3 & 0 \\
\hline & $\begin{array}{l}\text { Organización } \\
\text { personal }\end{array}$ & 0 & 2 & 2 & 3 & 2 & 2 & 2 \\
\hline \multirow{2}{*}{ Actitudes } & $\begin{array}{l}\text { Desarrollo } \\
\text { profesional }\end{array}$ & 1 & 2 & 2 & 3 & 2 & 2 & 1 \\
\hline & $\begin{array}{l}\text { Compromiso } \\
\text { profesional }\end{array}$ & 1 & 2 & 1 & 2 & 2 & 2 & 2 \\
\hline
\end{tabular}

Tabla 3.5: Correspondencia entre Competencias y Modalidades organizativas

\subsection{Proyectos de investigación internacionales so- bre adquisición de competencias en la Univer- sidad}

A continuación se describen brevemente los fundamentos de tres proyectos de investigación internacionales sobre adquisición de competencias en la Universidad. El Proyecto "Tuning Educational Structures in Europe" (TUNING), y los Proyectos "Careers After Higher Education - An European Research Study" (CHEERS) y "El Profesional Flexible en la Sociedad del Conocimiento: Nuevas Exigencias en la Educación Superior en Europa" (REFLEX). Ambos proyectos están basados en estudios de seguimiento de egresados, cuya importancia se ha incrementado notablemente en los últimos años como medio para cuantificar y evaluar la eficacia de los sistemas de gestión de las universidades (Conchado y Carot, 2009).

\subsubsection{El Proyecto "Tuning Educational Structures in Euro- pe" (TUNING)}

El proyecto TUNING "Tuning Educational Structures in Europe" fue financiado por la Comisión Europea en el marco del Programa Sócrates y dirigido por la Universidad de Deusto en España en colaboración con la Universidad de Groningen de los Países Bajos (González y Wagenaar, 2005).

El objetivo principal de este proyecto fue ofrecer un planteamiento concreto que posibilitara la aplicación del proceso de Bolonia en el ámbito de las disciplinas o áreas de estudio y en el de las Instituciones de Educación Superior. El enfoque Tuning se basa en una metodología con la que volver a diseñar, desarrollar, aplicar y evaluar los programas de estudio de cada uno de los ciclos del proceso de Bolonia.

La metodología del proyecto se ha diseñado de forma que facilite la com- 
prensión de los planes de estudio y su mutua comparación. Para organizar el análisis de las áreas de conocimiento se han planteado los siguientes enfoques:

- Competencias genéricas (Académicas de carácter general)

- Competencias específicas de cada área

- La función de ECTS como un sistema de acumulación

- Enfoques de aprendizaje, enseñanza y evaluación

- La función de la promoción de la calidad en el proceso educativo

Las tres primeras líneas de trabajo se desarrollaron durante la primera fase del proyecto $(2001 / 02)$ mientras que la cuarta y la quinta tuvieron una importancia clave en la segunda fase (2003/04). Durante la tercera fase se analizaron los estudios de Tercer ciclo (Posgrado y Doctorado) así como las estrategias necesarias para implantar el planteamiento Tuning en Instituciones de Educación Superior.

En la primera fase del proyecto se realizó una encuesta a gran escala a graduados, empleadores y profesores universitarios con el fin de identificar las competencias genéricas más relevantes de cada área de estudio. Los resultados mostraron que las competencias más importantes en algunos campos se consideraban irrelevantes en otros. A pesar de esto fue posible verificar la similitud en las respuestas obtenidas en las distintas áreas ya que en todas ellas se identificaron las competencias académicas tradicionales como las más importantes. Otras competencias genéricas fueron valoradas como muy importantes para la búsqueda de empleo. Entre ellas figuraban las competencias relacionadas con la aplicación práctica de los conocimientos, el trabajo en equipo y la comunicación.

Por otro lado, se identificaron las competencias específicas más relevantes en distintas áreas de estudio. Se analizaron las competencias específicas de titulaciones como Administración de Empresas, Química, Ciencias de la Educación, Estudios Europeos, Historia, Geología, Matemáticas, Enfermería y Física. Puesto que la estructura de estos estudios difiere por su naturaleza, es posible identificar diferencias en los planteamientos de cada análisis. No obstante todos siguen un procedimiento similar para la obtención de resultados y los documentos resultantes se han presentado como meros documentos de trabajo, sujetos a posibles revisiones y modificaciones a posteriori.

Este nuevo enfoque en base a las competencias es una de las novedades introducidas en el proyecto. En Tuning las competencias se describen como puntos de referencia para el diseño y la evaluación de los planes de estudio 
y no como rígidas estructuras. De esta forma se garantiza la flexibilidad y autonomía en el diseño de los planes de estudio así como la posibilidad de utilizar un lenguaje común mediante el cual poder describir sus objetivos. Una aplicación de la tipología de competencias propuesta en este proyecto puede encontrarse en Rodríguez y Vieira (2009) sobre una muestra de 1358 graduados en la Universidad entre los años 1997 y 2000.

Por otro lado cabe destacar el enfoque integrado que, desde el proyecto, se atribuye al concepto de competencia. Estas se entienden no sólo como conocer y comprender (conocimiento teórico de un campo académico) sino también como saber cómo actuar (aplicación práctica y operativa del conocimiento en ciertas situaciones) y saber cómo ser (valores como parte integrante de la forma de percibir a los otros y vivir un contexto social). Por tanto las competencias representan una combinación de atributos que describen el nivel o grado de suficiencia con que una persona es capaz de desempeñarlos.

Durante el proyecto se elaboraron dos cuestionarios: uno para la identificación de las competencias genéricas y su valoración por graduados y empleadores y otro para la evaluación por los académicos. Con el fin de preparar estos cuestionarios se elaboró una lista de 85 competencias diferentes seleccionadas por compañías privadas e instituciones de educación superior. Estas competencias se clasificaron posteriormente en tres grupos: instrumentales, interpersonales y sistémicas:

Competencias instrumentales: Competencias que tienen una función instrumental

Habilidades cognoscitivas: Capacidad de comprender y manipular ideas y pensamientos

Capacidades metodológicas para manipular el ambiente: Ser capaz de organizar el tiempo y las estrategias para el aprendizaje, tomar decisiones o resolver problemas

Destrezas tecnológicas relacionadas con el uso de maquinaria, computación y gerencia de la información

Destrezas lingüísticas como la comunicación oral y escrita o el conocimiento de una segunda lengua

Competencias interpersonales: Capacidades individuales relativas a la capacidad de expresar los propios sentimientos, habilidades críticas y de autocrítica así como destrezas sociales relacionadas con la capacidad de trabajar en equipo o la expresión de compromiso social o ético 
Competencias sistémicas: Son las destrezas y habilidades que conciernen a los sistemas como totalidad. Implican una combinación de la comprensión, la sensibilidad y el conocimiento que permiten al individuo ver cómo las partes de un todo se relacionan y se agrupan

El cuestionario definitivo contenía las siguientes 30 competencias:

1. Capacidad de análisis y síntesis

2. Capacidad de organizar y planificar

3. Conocimientos generales básicos

4. Conocimientos básicos de la profesión

5. Comunicación oral y escrita en la propia lengua

6. Conocimiento de una segunda lengua

7. Habilidades básicas de manejo del ordenador

8. Habilidades de gestión de la información

9. Resolución de problemas

10. Toma de decisiones

11. Capacidad crítica y autocrítica

12. Trabajo en equipo

13. Habilidades interpersonales

14. Capacidad de trabajar en un equipo interdisciplinar

15. Capacidad para comunicarse con expertos de otras áreas

16. Apreciación de la diversidad y multiculturalidad

17. Habilidad de trabajar en un contexto internacional

18. Compromiso ético

19. Capacidad de aplicar los conocimientos en la práctica

20. Habilidades de investigación

21. Capacidad de aprender

22. Capacidad para adaptarse a nuevas situaciones

23. Capacidad para generar nuevas ideas (creatividad)

24. Liderazgo

25. Conocimiento de culturas y costumbres de otros países

26. Habilidad para trabajar de forma autónoma 
27. Diseño y gestión de proyectos

28. Iniciativa y espíritu emprendedor

29. Preocupación por la calidad

30. Motivación de logro

\subsubsection{El Proyecto "Careers After Higher Education - An European Research Study" (CHEERS)}

El Proyecto CHEERS "Careers After Higher Education - An European Research Study" se desarrolló entre los años 1998 y 2000 y se fundamentó en las respuestas de más de 36,000 graduados universitarios. En él participaron universidades de Austria, República Checa, Finlandia, Francia, Alemania, Italia, Países Bajos, Noruega, España, Suecia, Reino Unido y Japón. Los graduados seleccionados obtuvieron la titulación durante el año académico 1994/95 por lo que en el momento de la entrevista habían transcurrido cuatro años desde su graduación. Los encuestados respondieron a diversas preguntas relativas a su entorno socioeconómico, trayectoria profesional, transición de la universidad al mercado laboral, primeros años de trabajo y relación entre los estudios y el empleo. Igualmente se les preguntó por el nivel de satisfacción con su trabajo y la evaluación de sus estudios de educación superior.

El objetivo fundamental del proyecto se plantea como el análisis de la experiencia en el mercado laboral de los graduados en Instituciones de Educación Superior durante los primeros años después de la graduación. Este objetivo genérico puede desglosarse en el análisis y estudio de las siguientes cuestiones específicas sobre los graduados universitarios:

- Cuestiones planteadas recientemente relacionadas con la Educación Superior y el Mercado Laboral

- Entorno socio-biográfico y carreras profesionales

- Movilidad internacional

- Identificación de las carreras profesionales de los graduados durante los primeros años

- Identificación del impacto de la Educación Superior

- Mejoras teóricas y metodológicas

- Estudio preliminar para la creación de una base de datos sobre la empleabilidad de los graduados universitarios en Europa 
La principal herramienta de trabajo fue un cuestionario estándar enviado a cada uno de los graduados seleccionados entre la población de estudio. Mediante esta herramienta fue posible encuestar a un gran número de graduados de cada país, así como obtener información representativa de la situación de los graduados en el mercado laboral. El desarrollo del cuestionario se basó en parte en los enfoques y experiencias de investigaciones precedentes en países europeos y también en nuevas cuestiones y planteamientos novedosos.

El cuestionario se dividió en los siguientes apartados:

1. Historial académico previo al ingreso en la universidad

2. Carreras universitarias

3. Búsqueda de trabajo e historial de actividades profesionales

4. Trabajo actual

5. Competencias profesionales y su aplicación

6. Relaciones entre la educación superior y el trabajo actual

7. Valoración y satisfacción del trabajo

8. Ampliación de estudios y formación

9. Datos socio-biográficos

El apartado relativo a las competencias y su aplicación es el más relevante para este estudio. En este apartado se pedía al encuestado que indicara hasta qué punto poseía cada una de las competencias mostradas a continuación. Esta evaluación debía referirse tanto al momento de la graduación en 1994/95 como las requeridas en el actual trabajo del encuestado en el momento de la entrevista (1998/99). Las competencias incluidas en el cuestionario eran:

\section{Habilidad para resolver problemas}

2. Capacidad de análisis

3. Habilidad para el aprendizaje

4. Capacidad reflexiva sobre su propio trabajo

5. Creatividad

6. Trabajar bajo presión

7. Exactitud, atención al detalle

8. Administración del tiempo 
9. Capacidad de negociación

10. Adecuación física al puesto de trabajo

11. Habilidades manuales

12. Trabajar independientemente

13. Trabajar en equipo

14. Iniciativa

15. Adaptabilidad

16. Firmeza, resolución, persistencia

17. Poder de concentración

18. Predisposición a involucrarse personalmente en el trabajo

19. Lealtad, honestidad

20. Pensamiento crítico

21. Habilidad en comunicación oral

22. Habilidad en comunicación escrita

23. Tolerancia, capacidad de apreciar los diferentes puntos de vista

24. Capacidad de liderazgo

25. Asumir responsabilidades, tomar decisiones

Los resultados revelaron una marcada diversidad entre los diferentes países en las cuestiones referentes a la Educación Superior y a los vínculos entre la Universidad y los puestos de trabajo que ocupan los graduados universitarios (Schomburg y Teichler, 2006).

\subsubsection{El Proyecto "El Profesional Flexible en la Sociedad del Conocimiento: Nuevas Exigencias en la Educación Superior en Europa" (REFLEX)}

El proyecto de investigación REFLEX "El Profesional Flexible en la Sociedad del Conocimiento: Nuevas Exigencias en la Educación Superior en Europa" más conocido como proyecto REFLEX es una iniciativa que forma parte del Sexto Programa Marco de la Unión Europea (Contrato $N^{\circ}$ : CIT2-CT-2004-506-352). En él han participado universidades de los siguientes países: Alemania, Austria, España, Finlandia, Francia, Italia, Noruega, Países Bajos, Reino Unido, Bélgica, República Checa, Portugal, Suiza, Japón y Estonia. A partir de la encuesta realizada en el marco de este proyecto se obtuvo una base de datos internacional con un total de 40.787 registros 
(Allen y Van der Velden, 2011). En la actualidad se está implantando la metodología del proyecto en Latinoamérica y Europa Oriental. La aplicación del proyecto REFLEX en Latinoamérica se realiza a través del Proyecto PROFLEX en el cual participan universidades de Chile, México, Colombia, Argentina, Puerto Rico, Honduras, Brasil, Bolivia y Uruguay (Mora, Carot y Conchado, 2007) mientras que el proyecto HEGESCO se basa en la implantación de la metodología del proyecto en Eslovenia, Hungría, Polonia, Lituania y Turquía (Allen y Van der Velden, 2009; Pavlin, 2009).

El proyecto intenta dar respuesta a tres cuestiones generales e interrelacionadas mediante aplicación de un cuestionario a los graduados universitarios que obtuvieron la titulación cinco años antes del momento de la entrevista. Estas cuestiones se enumeran a continuación:

- Las competencias que requieren los egresados para funcionar adecuadamente en la sociedad del conocimiento

- El papel de los centros de educación superior para ayudar a los graduados universitarios a desarrollar las competencias

- Las tensiones que surgen a medida que graduados universitarios, centros de educación superior, empresarios y otras figuras importantes se esfuerzan en alcanzar sus propios objetivos y formas de resolverlas

Esta selección se realizó considerando que la carrera profesional de cualquier graduado universitario sigue un patrón bien definido durante los primeros diez años. En primer lugar el graduado pasa por una fase inicial de transición desde la universidad al mercado laboral centralizada en la búsqueda de trabajo y la integración en el primer empleo. A continuación la segunda fase se asocian con el desarrollo de la experiencia profesional y la definición específica de la carrera profesional. Finalmente los graduados comienzan a asumir mayor responsabilidad durante la tercera fase debido a al elevado nivel de conocimientos y experiencia adquiridos durante las fases anteriores. Los puntos de transición entre la primera y segunda fase se establecieron entre el primer y segundo año después de la graduación mientras que la transición entre la segunda y la tercera fase se ubicó cinco años después de la graduación. Los coordinadores del proyecto acordaron que el momento más adecuado para realizar la entrevista debería corresponderse aproximadamente con las transiciones entre una y otra fase. En estos momentos se espera que los graduados tiendan a la reflexión sobre las experiencias pasadas y sus posibilidades futuras, así como sobre sus expectativas sobre la siguiente fase.

Puesto que la encuesta se realizó durante los años 2005 y 2006 los graduados seleccionados corresponden a la promoción del 1999/2000. Por tanto 
la población de estudio se definió como: Graduados universitarios que obtuvieron la titulación durante el curso académico 1999/2000 correspondiente al nivel ISCED 5A. En el caso de España este filtro se aplicó a todos los graduados universitarios que obtuvieron los títulos de Diplomatura, Ingeniería, Arquitectura Técnica, Licenciados, Arquitectos e Ingenieros. No se incluyeron los Doctorados ni otros tipos de Posgrado.

La principal herramienta de trabajo fue igualmente un cuestionario estándar que se podía completarse mediante tres tipos de entrevistas: online, por correo postal o entrevista telefónica. El cuestionario constaba de varios apartados referidos principalmente a la experiencia educativa del graduado durante la universidad así como su experiencia de transición al mercado laboral y características de su trabajo actual (Mora et al., 2007a).

- A - Carrera en la que te graduaste en 1999/2000

- B - Otras experiencias en el ámbito educativo

- $C$ - Transición de los estudios al trabajo

- D - Primer trabajo después de la graduación

- E - Historia laboral y situación actual

- F - Trabajo actual

- G - Organización en la que trabajas

- $H$ - Competencias

- I - Evaluación de la carrera

- $J$ - Valores y orientaciones

- $K$ - Información personal

La muestra mínima nacional se fijó en 2000 registros aunque se redefinió posteriormente en función de las tasas de respuesta de cada país. Para incrementar la eficiencia de la muestra se realizó un muestreo estratificado bietápico. Cada país seleccionó los estratos más adecuados a su contexto de trabajo pero básicamente se incluyeron categorías como tipo de grado, área de a estudio, región, etc. No obstante, a pesar de haber diseñado con detalle la muestra para cada país se aplicó una ponderación a los datos obtenidos para compensar las diferencias debidas a bajas tasas de respuesta. 


\section{Capítulo 4}

\section{El contexto académico y personal de los estudiantes de Educación Superior}

\subsection{Introducción}

Todos los procesos de enseñanza - aprendizaje constituyen un complejo sistema de factores que finalmente conducen a un determinado resultado académico. En el contexto de la Educación Superior estos sistemas adquieren todavía mayor complejidad en el sentido de que se incrementa en mayor medida el número de factores potencialmente influyentes en los resultados del aprendizaje, ya sea desde el entorno académico del estudiante o desde el personal. Evidentemente el efecto que estos factores tienen sobre el rendimiento de los estudiantes se produce de forma conjunta y simultánea, por lo que deben ser estudiados de forma conjunta y desde una perspectiva integradora, tal y como se expone en el capítulo 5.

No obstante para una adecuada comprensión de las relaciones que se producen en estos sistemas es necesario conocer las relaciones que se establecen entre los factores a nivel individual, tanto en lo que se refiere a la existencia de relación como a su intensidad. Asimismo el análisis de las relaciones de interacción y confusión entre variables permiten identificar posibles variables modificadoras del efecto, como ocurre en este capítulo con el Área de estudio. Por esta razón el objetivo de este capítulo es analizar las relaciones que se establecen entre variables y factores a nivel individual, así como en presencia de posibles variables modificadoras del efecto, causantes de relaciones de interacción y confusión entre variables. 


\subsection{Material y métodos}

Existen muy diversas técnicas estadísticas para abordar el objetivo planteado para este capítulo. Entre todas ellas se ha seleccionado el análisis factorial exploratorio mediante componentes principales y los valores-test asociados, y se han empleado técnicas de análisis de datos cualitativos como los contrastes de independencia, las medidas de asociación y el análisis de correspondencias múltiples.

\subsubsection{Análisis Factorial Exploratorio}

El análisis factorial es un método de análisis multivariante que intenta explicar, según un modelo lineal, un conjunto extenso de variables observables mediante un número reducido de variables hipotéticas, latentes o no observadas llamadas factores (Cuadras, 1991; Peña, 2002).

El objetivo principal del análisis factorial es la determinación de m nuevas variables llamadas factores que expliquen las $X_{1}, X_{2}, \ldots, X_{n}$ variables observables del modelo de forma que el número $m$ de nuevas variables sea inferior al número $n$ de variables observables del modelo. Entre estos factores se pueden distinguir dos tipos.

En primer lugar los $F_{1}, F_{2}, \ldots, F_{n}$ factores comunes que influyen en común sobre las $n$ variables observables. Asimismo los $U_{1}, U_{2}, \ldots, U_{n}$ factores únicos que influyen exclusivamente sobre cada una de las variables de forma individual.

$$
\begin{gathered}
X_{1}=a_{1} 1 \cdot F_{1}+\ldots+a_{1} m \cdot F_{m}+d_{1} \cdot U_{1} \\
X_{2}=a_{2} 1 \cdot F_{1}+\ldots+a_{2} m \cdot F_{m}+d_{2} \cdot U_{2} \\
\ldots \\
X_{n}=a_{n} 1 \cdot F_{1}+\ldots+a_{n} m \cdot F_{m}+d_{n} \cdot U_{n}
\end{gathered}
$$

El modelo factorial supone que el conjunto de las variables observables depende de los factores comunes con excepción de una parte de su variabilidad que sólo puede ser explicada por el factor único correspondiente.

El coeficiente $a_{i j}$, que recibe el nombre de Saturación, es el coeficiente de correlación entre $X_{i}$ y el factor común $j$ mientras que el coeficiente $a_{i j}^{2}$ se denomina Comunalidad. Este coeficiente indica la proporción de variabilidad de la variable $i$ que queda explicada por el factor correspondiente.

Estas relaciones pueden expresarse en notación matricial de la siguiente forma: 


$$
X=A \cdot F+D \cdot U
$$

Donde $A$ es la matriz de cargas factoriales, $F$ y $U$ los vectores columna que contienen los factores comunes y únicos, $D$ es la matriz diagonal con las saturaciones de los factores únicos y $X$ el vector que contiene las variables observables.

Los factores comunes son los únicos que tienen interés desde la perspectiva del investigador y además son susceptibles de interpretación experimental. La única razón por la cual se incluyen los factores únicos en el modelo es la imposibilidad de expresar $\mathrm{n}$ variables en función de un número reducido $m$ de factores comunes.

\section{Comunalidades}

Así, según Cuadras (2006), la variabilidad de cada variable observable $X_{i}$ se expresa como:

$$
\operatorname{var}\left(X_{i}\right)=a_{i 1}^{2}+a_{i 2}^{2}+a_{i m}^{2}+d_{i}^{2}
$$

Puesto que cada uno de los coeficientes $a_{i j}^{2}$ expresa la comunalidad de cada factor respecto a la variable $X_{i}$, entonces la comunalidad de la variable $X_{i}$ se define como la cantidad:

$$
h_{i}^{2}=a_{i 1}^{2}+a_{i 2}^{2}+a_{i m}^{2}
$$

La cantidad $d_{i}^{2}$ recibe el nombre de Unicidad y posibilita que la variabilidad de cada variable se exprese como la suma de su comunalidad y unicidad correspondiente.

Si las variables observables son reducidas entonces:

$$
1=h_{i}^{2}+d_{i}^{2}
$$

\section{Indeterminación del modelo factorial}

Los coeficientes de la matriz de cargas factoriales expresan la relación entre las variables y los factores comunes. Tal como señala Peña (2002), esta matriz se obtiene a partir de los coeficientes de correlación entre variables de la forma:

$$
R^{*}=A \cdot A^{\prime}
$$

Siendo $R^{*}$ la matriz de correlaciones reducida obtenida sustituyendo los unos de la diagonal principal de la matriz de correlaciones $\mathrm{R}$ por las comunalidades de las variables. Esta expresión recibe el nombre de Identidad 


\section{Fundamental.}

Pero esta relación es insuficiente en sí misma ya que hay infinitas matrices que la cumplen siendo soluciones válidas debido al problema de indeterminación del modelo factorial. Dicho problema se basa en que, puesto que no son observables ni la matriz de cargas factoriales ni los factores comunes, dos representaciones serán equivalentes si:

$$
A \cdot F=A^{\prime} \cdot F^{\prime}
$$

El hecho de que el número de ecuaciones sea inferior al número de cargas factoriales implica un problema general de indeterminación (Torrens-Ibern, 1972), lo cual conduce a dos indeterminaciones específicas. La primera de ellas es que un conjunto de datos puede expresarse con la misma precisión con factores correlados e incorrelados. Asimismo, los factores no están determinados de forma única.

La indeterminación de los modelos factoriales es inherente a los mismos. No debe considerarse como una característica negativa, ya que permite considerar ciertas condiciones que de otra forma no podrían reducirse a simples restricciones cuantitativas. La solución para poder estimar esta matriz $A$ es la imposición de restricciones. En función del tipo de restricción seleccionaremos diferentes métodos para la obtención de la matriz factorial $A$. Entre ellos el método de Componentes Principales prescinde de los factores únicos de cada variable y elige cada uno de los factores o componentes de modo que cada uno de ellos explique la mayor parte de la varianza de las variables.

\section{Rotación}

El análisis factorial proporciona la base del espacio de las columnas de la matriz de carga. Sin embargo, debido al problema de la indeterminación de esta matriz, es posible transformar esta base en otra del mismo espacio de forma que se facilite la interpretación del análisis. Esta transformación puede realizarse mediante el producto de la solución inicial por matrices ortogonales que equivalen a rotaciones. La facilidad de interpretación de los factores depende principalmente del grado de asociación entre los factores y las variables observadas. Si las columnas de la matriz de cargas factoriales contienen valores altos para determinadas variables y bajos para otras será fácil identificar el efecto de cada factor sobre las variables (Peña, 2002).

Los coeficientes de la matriz ortogonal que definen la rotación pueden obtenerse mediante la verificación de determinadas condiciones analíticas relativas al concepto de Estructura Simple de Thurstone (1931, 1935). Estas condiciones se obtienen mediante la minimización de una función objetivo 
que expresa la simplicidad de la representación tras la rotación.

Así se plantea el siguiente problema: dada la matriz de cargas factoriales $A$, hallar la matriz ortogonal $T$ de forma que:

$$
B=A \cdot T
$$

Sea $B$ la matriz de cargas factoriales formada por nuevos factores ortogonales y que verifica las condiciones fundamentales de estructura simple.

El método varimax expuesto por Kaiser es una modificación del criterio cuartimax que se aproxima mejor a la estructura simple. Kaiser define la simplicidad de un factor como la varianza de los cuadrados de sus cargas o saturaciones en las variables observables (Kaiser, 1958). La simplicidad del factor $F_{i}$ se define como:

$$
s_{i}^{2}=\frac{1}{n} \sum_{j=1}^{n}\left(a_{j i}^{2}\right)^{2}-\frac{1}{n^{2}}\left(\sum_{j=1}^{n} a_{j i}^{2}\right)^{2}
$$

Una vez definida la medida de simplicidad sobre un factor, Kaiser propone calcular $B=A \cdot T$ de forma que se maximice la suma de las simplicidades de todos los factores. El objetivo de este criterio es forzar a la matriz $B$ a tener cargas próximas a la unidad en detrimento de otras próximas a cero. La ventaja de este criterio de rotación es que no sólo obtiene una nueva matriz cuyos vectores son ortogonales sino que no varía los valores de las comunalidades.

\subsubsection{Análisis de Componentes Principales}

\section{Consideraciones generales}

El objetivo del análisis de componentes principales se fundamenta en la reducción de la dimensionalidad de un conjunto de $p$ variables a otro formado por un número $q$ menor de variables construidas como combinaciones lineales de las originales. Estas nuevas variables tienen la propiedad de explicar, por turnos, la máxima cantidad de varianza de las variables observables (Lebart, Morineau y Piron, 1995).

Como se ha expuesto anteriormente, el análisis factorial de forma genérica se basa en el modelo:

$$
X=A \cdot F+D \cdot U
$$

Existen varios métodos para obtener la matriz factorial $A$. Esta matriz verifica la relación $R^{*}=A \cdot A^{\prime}$, que es insuficiente en sí misma para deter- 
minarla ya que, por la propia indeterminación del modelo factorial, existen infinitas matrices que cumplen esta condición. Es necesario imponer otra condición para ello. Según el criterio seleccionado para definir esta condición obtendremos diversos métodos de extracción de factores.

Entre estos métodos el análisis de componentes principales se limita al estudio de los factores comunes prescindiendo de la influencia de los factores únicos:

$$
X=A \cdot F
$$

Las componentes principales se construyen para explicar las varianzas de las variables en contraste con el análisis factorial diseñado para explicar las covarianzas o correlaciones entre variables (Peña, 2002). Este análisis consiste en elegir $F_{1}$ (primera componente principal) de forma que explique la mayor parte de la varianza de las variables. Seguidamente se le resta a las variables esta primera componente $\mathrm{y}$, sobre la variabilidad restante, se elige $F_{2}$ (segunda componente principal) con el mismo criterio y así sucesivamente. Este procedimiento equivale al cálculo de los valores y vectores propios de la matriz de correlaciones (Cuadras, 1991). Este método se basa en el trabajo de Pearson (1901) con las adaptaciones específicas al análisis factorial sugeridas por Hotelling (1933).

\section{Factor tamaño}

En ocasiones al representar el conjunto de variables activas puede observarse que todas las variables se encuentran representadas a un mismo sector de los ejes factoriales. Esto es debido a que existe una fuerte correlación entre todas las variables de forma que, si para un determinado individuo una variable toma un valor elevado, ocurre lo mismo para todas las demás. Esta característica se da con mayor frecuencia en el primer eje factorial, y en tal caso se denomina como Factor Tamaño (Escofier y Pagès, 1992; Lebart et al., 1995).

La causa más común del factor tamaño es una elección inadecuada de las unidades en que se han medido las variables. La forma de eliminar el factor tamaño se basa en centrar la variable de la forma que se considere más adecuada. Aluja propone dividir el rango de la variable por su valor medio (Aluja y Morineau, 1999):

$$
x^{\prime}=\frac{x_{\max }-x_{\min }}{\bar{x}}
$$

Mientras que Erkel-Rousse (1995) propone simplemente calcular la diferencia entre la variable observada y su media de forma que se elimine el 
efecto más predominante .

$$
x^{\prime}=x-\bar{x}
$$

\section{Proyecciones de los puntos - individuo}

Existen dos formas de representar geométricamente un conjunto de $n$ valores de dos variables $x_{j}$ y $x_{k}$ en el espacio: mediante representación puntual y vectorial (Harman, 1980). El caso de la representación puntual se denomina también Diagrama de Dispersión y por medio de esta representación es posible representar por pares cada una de las $\mathrm{n}$ coordenadas correspondientes a cada variable: $\left(x_{j 1}, x_{k 1}\right),\left(x_{j 2}, x_{k 2}\right), \ldots,\left(x_{j n}, x_{k n}\right)$. En el caso de la representación vectorial las dos variables se representan por los vectores: $x_{j}:\left(x_{j 1}, x_{j 2}, \ldots, x_{j n}\right)$ y $x_{k}:\left(x_{k 1}, x_{k 2}, \ldots, x_{k n}\right)$.

Preferentemente se utilizará en este trabajo la representación puntual mediante la cual es posible asignar un punto en el plano para cada uno de los $n$ individuos. Cada uno de estos puntos está referido a un sistema de $p$ ejes de referencia - uno para cada variable. En el caso de poblaciones distribuidas normalmente los lugares geométricos de la nube formada por el conjunto de todos los puntos son elipsoides de $p$ dimensiones concéntricas y homotéticas cuyos ejes principales se toman como ejes de referencia (Yule y Kendall, 1969).

En este caso se considera la nube de puntos-fila o nube de los individuos está formada por $n$ individuos (tantos como filas) representados en un espacio $R^{p}$ de $p$ dimensiones (tantas como variables activas).

Tal como formulan Lebart et al. (1995), las componentes principales son las variables compuestas tal que:

$$
\begin{gathered}
\Psi_{1}=X \cdot u_{1} \\
\Psi_{2}=X \cdot u_{2} \\
\cdots \\
\Psi_{q}=X \cdot u_{q}
\end{gathered}
$$

Donde $u_{1}, u_{2}, \ldots, u_{q}$ son vectores ortonormales del subespacio $R^{q}$ tales que:

- La varianza de $\Psi_{1}$ es máxima condicionada a la restricción $u_{1}^{\prime} \cdot u_{1}=1$ 
- Entre todas las variables $\Psi$ tales que $\operatorname{cov}\left(\Psi_{1}, \Psi\right)=0$ la variable $\Psi_{2}$ es tal que su varianza es máxima condicionado a la restricción $u_{2}^{\prime} \cdot u_{2}=1$. Esto significa que la segunda componente principal está incorrelada con la primera y a su vez contribuye a un máximo de la varianza residual.

- $\Psi_{3}$ es una variable incorrelada con $\Psi_{1}$ y $\Psi_{2}$ con varianza máxima. Análogamente se define el resto de componentes principales

- El resto de variables se agregan sucesivamente hasta que finalmente es analizada la varianza total. La suma de la varianzas explicadas por las $n$ componentes principales es igual a la suma de las varianzas de las variables originales (Cuadras, 2006).

Si $U$ es la matriz $q x q$ :

$$
U=\left[u_{1}, u_{2}, \ldots, u_{\alpha}, \ldots, u_{q}\right]
$$

cuyas columnas contienen los vectores que definen las componentes principales entonces la transformación:

$$
\Psi_{\alpha}=X \cdot u_{\alpha}
$$

Se denomina transformación por componentes principales.

El subespacio de $q$ dimensiones que mejor se ajusta al espacio de $p$ dimensiones se genera por los q primeros vectores propios de la matriz simétrica $X^{\prime} \cdot X$ correspondientes a $\operatorname{los} q$ mayores valores propios. Por tanto para el cálculo de los vectores $u_{\alpha}$ se diagonalizará la matriz $X^{\prime} X$. Para el caso de un PCA normalizado esta matriz es igual a la matriz de correlaciones entre variables. Los vectores unitarios asociados a los mayores valores propios dan las direcciones de máxima inercia y se denominan ejes factoriales (Aluja y Morineau, 1999).

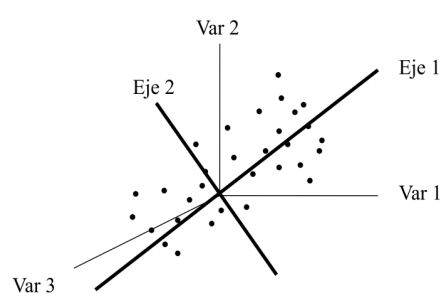

Figura 4.1: Subespacio engendrado por los dos primeros vectores de la matriz simétrica $X^{\prime} X$ en el espacio $R^{p}$ 
Los valores propios asociados a cada uno de los vectores propios dan las inercias proyectadas sobre cada una de las direcciones.

Las coordenadas de los $n$ puntos-individuo sobre el eje factorial $u_{\alpha}(\alpha-$ ésimo vector propio de la matriz de correlaciones asociado al autovalor $\lambda_{\alpha}$ ) son las $n$ componentes del vector:

$$
\Psi_{\alpha}=u_{1} \cdot x_{1}+\ldots+u_{p} \cdot x_{p}
$$

Los valores propios asociados a cada uno de los vectores propios dan las inercias proyectadas sobre cada una de las direcciones. Por consiguiente la varianza de cada eje factorial es igual a su correspondiente valor propio:

$$
\operatorname{var}\left(\Psi_{\alpha}\right)=\lambda_{\alpha}
$$

Y la variación total es:

$$
\operatorname{tra}(S)=\sum_{i=1}^{p} \lambda_{i}
$$

Puesto que los vectores propios $u_{\alpha}$ se ordenan en la matriz $U$ en función del valor de cada uno de los valores propios asociados se puede suponer que:

$$
\lambda_{1} \geq \lambda_{2} \geq \ldots \geq \lambda_{p}
$$

Por tanto:

- $\Psi_{i}$ contribuye con la cantidad $\lambda_{i}$ a la variación total $\operatorname{tra}(S)$

- Si $q<p, \Psi_{1}, \Psi_{2}, \ldots, \Psi(q)$ contribuyen con la cantidad $\sum_{i=1}^{q} \lambda_{i}$ a la variación total $\operatorname{tra}(S)$

- Según Cuadras (2006), el porcentaje de variabilidad explicada por las q componentes principales es:

$$
P_{m}=100 \frac{\lambda_{1}+\ldots+\lambda_{q}}{\lambda_{1}+\ldots+\lambda_{p}}
$$

\section{Proyecciones de los puntos - variable}

La nube de puntos-columna o variables está formada por $p$ variables (tantas como columnas) representados en un espacio $R^{n}$ de $n$ dimensiones (una por cada individuo).

Para el análisis de los puntos-columna se diagonaliza la matriz $X^{\prime} \cdot X$ y al igual que antes las direcciones definidas por los vectores propios $\mathrm{v}$ de esta 


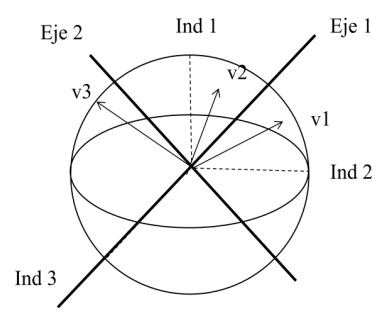

Figura 4.2: Subespacio engendrado por los dos primeros vectores de la matriz simétrica $X^{\prime} X$ en el espacio $R^{n}$

matriz son las de máxima inercia siendo la inercia proyectada sobre cada dirección igual a su valor propio asociado (Lebart et al., 1995).

La representación de las variables sobre cada uno de los ejes se obtiene proyectando cada uno de ellos sobre el vector unitario que define la dirección del eje. Así, la variable $j$ se proyecta sobre el eje $\alpha$ según la expresión:

$$
\varphi_{j \alpha}=\sum_{i=1}^{n} \frac{x_{i j}-\overline{x_{j}}}{s_{j}} \cdot v_{i j}
$$

Que en notación matricial equivale a:

$$
\varphi_{\alpha}=X^{\prime} \cdot v_{\alpha}
$$

Donde, como señalan Aluja y Morineau (1999), $\bar{x}_{j}$ es la media de la variable $j$ y la inercia sobre cada eje se calcula como:

$$
\sum_{j=1}^{p} \varphi_{j \alpha}^{2}=\lambda_{\alpha}
$$

\section{Relaciones de transición}

Entre los ejes factoriales de ambas nubes de puntos existen determinadas relaciones que permiten obtener las direcciones factoriales de un espacio conociendo previamente las del otro. Estas relaciones se denominan Relaciones de Transición. Por tanto en la práctica es suficiente con realizar un solo ajuste y obtener a partir de éste el otro. Puesto que la matriz de puntosfila tiene dimensiones más pequeñas generalmente ésta es la única matriz que se diagonaliza. La proyección de las variables en el espacio $R^{n}$ también se denomina matriz de componentes y se calcula posteriormente a partir de los vectores unitarios $u$ obtenidos en la diagonalización de la matriz de puntos-fila: 


$$
\varphi_{j \alpha}=\sqrt{\lambda_{\alpha}} \cdot u_{j \alpha}
$$

Igualmente conociendo los vectores unitarios v resultado de la diagonalización de la matriz de puntos-variable pueden obtenerse las proyecciones de los puntos-fila en el espacio $R^{p}$ también denominada Matriz de Puntuaciones Factoriales:

$$
\Psi_{j \alpha}=\sqrt{\lambda_{\alpha}} \cdot v_{j \alpha}
$$

Aunque resulta más habitual realizar el cálculo de las puntuaciones factoriales mediante la expresión:

$$
\Psi_{\alpha}=u_{1} \cdot x_{1}+\ldots+u_{p} \cdot x_{p}
$$

Que, según Lebart (1995), igualmente puede expresarse en notación matricial:

$$
\Psi_{\alpha}=X \cdot u_{\alpha}
$$

\section{Representación en la esfera de radio - unidad}

Todos los puntos-variable se encuentran situados a la distancia de la unidad del origen por lo que se considera que se ubican sobre una hiperesfera de radio unidad cuyo centro es el centro de gravedad de la nube de puntos. Después de diagonalizar la matriz $X X^{\prime}$ esta nube está representada por un conjunto de vectores con origen en el centro de la esfera y radio uno. El coeficiente de correlación entre dos variables coincide con el coseno del ángulo que forman sus respectivos vectores propios en el interior de la esfera.

Se puede definir una medida de distancia entre estas variables representadas por vectores en el interior de la esfera mediante la fórmula:

$$
d^{2}\left(j, j^{\prime}\right)=2\left(1-\operatorname{corr}\left(j, j^{\prime}\right)\right)
$$

Según esta expresión si dos variables tienen una correlación igual a la unidad la distancia que las separa es nula (están superpuestas) mientras que si su correlación toma el valor -1 entonces la distancia que las separa es máxima ya que encuentran en la misma dirección pero en sentidos contrarios en el interior de la esfera de radio unidad. Por el contrario si la correlación entre ambas variables es nula entonces los vectores asociados correspondientes son ortonormales (Aluja y Morineau, 1999). 


\section{Proyección de elementos suplementarios en el plano factorial}

\section{Individuos ilustrativos}

Los Elementos Ilustrativos son variables e individuos no incluidos en el análisis factorial pero cuyo posicionamiento en los planos factoriales resulta igualmente relevante por la información adicional que aportan (Lebart et al., 1995). Por el contrario, las variables e individuos que sí se consideran en el análisis factorial se denominan Elementos Activos. Tanto la información contenida en variables ilustrativas como individuos ilustrativos puede extraerse gracias a la técnica de Proyección en Suplementario.

La matriz de datos original $\mathrm{X}$, de dimensiones $n x p$, , puede completarse con otras dos matrices que contienen información complementaria. La matriz de variables suplementarias $X^{+}$tiene $n$ filas y $p s$ columnas aportando la información de $p s$ nuevas variables para los $n$ individuos anteriores. Por el contrario la información contenida la matriz de individuos $X^{+}$se refiere a las mismas $p$ variables activas pero referidas a un nuevo grupo de $n s$ individuos.

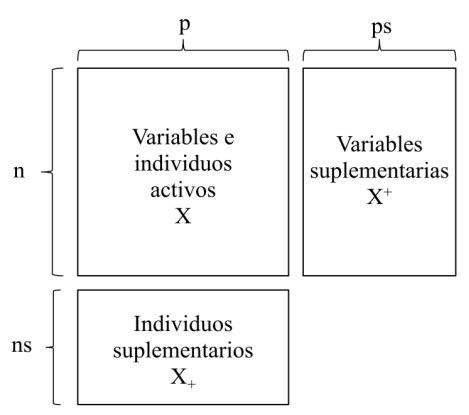

Figura 4.3: Distribución de variables e individuos activos y suplementarios en la base de datos original

El conjunto de los individuos suplementarios puede estar formado por una nueva muestra cuyas características resultan interesantes en relación con el resto de la muestra. Sin embargo en ocasiones los individuos suplementarios pueden representar determinadas características de referencia cuya posición respecto a los planos factoriales es interesante conocer en sí mismos.

La posición de las variables ilustrativas en el plano factorial permite visualizar la relación de éstas con el conjunto de variables activas a través de los ejes factoriales. Sin embargo no es posible definir distancias entre dos variables ilustrativas, o una variable activa y otra ilustrativa, ya que pueden 
estar medidas en diferentes unidades. En el caso de las variables activas sí se exigen determinadas condiciones de homogeneidad que posibilitan la comparación de distintas variables, pero no es necesario que estos requerimientos se apliquen a las variables ilustrativas. A pesar de esto, en la medida de que las estas variables estén bien representadas y se encuentren próximas entre sí, cabe esperar que la similitud de las correlaciones con los ejes sea consecuencia de fuertes correlaciones entre variables.

Tanto para individuos como para variables es necesario tipificar la matriz de datos con el fin de posicionarse en el centro de la nube de datos y homogeneizar las unidades en que se miden las variables.

\section{Individuos ilustrativos}

Las coordenadas de los nuevos individuos sobre el eje $\alpha$ son:

$$
\Psi_{+}^{\alpha}=X_{s} \cdot u_{\alpha}
$$

donde:

$$
X_{s}=\left(\begin{array}{c}
X \\
X_{+}
\end{array}\right)
$$

\section{Variables continuas ilustrativas}

Las coordenadas de las nuevas variables ilustrativas sobre el eje $\alpha$ se calculan de igual forma que las variables activas, es decir, como las componentes del vector:

$$
\varphi_{\alpha}^{+}=X^{\prime+} \cdot v_{\alpha}
$$

$\mathrm{Al}$ igual que ocurre con las variables activas cada una de las coordenadas se corresponde con el coeficiente de correlación entre la variable y el factor.

\section{Variables nominales ilustrativas}

En el caso de la variable sea nominal no es posible hacer la misma transformación sino que es preciso desagregar la matriz de variables activas en tantas matrices como categorías tenga la variable nominal. Así, cada matriz se corresponde con una determinada categoría de la variable nominal. De esta forma los valores de la matriz de variables activas se distribuyen entre las distintas matrices en función del valor que tome la variable nominal en cada caso. Seguidamente se calculan los promedios de las variables de cada una de las matrices y cada vector promedio se considera un nuevo individuo suplementario cuyas coordenadas se calculan como tal. 


\section{Representación simultánea}

Puesto que las nubes de individuos y variables se encuentran en dos subespacios distintos no tiene sentido representarlos simultáneamente mediante la superposición de los planos factoriales correspondientes. La nube de individuos se encuentra en el espacio $R^{p}$ cuyo origen es el centro de gravedad de los individuos y su base está formada por el conjunto de vectores $u_{\alpha}$. Por el contrario la nube de variables está en el espacio $R^{n}$ cuyo origen es el punto cero y la base de sus ejes factoriales está formada por el conjunto de vectores $v_{\alpha}$.

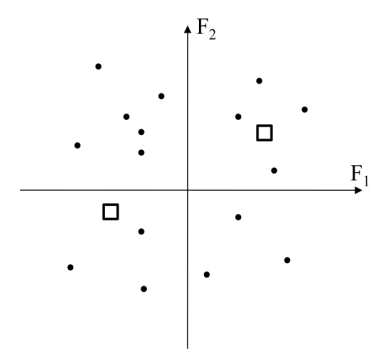

Figura 4.4: Nube de individuos en $R^{p}$. Representación de variables nominales ilustrativas

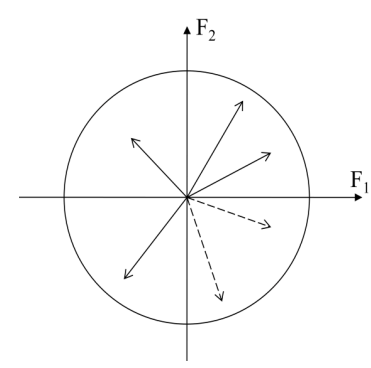

Figura 4.5: Nube de individuos en $R^{n}$. Representación de variables continuas ilustrativas

Sin embargo sí es posible representar simultáneamente la nube de individuos junto con los ejes de la antigua base $e_{j}$ de las variables originales. Cada antiguo eje $j$ que define la dirección de la j-ésima variable se considera como un nuevo ïndividuo. ${ }^{\text {en }}$ el espacio $R^{p}$. Este nuevo individuo tiene coordenada igual a la unidad en el j-ésimo eje y nula en el resto de los ejes. De esta forma es posible mostrar las direcciones de las $p$ variables situadas 
en el centro de la nube de individuos. Esta transformación equivale a una traslación del conjunto de las variables activas desde el punto cero hasta el centro de gravedad de la nube de individuos.

Los vectores que definen la base de las variables originales son:

$$
(1,0, \ldots, 0),(0,1, \ldots, 0), \ldots,(0,0, \ldots, 1)
$$

La proyección del vector $e_{j}$ sobre la nueva base definida por los $u_{\alpha}$ se define como:

$$
e_{j}^{\prime} \cdot u_{\alpha}=u_{j \alpha}
$$

Por lo que la proyección de los antiguos ejes factoriales, que proporciona las direcciones de las variables activas sobre la nueva base, está definida por los vectores propios $u$ resultantes del análisis de la nube de individuos. Por otro lado, puesto que sólo interesan las direcciones de las variables activas, los vectores asociados a éstas pueden dilatarse con el fin de facilitar la comprensión de la representación.

Esta representación de las direcciones de las variables activas difiere de la representación en el espacio de $R^{n}$. En la representación simultánea los vectores son las proyecciones de las variables ortogonales en los planos factoriales, mientras que en la representación en el espacio $R^{n}$ los ángulos entre distintas variables son una medida de la correlación entre ellas.

Asimismo en la representación en el espacio $R^{n}$ la coordenada de la variable activa $j$ sobre el eje $\alpha$ es:

$$
\varphi_{j \alpha}=\sqrt{\lambda_{\alpha}} \cdot u_{j \alpha}
$$

Mientras que la coordenada del antiguo eje unitario que representa la variable $j$ sobre el eje $\alpha$ en la representación simultánea se formula como:

$$
\varphi_{j \alpha}^{*}=u_{j \alpha}
$$

Por tanto esta expresión se utiliza simplemente como referencia ya que, como se ha indicado, las longitudes de los vectores que representan las direcciones de crecimiento de las variables activas en la representación simultánea se modifican habitualmente con el fin de facilitar la comprensión del gráfico (Aluja y Morineau, 1999).

En la representación simultánea no es posible la inclusión de variables continuas suplementarias como parte de la base de datos original para la nube de puntos - individuo. Sin embargo sí pueden incluirse variables nominales suplementarias ya que se consideran similares a individuos suplementarios 
(Lebart et al., 1995).

\section{Caracterización estadística de variables ilustrativas mediante valores - test}

El Valor - Test es un parámetro que permite evaluar en qué medida las categorías de una variable nominal presentan un comportamiento característico en el plano factorial. La proyección de cada categoría se interpreta como la posición promedio de todos los individuos que la integran. La proximidad de dicha posición al centro de gravedad de la nube de individuos sugiere cierta similitud entre los individuos que pertenecen a la categoría y el resto de la población. En cambio el hecho de que se interponga cierta distancia entre la posición promedio del grupo y el centro de gravedad indica un comportamiento claramente diferenciado del resto de la población. Así, el valor - test (v-test) permite definir la distancia en número de desviaciones típicas entre el centro de gravedad y la posición promedio de los individuos de cada categoría de la variable nominal (Aluja y Morineau, 1999).

Este concepto del valor test se plantea como las pruebas de hipótesis clásicas. En este caso la hipótesis nula $H_{0}$ se basa en la probabilidad crítica de la extracción al azar de los $n_{k}$ individuos con valores no nulos en la categoría de la variable nominal considerada. Estos individuos forman parte de una población total de $n$ elementos y la extracción se considera sin reposición puesto que cada valor corresponde a una y sólo una observación. Los elementos más característicos son aquellos con una probabilidad crítica menor, puesto que cuanto más débil es la probabilidad, más improbable resulta la hipótesis de extracción al azar.

\section{Caracterización de grupos según variables continuas ilustrati-} vas

Sea $m$ el promedio de las coordenadas del conjunto total $n$ de individuos y $s^{2}$ su varianza. Asimismo se define $m_{k}$ como el promedio de las coordenadas de los individuos de la categoría de estudio y $M_{k}$ la variable aleatoria Media de las $n_{k}$ extracciones.

Bajo la hipótesis nula anterior, y en condiciones de extracción uniforme y sin reposición se obtiene:

$$
\begin{gathered}
E_{H_{0}}\left[M_{k}\right]=m=0 \\
\operatorname{Var}_{H_{0}}=s_{k}^{2}=s^{2} \frac{\left(n-n_{k}\right)}{(n-1) \cdot n_{k}}
\end{gathered}
$$


En este caso la media de las coordenadas es nula ya que la nube de variables se sitúa sobre el punto cero y la varianza es igual al valor propio del eje correspondiente dividido por el número de observaciones del eje $k \mathrm{y}$ corregido por el factor $\frac{n-n_{k}}{n-1}$.

Si el número total de individuos $n$ y el número de individuos del grupo $n_{k}$ no es pequeño, entonces puede aplicarse el Teorema Central del Límite, según el cual en condiciones generales la distribución de la suma de variables aleatorias independientes tiende a una distribución normal. Por tanto en este caso la variable:

$$
U=\frac{M_{k}-m}{s_{k}}
$$

Sigue aproximadamente una distribución normal centrada y reducida. La probabilidad crítica asociada a esta variable es pues la probabilidad en una distribución normal de superar el valor numérico $u$ en $\operatorname{los} n$ individuos para la variable $U$ definida anteriormente.

Las modalidades más características se obtienen seleccionando aquellas con probabilidades críticas menores y, por consiguiente, las que tienen mayores valores tomados por la variable normal $u$ :

$$
u=\frac{m_{k}-m}{s_{k}}
$$

La formulación de este parámetro corresponde al valor - test y expresa la diferencia estadística entre las coordenadas promedio del grupo $k$ y la media general en número de desviaciones típicas de una distribución normal. Cuanto más elevado sea el valor absoluto del v-test más evidente será la diferencia del grupo respecto al resto de individuos (Lebart, Morineau y Warwick, 1984).

En la práctica se utiliza el umbral de 2 desviaciones típicas, procedente de una aproximación del valor exacto 1,96 correspondiente a un nivel de significación de $5 \%$, para considerar si la variable es significativa. De esta forma, se considera que valores superiores a 2 indican valores poco probables bajo la hipótesis de extracción al azar (Morineau, 1984).

\section{Caracterización de grupos según variables nominales ilustrati-} vas

Sea $k$ un grupo de $n_{k}$ individuos y $j$ una categoría de una de las variables nominales. Para determinar si esta categoría es característica de un grupo se debe analizar si su número de elementos es significativamente mayor en 
el grupo $k$ que entre el resto de los individuos.

Los elementos del problema siguen la siguiente nomenclatura:

\begin{tabular}{|l|c|c|c|}
\hline & En el grupo $k$ & Fuera del grupo $k$ & Total \\
\hline En la categoría $j$ & $n_{j k}$ & $n_{j \bar{k}}$ & $n_{j}$ \\
Fuera de la categoría $j$ & $n_{\bar{j} k}$ & $n_{\bar{j} \bar{k}}$ & $n_{j}$ \\
Total & $n_{k}$ & $n_{\bar{k}}$ & $n$ \\
\hline
\end{tabular}

Tabla 4.1: Elementos ilustrativos, por categoría y grupo

Al igual que antes se procede como en un test de hipótesis clásico donde la hipótesis nula $H_{0}$ es la extracción al azar (sin reposición) de los $n_{k}$ individuos entre los $n$ individuos de la población. Esta hipótesis asegura la equivalencia de las proporciones $\frac{n_{j k}}{n_{k}}$ y $\frac{n_{j}}{n}$. La hipótesis alternativa se basa en una proporción anormalmente elevada de $j$ entre los $n_{k}$ individuos.

Sea $\mathrm{N}$ la variable aleatoria Extracción de individuo de la categoría $j$ en el grupo $k$. Según la tabla anterior esta variable toma el valor:

$$
N=n_{j k}
$$

Bajo la hipótesis $H_{0}$ la variable $N$ sigue una distribución hipergeométrica cuyos tres parámetros son conocidos (Tamaño de población $n$, Tamaño de muestra $n_{k}$ y Cantidad de elementos que cumplen la característica deseada $\left.n_{j k}\right)$. Mediante dicha distribución es posible analizar el número de individuos pertenecientes a la categoría $j$ en una muestra de tamaño $n_{k}$ extraída al azar y sin reposición entre $n$ individuos. Concretamente se analiza:

$$
p_{k}(j)=\operatorname{Prob}_{H_{0}}\left[N \geq n_{j k}\right]
$$

Cuanto más elevado es el valor de $n_{j k}$ menor es la probabilidad asociada a la distribución hipergeométrica y por tanto más cuestionable es la hipótesis nula de extracción al azar. De esta forma pueden utilizarse las probabilidades críticas $p_{k}(j)$ para clasificar cada una de las $j$ categorías de todas las variables nominales. Aquellas categorías con menores probabilidades críticas serán las más que presenten un comportamiento más característico respecto al resto.

A partir de esta definición de probabilidad crítica, se formula el valor - test para variables nominales ilustrativas como el valor de una variable normal con la misma probailidad $p_{k}(j)$ de ser superado que la categoría $j$ en el grupo $k$. Cuanto más pequeña sea la probabilidad crítica más elevado será el valor - test correspondiente. Por tanto el valor - test se utiliza como 
criterio para comparar y seleccionar por orden de importancia las categorías características de un grupo de individuos.

\section{Caracterización de ejes factoriales}

Concretamente, en este caso se trata de identificar los grupos de individuos (pertenecientes a la categoría $j$ ) que mejor caracterizan cada factor $\alpha$.

Se trata de calcular la probabilidad crítica correspondiente al siguiente test de hipótesis:

$$
\begin{aligned}
& H_{0}: \bar{\Psi}_{k \alpha}=0 \\
& H_{1}: \bar{\Psi}_{k \alpha}>0
\end{aligned}
$$

Bajo la suposición de extracción al azar sin reposición de los individuos de la categoría $k$ se obtiene:

$$
\begin{gathered}
E\left[\bar{\Psi}_{k \alpha}\right]=0 \\
\operatorname{Var}\left[\bar{\Psi}_{k \alpha}\right]=\frac{n-n_{k}}{n_{k}-1} \cdot \frac{\lambda_{\alpha}}{n_{k}}
\end{gathered}
$$

Por el teorema central del límite la variable $\bar{\Psi}_{k \alpha}$ tiende a una distribución normal. El valor - test corresponde por tanto al valor de la variable normal centrada y reducida con la misma probabilidad crítica de la hipótesis $H_{0}$ de ser superado.

$$
v_{k \alpha}=\frac{\bar{\Psi}_{k \alpha}}{\sqrt{\frac{n-n_{k}}{n_{k}-1}} \cdot \frac{\lambda_{\alpha}}{n_{k}}}
$$

Puesto que el valor test también sigue una distribución normal se declaran características las modalidades cuyo valor test es superior a $1.96 \mathrm{o}$ inferiores a -1.96 , umbral que en la práctica se aproxima a 2 (Morineau, 1984).

\section{Aplicaciones de los valores test}

Habitualmente los valores test se emplean para el análisis de grandes cantidades de datos. El hecho de que los valores test ordenen las distintas categorías en función de su nivel de significación permite identificar a simple vista los grupos más característicos de una población. Esta es la aplicación de los valores test más habitual (Alevizos y Morineau, 1992; Sleiman, 2001). 
Otra aplicación muy frecuente es el análisis de datos textuales en preguntas abiertas incluidas en cuestionarios. Para identificar las palabras o grupos de palabras que aparecen con mayor frecuencia se asocia a cada conjunto de palabras un v-test que mide la diferencia entre la frecuencia de dicho conjunto en el grupo y la misma frecuencia en la muestra total. Dicho valor test está normalizado, de forma que se considera una variable normal centrada y reducida. Igualmente pueden encontrarse numerosos ejemplos de esta aplicación de los valores test (Bécue, Lebart y Rajadell, 1992; GuérinPace, 1997; Lebart, Salem y Perry, 1998; Lebart, Salem y Bécue, 2000).

\subsubsection{Análisis de Datos Cualitatitivos}

\section{Contrastes de independencia}

\section{Contrastes de independencia en tablas bidimensionales}

En la mayoría de los casos no podemos disponer de la información sobre estos factores para todo el conjunto de la población objeto de estudio, y se trabaja con una muestra aleatoria simple extraída a partir de la misma. Sobre esta muestra se plantea la hipótesis nula $H_{0}$ : "Independencia poblacional entre ambos factores", fijado un determinado nivel de significación. A continuación se obtiene la tabla de contingencia $2 x 2$ entre los factores $A$ y $B$, que en ambos casos sólo disponen con dos modalidades, niveles o categorías. Seguidamente se calcula el valor del estadístico $\chi^{2}$ a partir de los datos de la tabla de contingencia y bajo la hipótesis nula $H_{0}$ según la expresión:

$$
\chi^{2}=\sum_{i} \sum_{j} \frac{\left(n_{i j}-\widehat{E}_{i j}\right)^{2}}{\widehat{E}_{i j}}
$$

Donde $n_{i j}$ son las frecuencias observadas en cada uno de los niveles de los factores $A$ y $B: a, b, c$ y $d$ y $\widehat{E}_{i j}$ constituyen las estimaciones de las frecuencias observadas (Aguilera, 2001; Ruiz-Maya, Martín-Pliego, López, Montero y Uriz, 1990).

En muestras de tamaño elevado la distribución del estadístico $\chi^{2}$ se aproxima muy bien a la distribución asintótica $\chi^{2} \operatorname{con}(r-1) \cdot(c-1)$ grados de libertad.

A partir de este estadístico es posible calcular el valor $C$ tal que $P\left[\chi_{(r-1)(c-1)}^{2}>\right.$ $C]=\alpha$ que, en el caso de tablas $2 x 2$ resulta $P\left[\chi_{1}^{2}>C\right]$ ya que tanto $r$ como $c$ toman el valor 2 como niveles de los factores.

Si el valor del estadístico $\chi^{2}$ es superior a $C$, se rechaza la hipótesis nula $H_{0}$ con un nivel $\alpha$ de significación. Es decir, se considera que los factores 
$A$ y $B$ no actúan de forma independiente. Si el valor del estadístico $\chi^{2}$ es inferior a $C$ se acepta la hipótesis nula $H_{0}$ con un nivel $\alpha$ de significación, lo cual equivale a aceptar que los factores A y B actúan de forma independiente.

En otras palabras, se rechaza $H_{0}$ al nivel de significación $\alpha$ si el p-valor el test es menor o igual que el nivel de significación, habiendo definido este p-valor como la probabilidad bajo $H_{0}$ de que el valor tabular de la distribución $\chi^{2}$ sea inferior o igual al valor observado del estadístico $\chi^{2}$.

Puesto que el cálculo del estadístico $\chi^{2}$ es laborioso y además exige el cálculo de las estimaciones de las frecuencias esperadas $\widehat{E}_{i j k}$, es posible utilizar una expresión más operativa para el caso específico de tablas de contingencia $2 x 2$ en función de las frecuencias observadas, de acuerdo con la siguiente expresión:

$$
\chi^{2}=\frac{N(a \cdot d-b \cdot c)^{2}}{(a+b)(a+c)(b+d)(c+d)}
$$

Que constituye el cociente entre el cuadrado de la diferencia de los productos de las frecuencias de las diagonales y los productos de las frecuencias marginales, todo ello multiplicado por el número de observaciones. Para definir esta expresión se ha utilizado una distribución continua (la distribución $\chi^{2}$ ) como aproximación a la distribución de probabilidad discreta multinomial de las e frecuencias observadas.

Donde los valores $a, b, c$ y $d$ corresponden a las frecuencias observadas en cada una de las celdas de una tabla $2 x 2$ según la siguiente tabla (Everitt, 1977):

\begin{tabular}{|l|c|c|c|}
\hline & A:Nivel 1 & A:Nivel 2 & Total \\
\hline B: Nivel 1 & $a$ & $b$ & $a+b$ \\
B: Nivel 2 & $c$ & $d$ & $c+d$ \\
\hline Total & $a+c$ & $b+d$ & $\mathrm{~N}$ \\
\hline
\end{tabular}

Tabla 4.2: Elementos de la tabla de contingencia estándar

Este resultado fue propuesto por Pearson (1904) y se formula mediante la definición de una variable aleatoria $\left(N_{1}, \ldots, N_{I}\right)$ con distribución multinomial $\left(n ; p_{1}, \ldots, p_{I}\right)$ tal que $\sum_{i=1}^{I} p_{i}=1$. Entonces, a partir de esta premisa se define la variable aleatoria:

$$
\sum_{i=1}^{I} \frac{\left(N_{i}-n p_{i}\right)^{2}}{n p_{i}}
$$


Que se distribuye asintóticamente cuando $n \rightarrow \infty$ como una variable aleatoria $X_{I-1}^{2}$, resultado que se encuentra reflejado y demostrado en (Agresti, 1996).

Así, el estadístico $\chi^{2}$ refleja la distancia entre las frecuencias observadas y su correspondiente estimación esperada bajo $H_{0}$. A medida que las frecuencias observadas aumentan su discrepancia respecto a las esperadas se incrementa el valor de este estadístico, considerando un tamaño muestral fijo. Por tanto, para tamaños muestrales grandes, se rechaza $H_{0}$ al nivel de significación $\alpha$ si:

$$
\chi^{2} \geq \chi_{I-1, \alpha}^{2}
$$

Siendo $\chi_{I-1, \alpha}^{2}$ el cuantil de orden $(1-\alpha)$ de la distribución $\chi_{I-1, \alpha}^{2}$.

En los casos en que las probabilidades que definen a la multinomial sean función de $p_{i}$ parámetros desconocidos, pero con estimadores máximo verosímilitud $\hat{p}_{i}$ definidos para cada uno de ellos, entonces el estadístico

$$
\sum_{i=1}^{I} \frac{\left(N_{i}-n \hat{p}_{i}\right)^{2}}{n \hat{p}_{i}}
$$

Se distribuye asintóticamente cuando $n \rightarrow \infty$ como una variable aleatoria $X_{I-p-1}^{2}$.

Puesto que los contrastes de independencia basados en la distribución $\chi^{2}$ recurren a esta distribución continua para aproximar una distribución discreta, correspondiente al estadístico de contraste $\chi^{2}$ se produce un error considerable cuando el tamaño muestral no es muy elevado (Ruiz-Maya et al., 1990).

Sin embargo Yates propone una corrección en este estadístico, denominada Corrección por Continuidad, consistente en restar 0.5 a las desviaciones positivas, y sumar 0.5 a las desviaciones positivas, entre las frecuencias observadas $n_{i j}$ y las estimaciones de las frecuencias esperadas $\hat{E}_{i j k}$ (Yates, 1934). De este modo se logra fijar las frecuencias marginales de filas y columnas, logrando que las colas correspondan a las de una distribución hipergeométrica.

Así el estadístico $\chi_{C}^{2}$ corregido por continuidad considera la diferencia entre el valor absoluto de las discrepancias entre las frecuencias esperadas y observadas de acuerdo con la siguiente expresión para tablas $2 x 2$ :

$$
\chi_{C}^{2}=\frac{N(|a \cdot d-b \cdot c|-0,5 \cdot N)^{2}}{(a+b)(a+c)(b+d)(c+d)}
$$

Siendo su expresión generalizada para tablas $r x c$ : 


$$
\chi_{C}^{2}=\sum_{i} \sum_{j} \frac{\left(\left|N_{i j}-\hat{E}_{i j}\right|-0,5\right)^{2}}{\hat{E i j}}
$$

En general esta expresión sólo se utiliza cuando el número de grados de libertad es igual a la unidad. En muestras de tamaño elevado produce prácticamente los mismos resultados que el estadístico no corregido, aunque pueden variar los valores críticos. Su aplicación principal se encuentra en muestras pequeñas, en las que el número de frecuencias observadas en alguna combinación de factores $A$ y $B$ oscila entre 5 y 10. En estos casos se recomienda comparar los resultados obtenidos por uno y otro estadístico, y aumentar el tamaño de la muestra si no coinciden, o incluso emplear otros métodos que incluyan la distribución multinomial.

Otra propuesta de mejora de este estadístico $\chi^{2}$ corresponde al Test Exacto de Fisher (Fisher, 1922, 1924, 1925), aplicable a aquellos casos con frecuencias esperadas excesivamente pequeñas (inferiores a 5). De hecho, la aproximación del estadístico $\chi^{2}$ a una distribución $\chi^{2}$ con un grado de libertad sólo puede considerarse aceptable si las frecuencias esperadas superan este límite inferior.

El test exacto de Fisher se caracteriza por no utilizar una aproximación de probabilidad, sino la distribución de probabilidad exacta de las frecuencias observadas. Puesto que, considerando frecuencias marginales fijas, la distribución de probabilidad de las frecuencias observadas sigue una ley hipergeométrica, en caso de que ambos factores $A$ y $B$ actúen de forma independiente, la probabilidad $P$ de obtener cualquier disposición de frecuencias en tablas $2 x 2 a, b, c$ y $d$ se obtiene según la expresión:

$$
P=\frac{(a+b) !(c+d) !(a+c) !(b+d) !}{a ! b ! c ! d ! N !}
$$

\section{Contrastes de independencia en tablas rxc}

En tablas de contingencia de orden $R x C$, donde $R, C$, o ambas son superiores a 2 el contraste $\chi^{2}$ de independencia no tiene una interpretación tan obvia, ni proporciona en sí mismo la misma cantidad de información para extraer conclusiones acerca de las relaciones de dependencia entre variables (Ruiz-Maya et al., 1990).

Sin embargo, es posible plantear igualmente la cuestión de contrastar la existencia de independencia entre dos factores $A$ y $B$, cada uno de los cuales consta de $r$ y $c$ categorías o niveles, respectivamente. La hipótesis nula de independencia $H_{0}$ se expresa en términos de probabilidad conjunta para 
cada par de niveles $p_{i j}$ calculadas como el producto de las probabilidades marginales de ambos niveles $p_{i}$ y $p_{\cdot j}$. Así, el contraste se plantea como:

$$
\begin{aligned}
& H_{0}: p_{i j}=p_{i \cdot p_{\cdot j}} \forall i, j \\
& H_{1}: p_{i j} \neq p_{i \cdot p_{\cdot j}} \forall i, j
\end{aligned}
$$

A partir de ambas hipótesis se formula:

$$
\chi^{2}=\sum_{i=1}^{r} \sum_{j=1}^{c} \frac{\left(n_{i j}-\hat{E}_{i j}\right)^{2}}{\hat{E}_{i j}}
$$

Donde $n_{i j}$ son las frecuencias observadas y $\hat{E_{i j}}$ es la estimación de las frecuencias esperadas en cada celda de la tabla bajo la hipótesis nula de independencia. Al igual que ocurría en tablas $2 x 2$, en muestras de tamaño elevado la distribución de este estadístico $\chi^{2}$ tiende a una distribución $\chi^{2}$ con $(r-1)(c-1)$ grados de libertad.

En este caso la estimación de las frecuencias esperadas se obtiene, bajo la hipótesis nula, mediante la siguiente expresión:

$$
\hat{E}_{i j}=N \cdot p_{i j}=N \cdot \hat{p}_{i \cdot} \cdot \hat{p}_{\cdot j}=N \frac{n_{i} \cdot \frac{n_{\cdot j}}{N}}{N}
$$

Según Agresti (2002) el contraste a realizar en este caso consiste en comparar el valor del estadístico $\chi^{2}$ obtenido frente al valor tabulado para la distribución $\chi^{2}$ con $(r-1)(c-1)$ grados de libertad y un nivel de significación $\alpha$ de forma que:

$$
\begin{aligned}
& \text { Si } \chi^{2}<\chi_{(r-1)(c-1) ; \alpha}^{2} \text { se acepta } H_{0} \\
& \text { Si } \chi^{2}>\chi_{(r-1)(c-1) ; \alpha}^{2} \text { se rechaza } H_{0}
\end{aligned}
$$

\section{Contrastes de independencia en tablas multidimensionales}

La importancia de las tablas de contingencia n-dimensionales queda manifiesta simplemente mediante el enunciado de la Paradoja de Simpson, que evidencia un conocimiento generalizado, aunque formulado de forma intuitiva. El planteamiento de este resultado afirma que la asociación marginal entre dos variables implicadas en una tabla de contingencia puede ser de dirección y magnitud muy diferente a la asociación en las tablas parciales que se obtienen para estas dos variables controlando una tercera (Simpson, 1951). Esta paradoja ya fue discutida anteriormente por Yule (1903).

Aunque el análisis de tablas tridimensionales plantea nuevos problemas respecto del análisis de tablas bidimensionales, su generalización a cuatro, 
cinco o $\mathrm{n}$ dimensiones no añade mayor complejidad que la analítica (RuizMaya et al., 1990). Por ello, y dado que el análisis planteado en este trabajo no exige el estudio de tablas de contingencia con mayor número de dimensiones, se plantearán únicamente los contrastes de independencia asociados a tres variables $A, B$ y $C$, con $r, c$ y $s$ niveles, respectivamente.

Las frecuencias marginales unidimensionales se calculan de la forma habitual, independientemente del número de factores:

$$
\begin{aligned}
& n_{i . .}=\sum_{j=1}^{c} \sum_{k=1}^{s} n_{i j k} \\
& n_{. j .}=\sum_{i=1}^{r} \sum_{k=1}^{s} n_{i j k} \\
& n_{. . k}=\sum_{i=1}^{r} \sum_{j=1}^{c} n_{i j k}
\end{aligned}
$$

Mientras que las frecuencias marginales bidimensionales se calculan como:

$$
\begin{aligned}
& n_{i j .}=\sum_{k=1}^{s} n_{i j k} \\
& n_{i \cdot k}=\sum_{j=1}^{c} n_{i j k} \\
& n_{\cdot j k}=\sum_{i=1}^{r} n_{i j k}
\end{aligned}
$$

Contrastes de independencia global

A pesar de que en tablas de contingencia bidimensionales existe un único modelo de independencia, en tablas tridimensionales existen al menos siete modelos de independencia de interés. Considerando 3 factores $A, B$ y $C$, con $r, c$ y $s$ niveles respectivamente y correspondientes a las filas, columnas y estratos de la tabla tridimensional, pueden presentarse los siguientes modelos de independencia formulados jerárquicamente en tres niveles (Cox y Snell, 1990):

\section{Independencia global}

$A, B$ y $C$ son independientes; Las filas, columnas y estratos de la tabla son independientes 


\section{Independencia parcial}

1. $A$ es independiente respecto de $B$ y $C$ : Las filas son independientes de las columnas y los estratos, pero no necesariamente ambos son independientes entre sí

2. $B$ es independiente respecto de $A$ y $C$ : Las columnas son independientes de las filas y los estratos, pero no necesariamente ambos son independientes entre sí

3. $C$ es independiente respecto de $A$ y $B$ : Los estratos son independientes de las filas y las columnas, pero no necesariamente ambos son independientes entre sí

\section{Independencia condicional}

1. $A$ y $B$ son independientes, dado un nivel de $C$ : Dado cualquier estrato, las filas y las columnas son independientes

2. $A$ y $C$ son independientes, dado un nivel de $B$ : Dada cualquier columna, las filas y los estratos son independientes

3. $B$ y $C$ son independientes, dado un nivel de $A$ : Dada cualquier fila, las columnas y los estratos son independientes

La hipótesis más sencilla que puede plantearse en tablas multidimensionales es la relativa a la independencia global o conjunta entre los distintos factores implicados. En tablas tridimensionales esta hipótesis de independencia global se formula en términos de probabilidad, de la siguiente manera (Everitt, 1977):

$$
H_{0(1)}: p_{i j k}=p_{i . .} \cdot p_{\cdot j \cdot} \cdot . \cdot k
$$

Donde $p_{i j k}$ es la probabilidad de una observación de pertenecer a la celda $i j k$ y $p_{i . .}, p_{. j}$. y $p_{. . k}$ son las probabilidades marginales unidimensionales correspondientes a cada uno de los niveles de los factores $A, B$ y $C$.

Así, el contraste de la hipótesis nula de independencia global entre los factores $A, B$ y $C$, dado un determinado nivel de significación $\alpha$, implica en primer lugar el cálculo de las estimaciones de las frecuencias esperadas, de acuerdo con la siguiente expresión:

$$
\hat{E}_{i j k}=N \hat{p}_{i . .} \hat{p}_{\cdot j \cdot \hat{p}_{. . k}}
$$


Donde los estimadores de máxima verosimilitud de las probabilidades marginales unidimensionales se obtienen mediante:

$$
\begin{gathered}
\hat{p}_{i \cdot .}=\frac{n_{i \cdot .}}{N} \\
\hat{p}_{\cdot j \cdot}=\frac{n_{\cdot j \cdot}}{N} \\
\hat{p}_{\cdot \cdot k}=\frac{n_{\cdot \cdot k}}{N}
\end{gathered}
$$

Por tanto:

$$
\hat{E}_{i j k}=N \frac{n_{i \cdot .}}{N} \frac{n_{\cdot j \cdot j}}{N} \frac{n_{. \cdot k}}{N}=\frac{n_{i \cdot .} n_{\cdot j \cdot} n_{\cdot . k}}{N^{2}}
$$

Seguidamente se calcula el estadístico $\chi^{2}$ según la expresión (Pearson, 1904):

$$
\chi^{2}=\sum_{i=1}^{r} \sum_{j=1}^{c} \sum_{k=1}^{s} \frac{\left(n_{i j k}-\hat{E}_{i j k}\right)^{2}}{\hat{E}_{i j k}}
$$

Finalmente se compara el valor de este estadístico $\chi^{2}$ con el valor crítico de una distribución $X^{2}$ con $r c s-r-c-s+2$ grados de libertad, al nivel de significación $\alpha$ siendo r,c y s el número de niveles de los factores $A, B$ y $C$, respectivamente. En tal caso se rechaza la hipótesis nula de independencia $H_{0}$ si el valor del estadístico $\chi^{2}$ es inferior al valor crítico tabulado según la distribución $\chi^{2}$. Por el contrario, se acepta que los factores $A, B$ y $C$ actúan como variables independientes si el valor del estadístico $\chi^{2}$ resulta superior al dicho valor crítico.

\section{Contrastes de independencia parcial}

En caso de que se rechace la hipótesis de independencia global cabe plantearse la posible existencia de asociaciones parciales entre las variables implicadas. De este modo, la evidencia de relaciones de dependencia a nivel global da lugar a análisis de las relaciones parciales entre variables. Se considera que existe independencia parcial entre una variable $A$ y otros dos factores $B$ y $C$ cuando $A$ es independiente respecto de $B$ y $C$, pero ambas variables no necesariamente lo son entre sí. En otras palabras, un factor es independiente respecto de los otros dos.

En una tabla tridimensional pueden darse tres posibles situaciones de independencia parcial: 
1. Independencia parcial de $A$ respecto de $\mathrm{B}$ y $\mathrm{C}$, o también A es conjuntamente o mutuamente independiente de $B$ y $C$

$$
H_{0(2)}: p_{i j k}=p_{i . .} p_{\cdot j k}
$$

2. Independencia parcial de $B$ respecto de $A$ y $C$, o también $B$ es conjuntamente o mutuamente independiente de $A$ y $C$

$$
H_{0(3)}: p_{i j k}=p_{\cdot j \cdot} \cdot p_{i \cdot k}
$$

3. Independencia parcial de $C$ respecto de $A$ y $B$, o también $C$ es conjuntamente o mutuamente independiente de $A$ y $B$

$$
H_{0(4)}: p_{i j k}=p_{. . k} p_{i j} .
$$

Mientras que las hipótesis alternativas pueden expresarse, en general como:

1. Independencia parcial de $A$ respecto de $B$ y $C$

$$
H_{1(2)}: p_{i j k} \neq p_{i . .} p_{\cdot j k}
$$

2. Independencia parcial de $B$ respecto de $A$ y $C$

$$
H_{1(3)}: p_{i j k} \neq p_{\cdot j \cdot} \cdot p_{i \cdot k}
$$

3. Independencia parcial de $C$ respecto de $A$ y $B$

$$
H_{1(4)}: p_{i j k} \neq p_{. . k} p_{i j} \text {. }
$$

En el caso de la primera hipótesis nula planteada, el contraste de independencia parcial se formula como:

$$
\begin{aligned}
& H_{0(2)}: p_{i j k}=p_{i . . p} \cdot \cdot j k \\
& H_{1(2)}: p_{i j k} \neq p_{i . .} \cdot p_{\cdot j k}
\end{aligned}
$$

Donde la hipótesis nula establece que el primer factor $A$ es independiente de $B$ y $C$, mientras que estos dos últimos no son necesariamente independientes entre sí. Para ello la hipótesis nula define la probabilidad de que una observación pertenezca a la celda $i j k$ como el producto de la probabilidad de la frecuencia marginal de la i-ésima categoría del factor $A$ y la probabilidad de la frecuencia marginal de las jk-ésimas categorías de los factores $B$ y $C$. Para realizar este contraste bajo la hipótesis de independencia parcial, la 
estimación de las frecuencias esperadas viene dada por la expresión:

$$
\hat{E}_{i j k}=N \hat{p}_{i . .} \hat{p}_{. j k}
$$

Donde $\hat{p}_{i . .}$ y $\hat{p}_{. j k}$ se definen como estimadores máximo - verosímiles de $p_{i . .}$ y $p_{. j}$. respectivamente, y se obtienen mediante las siguientes expresiones:

$$
\begin{gathered}
\hat{p}_{i \cdot .}=\frac{n_{i \cdot .}}{N} \\
\hat{p}_{\cdot j k}=\frac{n_{\cdot j k}}{N}
\end{gathered}
$$

De forma que:

$$
\hat{E}_{i j k}=N \frac{n_{i . .}}{N} \frac{n_{\cdot j k}}{N}=\frac{n_{i . .} \cdot \cdot j k}{N}
$$

El estadístico $\chi^{2}$ se calcula mediante la misma expresión utilizada en el contraste de independencia global:

$$
\chi^{2}=\sum_{i=1}^{r} \sum_{j=1}^{c} \sum_{k=1}^{s} \frac{\left(n_{i j k}-\hat{E}_{i j k}\right)^{2}}{\hat{E}_{i j k}}
$$

Estadístico que, como ocurría anteriormente, en muestras de tamaño elevado se distribuye aproximadamente como una distribución $\chi^{2}$ con $r c s-r-$ $c s+1$ grados de libertad.

Para las hipótesis $H_{0(3)}$ y $H_{0(4)}$ la obtención de las frecuencias esperadas se realiza de forma equivalente y este número de grados de libertad se calcula respectivamente mediante las expresiones $r c s-c-r s+1$ y $r c s-s-r c+1$.

\section{Contrastes de independencia condicional}

Como se ha comentado anteriormente, la evidencia de relaciones de dependencia global entre variables da pie a plantear otro tipo de hipótesis asociadas a posibles relaciones parciales entre variables, o relaciones condicionales como ocurre en este caso. Se considera que existe independencia condicional entre dos variables $A$ y $B$ cuando son independientes entre sí, para cada nivel de un tercer factor $C$, pudiendo estar, sin embargo, ambas variables asociadas con dicho factor.

Del mismo modo, como se ha expuesto en apartados anteriores, en una tabla tridimensional pueden darse tres posibles situaciones de independencia condicional: 
1. Independencia entre las variables $A$ y $B$ para cada nivel de la variable C

$$
H_{0(5)}: p_{i j k}=p_{i \cdot k} p_{\cdot j k}
$$

2. Independencia entre las variables $A$ y $C$ para cada nivel de la variable $B$

$$
H_{0(6)}: p_{i j k}=p_{i j} \cdot p_{\cdot j k}
$$

3. Independencia entre las variables $B$ y $C$ para cada nivel de la variable A

$$
H_{0(7)}: p_{i j k}=p_{i j} \cdot p_{i \cdot k}
$$

La hipótesis nula plantea que los factores $A$ y $B$ son independientes dado un determinado nivel del factor $C$, pero no necesariamente lo son entre sí. Así, la probabilidad de que una observación pertenezca a la celda $i j k$ se calcula como el producto de las probabilidades de clasificación en las celdas $i j$ y $i k$, que corresponden a las clasificaciones de los factores $A$ y $B$ y $A$ y $C$ respectivamente.

El estadístico $\chi^{2}$ se calcula mediante la misma expresión utilizada en los contrastes de independencia global y parcial:

$$
\chi^{2}=\sum_{i=1}^{r} \sum_{j=1}^{c} \sum_{k=1}^{s} \frac{\left(n_{i j k}-\hat{E}_{i j k}\right)^{2}}{\hat{E}_{i j k}}
$$

En muestras de tamaño elevado este estadístico se distribuye como una distribución $\chi^{2}$ con $r c s-r c-r s+1$ grados de libertad. Una vez obtenido este estadístico, se compara con el valor tabular correspondiente y se acepta o rechaza la hipótesis nula planteada según el procedimiento anterior.

Estos siete modelos de independencia se encuentran relacionados entre sí de forma jerárquica. En primer lugar existe independencia global entre los factores $A, B$ y $C$ si y solo sí existe independencia parcial entre cualquier factor y los otros dos. Es decir, la hipótesis $H_{0(1)}$ es cierta si y solo si se cumplen $H_{0(2)}, H_{0(3)}$ y $H_{0(4)}$. Del mismo modo, existe independencia global entre los factores $A, B$ y $C$ si y solo sí los tres pares de variables son condicionalmente independientes dado un tercer factor. En otras palabras, la hipótesis $H_{0(1)}$ es cierta si y solo si se cumplen $H_{0(5)}, H_{0(6)}$ y $H_{0(7)}$.

Contrastes para la identificación de variables modificadoras del efecto: Efectos de Interacción y Confusión

El concepto de Modificación del Efecto surge con Miettinen (1974) y aunque está relacionado con el concepto de Confusión, sus repercusiones 
sobre la orientación de la investigación son radicalmente distintas. Mientras que la confusión implica una distorsión de las medidas observadas cuyo efecto debe ser eliminado o controlado en el transcurso de la investigación, la presencia de interacciones enriquece la información que se obtiene de un proceso de forma que no sólo no debe eliminarse sino que aporta información complementaria sobre un determinado fenómeno en presencia de una tercera variable.

Se considera que existe interacción o modificación del efecto entre dos o más factores causales cuando existe interdependencia entre sus mecanismos de acción para causar el efecto esperado. Este concepto de modificación del efecto posee dos características fundamentales. En primer lugar cabe la posibilidad de concluir tanto la existencia de interacción como la inexistencia de este condicionante en función del tipo de escala escogida (aditiva o multiplicativa). Por esta razón, cuando se describe una modificación del efecto, es preciso especificar la escala en la que se mide el efecto (De Irala, Martínez-González y Guillén, 2001b). Estas modificaciones del efecto pueden ser cuantitativas si únicamente se modifica la intensidad de la relación entre las variables o cualitativas si únicamente se modifica la dirección de la asociación. En los casos en que se incrementa la intensidad de la asociación se considera que la interacción produce un Efecto Sinérgico. Por el contrario, si la intensidad de la asociación se reduce a causa de la interacción se habla de Antagonismo o Efecto Supresivo.

Para identificar la presencia de posibles relaciones de interacción entre variables es necesario definir y especificar conceptualmente los factores latentes implicados así como la posible existencia de relaciones causales entre ellos, descartando la posible presencia de relaciones de confusión (Bou - Llusar, Camisón-Zornoza y Escrig-Tena, 2001). Una vez realizados este estudio previo, se debe realizar un análisis estratificado para estimar el efecto de la variable causante sobre la receptora en cada estrato de la variable considerada modificadora.

Respecto a las desviaciones del efecto aditivo o multiplicativo en cada uno de estos estratos se pueden valorar mediante modelos basados en escalas multiplicativas, como la regresión logística que estima cocientes de ventajas, o la regresión de Cox que estima razones de tasas (Cox, 1984; Kleinbaum y Klein, 2010).

A diferencia del fenómeno de la confusión, que por su propia naturaleza constituye una distorsión de la medida observada a controlar o eliminar, la presencia de interacciones debe ser analizado y estudiado de forma exhaustiva con el fin de enriquecer la información descriptiva del funcionamiento del proceso. Para ello es posible mostrar tablas y gráficos estratificados que 
ayuden a comprender de forma simple de qué modo se está produciendo la modificación del efecto.

\section{La ausencia de interacción o ausencia de asociación parcial}

Barlett propuso en 1935 el Modelo de Ausencia de Interacción entre 3 factores $A, B$ y $C$ o Modelo de Asociación Parcial, formulado como la igualdad de los cocientes de ventajas de las tablas parciales asociadas a dos cualesquiera de los factores, coeficientes que se describen de forma detallada en apartados posteriores (Barlett, 1935). Esta prueba se conoce también como Prueba de Breslow - Day (Breslow y Day, 1975, 1980).

Los contrastes de ausencia de asociación parcial pueden ser de dos tipos: condicionales y no condicionales. Los contrastes condicionales asumen que la asociación es la misma en todas las tablas parciales, y contrastan la hipótesis de igualdad a cero de la medida ponderada de asociación parcial (o igual a la unidad en el caso del cociente de ventajas). Por otro lado, los contrastes no condicionales de ausencia de asociación parcial no asumen esta suposición de asociación parcial constante, y contrastan la igualdad a cero de todas las medidas de asociación de las tablas parciales frente a la alternativa de que al menos una sea distinta de cero. Los contrastes empleados en este trabajo, y descritos en los siguientes apartados, son de tipo condicional puesto que asumen el cumplimiento de la hipótesis de asociación parcial.

Esta suposición de ausencia de asociación parcial constante hace referencia a la ausencia de interacción entre factores y se formula en términos de los cocientes de ventajas de los 3 factores $A, B$ y $C$. Para enunciar esta hipótesis se fijan los niveles de uno de los factores, por ejemplo el factor $C$, y se impone que los cocientes de ventajas entre los otros dos factores $A$ y $B$ sean similares en todos los niveles de $C$.

$$
H_{0(8)}: \theta_{i j \mid 1}^{A B}=\theta_{i j \mid k}^{A B} \forall 2, \ldots, s
$$

Esta misma hipótesis podría enunciarse equivalentemente fijando los niveles de los factores $A$ o $B$ según las siguientes hipótesis:

$$
\begin{aligned}
& H_{0(9)}: \theta_{j k \mid 1}^{B C}=\theta_{j k \mid i}^{B C} \forall 2, \ldots, r \\
& H_{0(10)}: \theta_{i k \mid 1}^{A C}=\theta_{i k \mid j}^{A C} \forall 2, \ldots, c
\end{aligned}
$$

Operando sobre estas expresiones se ha demostrado que la caracterización de independencia condicional en términos de cocientes de ventajas condicionales implica ausencia de interacción entre los 3 factores implicados 
$A, B$ y $C$ (Aguilera, 2006).

\section{Contraste Condicional de Ausencia de Interacción sobre el Ries- go Ponderado $R_{p}$}

La formulación del Contraste Condicional de Ausencia de Interacción sobre el Riesgo Ponderado, se aplica sobre una tabla $2 x 2 x s$ sobre la que se plantea el contraste de cualquiera de las hipótesis $H_{0(8)}$. Sin embargo igualmente podría haberse planteado sobre tablas $r x 2 x 2$ o $2 x c x 2$ mediante la aplicación de las hipótesis $H_{0(9)}$ y $H_{0(10)}$. En el caso de tablas $2 x 2 x s$ se plantea la hipótesis de ausencia de interacción entre los 3 factores $A, B$ y $C$, equivalente a que los cocientes de ventajas de las $s$ tablas parciales $2 x 2$ asociadas sean iguales.

Woolf (1955) formula el Riesgo Ponderado $R_{p}$ como un estimador de la asociación parcial común entre dos variables binarias. El logaritmo de este estadístico constituye una media ponderada de los $s$ logaritmos de los cocientes de ventajas asociadas a las $s$ tablas parciales $2 x 2$.

$$
R_{p}=\frac{\sum_{k=1}^{s} w_{k} \cdot \ln \hat{\theta}_{k}}{\sum_{k=1}^{s} w_{k}}
$$

Definiendo los pesos como:

$$
\frac{1}{w_{k}}=\hat{\sigma}^{2}\left(\ln \left(\hat{\theta_{k}}\right)\right)=\frac{1}{n_{11 k}}+\frac{1}{n_{12 k}}+\frac{1}{n_{21 k}}+\frac{1}{n_{22 k}}
$$

En muestreos mediante Poisson y muestreos multinomiales esta expresión se conoce como Error Estándar Asintótico (ASE: Asymptotic Standard Error) (Agresti, 2002). En caso de que alguna de las frecuencias observadas sea nula se añadirá el valor 0.5 para posibilitar el cálculo de la función inversa.

Entonces, el estadístico del test $\chi^{2}$ de ausencia de interacción, en este caso planteado como la homogeneidad de cocientes de ventajas se formula como:

$$
\chi^{2}=\sum_{k} w_{k}\left(\ln \left(\hat{\theta_{k}}\right)-\ln \left(\hat{R_{p}}\right)\right)^{2}=\sum_{k} w_{k}\left(\hat{\theta_{k}}\right)^{2}-\sum_{k} w_{k}\left(\hat{R}_{p}\right)^{2}
$$

Que bajo la hipótesis nula de interacción:

$$
H_{0(8)}: \theta_{1}=\theta_{k} \forall k=2, \ldots, s
$$


Sigue una distribución asintótica $\chi_{s-1}^{2}$ por lo que se rechaza la hipótesis de homogeneidad al nivel de significación $\alpha$ si el estadístico $\chi^{2}$ superior al valor tabular de la distribución $\chi^{2}$ con $s-1$ grados de libertad:

$$
\chi^{2} \geq \chi_{s-1 ; \alpha}^{2}
$$

Puesto que la varianza asintótica de $\ln \hat{R}_{p}$ bajo muestreo multinomial completo es:

$$
\hat{\sigma}^{2}\left(\ln \hat{R}_{p}\right)=\frac{1}{\sum_{k} w_{k}}
$$

El intervalo de confianza aproximado para el valor poblacional del logaritmo del riesgo ponderado al nivel $1-\alpha$ se formula como:

$$
\ln \hat{R}_{p} \pm z_{\frac{\alpha}{2}} \hat{\sigma}^{2}\left(\ln \hat{R}_{p}\right)
$$

\section{Contraste condicional sobre el Cociente de Ventajas Común}

Una variable de confusión distorsiona la medida de asociación entre otras dos variables. El efecto de la presencia de este tipo de variables puede ser tanto la observación de un efecto que en realidad no existe (Relación Espúrea) o por el contrario, puede intensificar o atenuar una asociación real (Confusión Positiva o Negativa) e incluso una inversión del sentido de una asociación real (Efecto Paradójico) (De Irala, Martínez-González y Guillén, 2001a). Al igual que ocurre con las medidas de asociación, el área de investigación donde más se ha aplicado este concepto es en epidemiología y de hecho, Last define en su diccionario de Epidemiología el concepto confusión como "una situación en que la medida del efecto de una exposición sobre el riesgo es distorsionada por la asociación con la exposición con otro factor o factores que influyen sobre el resultado en estudio" (Last, 2001). En términos generales la exposición es la ocurrencia de una variable causante sobre otra resultante, que en epidemiología corresponde al riesgo de contraer o desarrollar cierta enfermedad.

Los factores de confusión aparecen con frecuencia a pesar de los esfuerzos por evitarlos en la fase de diseño de la investigación. No existe un criterio generalizado para la identificación de los factores que llevan a confusión, ya que una misma variable puede ser un factor de confusión en una investigación y no serlo en la otra.

Para considerar que una variable tiene un efecto de confusión en la relación entre otras dos variables, una causante y otra receptora, deben cumplirse diversas condiciones. En primer lugar debe estar asociada con la variable receptora, independientemente de su efecto de confusión en la relación, al tiempo que debe estar asociada con la variable causante, pero no ser un 
resultado de la misma. Por último no debe ser un eslabón causal intermedio entre las variables causante y receptora.

Asimismo debe existir una base teórica conceptual para poder suponer que el factor causante afecta al resultante y evidentemente la ocurrencia del factor de confusión debe anteceder a la variable resultante (Ducoing, 1998).

La relación entre una variable de confusión y las otras dos cuya asociación distorsiona se suele representar mediante el siguiente esquema:

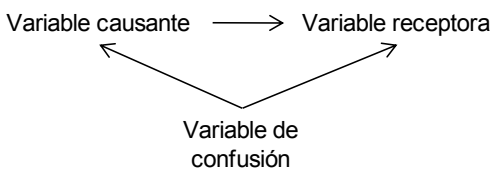

Figura 4.6: Relaciones establecidas por las variables de confusión

Según Greenland estos gráficos se denominan Gráficos acíclicos dirigidos ya que no forman un ciclo cerrado y las variables están unidas por flechas dirigidas (Greenland y Robins, 1999).

En términos generales, se considera que existe confusión cuando se dan diferencias importantes entre las estimaciones brutas de una asociación y las ajustadas por los posibles factores de confusión. Concretamente, si las diferencias entre ambas estimaciones superan el $10 \%$ del valor en las estimaciones brutas y ajustadas, es suficiente para considerar que el factor introduce un efecto de confusión significativo (De Irala y Fernández-Crehuet, 1999; Maldonado y Greenland, 1993; Rothman y Greenland, 1998; Sonis, 1998).

Puesto que los factores de confusión introducen un sesgo en las medidas de asociación por su propia naturaleza, es evidente que se debe prevenir y controlar dicho efecto antes de extraer conclusiones. En este sentido, en la fase de diseño de la investigación se suelen utilizar tres variantes metodológicas para evitar el efecto de confusión entre variables. En primer lugar la Aleatorización, consistente en asignar al azar a los sujetos de un estudio al azar a cada uno de los grupos que se pretende comparar. De este modo, cualquier posible factor de confusión se puede considerar distribuido de forma homogénea entre los distintos estratos. Otra posibilidad es realizar una labor de Restricción y Filtrado de Observaciones, de forma que sólo se admitan en el estudio a aquellos sujetos que estén en una de las categorías del factor de confusión. Asimismo cabe la posibilidad de realizar un Empare- 
jamiento (Matching), como modalidad de restricción empleada únicamente en estudios experimentales. En general se recomienda escoger esta opción cuando el factor de confusión puede tener un efecto potencial muy elevado sobre la variable resultante, así como cuando no es habitual la ocurrencia de la variable causante o en tamaños de muestra muy pequeños (GómezRestrepo y Okuda, 2006).

Por otro lado, en la fase de análisis existen otras dos opciones válidas para controlar el efecto de los factores de confusión: el Análisis Estratificado y el Análisis Multivariante. El primero consiste en estimar las medidas de asociación entre los distintos factores implicados para cada uno de los estratos de la variable de confusión. El análisis multivariante ofrece múltiples técnicas para el ajuste en las estimaciones de las medidas de asociación.

Con el fin de identificar posibles factores de confusión se aplica un segundo contraste, que permite el análisis de la ausencia de interacción y se formula nuevamente sobre una tabla tridimensional $2 x 2 x s$ y las $s$ tablas parciales $2 x 2$ asociadas, para la que se contrasta la hipótesis de independencia condicional entre $A$ y $B$ dado un determinado nivel de $C$. Esta hipótesis se formula en términos de probabilidades como:

$$
H_{0(5)}: p_{i j k}=p_{i \cdot k} p_{\cdot j k}
$$

Sin embargo, esta misma hipótesis puede ser formulada en términos de cocientes de ventajas como el hecho de que los cocientes de ventajas de las tablas parciales $\theta_{k}$ sean en su totalidad iguales a la unidad:

$$
H_{0(9)}: \theta_{k}=1 \forall k=2, \ldots, s
$$

Bajo la hipótesis nula de ausencia de interacción este contraste es equivalente al contraste de la hipótesis nula $\theta_{M H}=1$. La justificación para esta equivalencia se encuentra en que en condiciones de ausencia de interacción todos $\operatorname{los} \theta_{k}(k=1, \ldots, s)$ son iguales, por lo que la igualdad a uno del cociente de ventajas común $\theta_{M H}$ equivale a la igualdad a uno de todos ellos.

De este modo este test contrasta la independencia condicional entre dos factores asumiendo ausencia de interacción entre las tres variables. La ventaja de este contraste frente a otros contrastes condicionales como el anterior, basado en otras medidas de asociación parcial común como el riesgo ponderado, es que es válido incluso para tablas no equilibradas con tamaños muestrales pequeños en algunos estratos.

Este cociente de ventajas común $\theta_{M H}$ se define como la media ponderada de los cocientes de ventajas de las s tablas parciales: 


$$
\theta_{M H}=\sum_{k=1}^{s} \alpha_{k} \theta_{k}
$$

Donde las ponderaciones se definen como:

$$
\alpha_{k}=\frac{\frac{\hat{E}_{12 k} \hat{E}_{21 k}}{\hat{E}_{. k}}}{\sum_{l=1}^{s} \frac{\hat{E}_{12 l} \hat{E}_{21 l}}{\hat{E}_{. l l}}}
$$

Mantel y Haënszel (1959) propusieron para este contraste el estadístico $\chi_{M H}^{2}$, que bajo la hipótesis nula de ausencia de interacción se distribuye aproximadamente como una distribución $\chi^{2}$ con 1 grado de libertad:

$$
\chi_{M H}^{2}=\frac{\left[\sum_{k=1}^{S} \frac{\left(n_{11 k} n_{22 k}-n_{12 k} n_{21 k}\right)}{n_{\cdot . k}}\right]^{2}}{\sum_{k=1}^{S} \frac{\left(n_{1 \cdot k} n_{2 \cdot k} n_{\cdot 1 k} n_{\cdot 2 k}\right)}{n_{\cdot \cdot k}^{2}\left(n_{\cdot \cdot k}-1\right)}}
$$

El objetivo de la formulación de este estadístico enfatiza en la necesidad de considerar cada una de las subcategorías de un tercer factor, potencialmente influyente en el riesgo de enfermedad dado un determinado nivel de exposición al factor de riesgo. Posteriormente este estadístico, propuesto de forma genérica, se ha formulado específicamente para tablas $2 x 2$ (Mantel, 2011)

El estimador máximo verosímil propuesto por Mantel y Haënszel para el cociente de ventajas común a todas las tablas parciales $2 x 2$ se define como:

$$
\hat{\theta}_{M H}=\sum_{k=1}^{s} \hat{\alpha}_{k} \hat{\theta}_{k}=\frac{\sum_{k=1}^{s} \frac{n_{11 k} n_{22 k}}{n_{. k}}}{\sum_{l=1}^{s} \frac{n_{12 l} n_{21 l}}{n_{. . l}}}
$$

Este estadístico se distribuye según una distribución normal de media igual a la unidad y varianza $\hat{\sigma}^{2}\left(\theta_{M H}\right)$. Entonces, se ha demostrado que el estadístico $\chi_{M H}^{2}$ se puede obtener como el cuadrado de su valor tipificado:

$$
\chi_{M H}^{2}=\left(\frac{\hat{\theta}_{M H}-1}{\hat{\sigma}\left(\hat{\theta}_{M H}\right)}\right)^{2}
$$

A partir de este valor, se rechazará la hipótesis nula de independencia condicional al nivel de significación $\alpha$ cuando se verifique que es superior al valor tabular de la distribución $\chi^{2}$ con 1 grado de libertad y nivel $\alpha$.

$$
\chi_{M H}^{2} \geq \chi_{1 ; \alpha}^{2}
$$

El desarrollo de este test pone de manifiesto la necesidad de aplicar un análisis estratificado en aquellos casos en que las relaciones de asociación entre 2 variables pueden verse modificadas en función de los distintos estratos 
de una tercera variable (Molinero, 2004).

\section{Medidas de asociación \\ Concepto y propiedades}

Las Medidas de Asociación tienen una larga historia y se emplean fundamentalmente en la rama de epidemiología (Ahrens y Pigeot, 2001), como método de evaluación de la asociación entre distintas situaciones de exposición a factores de riesgo, y la probabilidad de desarrollar una determinada enfermedad, en lo que se conoce como estudios de casos y controles (Bullón, 2002). De hecho, no es de extrañar que el uso de esta metodología se encuentre tan generalizado en esta rama de las ciencias, considerando que las primeras aportaciones en este ámbito, realizadas por Fisher y Yule (Fisher, 1922; Yule, 1903, 1912), pertenecen al campo de la biología y la agricultura.

A pesar de la innegable importancia de dichas contribuciones seminales, lo cierto es que la gran parte de estas técnicas se desarrollaron a partir de los años sesenta, motivados por el incremento en el nivel de sofisticación de los métodos empleados en ciencias sociales y biomédicas (Agresti, 2002).

En este sentido resultaron determinantes los trabajos de Goodman y Kruskal $(1954,1959,1963,1972)$, en los que proporcionan diversos criterios para la evaluación de medidas de asociación y aporta nuevas medidas específicas para determinados contextos. En estos trabajos los investigadores señalan que desde un enfoque estricto, cada hipótesis de investigación debería desarrollar sus medidas de asociación específicas de acuerdo con los objetivos planteados. En la práctica esto no ocurre así, pero sí se han propuesto muy distintas medidas de asociación, hasta el punto en que es difícil no encontrar al menos una de ellas adecuadas al contexto del estudio. De hecho, excepto en el caso de tablas $2 x 2$, es muy improbable que una única función refleje el gran número de posibles asociaciones que pueden darse en una tabla, una vez descartada la independencia (Bishop, Fienberg, Holland, Light y Mosteller, 2007). Por esta razón se han propuesto una gran variadad de medidas de asociación con el fin de disponer de más opciones a la hora de escoger en un determinado contexto de investigación.

Por todo ello la dificultad del uso de las medidas de asociación no consiste en su desarrollo, sino en la correcta selección de la medida más adecuada. Para realizar dicha elección es conveniente conocer sus definiciones y características, así como la forma de clasificarse en grupos generales. Por motivos de simplicidad, en este trabajo se ha realizado exclusivamente una revisión de las medidas de asociación empleadas en la fase de análisis. Esta selección 
se realizó atendiendo a las características de las variables implicadas en el análisis así como a los objetivos de esta fase.

En los casos en que se rechazan las hipótesis de independencia entre distintos factores de una tabla de contingencia se plantea el análisis del grado y dirección de la asociación entre dichas variables. Para ello se estudian las medidas de asociación, definidas como parámetros poblacionales estimados a partir de las frecuencias observadas en la tabla de contingencia.

En ocasiones se confunde la aplicación que corresponde a contrastes de independencia y medidas de asociación. Los contrastes de independencia se utilizan para identificar la existencia de relaciones entre dos variables cualitativas, mientras que las medidas de asociación aportan información sobre la dirección y el grado de intensidad de dicha relación (Aguilera, 2001).

Se considera que existe Asociación Perfecta entre dos factores $A$ y $B$ de una tabla $2 x 2$ cuando al menos uno de los niveles de uno de los factores queda completamente determinado por uno de los niveles del otro factor, lo cual ocurre cuando existe algún cero en la tabla $2 x 2$. La asociación perfecta puede ser estricta o implícita, y a su vez esta última puede ser de tipo I o de tipo II.

La Asociación Perfecta Estricta se da cuando uno de los niveles de uno de los factores queda inmediatamente determinado por el nivel del otro factor. Esto ocurre cuando:

$$
n_{11}=n_{22}=0
$$

O bien:

$$
n_{21}=n_{12}=0
$$

Por otro lado la asociación implícita de tipo I no se da en tablas $2 x 2$ y se considera que existe cuando se puede determinar el nivel de un factor $B$ a partir del nivel de otro factor $A$, pero dado el nivel del factor $B$ no es posible determinar el nivel del factor $A$. Asimismo existe Asociación Perfecta Implícita de Tipo II si es posible determinar el nivel de un factor $B$ a partir del nivel de otro factor $A$, pero no es posible hacer lo mismo con otros niveles de los mismos factores. En definitiva, esto ocurre cuando alguna de las frecuencias observadas es nula.

Independientemente de los casos específicos de asociación perfecta, en el resto de los casos la asociación simplemente se clasifica como positiva o negativa. La asociación es Positiva cuando se cumple que el primer nivel del factor $A$ está asociado con el primer nivel del factor $B$ e igualmente ocurre lo mismo para el segundo nivel del factor $A$ respecto al segundo nivel del 
factor $B$. Por el contrario la asociación es Negativa cuando el primer nivel del factor $A$ está asociado con el segundo nivel del factor $B$ y el segundo nivel del factor A está asociado con el primer nivel del factor $B$.

El siguiente paso a las medidas de asociación son los Modelos LogLineales consistentes en un conjunto de técnicas para el análisis de las relaciones causales entre variables cualitativas. Estos modelos han sido desarrollados extensamente por muchos investigadores en este campo (Agresti, 1996; Cox y Snell, 1990; Fienberg, 2007; Ruiz-Maya et al., 1995) y aunque resulta una técnica de notable importancia en el ámbito del análisis de datos cualitativos, no se ha aplicado en este trabajo debido a que su empleo excede los objetivos de la investigación ya que abordan el planteamiento un modelo causal entre distintas variables cualitativas sin profundizar en las relaciones de independencia, interacción y confusión entre variables cualitativas, que constituyen el eje central de este apartado del análisis.

\section{Propiedades de las medidas de asociación}

En principio es deseable que las medidas de asociación cumplan tres propiedades fundamentales: interpretabilidad, simetría e invarianza (Aguilera, 2001; Haberman, 1978).

En primer lugar la Interpretabilidad hace referencia a la facilidad de interpretación de los resultados obtenidos mediante medidas de asocación. Con este objetivo suelen estar acotadas entre dos extremos inferior y superior, que indican asociación nula o independencia y asociación perfecto o ausencia de independencia, respectivamente. Con este objetivo, es habitual normalizar los valores de dichas medidas de asociación en los intervalos $[0,1]$ ó $[-1,1]$. La ventaja del primer intervalo respecto al segundo es que elimina el efecto de las diferencias en el número de filas y columnas, de forma que posibilita la comparación de medidas de asociación obtenidas en tablas con distintas dimensiones.

Aunque es habitual realizar la normalización de las medidas, no están especificadas explícitamente las razones de esta práctica. Goodman y Kruskal (1954) señalaron que la importancia de la normalización disminuye a medida que la interpretabilidad de las medidas se incrementa. Asimismo señalaron el riesgo de realizar las mismas interpretaciones en resultados procedentes de distintas medidas, todas ellas normalizadas, sin considerar el origen de dichos valores normalizados. Una ventaja de la normalización es que, en principio, existe la creencia generalizada de que puede utilizarse para eliminar el efecto de las frecuencias marginales desequilibradas, lo cual permite comparar medidas procedentes de distintas tablas de contingencia y con distintas dimensiones. No obstante, no se ha evidenciado que esta práctica garantice 
realmente la comparabilidad de medidas de asociación procedentes de tablas con distintas dimensiones (Bishop et al., 2007).

Por otro lado, el hecho de que una medida de asociación sea Simétrica implica que su valor no se modifica en función de si se considera variable explicada o explicativa. Por el contrario, las medidas asimétricas toman distintos valores en función de este criterio, y en los casos en que no puede distinguirse entre ambos tipos de variables, se define un promedio de los valores correspondientes en ambos casos a modo de aproximación a una medida de asociación simétrica. Este enfoque resulta más frecuente en experimentos en los que se controle el número de unidades que corresponden a cada categoría a nivel de frecuencias marginales. Las Medidas Basadas en el Estadístico $\chi^{2}$ pertenecen a la categoría de medidas simétricas mientras que las Medidas de Reducción Proporcional del Error y Proporción de Varianza Explicada se clasifican como asimétricas.

Finalmente la invarianza alude a que los valores de las medidas de asociación son robustos frente a posibles cambios de escala sobre filas y columnas. Estas transformaciones consisten fundamentalmente en multiplicar filas y columnas por constantes con el fin de transformar las frecuencias marginales de la tabla de contingencia. Concretamente las medidas de asociación invariantes a cambios de escala se definen en todos los casos como funciones de los cocientes de ventajas asociados a cada una de las subtablas $2 x 2$ de una tabla $r x c$.

En función de si se requiere o no la descomposición de la tabla de contingencia en distintas tablas $2 x 2$ para la obtención de la medida de asociación es posible caracterizarlas como parciales o globales. Las Medidas de Asociación Globales consisten en un índice simple que describe la intensidad de la asociación, y en variables ordinales la dirección de la misma. Las Medidas de Asociación Parciales constituyen un conjunto de índices construidos a partir de tantas tablas $2 x 2$ como grados de libertad tenga la tabla de contingencia, mediante el análisis de la asociación en cada una de las subtablas de forma independiente. Para efectuar esta partición es necesario fijar una celda de referencia que deberá aparecer en todas las subtablas. Esta forma de realizar la partición de la tabla global fue definida por (Lancaster, 1949), quien demuestra que el estadístico $\chi^{2}$ correspondiente a una tabla de contingencia $r x c$ puede ser segmentado en cualquier caso en tantas componentes como grados de libertad tenga la tabla original.

\section{Medidas de asociación para tablas 2x2}

En el caso de tablas $2 x 2$ se dispone únicamente de un grado de libertad para el análisis de las medidas de asociación e interacción. En consecuencia, 
se trata de un caso muy concreto que se considera aparte en el conjunto de las medidas de asociación en tablas $r x c$. Prácticamente todas las medidas se reducen a funciones derivadas del cociente de ventajas o el coeficiente de correlación de Pearson. Asimismo, cualquier tipo de distinción referente a medidas simétricas o asimétricas desaparece como es evidente en tablas $2 x 2$.

La selección de la medida de asociación más adecuada para tablas $2 x 2$ consiste fundamentalmente en la elección entre el cociente de ventajas o el coeficiente de correlación de Pearson, o alguna función relacionada con estas medidas (Bishop et al., 2007).

\section{Cociente de ventajas}

El Cociente de Ventajas o Razón de Productos Cruzados (Odds Ratio) se define como la el cociente entre la ventaja de la segunda columna para las observaciones de la segunda fila y la misma ventaja para los individuos de la primera fila (Aguilera, 2001). En general, la ventaja w se define como la probabilidad de que ocurra un suceso A con probabilidad p frente a la posibilidad de que no ocurra:

$$
w=\frac{p}{1-p}
$$

Aplicando esta definición al contexto de tablas $2 \times 2$ el Cociente de Ventajas Poblacional se formula como:

$$
\theta=\frac{w_{2}}{w_{1}}=\frac{\frac{p_{2 \mid 1}}{p_{1 \mid 1}}}{\frac{p_{2 \mid 2}}{p_{1 \mid 2}}}=\frac{\frac{p_{12}}{p_{11}}}{\frac{p_{22}}{p_{21}}}=\frac{p_{11} \cdot p_{22}}{p_{12} \cdot p_{21}}
$$

El rango de variación del cociente de ventajas $\theta$ se sitúa en el intervalo $[0, \infty)$. En este rango, cuando $\theta=11$ se considera que ambas variables son independientes. En los casos en que $\theta>1$ y $\theta<1$, se considera que las variables no son independientes y existen relaciones de asociación positiva y negativa, respectivamente (Agresti, 2002).

El cociente de ventajas es invariante ante el intercambio de filas o columnas, aunque un intercambio de solo filas o solo columnas implica que el valor $\theta$, se convierte en $\frac{1}{\theta}$. Dado que el campo de variación es el intervalo $[0, \infty)$, entonces $\theta$ y $\frac{1}{\theta}$ indican igual intensidad de la asociación, pero en direcciones opuestas.

Por otro lado también es invariante ante transformaciones relacionadas con productos de constante. En otras palabras es invariante a cambios de escala en filas, columnas y ambas, de modo que sus valores no se modifican si se multiplica por una constante no nula, una o parte de las filas o columnas, 
o incluso ambas. Si se multiplica la primera y segunda fila por $t_{1}>0 \mathrm{y}$ $t_{2}>0$, respectivamente, y la primera y segunda columna por $s_{1}>0 \mathrm{y}$ $s_{2}>0$, respectivamente y posteriormente se repite la normalización de la medida, resulta (Yule, 1900):

$$
\frac{\left(t_{1} s_{1} p_{11}\right)\left(t_{2} s_{2} p_{22}\right)}{\left(t_{1} s_{2} p_{12}\right)\left(t_{2} s_{1} p_{21}\right)}=\frac{p_{11} p_{22}}{p_{12} p_{21}}=\theta
$$

Asimismo alcanza sus valores extremos 0 y $\infty$, bajo asociación perfecta y estricta, y también bajo asociación perfecta e implícita de tipo II.

El estimador de máxima verosimilitud del cociente ventajas se denomina cociente de ventajas muestral $\hat{\theta}$ y se calcula como:

$$
\hat{\theta}=\frac{n_{11} n_{22}}{n_{12} n_{21}}
$$

Este estimador no es insesgado y, bajo muestreo multinomial completo, tiene la siguiente distribución asintótica normal multivariante:

$$
(\hat{\theta}-\theta) \rightarrow_{n \rightarrow \infty} N\left(0, \hat{\sigma}^{2}(\hat{\theta})\right)
$$

Donde $\hat{\sigma}^{2}(\hat{\theta})$ se obtiene como:

$$
\hat{\sigma}^{2}(\hat{\theta})=\hat{\theta}^{2}\left[\frac{1}{n_{11}}+\frac{1}{n_{12}}+\frac{1}{n_{21}}+\frac{1}{n_{22}}\right]
$$

La presencia de funciones inversas en las frecuencias observadas en la estimación de la varianza implica que esta expresión sólo es aplicable cuando todas las frecuencias observadas son positivas.

En los casos en que las frecuencias observadas en una de las celdas sean cero $n_{i j}=0$ entones el odds ratio toma el valor nulo o $\infty$, y resulta indefinido si esto ocurre en más de una celda. Con el fin de solventar esta dificultad Gart y Zweiful (1967) y Haldane (1955) propusieron el estimador (Gart, 1967; Haldane, 1955), obtenido mediante muestreo multinomial y a partir del modelo de distribución binomial con frecuencias marginales fijas:

$$
\hat{\theta}=\frac{\left(n_{11}+0,5\right)\left(n_{22}+0,5\right)}{\left(n_{12}+0,5\right)\left(n_{21}+0,5\right)}
$$

Este estimador tiene sesgo y error cuadrático medio menor que el estimador máxima verosimilitud $\hat{\theta}$. A partir de dicho estimador se construye el $\log \hat{\theta}$, que igualmente funciona bien en estas circunstancias. Este estimador $\hat{\theta}$ corregido se distribuye asintóticamente de forma normal, al igual que ocurría con el anterior, y el efecto resultante de la adición de la constante 0.5 a cada una de las celdas desaparece cuando $n \rightarrow \infty$ (Agresti, 2002). 
En ocasiones, con el fin de lograr una medida cuyo campo de variación sea simétrico se realiza la siguiente transformación:

$$
\hat{\theta}^{*}=\ln \theta
$$

Cuyos valores están comprendidos entre $(-\infty, \infty)$ de forma que $\hat{\theta}^{*}=0$ indica independencia y $\hat{\theta}^{*}>0$ y $\hat{\theta}^{*}<0$ implican asociación positiva y negativa.

Aunque este resultado se utiliza preferentemente en tablas $2 x 2$ es posible generalizarlo a tablas $r x c$ obteniendo los cocientes de ventajas parciales según la expresión:

$$
\theta=\frac{n_{i j} \cdot n_{r c}}{n_{i c} \cdot n_{r j}}
$$

Siendo

$$
\begin{aligned}
& i=1, \ldots, r-1 \\
& j=1, \ldots, c-1
\end{aligned}
$$

La interpretación de esta medida aplicada a tablas $r x c$ se realiza aplicando el mismo criterio definido para tablas $2 x 2$.

A partir del cociente de ventajas se ha propuesto otras medidas de asociación para tablas $2 x 2$ mediante la aplicación de funciones monótonas crecientes o decrecientes. Puesto que el número de medidas de asociación propuestas siguiendo este criterio es muy elevado, resulta adecuado formular una expresión general para sus varianzas asintóticas.

Si $f(\theta)$ es una función monótona positiva creciente de $\theta$ tal que $f(1)=1$ entonces una medida normalizado de asociación basada en $f(\theta)$ con valor máximo absoluto igual a la unidad se formula como:

$$
g(\theta)=\frac{f(\theta)-1}{f(\theta)+1}
$$

$\mathrm{Su}$ varianza asintótica se obtiene como:

$$
\sigma_{\infty}^{2}[g(\hat{\theta})]=\frac{[1-g(\theta)]^{4}\left[f^{\prime}(\theta)\right]^{2}}{4} \sigma_{\infty}^{2}(\hat{\theta})
$$

Donde $\sigma_{\infty}^{2}(\hat{\theta})$ es la varianza asintótica de $\hat{\theta}$ y $f^{\prime}(\theta)$ es la derivada respecto de $\theta$. 
Medida de asociación general: $Q$ de Yule

La medida de asociación $Q$ de Yule fue propuesta por Yule (1900) en honor al estadístico Quetelet. Su valor poblacional se formula como:

$$
Q=\frac{p_{11} p_{22}-p_{12} p_{21}}{p_{11} p_{22}+p_{12} p_{21}}=\frac{\theta-1}{\theta+1}
$$

El rango de variación de esta medida es el intervalo $[-1,1]$ y toma el valor nulo cuando las variables $A$ y $B$ son independientes. Si y solo si $Q>0$ se considera que existe asociación positiva, y lo mismo ocurre con $Q<0$ en cuanto a la asociación negativa. En los casos en que haya asociación perfecta estricta entonces $Q=1$ si es positiva y $Q=-1$ si es negativa. Sin embargo $Q$ puede tomar ambos valores si se da la circunstancia de que alguna de las probabilidades poblacionales resulta nula.

La $\hat{Q}$ de Yule muestral es el estimador máximo verosímil de la $Q$ de Yule, que se obtiene mediante la expresión:

$$
\hat{Q}=\frac{n_{11} n_{22}-n_{12} n_{21}}{n_{11} n_{22}+n_{12} n_{21}}=\frac{\hat{\theta}-1}{\hat{\theta}+1}
$$

La estimación de su desviación típica asintótica viene dada por:

$$
\hat{\sigma}(\hat{Q})=\frac{1}{2}\left(1-\hat{Q}^{2}\right) \sqrt{\frac{1}{n_{11}}+\frac{1}{n_{12}}+\frac{1}{n_{21}}+\frac{1}{n_{22}}}
$$

Coligación de Yule

A partir de la medida de asociación general formulada anteriormente por Yule, se propone la Medida de Coligación de Yule (1912) como:

$$
Y=\frac{\sqrt{p_{11} p_{22}}-\sqrt{p_{12} p_{21}}}{\sqrt{p_{11} p_{22}}+\sqrt{p_{12} p_{21}}}=\frac{\sqrt{\theta}-1}{\sqrt{\theta}+1}
$$

El rango de variación de esta medida de asociación es el mismo que el de la $Q$ de Yule, el intervalo $[-1,1]$ y su interpretación es similar. Asimismo la estimación de su desviación típica viene dada por:

$$
\hat{\sigma}\left(\hat{Y}^{2}\right)=\frac{1}{2}\left(1-\hat{Y}^{2}\right) \sqrt{\frac{1}{n_{11}}+\frac{1}{n_{12}}+\frac{1}{n_{21}}+\frac{1}{n_{22}}}
$$

En muestras de tamaño elevado tanto $\hat{Q}$ como $\hat{Y}$ se distribuyen de forma normal con media $Q$ y Y respectivamente, de modo que es posible emplear tanto $\hat{\sigma}(\tilde{Q})$ como $\hat{\sigma}(\tilde{Y})$ para obtener los intervalos de confianza para $Q$ y $Y$. Ambas medidas son consistentes puesto que si $\hat{Q}_{1}>\hat{Q}_{2}$ para un par de tablas entonces $\hat{Y}_{1}>\hat{Y}_{2}$. 
En este sentido Goodman y Kruskal (1959) propusieron una medida de asociación $\gamma$ aplicable a tablas $r x c$ en el caso de que ambas variables sean ordinales y se desee conocer la probabilidad de obtener dicho orden en un par de observaciones escogidas aleatoriamente en el conjunto de la población. Esta medida es idéntica a la $\hat{Q}$ de Yule en tablas $2 x 2$ y, de hecho, su interpretación se fundamenta en esta medida de asociación genérica (Bishop et al., 2007).

\section{Coeficiente de correlación de Pearson}

El Coeficiente de Correlación Lineal se define como una medida de asociación lineal entre dos factores dicotómicos, codificados en el intervalo $[0,1]$. Este coeficiente de correlación lineal se define como el cociente entre la covarianza de los factores $A$ y $B\left(S_{A B}\right)$ y el producto de sus desviaciones típicas $S_{A}$ y $S_{B}$.

$$
\hat{r}=\frac{S_{A B}}{S_{A} S_{B}}=\frac{\frac{n_{11} n_{22}-n_{21} n_{12}}{N^{2}}}{\frac{\sqrt{n_{1 \cdot} \cdot n_{2}}}{N} \frac{\sqrt{n_{1} n_{\cdot 2}}}{N}}=\frac{n_{11} n_{22}-n_{21} n_{12}}{\sqrt{n_{1} \cdot n_{2} \cdot} \sqrt{n_{1} n_{\cdot 2}}}
$$

El rango de variación del coeficiente de correlación lineal $r$ oscila en el intervalo $[-1,1]$ de forma que $r=-1$ y $r=1$ indican asociación lineal perfecta y estricta negativa y positiva, respectivamente. En el primer caso este valor implica que $n_{11}=n_{22}=0$ mientras que en el segundo $n_{12}=n_{21}=0$. . Por el contrario, en caso de que $r=0$, se considera que hay ausencia de asociación lineal y por tanto cabe esperar igualmente ausencia de incorrelación lineal.

Como principales características cabe destacar que es invariante en cuanto a la intensidad de la relación ante posibles intercambios de distintos niveles de un mismo factor entre sí, aunque sí cambia la dirección de la misma en coherencia con el cambio. Del mismo modo es invariante ante intercambios de filas y columnas, e igualmente cambia el signo de la dirección si únicamente se cambian sólo filas o sólo columnas (Bishop, 2007). No obstante, no resulta robusto a la presencia de valores anómalos, de modo que en estas circunstancias puede aportar medidas poco representativas de la asociación entre variables. Tampoco aporta buenos resultados en el caso de que la distribución de los puntos no sea lineal. Del mismo modo se ha demostrado que una limitación en el rango de variación de uno de los factores puede tener un efecto contraproducente, en el sentido de que puede incrementar injustificadamente el valor absoluto del coeficiente (Wilcox, 2010).

Adicionalmente es sensible a frecuencias marginales desequilibradas, lo cual puede comprobarse comparando los valores de esta medida en distin- 
tas tablas $2 x 2$ con frecuencias marginales distintas y similares. Finalmente, en términos generales alcanza valores inferiores a los de la $\hat{Q}$ de Yule, con excepción de los casos en que los factores son independientes o están perfectamente asociados. Concretamente Yule señala que, si se estandariza la tabla $2 x 2$, entonces se eliminan los efectos de las frecuencias marginales desequilibradas y los resultados son equivalentes a los obtenidos mediante medidas de asociación formuladas a partir de cociente de ventajas (Yule, 1912). En este aspecto, si no se realiza esta estandarización y distintas tablas $2 x 2$ presenta el mismo cociente de ventajas, la tabla con mayor desequilibrio en sus frecuencias marginales muestra el menor coeficiente de correlación.

El coeficiente $r$ tal y como se ha formulado anteriormente corresponde al estimador de máxima verosimilitud bajo muestreo multinomial. La varianza asintótica de este estimador se formula como:

$\sigma^{2}(\hat{r})=\frac{1}{N}\left[1-\hat{r}^{2}+\left(\hat{r}+\frac{1}{2} \hat{r}^{3}\right) \frac{\left(n_{1 \cdot}-n_{2 \cdot}\right)\left(n_{\cdot 1}-n_{\cdot 2}\right)}{\sqrt{n_{1} \cdot n_{2} \cdot n_{\cdot 1} n_{\cdot}}}-\frac{3}{4} \hat{r}^{2}\left(\frac{\left(n_{1} \cdot-n_{2 \cdot}\right)^{2}}{n_{1} \cdot n_{2} \cdot}+\frac{\left(n_{\cdot 1}-n_{\cdot 2}\right)^{2}}{n_{\cdot 1} n_{\cdot 2}}\right)\right]$

Esta expresión fue obtenida por Yule (1912). Bajo la hipótesis nula de independencia en filas y columnas, esta expresión se reduce a $\frac{1}{N}$. No obstante, esta expresión resulta más adecuada para el cálculo de los intervalos de confianza del estimador $\hat{r}$ En caso de la tabla sea completamente simétrica, de forma que $p_{1} .=p_{2} .=p_{\cdot 1}=p_{.2}$ entonces la expresión para la varianza asintótica del estimador $\hat{r}$ se reduce a:

$$
\sigma^{2}(\hat{r})=\frac{1-\hat{r}^{2}}{N}
$$

\section{Medidas de asociación basadas en el estadístico $\chi^{2}$}

Generalmente se evita utilizar el estadístico $\chi^{2}$ como medida de asociación entre dos factores $A$ y $B$ debido a que su valor es muy sensible al tamaño de la muestra. En lugar de este estadístico se emplean otros derivados que guardan relación con el mismo, de donde proviene el nombre de estas medidas. A pesar de que estas medidas sirven para comparar la magnitud de la asociación entre distintas tablas, también presentan determinados inconvenientes como sensibilidad a la existencia de frecuencias marginales desequilibradas, dependencia de las dimensiones de la tabla así como cierta dificultad de interpretación en términos probabilísticos. Por todo ello existe la tendencia de emplearlas exclusivamente como aproximaciones al coeficiente de correlación lineal y sustituirlas por las medidas basadas en la reducción proporcional del error.

Las distintas Medidas de Asociación Basadas en el Estadístico $\chi^{2}$ pueden interpretarse en términos de varianza entre las frecuencias observadas y 
esperadas, medida en el intervalo [0,1]. Esta interpretación resulta especialmente útil para comparar distintas tablas.

Medida $\Phi^{2}$ de Pearson

El Coeficiente $\Phi^{2}$ de Pearson (1900) se define como:

$$
\Phi^{2}=\frac{\chi^{2}}{N}
$$

Cuyo rango de variación se encuentra en el intervalo [0,1] de modo que $\Phi^{2}=0$ indica independencia entre ambos factores y $\Phi^{2}=1$ indica asociación perfecta y estricta. Al estar limitados sus valores a este rango de variación no proporciona la dirección de la asociación. Asimismo únicamente se alcanza el valor de la unidad en condiciones de asociación perfecta estricta, pero no lo hace cuando existe asociación perfecta implícita de tipo I o II. Es decir, que nunca podrá alcanzar el límite superior fijado en la unidad en caso de no aplicarse sobre tablas cuadradas.

Por otro lado, en tablas $2 x 2$ coincide con el cuadrado del coeficiente de correlación lineal descrito anteriormente y de hecho, es una medida muy sensible a frecuencias marginales desequilibradas tal y como ocurría con este coeficiente. Esto implica que, bajo estas circunstancias, los resultados pueden verse distorsionados de modo que no resultan representativos del grado de asociación entre variables.

En contraste con estas limitaciones, al tratarse de una medida simétrica, su valor no depende de qué factor se considere explicado y explicativo. Esta característica es una de las principales ventajas de este coeficiente, así como su facilidad de cálculo.

\section{Coeficiente de contingencia}

El Coeficiente de Contingencia se propuso como forma de solventar los inconvenientes asociados al coeficiente $\Phi^{2}=1$ de Pearson (1904). Este coeficiente de contingencia se define como:

$$
C=\sqrt{\frac{\chi^{2}}{\chi^{2}+N}}=\sqrt{\frac{\frac{\chi^{2}}{N}}{\frac{\chi^{2}}{N}+1}}=\sqrt{\frac{\Phi^{2}}{\Phi^{2}+N}}
$$

El rango de variación de este coeficiente también se ubica en el intervalo $[0,1]$ de modo que tampoco alcanza el valor máximo aunque exista asociación perfecta si no se aplica a tablas cuadradas. Su valor depende el número de filas y columnas de la tabla y cuando ésta es cuadrada el máximo valor que 
puede alcanzar viene dado por $\sqrt{\frac{r-1}{r}}$, siendo este límite superior desconocido en tablas rectangulares. Por esta razón se define un nuevo valor de esta medida, denominado coeficiente de contingencia ajustado. La ventaja de este coeficiente es que proporciona una idea del verdadero grado de asociación, al evaluar la discrepancia entre el valor obtenido y el máximo potencialmente alcanzable. Este coeficiente ajustado se formula mediante la expresión:

$$
C_{A}=\frac{C}{C_{\max }}
$$

\section{$T$ de Tshuprow}

En cambio, aunque la medida $T$ de Tshuprow oscila igualmente en el intervalo $[0,1]$ y en ningún caso alcanza el valor máximo en tablas rectangulares en las que se dé asociación implícita, sí lo alcanza en tablas cuadradas en condiciones de asociación perfecta implícita. Esta medida se formula como:

$$
T=\sqrt{\frac{\frac{\chi^{2}}{N}}{\sqrt{(r-1)(c-1)}}}=\sqrt{\frac{\Phi^{2}}{\sqrt{(r-1)(c-1)}}}
$$

En tablas $r x c$ el máximo valor que puede alcanzar $\Phi^{2}$ viene dado por $\min ((r-1)(c-1))$

Y puesto que

$$
\sqrt{(r-1)(c-1)} \geq \min ((r-1)(c-1))
$$

Siempre ocurrirá que $T<1$ (Bishop et al., 2007).

Esta medida está relacionada con la siguiente, V de Cramer (Cramer, 1946), mediante la expresión:

$$
T^{2}=\frac{C^{2}}{\left(1-C^{2}\right) \sqrt{(r-1)(c-1)}}
$$

\section{$V$ de Cramer}

Esta medida se formula como:

$$
V=\sqrt{\frac{\Phi^{2}}{m}}=\sqrt{\frac{\chi^{2}}{N \cdot m}}
$$

Donde $m=\min (r-1 ; c-1)$

Esta medida alcanza la unidad cuando existe asociación perfecta, independientemente del número de filas y columnas de la tabla, es decir, tanto 
en el caso de asociación perfecta estricta como en el de asociación perfecta implícita. Asimismo $V=0$ si y solo si $A$ y $B$ son independientes. Adicionalmente si la tabla es cuadrada $V=T$ y si no se dan estas condiciones, entonces $V>T$. En tablas $2 x 2$ se verifica que el valor de esta medida elevada al cuadrado coincide con el cuadrado del coeficiente de correlación lineal.

Asimismo Cramer propone una normalización de esta medida mediante la expresión:

$$
V=\sqrt{\frac{\Phi^{2}}{\min ((r-1)(c-1))}}
$$

Esta normalización se propone con el fin de definir una medida que alcance el valor de la unidad en condiciones de asociación perfecta en cualquier tabla $r x c$, no necesariamente cuadrada.

Igualmente esta medida se relaciona con la anterior, mediante la expresión:

$$
C^{2}=\frac{T^{2} \sqrt{(r-1)(c-1)}}{1+T^{2} \sqrt{(r-1)(c-1)}}
$$

En los casos en que $r>2$ y $c>2$ ocurre que $V \geq T$

\section{Medidas de asociación basadas en la Reducción Proporcional del Error (PRE)}

La interpretación de las medidas de asociación basadas en el estadístico $\chi^{2}$ no resulta muy intuitiva ya que aporta información similar a la que se obtendría mediante números índice sobre el grado de asociación entre distintas tablas de contingencia, de forma relativa. Por ello Goodman y Kruskal propusieron las Medidas De Asociación Basadas en la Reducción Proporcional del Error (PRE) como medida con una interpretación más clara en términos de probabilidades.

Estas medidas, propuestas por Goodman y Kruskall (1954, 1959, 1963, 1972), son asimétricas y se basan en la capacidad de una variable para predecir los niveles de la otra. Considerando que $A$ es el factor explicativo y $B$ el factor explicado, es posible realizar esta predicción de dos formas. La primera de ellas es al azar, o suponiendo que no se dispone de información sobre la posible asociación entre ambas variables, es decir, asumiendo que son estadísticamente independientes por lo que la única forma de realizar la predicción es sin tener en consideración el nivel de clasificación del factor $A$. Se denota como $p_{1}$ a la probabilidad de error mediante esta predicción de este modo. Por el contrario, si se dispone de información previa sobre la 
relación entre $A$ y $B$, es posible tener en consideración el nivel del clasificación en el factor $A$ para predecir el nivel que le corresponde a la observación en el factor $B$. Se denota como $p_{2}$ a la probabilidad de error mediante esta predicción.

A partir de ambas probabilidades se define la medida de reducción proporcional del error como:

$$
P R E=\frac{p_{1}-p_{2}}{p_{1}}
$$

De modo que se mide la reducción en el error producida al tener en cuenta la información previa sobre la relación entre $A$ y $B$. Por tanto, esta medida representa la mejora relativa en la predicción del nivel del factor $B$ cuando la categoría del nivel del factor $A$ es conocida, respecto a cuando es desconocida. Es decir, cuánto disminuye la probabilidad de cometer error cuando la predicción se realiza con información previa sobre los niveles del factor $A$ respecto a cuando se realizar al azar.

Cuando los factores $A$ y $B$ son independientes, se deduce que $A$ no proporciona ninguna información sobre $B$, por lo que $p_{1}>p_{2}$ resultando que $0 \leq P R E \leq 1$. Concretamente cuando los factores $A$ y $B$ están perfectamente asociados $p_{2}=0$ y por tanto $P R E=1$.

Así, definiendo el factor $B$ como factor explicado y el factor $A$ como explicativo, se define el índice:

$$
\lambda_{B}=\frac{\sum_{i=1}^{r} \max _{j} \cdot n_{i j}-\max _{j} \cdot n_{\cdot j}}{N-\max _{j} \cdot n_{\cdot j}}
$$

Del mismo modo, considerando el factor $A$ como factor explicado y el factor $B$ como explicativo, se define el índice:

$$
\lambda_{A}=\frac{\sum_{j=1}^{c} \max _{i} \cdot n_{i j}-\max _{i} \cdot n_{i}}{N-\max _{i} \cdot n_{i} .}
$$

En el primer caso, definiendo el factor $B$ como factor explicado y el factor $A$ como explicativo, $\lambda_{B}=0$ si y solo sí no es posible determinar el valor del factor $B$ a partir del factor $A$, mientras que $\lambda_{B}=1$ si y solo sí el valor del factor $A$ especifica completamente el valor del factor $B$. Asimismo esta medida es invariante frente a permutaciones entre las distintas categorías de cada uno de los factores.

Por su propia definición ambas medidas son asimétricas, por lo que resultan poco adecuadas cuando no se ha definido qué variables son explicativas y explicadas sino que simplemente se pretende analizar la dirección y el grado de asociación entre los factores. Esta limitación se ha solventando fácilmente 
mediante la definición de una versión simétrica de esta medida mediante la siguiente expresión que recibe el nombre de Medida Lambda de Goodman y Kruskal simétrica:

$$
\lambda=\frac{\sum_{i=1}^{r} \max _{j} \cdot n_{i j}+\sum_{j=}^{c} \max _{i} \cdot n_{i j}-\max _{i} \cdot n_{i \cdot}-\max _{j} \cdot n_{\cdot j}}{2 N-\max _{i} \cdot n_{i \cdot}-\max _{j} \cdot n_{\cdot j}}
$$

La principal ventaja de este tipo de medidas es la facilidad de interpretación y la posibilidad de emplear medidas asimétricas en determinados contextos, por ejemplo cuando se plantean relaciones temporales o causales entre variables.

\section{Medidas de asociación basadas en la Proporción de Varianza Explicada}

Propuestas por Light y Margolin (1971). Este tipo de medidas son asimétricas por su propia definición, y describen la reducción proporcional en varianza desde la distribución marginal de la respuesta a sus distribuciones condicionales respecto de los niveles de la variable explicativa.

Considerando dos factores $A$ y $B$, donde $B$ es la variable explicada y $A$ la variable explicativa, entonces $V(B)$ se define como una medida de variación para la distribución marginal $p_{\cdot j}: j=1, \ldots, c$ de $B$ y $V\left(B \mid A_{i}\right)$ la misma medida de variación para la distribución condicional $p_{j \mid i}: j=1, \ldots, c$ de $B$ al nivel $A_{i}$ de la variable explicativa $A$. En términos generales, una medida de reducción de proporción de varianza explicada se define como:

$$
\frac{V(B)-E[V(B \mid A)]}{V(B)}
$$

Donde $E[V(B \mid A)]$ es la esperanza de la variación condicional respecto a la distribución marginal $p_{i}: j=1, \ldots, r$ dada por:

$$
E[V(B \mid A)]=\sum_{i} V\left(B \mid A_{i}\right) p_{i}
$$

De forma que la medida de variación utilizada determinará la medida de proporción de varianza explicada en la relación entre los factores $A$ y $B$. Gini (1912) definió la variación total para una variable cualitativa $X$ con $I$ categorías posibles y tamaño de muestra $N$ :

$$
\text { Variacion }=\frac{N}{2}-\frac{1}{2 N} \sum_{i=1}^{I} x_{i}^{2}
$$


Donde $x_{i}$ es el número de observaciones en la categoría i-ésima tal que $i=1, \ldots, I$ y $\sum_{i} x_{i}=N$

Light y Margolin (1971) señalaron que esta medida de variación propuesta por Gini tenía dos propiedades fundamentales. En primer lugar, la variación se minimiza hasta alcanzar el valor nulo si y solo si el conjunto de las $\mathrm{N}$ observaciones cae en la misma categoría. Asimismo, la variación se maximiza cuando las respuestas se distribuyen entre todas las categorías tan equilibradamente como sea posible. Una tercer propiedad que deriva directamente de su propia definición es que la agregación de dos categorías en una nueva no puede incrementar el nivel de variación, y de hecho lo reduce a menos que una de las categorías combinadas no corresponda a ninguna respuesta (Bishop et al., 2007).

Principalmente existen dos medidas, ambas de carácter asimétrico: La medida Tau de Goodman y Kruskal asimétrica y el coeficiente de incertidumbre asimétrico. Este último coeficiente se ha seleccionado como medida más representativa entre las medidas pertenecientes a este grupo por su facilidad de cálculo mediante el paquete estadístico SPSS (versión 16) y la equivalencia de los resultados que aporta respecto a las restantes.

\section{Coeficientes de incertidumbre}

Propuesto por Theil (1970), se formula como:

$$
V(B)=\sum_{j} p_{\cdot j} \log p_{\cdot j}
$$

La variación condicional en cada fila resulta:

$$
V\left(B \mid A_{i}\right)=\sum_{j} p_{\cdot j \mid i} \log p_{\cdot j \mid i}
$$

A partir de ambas expresiones se obtienen las expresiones de los coeficientes de incertidumbre asimétricos considerando cada uno de los factores $A$ y $B$ como factores explicativos.

$$
\begin{aligned}
U_{B} & =-\sum_{j} \frac{n_{\cdot j}}{n} \log \frac{n_{\cdot j}}{n} \\
U_{A} & =-\sum_{j} \frac{n_{i \cdot}}{n} \log \frac{n_{i} \cdot}{n}
\end{aligned}
$$

Asimismo, para los casos en que no es posible distinguir entre variables explicadas y explicativas, se define una combinación de ambas medidas de incertidumbre asimétricas: 


$$
\hat{U}_{B \mid A}=\frac{U_{B}-U_{A}-U_{A B}}{\frac{U_{B}+U_{A}}{2}}
$$

Esta expresión también puede presentarse sin la normalización $\frac{1}{2}$ en el denominador, incluida en este caso para que el coeficiente oscile entre 0 y 1 , al igual que los coeficientes de incertidumbres asimétricos.

En términos generales, el principal inconveniente de este tipo de medidas es determinar a partir de qué punto su valor se considera suficientemente elevado como para indicar que éste indica asociación fuerte, ya que su valor tiende a disminuir al incrementarse el número de categorías de la variable explicada.

En resumen, las medidas basadas en el estadístico $\chi^{2}$ son simétricas y tienen la ventaja de que evalúan el nivel de significación de la asociación entre las variables. Como contrapartida resultan más difíciles de interpretar. Por otro lado las medidas basadas en la reducción proporcional del error son útiles para analizar un tipo diferente de asociación, la predictibilidad en sentido asimétrico. Aunque dos variables no sean independientes, puede que una de ellas capacidad nula de predecir los valores de la otra. De hecho, es posible que una pueda predecir el valor de la otra, pero no suceda lo mismo al contrario. Por ello no se puede definir un valor del estadístico $\chi^{2}$ común para ambas relaciones. Las medidas de proporción de varianza explicada aportan otra perspectiva a la interpretación de resultados, al focalizarse sobre la varianza explicada, aunque dicha interpretación está relacionada igualmente con la predicción que realizan las medidas anteriores.

Así, no existe una única medida superior al resto y aplicable en cualquier circunstancia. Cada una de ellas ha sido formulada con diferentes objetivos y su selección dependerá del objetivo de investigación planteado.

\section{Análisis de correspondencias}

El objetivo de Análisis de Correspondencias es la reducción de la dimensión de los datos conservando la máxima información posible. Se trata de una técnica de análisis muy similar al Escalamiento Multidimensional, aunque existe una diferencia fundamental entre ambos métodos de análisis. Mientras que el escalamiento multidimensional el mapa perceptual obtenido mediante escalamiento multidimensional muestra las distancias percibidas entre diversos sujetos u objetos, el mapa obtenido mediante análisis de correspondencias muestra las distancias entre los distintos niveles de dos o más variables no métricas (Uriel y Aldás, 2005). 
Su máximo desarrollo se producto gracias al trabajo del investigador francés Benzécri $(1980,1992)$. Sin embargo su aplicación en el ámbito de la investigación educativa todavía es muy reducida y se encuentran localizadas en publicaciones muy concretas (Aliaga, 1999).

\section{Consideraciones generales}

\section{Perfiles}

El Análisis de Correspondencias Simple se plantea sobre una tabla de contingencia bidimensional $K(n x p)$ y permite comprender los principios básicos aplicados posteriormente en el Análisis de Correspondencias Múltiple. Entre las diversas notaciones para la descripción de las relaciones características en esta técnica multivariante, se ha optado por trabajar con la definida por Lebart, Morineau y Warwick (1984) y Lebart et al. (1995). Esta notación designa cada uno de los elementos de la tabla de contingencia original como se muestra a continuación:

\begin{tabular}{c|ccccc} 
& 1 & $\ldots$ & $j$ & $\ldots$ & $J$ \\
\hline 1 & $k_{11}$ & $\ldots$ & $k_{1 j}$ & $\ldots$ & $k_{1 J}$ \\
$\ldots$ & $\ldots$ & $\ldots$ & $\ldots$ & $\ldots$ & $\ldots$ \\
$i$ & $k_{i 1}$ & $\ldots$ & $k_{i j}$ & $\ldots$ & $k_{i J}$ \\
$\ldots$ & $\ldots$ & $\ldots$ & $\ldots$ & $\ldots$ & $\ldots$ \\
$I$ & $k_{I 1}$ & $\ldots$ & $k_{I j}$ & $\ldots$ & $k_{I J}$
\end{tabular}

Las distribuciones marginales por filas y columnas se expresan como:

$$
\begin{aligned}
& K(i)=\sum K(i, j), j \epsilon p \\
& K(j)=\sum K(i, j), i \epsilon n
\end{aligned}
$$

Ambas distribuciones marginales equivalen a las masas de los puntos - fila $K(i)$ y puntos - columna $K(j)$. La masa asociada a cada elemento (categoría de la variable fila o de la variable columna) mide la importancia relativa de dicho elemento. En términos probabilísticos se expresan como:

$$
\begin{aligned}
& f_{i}=\frac{K(i)}{K}, \forall i \epsilon n \\
& f_{j}=\frac{K(j)}{K}, \forall j \epsilon p
\end{aligned}
$$

A partir de las cuales se definen los Sistemas de Masas:

$$
\begin{aligned}
& f_{n}=f_{i}, i \epsilon n \\
& f_{p}=f_{j}, j \epsilon p
\end{aligned}
$$


La suma de las casillas de la tabla cumple la propiedad:

$$
K=\sum K(i, j), i \epsilon n, j \epsilon p=\sum K(i), i \epsilon n=\sum K(j), j \epsilon p
$$

A partir de la cual se define la expresión de las frecuencias relativas:

$$
f_{i j}=\frac{K(i, j)}{K}
$$

Así como las expresiones de los Perfiles - Fila y Perfiles - Columna:

$$
\begin{gathered}
\text { Perfil-fila }: f_{\cdot j}=\sum_{i=1}^{n} f_{i j}=\frac{K(i, j)}{K(j)} \\
\text { Perfil-columna }: f_{i} .=\sum_{j=1}^{p} f_{i j}=\frac{K(i, j)}{K(i)}
\end{gathered}
$$

La formulación de estos perfiles se debe a que en la práctica resulta más práctico trabajar con la matriz de frecuencias relativas $F$, cuyo elemento genérico se representa por $f_{i j}$, y que consta de las mismas dimensiones $n x p$ de la matriz de frecuencias absolutas $K$.

\section{Hipótesis de independencia}

Como se ha expuesto anteriormente en la sección sobre análisis de datos cualitativos, el contraste de independencia clásico basado en el estadísto $\chi^{2}$ propuesto por Pearson (1900) para tablas de contingencia bidimensionales permite evaluar las diferencias entre proporciones según las hipótesis:

$$
\begin{aligned}
& H_{0}: p_{i j}=p_{i \cdot p \cdot j}, \forall i y j \\
& H_{1}: p_{i j} \neq p_{i \cdot p_{\cdot j}, \not \forall i y j}
\end{aligned}
$$

Hipótesis que pueden expresarse en términos de los perfiles - fila y perfiles - columna.

En efecto, dado j el perfil - fila se puede formular como:

$$
f_{\cdot j}=\frac{f_{i j}}{f_{i}}
$$

El Perfil Medio de Filas es la media ponderada de los perfiles fila, donde los pesos son precisamente las masas de cada perfil fila. Y del mismo modo, dado i, se puede formular el Perfil Medio de Columnas como la media ponderada de los perfiles columna, donde los pesos son precisamente las masas de cada perfil columna: 


$$
f_{i \cdot}=\frac{f_{i j}}{f_{\cdot j}}
$$

Así, el análisis de las similitudes entre perfiles permite estudiar la relación de dependencia o independencia entre las dos variables nominales de la tabla de contingencia, de forma similar al planteamiento del contraste de independencia $\chi^{2}$.

\section{Definición de subespacios}

El conjunto de todos los puntos - fila forma una nube de $n$ puntos en el espacio de las $p$ columnas. Las coordenadas de cada punto - fila $i$ en el espacio $R^{p}$ vienen dadas por la expresión:

$$
\frac{f_{i j}}{f_{i} .}, j=1,2, \ldots, p
$$

Puesto que $\sum_{j=1}^{n} \frac{f_{i j}}{f_{i .}}=1$, los $n$ puntos - fila se sitúan en un subespacio formado por $p-1$ dimensiones. El centro de gravedad de esta nube se sitúa en el punto medio de los puntos - fila y coincide con el perfil medio de filas:

$$
\sum_{j=1}^{n} f_{i} \cdot \frac{f_{i j}}{f_{i} .}=f_{\cdot j}
$$

Del mismo modo, el conjunto de los $p$ puntos - columna constituye una nube de $p$ puntos en el espacio de las $n$ líneas. Las coordenadas de cada punto - columna j en el espacio $R^{n}$ vienen dadas por la expresión:

$$
\frac{f_{i j}}{f_{\cdot j}}, i=1,2, \ldots, n
$$

Puesto que $\sum_{i=1}^{p} \frac{f_{i j}}{f_{\cdot j}}=1, \operatorname{los} n$ puntos - columna se sitúan en un subespacio formado por $n-1$ dimensiones. El centro de gravedad de esta nube se sitúa en el punto medio de los puntos - columna y coincide con el perfil columna medio:

$$
\sum_{i=1}^{p} f_{i} \cdot \frac{f_{i j}}{f_{\cdot j}}=f_{i} .
$$

En la definición de ambos subespacios $R^{p}$ y $R^{n}$ la expresión de las coordenadas asigna la misma importancia a todas las categorías de las variables nominales. Por ello, es necesario dividir dichas coordenadas por la masa correspondiente a cada punto con el fin de ponderar cada una de las categorías en función de sus frecuencias en la tabla de frecuencias absolutas. 


\section{Análisis de Correspondencias Simples}

\section{Selección de la medida de distancia}

Una vez definidos los subespacios en los que se proyectan los puntos - fila y puntos - columna se plantea cuál es la medida de distancia más adecuada para medir distancias entre puntos. El empleo de la distancia euclídea habitual no resulta apropiada en este caso ya que no incluye en su formulación las frecuencias totales de cada categoría. Por ello, en caso de utilizarla para evaluar las distancias entre puntos pertenecientes a las filas $i$ e $i^{\prime}$ únicamente reflejaría la diferencia entre las frecuencias marginales $K(i)$ y $K\left(i^{\prime}\right)$. Es decir, que sus resultados estarían afectados por el efecto tamaño de las frecuencias marginales. Esta distancia se formula para los puntos fila mediante la expresión:

$$
d^{2}\left(i, i^{\prime}\right)=\sum_{j=1}^{p}\left(\frac{f_{i j}}{f_{i} .}-\frac{i^{\prime} j}{f_{i^{\prime}} .}\right)^{2}
$$

Por su propia definición el empleo de esta distancia no resulta apropiada en este análisis ya que considera que todas las columnas tienen la misma importancia. Esta equivalencia de pesos no es real ya que el perfil de cada columna está referido a un total marginal diferente. Por ello, la medida seleccionada para la representación de los perfiles debe asignar mayor importancia a aquellos que tengan mayor masa. Igualmente se aplica con la medida de distancia utilizada para medir las distancias entre puntos - fila.

Por el contrario la distancia $\chi^{2}$ entre dos perfiles - fila, es equivalente a calcular la distancia euclídea ordinaria entre esos dos perfiles, pero ponderando cada una de las componentes por el perfil medio de filas $f_{\cdot j}$.

La distancia $\chi^{2}$ para los puntos - columna se define equivalentemente como:

$$
d^{2}\left(i, i^{\prime}\right)=\sum_{i=1}^{n} \frac{1}{f_{i} \cdot}\left(\frac{f_{i j}}{f_{j} .}-\frac{i j^{\prime}}{f_{\cdot j^{\prime}}}\right)^{2} ; j, j^{\prime} \epsilon p
$$

Del mismo modo, calcular la distancia $\chi^{2}$ entre dos perfiles - columna, es equivalente a calcular la distancia euclídea ordinaria entre esos dos perfiles, pero ponderando cada una de las componentes por el perfil medio de columnas $f_{i}$.

De esta forma se da la misma importancia a todas las columnas en la distancia $\chi^{2}$ definida para los puntos - fila, y en todas las filas en la distancia $\chi^{2}$ definida para los puntos - columna. Esta definición nos permite trabajar 
con perfiles así como tener en cuenta la ponderación de las dimensiones del espacio.

Así, cuando este estadístico toma un valor de magnitud no significativo no existen evidencias para rechazar la hipótesis nula de independencia, planteada de forma similar al contraste de independencia original. En tal no hay razones para pensar en una heterogeneidad entre los perfiles en relación a la otra variable. Por tanto los perfiles son similares y consecuentemente también son similares a su perfil medio, ya sea de filas o de columnas. En otras palabras, cuando los perfiles sean similares la distancia $\chi^{2}$ entre ellos y entre el perfil medio será pequeña, por lo que se aceptará la hipótesis nula de homogeneidad (Peña, 2002).

La distancia $\chi^{2}$ posee la propiedad de la equivalencia distribucional, consistente en que las distancias entre filas no se alteran si se fusionan las columnas $j$ y $j^{\prime}$ de perfil semejante (Joaristi y Lizasoain, 2000). Así, la propiedad de equivalencia distribucional permite agregar dos categorías de una misma variable con dos perfiles idénticos en una nueva categoría cuya masa sea la suma de las dos anteriores, sin que ello implique alterar las categorías de ninguna de las variables implicadas. La principal aportación de esta propiedad es que aporta robustez frente a posibles arbitrariedades en la codificación de las categorías de las variables. Esto implica que el hecho de fusionar o desagregar varias categorías de una misma variable nominal, asociadas a un mismo fenómeno, y por tanto fuertemente correlacionadas, no altera los resultados del análisis. En contraposición, la distancia euclídea no posee esta propiedad y de hecho, si calculáramos las distancias entre categorías a partir de la tabla original de frecuencias absolutas $K(i, j)$ aplicando la formula de la distancia euclídea usual, las modalidades correlacionadas de una variable aportarían varias veces la misma información a las distancias entre las categorías de la otra variable. Esta propiedad es especialmente útil en el análisis de tablas de grandes dimensiones, en las que una reorganización de las categorías de las variables contribuye a facilitar la interpretación de los resultados (Clausen, 1988).

\section{Análisis de la tabla de contingencia simple}

El objetivo de esta fase es encontrar los ejes principales, o de máxima inercia en ambas nubes de puntos para representar gráficamente las proximidades entre puntos respecto a dichos ejes. Por su propia definición los ejes principales de inercia absorben el máximo de inercia de cada nube de puntos. Así, el principal objetivo de esta técnica multivariante es explicar la máxima inercia posible a través del primer eje. El segundo eje explica el máximo de la inercia restante y así sucesivamente (Greenacre, 2008). 
Para ello se toman como referencia los centros de gravedad de cada una de las nubes de puntos, pero descartando el eje factorial que relaciona el centro de gravedad con el origen. Este eje se asocia con el valor propio igual a la unidad, denominado Valor Propio Trivial. Esta forma de proceder es equivalente al análisis tomando directamente el origen como punto de referencia, siempre y cuando se tenga en cuenta la consideración anterior (Lebart et al., 1995).

Se definen las siguientes matrices:

1. Matriz diagonal de la distribución marginal de las filas: $D_{n}=\left[f_{i}\right]$

2. Matriz diagonal de la distribución marginal de las columnas: $D_{p}=\left[f_{j}\right]$

Como señala Lebart, en primer lugar se calcula el eje de inercia máximo de la nube de puntos - fila en el espacio de los puntos - columna $R^{p}$ Este eje de inercia pasa por el origen $O$ y se genera por un vector unitario $u$ a través de la métrica $D_{p}^{-1}$ Esto implica maximizar la suma ponderada de los cuadrados de las proyecciones sobre el eje:

$$
\operatorname{Max}_{u} \sum_{i} f_{i} \cdot d^{2}(i, O)
$$

Lo cual implica maximizar la expresión:

$$
u^{\prime} D_{p}^{-1} F^{\prime} D_{n}^{-1} F D_{p}^{-1}
$$

Con la restricción:

$$
u^{\prime} D_{p}^{-1} u=1
$$

$u$ es entonces el vector propio asociado al mayor autovalor distinto de la unidad de la matriz:

$$
S=F^{\prime} D_{n}^{-1} F D_{p}^{-1}
$$

Del mismo modo, en el espacio de los puntos - fila $R^{n}$ se maximiza la expresión:

$$
v^{\prime} D_{n}^{-1} F D_{p}^{-1} F^{\prime} D_{n}^{-1} v
$$

Con la restricción:

$$
v^{\prime} D_{n}^{-1} v=1
$$

$v$ es entonces el vector propio asociado al mayor autovalor distinto de la unidad de la matriz: 


$$
T=F^{\prime} D_{p}^{-1} F D_{n}^{-1}
$$

Tanto en el conjunto de autovalores obtenidos en el espacio de los puntos - fila como en el de los puntos - columna se descarta el autovalor trivial de valor igual a la unidad y su vector propio asociado. De este modo se analizan los autovalores restantes no nulos y sus vectores propios correspondientes.

Los ejes factoriales en el espacio de los puntos - columna $R^{p}$ los ejes factoriales vienen dados por la expresión:

$$
T v_{\alpha}=\lambda_{\alpha} v_{\alpha}
$$

A partir de estas expresiones se obtienen las coordenadas factoriales, que en el espacio de los puntos - columna $R^{p}$ se expresan en la matriz $\Psi_{\alpha}$ :

A partir de estas expresiones se obtienen las coordenadas factoriales, que en el espacio de los puntos - columna $R^{p}$ se expresan en la matriz $\Phi_{\alpha}$ :

$$
\Psi_{\alpha}=D_{n}^{-1} F D_{p}^{-1} u_{\alpha}
$$

Cuyo término genérico viene dado por:

$$
\psi_{\alpha i}=\sum_{j=1}^{p} \frac{f_{i j}}{f_{i \cdot} \cdot f_{\cdot j}} u_{\alpha j}
$$

Equivalentemente las coordenadas factoriales en el espacio de los puntos - fila $R^{n}$ se obtienen como:

$$
\Phi_{\alpha}=D_{p}^{-1} F^{\prime} D_{n}^{-1} v_{\alpha}
$$

Cuyo término genérico viene dado por:

$$
\phi_{\alpha j}=\sum_{i=1}^{n} \frac{f_{i j}}{f_{i \cdot f} \cdot j} v_{\alpha i}
$$

Mediante el cálculo de la esperanza matemática, se obtiene que las coordenadas factoriales están centradas en el origen:

$$
E(\Psi)=E(\Phi)=\sum_{i=1}^{n} f_{i} \Psi_{\alpha i}=\sum_{j=1}^{p} f_{\cdot j} \Phi_{\alpha j}=0
$$

Y tienen una varianza igual a $\lambda_{\alpha}$

$$
\operatorname{Var}(\Psi)=\operatorname{Var}(\Phi)=\sum_{i=1}^{n} f_{i} \Psi_{\alpha i}^{2}=\sum_{j=1}^{p} f_{\cdot j} \Phi_{\alpha j}^{2}=\lambda_{\alpha}
$$

La varianza se interpreta en la mecánica newtoniana como la inercia de los puntos respecto del origen de coordenadas, calculada como una suma 
ponderada de las distancias de los puntos a otro de referencia, en este caso el origen.

\section{Inercia}

Se define la Inercia, o Varianza, como la suma ponderada de la masa de cada punto por su distancia al punto de referencia elevada al cuadrado:

$$
I=\sum_{i=1}^{n} f_{i} \cdot d^{2}(i, G)=\sum_{j=1}^{p} f_{\cdot j} d^{2}(i, G)=\sum_{j=1}^{p} \sum_{i=1}^{n}\left(\frac{f_{i j}-f_{i \cdot} \cdot f_{\cdot j}}{f_{i \cdot} \cdot f_{\cdot j}}\right)^{2}
$$

Se puede interpretar como la media ponderada de las distancias $\chi^{2}$ entre los perfiles fila y su perfil medio. O, alternativamente, se puede definir del mismo modo para los perfiles columna.

La inercia aporta información sobre el grado de asociación entre variables de forma que si la inercia es baja se considera que existe poca asociación y si es alta si el grado de asociación es elevado. Así, constituye un indicador de la dispersión de la nube y mide el grado de relación entre variables. De este modo la inercia será alta cuando los perfiles presenten grandes desviaciones en relación a su perfil medio mientras que será baja cuando se encuentren cerca del perfil medio. Si todos los perfiles fueran idénticos, y por tanto todos se hallaran situados sobre el perfil medio, las distancias $\chi^{2}$ serían nulas y también lo sería la inercia total. Por otro lado, la inercia máxima se obtendría cuando todos los perfiles se hallaran exactamente en los vértices del espacio de punto, es decir, cuando la dispersión entre los puntos fuera máxima. En tal caso, la inercia máxima sería igual a la dimensionalidad del espacio (Greenacre, 2008). No obstante, suele resultar más interesante la identificación de direcciones preferentes a través de la inercia que el análisis de la dispersión de la nube.

El producto $N \cdot I$ se distribuye asintóticamente como una distribución $\chi^{2}$ con $(n-1)(p-1)$ grados de libertad, bajo la hipótesis de independencia. Por ello la inercia puede expresarse como una función de la $\chi^{2}$ :

$$
I=\frac{\chi^{2}}{N}
$$

O equivalentemente:

$$
I=\sum_{\alpha=1}^{p-1} \lambda_{\alpha}
$$

Donde $\lambda_{\alpha}$ son los autovalores no triviales obtenidos en la diagonalización de la matriz objetivo descrita en los siguientes apartados. 


\section{Relaciones de transición}

Anteriormente se ha visto que las matrices $S$ y $T$ poseen los mismos valores propios no nulos $\lambda_{\alpha}$ y que tanto el vector propio unitario $u_{\alpha}$ asociado a $S$ como el vector propio unitario $v_{\alpha}$ asociado a $T$ se encuentran relacionados con el mismo valor propio. Por ello es posible definir las Relaciones de Transición (Lebart et al., 1995):

$$
\begin{aligned}
& v_{\alpha}=\frac{1}{\sqrt{\lambda_{\alpha}}} F D_{p}^{-1} u_{\alpha} \\
& u_{\alpha}=\frac{1}{\sqrt{\lambda_{\alpha}}} F^{\prime} D_{n}^{-1} v_{\alpha}
\end{aligned}
$$

Estas relaciones de transición modifican las expresiones de las coordenadas factoriales:

$$
\begin{aligned}
& \Psi_{\alpha}=D_{n}^{-1} F D_{p}^{-1} u_{\alpha}=\sqrt{\lambda_{\alpha}} D_{n}^{-1} v_{\alpha} \\
& \Phi_{\alpha}=D_{p}^{-1} F^{\prime} D_{n}^{-1} v_{\alpha}=\sqrt{\lambda_{\alpha}} D_{p}^{-1} u_{\alpha}
\end{aligned}
$$

De modo que el término genérico de ambas expresiones resulta:

$$
\begin{aligned}
\psi_{\alpha i} & =\frac{\sqrt{\lambda_{\alpha}}}{f_{i \cdot}} v_{\alpha i} \\
\phi_{\alpha j} & =\frac{\sqrt{\lambda_{\alpha}}}{f_{\cdot j}} u_{\alpha j}
\end{aligned}
$$

Operando con las expresiones anteriores es posible formular las coordenadas factoriales de los puntos - fila en función de las coordenadas de los puntos - columna, y viceversa.

$$
\begin{aligned}
& \psi_{\alpha i}=\frac{1}{\sqrt{\lambda_{\alpha}}} \sum_{j=1}^{p} \frac{f_{i j}}{f_{i \cdot}} \phi_{\alpha j} \\
& \phi_{\alpha j}=\frac{1}{\sqrt{\lambda_{\alpha}}} \sum_{i=1}^{n} \frac{f_{i j}}{f_{\cdot j}} \psi_{\alpha i}
\end{aligned}
$$

Siendo $\frac{1}{\sqrt{\lambda_{\alpha}}}$ el coeficiente de dilatación que permite la representación simultánea de ambas nubes de puntos, mientras que $\frac{f_{i j}}{f_{i}}$ y $\frac{f_{i j}}{f_{\cdot j}}$ son los términos genéricos de la matriz de perfiles - fila y perfiles - columna respectivamente.

Las relaciones de transición se plantean como la relación entre las medias ponderadas entre coordenadas de filas y columnas, ponderadas con los elementos de los perfiles (de filas o columnas según el caso) (Greenacre, 2008). 
Así, la coordenada de la categoría $i$ de una de las variables es la media de las $j$ categorías de la otra variable, ponderadas por las frecuencias condicionales del perfil de las filas. Equivalentemente, la coordenadas de la categoría $j$ es la media del conjunto de las categorías $i$ ponderadas por las frecuencias condicionales del perfil de las columnas.

Joaristi: Estas fórmulas permiten representar los puntos de un espacio en función de las coordenadas de los puntos del otro. En estas circunstancias es posible obtener una representación simultánea de las dos nubes de puntos. No obstante, aunque la coordenada de cada fila es el centro de gravedad de las columnas se encuentra ponderada por el factor de escala $\frac{1}{\sqrt{\lambda_{\alpha}}}$ y por esta razón se consideran relaciones cuasi - baricéntricas, y no baricéntricas.

\section{Relación entre subespacios}

Los Baricentros o Centros de Gravedad de ambas nubes de puntos se definen como:

$$
\begin{aligned}
& N(n): \sum f_{i} \cdot f_{\cdot j}, i \epsilon n=f_{p}=i_{g} \\
& N(p): \sum f_{j} \cdot f_{i \cdot}, j \epsilon p=f_{n}=j_{g}
\end{aligned}
$$

Que en ambos casos coinciden con los centros de gravedad de los sistemas de masas correspondientes, que ocupan la posición central de la nube de puntos. Tienden a estar más cerca de aquellos perfiles que tienen masas más grandes, y por consiguiente más alejados de los que tienen masas más pequeñas.

En la expresión de los perfiles - fila se aprecia que hacen referencia a la distribución de las columnas condicionada a las filas. Del mismo modo la expresión de los perfiles - columna representa la distribución de la filas condicionada a las columnas. Es decir, el baricentro de la nube de puntos fila $N(n)$ o perfil medio de las filas $i$ es el sistema de masas del conjunto $p$, al igual que el baricentro de la nube de puntos - columna $N(p)$ es el sistema de masas del conjunto $n$.

A partir de la información mostrada en los apartados anteriores se podría plantear analizar cada una de las dos nubes de puntos (puntos - fila y puntos - columna) en su subespacio correspondiente e interpretar los planos factoriales del mismo modo que en análisis de componentes principales. Sin embargo, gracias a la definición de las relaciones baricéntricas es posible obtener representaciones simultáneas de las categorías de ambas variables y extraer conclusiones sobre su nivel de relación en base a la posición que ocupan. Así, puede darse el caso de que la cercanía o alejamiento de dos 
categorías de una misma variable queden eventualmente explicadas por las categorías de la otra. No obstante, no tiene sentido evaluar la distancia entre un punto - fila y un punto - columna ya que pertenecen a espacios diferentes.

Es posible posicionar cada punto de una nube en el conjunto de puntos de la nube alternativa. Así, puesto que cada perfil - fila constituye el baricentro del conjunto de los puntos - columna, del mismo modo que cada perfil - columna es el baricentro de la nube de puntos fila es posible obtener dos posibles representaciones simultáneas de las distintas categorías pertenecientes a ambas variables.

Sin embargo, es suficiente con disponer de una única representación simultánea de las dos nubes de puntos y de hecho, en la mayoría de los casos se prefiere obtener una sola representación gráfica que sintetice las relaciones entre categorías. En principio esto no es posible por la propia definición de los baricentros y las relaciones baricéntricas, ya que en tal caso cada conjunto de datos debería estar incluido en el otro. Pero sí es posible forzar esta representación mediante la dilatación de la posición de los centros de gravedad en cada eje hasta obtener un conjunto único de ejes sobre los que representar las distintas categorías. En estas condiciones se considera que las relaciones no son baricéntricas, sino quasibaricéntricas, pero igualmente válidas para la representación simultánea de los datos.

\section{Reconstrucción de los datos}

Los cálculos anteriores pueden aplicarse igualmente para recalcular los valores de la tabla inicial en función de las frecuencias marginales y los factores (Escofier y Pagès, 1992). En la mayoría de los casos, cuando se examinan los resultados de un AFC la interpretación se limita a los primeros factores, lo cual lleva a considerar no la tabla de datos original sino la aproximación obtenida mediante los primeros vectores propios.

$$
f_{i j}=f_{i \cdot} f_{\cdot j} \sum_{\alpha=1}^{p} \sqrt{\lambda_{\alpha}} \hat{\Phi}_{\alpha j} \hat{\Psi}_{\alpha i}
$$

Por ello podemos definir el análisis de correspondencias como una modelo de regresión bilineal, puesto que es posible recuperar los datos originales a partir de un modelo lineal de productos de coordenadas de filas y columnas.

\section{Contribuciones absolutas y relativas}

Es habitual realizar la interpretación de los factores observando las categorías que más asocian a cada uno de ellos, siendo estas categorías las 
que ocupan una posición más próxima al factor y más alejadas del origen. En realidad este procedimiento aporta una buena orientación pero no resulta estrictamente correcto, puesto que la selección de las categorías más representativas de cada eje debería realizarse en base a su inercia y sus coordenadas factoriales. Para ello se definen dos tipos de coeficientes que aportan información adicional sobre las coordenadas factoriales: las contribuciones absolutas y relativas (Joaristi y Lizasoain, 2000).

Cada eje factorial explica una parte de la inercia de la nube de puntos, que es precisamente la inercia de la nube proyectada sobre este eje. Esta inercia se puede descomponer aditivamente punto por punto, y posteriormente generalizarla a un conjunto de elementos, de modo que la contribución a la inercia de un conjunto de elementos es la suma de las contribuciones de los elementos que lo componen. Estas relaciones resultan especialmente útiles para identificar subconjuntos de elementos, o categorías de variables, con mayor importancia en la construcción de los ejes y por tanto determinantes en su interpretación.

Se define la Contribución Absoluta del elemento $i$ al eje $\alpha$ - ésimo como:

$$
C_{r_{\alpha(i)}}=\frac{f_{i} . \Psi_{\alpha i}^{2}}{\lambda_{\alpha}}
$$

Que representa la parte correspondiente al elemento $i$ en la varianza del eje $\alpha$ y permite analizar en qué proporción dicho punto $i$ contribuye a la inercia $\lambda_{\alpha}$ de la nube proyectada sobre el eje $\alpha$.

Para cualquier eje factorial $\alpha$ se cumple que:

$$
\sum_{i=1}^{n} C_{r_{\alpha(i)}}=1
$$

Del mismo modo se define la contribución absoluta del elemento $j$ al eje $\alpha$ como:

$$
C_{r_{\alpha(j)}}=\frac{f_{\cdot j} \Phi_{\alpha j}^{2}}{\lambda_{\alpha}}
$$

E igualmente para cualquier eje factorial $\alpha$ se cumple que:

$$
\sum_{j=1}^{p} C_{r_{\alpha(j)}}=1
$$

Estas expresiones permiten identificar los puntos con mayor contribución a cada uno de los ejes factoriales, y determinan la posición del eje (en el espacio $R^{p}$ para los puntos - fila $i$ y en el espacio $R^{n}$ para los puntos - columna $j$ ). 
Las contribuciones absolutas permiten diagnosticar qué filas o columnas han tenido mayor importancia en la determinación de la orientación de los ejes principales, lo cual facilita la interpretación de estos ejes. No obstante, estas contribuciones no representan adecuadamente la importancia de un punto en la construcción del eje factorial, por lo que se han definido las Contribuciones Relativas. Estas contribuciones relativas permiten analizar la posición de los perfiles en relación a los ejes y si están bien representados en el mapa. En tal caso se podrán interpretar con seguridad, mientras que si están pobremente representados sus posiciones deberán interpretarse con cautela (Greenacre, 2008).

La distancia de un punto - fila $i$ al centro de gravedad $G$ perteneciente al espacio $R^{p}$ se formula como:

$$
d^{2}(i, G)=\sum_{j=1}^{p} \frac{1}{f_{\cdot j}}\left(\frac{f_{i j}}{f_{i \cdot}}-f_{\cdot j}\right)^{2}
$$

A partir de esta distancia se define la contribución relativa de dicho punto - fila $i$ al eje factorial $\alpha$, que permite evaluar la "calidad" de la representación del punto este eje mediante el coseno del ángulo entre dicho eje factorial $\alpha$ y el vector que une dicho centro de gravedad con el punto - fila $i$.

$$
\cos _{\alpha}^{2}(i)=\frac{d_{\alpha}^{2}(i, G)}{d^{2}(i, G)}=\frac{\Psi_{\alpha i}^{2}}{d^{2}(i, G)}
$$

Esta expresión, denominada Coseno Cuadrado, representa la parte de la distancia desde el punto - fila $i$ al centro de gravedad $G$ en la dirección del eje factorial $\alpha$. Así, cuanto más se aproxime este término a la unidad, más próxima estará la proyección de la posición del punto observado de la posición real del punto en el espacio.

Para cualquier punto - fila $i$ se cumple que:

$$
\sum_{\alpha=1}^{p-1} \cos _{\alpha}^{2}(i)=1
$$

Del mismo modo es posible medir la contribución relativa de los puntos - columna $j$ al eje factorial $\alpha$ como:

$$
\cos _{\alpha}^{2}(j)=\frac{d_{\alpha}^{2}(j, G)}{d^{2}(j, G)}=\frac{\Phi_{\alpha j}^{2}}{d^{2}(j, G)}
$$

E igualmente para cualquier punto - columna $j$ se cumple que: 


$$
\sum_{\alpha=1}^{p-1} \cos _{\alpha}^{2}(j)=1
$$

A partir de estas definiciones se entiende por çalidad" de la reconstitución de una categoría por medio de los primeros ejes factoriales como la suma de las contribuciones relativas de esos primeros ejes sobre tal categoría. Concretamente, para interpretar un factor adecuadamente es conveniente seleccionar un conjunto reducido de elementos agrupados en categorías de las variables, cuya contribución relativa a la inercia del factor sea elevada (Joaristi y Lizasoain, 2000).

\section{Elementos suplementarios y valores - test}

Paralelamente a su definición en la sección sobre análisis de componentes principales, en análisis de correspondencias se denominan Puntos Activos a las filas y columnas que determinan la orientación de los ejes principales de inercia, y por tanto contribuyen a la construcción de los planos factoriales. Estos elementos deben constituir un conjunto homogéneo, exhaustivo y referido al mismo tema, de modo que las distancias entre elementos resulten fácilmente interpretables. Por el contrario los Puntos Adicionales o Ilustrativos no participan en la configuración del mapa, pero constan de verdaderos perfiles que pueden ser proyectados sobre el mismo con el fin de interpretar sus posiciones en relación a los puntos activos. Por su propia naturaleza son distintos de los activos o simplemente se han recogido en distintas condiciones u otro momento del análisis. Los elementos suplementarios no intervienen en la contribución de la nube de puntos por lo que se considera que tienen un peso nulo y una contribución nula a la definición de los ejes factoriales (Cazes, 1982).

En este sentido se define el perfil de la coordenada $i$ - ésima en la $j$ ésima columna suplementaria $k_{i j}^{+}$como:

$$
\frac{k_{i j}^{+}}{k_{\cdot j}^{+}}, i=1,2, \ldots, n
$$

Donde:

$$
k_{\cdot j}^{+}=\sum_{i=1}^{n} k_{i j}^{+}
$$

Esta columna suplementaria $j$ se proyecta sobre el eje factorial $\alpha$ mediante la misma fórmula de transición que para las columnas de la tabla de contingencia: 


$$
\begin{aligned}
& \phi_{\alpha j}^{+}=\frac{1}{\sqrt{\lambda_{\alpha}}} \sum_{i=1}^{n} \frac{k_{i j}^{+}}{k_{\cdot j}^{+}} \phi_{\alpha i} \\
& \psi_{\alpha i}^{+}=\frac{1}{\sqrt{\lambda_{\alpha}}} \sum_{j=1}^{p} \frac{k_{i j}^{+}}{k_{i \cdot}^{+}} \psi_{\alpha j}
\end{aligned}
$$

Así, las categorías suplementarias se calculan e interpretan considerando nuevamente las relaciones cuasi - baricéntricas. A pesar de que las contribuciones relativas de los elementos suplementarios al factor sean nulas, las contribuciones de factor al elemento sí pueden utilizarse para interpretar con mayor facilidad los resultados del análisis de correspondencias.

Con el fin de evaluar la significación de un elemento suplementario respecto a un eje factorial se define el Valor - Test. Este valor analiza si los individuos vinculados a una categoría de la variable suplementaria están aleatoriamente repartidos en la población, o si se encuentran significativamente alejados del centro de gravedad. Para ello mide la distancia entre los centros de gravedad y transforma esta medida en una variable normal típica. De este modo es posible determinar que el elemento suplementario está relacionado con el factor en los casos en que el valor absoluto del valor - test supere el valor 1.96, valor definido en función del nivel de confianza fijado por el investigador.

\section{Análisis de correspondencias Múltiples}

En el caso bidimensional en que únicamente dos variables se encuentren implicadas en el análisis es suficiente con aplicar Análisis de Correspondencias Simple. Sin embargo, si el objetivo es analizar un conjunto de variables cualitativas, cada una de las cuales consta de dos o más categorías, es necesario recurrir al Análisis de Correspondencias Múltiple. En este caso los datos ya no vienen dados en forma de tabla de contingencia, sino que se almacenan en una matriz en que cada fila representa un individuo u observación y cada columna una variable. Esta técnica analiza las relaciones entre un conjunto de variables, en general planteadas como variables homogéneas en cuanto a que hacen referencia a un mismo tema, siendo además las escalas de respuesta iguales (Greenacre, 2008).

\section{La matriz disyuntiva y la matriz de Burt}

Es habitual en Análisis de Correspondencias Múltiples representar los datos mediante una tabla cuyas filas representan a los individuos y las columnas representan a las categorías de las variables. De este modo la interacción de la fila $i$ con la columna $j$ contiene $x_{i j}$ y toma el valor 1 o 0 en función 
de si el individuo $i$ posee o no la categoría $j$. Esta tabla recibe el nombre de Matriz Disyuntiva Completa $Z$ ya que el conjunto de valores $x_{i j}$ de un mismo individuo para las categorías de una misma variable está formado por el valor 1 una vez (completa) y una sola vez (disyuntiva) (Escofier y Pagès, 1992).

Sea $n$ el número de observaciones y $p$ el número de categorías incluidas en $s$ variables cualitativas, entonces según Lebart et al. (1995):

$$
p=\sum_{q=1}^{s} p_{q}
$$

La matriz disyuntiva completa $Z$ se construye a partir de la matriz de datos original de forma que consta de $n$ líneas y $p$ columnas que describen las $s$ respuestas de los $n$ individuos mediante un código binario. La matriz $Z$ está formada por la yuxtaposición de las $s$ subtablas:

$$
Z=\left[Z_{1}, \ldots, Z_{q}, \ldots, Z_{s}\right]
$$

La subtabla $Z_{q} n$ líneas y $p$ columnas y se define de modo que su i -ésima línea contiene $p_{q}-1$ veces el valor 0 y una única vez el valor 1 en la columna correspondiente a la categoría de la variable $q$ escogida por el individuo $i$. En otras palabras, la submatriz $Z_{q}$ contiene las respuestas en código binario de los $n$ individuos a las distintas categorías de la variable $q$.

El término genérico de esta matriz se escribe como:

$$
\begin{aligned}
& z_{i j}=1 \\
& z_{i j}=0
\end{aligned}
$$

En función de si el individuo $i$ ha seleccionado la categoría $j$ de la variable $q$ o no.

Las frecuencias marginales por línea de la matriz disyuntiva completa $Z$ son constantes e iguales al número $s$ de variables:

$$
z_{i .}=\sum_{j=1}^{p} z_{i j}=s
$$

Por el contrario las frecuencias marginales por columna de la matriz disyuntiva completa $Z$ corresponden al número de individuos $n$ que han escogido la categoría $j$ de la variable $q$ :

$$
z_{\cdot j}=\sum_{i=1}^{n} z_{i j}
$$


Por tanto las frecuencias marginales considerando todas y cada una de las subtablas constituyen el total de $\operatorname{los} n$ individuos:

$$
z_{q}=\sum_{j \in q} z_{\cdot j}=n
$$

De igual modo la suma de las frecuencias marginales por fila y columna es:

$$
\sum_{i=1}^{n} \sum_{j=1}^{p} z_{i j}=n s
$$

A partir de la Matriz disyuntiva Completa es posible construir la Matriz Simétrica $B$ de dimensiones $p x p$ que contiene las frecuencias cruzadas de todas las variables implicadas en el análisis por pares:

$$
B=Z^{\prime} Z
$$

Esta matriz recibe el nombre de Tabla de Contingencia de Burt y su término genérico se expresa como:

$$
b_{j j^{\prime}}=\sum_{i=1}^{n} z_{i j} z_{i j^{\prime}}
$$

Las frecuencias marginales de esta tabla son:

$$
b_{j}=\sum_{j}^{p} b_{j j^{\prime}}=s \cdot z_{\cdot j}
$$

Mientras que la frecuencia total $b$ toma el valor:

$$
b=s^{2} n
$$

Esta tabla de contingencia $B$ está compuesta por $s^{2}$ bloques entre los cuales es posible distinguir:

- El bloque $Z_{q}^{\prime} Z_{q^{\prime}}$ correspondiente a las variables $q$ y $q^{\prime}$ de dimensiones $p_{q} p_{q^{\prime}}$

- El q - ésimo bloque cuadrado $Z_{q}^{\prime} Z_{q}$ obtenido mediante el cruce de una variable con ella misma. Es una matriz diagonal de orden $p_{q} p_{q}$ cuyos términos en la diagonal son las frecuencias de las categorías de la variable $q$

Se define $D$ como la matriz diagonal de orden $p x p$ con los mismos elementos en la diagonal que $B$, correspondientes a cada una de las categorías.

$$
d_{j j}=b_{j j}=z \cdot j
$$




$$
d_{j j}=0, \forall j^{\prime} \neq j
$$

Análisis de la matriz disyuntiva completa

El análisis de correspondencias múltiple es equivalente al análisis de correspondencias de una matriz disyuntiva completa. Así, según Lebart es posible extrapolar ciertos principios definidos en análisis de correspondencias simples como:

- Idénticas transformaciones de la matriz de datos original en perfiles línea y perfiles - columna

- Idéntico criterio de ajuste con ponderación de los puntos por sus perfiles marginales

- Idéntica selección de distancia $\left(\chi^{2}\right)$

Todos los individuos tienen idéntica masa:

$$
m_{i}=\frac{1}{n}
$$

Y cada una de las categorías $j$ está ponderada por su frecuencia:

$$
m_{j}=\frac{z \cdot j}{n s}
$$

La medida de distancia seleccionada para medir las distancias entre puntos continúa siendo la $\chi^{2}$, lo cual implica que en el espacio $R^{n}$ la distancia entre categorías se escribe como:

$$
d^{2}\left(j, j^{\prime}\right)=\sum_{i=1}^{n} n\left(\frac{z_{i j}}{z_{\cdot j}}-\frac{z_{i j^{\prime}}}{z_{\cdot j^{\prime}}}\right)^{2}
$$

Así, dos categorías escogidas por los mismos individuos coinciden. Por el contrario, las categorías con frecuencias débiles se encuentran alejadas de otras categorías. En el espacio $R^{p}$ la distancia entre dos individuos $i$ e $i^{\prime}$ se expresa como:

$$
d^{2}\left(i, i^{\prime}\right)=\frac{1}{s} \sum_{j=1}^{p} \frac{n}{z_{\cdot j}}\left(z_{i j}-z_{i^{\prime} j}\right)^{2}
$$

Así, dos individuos se encuentran próximos si ambos han escogido las mismas categorías. Por el contrario se encuentran alejados si no han respondido de igual forma.

Siguiendo la notación empleada para Análisis de correspondencias simples se definen las siguientes matrices: 
1. $F=\frac{1}{n s} Z$

2. $D_{p}=\frac{1}{n s} D$

3. $D_{n}=\frac{1}{n} I_{n}$

y sus correspondientes términos genéricos:

1. $f_{i j}=\frac{z_{i j}}{n s}$

2. $f_{\cdot j}=\delta_{i j} \frac{z \cdot j}{n s}$

3. $f_{i}=\frac{\delta_{i j}}{n}$

Siendo $I_{n}$ la matriz de los $n$ individuos contenidos en las filas.

Para encontrar los ejes factoriales $u_{\alpha}$ en $R^{p}$ se diagonaliza la misma matriz que en análisis de correspondencias simples, aunque en este caso se puede expresar en términos de la matriz $Z$ :

$$
S=F^{\prime} D_{n}^{-1} F D_{p}^{-1}=\frac{1}{s} Z^{\prime} Z D^{-1}
$$

De forma que la expresión del $\alpha$ - ésimo eje factorial $u_{\alpha}$ viene dada por:

$$
\frac{1}{s} Z^{\prime} Z D^{-1} u_{\alpha}=\lambda_{\alpha} u_{\alpha}
$$

Por lo que su proyección sobre el $\alpha$ - ésimo eje factorial $\hat{\phi}_{\alpha}=D^{-1} u_{\alpha}$ resulta:

$$
\frac{1}{s} D^{-1} Z^{\prime} Z \hat{\phi}_{\alpha}=\lambda_{\alpha} \hat{\phi}_{\alpha}
$$

Asimismo la expresión equivalente en el espacio $R^{n}$ se escribe:

$$
\frac{1}{s} Z D^{-1} Z^{\prime} \hat{\psi}_{\alpha}=\lambda_{\alpha} \hat{\psi}_{\alpha}
$$

$\mathrm{Al}$ igual que antes, los factores $\phi_{\alpha}$ y $\psi_{\alpha}\left(\right.$ con norma $\left.\left.\lambda_{\alpha}\right)\right)$ representan las coordenadas de los puntos - línea y los puntos - columna sobre el eje factorial $\alpha$.

Las relaciones de transición entre ambos factores $\phi_{\alpha}$ y $\psi_{\alpha}$ se formulan como:

$$
\begin{gathered}
\phi_{\alpha}=\frac{1}{\sqrt{\lambda_{\alpha}}} D^{-1} Z^{\prime} \psi_{\alpha} \\
\psi_{\alpha}=\frac{1}{s \sqrt{\lambda_{\alpha}}} Z \phi_{\alpha}
\end{gathered}
$$


A partir de ambas relaciones de transición se obtienen las coordenadas factoriales del individuo $i$ sobre el eje factorial $\alpha$ como:

$$
\begin{aligned}
& \phi_{\alpha j}=\frac{1}{\sqrt{\lambda_{\alpha}}} \sum_{i=1}^{n} \frac{z_{i j}}{z_{\cdot j}} \psi_{\alpha i} \\
& \psi_{\alpha i}=\frac{1}{\sqrt{\lambda_{\alpha}}} \sum_{j=1}^{p} \frac{z_{i j}}{z_{i}} \phi_{\alpha j}
\end{aligned}
$$

De este modo, en caso de que no se realizara la ponderación por el coeficiente $\frac{1}{\sqrt{\lambda_{\alpha}}}$ la categoría $j$ se encontraría en el punto medio de la nube de individuos que la hubieran escogido como respuesta.

La representación de los resultados del análisis de correspondencias múltiples puede formularse de modo particular debido a la codificación específica de la matriz disyuntiva completa. Para ello es necesario identificar un eje factorial tal que la coordenada de un individuo $i$ sea la media aritmética de las coordenadas de las categorías que ha escogido. Asimismo la coordenada de una categoría j vendrá dada por la media aritmética de las coordenadas de los individuos que la han escogido. De este modo, se obtienen las relaciones cuasi - baricéntricas resultantes del análisis de la matriz disyuntiva completa $Z$ con un valor mínimo $\beta=\frac{1}{\sqrt{\lambda}}$ para el coeficiente de dilatación.

En cualquier caso, a pesar de la importancia de la representación simultánea, se utiliza como tal en muy pocos casos debido al elevado número de individuos, que en la mayoría de los casos son anónimos. La principal aportación de este posible análisis es la información proporcionada por el individuo promedio, que representa la característica concreta de la variable a estudiar.

La distancia entre la categoría $j$ y el centro de gravedad de la nube $G$ se formula como:

$$
d^{2}(j, G)=n \sum_{i=1}^{n}\left(\frac{z_{i j}}{z_{\cdot j}}-\frac{1}{n}\right)^{2}=\frac{n}{z_{\cdot j}}-1
$$

En esta expresión se aprecia que la distancia de una categoría al centro de gravedad se incrementa a medida que disminuye su frecuencia $z \cdot j$, al igual que se aproxima al centro de gravedad a medida que aumenta la frecuencia $z \cdot j$.

La Inercia $I(j)$ de una Modalidad $j$ se formula como:

$$
I(j)=m_{j} d^{2}(j, G)
$$




$$
m_{j}=\frac{z \cdot j}{n s}
$$

Por tanto,

$$
I(j)=\frac{1}{s}\left(1-\frac{z \cdot j}{n}\right)
$$

Igualmente la parte de inercia debida a una categoría determinada de respuesta se incrementa a medida que la frecuencia de dicha modalidad disminuye.

El valor máximo $\frac{1}{s}$ se alcanza únicamente en el caso de que la categoría tenga un peso nulo por lo que, en términos generales, deben evitarse las categorías con frecuencias muy reducidas que puedan distorsionar las direcciones de los ejes principales de inercia.

La Inercia $I(q)$ de una Variable $q$ viene dada por:

$$
I(q)=\sum_{j=1}^{p_{q}} I(j)=\frac{1}{s}\left(p_{q}-1\right)
$$

El valor mínimo $\frac{1}{s}$ se alcanza únicamente en el caso de variables binarias por lo que resulta conveniente equilibrar la estructura en categorías de las variables con el fin de asignar la misma importancia a todas las preguntas.

Por tanto se deduce que la Inercia Total I viene dada por:

$$
I=\sum_{q} I(q)=\sum_{j=1}^{p} \frac{z \cdot j}{n s} d^{2}(j, G)
$$

De donde se obtiene que:

$$
I=\frac{p}{s}-1
$$

En el caso particular de que todas las variables sean binarias alcanza su valor máximo igual a la unidad. En este caso concreto el análisis de correspondencias múltiples y el análisis de componentes principales aportan los mismos resultados.

La inercia total depende únicamente del número de variables, de las categorías y del número de relaciones entre variable. Este valor no posee ningún significado estadístico en el ámbito del análisis de correspondencias múltiples. 
En estas expresiones, así como en las relativas a las distancias entre puntos, se observa que las categorías con frecuencias absolutas muy pequeñas dan lugar a distancias e inercias muy elevadas, introduciendo cierta inestabilidad en los resultados. En este sentido hay que estar alerta frente a la presencia de observaciones atípicas de poca masa, que puede tener una gran influencia en la solución (Greenacre, 2008). Es por ello que los programas estadísticos que realizan este tipo de análisis ofrecen también la posibilidad de evaluar el impacto de estas categorías y eliminarlas si es necesario. Habitualmente los programas identifican estas categorías mediante el cálculo del porcentaje de sus frecuencias respecto al total y su comparación con una cota superior fijada en el $1 \%$ o $2 \%$. También cabe la posibilidad de proyectarlos como variables suplementarias o combinarlos con otras categorías, siempre y cuando se trata de transformaciones con sentido.

En general, el empleo de tasas de inercia como indicador para la evaluación global de la calidad de una representación obtenida mediante análisis de correspondencias múltiples no resulta apropiado. Las tasas de inercia son medidas excesivamente pesimistas de la "calidad" de una representación, al contrario de lo que ocurre con los coeficientes de correlación múltiple que son medidas optimistas de la calidad de una regresión. Esto se debe a que la varianza bruta inicial no constituye en general una medida de referencia adecuada por lo que no resulta adecuado hacer referencia a tasas de inercia respecto a esta cantidad inicial (Lebart et al., 1995).

Por ello Benzécri propuso una expresión para el cálculo de las tasas de inercia a partir de pseudo - valores propios $\rho(\lambda)$ mediante la expresión (Benzécri, 1979):

$$
\rho(\lambda)=\left(\frac{s}{s-1}\right)^{2}\left(\lambda-\frac{1}{s}\right)^{2}, \lambda>\frac{1}{s}
$$

Donde $s$ representa el número de variables y $\lambda$ representa el valor propio obtenido a partir del análisis de la matriz disyuntiva completa.

\section{Análisis de la matriz de Burt}

El análisis de correspondencias aplicado a la matriz disyuntiva completa $Z$ es equivalente al análisis de la matriz de Burt $B$ y da lugar a los mismos factores. El análisis de esta matriz $B$, simétrica de orden $p$, se reduce al análisis de una nube de $p$ puntos, genéricos (fila o columna), en el espacio $R^{p}$ Las frecuencias marginales de esta matriz, tanto en filas como en columnas, son los elementos de la diagonal en la matriz $D$ (Lebart et al., 1995).

Partiendo de la ecuación mostrada anteriormente los ejes factoriales $u_{\alpha}$ en $R^{p}$ se diagonaliza la matriz $S$, expresada en términos de la matriz $Z$ y 
en este caso también en términos de la matriz $B$ :

$$
S=F^{\prime} D_{n}^{-1} F D_{p}^{-1}=\frac{1}{s} D^{-1} Z^{\prime} Z=\frac{1}{s} D^{-1} B
$$

La matriz de frecuencias relativas $F$ se construye a partir de la matriz $B$ :

$$
F=\frac{1}{n s^{2}} B
$$

Y también:

$$
D_{p}=D_{n}=\frac{1}{n s} B
$$

El análisis de la matriz $B$ implica diagonalizar la matriz:

$$
S^{*}=\frac{1}{s^{2}} D^{-1} B D^{-1} B
$$

De donde se obtiene:

$$
S^{*}=S^{2}
$$

Igualmente, multiplicando la expresión anterior para el cálculo de las coordenadas factoriales por $\frac{1}{s} D^{-1} B$ se obtiene:

$$
\frac{1}{s} D^{-1} B D^{-1} B \hat{\phi}_{\alpha}=\lambda_{\alpha}^{2} \hat{\phi}_{\alpha}
$$

Los factores resultantes de los dos análisis son por tanto colineales en el espacio $R^{p}$, aunque los valores propios son diferentes. Sin embargo los valores propios resultantes del análisis de la matriz $B$ se obtienen mediante la raíz de los valores propios obtenidos a partir de la matriz disyuntiva completa.

$$
\lambda_{B}=\lambda^{2}
$$

Esta es la principal diferencia entre realizar el análisis sobre la matriz disyuntiva completa o sobre la matriz de Burt. Puesto que la inercia resultante del análisis de la matriz de Burt es el cuadrado de la obtenida mediante el análisis sobre la matriz disyuntiva completa, los porcentajes de inercia del análisis de Burt son siempre más elevados que los del análisis binario. Sin embargo, la codificación de los datos originales hace que los porcentajes de inercia sean artificialmente bajos, lo que conlleva la subestimación de la çalidad" de los datos comentada anteriormente (Greenacre, 2008).

La relación entre las coordenadas factoriales obtenidas a partir de la matriz disyuntiva completa y la matriz de Burt se expresa como:

$$
\phi_{B \alpha}=\phi_{\alpha} \sqrt{\lambda_{\alpha}}
$$


Elementos suplementarios y valores - test

El empleo de elementos suplementarios en análisis de correspondencias múltiple permite introducir nuevas categorías que facilitan la interpretación de los factores. Así, este análisis aporta la posibilidad de incrementar la información referida a los factores mediante variables que en principio no habían sido incluidas en la construcción de los ejes. Asimismo permite conocer la posición de las variables suplementarias en el espacio previamente definido por las variables activas (Lebart et al., 1995).

La coordenada factorial $\phi_{\alpha j}$ de una categoría $j$ sobre un eje $\alpha$, ya sea una categoría activa o suplementaria, se obtiene como el producto del coeficiente $\frac{1}{\sqrt{\lambda_{\alpha}}}$ y la media aritmética de las coordenadas $\psi_{\alpha i}$ del conjunto de individuos que hayan escogido la categoría $j, I(j)$ :

$$
\phi_{\alpha j}=\frac{1}{z_{\cdot j} \sqrt{\lambda_{\alpha}}} \sum_{i \in I(j)} \psi_{\alpha i}
$$

El número total de individuos que han escogido la categoría j es $z_{\cdot j}=n_{j}$ mientras que el número total de individuos se representa como $n$. Partiendo de estos parámetros es posible plantear un contraste estadístico basado en la hipótesis de independencia $H_{0}: n_{j}$ escogidos al azar entre $n$ individuos, suponiendo en todos los casos extracción sin reemplazamiento. La coordenada media de $\operatorname{los} n_{j}$ individuos extraídos al azar de la población $n$, cuyas coordenadas se expresan como $\phi_{\alpha i}$ es una variable aleatoria $X_{\alpha j}$, cuya esperanza matemática y varianzas son:

$$
\begin{gathered}
E\left(X_{\alpha j}\right)=0 \\
\operatorname{Var}\left(X_{\alpha j}\right)=\frac{n-n_{j}}{n-1} \frac{\lambda_{\alpha}}{n_{j}}
\end{gathered}
$$

La coordenada $\phi_{\alpha j}$ de la categoría suplementaria se vincula a la variable aleatoria $X_{\alpha j}$ mediante la relación:

$$
\phi_{\alpha j}=\frac{1}{\sqrt{\lambda_{\alpha}}} X_{\alpha j}
$$

Por tanto,

$$
\begin{gathered}
E\left(\phi_{\alpha j}\right)=0 \\
\operatorname{Var}\left(\phi_{\alpha j}\right)=\frac{n-n_{j}}{n-1} \frac{1}{n_{j}}
\end{gathered}
$$

La siguiente expresión mide en número de desviaciones típicas la distancia entre el baricentro de los $n_{j}$ individuos que han escogido la categoría $j$ 
y el origen del eje factorial $\alpha$. Este parámetro recibe el mismo nombre que su equivalente en análisis de componentes principales, el Valor - Test:

$$
t_{\alpha j}=\sqrt{n \frac{n-1}{n-n_{j}}} \phi_{\alpha j}
$$

Aplicando el teorema central del límite, la distribución del valor - test sigue una distribución de Laplace - Gauss reducida. De este modo, se considera que una categoría ocupa una posición significativa en una dirección si el subconjunto de puntos asociados a dicha categoría posee baja dispersión y además se encuentra alejada del centro de gravedad de la nube de individuos general.

En términos generales se considera que una categoría ocupa una posición significativa si su valor - test en términos absolutos supera el valor 1.96. Este contraste se aplica de forma individual a cada una de las variables proyectadas sobre los ejes, y en principio no ofrece la posibilidad de realizar otro tipo de análisis que consideren las variables de forma simultánea.

El cálculo de los valores - test se aplica fundamentalmente a variables nominales ilustrativas. No obstante, es igualmente posible proyectar variables continuas ilustrativas si previamente se realiza una categorización de los valores en intervalos o categorías. En cualquier caso este análisis no puede aplicarse a variables continuas con valores negativos o variables cuyos perfiles no tengan sentido en el contexto de los datos (Greenacre, 2008).

\subsection{Resultados y discusión}

\subsubsection{Análisis descriptivo}

Las variables implicadas en los análisis de este trabajo se han clasificado en cuatro grupos, tal y como se describe en el Anexo A. En la sección relativa al contexto académico del estudiante se han incluido las variables relativas a las Características de los estudios y los Métodos de enseñanza - aprendizaje, mientras que las variables referentes a la Gestión del tiempo durante los estudios y las Características personales de los encuestados se engloban como parte de su contexto personal. Como se verá más adelante el área de estudio constituye en este trabajo una variable mediadora que debe ser considerada de forma individual. Por último, las Competencias adquiridas en la Universidad constituyen el resultado de los procesos de enseñanza - aprendizaje y el Área de estudio hace referencia a la agrupación de las titulaciones académicas de los encuestados en ocho campos de estudio, cuyo 
detalle puede consultarse en el Anexo B.

Contexto académico: Incluye las Características de los estudios y los Métodos de enseñanza - aprendizaje

Contexto personal: Incluye la Gestión del tiempo y las Características personales

Área de estudio : Educación, Humanidades, Ciencias Sociales, Economía y empresa, Derecho, Técnicas, Salud y Ciencias

Competencias adquiridas en la Universidad : 19 competencias medidas en escala Likert ( $1=$ Nada, $7=$ Mucho)

En cuanto a las Características de los Estudios y los Métodos de Enseñanza - Aprendizaje las preguntas del cuestionario se formularon como "En tu carrera..." en el primer caso y "¿En qué medida se hacía énfasis en tu carrera en los siguientes métodos de enseñanza y aprendizaje?" en el segundo. En ambos casos las respuestas se midieron mediante una escala Likert de 5 puntos donde $1=$ Nada y $5=$ Mucho.

Puesto que por motivos de espacio no es posible incluir en las tablas siguientes el nombre completo de las variables se muestra a continuación la codificación empleada para la denominación de cada una de las variables. Concretamente el ítem Realización de pruebas tipo test se ha codificado sin numeración, como se ha hecho con el resto de ítems, debido a que se ha descartado del conjunto de variables de estudio en análisis posteriores. En el Anexo E es posible consultar el listado de todas las abreviaturas empleadas en el trabajo. 
Características de los estudios

C1 En tu carrera había que trabajar mucho para aprobar

C2 En tu carrera el contenido era conocido por los empleadores

C3 En tu carrera podías elegir las asignaturas que querías cursar

C4 En tu carrera el enfoque era generalista

C5 En tu carrera el enfoque era especializado

C6 Tu carrera era una carrera con prestigio académico Métodos de enseñanza - aprendizaje

V1 Asistencia a clase

V2 Trabajos en grupo

V3 Participación en proyectos de investigación

V4 Prácticas en empresas, instituciones o similares

V5 Conocimientos prácticos y metodológicos

V6 Teorías, conceptos y paradigmas

V7 El profesor era la principal fuente de información

V8 Aprendizaje basado en proyectos o problemas

V9 Trabajos escritos

V10 Exposiciones orales

VT Realización de pruebas tipo test

Gestión del tiempo

G1 Dedicación exclusiva a los estudio

G2 Prácticas en empresa

G3 Nota obtenida en los estudios

G4 Dedicación de tiempo semanal a los estudios

G5 Estancia en el extranjero

\begin{tabular}{|l|l|}
\hline \multicolumn{2}{|c|}{ Características personales } \\
\hline P1 & Sexo \\
P2 & Edad \\
P3 & Situación de convivencia \\
P4 & Tiene hijos \\
P5 & Nivel de estudios del padre \\
P6 & Nivel de estudios de la madre \\
\hline \multicolumn{2}{|c|}{ Área y nivel de estudios } \\
\hline A & Área de estudios \\
C & Nivel de estudios \\
\hline
\end{tabular}

Tabla 4.3: Variables contextuales del entorno académico y personal de los encuestados. Abreviaturas 
Del mismo modo se muestra la descripción de cada uno de los ítems referidos a las Competencias adquiridas en la Universidad. Dichas abreviaturas se muestran igualmente en el Anexo E que contiene todas las utilizadas en el trabajo. También en este caso el ítem Capacidad para escribir y hablar en idiomas extranjeros se han codificado sin numeración, por haberse descartado del estudio en análisis posteriores:

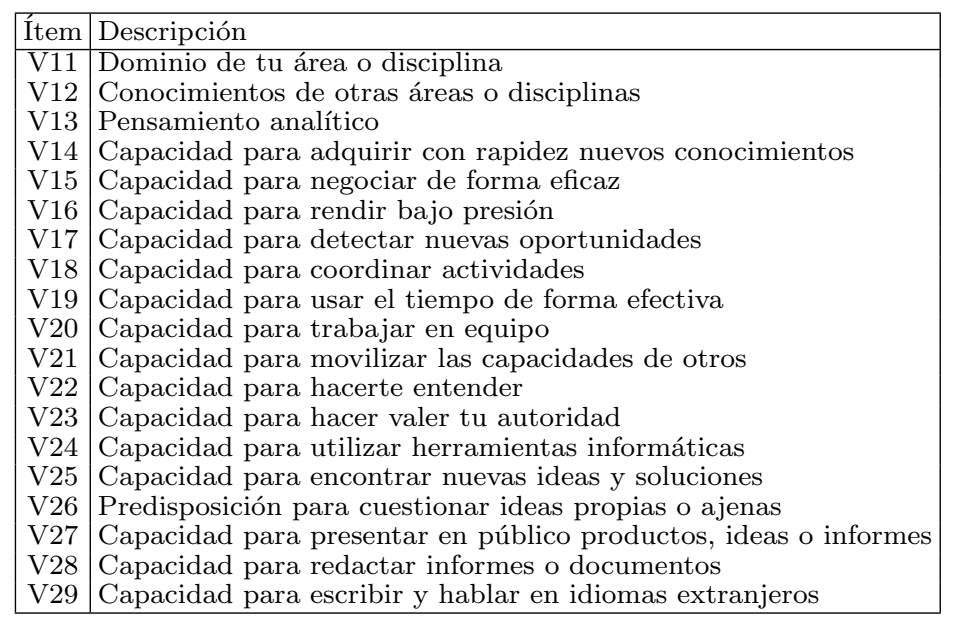

Tabla 4.4: Descripción de los ítems incluidos en la matriz de correlaciones. Competencias adquiridas en la Universidad

En la Tabla 4.5 se observa que los métodos más empleados en las universidades españolas son las Teorías, conceptos y paradigmas y El profesor como principal fuente de información. Asimismo, entre las características de los estudios más valoradas, predominan En tu carrera había que trabajar mucho para aprobar y En tu carrera el enfoque era generalista, lo cual evidencia que una parte considerable de los graduados encuestados opina que sus carreras eran teóricas y de enfoque generalista. Por el contrario los métodos menos empleados en los estudios universitarios, según los encuestados, resultaron ser las Prácticas en empresa y los Proyectos de investigación. 


\begin{tabular}{|l|c|c|c|}
\hline Descripción & $\mathrm{N}$ & Media & D. Típica \\
\hline Teorías, conceptos y paradigmas & 5215 & 3.81 & 0.92 \\
El profesor fuente de información & 5226 & 3.74 & 0.89 \\
Había que trabajar mucho para aprobar & 5438 & 3.68 & 0.87 \\
Enfoque era generalista & 5390 & 3.53 & 1.01 \\
Contenido conocido por empleadores & 5063 & 3.19 & 0.99 \\
Trabajos escritos & 5226 & 3.08 & 1.11 \\
Prestigio académico & 5396 & 2.99 & 1.18 \\
Asistencia a clase & 5230 & 2.96 & 1.22 \\
Trabajos en grupo & 5234 & 2.91 & 1.24 \\
Conocimientos prácticos & 5228 & 2.90 & 1.10 \\
Podías elegir las asignaturas & 5203 & 2.88 & 1.17 \\
Realización de pruebas tipo test & 5226 & 2.80 & 1.25 \\
Enfoque era especializado & 5386 & 2.70 & 1.06 \\
Proyectos o problemas & 5221 & 2.63 & 1.05 \\
Exposiciones orales & 5228 & 2.40 & 1.15 \\
Practicas en empresas & 5228 & 2.35 & 1.31 \\
Proyectos de investigación & 5226 & 1.87 & 1.03 \\
\hline
\end{tabular}

Tabla 4.5: Análisis descriptivo. Características de los estudios y Métodos de enseñanza - aprendizaje

Seguidamente se muestran los estadísticos descriptivos de las variables contextuales referidas a la gestión del tiempo de los estudiantes universitarios. En la Tabla 4.6 se observa que la mayoría de los encuestados señalaron dedicarse de forma exclusiva a sus estudios en la Universidad (82,8\%) y más de la mitad realizaron alguna práctica en empresa como parte de sus estudios $(56,0 \%)$. Respecto a la dedicación de tiempo al estudio son pocos los que afirmaron dedicar menos de 15 horas semanales (13,6\%) mientras que una gran parte manifestó dedicar entre 30 y 45 horas semanales $(37,6 \%)$. La gran mayoría de los encuestados afirmó haber obtenido una nota de aprobado $(46,2 \%)$ o notable $(48,4 \%)$ en sus estudios, mientras que el porcentaje de los encuestados que ha realizado alguna estancia en el extranjero, ya sea durante los estudios o con posterioridad y por motivos tanto de estudio como de trabajo no resultó ser muy elevado $(12,2 \%)$.

\begin{tabular}{|l|c|c|}
\hline Categorías de respuesta & $\mathrm{N}$ & $\%$ \\
\hline Los estudios eran mi actividad principal & 4316 & $82,8 \%$ \\
Los estudios no eran mi actividad principal & 899 & $17,2 \%$ \\
\hline Prácticas en empresa & 3044 & $56,0 \%$ \\
No prácticas en empresa & 2388 & $44,0 \%$ \\
\hline Más de 15 horas & 730 & $13,6 \%$ \\
$15-30$ horas & 1142 & $21,8 \%$ \\
$30-45$ horas & 2021 & $37,6 \%$ \\
Más de 45 horas & 1480 & $27,5 \%$ \\
\hline Aprobado & 2366 & $46,2 \%$ \\
Notable & 2476 & $48,4 \%$ \\
Sobresaliente & 257 & $5,0 \%$ \\
Matrícula de Honor & 20 & $0,4 \%$ \\
\hline Estancia en el extranjero & 675 & $12,4 \%$ \\
No estancia en el extranjero & 4781 & $87,6 \%$ \\
\hline
\end{tabular}

Tabla 4.6: Análisis descriptivo. Gestión del tiempo 
Respecto a las variables contextuales sobre las circunstancias personales del titulado en el momento de la entrevista se observa que el porcentaje de mujeres $(66,2 \%)$ es superior al de hombres, del mismo modo que los titulados con edades comprendidas entre 26 y 30 años son mayoría (61,6\%). En el momento de la entrevista, en principio cinco años después del momento de la graduación, casi la mitad de los titulados vive con sus padres $(43,4 \%)$ y constituyen una minoría los graduados con hijos (10,4\%). El porcentaje de encuestados cuyos padres tienen estudios de Educación Superior alcanza el $26,6 \%$ para el padre, y se reduce hasta el $15,2 \%$ para la madre.

\begin{tabular}{|l|c|c|}
\hline Categorías de respuesta & $\mathrm{N}$ & $\%$ \\
\hline Hombre & 1790 & $33,8 \%$ \\
Mujer & 3506 & $66,2 \%$ \\
\hline 26-30 años & 3110 & $61,6 \%$ \\
30-35 años & 1712 & $33,9 \%$ \\
Más de 35 años & 228 & $4,5 \%$ \\
\hline Vive solo & 720 & $14,0 \%$ \\
Vive con padres & 2240 & $43,4 \%$ \\
Vive en pareja & 1893 & $36,7 \%$ \\
Otros & 304 & $5,9 \%$ \\
\hline Sí hijos & 553 & $10,4 \%$ \\
No hijos & 4746 & $89,6 \%$ \\
\hline Padre sin Educación Superior & 1452 & $26,6 \%$ \\
Padre con Educación Superior & 4004 & $73,4 \%$ \\
\hline Madre sin Educación Superior & 827 & $15,2 \%$ \\
Madre con Educación Superior & 4629 & $84,8 \%$ \\
\hline
\end{tabular}

Tabla 4.7: Análisis descriptivo. Características personales

En cuanto al área de estudio se han definido ocho categorías en las que se han clasificado todas las titulaciones de los graduados que han respondido a la encuesta. En la mayoría de los casos los datos de cada grupo se han segmentado en otros dos subgrupos en función del nivel de estudios. Así, en la categoría Ciclo Corto se han incluido los estudios de Diplomatura, Ingeniería Técnica y Arquitectura Técnica, mientras que los estudios de Licenciatura, Ingeniería y Arquitectura se han clasificado en la categoría Ciclo Largo. En los casos en que el número de titulaciones de ciclo corto es insuficiente para desagregar por estratos se ha optado por presentar los resultados sin esta segmentación como ocurre en las áreas de Humanidades, Derecho y Ciencias. De este modo, en la Tabla 4.8 se observa que la distribución de titulados por áreas de estudio está relativamente equilibrada, en relación con las promociones anuales de titulados en las universidades españolas. 


\begin{tabular}{|l|c|c|}
\hline Categorías de respuesta & $\mathrm{N}$ & $\%$ \\
\hline Educación - Ciclo Largo & 113 & $2,1 \%$ \\
Educación - Ciclo Corto & 518 & $9,6 \%$ \\
\hline Humanidades & 532 & $9,9 \%$ \\
\hline Ciencias Sociales - Ciclo Largo & 389 & $7,2 \%$ \\
Ciencias Sociales - Ciclo Corto & 230 & $4,3 \%$ \\
\hline Economía y empresa - Ciclo Largo & 537 & $10,0 \%$ \\
Economía y empresa - Ciclo Corto & 520 & $9,7 \%$ \\
\hline Derecho & 341 & $6,3 \%$ \\
\hline Técnicas - Ciclo Largo & 378 & $7,0 \%$ \\
Técnicas - Ciclo Corto & 669 & $12,4 \%$ \\
\hline Salud - Ciclo Largo & 265 & $4,9 \%$ \\
Salud - Ciclo Corto & 278 & $5,2 \%$ \\
\hline Ciencias & 610 & $11,3 \%$ \\
\hline Total & 5380 & $100,0 \%$ \\
\hline
\end{tabular}

Tabla 4.8: Análisis descriptivo. Área y Nivel de estudios

Respecto a las Competencias adquiridas en la Universidad la pregunta específica del cuestionario para este apartado se formula como: "¿En qué medida ha contribuido la carrera en que te graduaste en 1999/00 al desarrollo de estas competencias?", cuyas respuestas se midieron mediante una escala Likert de 7 puntos donde $1=$ Muy bajo y $7=$ Muy alto.

Los ítems que obtuvieron las puntuaciones más elevadas fueron Adquirir con rapidez nuevos conocimientos, Trabajar en equipo y Redactar informes y documentos. Otras competencias como Escribir y hablar en idiomas extranjeros, Negociar de forma eficaz y Hacer valer tu autoridad obtuvieron las puntuaciones más bajas de todo el listado mostrado en el cuestionario. En esta tabla destaca la disminución en las frecuencias observadas respecto a la tabla anterior, debida a las elevadas tasas de abandono de los encuestados en la cumplimentación del cuestionario. Así, mientras que las cuestiones relativas a las Características de los estudios y Métodos de enseñanza aprendizaje se ubican en las primeras secciones del cuestionario, las preguntas sobre Competencias adquiridas en la Universidad se encuentran en la parte final del cuestionario, por lo que resulta comprensible que la tasa de respuesta haya disminuido en estas preguntas. 


\begin{tabular}{|l|c|c|c|}
\hline Descripción & $\mathrm{N}$ & Media & Desv. típ. \\
\hline Adquirir con rapidez nuevos conocimientos & 4704 & 4.40 & 1.62 \\
Trabajar en equipo & 4717 & 4.26 & 1.78 \\
Redactar informes o documentos & 4710 & 4.12 & 1.74 \\
Pensamiento analítico & 4692 & 4.08 & 1.62 \\
Dominio de tu área o disciplina & 4754 & 4.04 & 1.72 \\
Hacerte entender & 4711 & 3.94 & 1.67 \\
Usar el tiempo de forma efectiva & 4721 & 3.89 & 1.70 \\
Predisposición para cuestionar ideas propias o ajenas & 4703 & 3.72 & 1.67 \\
Encontrar nuevas ideas y soluciones & 4699 & 3.66 & 1.63 \\
Coordinar actividades & 4706 & 3.64 & 1.66 \\
Presentar en público productos, ideas o informes & 4704 & 3.62 & 1.76 \\
Rendir bajo presión & 4718 & 3.60 & 1.81 \\
Conocimientos de otras áreas o disciplinas & 4721 & 3.25 & 1.59 \\
Movilizar las capacidades de otros & 4699 & 3.23 & 1.62 \\
Utilizar herramientas informáticas & 4715 & 3.15 & 1.81 \\
Detectar nuevas oportunidades & 4669 & 3.00 & 1.54 \\
Hacer valer tu autoridad & 4701 & 2.96 & 1.58 \\
Negociar de forma eficaz & 4687 & 2.82 & 1.58 \\
Escribir y hablar en idiomas extranjeros & 4633 & 2.21 & 1.54 \\
\hline
\end{tabular}

Tabla 4.9: Análisis descriptivo. Competencias adquiridas en la Universidad

\subsubsection{Metodologías docentes empleadas en la Universidad y adquisición de competencias}

\section{Supuestos estadísticos}

La medida de adecuación muestral de Kaiser - Meyer - Olkin alcanzó el valor de 0.779 para el conjunto de ítems referidos a las Características de los estudios y Métodos de enseñanza - aprendizaje y se incrementó hasta 0.940 para las Competencias adquiridas en la Universidad. Asimismo el contraste de esfericidad de Barlett arrojó resultados significativos indicando la adecuación de los datos a la realización del análisis factorial exploratorio.

\begin{tabular}{|c|c|}
\hline$\chi^{2}$ & 19330.4 \\
g.l. & 136 \\
Sig. & 0.000 \\
\hline
\end{tabular}

Tabla 4.10: Contraste de esfericidad de Barlett. Características de los estudios y Métodos de enseñanza - aprendizaje

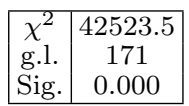

Tabla 4.11: Contraste de esfericidad de Barlett. Competencias adquiridas en la Universidad

Asimismo, el análisis de la matriz de correlaciones observada revela que es apropiado aplicar este análisis a los datos sobre Características de los estudios y Métodos de enseñanza - aprendizaje ya que una parte considera- 
ble de sus valores superan el umbral de 0.30. Adicionalmente los elementos de la matriz de diferencias entre esta matriz de correlaciones observada y su homóloga reproducida tomaron valores muy próximos a cero, siendo los valores máximos en valor absoluto 0.21 y 0.15 .

\begin{tabular}{|c|cccccc|cccccccccccc|}
\hline & C1 & C2 & C3 & C4 & C5 & C6 & V1 & V2 & V3 & V4 & V5 & V6 & V7 & V8 & V9 & V10 & VT \\
\hline C1 & 1.00 & 0.16 & -0.11 & -0.03 & 0.11 & 0.48 & 0.01 & -0.11 & 0.01 & -0.05 & 0.03 & 0.14 & 0.04 & 0.19 & -0.13 & -0.10 & 0.00 \\
C2 & 0.16 & 1.00 & 0.07 & -0.09 & 0.22 & 0.25 & 0.05 & 0.05 & 0.08 & 0.15 & 0.18 & 0.03 & 0.04 & 0.15 & 0.01 & 0.06 & 0.07 \\
C3 & -0.11 & 0.07 & 1.00 & -0.03 & 0.17 & -0.11 & 0.06 & 0.22 & 0.17 & 0.13 & 0.15 & 0.01 & 0.00 & 0.09 & 0.22 & 0.14 & 0.03 \\
C4 & -0.03 & -0.09 & -0.03 & 1.00 & -0.65 & -0.04 & -0.05 & -0.09 & -0.13 & -0.09 & -0.20 & 0.12 & 0.08 & -0.15 & -0.06 & -0.06 & 0.04 \\
C5 & 0.11 & 0.22 & 0.17 & -0.65 & 1.00 & 0.15 & 0.12 & 0.19 & 0.23 & 0.19 & 0.33 & -0.03 & -0.02 & 0.26 & 0.13 & 0.14 & -0.01 \\
C6 & 0.48 & 0.25 & -0.11 & -0.04 & 0.15 & 1.00 & -0.04 & -0.17 & 0.00 & -0.01 & 0.08 & 0.12 & 0.04 & 0.21 & -0.22 & -0.16 & 0.06 \\
\hline V1 & 0.01 & 0.05 & 0.06 & -0.05 & 0.12 & -0.04 & 1.00 & 0.44 & 0.24 & 0.24 & 0.25 & 0.00 & 0.00 & 0.16 & 0.31 & 0.33 & 0.01 \\
V2 & -0.11 & 0.05 & 0.22 & -0.09 & 0.19 & -0.17 & 0.44 & 1.00 & 0.48 & 0.40 & 0.41 & -0.01 & -0.08 & 0.28 & 0.61 & 0.56 & 0.09 \\
V3 & 0.01 & 0.08 & 0.17 & -0.13 & 0.23 & 0.00 & 0.24 & 0.48 & 1.00 & 0.31 & 0.39 & 0.01 & -0.13 & 0.33 & 0.34 & 0.39 & 0.05 \\
V4 & -0.05 & 0.15 & 0.13 & -0.09 & 0.19 & -0.01 & 0.24 & 0.40 & 0.31 & 1.00 & 0.49 & -0.05 & -0.07 & 0.23 & 0.22 & 0.29 & 0.24 \\
V5 & 0.03 & 0.18 & 0.15 & -0.20 & 0.33 & 0.08 & 0.25 & 0.41 & 0.39 & 0.49 & 1.00 & 0.04 & -0.06 & 0.40 & 0.25 & 0.28 & 0.12 \\
V6 & 0.14 & 0.03 & 0.01 & 0.12 & -0.03 & 0.12 & 0.00 & -0.01 & 0.01 & -0.05 & 0.04 & 1.00 & 0.23 & 0.02 & 0.05 & 0.01 & 0.08 \\
V7 & 0.04 & 0.04 & 0.00 & 0.08 & -0.02 & 0.04 & 0.00 & -0.08 & -0.13 & -0.07 & -0.06 & 0.23 & 1.00 & -0.01 & -0.03 & -0.09 & 0.05 \\
V8 & 0.19 & 0.15 & 0.09 & -0.15 & 0.26 & 0.21 & 0.16 & 0.28 & 0.33 & 0.23 & 0.40 & 0.02 & -0.01 & 1.00 & 0.17 & 0.14 & 0.02 \\
V9 & -0.13 & 0.01 & 0.22 & -0.06 & 0.13 & -0.22 & 0.31 & 0.61 & 0.34 & 0.22 & 0.25 & 0.05 & -0.03 & 0.17 & 1.00 & 0.56 & 0.01 \\
V10 & -0.10 & 0.06 & 0.14 & -0.06 & 0.14 & -0.16 & 0.33 & 0.56 & 0.39 & 0.29 & 0.28 & 0.01 & -0.09 & 0.14 & 0.56 & 1.00 & 0.07 \\
VT & 0.00 & 0.07 & 0.03 & 0.04 & -0.01 & 0.06 & 0.01 & 0.09 & 0.05 & 0.24 & 0.12 & 0.08 & 0.05 & 0.02 & 0.01 & 0.07 & 1.00 \\
\hline
\end{tabular}

Tabla 4.12: Matriz de correlaciones observadas. Características de los estudios y Métodos de enseñanza - aprendizaje

Estas correlaciones superiores al valor de 0.30 se evidencian en mayor medida en la matriz de correlaciones correspondiente a las Competencias adquiridas en la Universidad. En este caso se puede observar que la mayor parte de los datos sí superan este límite inferior indicando la adecuación de los datos a la realización del análisis. Del mismo modo, los valores de la matriz de diferencias entre esta matriz de correlaciones observada y su homóloga reproducida tomaron valores muy próximos a cero, resultando en este caso el valor máximo en valor absoluto igual a 0.15 .

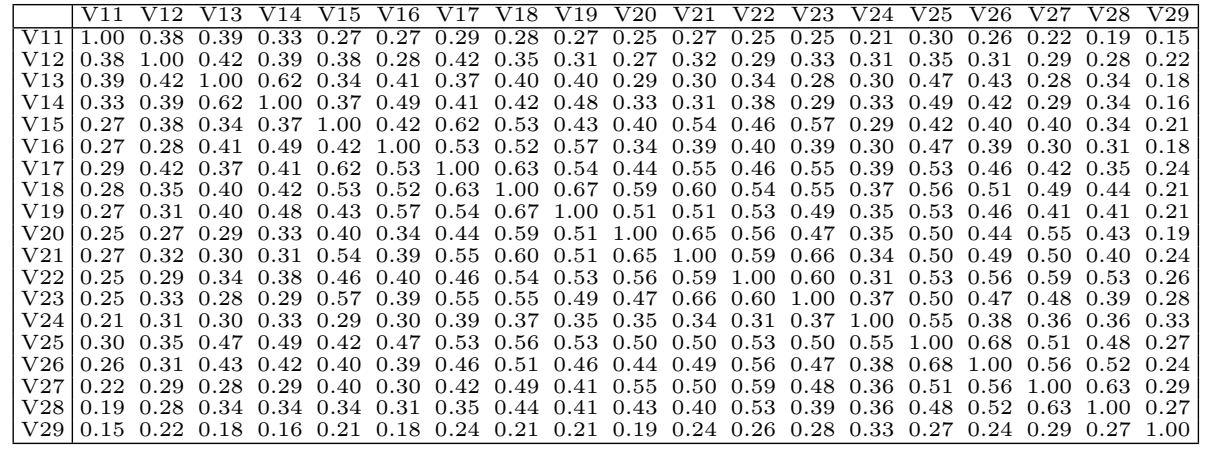

Tabla 4.13: Matriz de correlaciones observadas. Competencias adquiridas en la Universidad 


\section{Análisis de consistencia interna y validez del cuestionario}

La mayoría de los estudios relativos a la fiabilidad y consistencia interna de cuestionarios se basan en el valor del coeficiente Alfa de Cronbach. Los valores de este coeficiente oscilan entre 0 y 1 y se considera que la consistencia interna del cuestionario es mayor a medida que se aproxima a la unidad. Posteriormente se expondrán las razones que desaconsejan su uso para el análisis de la consistencia interna de conjuntos de variables en contextos multidimensionales. Sin embargo, puesto que la gran mayoría de los estudios sobre fiabilidad de cuestionarios y validación de escalas se basan en este parámetro, se ha calculado igualmente con el fin de valorar esta resultado como referencia para posteriores análisis de fiabilidad.

El coeficiente Alfa de Cronbach tomó el valor de 0.673 para los 17 ítems correspondientes a las variables sobre Características de los estudios y Métodos de enseñanza - aprendizaje mientras que alcanzó el valor 0.926 para los 19 ítems referidos a las Competencias adquiridas en la Universidad. Estos valores se pueden considerar aceptables en el primer caso y bastante buenos en el segundo.

Asimismo, se ha aplicado otro criterio para la evaluación de la consistencia interna de los cuestionarios consistente en la obtención del mismo coeficiente Alfa de Cronbach, aunque eliminando cada uno de los ítems planteados en el cuestionario. Así se evalúa la contribución individual de cada ítem a la explicación de la variabilidad del constructo. En el caso de los ítems referidos a Características de los estudios y Métodos de enseñanza - aprendizaje oscilaron en torno a los valores 0.6 y 0.7 lo cual no supone una variación excesiva respecto al valor de referencia 0.673 .

Respecto a los ítems referidos a las Competencias adquiridas en la Universidad tomaron valores alrededor de 0.92 , lo cual supone una variabilidad todavía más ajustada que en el caso anterior, indicando que la contribución de todos los ítems es similar en todo el conjunto. 


\begin{tabular}{|l|c|}
\hline Descripción & $\alpha$ Cronbach \\
\hline En tu carrera el enfoque era generalista & 0.709 \\
El profesor era la principal fuente de información & 0.687 \\
Tu carrera era una carrera con prestigio académico & 0.685 \\
En tu carrera había que trabajar mucho para aprobar & 0.677 \\
Realización de pruebas tipo test & 0.676 \\
Teorías, conceptos y paradigmas & 0.676 \\
En tu carrera podías elegir las asignaturas que querías cursar & 0.669 \\
En tu carrera el enfoque era especializado & 0.662 \\
En tu carrera el contenido era conocido por los empleadores & 0.661 \\
Asistencia a clase & 0.648 \\
Aprendizaje basado en proyectos o problemas & 0.644 \\
Trabajos escritos & 0.642 \\
Exposiciones orales & 0.638 \\
Participación en proyectos de investigación & 0.636 \\
Prácticas en empresas, instituciones o similares & 0.634 \\
Conocimientos prácticos y metodológicos & 0.627 \\
Trabajos en grupo & 0.618 \\
\hline
\end{tabular}

Tabla 4.14: $\alpha$ Cronbach con exclusión de cada uno de los ítems. Características de los estudios y Métodos de enseñanza - aprendizaje

\begin{tabular}{|l|c|}
\hline Descripción & $\alpha$ Cronbach \\
\hline Escribir y hablar en idiomas extranjeros & 0.928 \\
Dominio de tu área o disciplina & 0.927 \\
Conocimientos de otras áreas o disciplinas & 0.925 \\
Utilizar herramientas informáticas & 0.925 \\
Pensamiento analítico & 0.924 \\
Rendir bajo presión & 0.923 \\
Adquirir con rapidez nuevos conocimientos & 0.923 \\
Redactar informes o documentos & 0.923 \\
Negociar de forma eficaz & 0.922 \\
Trabajar en equipo & 0.922 \\
Presentar en público productos, ideas o informes & 0.922 \\
Hacer valer tu autoridad & 0.921 \\
Predisposición para cuestionar ideas propias o ajenas & 0.921 \\
Usar el tiempo de forma efectiva & 0.921 \\
Movilizar las capacidades de otros & 0.921 \\
Detectar nuevas oportunidades & 0.921 \\
Hacerte entender & 0.921 \\
Coordinar actividades & 0.92 \\
Encontrar nuevas ideas y soluciones & 0.92 \\
\hline
\end{tabular}

Tabla 4.15: $\alpha$ Cronbach con exclusión de cada uno de los ítems. Competencias adquiridas en la Universidad

\section{Determinación de la dimensionalidad de metodologías docentes y competencias adquiridas en la Universidad}

En la fase de determinación de la dimensionalidad se detectaron ciertos ítems cuya comunalidad resultó ser excesivamente baja respecto al resto. Concretamente las variables 2 y 3 - En tu carrera el contenido era conocido por los empleadores y En tu carrera podías elegir las asignaturas que querías cursar - obtuvieron valores en torno a 0.3 mientras que la comunalidad del ítem 17 - Realización de pruebas tipo test - obtuvo un valor de 0.627, superior a los anteriores. Estos tres ítems fueron excluidos del análisis factorial 
por sus bajos valores de comunalidad, como es evidente en los ítems 2 y 3 , pero también por su propia definición, más alejada del constructo que se intenta representar. Esta última razón se aplica en mayor medida al ítem 17, que según la teoría debería ser clasificado como método de evaluación y no como posible Característica de los estudios o Método de enseñanza aprendizaje.

\begin{tabular}{|l|c|}
\hline Descripción & Comunalidad \\
\hline En tu carrera el contenido era conocido por los empleadores & 0.300 \\
En tu carrera podías elegir las asignaturas que querías cursar & 0.299 \\
Realización de pruebas tipo test & 0.627 \\
\hline
\end{tabular}

Tabla 4.16: Comunalidades de los ítems excluidos del análisis. Características de los estudios y Métodos de enseñanza - aprendizaje

El resto de los ítems obtuvieron valores superiores a los anteriores y/o se consideraron relevantes para la definición del constructo subyacente a los ítems restantes, por lo que finalmente fueron incluidos en el análisis factorial.

\begin{tabular}{|l|c|}
\hline Descripción & Comunalidad \\
\hline En tu carrera había que trabajar mucho para aprobar & 0.700 \\
En tu carrera el enfoque era generalista & 0.850 \\
En tu carrera el enfoque era especializado & 0.824 \\
Tu carrera era una carrera con prestigio académico & 0.682 \\
\hline Asistencia a clase & 0.350 \\
Trabajos en grupo & 0.72 \\
Participación en proyectos de investigación & 0.467 \\
Prácticas en empresas, instituciones o similares & 0.680 \\
Conocimientos prácticos y metodológicos & 0.680 \\
Teorías, conceptos y paradigmas & 0.600 \\
El profesor era la principal fuente de información & 0.741 \\
Aprendizaje basado en proyectos o problemas & 0.466 \\
Trabajos escritos & 0.693 \\
Exposiciones orales & 0.645 \\
\hline
\end{tabular}

Tabla 4.17: Comunalidades de los ítems incluidos en el análisis. Características de los estudios y Métodos de enseñanza - aprendizaje

En el caso de las variables referidas a las Competencias adquiridas en la Universidad todos los ítems obtuvieron valores de comunalidad aproximadamente similares. Asimismo se consideró que todas las descripciones de los ítems hacen referencia a algún tipo de competencia definida previamente según la teoría. Por tanto, ninguno de ellos fue excluido en la aplicación del análisis factorial. 


\begin{tabular}{|l|c|}
\hline Descripción & Comunalidad \\
\hline Dominio de tu área o disciplina & 0.453 \\
Conocimientos de otras áreas o disciplinas & 0.430 \\
Pensamiento analítico & 0.569 \\
Adquirir con rapidez nuevos conocimientos & 0.541 \\
Negociar de forma eficaz & 0.528 \\
Rendir bajo presión & 0.492 \\
Detectar nuevas oportunidades & 0.511 \\
Coordinar actividades & 0.431 \\
Usar el tiempo de forma efectiva & 0.560 \\
Trabajar en equipo & 0.677 \\
Movilizar las capacidades de otros & 0.560 \\
Hacerte entender & 0.465 \\
Hacer valer tu autoridad & 0.455 \\
Utilizar herramientas informáticas & 0.661 \\
Encontrar nuevas ideas y soluciones & 0.691 \\
Predisposición para cuestionar ideas propias o ajenas & 0.604 \\
Presentar en público productos, ideas o informes & 0.507 \\
Redactar informes o documentos & 0.598 \\
Escribir y hablar en idiomas extranjeros & 0.536 \\
\hline
\end{tabular}

Tabla 4.18: Comunalidades de los ítems incluidos en el análisis. Competencias adquiridas en la Universidad

En la extracción de las componentes principales de las variables referidas a las Características de los estudios y Métodos de enseñanza - aprendizaje se seleccionaron las 5 primeras componentes, que explican el $65 \%$ de la varianza de los datos. Según el criterio de Kaiser deberían haberse seleccionado únicamente aquellos ítems cuyo autovalor fuera superior a la unidad, de modo que, considerando exclusivamente este criterio, el quinto factor ya no se habría incluido. Asimismo el porcentaje de varianza explicada por los 4 primeros factores se encuentra muy próximo al umbral de referencia del $60 \%$ definido para aplicaciones de este análisis en el área de las ciencias sociales. Sin embargo, puesto que el autovalor correspondiente a este quinto factor no se encuentra muy alejado de la unidad, y por motivos de interpretabilidad de la solución obtenida, se consideró oportuno incluir también esta componente en la solución final. Así, se ha reducido el conjunto inicial de las 14 variables a un nuevo conjunto de 5 dimensiones o variables latentes logrando un porcentaje de varianza explicada del $65 \%$. 


\begin{tabular}{|c|c|c|}
\hline & Autovalores & \% Var. Acum. \\
\hline 1 & 3.563 & $25.5 \%$ \\
2 & 1.986 & $39.6 \%$ \\
3 & 1.466 & $50.1 \%$ \\
4 & 1.121 & $58.1 \%$ \\
5 & 0.962 & $65.0 \%$ \\
6 & 0.831 & $70.9 \%$ \\
7 & 0.731 & $76.1 \%$ \\
8 & 0.654 & $80.8 \%$ \\
9 & 0.615 & $85.2 \%$ \\
10 & 0.506 & $88.8 \%$ \\
11 & 0.471 & $92.2 \%$ \\
12 & 0.439 & $95.3 \%$ \\
13 & 0.339 & $97.7 \%$ \\
14 & 0.316 & $100 \%$ \\
\hline
\end{tabular}

Tabla 4.19: Autovalores y porcentaje de varianza acumulada. Características de los estudios y Métodos de enseñanza - aprendizaje

El gráfico de sedimentación y la representación gráfica del porcentaje de varianza acumulada muestran claramente que las diferencias entre los factores 4 y 5 son muy reducidas por lo que ambos factores son candidatos para definir el número de componentes finalmente seleccionadas.

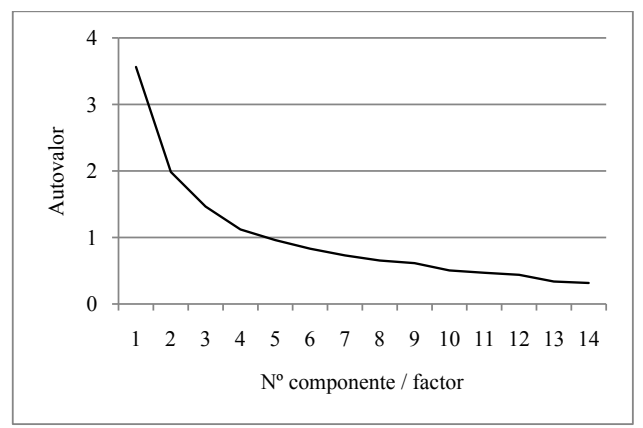

Figura 4.7: Autovalores. Características de los estudios y Métodos de enseñanza - aprendizaje 


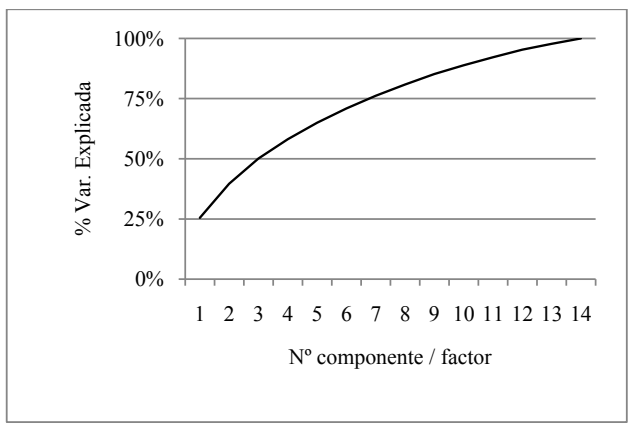

Figura 4.8: Porcentaje de varianza acumulada. Características de los estudios y Métodos de enseñanza - aprendizaje

Respecto a las Competencias adquiridas en la Universidad, tanto en los resultados de los autovalores y porcentajes de varianza explicada, como en resultados posteriores relativos a la matriz de cargas factoriales se observa la presencia del llamado factor tamaño. Concretamente en la Tabla 4.20 se observa que los datos originales, con factor tamaño, existe un primer factor cuyo autovalor es relativamente mucho más elevado a los restantes $(8,396)$. Asimismo el porcentaje de varianza explicada por este autovalor $(44,2 \%)$ es mucho mayor a la aportación de los siguientes factores. Este primer factor resultante del análisis factorial explica la mayor parte de la varianza asociada a las variables originales. Tal y como se ha indicado anteriormente este efecto es consecuencia de la alta correlación existente entre variables de forma que constituyen un haz homogéneo de competencias en torno al primer factor. Así, si un individuo asigna una puntuación alta a alguna competencia, también lo hace con el resto de competencias. El hecho de que este fenómeno se repita de forma generalizada en el conjunto de datos implica que se genere un primer factor dominante que elimina la utilidad del análisis aplicado.

Para la eliminación del factor tamaño se aplica la transformación sobre las variables, propuesta por Aluja (1999), consistente en restar y dividir cada uno de los valores por el correspondiente valor promedio del individuo.

Una vez aplicada esta transformación, la media de cada una de estas nuevas variables expresa la diferencia entre la contribución media de los estudios al desarrollo de competencias en general y la contribución individual correspondiente a cada una de ellas. Como se observa en la Tabla 4.20, la aplicación del análisis de componentes principales sobre las variables transformadas da como resultado valores más equilibrados en las distintas componentes extraídas, tanto en los autovalores como en los porcentajes de varianza explicada. 


\begin{tabular}{|c|c|c|c|}
\hline \multicolumn{2}{|c|}{ Con Factor tamaño } & \multicolumn{2}{|c|}{ Sin Factor tamaño } \\
\hline Autovalores & \% Var. Acum. & Autovalores & \% Var. Acum. \\
\hline 8.396 & $44.2 \%$ & 2.620 & $13.8 \%$ \\
1.456 & $51.9 \%$ & 2.180 & $25.3 \%$ \\
1.145 & $57.9 \%$ & 1.580 & $33.6 \%$ \\
0.987 & $63.1 \%$ & 1.440 & $41.2 \%$ \\
0.886 & $67.7 \%$ & 1.240 & $47.7 \%$ \\
0.708 & $71.5 \%$ & 1.200 & $54.0 \%$ \\
0.693 & $75.1 \%$ & 1.010 & $59.4 \%$ \\
0.598 & $78.3 \%$ & 0.930 & $64.3 \%$ \\
0.564 & $81.2 \%$ & 0.850 & $68.8 \%$ \\
0.502 & $83.9 \%$ & 0.830 & $73.1 \%$ \\
0.469 & $86.3 \%$ & 0.750 & $77.1 \%$ \\
0.423 & $88.6 \%$ & 0.720 & $80.9 \%$ \\
0.379 & $90.6 \%$ & 0.670 & $84.4 \%$ \\
0.328 & $92.3 \%$ & 0.650 & $87.9 \%$ \\
0.326 & $94.0 \%$ & 0.620 & $91.1 \%$ \\
0.321 & $95.7 \%$ & 0.610 & $94.4 \%$ \\
0.288 & $97.2 \%$ & 0.560 & $97.3 \%$ \\
0.285 & $98.7 \%$ & 0.520 & $100.0 \%$ \\
0.245 & $100.0 \%$ & 0.000 & $100.0 \%$ \\
\hline
\end{tabular}

Tabla 4.20: Autovalores y porcentaje de varianza acumulada con y sin eliminación del factor tamaño. Competencias adquiridas en la Universidad

En este caso el gráfico de sedimentación y el porcentaje de varianza explicada por las componentes extraídas incluyen tanto los resultados con factor tamaño como los obtenidos tras su eliminación. En la Figura 4.9 se observa la enorme diferencia entre el primer autovalor y los restantes en los autovalores obtenidos sobre los datos con factor tamaño. Igualmente la pendiente de la curva de porcentaje de varianza resulta mucho elevada en el caso de los datos originales, sin eliminación del factor tamaño.

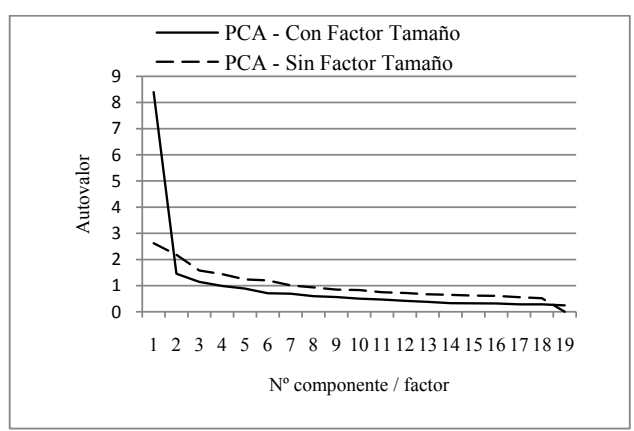

Figura 4.9: Autovalores con y sin eliminación del factor tamaño. Competencias adquiridas en la Universidad 


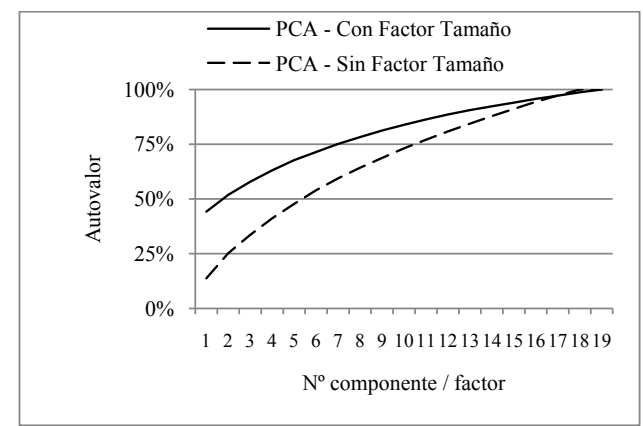

Figura 4.10: Porcentaje de varianza acumulada con y sin eliminación del factor tamaño. Competencias adquiridas en la Universidad

La matriz de cargas factoriales, obtenida a partir del análisis de componentes principales sobre los datos originales de Competencias adquiridas en la Universidad, confirma igualmente la conveniencia de realizar esta transformación. En la Tabla 4.21 se observa que la mayoría de las saturaciones con valores elevados recaen sobre el primer factor y disminuye a medida que se incrementa el número de componente. Así, mientras que la primera componente consta de 11 ítems con saturaciones significativas, este número disminuye a 9 para la segunda componente, 8 para la tercera y 4 para la cuarta. El número de ítems correspondientes a las componentes 5 y 6 no supera las dos unidades. Independientemente de la imposibilidad de realizar ninguna interpretación de estos resultados según la teoría, esta distribución de saturaciones no resulta recomendable, confirmando nuevamente la necesidad de aplicar la transformación a los datos originales. 


\begin{tabular}{|l|c|c|c|c|c|c|}
\hline Descripción & 1 & 2 & 3 & 4 & 5 & 6 \\
\hline Dominio de tu área o disciplina & 0.164 & 0.108 & 0.134 & 0.774 & 0.042 & -0.049 \\
Conocimientos de otras áreas o disciplinas & 0.240 & 0.090 & 0.167 & 0.714 & 0.121 & 0.178 \\
Pensamiento analítico & 0.023 & 0.246 & 0.646 & 0.465 & 0.146 & 0.002 \\
Adquirir con rapidez nuevos conocimientos & 0.108 & 0.209 & 0.739 & 0.321 & 0.138 & 0.003 \\
Negociar de forma eficaz & 0.698 & 0.146 & 0.183 & 0.283 & 0.003 & 0.145 \\
Rendir bajo presión & 0.454 & 0.061 & 0.700 & 0.002 & 0.054 & 0.108 \\
Detectar nuevas oportunidades & 0.698 & 0.100 & 0.338 & 0.201 & 0.188 & 0.131 \\
Coordinar actividades & 0.654 & 0.313 & 0.380 & 0.065 & 0.156 & -0.021 \\
Usar el tiempo de forma efectiva & 0.538 & 0.272 & 0.545 & -0.007 & 0.112 & 0.024 \\
Trabajar en equipo & 0.531 & 0.518 & 0.080 & 0.081 & 0.242 & -0.171 \\
Movilizar las capacidades de otros & 0.719 & 0.400 & 0.038 & 0.151 & 0.168 & -0.026 \\
Hacerte entender & 0.479 & 0.654 & 0.183 & 0.094 & -0.005 & 0.064 \\
Hacer valer tu autoridad & 0.714 & 0.317 & 0.037 & 0.163 & 0.156 & 0.146 \\
Utilizar herramientas informáticas & 0.197 & 0.159 & 0.135 & 0.120 & 0.863 & 0.193 \\
Encontrar nuevas ideas y soluciones & 0.311 & 0.440 & 0.376 & 0.148 & 0.545 & 0.018 \\
Predisposición para cuestionar ideas propias o ajenas & 0.234 & 0.620 & 0.323 & 0.129 & 0.290 & 0.033 \\
Presentar en público productos, ideas o informes & 0.311 & 0.777 & 0.049 & 0.120 & 0.112 & 0.121 \\
Redactar informes o documentos & 0.127 & 0.773 & 0.222 & 0.092 & 0.083 & 0.198 \\
Escribir y hablar en idiomas extranjeros & 0.125 & 0.189 & 0.048 & 0.079 & 0.162 & 0.897 \\
\hline
\end{tabular}

Tabla 4.21: Matriz de componentes sin eliminación de factor tamaño. Competencias adquiridas en la Universidad

Como se observa en la Tabla 4.22, en los ítems referentes a las Características de los estudios y Métodos de enseñanza - aprendizaje no existe ningún indicio de la existencia de este factor. En los resultados anteriores se ha comprobado que los autovalores y porcentajes de varianza explicada se distribuyen sin cambios bruscos notables en sus valores entre las distintas componentes. Acorde con estos resultados, la distribución de las saturaciones de los ítems se realiza de forma equilibrada entre las 5 componentes seleccionadas.

\begin{tabular}{|l|c|c|c|c|c|}
\hline Descripción & 1 & 2 & 3 & 4 & 5 \\
\hline En tu carrera había que trabajar mucho para aprobar & -0.02 & 0.04 & -0.05 & 0.83 & 0.05 \\
En tu carrera el enfoque era generalista & -0.02 & -0.92 & -0.04 & 0.01 & 0.10 \\
En tu carrera el enfoque era especializado & 0.12 & 0.87 & 0.19 & 0.14 & 0.02 \\
Tu carrera era una carrera con prestigio académico & -0.18 & 0.06 & 0.12 & 0.79 & 0.04 \\
Asistencia a clase & 0.56 & 0.02 & 0.18 & 0.05 & 0.03 \\
Trabajos en grupo & 0.78 & 0.05 & 0.31 & -0.09 & -0.03 \\
Participación en proyectos de investigación & 0.52 & 0.09 & 0.39 & 0.14 & -0.13 \\
Prácticas en empresas, instituciones o similares & 0.18 & -0.02 & 0.79 & -0.12 & -0.06 \\
Conocimientos prácticos y metodológicos & 0.23 & 0.18 & 0.76 & 0.08 & 0.03 \\
Teorías, conceptos y paradigmas & 0.12 & -0.10 & -0.09 & 0.28 & 0.70 \\
El profesor era la principal fuente de información & -0.14 & 0.01 & 0.04 & -0.12 & 0.84 \\
Aprendizaje basado en proyectos o problemas & 0.20 & 0.16 & 0.51 & 0.38 & 0.02 \\
Trabajos escritos & 0.81 & 0.06 & 0.02 & -0.15 & 0.07 \\
Exposiciones orales & 0.79 & 0.04 & 0.08 & -0.08 & -0.06 \\
\hline
\end{tabular}

Tabla 4.22: Matriz de componentes y rotación Varimax. Características de los estudios y Métodos de enseñanza - aprendizaje 
En la Tabla 4.23 se observa que el primer factor Estudio y trabajo en grupo, está compuesto por los ítems Asistencia a clase, Trabajos en grupo, Participación en proyectos de investigación, Trabajos escritos y Exposiciones orales, cuyo Alfa de Cronbach alcanza el valor de 0.787. No ocurre los mismo con otros factores formados por un número inferior de ítems. Así, el segundo factor Especialización, compuesto por el ítem de carácter opuesto En tu carrera el enfoque era especializado y En tu carrera el enfoque era generalista únicamente no aporta un valor válido para este parámetro. El tercer factor Clases prácticas y Prácticas externas comprende los métodos relativos a Prácticas en empresa, instituciones o similares, Conocimientos prácticos y metodológicos y Aprendizaje basado en proyectos o problemas, de modo que el Alfa de Cronbach asciende hasta el valor 0.631. El cuarto factor Esfuerzo y Prestigio está compuesto por dos aspectos tradicionalmente muy vinculados como son las percepciones de los ítems En tu carrera habia que trabajar mucho para aprobar al tiempo que Tu carrera era una carrera con prestigio académico. En este caso el Alfa de Cronbach toma el valor 0.626. Por último el quinto factor Clases Teóricas hace referencia a las metodologías tradicionales, como son las Teorías, conceptos y paradigmas y El profesor era la principal fuente de información. En este factor destaca el hecho de que, puesto que sólo se encuentra formado por dos ítems, el Alfa de Cronbach se limita al valor 0.364 .

Por otro lado en la matriz de cargas factoriales de los ítems referidos a las Competencias adquiridas en la Universidad, reflejada en la Tabla 4.24, puede observarse que determinados factores contienen altas saturaciones con signos positivos y negativos. En este caso es posible agrupar las competencias en función de su signo ya que existe cierta relación lógica entre competencias del mismo signo y al mismo tiempo contrapuesta al conjunto de competencias con signo opuesto. Por esta razón es posible considerar estos factores como una combinación de ambas competencias genéricas, a su vez opuestas y excluyentes. 


\begin{tabular}{|l|l|l|}
\hline Factor & $\alpha$ & Ítems \\
\hline 1-Estudio y trabajo en grupo & $\alpha=0,787$ & $\begin{array}{l}\text { Asistencia a clase } \\
\text { Trabajos en grupo } \\
\text { Proyectos de investigación } \\
\text { Trabajos escritos } \\
\text { Exposiciones orales }\end{array}$ \\
\hline 2-Especialización & - & $\begin{array}{l}\text { Enfoque generalista } \\
\text { Enfoque especializado }\end{array}$ \\
\hline 3-Clases prácticas y Prácticas externas & $\alpha=0,631$ & $\begin{array}{l}\text { Prácticas en empresas } \\
\text { Conocimientos prácticos y metodológicos } \\
\text { Proyectos o problemas }\end{array}$ \\
\hline 4-Esfuerzo y prestigio & $\alpha=0,626$ & $\begin{array}{l}\text { Había que trabajar mucho para aprobar } \\
\text { Prestigio académico }\end{array}$ \\
\hline 5-Clases teóricas & $\alpha=0,364$ & $\begin{array}{l}\text { Teorías, conceptos y paradigmas } \\
\text { El profesor era la principal fuente de información }\end{array}$ \\
\hline
\end{tabular}

Tabla 4.23: Interpretación de las componentes principales. Características de los estudios y Métodos de enseñanza - aprendizaje

\begin{tabular}{|c|l|c|c|c|c|c|c|}
\hline Ítem & Descripción & 1 & 2 & 3 & 4 & 5 & 6 \\
\hline 1 & Dominio de tu área o disciplina & -0.24 & -0.31 & -0.37 & -0.01 & -0.40 & 0.07 \\
2 & Conocimientos de otras áreas o disciplinas & 0.00 & -0.27 & -0.43 & -0.20 & -0.36 & -0.03 \\
3 & Pensamiento analítico & -0.48 & -0.38 & -0.24 & -0.26 & -0.03 & 0.26 \\
4 & Adquirir con rapidez nuevos conocimientos & -0.49 & -0.34 & -0.05 & -0.31 & -0.07 & 0.27 \\
5 & Negociar de forma eficaz & 0.64 & -0.13 & -0.09 & -0.17 & -0.15 & 0.21 \\
6 & Rendir bajo presión & 0.00 & -0.19 & 0.55 & -0.38 & -0.05 & 0.10 \\
7 & Detectar nuevas oportunidades & 0.58 & -0.26 & 0.24 & -0.21 & -0.02 & -0.03 \\
8 & Coordinar actividades & 0.17 & -0.11 & 0.58 & 0.21 & -0.03 & 0.06 \\
9 & Usar el tiempo de forma efectiva & -0.13 & -0.07 & 0.72 & 0.01 & -0.11 & 0.06 \\
10 & Trabajar en equipo & -0.12 & 0.10 & 0.11 & 0.80 & -0.05 & 0.02 \\
11 & Movilizar las capacidades de otros & 0.42 & -0.03 & 0.01 & 0.61 & -0.02 & 0.11 \\
12 & Hacerte entender & 0.10 & 0.51 & -0.02 & 0.22 & 0.09 & 0.37 \\
13 & Hacer valer tu autoridad & 0.64 & 0.04 & -0.07 & 0.18 & 0.05 & 0.06 \\
14 & Utilizar herramientas informáticas & -0.10 & -0.17 & -0.12 & 0.08 & 0.25 & -0.73 \\
15 & Encontrar nuevas ideas y soluciones & -0.06 & -0.17 & -0.04 & 0.01 & 0.79 & -0.19 \\
16 & Predisposición para cuestionar ideas propias o ajenas & -0.04 & 0.23 & -0.16 & -0.13 & 0.69 & 0.19 \\
17 & Presentar en público productos, ideas o informes & 0.02 & 0.68 & -0.14 & 0.16 & 0.04 & -0.03 \\
18 & Redactar informes o documentos & -0.25 & 0.69 & -0.07 & -0.22 & -0.01 & -0.06 \\
19 & Escribir y hablar en idiomas extranjeros & 0.01 & 0.25 & -0.13 & -0.18 & -0.27 & -0.59 \\
\hline
\end{tabular}

Tabla 4.24: Matriz de componentes con eliminación de factor tamaño y rotación Varimax. Competencias adquiridas en la Universidad 
A partir de los resultados obtenidos en esta matriz de cargas factoriales se ha elaborado la siguiente tabla resumen en la que se muestra la interpretación de cada uno de los factores así como las competencias que los componen y sus correspondientes Alfa de Cronbach verificando la fiabilidad de las escalas.

\begin{tabular}{|l|c|l|}
\hline Factor & $\alpha$ & Ítems \\
\hline 1-Liderazgo (+) - Pensamiento metacognitivo (-) & $\alpha=0,830$ & $\begin{array}{l}\text { Negociar de forma eficaz } \\
\text { Detectar nuevas oportunidades } \\
\text { Movilizar las capacidades de otros } \\
\text { Hacer valer tu autoridad } \\
\text { Pensamiento analítico } \\
\text { Adquirir con rapidez nuevos conocimientos }\end{array}$ \\
\hline 2-Comunicación (+) & $\alpha=0,809$ & $\begin{array}{l}\text { Hacerte entender } \\
\text { Presentar en público productos, ideas o informes } \\
\text { Redactar informes o documentos }\end{array}$ \\
\hline 3-Organización personal (+) - Conocimientos (-) & $\alpha=0,762$ & $\begin{array}{l}\text { Rendir bajo presión } \\
\text { Coordinar actividades } \\
\text { Movilizar las capacidades de otros } \\
\text { Usar el tiempo de forma efectiva } \\
\text { Dominio de tu área o disciplina } \\
\text { Conocimientos de otras áreas o disciplinas }\end{array}$ \\
\hline 4-Cooperación (+) & $\alpha=0,784$ & $\begin{array}{l}\text { Trabajar en equipo } \\
\text { Movilizar las capacidades de otros }\end{array}$ \\
\hline 5-Innovación (+) - Conocimientos (-) & $\alpha=0,708$ & $\begin{array}{l}\text { Encontrar nuevas ideas y soluciones } \\
\text { Predisposición para cuestionar ideas propias o ajenas } \\
\text { Dominio de tu área o disciplina } \\
\text { Conocimientos de otras áreas o disciplinas }\end{array}$ \\
\hline 6-Instrumental (-) & $\alpha=0,487$ & $\begin{array}{l}\text { Utilizar herramientas informáticas } \\
\text { Escribir y hablar en idiomas extranjeros }\end{array}$ \\
\hline
\end{tabular}

Tabla 4.25: Interpretación de las componentes principales con eliminación del factor tamaño. Competencias adquiridas en la Universidad

Esta doble interpretación puede aplicarse al primer factor en el que las competencias relacionadas con el Liderazgo se contraponen al Pensamiento metacognitivo, asociadas a las capacidades de aprendizaje y pensamiento analítico. En este caso el factor Liderazgo está compuesto por los ítems Negociar de forma eficaz, Detectar nuevas oportunidades, Movilizar las capacidades de otros y Hacer valer tu autoridad mientras que el Pensamiento metacognitivo incluye los ítems relacionados con el Pensamiento analítico y Adquirir con rapidez nuevos conocimientos. Así, la bipolaridad de este factor implica que individuos con saturaciones altas positivas muestran mayor probabilidad de haber cursado estudios que fomentan el desarrollo del $L i$ derazgo pero no de Pensamiento metacognitivo. Lo mismo ocurre para los individuos con saturaciones altas negativas. Presentan mayor probabilidad de haber estudiado carreras universitarias en las que se desarrolla el Pensamiento metacognitivo pero no el Liderazgo.

Esta bipolaridad se aplica igualmente a los factores tres y cinco. En ambos casos los Conocimientos, definidos como Dominio de tu área o disci- 
plina y Conocimientos de otras áreas o disciplinas, se oponen al desarrollo de otro tipo de competencias. En el caso del tercer factor estos Conocimientos se contraponen al desarrollo de la capacidad de Organización personal, compuesta por los ítems Rendir bajo presión, Coordinar actividades y Usar el tiempo de forma efectiva, mientras que en el quinto factor se opone al desarrollo de la capacidad de Innovación, formada por los ítems Encontrar nuevas ideas y soluciones y Predisposición para cuestionar ideas propias o ajenas.

Por el contrario el segundo y cuarto factor únicamente muestran cargas factoriales significativas positivas, por lo que no resulta aplicable la doble interpretación comentada para los factores anteriores. En el caso del segundo factor Comunicación, se encuentra formado por los ítems, Hacerte entender, Presentar en público productos, ideas o informes y Redactar informes o documentos, mientras que el cuarto factor Cooperación lo componen los ítems Trabajar en equipo y Movilizar las capacidades de otros.

Estos mismos resultados pueden representarse de forma gráfica mediante los correspondientes círculos de variables activas. En la Figura 4.11 se observa cómo la Especialización de los estudios se opone al Enfoque Generalista así como la agrupación de ítems relativos a las metodologías basadas en el Estudio y trabajo en grupo. La Figura 4.12 muestra la asociación entre la percepción de que los estudios requieren mucho Esfuerzo para aprobar, y que al mismo tiempo gozan de cierto Prestigio. Por último la Figura 4.13 muestra con claridad el quinto factor, correspondiente a las Clases teóricas, integrado por los ítems relativos a las Teorías, conceptos y paradigmas y El profesor como principal fuente de información. 


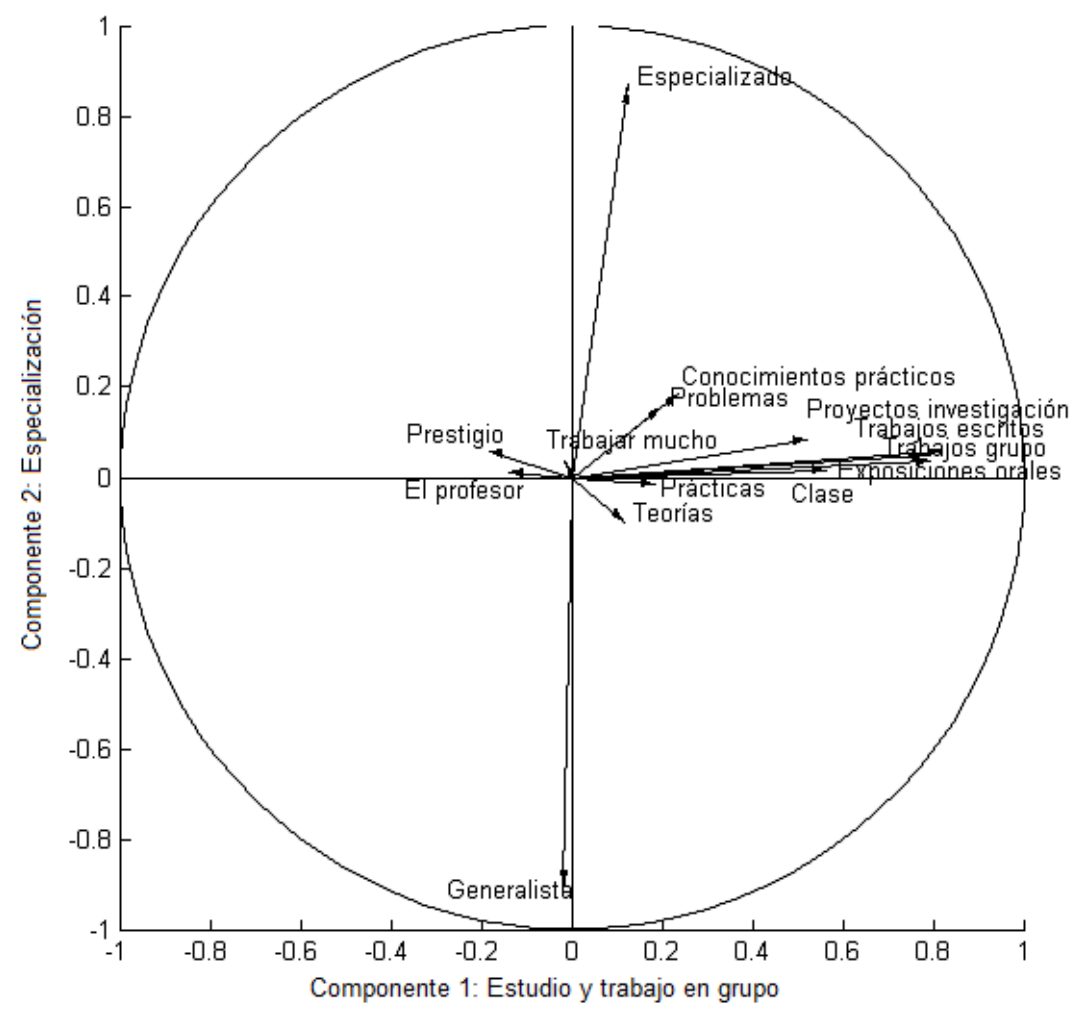

Figura 4.11: Círculo de variables activas en las componentes 1 y 2. Características de los estudios y Métodos de enseñanza - aprendizaje 


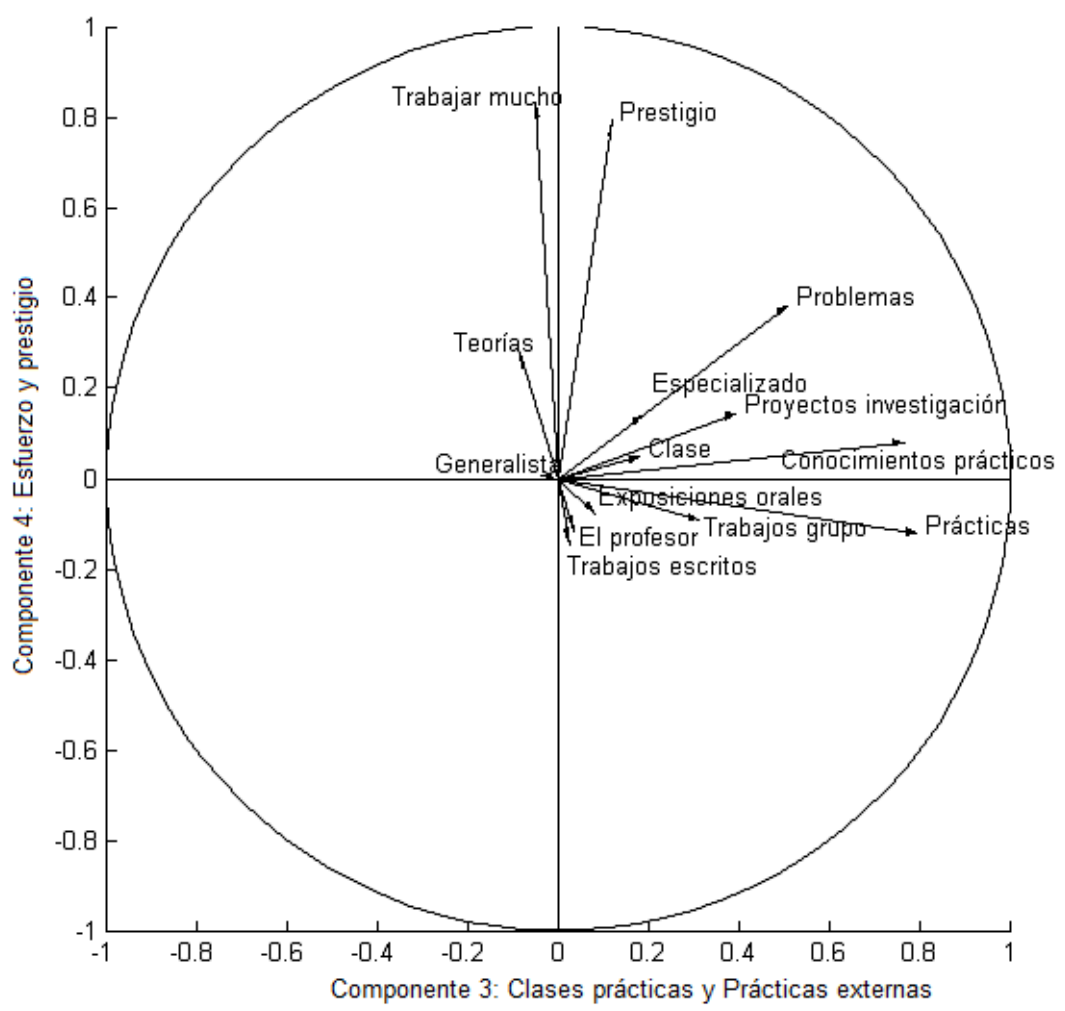

Figura 4.12: Círculo de variables activas en las componentes 3 y 4. Características de los estudios y Métodos de enseñanza - aprendizaje 


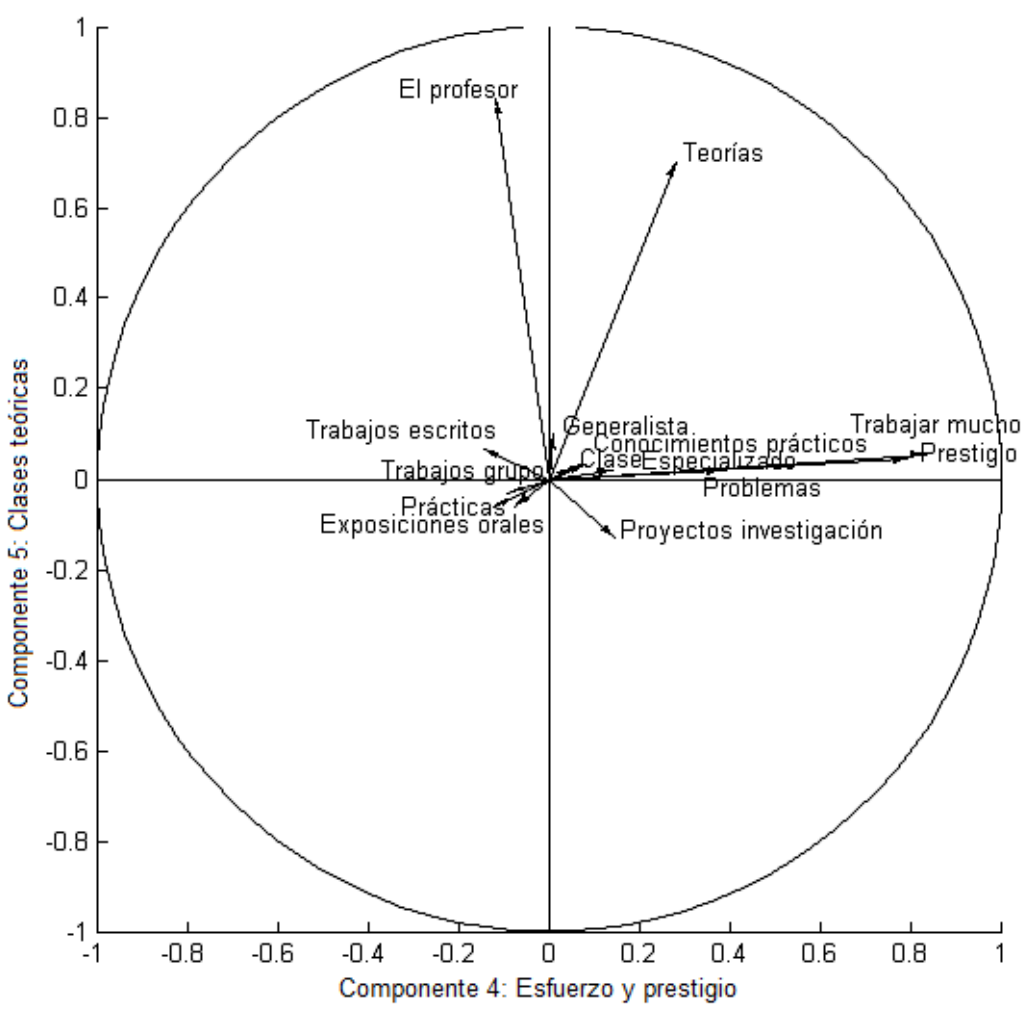

Figura 4.13: Círculo de variables activas en las componentes 5 y 6 . Características de los estudios y Métodos de enseñanza - aprendizaje

En el caso de las Competencias adquiridas en la Universidad la Figura 4.14 muestra las dimensiones relativas a la Comunicación y a la componente negativa sobre competencia Instrumental. Las variables relacionadas con esta componente se ubican en la mitad inferior del gráfico ya que sus cargas factoriales, aunque elevadas, resultaron ser negativas. La Figura 4.15 muestra la distribución de los ítems asociados con las capacidades de Cooperación y Organización personal mientras que la Figura 4.16 representa las componentes relativas a la Innovación, el Pensamiento analítico y el Liderazgo. 


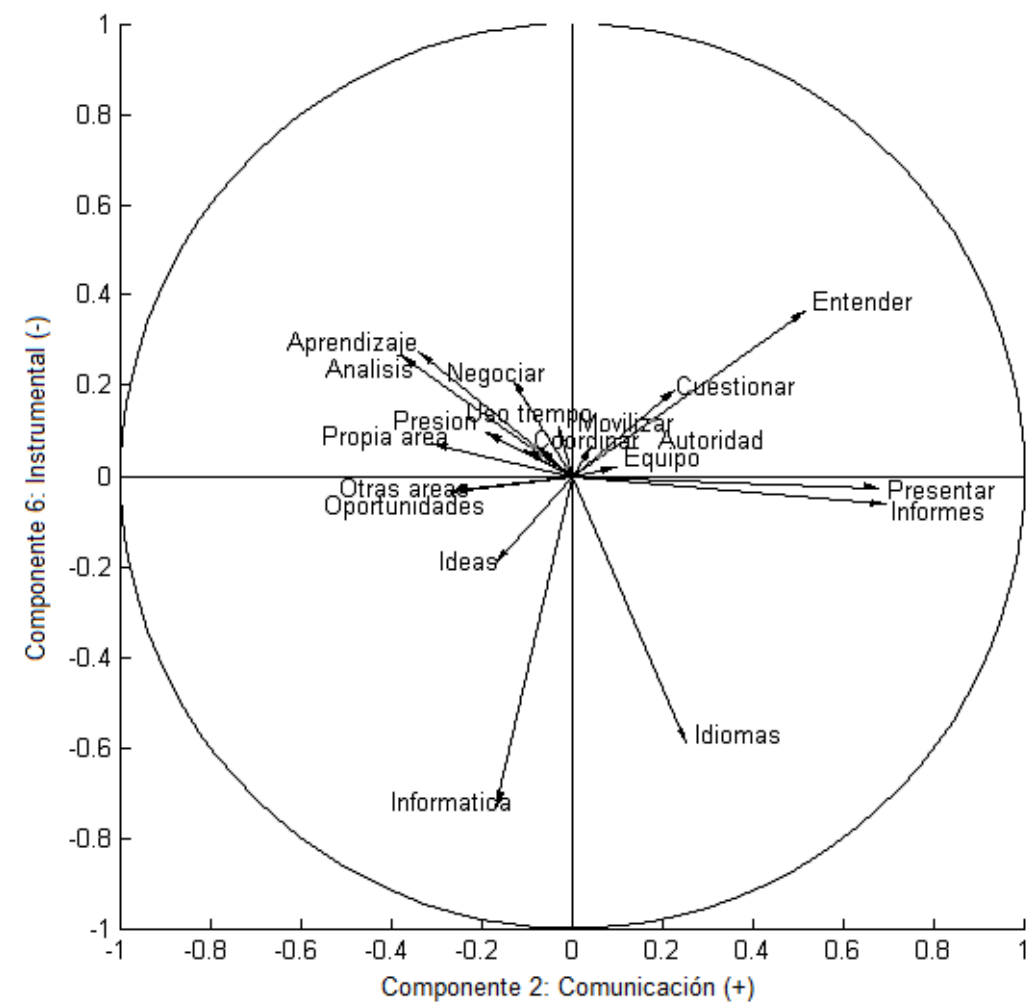

Figura 4.14: Círculo de variables activas en las componentes 2 y 6 . Competencias adquiridas en la Universidad 


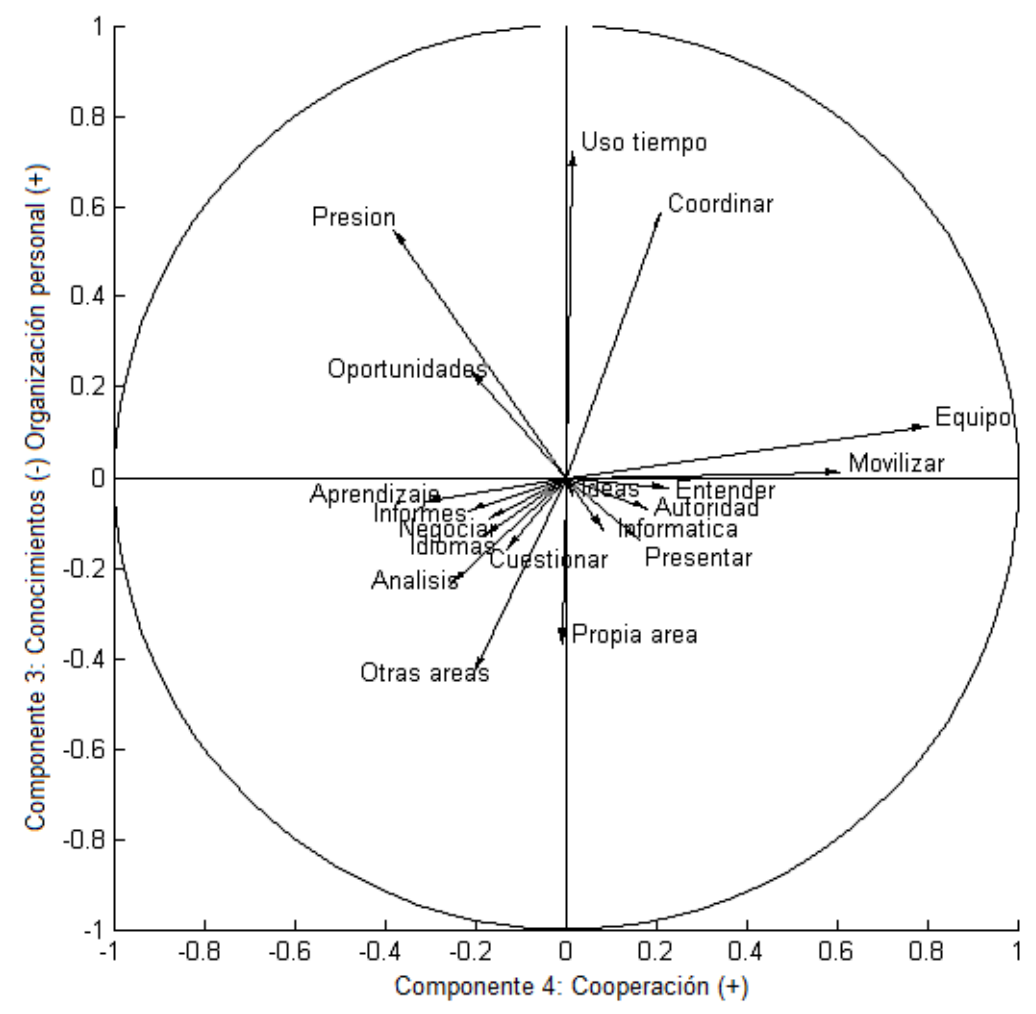

Figura 4.15: Círculo de variables activas en las componentes 4 y 3. Competencias adquiridas en la Universidad 


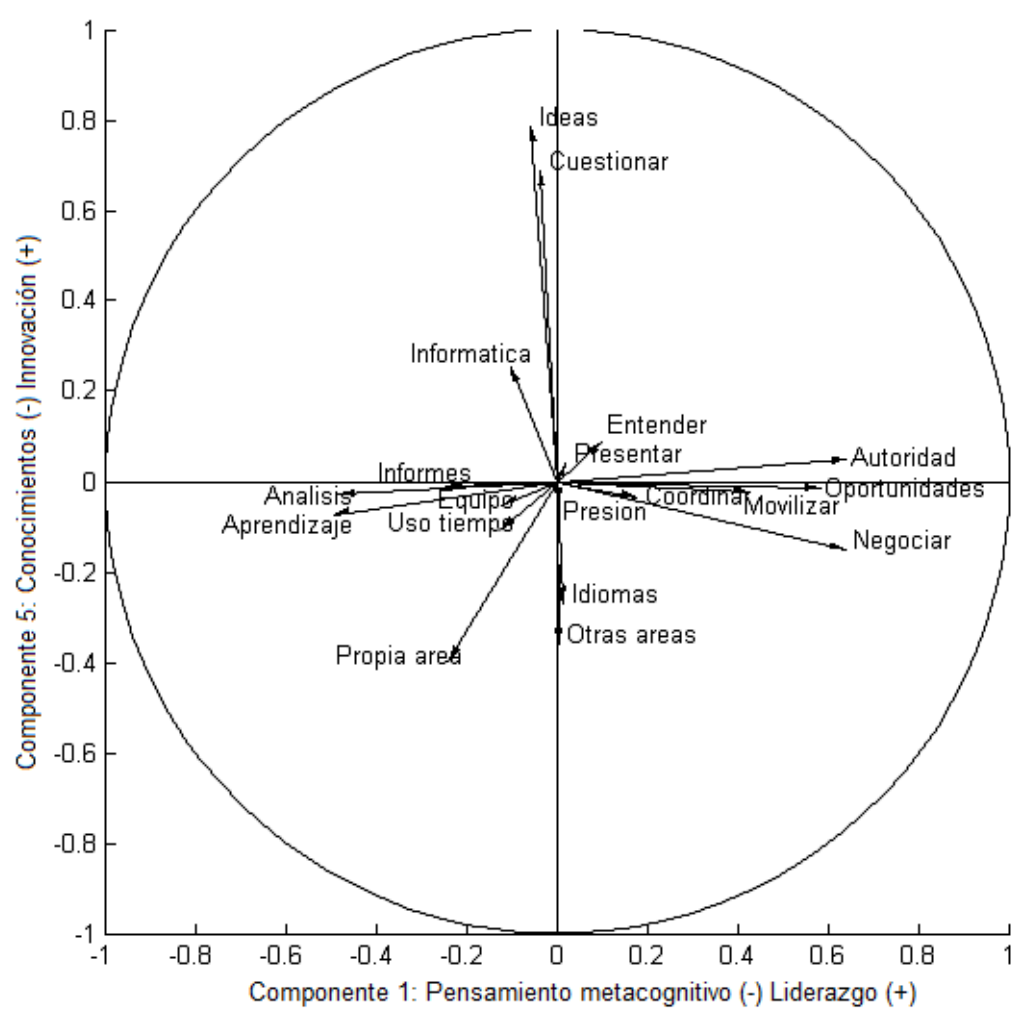

Figura 4.16: Círculo de variables activas en las componentes 1 y 5. Competencias adquiridas en la Universidad

\subsubsection{El contexto académico y personal de los estudiantes}

En este apartado se describen las relaciones de dependencia y asociación entre las distintas variables pertenecientes a los grupos definidos inicialmente. En primer lugar el Contexto académico, formado por las Características de la carrera y los Métodos de enseñanza - aprendizaje, y a continuación el Contexto personal, integrado por las variables relativas a la Gestión del tiempo y las Características personales. 


\section{Análisis bidimensional}

\section{Contrastes de independencia y medidas de asociación en el con- texto bidimensional}

Las variables clasificadas en como ítems relativos al entorno académico del encuestado son de tipo ordinal pero se han transformado a variables binarias con el fin de aplicar el mismo análisis de datos cualitativos a todas las variables. Esta transformación ha consistido en la agregación de las categorías superiores al promedio frente al resto, de modo que dichas variables inicialmente ordinales, se tratan en este análisis como variables cualitativas dicotómicas. Puesto que la escala de medida de estas variables oscila es una escala Likert de 1 a 5 se han agrupado las categorías 4 y 5 frente al resto, correspondiendo la primera opción a la presencia explícita de la característica enunciada frente a su ausencia o indeterminación.

Así, las variables clasificadas como ítems referentes a las Características de los estudios y los Métodos de enseñanza - aprendizaje se tratan en esta fase del análisis como dicotómicas mientras que las variables de otros grupos no necesariamente pertenecen a esta categoría. Concretamente las variables relativas a la edad del encuestado y su situación de convivencia constan respectivamente tres y cuatro categorías. El análisis de estas variables implica la utilización de los contrastes de independencia y medidas de asociación vinculados a tablas $r x c$. Por esta razón, no se han aplicado las técnicas expuestas para tablas $2 x 2$ en los bloques de variables que las contienen (comportamiento y características del encuestado).

Planteada la hipótesis nula de independencia entre todos los posibles pares de variables, el contraste de independencia para tablas $2 x 2$ verifica si efectivamente no existe relación entre el número de observaciones correspondientes a cada combinación de categorías. El objetivo de esta fase del análisis es descartar aquellas relaciones que no muestren indicios de dependencia con el fin de ser excluidas en posteriores análisis sobre la intensidad de dichas asociaciones. Es decir, que la aplicación del contraste de independencia a todos los posibles pares de variables se realiza con el fin de descartar en análisis posteriores aquellos cuya relación no resulta significativa.

Por motivos de espacio no es posible mostrar en una única tabla los resultados obtenidos para todos y cada uno de los pares de variables planteados. Por ello se mostrarán dichos resultados segmentados en tres subtablas según el esquema:

1. Relación entre los ítems referidos al entorno académico (Características de los estudios y Métodos de enseñanza - aprendizaje) 
2. Relación entre los ítems referidos al entorno académico y personal (Características de los estudios y Métodos de enseñanza - aprendizaje frente a Gestión del tiempo y Características personales)

3. Relación entre los ítems referidos al entorno personal (Gestión del tiempo y Características personales)

Las Tablas 4.26, 4.27 y 4.28 muestran el p-valor asociado a dicho contraste donde se observa que en la gran mayoría de los casos dicho p-valor resulta inferior al nivel de significación $\alpha=5 \%$ considerado habitualmente en este tipo de análisis. En estas tablas se han señalado en gris los p-valores superiores al umbral de 0.05 especificado para este nivel de significación. No obstante, la mayoría de estos p-valores observados en tablas resultan incluso significativos considerando un nivel de significación del 1\%. Así, en términos generales se observa un elevado número de relaciones de dependencia entre los ítems relativos al entorno académico del alumno, tanto en los subgrupos de variables sobre Características de los estudios y sobre Métodos de enseñanza - aprendizaje como en las relaciones entre ítems pertenecientes a ambos grupos.

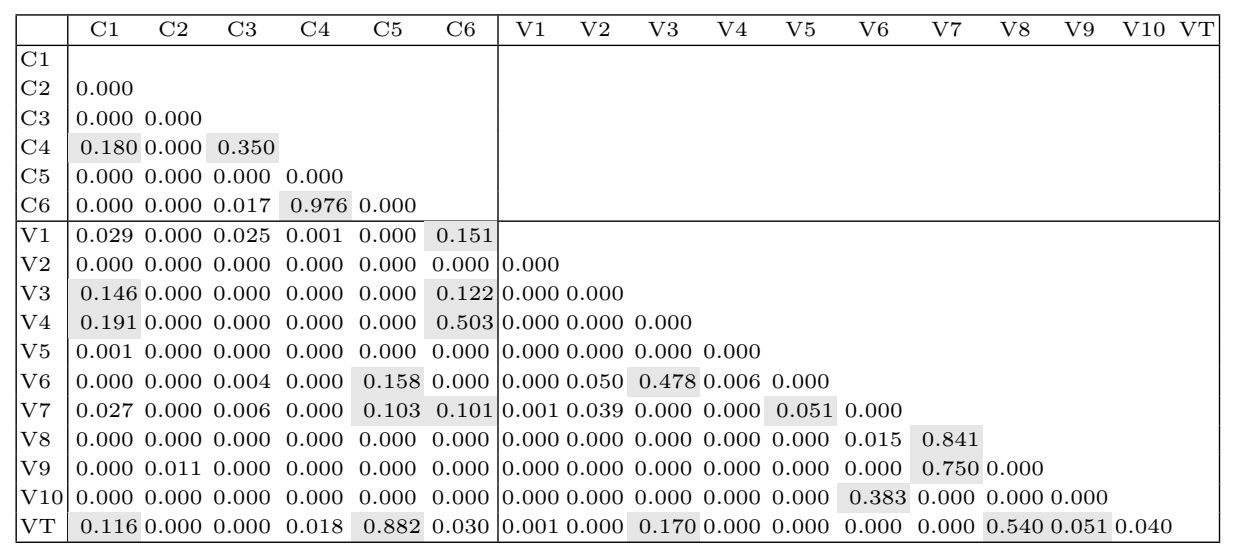

Tabla 4.26: p-valor del Contraste $\chi^{2}$ de independencia. Características de la carrera y Métodos de enseñanza - aprendizaje

En el caso de las relaciones entre los entornos académico y personal del encuestado es fácil observar que el número de relaciones de dependencia resulta inferior al caso anterior. Concretamente se comprueba que la mayoría de relaciones de dependencia se establecen entre el entorno académico y la gestión del tiempo, y no entre este entorno académico y las características personales. De hecho, puesto que en principio no cabe esperar ningún de tipo de relación entre las variables pertenecientes a ambos grupos, se deduce que las posibles relaciones observadas en este caso obedecen a alguna 
modificación del efecto, ya sea de interacción o confusión.

\begin{tabular}{|c|c|c|c|c|c|c|c|c|c|c|c|c|c|c|c|c|c|}
\hline & C1 & $\mathrm{C} 2$ & C3 & $\mathrm{C} 4$ & C5 & C6 & V1 & V2 & V3 & $\mathrm{V} 4$ & V5 & V6 & V7 & V8 & V9 & V10 & VT \\
\hline G1 & 0.003 & 0.007 & 0.134 & 0.711 & 0.042 & 0.480 & 0.070 & 0.000 & 0.712 & 0.000 & 0.001 & 0.047 & 0.013 & 0.510 & 0.003 & 0.007 & 0.001 \\
\hline $\mathrm{G} 2$ & 0.000 & 0.035 & 0.005 & 0.008 & 0.000 & 0.000 & 0.000 & 0.000 & 0.000 & 0.000 & 0.000 & 0.471 & 0.383 & 0.002 & 0.000 & 0.000 & 0.000 \\
\hline G3 & 0.000 & 0.570 & 0.065 & 0.040 & 0.055 & 0.000 & 0.000 & 0.000 & 0.000 & 0.000 & 0.000 & 0.189 & 0.850 & 0.139 & 0.000 & 0.000 & 0.150 \\
\hline $\mathrm{G} 4$ & 0.000 & 0.474 & 0.110 & 0.006 & 0.004 & 0.000 & 0.000 & 0.001 & 0.358 & 0.039 & 0.002 & 0.188 & 0.205 & 0.000 & 0.000 & 0.024 & 0.141 \\
\hline G5 & 0.857 & 0.970 & 0.635 & 0.013 & 0.158 & 0.010 & 0.659 & 0.001 & 0.021 & 0.000 & 0.019 & 0.005 & 0.031 & 0.306 & 0.985 & 0.902 & 0.000 \\
\hline $\mathrm{P} 1$ & 0.001 & 0.117 & 0.396 & 0.043 & 0.462 & 0.000 & 0.000 & 0.000 & 0.002 & 0.000 & 0.000 & 0.156 & 0.046 & 0.038 & 0.000 & 0.000 & 0.000 \\
\hline $\mathrm{P} 2$ & 0.000 & 0.002 & 0.002 & 0.028 & 0.987 & 0.000 & 0.006 & 0.000 & 0.175 & 0.000 & 0.003 & 0.000 & 0.001 & 0.058 & 0.000 & 0.000 & 0.017 \\
\hline P3 & 0.000 & 0.799 & 0.594 & 0.019 & 0.179 & 0.000 & 0.59 & 0.197 & 0.711 & 0.007 & 0.199 & 0.453 & 0.156 & 0.022 & 0.023 & 0.032 & 0.462 \\
\hline P4 & 0.280 & 0.490 & 0.180 & 0.702 & 0.102 & 0.000 & 0.99 & 0.950 & 0.496 & 0.876 & 0.714 & 0.173 & 0.031 & 0.295 & 0.314 & 0.467 & 0.165 \\
\hline P5 & 0.135 & 0.117 & 0.031 & 0.149 & 0.798 & 0.000 & 0.821 & 0.025 & 0.398 & 0.030 & 0.160 & 0.320 & 0.004 & 0.945 & 0.033 & 0.255 & 0.046 \\
\hline P6 & 0.726 & 0.802 & 0.191 & 0.301 & 0.970 & 0.000 & 0.599 & 0.008 & 0.985 & 0.011 & 0.124 & 0.032 & 0.019 & 0.167 & 0.029 & 0.219 & 0.064 \\
\hline A & 0.000 & 0.000 & 0.000 & 0.000 & 0.000 & 0.000 & 0.000 & 0.000 & 0.000 & 0.000 & 0.000 & 0.000 & 0.000 & 0.000 & 0.000 & 0.000 & 0.000 \\
\hline & & & & & & & & & & & & & & & & & \\
\hline
\end{tabular}

Tabla 4.27: p-valor del Contraste $\chi^{2}$ de independencia. Características de la carrera y Métodos de enseñanza - aprendizaje frente a Gestión del tiempo y Características personales

En cuanto a los ítems referidos al entorno personal del individuo, el número de relaciones de dependencia vuelve a ser más elevado. Este resultado resulta coherente puesto que las respuestas a estas variables suelen estar asociadas por la propia situación personal de los encuestados. Por ejemplo, es fácil que exista relación entre la edad del encuestado, su situación de convivencia familiar y el hecho de tener hijos, como se verifica en la Tabla 4.28.

\begin{tabular}{|c|c|c|c|c|c|c|c|c|c|c|c|}
\hline & G1 & G2 & G3 & G4 & G5 & P1 & P3 & $\mathrm{P} 4$ & P5 & P6 & A C \\
\hline G1 & & & & & & & & & & & \\
\hline G2 & 0.000 & & & & & & & & & & \\
\hline G3 & 0.000 & 0.000 & & & & & & & & & \\
\hline G4 & 0.000 & 0.155 & 0.000 & & & & & & & & \\
\hline G5 & 0.180 & 0.010 & 0.000 & 0.898 & & & & & & & \\
\hline $\mathrm{P} 1$ & 0.000 & 0.000 & 0.001 & 0.881 & 0.984 & & & & & & \\
\hline P2 & 0.000 & 0.000 & 0.000 & 0.000 & 0.017 & 0.000 & & & & & \\
\hline P3 & 0.000 & 0.028 & 0.089 & 0.085 & 0.000 & 0.000 & 0.000 & & & & \\
\hline P4 & 0.000 & 0.589 & 0.008 & 0.011 & 0.006 & 0.786 & 0.0000 .000 & & & & \\
\hline P5 & 0.001 & 0.260 & 0.000 & 0.220 & 0.000 & 0.589 & 0.0010 .000 & 0.071 & & & \\
\hline P6 & 0.001 & 0.192 & 0.000 & 0.069 & 0.000 & 0.680 & 0.0090 .019 & 0.207 & 0.000 & & \\
\hline A & 0.000 & 0.000 & 0.000 & 0.000 & 0.000 & 0.000 & 0.0000 .000 & 0.411 & 0.000 & 0.000 & \\
\hline C & 0.001 & 0.000 & 0.085 & 0.071 & 0.000 & 0.843 & 0.0000 .006 & 0.758 & 0.000 & 0.000 & \\
\hline
\end{tabular}

Tabla 4.28: p-valor del Contraste $\chi^{2}$ de independencia. Gestión del tiempo y Características personales

De este modo se han analizado las relaciones para todos y cada uno de los posibles pares de variables planteados en el estudio y se ha comprobado que efectivamente existe mayor número de relaciones de dependencia entre ítems pertenecientes a la misma clasificación de variables, ya sean relativas al entorno académico o al entorno personal. Asimismo en las dos últimas subtablas se observa que existe una única variable vinculada a todas y cada 
una de las variables restantes, independientemente del entorno en que se hayan clasificado. Esta variable es el Área de estudio, cuya asociación con el resto de variables resulta evidente.

Una vez analizadas las relaciones de dependencia entre todos los posibles pares de variables potencialmente vinculados, se plantea el análisis de la intensidad de dichas relaciones mediante el cálculo de las medidas de asociación correspondientes. En este caso se descartan aquellas relaciones entre variables que en el análisis anterior resultaron independientes al obtener valores de significación inferiores al nivel especificado. Puesto que no se ha planteado la presencia de posibles relaciones causa - efecto entre variables se han analizado exclusivamente los resultados obtenidos en medidas de asociación simétricas y no se han considerado las asimétricas.

Como se ha expuesto anteriormente es posible calcular el cociente de ventajas para tablas $r x c$ segmentando la tabla original en diversas tablas $2 x 2$ a partir de la definición de una de las celdas como referencia. Dichos cocientes de ventaja parciales aportan información relativa a la vinculación entre las distintas categorías de las variables implicadas en la tabla de contingencia. No obstante, la aplicación de esta medida de asociación parcial no resulta adecuada en este caso ya que no aporta información comparable entre las distintas variables analizadas e incluidas en tablas de distintas dimensiones.

En condiciones de independencia esta medida toma valores próximos a la unidad y de hecho, los cocientes correspondientes a los pares de variables identificados anteriormente como independientes oscilaron en torno a este valor. Estos valores no se mostrarán en adelante ya que el objetivo de este apartado es el análisis de las relaciones de dependencia entre variables. En aquellos casos en que el valor del cociente de ventajas se aleja de la unidad caben dos posibilidades. En primer lugar si se trata de una asociación positiva este valor no tiene una cota superior de modo que se considera que incrementa la intensidad de la asociación a medida que aumenta su valor. Por el contrario en los casos de asociación negativa sí existe una cota inferior, establecida en el valor nulo. Así, se considera que cuanto más pequeño es este cociente alejándose de la unidad y aproximándose a cero, se incrementa la intensidad de la asociación negativa.

En la Tabla 4.29 se observa la presencia de fuertes asociaciones positivas entre las percepciones de los encuestados en el empleo de distintos Métodos de enseñanza - aprendizaje por el profesorado y las Características de los estudios. Concretamente se observan fuertes asociaciones positivas entre la utilización de Exposiciones orales (V10) y Trabajos en grupo (V2) ( $\mathrm{OR}=13.060)$, Trabajos escritos (V9) y Trabajos en grupo (V2) $(\mathrm{OR}=12.562)$, así como entre Exposiciones orales (V10) y Trabajos escritos 
(V9) $(\mathrm{OR}=10.641)$. Por tanto, parece existir cierta relación entre el uso de estas metodologías en los estudios universitarios. Del mismo modo es posible identificar otras relaciones de este tipo como la Participación en proyectos de investigación (V3) y los Trabajos en grupo (V2) ( $\mathrm{OR}=8.498)$ así como entre la Realización de prácticas en empresa (V4) y los Conocimientos prácticos (V5) $(\mathrm{OR}=7.068)$. Entre los ítems relativos a las características de la carrera destaca la relación entre la percepción del Esfuerzo requerido por los estudios (C1) y el Prestigio de dichos estudios (C6) (OR=5.755). Por el contrario también es posible observar casos de fuertes asociaciones negativas, como la encontrada entre el Enfoque generalista (C4) y el Enfoque especializado (C5) $(\mathrm{OR}=0.119)$, donde evidentemente se verifica que la presencia de uno implica la ausencia del contrario.

\begin{tabular}{|c|c|c|c|c|c|c|c|c|c|c|c|c|c|c|c|}
\hline & $\mathrm{C} 2$ & C3 & $\mathrm{C} 4$ & C5 & C6 & V1 & V2 & V3 & V4 & V5 & V6 & V7 & V8 & V9 & V10 VT \\
\hline $\mathrm{C} 1$ & & & & & & & & & & & & & & & \\
\hline $\mathrm{C} 2$ & 1.650 & & & & & & & & & & & & & & \\
\hline C3 & 0.8091 .585 & & & & & & & & & & & & & & \\
\hline $\mathrm{C} 4$ & 0.764 & & & & & & & & & & & & & & \\
\hline C5 & 1.4781 .784 & 1.553 & 0.119 & & & & & & & & & & & & \\
\hline C6 & 5.7551 .847 & 0.860 & & 1.546 & & & & & & & & & & & \\
\hline V1 & 1.1401 .307 & 1.150 & 0.829 & 1.577 & & & & & & & & & & & \\
\hline $\mathrm{V} 2$ & $\begin{array}{lll}0.676 & 1.267\end{array}$ & 1.715 & 0.762 & 1.988 & 0.532 & 4.630 & & & & & & & & & \\
\hline V3 & 1.488 & 1.890 & 0.676 & 2.601 & & 2.355 & 8.498 & & & & & & & & \\
\hline V4 & 1.775 & 1.564 & 0.740 & 1.891 & & 2.407 & 4.162 & 4.392 & & & & & & & \\
\hline V5 & 1.985 & 1.709 & 0.531 & 3.305 & 1.290 & 2.407 & 3.473 & 5.338 & 7.068 & & & & & & \\
\hline V6 & 1.5991 .462 & 1.190 & 1.439 & & 1.561 & 1.280 & 1.130 & & 0.830 & 1.481 & & & & & \\
\hline V7 & 1.1321 .385 & 1.180 & 1.253 & & & 1.220 & 0.885 & 0.576 & 0.771 & 0.890 & 2.454 & & & & \\
\hline V8 & 2.3641 .585 & 1.470 & 0.686 & 2.427 & 1.973 & 1.612 & 2.079 & 4.143 & 2.304 & 4.032 & 1.190 & & & & \\
\hline V9 & 0.7141 .160 & 1.932 & 0.717 & 1.658 & 0.468 & 2.688 & 10.641 & 4.729 & 2.414 & 2.419 & 1.419 & & 1.724 & & \\
\hline V10 & 0.7401 .349 & 1.661 & 0.761 & 1.865 & 0.525 & 3.585 & 13.060 & 6.578 & 3.244 & 2.841 & & 0.725 & 1.917 & 2.562 & \\
\hline VT & 1.274 & 1.305 & 1.153 & & 1.146 & 1.220 & 1.281 & & 2.018 & 1.576 & 1.682 & 1.366 & & & 1.169 \\
\hline
\end{tabular}

Tabla 4.29: Cociente de ventajas. Características de la carrera y Métodos de enseñanza - aprendizaje

Analizando los resultados del coeficiente de correlación de Pearson se extraen conclusiones similares a las anteriores. La principal asociación negativa resulta igualmente la encontrada entre el Enfoque generalista (C4) y el Enfoque especializado (C5) ( $\mathrm{r}=-0.646)$, mientras que las asociaciones positivas más fuertes se dan entre las distintas variables relativas a las Exposiciones orales (V10) y Trabajos en grupo (V2) ( $\mathrm{r}=0.559)$, Trabajos escritos (V9) y Trabajos en grupo (V2) ( $\mathrm{r}=0.598)$, así como las Exposiciones orales (V10) y Trabajos escritos (V9) ( $\mathrm{r}=0.565)$. En este punto hay que señalar que, aunque estos valores no se encuentren muy próximos a la unidad, la identificación de las relaciones de asociación más fuertes debe enfocarse en términos relativos y no absolutos, de modo que no se trata de especificar un umbral por encima del cual los coeficientes de correlación se consideren suficientemente significativos, sino de seleccionar las relaciones cuyos coeficientes sean más elevados. 


\begin{tabular}{|c|c|c|c|c|c|c|c|c|c|c|c|c|c|c|c|c|c|}
\hline & C1 & $\mathrm{C} 2$ & C3 & $\mathrm{C} 4$ & C5 & C6 & V1 & V2 & V3 & V4 & V5 & V6 & V7 & V8 & V9 & V10 & VT \\
\hline C1 & 1 & & & & & & & & & & & & & & & & \\
\hline $\mathrm{C} 2$ & 0.162 & 1 & & & & & & & & & & & & & & & \\
\hline C3 & -0.108 & 0.067 & 1 & & & & & & & & & & & & & & \\
\hline $\mathrm{C} 4$ & & -0.087 & & 1 & & & & & & & & & & & & & \\
\hline C5 & 0.102 & 0.227 & 0.160 & -0.646 & 1 & & & & & & & & & & & & \\
\hline C6 & 0.476 & 0.242 & -0.107 & & 0.150 & 1 & & & & & & & & & & & \\
\hline V1 & 0.030 & 0.053 & 0.062 & -0.038 & 0.125 & & 1 & & & & & & & & & & \\
\hline V2 & -0.108 & 0.050 & 0.220 & -0.089 & 0.191 & -0.176 & 0.440 & 1 & & & & & & & & & \\
\hline V3 & & 0.082 & 0.170 & -0.122 & 0.230 & & 0.241 & 0.480 & 1 & & & & & & & & \\
\hline V4 & & 0.151 & 0.128 & -0.091 & 0.189 & & 0.241 & 0.404 & 0.313 & 1 & & & & & & & \\
\hline V5 & 0.046 & 0.184 & 0.150 & -0.201 & 0.329 & 0.071 & 0.251 & 0.405 & 0.394 & 0.484 & 1 & & & & & & \\
\hline V6 & 0.143 & 0.032 & 0.038 & 0.123 & & 0.124 & 0.055 & 0.275 & & -0.055 & 0.040 & 1 & & & & & \\
\hline V7 & 0.041 & 0.043 & 0.037 & 0.075 & & & 0.044 & -0.077 & -0.120 & -0.065 & -0.060 & 0.223 & 1 & & & & \\
\hline V8 & 0.180 & 0.143 & 0.081 & -0.158 & 0.260 & 0.205 & 0.150 & 0.275 & 0.328 & 0.228 & 0.392 & 1.190 & & 1 & & & \\
\hline V9 & -0.122 & 0.034 & 0.216 & -0.070 & 0.144 & -0.219 & 0.299 & 0.598 & 0.336 & 0.222 & 0.252 & 0.056 & & 0.170 & 1 & & \\
\hline V10 & -0.099 & 0.061 & 0.140 & -0.058 & 0.146 & -0.162 & 0.328 & 0.559 & 0.396 & 0.288 & 0.285 & & -0.086 & 0.144 & 0.565 & 1 & \\
\hline VT & & 0.071 & 0.028 & 0.045 & & 0.068 & 0.043 & 0.084 & 0.045 & 0.235 & 0.114 & 0.086 & 0.052 & & & 0.055 & 1 \\
\hline
\end{tabular}

Tabla 4.30: Coeficiente de correlación de Pearson. Características de la carrera y Métodos de enseñanza - aprendizaje

El coeficiente $\Phi^{2}$ de Pearson aplicado a las variables clasificadas en el entorno académico del estudiante aporta información sobre la intensidad de la asociación, pero no sobre su dirección ya que únicamente oscilan en el intervalo [0,1]. Así, los resultados mostrados en la Tabla 4.31 indican que la asociación entre el Enfoque generalista (C4) y el Enfoque especializado (C5) $\Phi^{2}=0,151$ es del orden de la encontrada para la percepción del Esfuerzo requerido por los estudios (C1) y el Prestigio de dichos estudios (C6) $\Phi^{2}=0,135$. Sin embargo, no permiten distinguir entre el tipo de asociación (positiva o negativa) entre ambas variables. Respecto a las variables referentes a Exposiciones orales, Trabajos en grupo y Trabajos escritos presentan elevados valores de este coeficiente. De este modo, aunque este coeficiente no facilita la identificación del tipo de asociación que vincula a las variables, sí permite establecer un rango de variación en términos relativos para identificar por ejemplo, que las relaciones establecidas entre estos Métodos de enseñanza - aprendizaje tienen una intensidad superior a las comentadas anteriormente sobre el enfoque de los estudios o la percepción del esfuerzo y el prestigio. 


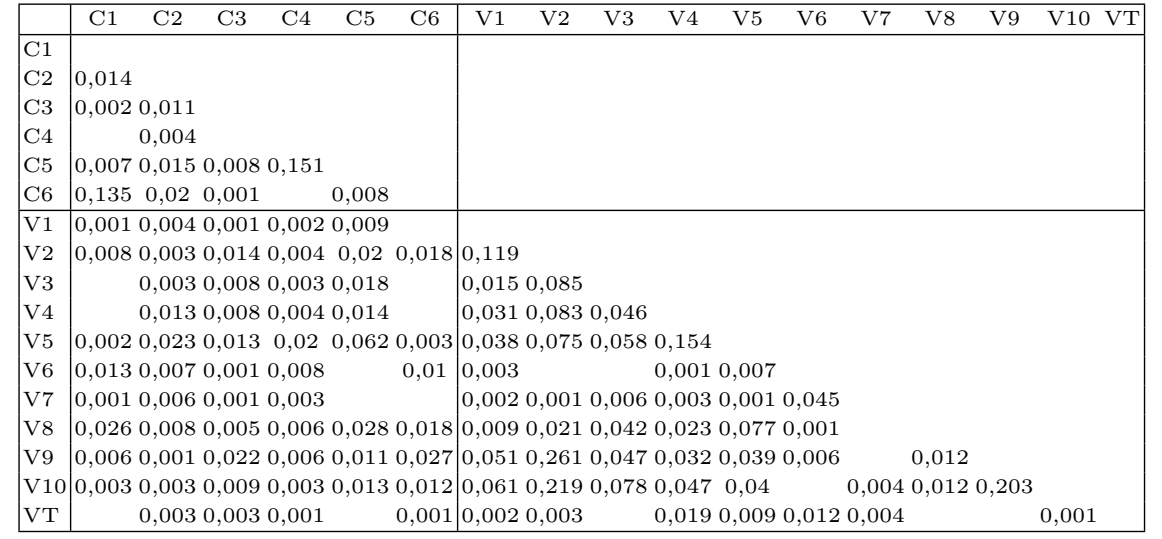

Tabla 4.31: Medida $\Phi^{2}$ de Pearson. Características de la carrera y Métodos de enseñanza - aprendizaje

En el caso de las relaciones entre ítems del entorno académico y personal del estudiante, destaca la fuerte asociación del Área de estudio con la práctica totalidad de las variables consideradas. Concretamente esta variable se encuentra muy vinculada al empleo de determinados Métodos de enseñan$z a$ - aprendizaje como Trabajos en grupo (V2) $\Phi^{2}=0,203$, Realización de prácticas en empresa (V4) $\Phi^{2}=0,137$, Trabajos escritos (V9) $\Phi^{2}=0,187$, Exposiciones orales (V10) $\Phi^{2}=0,135$, y Realización de pruebas tipo test (VT) $\Phi^{2}=0,160$. En este orden de magnitud únicamente se encuentra la relación directa entre la variable relativa a las Prácticas en empresa (G2), como parte de los ítems referidos al comportamiento del estudiante y el empleo de metodologías docentes basadas en la Realización de prácticas en empresa (V4) $\Phi^{2}=0,152$.

\begin{tabular}{|c|c|c|c|c|c|c|c|c|c|c|c|c|c|c|c|c|}
\hline & $\mathrm{C} 2$ & C3 & $\mathrm{C} 4$ & C5 & C6 & V1 & V2 & V3 & V4 & V5 & V6 & V7 & V8 & V9 & V10 & VT \\
\hline G1 & 0.0020 .001 & & & 0.001 & & & 0.002 & & 0.004 & 0.002 & 0.001 & 0.001 & & 0.002 & 0.001 & 0.002 \\
\hline $\mathrm{G} 2$ & 0.0100 .001 & 0.001 & 0.001 & 0.003 & 0.005 & 0.013 & 0.070 & 0.005 & 50.152 & 0.027 & & & 0.002 & 0.018 & 0.022 & 0.027 \\
\hline G3 & 0.005 & & 0.001 & & 0.007 & 0.013 & 0.031 & 0.009 & 0.025 & 0.016 & & & & 0.025 & 0.028 & \\
\hline G4 & 0.057 & & 0.002 & 0.002 & 0.022 & 0.004 & 0.003 & & 0.002 & 0.003 & & & 0.008 & 0.003 & 0.002 & \\
\hline G5 & & & 0.001 & & 0.001 & & 0.002 & 0.001 & 0.003 & 0.001 & 0.001 & 0.001 & & & & 0.003 \\
\hline P1 & 0.002 & & 0.001 & & 0.021 & 0.010 & 0.023 & 0.002 & 0.014 & 0.008 & & 0.001 & 0.001 & 0.019 & 0.013 & 0.005 \\
\hline $\mathrm{P} 2$ & 0.0150 .003 & 0.003 & 0.001 & & 0.016 & 0.002 & 0.011 & & 0.011 & 0.002 & 0.003 & 0.003 & 0.000 & 0.009 & 0.004 & 0.002 \\
\hline P3 & 0.004 & & 0.002 & & 0.007 & & & & 0.002 & & & & 0.002 & 0.002 & 0.002 & \\
\hline P4 & & & & & 0.002 & & & & & & & 0.001 & & & & \\
\hline P5 & & 0.001 & & & 0.007 & & 0.001 & & 0.001 & & & 0.002 & & 0.001 & & 0.001 \\
\hline P6 & & & & & 0.004 & & 0.001 & & 0.001 & & 0.001 & 0.001 & & 0.001 & & \\
\hline A & 0.1670 .008 & 0.029 & 0.029 & 0.029 & 0.184 & 0.044 & 0.203 & 0.041 & 0.137 & 0.071 & 0.009 & 0.010 & 0.07 & 0.187 & & 0.160 \\
\hline C & 0.004 & & 0.009 & 0.007 & 0.014 & 0.008 & 0.019 & 0.001 & 0.059 & 0.018 & 0.019 & 0.001 & 0.013 & 0.003 & 0.006 & \\
\hline
\end{tabular}

Tabla 4.32: Medida $\Phi^{2}$ de Pearson. Características de la carrera y Métodos de enseñanza - aprendizaje frente a Gestión del tiempo, Características personales y Área y Nivel de estudios 
En el ámbito de las variables relativas al entorno personal del estudiante destaca la relación entre tener Padre (P5) y Madre (P6) con estudios universitarios. Es decir, que existe una fuerte asociación entre el hecho de que el padre y la madre hayan estudiado en la Universidad $\Phi^{2}=0,493$. Esta relación detectada, que en principio no forma parte de los objetivos de la investigación, sirve para contrastar que las medidas de asociación están aportando información coherente con la realidad del entorno de los estudiantes. En otro orden de magnitud, pero igualmente significativas, son las relaciones detectadas entre la Edad de los encuestados y Tener hijos $\Phi^{2}=0,120$ así como la asociación entre la Edad y Compaginar los estudios con el trabajo $\Phi^{2}=0,163$ y la Edad y la Situación de convivencia familiar $(\mathrm{P} 3) \Phi^{2}=0,123$.

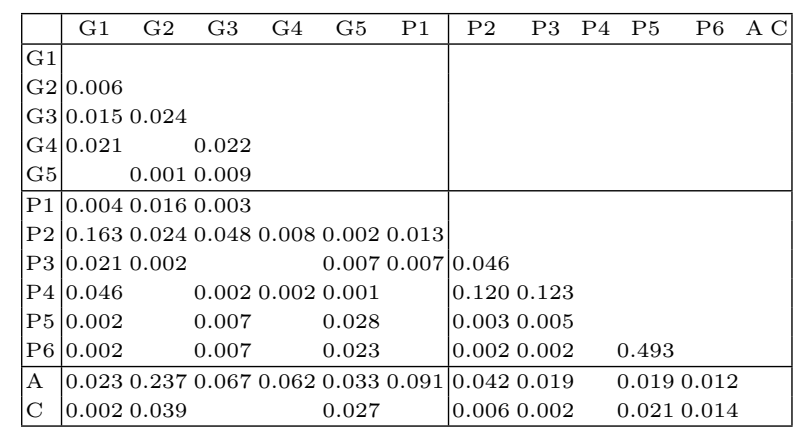

Tabla 4.33: Medida $\Phi^{2}$ de Pearson. Gestión del tiempo, Características personales y Área y Nivel de estudios

Estos resultados son muy similares a los obtenidos mediante el cálculo de otras medidas de asociación basadas en el estadístico $\chi^{2}$ como el coeficiente de contingencia o la $\mathrm{V}$ de Cramer y que pueden consultarse en el Anexo C. Asimismo en este anexo se muestran los resultados correspondientes a la medida lambda propuesta por Goodman y Kruskal, basada en la reducción proporcional del error así como el coeficiente de incertidumbre asimétrico, medida basada en la proporción de varianza explicada.

\section{Análisis tridimensional}

El objetivo de esta fase del análisis es comprobar si las relaciones de dependencia cuya asociación ha resultado significativa, interactúan o se encuentran confundidas por la variable Área de estudio, cuya vinculada de forma generalizada al conjunto de todas las variables como se ha mostrado en el apartado anterior. Puesto que la relación de dicha variable con los ítems referidos al entorno académico del estudiante es más fuerte que la establecida con los ítems del entorno personal, el análisis se ha planteado exclusivamente 
sobre estas variables. De este modo se han descartando las variables relativas al entorno personal del estudiante, entre las cuales se habían clasificado dos con más de dos categorías (Edad y Situación de convivencia). Por ello el análisis de relaciones a nivel tridimensional se aplica a la modificación del efecto producida por el Área de estudio, con 8 categorías, sobre las relaciones establecidas entre las distintas variables del entorno académico del estudiante, todas ellas analizadas mediante tablas de contingencia $2 x 2$. Por tanto se han construido tantas tablas $2 x 2 x 8$ como relaciones de dependencia significativas se han identificado anteriormente.

De acuerdo con las expresiones formuladas para cada contraste de independencia (global, parcial y condicional) se han calculado para cada una de estas tablas $2 x 2 x 8$ las frecuencias esperadas en cada celda, a partir de las cuales se ha obtenido el valor global del estadístico $\chi^{2}$. El número de grados de libertad para cada contraste se ha calculado mediante la formulación correspondiente, resultando 22 para el contraste de independencia global, 21 para el parcial y únicamente uno para el condicional. Dicho cálculo resulta aplicable a todas las tablas analizadas, ya que todas ellas tienen las mismas dimensiones. De este modo, considerando el valor de los estadísticos $\chi^{2}$ obtenidos en cada caso y el número de grados de libertad correspondiente a cada contraste, se han estudiado las relaciones de dependencia entre variables considerando la posible modificación del efecto a causa de la variable Área de estudio.

\section{Contraste de Independencia Global}

La Tabla 4.34 muestra los valores del estadístico $\chi^{2}$ para el Contraste de Independencia Global entre los pares de variables clasificadas en el entorno académico del estudiante e identificados como dependientes en presencia de la variable Área de estudio. El número de grados de libertad correspondiente a este contraste y para tablas $2 x 2 x 8$ es 22 .

$$
g l=r c s-r-c-s+2=8 \cdot 2 \cdot 2-8-2-2+2=22
$$

Por tanto el valor tabular de la distribución $\chi^{2}$ con un nivel del significación del $5 \%$ resulta 12.34 . En los casos en que el valor del estadístico $\chi^{2}$ calculado para cada una de las tablas $2 x 2 x 8$ supere este valor de referencia se considera que existen relaciones de dependencia globales entre las tres variables implicadas. Como se observa en la Tabla 4.34 esta situación se da en todos los pares de variables estudiados, por lo que puede concluirse que todas las variables clasificadas en el entorno académico del estudiante son dependientes a nivel global en presencia del Área de estudio. 


\begin{tabular}{|c|c|c|c|c|c|c|c|c|c|c|c|c|c|c|c|c|}
\hline & $\mathrm{C} 1$ & $\mathrm{C} 2$ & $\mathrm{C} 3$ & C4 & C5 & C6 & V1 & V2 & V3 & V4 & V5 & V6 & V7 & V8 & V9 & $\mathrm{V} 10 \mathrm{VT}$ \\
\hline C1 & & & & & & & & & & & & & & & & \\
\hline $\mathrm{C} 2$ & 1013 & & & & & & & & & & & & & & & \\
\hline C3 & 1026 & 261 & & & & & & & & & & & & & & \\
\hline $\mathrm{C} 4$ & & 225 & & & & & & & & & & & & & & \\
\hline C5 & 1088 & 291 & 343 & 1122 & & & & & & & & & & & & \\
\hline C6 & 2531 & 1112 & 1088 & & 1145 & & & & & & & & & & & \\
\hline V1 & 1160 & 339 & 404 & 419 & 454 & & & & & & & & & & & \\
\hline $\mathrm{V} 2$ & 2046 & 1086 & 1227 & 1188 & 1278 & 2197 & 1981 & & & & & & & & & \\
\hline V3 & & 280 & 402 & 353 & 431 & & 548 & 1757 & & & & & & & & \\
\hline V4 & & 986 & 1070 & 1004 & 1090 & & 1366 & 2389 & 1355 & & & & & & & \\
\hline V5 & 1307 & 602 & 640 & 657 & 874 & 1372 & 916 & 1810 & 946 & 2569 & & & & & & \\
\hline V6 & 1019 & 169 & 219 & 268 & & 1051 & 329 & 1077 & & 915 & 512 & & & & & \\
\hline V7 & 923 & 143 & 199 & 221 & & & 308 & 1059 & 297 & 897 & 468 & 367 & & & & \\
\hline V8 & 1444 & 458 & 532 & 548 & 655 & 1481 & 697 & 1515 & 814 & 1334 & 1199 & 459,4 & & & & \\
\hline V9 & 1954 & 1034 & 1231 & 1171 & 1192 & 2154 & 1615 & 3638 & 1465 & 2134 & 1607 & 1071 & & 1434 & & \\
\hline V10 & 1659 & 783 & 886 & 868 & 943 & 1801 & 1435 & 3386 & 1491 & 2029 & 1391 & & 757 & 1193 & 3127 & \\
\hline $\mathrm{VT}$ & & 959 & 1125 & 1025 & & 2016 & 1138 & 1931 & & 2051 & 1425 & 1002 & 934 & & & 1582 \\
\hline
\end{tabular}

Tabla 4.34: Valor del estadístico $\chi^{2}$ para el Contraste de independencia global. Características de la carrera y Métodos de enseñanza - aprendizaje

\section{Contraste de Independencia Parcial}

Una vez verificada la existencia de relaciones de dependencia a nivel global resulta pertinente plantear el estudio detallado de dichas relaciones, mediante los contrastes de independencia parcial y condicional. En este caso el contraste de independencia parcial se ha planteado para estudiar la independencia del Área de estudio dada la presencia de los otros dos factores, correspondientes a los distintos pares de variables, pertenecientes al contexto académico e identificadas previamente como dependientes. Aunque podrían haberse planteado otros análisis equivalentes, como la independencia de uno de los factores considerando la presencia del otro factor y la variable Área de estudio, este planteamiento no resulta interesante en este caso, ya que el objetivo de esta fase del análisis es comprobar en qué medida el Área de estudio es una variable modificadora del efecto sobre las relaciones entre otras variables. Una revisión exhaustiva de todas las posibles relaciones de dependencia entre las tres variables implicadas en cada caso resultaría excesivamente profunda y no aportaría información relevante.

$$
g l=r c s-r-c s+1=8 \cdot 2 \cdot 2-8-2 \cdot 2+1=21
$$

El número de grados de libertad para este contraste de independencia y tablas $2 x 2 x 8$ es 21 , por lo que el valor tabular de la distribución $\chi^{2}$ con un nivel del significación del $5 \%$ toma el valor 11.59. Al igual que antes, en los casos en que el valor del estadístico $\chi^{2}$ calculado para cada tabla $2 x 2 x 8$ supere este valor de referencia se puede considerar que la variable Área de estudio es dependiente de los otros dos factores. Como se observa en la Tabla 4.35 , esto ocurre en todos los casos por lo que es posible afirmar que el Área 
de estudio establece relaciones de dependencia parcial con los distintos pares de variables planteados en esta categoría. En otras palabras, el Área de estudio no es independiente dada la presencia de cualquier par de variables pertenecientes al contexto académico del individuo.

\begin{tabular}{|c|c|c|c|c|c|c|c|c|c|c|c|c|c|c|c|c|}
\hline & C1 & $\mathrm{C} 2$ & C3 & $\mathrm{C} 4$ & C5 & C6 & V1 & V2 & V3 & V4 & V5 & V6 & V7 & V8 & V9 & $\mathrm{V} 10 \mathrm{VT}$ \\
\hline & & & & & & & & & & & & & & & & \\
\hline $\mathrm{C} 2$ & 916 & & & & & & & & & & & & & & & \\
\hline C3 & 996 & 219 & & & & & & & & & & & & & & \\
\hline $\mathrm{C} 4$ & & 200 & & & & & & & & & & & & & & \\
\hline C5 & 1055 & 207 & 297 & 236 & & & & & & & & & & & & \\
\hline C6 & 1369 & 963 & 1072 & 1095 & 1104 & & & & & & & & & & & \\
\hline V1 & 1170 & 318 & 400 & 400 & 400 & & & & & & & & & & & \\
\hline $\mathrm{V} 2$ & 1889 & 1085 & 1140 & 1162 & 1170 & 1948 & 1139 & & & & & & & & & \\
\hline V3 & & 273 & 329 & 342 & 357 & & 426 & 1088 & & & & & & & & \\
\hline V4 & & 883 & 1054 & 982 & 990 & & 999 & 1771 & 1041 & & & & & & & \\
\hline V5 & 1288 & 462 & 595 & 534 & 510 & 1353 & 620 & 1392 & 591 & 1141 & & & & & & \\
\hline V6 & 918 & 133 & 215 & 217 & & 974 & 326 & 1078 & & 894 & 487 & & & & & \\
\hline V7 & 915 & 119 & 194 & 202 & & & 306 & 1045 & 233 & 873 & & 123 & & & & \\
\hline V8 & 1186 & 414 & 517 & 511 & 510 & 1268 & 690 & 1482 & 586 & 1279 & 821 & 440 & & & & \\
\hline V9 & 1815 & 1037 & 1078 & 1124 & 1122 & 1820 & 1170 & 1537 & 1078 & 1922 & 1418 & 1061 & & 1431 & & \\
\hline V10 & 1571 & 768 & 11 & 839 & 849 & 1590 & 838 & 1325 & 595 & 1525 & 1084 & & 724 & 1167 & 1233 & \\
\hline VT & & 927 & 1128 & 1020 & & 1995 & 1119 & 1926 & & 1666 & 1287 & 966 & 915 & & & 1588 \\
\hline
\end{tabular}

Tabla 4.35: Valor del estadístico $\chi^{2}$ para el Contraste de independencia parcial. Características de la carrera y Métodos de enseñanza - aprendizaje

\section{Contraste de Independencia Condicional}

Seguidamente se han estudiado las posibles relaciones de dependencia condicional entre los distintos pares de variables del contexto académico, dado un determinado nivel del Área de estudio. Este planteamiento implica que se han revisado nuevamente las relaciones entre factores, considerando las diferencias entre las distintas áreas de estudio. Al igual que antes, cabía la posibilidad de haber realizado planteamientos similares para todas las posibles combinaciones de variables. Sin embargo, este análisis hubiera resultado excesivamente exhaustivo y no hubiera aportado información de interés, ya que el objetivo sigue siendo comprobar en qué medida el Área de estudio es responsable de la modificación de las relaciones entre variables del contexto académico. El número de grados de libertad correspondiente a este contraste para tablas $2 x 2 x 8$ resulta igual a la unidad.

$$
g l=r c s-r c-r s+1=8 \cdot 2 \cdot 2-8 \cdot 2-8 \cdot 2+1=1
$$

El valor tabular de la distribución $\chi^{2}$ con un grado de libertad, y considerando un nivel de significación del $5 \%$ resulta 3.84 . Por ello en los casos en que los valores calculados de este estadístico superan este valor de referencia se considera que se dan relaciones de dependencia entre las tres variables implicadas. Como se observa en la Tabla 4.36 esto ocurre en todos los casos, 
de modo que es posible concluir que las variables del contexto académico son dependientes siempre y cuando se tomen en consideración las distintas áreas de estudio de los encuestados.

\begin{tabular}{|c|c|c|c|c|c|c|c|c|c|c|c|c|c|c|c|}
\hline & C1 & $\mathrm{C} 2$ & C3 & $\mathrm{C} 4$ & C5 & C6 & V1 V2 & $2 \mathrm{~V} 3$ & $\mathrm{~V} 4$ & V5 & V6 & V7 & V8 & V9 & V10 VT \\
\hline $\mathrm{C} 1$ & & & & & & & & & & & & & & & \\
\hline $\mathrm{C} 2$ & 44 & & & & & & & & & & & & & & \\
\hline C3 & 138 & 168 & & & & & & & & & & & & & \\
\hline $\mathrm{C} 4$ & & 146 & & & & & & & & & & & & & \\
\hline C5 & 168 & 152 & 150 & 101 & & & & & & & & & & & \\
\hline $\mathrm{C} 6$ & 558 & 906 & 921 & & 938 & & & & & & & & & & \\
\hline V1 & 303 & 259 & 256 & 257 & 250 & & & & & & & & & & \\
\hline $\mathrm{V} 2$ & 985 & 1020 & 989 & 1006 & 990 & 927 & 863 & & & & & & & & \\
\hline V3 & & 215 & 182 & 196 & 199 & & 17373 & & & & & & & & \\
\hline V4 & & 824 & 855 & 836 & 823 & & 730756 & 6837 & & & & & & & \\
\hline V5 & 404 & 403 & 425 & 390 & 362 & 432 & 350365 & 5389 & 255 & & & & & & \\
\hline V6 & 55 & 79 & 73 & 75 & & 67 & $80 \quad 91$ & & 75 & 89 & & & & & \\
\hline V7 & 51 & 67 & 52 & 54 & & & $\begin{array}{ll}59 & 59\end{array}$ & 44 & 57 & 61 & 62 & & & & \\
\hline V8 & 273 & 362 & 366 & 357 & 347 & 299 & 411431 & 1391 & 440 & 393 & 378 & & & & \\
\hline V9 & 969 & 983 & 929 & 954 & 953 & 855 & 869515 & 5896 & 927 & 948 & 999 & & 1032.7 & & \\
\hline V10 & 736 & 710 & 697 & 682 & 686 & 624 & 559269 & 9607 & 599 & 645 & & 689 & 776 & 252 & \\
\hline VT & & 854 & 908 & 864 & & 885 & 859875 & & 766 & 833 & 888 & 872 & & & 875 \\
\hline
\end{tabular}

Tabla 4.36: Valor del estadístico $\chi^{2}$ para el Contraste de independencia condicional. Características de la carrera y Métodos de enseñanza - aprendizaje

Por tanto, la aplicación de los contrastes de independencia a nivel global, parcial y condicional sobre distintos pares de variables del contexto académico, ha permitido comprobar que el Área de estudio establece relaciones de dependencia en todos los casos y a todos los niveles. Este resultado indica la posibilidad de que dicha variable sea responsable de la introducción de algún tipo de modificación del efecto, ya sea de interacción o de confusión entre las variables implicadas. En cualquier caso, el estudio de ambas circunstancias debe realizarse mediante la aplicación de contrastes específicos, como se detalla a continuación.

\section{Contraste condicional de ausencia de interacción o ausencia de asociación parcial sobre el Riesgo Ponderado}

El Contraste de Ausencia de Interacción o Ausencia de Asociación Parcial plantea la igualdad de los cocientes de ventajas obtenidos en una tabla de contingencia bidimensional para cada uno de los estratos de una tercera variable. En este caso la aplicación de este contraste supone la estimación de los cocientes de ventajas entre los pares de variables planteados anteriormente, para cada una de las ocho áreas de estudio. A partir de este cálculo se obtienen los pesos $w_{k}$ para la ponderación de dichos coeficientes así como el riesgo correspondiente a cada estrato. Mediante la aplicación de estos pesos se obtiene el valor del estadístico $\chi^{2}$ y el riesgo ponderado correspondientes a cada tabla $2 x 2 x 8$ tal y como se muestra en las Tablas 
4.38 y 4.39, respectivamente. El número de grados de libertad correspondiente a este caso se calcula como el número de estratos menos la unidad por lo que toma el valor 7 . El valor tabular de la distribución $\chi^{2}$ con dicho número de grados de libertad y nivel de significación del $5 \%$ es 14.07. Únicamente en los casos en que se supere este valor de referencia se considera que existe el Área de estudio interactúa en la relación entre los distintos pares de variables. En la Tabla 4.37 se han sombreado estos casos ya que en esta ocasión las relaciones de interacción no se dan de forma generalizada.

\begin{tabular}{|l|cccccc|cccccccccc|}
\hline & C1 & C2 & C3 & C4 & C5 & C6 & V1 & V2 & V3 & V4 & V5 & V6 & V7 & V8 & V9 & V10 VT \\
\hline C1 & & & & & & & & & & & & & & & & \\
C2 & 8.7 & & & & & & & & & & & & & & \\
C3 & 9.9 & 13.2 & & & & & & & & & & & & \\
C4 & & 6.6 & & & & & & & & & & & & & \\
C5 & 15 & 7 & 5.5 & 19.8 & & & & & & & & & & & & \\
C6 & 9.4 & 12.3 & 19.7 & & 18.1 & & & & & & & & & & & \\
\hline V1 & 18.2 & 8.5 & 5.8 & 12.1 & 14.6 & & & & & & & & & & & \\
V2 & 24.1 & 5 & 5.6 & 16.6 & 10.3 & 40.1 & 26 & & & & & & & & \\
V3 & & 19 & 6.9 & 10.6 & 19.1 & & 12.6 & 10.3 & & & & & & & \\
V4 & & 14.1 & 7.9 & 17 & 11.8 & & 11.5 & 23.7 & 8.9 & & & & & & & \\
V5 & 7.7 & 15.7 & 8.1 & 16.1 & 12.2 & 23.2 & 17.6 & 13.7 & 16.4 & 23.1 & & & & & & \\
V6 & 10.4 & 16.1 & 11.5 & 20.9 & 1.2 & 17.4 & 10.6 & 24.3 & & 20 & 19.4 & & & & & \\
V7 & 8.5 & 23 & 5.2 & 10.1 & 10.8 & & & 19.7 & 5.9 & 6.4 & & 25.4 & & & & \\
V8 & 15.3 & 5.9 & 5.6 & 6.9 & 11.3 & 10.6 & 35.2 & 37.7 & 21.2 & 8.1 & 14.7 & 32.6 & & & & \\
V9 & 19.6 & 12.3 & 7.6 & 7.7 & 8.4 & 35.2 & 23.8 & 20.2 & 7.5 & 8.3 & 16.9 & 14.1 & & 23.6 & & \\
V10 & 18.6 & 9 & 2.2 & 5.4 & 23.8 & 16.9 & 5.3 & 16.1 & 13.1 & 6.5 & 5.6 & & 9.7 & 15.9 & 17.5 \\
VT & & 4.8 & 29.5 & 7.7 & 6.5 & & 7.6 & 10.8 & & 3.6 & 9.1 & 19.8 & 14.5 & & 10.3 \\
\hline
\end{tabular}

Tabla 4.37: Valor del estadístico $\chi^{2}$ para el Contraste de asociación parcial no condicional. Características de la carrera y Métodos de enseñanza aprendizaje

La Tabla 4.38 muestra el riesgo ponderado calculado para cada una de las relaciones de interacción identificadas como significativa. Para los pares de variables cuya relación de interacción con el Área de estudio ha resultado no significativa no se ha obtenido el valor de esta medida. Como se ha expuesto anteriormente, esta medida constituye un estimador de la asociación parcial común entre dos variables binarias. Su interpretación es similar a la del cociente de ventajas y de hecho es fácil observar cómo las variables Enfoque generalizado (C4) y Enfoque especializado (C5) que anteriormente presentaban asociación negativa también lo hacen en condiciones de interacción con el Área de estudio $\left(R_{p}=0.130\right)$ mientras que las variables relativas a determinadas metodologías docentes muestran fuertes asociaciones positivas, de forma coherente con los resultados anteriores. Así, los valores de riesgo ponderado más elevados se alcanzan en las relaciones entre Exposiciones orales (V10) y Trabajos en grupo (V2) $\left(R_{p}=10.383\right)$, Exposiciones orales (V10) y Trabajos escritos (V9) $\left(R_{p}=9.206\right)$ y Trabajos escritos (V9) y Trabajos en grupo (V2) $\left(R_{p}=7.930\right)$. 


\begin{tabular}{|c|c|c|c|c|c|c|c|c|c|c|c|c|c|c|c|}
\hline & C1 & $\mathrm{C} 2$ & C3 & $\mathrm{C} 4$ & $\mathrm{C} 5$ & C6 & V1 & V2 & V3 & $\mathrm{V} 4$ & V5 & V6 & V7 & V8 & V10 VT \\
\hline \multicolumn{16}{|l|}{$\mathrm{C} 1$} \\
\hline \multicolumn{16}{|l|}{$\mathrm{C} 2$} \\
\hline \multicolumn{16}{|l|}{ C3 } \\
\hline \multicolumn{16}{|l|}{$\mathrm{C} 4$} \\
\hline \multicolumn{16}{|c|}{1.648} \\
\hline C6 & & & 1.151 & & 1.964 & & & & & & & & & & \\
\hline V1 & 1.492 & & & & 1.405 & & & & & & & & & & \\
\hline $\mathrm{V} 2$ & 1.191 & & & 0.841 & & 1.008 & 4.066 & & & & & & & & \\
\hline V3 & & 1.565 & & & 2.274 & & & & & & & & & & \\
\hline V4 & & 1.749 & & 0.8 & & & & 3.817 & & & & & & & \\
\hline V5 & & 1.935 & & 0.597 & & 1.523 & 2.104 & & 4.599 & 6.578 & & & & & \\
\hline V6 & & 1.471 & & 1.438 & & 1.619 & & 1.205 & & 0.841 & 1.562 & & & & \\
\hline V7 & & 1.306 & & & & & & 0.93 & & & & 2.393 & & & \\
\hline V8 & 1.929 & & & & & & 1.82 & 2.619 & 4.607 & & 4.567 & 1.162 & & & \\
\hline V9 & 1.352 & & & & & 0.934 & 2.254 & 7.93 & & & 2.365 & 1.657 & & 2.238 & \\
\hline V10 & 1.516 & & & & 1.796 & 1.182 & & 10.383 & & & & & & 3.0519 .206 & \\
\hline VT & & & 1.5 & & & & & & & & & 1.682 & 1.285 & & \\
\hline
\end{tabular}

Tabla 4.38: Riesgo ponderado para el Contraste de asociación parcial no condicional. Características de la carrera y Métodos de enseñanza - aprendizaje

\section{Contraste condicional sobre el Cociente de Ventajas Común}

Los resultados obtenidos mediante el contraste condicional de ausencia de asociación parcial sobre el riesgo ponderado resultan menos restrictivos que los correspondientes a este contraste, que no solo plantea la igualdad de los cocientes de ventajas en los diferentes estratos sino que considera la igualdad de todos sus valores a la unidad. Al igual que antes, la aplicación de este contraste supone la estimación de los cocientes de ventajas entre los pares de variables planteados anteriormente, para cada una de las ocho áreas de estudio. Así, la estimación del estadístico $\chi^{2}$ se realiza nuevamente a partir del cálculo de los cocientes de ventajas correspondientes a cada estrato. No obstante, en este caso se obtiene un nuevo valor de este estadístico, que se compara al valor tabular de la distribución $\chi^{2}$ con un solo grado de libertad y un nivel de significación del $5 \%$, que corresponde al valor 3.84. Únicamente en los casos en que se supera este valor de referencia se considera que el Área de estudio interactúa en la relación entre los distintos pares de variables. Los casos en que este estadístico ha resultado significativo, y por tanto se ha rechazado la hipótesis nula de ausencia de interacción, se han sombreado en la Tabla 4.39.

Se han empleado dos contrastes distintos para verificar la hipótesis de ausencia de interacción entre variables, ambos basados en las diferencias entre los cocientes de ventajas correspondientes a cada estrato. No obstante, la formulación matemática de estos contrastes difiere en determinados aspectos que dan lugar a aparentes incoherencias entre los resultados obtenidos con ambos contrastes. Sin embargo, la causa de estas discrepancias no son 


\begin{tabular}{|c|c|c|c|c|c|c|c|c|c|c|c|c|c|c|c|c|}
\hline & C1 & $\mathrm{C} 2$ & C3 & $\mathrm{C} 4$ & C5 & C6 & V1 & V2 & V3 & V4 & V5 & V6 & V7 & V8 & V9 & V10 VT \\
\hline & & & & & & & & & & & & & & & & \\
\hline $\mathrm{C} 2$ & 9.4 & & & & & & & & & & & & & & & \\
\hline C3 & 1.2 & 11.3 & & & & & & & & & & & & & & \\
\hline $\mathrm{C} 4$ & & 3.6 & & & & & & & & & & & & & & \\
\hline C5 & 7.6 & 10.8 & 6.1 & 113.3 & & & & & & & & & & & & \\
\hline C6 & 58.9 & 17.1 & 2.2 & & 10.9 & & & & & & & & & & & \\
\hline V1 & 7.7 & 3.3 & 1.0 & 1.9 & 4.9 & & & & & & & & & & & \\
\hline $\mathrm{V} 2$ & 4.2 & 5.5 & 8.5 & 2.8 & 9.8 & 5.8 & 62.0 & & & & & & & & & \\
\hline V3 & & 4.8 & 6.0 & 2.6 & 9.3 & & 8.2 & 42.5 & & & & & & & & \\
\hline V4 & & 12.5 & 11.3 & 4.7 & 11.1 & & 15.5 & 45.5 & 34.8 & & & & & & & \\
\hline V5 & 3.1 & 18.3 & 10.9 & 11.9 & 36.7 & 8.4 & 19.4 & 42.4 & 37.3 & 105.1 & & & & & & \\
\hline V6 & 11.2 & 5.8 & 2.4 & 10.5 & & 10.7 & 3.7 & 4.3 & & 4.2 & 8.7 & & & & & \\
\hline V7 & 0.9 & 4.4 & 1.8 & 3.9 & & & 2.5 & 2.7 & 6.6 & 3.9 & & 30.3 & & & & \\
\hline V8 & 12.5 & 8.7 & 4.5 & 6.9 & 18.5 & 10.6 & 10.6 & 22.5 & 35.1 & 32.2 & 66.2 & 4.9 & & & & \\
\hline V9 & 6.0 & 3.9 & 8.7 & 1.9 & 4.1 & 5.0 & 22.9 & 127.4 & 16.8 & 19.5 & 19.9 & 8.9 & & 17.4 & & \\
\hline V10 & 7.5 & 6.4 & 0.0 & 1.7 & 7.3 & 2.6 & 31.5 & 108.3 & 41.7 & 31.8 & 25.6 & & 2.2 & 24.9 & 93.8 & \\
\hline VT & & 2.0 & 10.1 & 1.1 & & & 1.4 & 3.2 & & 2.1 & 5.1 & 9.3 & 3.6 & & & 2.7 \\
\hline
\end{tabular}

Tabla 4.39: Valor del estadístico $\chi^{2}$ para el Contraste de asociación condicional. Características de la carrera y Métodos de enseñanza - aprendizaje

posibles errores que se hayan podido cometer en la formulación de los contrastes, sino la aportación de información desde distintos enfoques.

Así, los estadísticos $\chi^{2}$ obtenidos mediante el contraste condicional sobre el cociente de ventajas común significativos e indican que al menos en un caso algún cociente de ventajas en un estrato ha resultado distinto a la unidad, y por tanto distinto al resto de cocientes de ventajas obtenidos en los estratos restantes. En la mayoría de los casos la identificación de estos casos puede realizarse equivalentemente mediante el contraste condicional sobre el riesgo ponderado, que por su propia definición es menos restrictivo que el anterior, ya que únicamente plantea la igualdad de los cocientes de ventajas en los distintos estratos. En esta situación cabe esperar diferencias notables entre los cocientes de ventajas en cada estrato y que al menos uno de ellos se aleje significativamente de la unidad. Los casos en que se dan estas circunstancias se han señalado en la Tabla 4.40 con la notación (1).

Al mismo tiempo cabe la posibilidad de que, aun habiendo resultado significativo el estadístico $\chi^{2}$ obtenido mediante el contraste condicional sobre el cociente de ventajas común, no ocurra lo mismo con el el estadístico $\chi^{2}$ obtenido mediante el contraste sobre el riesgo ponderado. Esto implica que, aunque se haya detectado al menos un cociente de ventajas en los estratos distinto a la unidad, y por tanto distinto al resto, no se ha encontrado evidencia de que al menos en un caso algún cociente de ventajas sea distinto al resto. Esta situación se da en los casos en que no existen grandes diferencias entre los cocientes de ventajas en los estratos, pero al menos uno de ellos sí se aleja significativamente de la unidad. Estos casos se han etiquetado en la Tabla 4.40 con la notación (2). 
Por último, el caso menos frecuente es aquel en que el estadístico $\chi^{2}$ obtenido mediante el contraste condicional sobre el riesgo ponderado ha resultado significativo, pero no el obtenido mediante el contraste sobre el cociente de ventajas común. Esta situación supone que no existe evidencia de que al menos un cociente de ventajas en los estratos es significativamente distinto a la unidad, y por tanto distinto al resto, pero sí la hay en cuanto a la existencia de diferencias significativas entre los cocientes de ventajas en los estratos. Esta situación se interpreta como que existen diferencias significativas entre los cocientes de ventajas pertenecientes a cada estrato, pero puesto que todos ellos oscilan en torno a la unidad, no es posible afirmar que alguno de ellos se encuentre significativamente alejado de este valor. Los casos en que se da esta situación minoritaria se han señalado en la Tabla 4.40 con la notación (3).

Los pares de variables señalados con la notación (1) constituyen los casos en que la interacción entre variables cobra mayor intensidad. En estos casos no sólo existe evidencia de diferencias significativas entre los cocientes de ventajas en los estratos sino que se verifica la presencia de algún cociente de ventajas alejado de la unidad. Estos casos alcanzan el máximo nivel de significatividad en los contrastes de interacción por lo que deberían ser considerados en primer lugar en futuros análisis más detallados sobre relaciones de interacción.

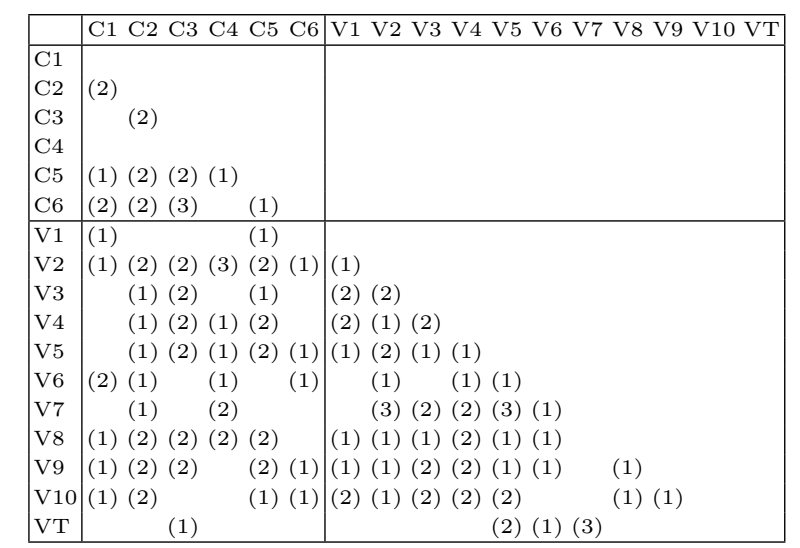

Tabla 4.40: Interacción y confusión entre variables. Características de los estudios y Métodos de enseñanza - aprendizaje

De este modo, se ha realizado una revisión exhaustiva de todas las posibles situaciones de interacción entre el Área de estudio y los distintos pares de variables pertenecientes al contexto académico y cuyas relaciones de de- 
pendencia global, parcial y condicional se han identificado previamente.

Independientemente de este análisis sobre la interacción entre variables, es posible plantear otro estudio enfocado a la identificación de posibles efectos de confusión debidos al Área de estudio, a partir de los resultados obtenidos para el contraste condicional sobre el cociente de ventajas común. Para ello se muestran en la Tabla 4.41 los cocientes de ventajas globales para cada tabla de contingencia. En cada caso se muestran dos cocientes de ventajas. El primero de ellos, denotado OR, corresponde al cociente de ventajas global independientemente del Área de estudio mientras que el cociente $\mathrm{OR}(\mathrm{MH})$ es el cociente de de ventajas global asociado al contraste condicional desarrollado por Mantel - Haënszel, calculado como una ponderación de los cocientes de ventajas en cada estrato o área de estudio. De este modo se dispone de dos coeficientes para el examen del efecto introducido por el Área de estudio en las relaciones entre variables. Nuevamente se han descartado en esta tabla aquellas relaciones identificadas previamente como independientes, a las cuales se les ha asignado simplemente un espacio en blanco.

Las diferencias entre ambos coeficientes $\mathrm{OR}, \mathrm{y}$ OR $(\mathrm{MH})$ se muestran en la Tabla 4.42. Como se ha expuesto en la teoría los posibles efectos que el Área de estudio puede tener sobre las relaciones entre variables son tanto la amplificación de la intensidad de las asociaciones como su atenuación. Asimismo cabe la posibilidad de una inversión del efecto en aquellos casos en que las diferencias entre ambos cocientes muestren un cambio en la dirección de la asociación. Por último, en los casos en que la presencia del Área de estudio suponga que el cociente de ventajas global deje de ser significativo, se considera que esta variable es responsable de mostrar una relación espúrea. Del mismo modo, si la presencia del área de estudio revela que en realidad el cociente de ventajas sí es significativo queda manifiesta una relación de enmascaramiento entre las variables. 


\begin{tabular}{|c|c|c|c|c|c|c|c|c|c|c|c|c|c|}
\hline & $\mathrm{C} 1 \quad \mathrm{C} 2$ & $\mathrm{C} 3$ & $\mathrm{C} 4$ & C5 & C6 & V1 V2 & V3 & V4 & V5 & V6 & V7 & V8 V9 & $\mathrm{V} 10 \mathrm{VT}$ \\
\hline \multicolumn{14}{|l|}{$\begin{array}{l}\text { C1-OR } \\
\text { C1-OR(MH) }\end{array}$} \\
\hline $\mathrm{C} 2-\mathrm{OR}$ & 1.65 & & & & & & & & & & & & \\
\hline $\mathrm{C} 2-\mathrm{OR}(\mathrm{MH})$ & 1.67 & & & & & & & & & & & & \\
\hline C3-OR & 0.811 .59 & & & & & & & & & & & & \\
\hline C3-OR(MH) & $0.94 \quad 1.68$ & & & & & & & & & & & & \\
\hline C4-OR & 0.76 & & & & & & & & & & & & \\
\hline $\mathrm{C} 4-\mathrm{OR}(\mathrm{MH})$ & 0.76 & & & & & & & & & & & & \\
\hline C5-OR & $1.48 \quad 1.78$ & 31.55 & 0.12 & & & & & & & & & & \\
\hline C5-OR(MH) & 1.651 .75 & 51.51 & 0.13 & & & & & & & & & & \\
\hline C6-OR & $5.76 \quad 1.85$ & 50.86 & & 1.55 & & & & & & & & & \\
\hline C6-OR(MH) & 3.871 .91 & 1.15 & & 1.94 & & & & & & & & & \\
\hline V1-OR & 1.141 .31 & 1.15 & 0.83 & 1.58 & & & & & & & & & \\
\hline V1-OR(MH) & 1.491 .30 & 1.07 & 0.89 & 1.40 & & & & & & & & & \\
\hline V2-OR & $0.68 \quad 1.27$ & 71.72 & 0.76 & 1.99 & 0.53 & 4.63 & & & & & & & \\
\hline V2-OR(MH) & 1.201 .50 & 1.61 & 0.84 & 1.78 & 1.01 & 4.08 & & & & & & & \\
\hline V3-OR & 1.49 & 1.89 & 0.68 & 2.6 & & 2.368 .5 & & & & & & & \\
\hline V3-OR(MH) & 1.55 & 1.7 & 0.71 & 2.21 & & 1.956 .75 & & & & & & & \\
\hline V4-OR & 1.78 & 31.56 & 0.74 & 1.89 & & 2.414 .16 & 4.39 & & & & & & \\
\hline $\mathrm{V} 4-\mathrm{OR}(\mathrm{MH})$ & 1.77 & 1.79 & 0.8 & 1.79 & & 1.963 .85 & 4.24 & & & & & & \\
\hline V5-OR & 1.231 .99 & 1.71 & 0.53 & 3.31 & 1.29 & 2.413 .47 & 5.34 & 7.07 & & & & & \\
\hline V5-OR(MH) & $1.32 \quad 1.93$ & 1.77 & 0.6 & 2.86 & 1.51 & 2.113 .41 & 4.46 & 6.7 & & & & & \\
\hline V6-OR & $\begin{array}{|ll|}1.6 & 1.46 \\
\end{array}$ & 1.19 & 1.44 & & 1.56 & 1.281 .13 & & 0.83 & 1.48 & & & & \\
\hline V6-OR(MH) & 1.691 .48 & 31.19 & 1.44 & & 1.63 & $1.32 \quad 1.21$ & & 0.85 & 1.57 & & & & \\
\hline V7-OR & 1.131 .39 & 1.18 & 1.25 & & & 1.220 .89 & 0.58 & 0.77 & 0.89 & 2.45 & & & \\
\hline V7-OR $(\mathrm{MH})$ & 1.011 .31 & 1.2 & 1.28 & & & 1.250 .93 & 0.59 & 0.75 & 0.87 & 2.39 & & & \\
\hline V8-OR & $\begin{array}{|ll|}2.36 & 1.59\end{array}$ & 1.47 & 0.69 & 2.43 & 1.97 & 1.612 .08 & 4.14 & 2.3 & 4.03 & 1.19 & & & \\
\hline V8-OR(MH) & 1.961 .62 & 21.5 & 0.71 & 2.24 & 1.67 & $1.8 \quad 2.67$ & 4.39 & 3.29 & 4.57 & 1.18 & & & \\
\hline V9-OR & 0.711 .16 & 1.93 & 0.72 & 1.66 & 0.47 & 2.6910 .6 & 4.73 & 2.41 & 2.42 & 1.42 & & 1.72 & \\
\hline V9-OR(MH) & $1.36 \quad 1.3$ & 1.68 & 0.83 & 1.45 & 0.93 & 2.267 .76 & 3.12 & 2.37 & 2.33 & 1.66 & & 2.26 & \\
\hline V10-OR & 0.741 .35 & 51.66 & 0.76 & 1.87 & 0.53 & 3.5913 .1 & 6.58 & 3.24 & 2.84 & & 0.73 & 1.9212 .6 & \\
\hline $\mathrm{V} 10-\mathrm{OR}(\mathrm{MH})$ & 1.521 .51 & 1.57 & 0.81 & 1.75 & 1.17 & 2.9410 .5 & 5.03 & 3.05 & 2.78 & & 0.81 & 3.018 .95 & \\
\hline VT-OR & 1.27 & 1.31 & 1.15 & & 1.15 & 1.221 .28 & & 2.02 & 1.58 & 1.68 & 1.37 & & 1.17 \\
\hline VT-OR(MH) & 1.22 & 1.49 & 1.09 & & 1.12 & 1.121 .41 & & 1.25 & 1.44 & 1.7 & 1.29 & & 1.37 \\
\hline
\end{tabular}

Tabla 4.41: Cociente de ventajas para el Contraste de asociación parcial no condicional, con y sin segmentación. Características de la carrera y Métodos de enseñanza - aprendizaje

En la Tabla 4.43 se muestran las relaciones cuyas diferencias entre los cocientes de ventajas $\mathrm{OR}$, y $\mathrm{OR}(\mathrm{MH})$ son superiores a la unidad en valor absoluto. En estos casos cabe esperar la presencia de algún tipo de efecto de confusión, ya que las diferencias entre coeficientes alcanzan su valor máximo. De este modo se muestran en la tabla los valores de ambos cocientes, sus diferencias y los correspondientes a cada uno de los ocho estratos contenidos en el Área de estudio. En gris se han sombreado aquellos valores superiores al cociente ponderado $\mathrm{OR}(\mathrm{MH})$ con el fin de facilitar la identificación de las variaciones en los valores. En los 7 primeros casos el $\mathrm{OR}(\mathrm{MH})$ es inferior al OR, lo que indica que el Área de estudio produce un efecto de atenuación en la intensidad de la asociación en estas variables. Por el contrario en el octavo caso el valor del cociente $\mathrm{OR}(\mathrm{MH})$ resulta superior al del OR, señalando que el Área de estudio origina un efecto de ampliación de la intensidad entre las variables implicadas: Exposiciones orales (V10) y Aprendizaje basado en problemas (V8). En ningún caso se han dado situaciones de enmascaramien- 


\begin{tabular}{|c|c|c|c|c|c|c|c|c|c|c|c|c|c|c|c|c|}
\hline & C1 & $\mathrm{C} 2$ & C3 & $\mathrm{C} 4$ & C5 & C6 & V1 & V2 & V3 & V4 & V5 & V6 & V7 & V8 & V9 & $\begin{array}{ll}\text { V10 } & \text { VT } \\
\end{array}$ \\
\hline C1 & & & & & & & & & & & & & & & & \\
\hline $\mathrm{C} 2$ & -0.018 & & & & & & & & & & & & & & & \\
\hline C3 & -0.132 & -0.091 & & & & & & & & & & & & & & \\
\hline $\mathrm{C} 4$ & & 0 & & & & & & & & & & & & & & \\
\hline C5 & -0.172 & 0.032 & 0.043 & -0.009 & & & & & & & & & & & & \\
\hline C6 & 1.88 & -0.063 & -0.285 & & -0.393 & & & & & & & & & & & \\
\hline V1 & -0.355 & 0.01 & 0.084 & -0.066 & 0.181 & & & & & & & & & & & \\
\hline $\mathrm{V} 2$ & -0.526 & -0.231 & 0.102 & -0.08 & 0.208 & -0.475 & 0.554 & & & & & & & & & \\
\hline V3 & & -0.058 & 0.195 & -0.032 & 0.391 & & 0.401 & 1.749 & & & & & & & & \\
\hline V4 & & 0.001 & -0.226 & -0.06 & 0.103 & & 0.448 & 0.316 & 0.152 & & & & & & & \\
\hline V5 & -0.089 & 0.053 & -0.062 & -0.067 & 0.447 & -0.222 & 0.3 & 0.06 & 0.875 & 0.364 & & & & & & \\
\hline V6 & -0.09 & -0.016 & 0.001 & 0.001 & & -0.065 & -0.037 & -0.082 & & -0.016 & -0.094 & & & & & \\
\hline V7 & 0.122 & 0.074 & -0.012 & -0.026 & & & -0.036 & -0.047 & -0.009 & 0.021 & 0.02 & 0.062 & & & & \\
\hline V8 & 0.404 & -0.032 & -0.03 & -0.024 & 0.187 & 0.308 & -0.189 & -0.592 & -0.247 & -0.987 & -0.537 & 0.013 & & & & \\
\hline V9 & -0.645 & -0.135 & 0.256 & -0.112 & 0.208 & -0.463 & 0.428 & 2.886 & 1.606 & 0.046 & 0.091 & -0.237 & & -0.537 & & \\
\hline V10 & -0.781 & -0.156 & 0.091 & -0.045 & 0.114 & -0.645 & 0.649 & 2.551 & 1.548 & 0.193 & 0.065 & & -0.086 & -1.096 & 3.612 & \\
\hline VT & & 0.052 & -0.182 & 0.059 & & 0.026 & 0.102 & -0.133 & & 0.764 & 0.139 & -0.022 & 0.08 & & & -0.204 \\
\hline
\end{tabular}

Tabla 4.42: Diferencias entre los cocientes de ventajas para el Contraste de asociación parcial no condicional, con y sin segmentación. Características de la carrera y Métodos de enseñanza - aprendizaje

to, relaciones espúreas o incluso inversión del efecto. Por tanto, aunque la presencia del Área de estudio modifica en todos los casos la intensidad de las relaciones entre variables pertenecientes al contexto académico, esta influencia no llega a distorsionar la dirección o la significatividad de dichas relaciones, hasta el extremo de perturbar en exceso las conclusiones del análisis. En cualquier caso, deberá tenerse en consideración el efecto modificador de la variable Área de estudio sobre la intensidad de las relaciones entre variables.

\begin{tabular}{|c|cc|ccc|cccccccc|}
\hline Caso & Var. 1 & Var. 2 & OR - OR $(\mathrm{MH})$ & OR & OR(MH) & Educ. & Hum. & CSoc. & Econ. & Der. & Tec. & Sal. & Cien. \\
\hline 1 & V10 & V9 & 3.612 & 12,562 & 8,949 & 6,620 & 6,584 & 10,285 & 14,347 & 6,500 & 6,058 & 18,226 & 14,738 \\
2 & V9 & V2 & 2.886 & 10,641 & 7,756 & 7,611 & 6,143 & 6,159 & 11,947 & 25,371 & 5,865 & 13,132 & 8,242 \\
3 & V10 & V2 & 2.551 & 13,060 & 10,509 & 12,833 & 6,913 & 10,220 & 21,694 & 9,289 & 6,347 & 13,801 & 10,296 \\
4 & C6 & C1 & 1.88 & 5,755 & 3,874 & 2,667 & 2,578 & 4,421 & 4,119 & 3,266 & 4,334 & 3,208 & 6,019 \\
5 & V3 & V2 & 1.749 & 8,498 & 6,749 & 12,812 & 5,056 & 8,750 & 15,221 & 14,267 & 4,941 & 5,043 & 5,028 \\
6 & V9 & V3 & 1.606 & 4,729 & 3,123 & 2,629 & 3,130 & 3,809 & 6,304 & & 2,291 & 2,739 & 2,297 \\
7 & V10 & V3 & 1.548 & 6,578 & 5,030 & 5,544 & 3,720 & 4,566 & 16,011 & 4,667 & 4,407 & 6,659 & 3,595 \\
8 & V10 & V8 & -1.096 & 1,917 & 3,013 & 2,861 & 3,334 & 4,624 & 3,496 & 11,566 & 2,196 & 2,552 & 1,521 \\
\hline
\end{tabular}

Tabla 4.43: Máximas diferencias de los cocientes de ventajas para el Contraste de asociación parcial no condicional, con y sin segmentación, por área de estudio. Características de la carrera y Métodos de enseñanza - aprendizaje

\section{Análisis multidimensional: Identificación de perfiles de estudiantes universitarios}

Como se ha expuesto en el apartado correspondiente, el análisis de correspondencias múltiples es una técnica multivariante de carácter exploratorio aplicada al análisis de las relaciones entre un conjunto de variables, gene- 
ralmente nominales, que hacen referencia a un mismo tema. En este caso se ha planteado aplicar este análisis al conjunto de variables del entorno personal del estudiante en la Universidad. Así, se hace referencia tanto a sus pautas de gestión del tiempo (compaginar los estudios con el trabajo, nota obtenida en los estudios, posibles estancias en el extranjero, etc.) como a las características personales propias del encuestado (género, edad, situación de convivencia en el momento de la entrevista, etc.). A partir de las relaciones entre estas variables se ha obtenido un diagrama o biplot en el que se representan mediante puntos las distintas categorías de respuesta de las preguntas. En este diagrama la proximidad entre los puntos representados se relaciona con el nivel de asociación entre las distintas categorías de respuesta.

Así, la aplicación del análisis de la matriz de Burt al conjunto de las once preguntas del cuestionario sobre el contexto personal de los estudiantes ha permitido simplificar las respuestas de los encuestados mediante la definición de dos ejes principales. La varianza explicada por el primer eje corresponde al $11.36 \%$ de la varianza contenida en el conjunto de las respuestas de todos los encuestados mientras que el segundo contribuye al $10.47 \%$, sumando un porcentaje de inercia acumulado entre ambos ejes del $21.83 \%$. En cualquier caso esta medida de varianza explicada por los ejes no constituye una medida de referencia adecuada ya que se ha obtenido a partir de un análisis de correspondencias múltiples, y como se ha expuesto previamente no resulta procedente emplear estos porcentajes como indicador de la calidad global de la representación.

\begin{tabular}{|c|c|c|c|}
\hline Núm. & $\begin{array}{r}\text { Valor } \\
\text { propio }\end{array}$ & \% Inercia & $\begin{array}{c}\text { \% Inercia } \\
\text { Acumulado }\end{array}$ \\
\hline 1 & 0.1756 & $11.36 \%$ & $11.36 \%$ \\
2 & 0.1617 & $10.47 \%$ & $21.83 \%$ \\
\hline
\end{tabular}

Tabla 4.44: Análisis de correspondencias múltiple. Valores propios y porcentajes de inercia en los dos primeros factores

En la Tabla 4.45 se muestra tanto el peso relativo de cada categoría como su distancia al origen. Asimismo se muestran las coordenadas factoriales y las contribuciones absolutas y relativas de las distintas categorías. Las categorías con menor peso relativo son aquellas cuyas frecuencias han resultado más pequeñas respecto a las frecuencias totales. Las categorías incluidas en este grupo incluyen haber obtenido Sobresaliente o Matrícula de honor en los estudios (0.50), la opción Otros en la variable Situación de convivencia (0.54) o los encuestados con más de 35 años en el momento de la entrevista (0.38). Concretamente en el caso de la nota obtenida en los estudios, la categoría Sobresaliente o Matrícula de honor representa el $5.5 \%$ respecto 
a la suma de los pesos de categorías restantes (Aprobado o Notable). Asimismo la categoría Otros de la variable Situación de convivencia supone el $6 \%$ mientras que la categoría Más de 35 años representa el $4.2 \%$ del peso. Según este razonamiento cabría plantearse eliminar o agrupar la categoría Más de 35 años con otras similares, como podría ser 30 - 35 años, ya que adicionalmente la categoría Más de 35 años alcanza la distancia más elevada respecto al origen (22.70). Sin embargo, se ha considerado apropiado mantener esta división de grupos en el límite de edad de 35 años, ya que constituye una franja de edad interesante en la descripción de los distintos perfiles entre encuestados.

Entre las contribuciones relativas de las categorías destacan las variables relativas al Nivel de estudios de los padres y las Estancias en el extranjero como ítems determinantes del segundo factor. Por el contrario la gran mayoría de las variables restantes alcanzan elevadas contribuciones en el primer factor. Así, el sector positivo del primer factor queda determinado por categorías como Dedicación exclusiva a los estudios (40\%), Edad entre 26 y 30 años (39\%), Convivencia con los padres en el momento de la entrevista $(12 \%)$ y No tener hijos (35\%). Por el contrario, el sector negativo de este primer factor está determinado por categorías de variables como Edad superior a 35 años (29\%) o Entre 30 y 35 años (17\%), Tener hijos (35\%), Compaginar estudios y trabajo (40\%), Convivencia en pareja en el momento de la entrevista (17\%) o haber obtenido una nota de Aprobado en los estudios (13\%). Por tanto, se han identificado dos direcciones principales de inercia. La primera de ellas diferencia fundamentalmente a los encuestados en función de su situación personal en cuanto a la edad, los hijos, el trabajo y la situación de convivencia familiar. Por otro lado el segundo factor distingue a los encuestados cuyos Padres tienen estudios universitarios o han realizado alguna Estancia en el extranjero, bien durante los estudios o al finalizarlos. En resumidas cuentas, se puede considerar que este segundo factor hace referencia indirectamente al poder adquisitivo de la familia del encuestado. 


\begin{tabular}{|c|c|c|c|c|c|c|c|c|}
\hline \multirow[t]{2}{*}{ Descripción } & \multirow{2}{*}{\multicolumn{2}{|c|}{\begin{tabular}{cc|} 
Peso Distancia \\
Relativo al origen
\end{tabular}}} & \multicolumn{2}{|c|}{ Coord. factoriales } & \multicolumn{2}{|c|}{ Cont. absolutas } & \multicolumn{2}{|c|}{ Cont. relativas $\cos ^{2}$} \\
\hline & & & Eje 1 & Eje 2 & Eje 1 & Eje 2 & Eje 1 & Eje 2 \\
\hline Dedicación exclusiva a estudios & 7.63 & 0.19 & 0.28 & -0.07 & 3.29 & 0.21 & 0.40 & 0.02 \\
\hline Estudios y trabajo & 1.46 & 5.23 & -1.44 & 0.35 & 17.24 & 1.09 & 0.40 & 0.02 \\
\hline Prácticas & 5.10 & 0.78 & 0.20 & -0.14 & 1.11 & 0.65 & 0.05 & 0.03 \\
\hline No Prácticas & 3.99 & 1.28 & -0.25 & 0.18 & 1.42 & 0.83 & 0.05 & 0.03 \\
\hline Hombre & 3.13 & 1.90 & -0.19 & 0.16 & 0.66 & 0.49 & 0.02 & 0.01 \\
\hline Mujer & 5.96 & 0.52 & 0.10 & -0.08 & 0.34 & 0.26 & 0.02 & 0.01 \\
\hline Aprobado & 4.21 & 1.16 & -0.39 & 0.06 & 3.55 & 0.09 & 0.13 & 0.00 \\
\hline Notable & 4.38 & 1.07 & 0.30 & -0.12 & 2.22 & 0.42 & 0.08 & 0.01 \\
\hline Sobresaliente o M. de Honor & 0.50 & 17.22 & 0.62 & 0.59 & 1.11 & 1.08 & 0.02 & 0.02 \\
\hline Vive solo & 1.27 & 6.16 & 0.14 & 0.28 & 0.14 & 0.62 & 0.00 & 0.01 \\
\hline Vive con pareja & 3.92 & 1.32 & -0.48 & 0.27 & 5.10 & 1.72 & 0.17 & 0.05 \\
\hline Vive con padres & 3.36 & 1.71 & 0.46 & -0.38 & 4.07 & 3.01 & 0.12 & 0.08 \\
\hline Otros & 0.54 & 15.95 & 0.28 & -0.23 & 0.23 & 0.18 & 0.00 & 0.00 \\
\hline Hijos & 0.92 & 8.90 & -1.76 & 0.60 & 16.21 & 2.05 & 0.35 & 0.04 \\
\hline No Hijos & 8.17 & 0.11 & 0.20 & -0.07 & 1.82 & 0.23 & 0.35 & 0.04 \\
\hline Menos de 15 horas de estudio & 1.21 & 6.50 & -0.45 & 0.04 & 1.39 & 0.01 & 0.03 & 0.00 \\
\hline 15-30 horas de estudio & 1.90 & 3.77 & -0.20 & 0.13 & 0.46 & 0.20 & 0.01 & 0.00 \\
\hline $30-45$ horas de estudio & 3.42 & 1.66 & 0.18 & -0.09 & 0.66 & 0.17 & 0.02 & 0.00 \\
\hline Más de 45 horas de estudio & 2.56 & 2.55 & 0.12 & 0.00 & 0.21 & 0.00 & 0.01 & 0.00 \\
\hline $26-30$ años & 5.63 & 0.61 & 0.49 & -0.18 & 7.63 & 1.09 & 0.39 & 0.05 \\
\hline 30-35 años & 3.07 & 1.96 & -0.57 & 0.27 & 5.76 & 1.34 & 0.17 & 0.04 \\
\hline Más de 35 años & 0.38 & 22.70 & -2.56 & 0.47 & 14.37 & 0.52 & 0.29 & 0.01 \\
\hline Padre con Ed. Superior & 2.56 & 2.55 & 0.49 & 1.34 & 3.50 & 28.23 & 0.09 & 0.70 \\
\hline No Padre con Ed. Superior & 6.53 & 0.39 & -0.19 & -0.52 & 1.37 & 11.05 & 0.09 & 0.70 \\
\hline Madre con Ed. Superior & 1.45 & 5.26 & 0.71 & 1.89 & 4.13 & 32.04 & 0.09 & 0.68 \\
\hline No Madre con Ed. Superior & 7.64 & 0.19 & -0.13 & -0.36 & 0.79 & 6.09 & 0.09 & 0.68 \\
\hline Estancia en extranjero & 1.16 & 6.86 & 0.41 & 0.88 & 1.08 & 5.54 & 0.02 & 0.11 \\
\hline No estancia en extranjero & 7.93 & 0.15 & -0.06 & -0.13 & 0.16 & 0.81 & 0.02 & 0.11 \\
\hline
\end{tabular}

Tabla 4.45: Coordenadas factoriales, contribuciones absolutas y contribuciones relativas. Gestión del tiempo y Características personales

En la Tabla 4.46 se muestran los valores - test de las variables activas que indican el nivel de significatividad de las posiciones que ocupan en los planos factoriales. En ella se observa que prácticamente todas las categorías de respuesta son significativas así como que el valor - test correspondiente a cada una de ellas está muy relacionado con esta posición, en lo que respecta a la dirección, reflejado en el signo, y la distancia respecto al origen, asociada al valor absoluto del valor -test.

Sobre este mismo diagrama es posible representar otros datos que no hayan tomado parte en la construcción de los planos factoriales, denominados elementos suplementarios o ilustrativos, que se consideran de forma independiente por tratarse de observaciones de distinta naturaleza al conjunto principal de variables activas. En este estudio se ha utilizado este método para completar la información con datos sobre el área y el nivel de los estudios.

En la Tabla 4.47 y la Figura 4.17 se observa que determinadas áreas de estudio, como Derecho y Humanidades, ocupan posiciones significativas respecto al segundo factor, de forma existe cierta asociación entre la elección de ambas áreas de estudio (tradicionalmente estudios de Ciclo Largo), y el nivel de estudios de los padres y las estancias en el extranjero. En este aspecto 


\begin{tabular}{|c|c|c|c|c|c|}
\hline \multirow[t]{2}{*}{ Descripción } & \multirow[t]{2}{*}{$\mathrm{N}$} & \multirow{2}{*}{$\begin{array}{c}\text { Peso } \\
\text { absoluto }\end{array}$} & \multirow{2}{*}{$\begin{array}{l}\text { Distancia } \\
\text { al origen }\end{array}$} & \multicolumn{2}{|c|}{ Valores - test } \\
\hline & & & & Eje 1 & Eje 2 \\
\hline Dedicación exclusiva estudios & 3900 & 3900 & 0,19 & 42,92 & $-10,34$ \\
\hline Estudios y trabajo & 745 & 745 & 5,23 & $-42,92$ & 10,34 \\
\hline Prácticas & 2605 & 2605 & 0,78 & 15,08 & $-11,06$ \\
\hline No Prácticas & 2040 & 2040 & 1,28 & $-15,08$ & 11,06 \\
\hline Hombre & 1599 & 1599 & 1,9 & $-9,47$ & 7,85 \\
\hline Mujer & 3046 & 3046 & 0,52 & 9,47 & $-7,85$ \\
\hline Aprobado & 2150 & 2150 & 1,16 & $-24,36$ & 3,73 \\
\hline Notable & 2240 & 2240 & 1,07 & 19,63 & $-8,15$ \\
\hline Sobresaliente o M. Honor & 255 & 255 & 17,22 & 10,25 & 9,72 \\
\hline Vive solo & 649 & 649 & 6,16 & 3,82 & 7,73 \\
\hline Vive & 2005 & 2005 & 1,32 & $-28,36$ & 15,82 \\
\hline Vive co & 1717 & 1717 & 1,71 & 24,06 & $-19,85$ \\
\hline Otros & 274 & 274 & 15,95 & 4,71 & $-3,96$ \\
\hline Hijos & 469 & 469 & 8,9 & $-40,22$ & 13,71 \\
\hline No Hijos & 4176 & 4176 & 0,11 & 40,22 & $-13,71$ \\
\hline Menos de 15 horas & 619 & 619 & 6,5 & $-11,98$ & 1,19 \\
\hline $15-30$ horas & 973 & 973 & 3,77 & $-7,19$ & 4,58 \\
\hline $30-45$ horas & 1745 & 1745 & 1,66 & 9,71 & $-4,72$ \\
\hline Más de 45 horas & 1308 & 1308 & 2,55 & 5,09 & 0,04 \\
\hline 26-30 años & 2879 & 2879 & 0,61 & 42,42 & $-15,37$ \\
\hline & 1570 & 1570 & 1,96 & $-27,95$ & 12,92 \\
\hline Más de 35 años & 196 & 196 & 22,7 & $-36,68$ & 6,71 \\
\hline Padre con E. Superior & 1307 & 1307 & 2,55 & 20,91 & 56,97 \\
\hline No Padre con E. Superior & 3338 & 3338 & 0,39 & $-20,91$ & $-56,97$ \\
\hline Madre con E. Superior & 742 & 742 & 5,26 & 21 & 56,13 \\
\hline No Madre con E. Superior & 3903 & 3903 & 0,19 & -21 & $-56,13$ \\
\hline Extranjero & 591 & 591 & 6,86 & 10,54 & 22,9 \\
\hline No Extranjero & 4054 & 4054 & 0,15 & $-10,54$ & $-22,9$ \\
\hline
\end{tabular}

Tabla 4.46: Valores - test. Gestión del tiempo y Características personales

debe tenerse en consideración que una parte considerable de la relación entre esta última variable y los estudios de Humanidades, es la alta proporción de titulados en diversas modalidades de Filología. Esta misma conclusión puede extraerse mediante el análisis de la posición ocupada por los estudios de Ciclo Largo y Ciclo Corto. Al igual que en el caso anterior se observan relaciones significativas entre la selección de estudios de Ciclo Largo y Tener padres con estudios universitarios y las Estancias en el extranjero. Por el contrario los estudios de Ciclo Corto se sitúan en el extremo inferior del gráfico, indicando cierta asociación con las categorías relativas a los padres sin estudios universitarios, que en principio ocupan posiciones no significativas en el plano factorial. Igualmente los estudios de Educación y Ciencias Sociales ocupan posiciones similares en este sector del plano factorial.

Respecto al primer factor se observa que los titulados en estudios del área de Salud se sitúan en el sector derecho del gráfico, indicando cierta relación con la dedicación exclusiva a los estudios, tener edades comprendidas entre 26 y 30 años, convivir con los padres en el momento de la entrevista o no tener hijos. Por el contrario otras áreas de estudio como Economía, Técnicas y Ciencias se relacionan en mayor medida con las categorías de variables que indican mayor independencia del encuestado, como son tener hijos, compaginar los estudios con el trabajo o convivir con la pareja en el 
momento de la entrevista. También se incluye en este grupo haber obtenido una nota de aprobado en los estudios, aunque la contribución de esta categoría a la construcción del eje es inferior.

\begin{tabular}{|l|c|c|c|cc|cc|}
\hline Descripción & $\mathrm{N}$ & $\begin{array}{c}\text { Peso } \\
\text { absoluto }\end{array}$ & $\begin{array}{c}\text { Distancia } \\
\text { al origen }\end{array}$ & \multicolumn{2}{|c|}{ Coordenadas factoriales } & \multicolumn{2}{|c|}{ Ealores - test } \\
Eje 1 & Eje 2 \\
\hline Educación & 528 & 528 & 7,80 & 0,21 & $-0,40$ & 5,21 & $-9,67$ \\
Humanidades & 438 & 438 & 9,61 & 0,05 & 0,13 & 1,06 & 2,85 \\
Ciencias Sociales & 528 & 528 & 7,80 & 0,08 & $-0,21$ & 2,01 & $-5,09$ \\
Economía & 895 & 895 & 4,19 & $-0,14$ & $-0,11$ & $-4,50$ & $-3,74$ \\
Derecho & 257 & 257 & 17,07 & 0,11 & 0,29 & 1,80 & 4,76 \\
Técnicas & 930 & 930 & 3,99 & $-0,21$ & 0,19 & $-7,09$ & 6,62 \\
Salud & 482 & 482 & 8,64 & 0,33 & 0,03 & 7,60 & 0,58 \\
Ciencias & 525 & 525 & 7,85 & $-0,10$ & 0,16 & $-2,44$ & 3,83 \\
Ciclo Largo & 2666 & 2666 & 0,74 & 0,01 & 0,17 & 0,65 & 13,13 \\
Ciclo Corto & 1965 & 1965 & 1,36 & $-0,01$ & $-0,23$ & $-0,48$ & $-13,18$ \\
\hline
\end{tabular}

Tabla 4.47: Coordenadas factoriales y valores - test de la proyección en suplementario. Area y Nivel de estudios

La Figura 4.17 contiene la misma información que las tablas anteriores, aunque su interpretación resulta más intuitiva visualmente. Puesto que prácticamente todas las categorías de las variables activas ocupan posiciones significativas en el plano factorial, como se ha visto en la tabla de valores test correspondiente, se ha optado por destacar aquellas con mayor contribución relativa a la inercia de cada eje. Así, se ha señalado en color azul las categorías de las variables activas cuya contribución relativa a la inercia del primer factor ha sido mayor, mientras que las categorías cuya contribución relativa a la inercia del primer factor ha sido más significativa se ha señalado en rojo. Del mismo modo, se ha marcado con color azul las categorías de las variables ilustrativas (Área de estudio y Nivel de estudio) en posiciones significativas en el primer eje según su valor - test, mientras que el color rojo representa las categorías en posiciones significativas respecto al segundo. Como es evidente, las categorías destacadas en color morado indican la significatividad de la posición respecto a ambos ejes. 


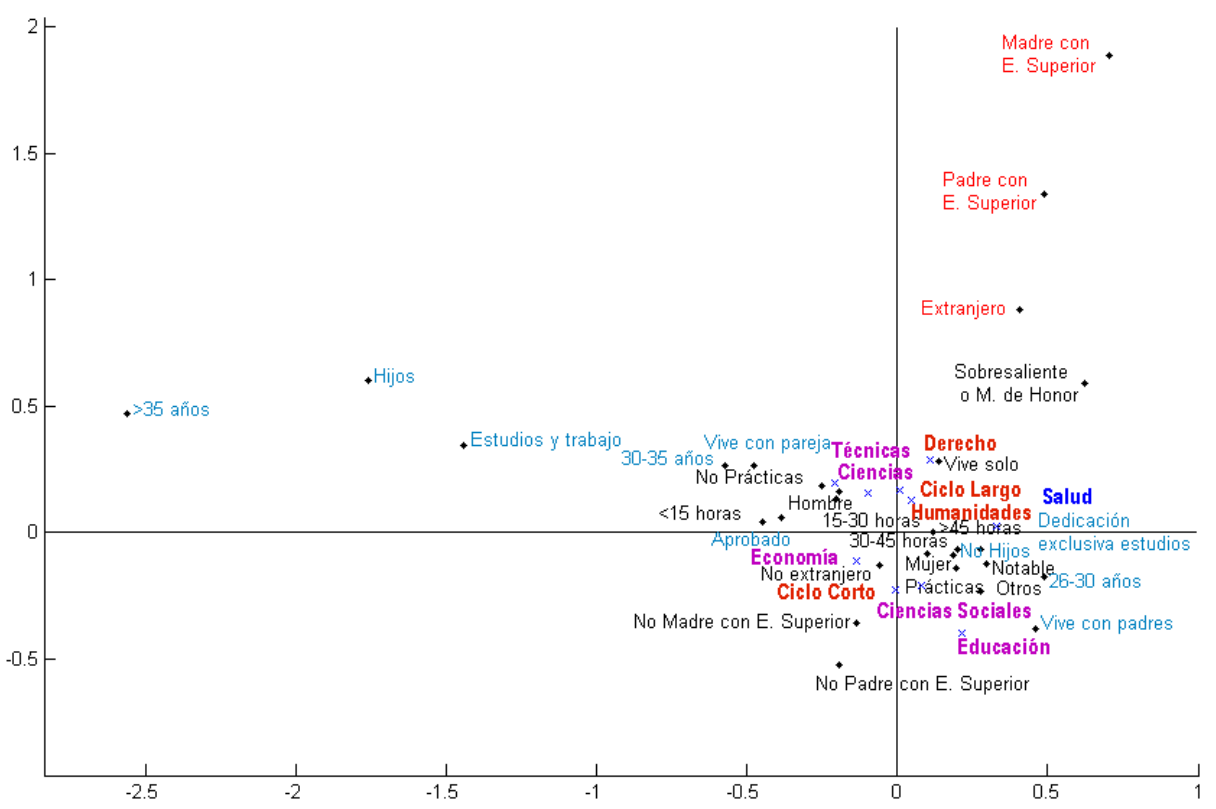

Figura 4.17: Proyección del Área y nivel de estudios sobre los ejes definidos sobre Gestión del tiempo y Características personales. Factores 1 y 2 


\subsubsection{Relaciones entre el contexto de los estudiantes y la adquisición de competencias en la Universidad}

\section{Relaciones entre variables contextuales y factores competenciales}

El objetivo de este apartado es analizar cada una de las relaciones individuales establecidas entre las variables pertenecientes al contexto académico y personal de los estudiantes, denominadas a partir de ahora Variables contexuales de forma genérica, y la adquisición de los factores competenciales identificados anteriormente. Para ello se han proyectado dichas variables contextuales sobre las componentes principales obtenidas a partir de las valoraciones sobre Competencias adquiridas en la Universidad. Posteriormente se han calculado los valores - test correspondientes a cada combinación de variable contextual y factor competencial con el fin de conocer el nivel de significación asociado a cada relación. Estas variables se han considerado como variables nominales ilustrativas, proyectadas sobre los factores competenciales. Este estudio podría haberse realizado de forma directa con el programa estadístico $S P A D$ (versión 6.0) que ofrece múltiples opciones para la ejecución de este tipo de análisis. Sin embargo, este programa no contempla la posibilidad de aplicar rotaciones sobre los componentes extraídos y por tanto no pudo emplearse en este apartado.

Con el fin de solventar esta limitación se optó por programar en Matlab (Versión 6.5) el procedimiento de cálculo para la obtención de las coordenadas de las variables contextuales en los planos factoriales rotados y sus valores - test asociados. El procedimiento para la ejecución de estos cálculos se estructuró en las siguientes fases detalladas a continuación.

En primer lugar se tipifican las variables de la matriz de puntuaciones asignadas por los titulados a cada una de las 19 competencias listadas en el cuestionario. De este modo cada una de las columnas de la matriz de datos original (a la que previamente se le ha aplicado la transformación para la eliminación del factor tamaño) resulta:

$$
C_{5474 x 1}^{\prime}=\frac{C_{5474 x 1}-\bar{x}_{i}}{\sigma_{i}}, i=1, \ldots, 19
$$

A continuación se realiza una desagregación de la matriz de puntuaciones tipificada en tantas submatrices como k categorías posean las variables contextuales implicadas. Así, al considerar todas y cada una de estas n categorías, cada columna de la matriz de datos tipificada $C_{5474 x 1}^{\prime}$ se transforma en una matriz de dimensiones $C_{5474 x k}$.

A partir de estas submatrices es posible calcular las puntuaciones promedio correspondientes a cada competencia y a cada categoría de las variables 
contextuales, que constituyen las coordenadas de cada categoría en el espacio de 19 dimensiones de las competencias. Seleccionando exclusivamente las categorías de interés para el análisis (presencia de la variable enunciada, descartando la no presencia) se obtienen $n$ vectores $\bar{\Psi}_{1 x 19}$ con dichas puntuaciones.

En la Tabla 4.48 se muestra la matriz que contiene los antiguos ejes rotados de los factores competenciales. Estos valores se obtienen directamente a partir de los autovectores calculados en la extracción de los componentes principales, como se ha expuesto en la teoría. Su empleo se justifica por la necesidad de representar en el espacio definido por los factores competenciales las coordenadas de las variables contextuales, consideradas en este análisis como ilustrativas y por tanto como un promedio equivalente a un nuevo individuo ilustrativo. De este modo, es posible representar de forma simultánea las coordenadas de estas variables junto con las direcciones de las variables activas, contenidas en las coordenadas de los antiguos ejes rotados.

\begin{tabular}{|c|c|c|c|c|c|c|}
\hline & F1 & F2 & F3 & F4 & F5 & F6 \\
\hline Dominio de tu área o disciplina & -0.146 & -0.206 & -0.299 & 0.053 & -0.300 & 0.039 \\
\hline Conocimientos de otras áreas o disciplinas & 0.046 & -0.183 & -0.342 & -0.109 & -0.239 & -0.060 \\
\hline Pensamiento analítico & -0.308 & -0.244 & -0.184 & -0.134 & 0.001 & 0.249 \\
\hline Adquirir con rapidez nuevos conocimientos & -0.324 & $4-0.207$ & -0.032 & -0.177 & -0.044 & 0.245 \\
\hline Negociar de forma eficaz & 0.489 & -0.091 & -0.106 & -0.191 & -0.065 & 0.154 \\
\hline Rendir bajo presión & 0.002 & -0.080 & 0.418 & -0.319 & -0.037 & 0.076 \\
\hline Detectar nuevas oportunidades & 0.420 & -0.172 & 0.159 & -0.214 & 0.013 & -0.074 \\
\hline Coordinar actividades & 0.075 & -0.069 & 0.441 & 0.124 & -0.058 & 0.030 \\
\hline Usar el tiempo de forma efectiva & -0.141 & -0.025 & 0.555 & 0.012 & -0.124 & 0.040 \\
\hline Trabajar en equipo & -0.159 & 0.028 & 0.074 & 0.632 & -0.119 & 0.018 \\
\hline Movilizar las capacidades de otros & 0.257 & -0.084 & -0.037 & 0.459 & -0.026 & 0.098 \\
\hline Hacerte entender & 0.062 & 0.337 & -0.042 & 0.128 & 0.052 & 0.338 \\
\hline Hacer valer tu autoridad & 0.452 & -0.019 & -0.100 & 0.107 & 0.082 & 0.061 \\
\hline Utilizar herramientas informáticas & -0.094 & $1-0.154$ & -0.029 & 0.093 & 0.197 & -0.630 \\
\hline Encontrar nuevas ideas y soluciones & -0.079 & -0.189 & 0.012 & 0.056 & 0.627 & -0.145 \\
\hline Predisposición para cuestionar ideas propias o ajenas & -0.023 & 0.112 & -0.126 & -0.091 & 0.565 & 0.202 \\
\hline Presentar en público productos, ideas o informes & 0.019 & 0.493 & -0.077 & 0.042 & -0.001 & -0.034 \\
\hline Redactar informes o documentos & -0.138 & 0.543 & -0.017 & -0.231 & -0.022 & -0.029 \\
\hline Escribir y hablar en idiomas extranjeros & 0.033 & 0.202 & -0.069 & -0.150 & -0.224 & -0.497 \\
\hline
\end{tabular}

Tabla 4.48: Coordenadas de los antiguos ejes rotados. Competencias adquiridas en la Universidad

Una vez obtenidas las puntuaciones promedio $\bar{\Psi}_{1 x 19}$, , para cada combinación de categoría de variable contextual y factor competencial, es posible realizar su transformación al espacio rotado de los 6 factores competenciales a través del producto con la matriz que contiene los antiguos ejes rotados. La transformación de dichas coordenadas al espacio rotado formado por 6 factores competenciales se realiza a través del producto con la matriz que contiene los antiguos ejes rotados en el espacio rotado. En la Tabla 4.49 se muestran los valores correspondientes a las variables del contexto académico en cada una de las componentes de competencias. 


$$
\bar{\Psi}_{1 x 6}^{r o t}=\bar{\Psi}_{1 x 19} \cdot u_{j \alpha}
$$

\begin{tabular}{|c|c|c|c|c|c|c|}
\hline & F1 & F2 & F3 & F4 & F5 & F6 \\
\hline En tu carrera había que trabajar mucho para aprobar & -0.160 & -0.120 & 0.087 & -0.144 & 0.032 & -0.016 \\
\hline En tu carrera el contenido era conocido por los empleadores & 0.009 & -0.059 & 0.015 & -0.010 & -0.040 & -0.025 \\
\hline En tu carrera podías elegir las asignaturas que querías cursar & 0.032 & 0.050 & -0.039 & 0.072 & 0.055 & -0.069 \\
\hline En tu carrera el enfoque era generalista & -0.066 & 0.035 & 0.024 & -0.047 & -0.001 & 0.024 \\
\hline En tu carrera el enfoque era especializado & 0.054 & 0.047 & 0.051 & 0.083 & -0.006 & -0.066 \\
\hline Tu carrera era una carrera con prestigio académico & -0.211 & -0.257 & 0.054 & -0.290 & 0.032 & -0.053 \\
\hline Asistencia a clase & 0.126 & 0.077 & 0.039 & 0.167 & -0.018 & -0.001 \\
\hline Trabajos en $g$ & 0.203 & 0.306 & 0.062 & 0.515 & 0.070 & -0.018 \\
\hline Participación en proyectos de investigación & 0.170 & 0.392 & 0.085 & 0.291 & 0.172 & -0.134 \\
\hline Prácticas en empresas, instituciones o similares & 0.223 & 0.119 & 0.019 & 0.317 & -0.054 & -0.020 \\
\hline Conocimientos prácticos y metodológicos & 0.048 & 0.057 & 0.052 & 0.176 & 0.047 & -0.082 \\
\hline Teorías, conceptos y paradigmas & -0.102 & -0.018 & 0.016 & -0.072 & 0.019 & 0.017 \\
\hline El profesor era la principal fuente de información & -0.038 & -0.054 & -0.007 & -0.033 & -0.017 & 0.021 \\
\hline Aprendizaje basado en proyectos o problemas & -0.162 & -0.066 & 0.179 & 0.016 & 0.212 & -0.138 \\
\hline Trabajos escritos & 0.107 & 0.359 & -0.001 & 0.324 & 0.044 & -0.033 \\
\hline Exposiciones orales & 0.235 & 0.592 & -0.007 & 0.476 & 0.082 & 0.035 \\
\hline Realización de pruebas tipo test & 0.100 & -0.061 & 0.018 & 0.072 & -0.093 & 0.097 \\
\hline
\end{tabular}

Tabla 4.49: Coordenadas sobre las componentes principales de Competencias adquiridas en la Universidad. Características de la carrera y Métodos de enseñanza - aprendizaje

El cálculo de los valores - test se aplica a las $k$ coordenadas anteriores en el espacio rotado de 6 factores competenciales $\bar{\Psi}_{k \alpha}$ siendo $\alpha=1, \ldots, 6$. Asimismo se incluyen en este cálculo el número de encuestados total $n \mathrm{y}$ el número de encuestados $n_{k}$ pertenecientes a cada categoría $k$. También la inercia de los ejes $\lambda_{\alpha}$ influye en la determinación de los valores - test, estimadas mediante los autovalores asociados a cada autovector obtenido en la extracción de las componentes principales. De este modo se obtiene la Tabla 4.50 que muestra los valores - test correspondientes a las variables del contexto académico. En gris claro se han sombreado los valores superiores al límite superior definido en 1.96 mientras que los valores inferiores al límite inferior establecido en -1.96 se han marcado en un tono de gris más oscuro.

En este punto del análisis cabe destacar que la representación simultánea de variables contextuales y factores competenciales no permite extraer conclusiones sobre relaciones de causalidad. Por esta razón es posible detectar relaciones significativas entre factores y variables pero no es posible determinar relaciones causa - efecto. Por otro lado, también debe señalarse que en esta fase del trabajo se analizarán las relaciones entre factores y variables de forma independiente. Aunque es obvio que tanto el contexto personal como el académico influyen simultáneamente en el proceso de adquisición de competencias en la Universidad, en esta primera fase del trabajo se analizarán por separado cada una de estas relaciones. La razón de este planteamiento es conocer qué factores tienen influencia en sí mismos en la formación en com- 


\begin{tabular}{|l|cccccc|}
\hline & $\mathrm{F} 1$ & $\mathrm{~F} 2$ & $\mathrm{~F} 3$ & $\mathrm{~F} 4$ & $\mathrm{~F} 5$ & $\mathrm{~F} 6$ \\
\hline En tu carrera había que trabajar mucho para aprobar & -8.31 & -6.79 & 5.78 & -10.02 & 2.38 & -1.25 \\
El contenido era conocido por los empleadores & 0.32 & -2.24 & 0.65 & -0.45 & -2.02 & -1.26 \\
Podías elegir las asignaturas que querías cursar & 0.96 & 1.62 & -1.50 & 2.91 & 2.39 & -3.05 \\
En tu carrera el enfoque era generalista & -3.11 & 1.80 & 1.43 & -2.93 & -0.07 & 1.67 \\
En tu carrera el enfoque era especializado & 1.38 & 1.32 & 1.65 & 2.86 & -0.21 & -2.47 \\
Tu carrera era una carrera con prestigio académico & -7.06 & -9.37 & 2.28 & -13.02 & 1.57 & -2.58 \\
\hline Asistencia a clase & 4.06 & 2.72 & 1.63 & 7.24 & -0.83 & -0.06 \\
Trabajos en grupo & 6.45 & 10.64 & 2.50 & 22.01 & 3.24 & -0.85 \\
Participación en proyectos de investigación & 2.38 & 6.00 & 1.51 & 5.48 & 3.50 & -2.77 \\
Prácticas en empresas, instituciones o similares & 5.23 & 3.05 & 0.56 & 10.00 & -1.86 & -0.70 \\
Conocimientos prácticos y metodológicos & 1.39 & 1.80 & 1.94 & 6.90 & 2.00 & -3.51 \\
Teorías, conceptos y paradigmas & -6.18 & -1.17 & 1.23 & -5.87 & 1.65 & 1.56 \\
El profesor era la principal fuente de información & -2.25 & -3.43 & -0.53 & -2.61 & -1.40 & 1.83 \\
Aprendizaje basado en proyectos o problemas & -3.69 & -1.63 & 5.20 & 0.49 & 7.03 & -4.65 \\
Trabajos escritos & 3.63 & 13.30 & -0.04 & 14.75 & 2.15 & -1.65 \\
Exposiciones orales & 5.10 & 14.03 & -0.19 & 13.89 & 2.57 & 1.12 \\
Realización de pruebas tipo test & 2.88 & -1.93 & 0.67 & 2.80 & -3.91 & 4.13 \\
\hline
\end{tabular}

Tabla 4.50: Valores-test sobre las componentes principales de Competencias adquiridas en la Universidad. Características de la carrera y Métodos de enseñanza - aprendizaje

petencias del universitario. El estudio posterior de relaciones simultáneas entre distintas variables se realiza tanto en los análisis de interacción y confusión precedentes como en la sección posterior sobre modelos estructurales.

La Figura 4.18 muestra la posición de las variables pertenecientes al contexto académico en el espacio factorial rotado de los dos primeros factores competenciales. Por razones de simplicidad se han omitido en este capítulo los resultados correspondientes a los factores restantes, aunque sí se encuentran disponibles en el Anexo D. Esta forma de proceder se ha aplicado en todas las etapas de este apartado del análisis.

En este gráfico, se han señalado en azul las categorías cuya posición respecto al primer factor ha resultado significativa. Asimismo se han marcado en rojo aquellas con posiciones significativas en el segundo factor, mientras que aquellas ocupan posiciones significativas en ambos factores se han señalado en morado.

El hecho de que se considere que los estudios cursados tienen Prestigio y que requieran un gran Esfuerzo para superarlos es la característica más relevante en cuanto al desarrollo del Pensamiento Metacognitivo. Sin embargo, estos factores parecen ser contraproducentes en cuanto al desarrollo de competencias relacionadas con la Comunicación. Los estudios con Enfoques Generalistas contribuyen igualmente al desarrollo del Pensamiento Metacognitivo aunque esta relación es menos significativa como puede observarse tanto en su posición, cercana al eje de ordenadas como en su valor test, inferior a los correspondientes a las características del Prestigio y el Esfuerzo. 
Respecto a los Métodos de enseñanza - aprendizaje, los más relacionados con el desarrollo del Pensamiento Metacognitivo han resultado ser las Teorías, conceptos y paradigmas y el Aprendizaje basado en problemas mientras que los Trabajos en grupo, las Prácticas en empresa y la Asistencia a clase parecen estar vinculados con el desarrollo del Liderazgo.

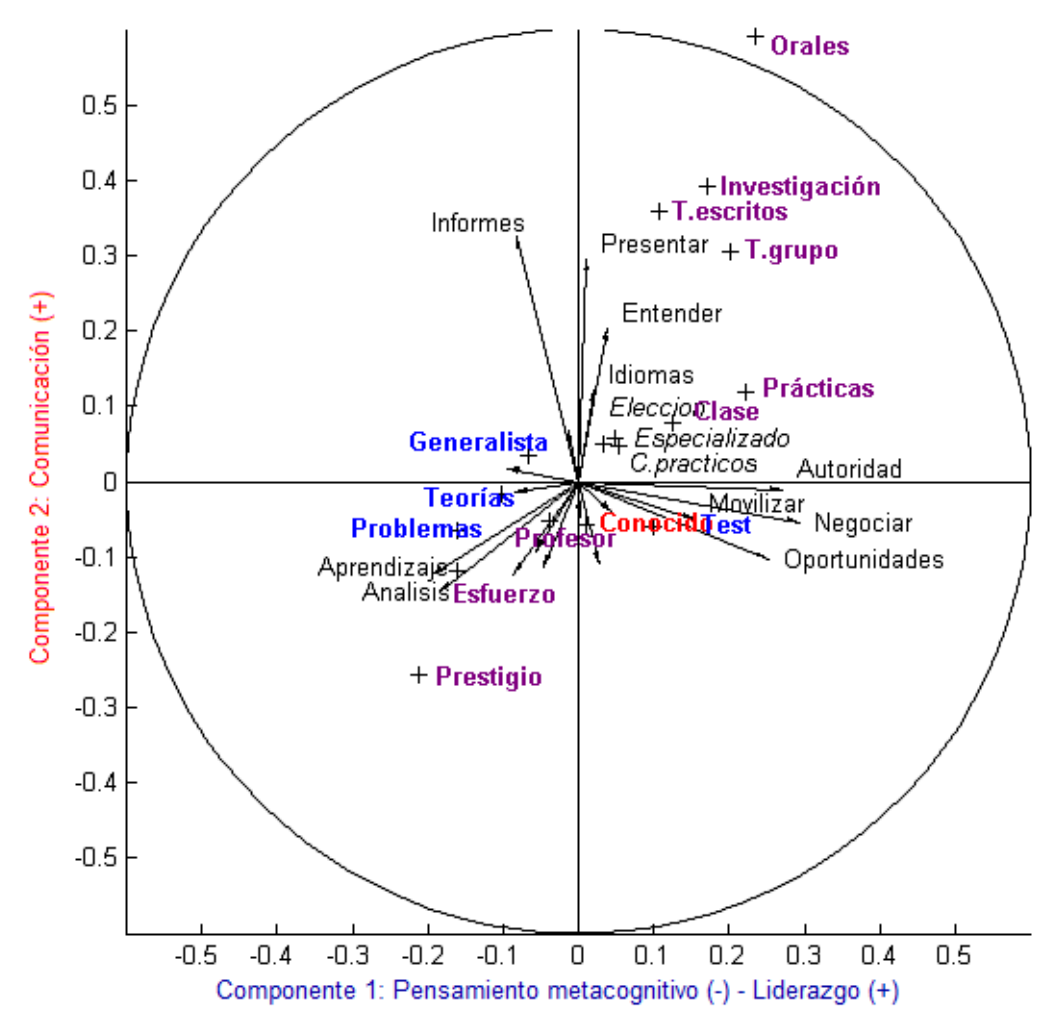

Figura 4.18: Representación simultánea sobre las componentes principales 1 y 2 de Competencias adquiridas en la Universidad. Características de los estudios y Métodos de enseñanza - aprendizaje

Asimismo en la Tabla 4.51 se muestran los valores - test correspondientes a las variables pertenecientes al contexto personal del estudiante. Al igual que antes, se han sombreado en gris claro los valores superiores 1.96 mientras que los valores inferiores a -1.96 se han marcado en un tono de gris más oscuro.

En la Figura 4.19 se observa que la Realización de prácticas en empresa 


\begin{tabular}{|l|cccccc|}
\hline & $\mathrm{F} 1$ & $\mathrm{~F} 2$ & $\mathrm{~F} 3$ & $\mathrm{~F} 4$ & $\mathrm{~F} 5$ & $\mathrm{~F} 6$ \\
\hline Trabajo durante los estudios & 1.72 & 0.25 & -2.29 & -3.66 & 0.00 & -1.71 \\
\hline Prácticas en empresa & 6.31 & 1.43 & 1.59 & 11.46 & -1.98 & -0.62 \\
\hline Aprobado & 0.80 & -5.00 & 0.16 & -3.18 & 2.13 & -2.52 \\
Notable & -0.30 & 4.52 & 0.31 & 4.52 & -0.05 & 3.14 \\
Sobresaliente & -2.31 & 2.54 & 0.04 & -0.79 & -2.39 & -0.63 \\
\hline i15 h & 1.89 & -1.41 & -3.07 & -0.24 & -0.91 & -1.56 \\
$15-30 \mathrm{~h}$ & 1.74 & 1.50 & -5.31 & -0.96 & 0.50 & 0.84 \\
$30-45 \mathrm{~h}$ & 3.71 & -2.76 & -6.00 & -0.47 & -1.78 & -3.05 \\
¿45 h & 2.07 & 1.78 & -6.30 & -1.14 & 0.59 & 1.00 \\
\hline Estancia en el extranjero & -2.95 & 2.69 & -4.00 & -7.82 & -3.46 & -7.03 \\
\hline Hombre & -2.38 & -1.75 & -1.74 & -3.52 & 2.26 & -3.24 \\
Mujer & 3.94 & 3.18 & 2.93 & 6.38 & -4.17 & 6.15 \\
\hline $26-30$ años & -0.09 & 4.41 & 1.67 & 7.81 & 0.05 & 1.80 \\
$30-35$ años & -0.93 & -4.69 & -0.14 & -6.61 & 1.12 & -2.07 \\
¿35 años & 2.07 & -1.09 & -2.96 & -4.84 & -0.37 & 0.58 \\
\hline Solo & -0.54 & -0.31 & -0.29 & -0.71 & -0.22 & 0.13 \\
Con padres & -0.70 & -0.51 & 1.15 & -0.24 & 0.71 & -0.98 \\
Con pareja & 1.40 & 1.26 & -0.55 & 1.74 & -0.24 & 0.64 \\
Otros & -0.13 & -0.13 & -0.10 & -0.09 & -0.11 & -0.03 \\
\hline Tiene hijos & 1.01 & -0.75 & 0.86 & -0.74 & 1.44 & 1.81 \\
\hline Padre con Ed. Superior & -5.01 & -1.38 & 0.02 & -5.27 & -1.81 & -0.09 \\
Madre con Ed. Superior & -4.37 & -2.67 & -0.51 & -6.47 & -0.39 & 1.01 \\
\hline
\end{tabular}

Tabla 4.51: Valores-test sobre las componentes principales de Competencias adquiridas en la Universidad. Gestión del tiempo y Características personales

parece tener una relación directa con el desarrollo de las competencias de Liderazgo así como el hecho de dedicar Más de 30 horas semanales a los estudios. Por otro lado otros factores como las Estancias en el extranjero parecen estar relacionados con el desarrollo del Pensamiento metacognitivo.

Asimismo una nota promedio de Notable en los estudios universitarios, o dicho de otra forma, las buenas calificaciones en la universidad y las Estancias en el extranjero están relacionados con la capacidad de Comunicación de los graduados. No ocurre lo mismo en los casos en que los graduados aprueban con un simple Aprobado.

Por otro lado, aunque el Sexo parece ser un factor determinante en cuanto al desarrollo de competencias es necesario revisar esta relación ya que el comportamiento de esta variable se encuentra confundido con la elección del área de estudio. Este argumento se ha revisado en los análisis de interacción y confusión entre variables, concluyendo que la elección del área de estudio que realizan hombres y mujeres es lo que realmente condiciona las competencias que unos y otros adquieren en la universidad.

En cuanto a las competencias de comunicación también parece estar relaciondo con el Sexo y la Edad puesto que quienes afirman haberla adquirido son las mujeres y los graduados con determinada Edad en el momento de la entrevista (cinco años después de la graduación). Igualmente se intuye que 
esta relación está confundida o interactúa con el efecto del Área de estudio puesto que un alto porcentaje de mujeres de la muestra cursaron estudios vinculados al desarrollo de competencias de Comunicación. Como se mostrará más adelante estos estudios corresponden a Educación, Humanidades, Ciencias Sociales y Derecho.

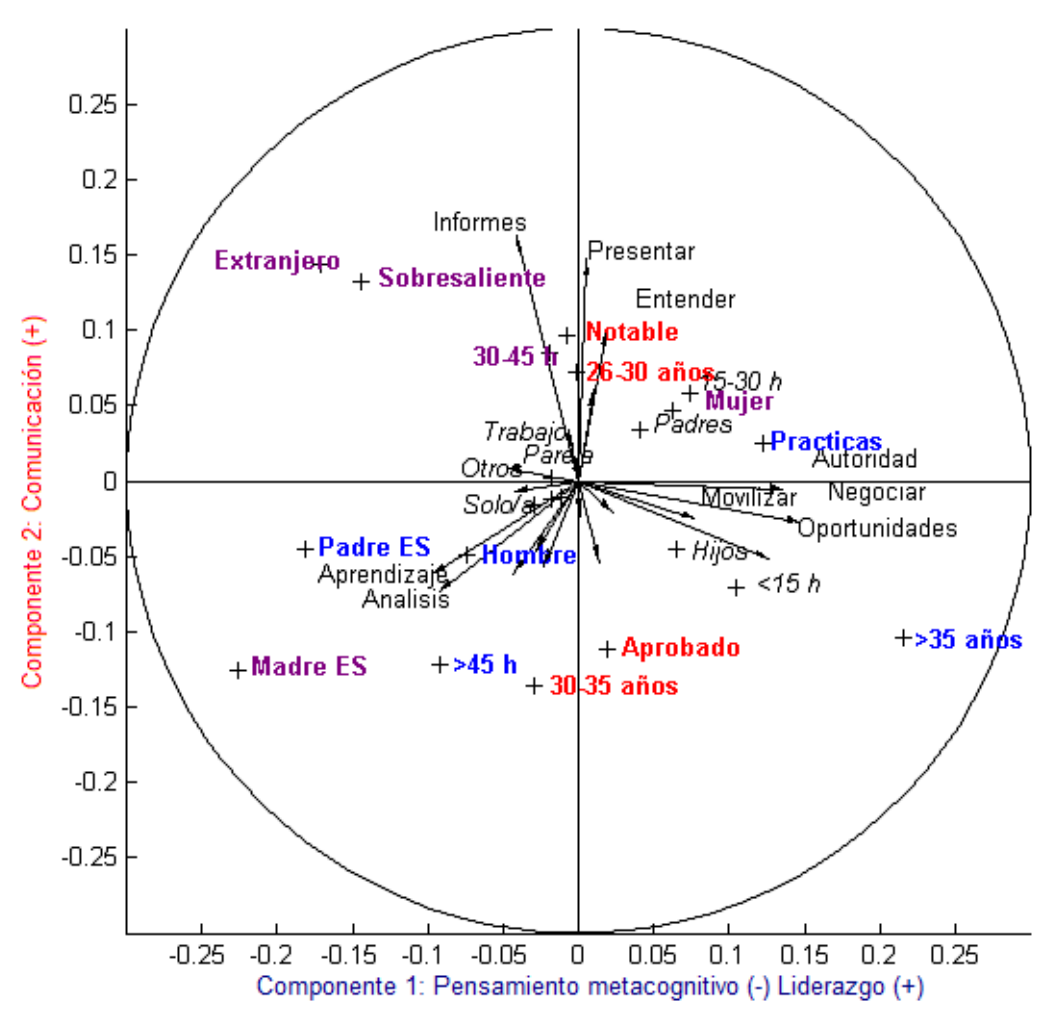

Figura 4.19: Representación simultánea sobre las componentes principales 1 y 2 de Competencias adquiridas en la Universidad. Gestión del tiempo y Características personales

Por último la Tabla 4.52 muestra los valores - test correspondientes al Área y Nivel de estudios de los titulados encuestados respecto a los factores competenciales. En este aspecto hay que recordar que, puesto que la encuesta se aplicó en el año 2005 cuando todavía no se habían implantado los estudios de Grado y Máster en la Universidad, la categoría Ciclo Corto incluye las antiguas Diplomaturas e Ingenierías Técnicas y Arquitectura Técnica mientras que la categoría Ciclo Largo abarca las Licenciaturas así como las Ingenierías Superiores y Arquitectura Superior, tal y como se detalla en el 
Anexo B.

\begin{tabular}{|l|cccccc|}
\hline & F1 & F2 & F3 & F4 & F5 & F6 \\
\hline Educación - Ciclo Largo & 1.1 & 5.26 & 2.48 & 7.32 & 2.12 & 3.26 \\
Educación - Ciclo Corto & 8.14 & 7.7 & -1.19 & 16.51 & -1.55 & -2.71 \\
\hline Humanidades & -1.87 & 11.78 & -5.79 & -5.46 & -2.41 & -6.16 \\
\hline Ciencias Sociales - Ciclo Largo & 3.94 & 5.22 & -1.27 & 6.84 & 4.93 & 7.66 \\
Ciencias Sociales - Ciclo Corto & 6.12 & 2.64 & -0.38 & 8.29 & 1.39 & 4.83 \\
\hline Economía y empresa - Ciclo Largo & -1.26 & -4.36 & -0.23 & -4.01 & -3.94 & 0.3 \\
Economía y empresa - Ciclo Corto & 6.4 & -2.79 & -0.55 & -0.27 & -3.01 & -2.15 \\
\hline Derecho & 0.44 & 5.06 & -0.46 & -12.42 & -2.75 & 12.74 \\
\hline Técnicas - Ciclo Largo & -10.55 & -8.32 & 3.53 & -8.89 & 7.09 & -6.62 \\
Técnicas - Ciclo Corto & -4.49 & -6.99 & 3.48 & -3.19 & 4.04 & -7.62 \\
\hline Salud - Ciclo Largo & -1.25 & -3.97 & -0.34 & -3.15 & -7.85 & 1.76 \\
Salud - Ciclo Corto & 3.23 & -1.46 & 0.26 & 6.07 & -4.23 & 3.73 \\
\hline Ciencias & -7.49 & -5.2 & 1.46 & -2.45 & 4.63 & 0.39 \\
\hline
\end{tabular}

Tabla 4.52: Valores-test sobre las componentes principales de Competencias adquiridas en la Universidad. Área y Nivel de estudios

En la Figura 4.20 se observa que las áreas de estudio más relacionadas con el desarrollo del Liderazgo son los Ciclos Cortos de Educación, Economía y Empresa, Ciencias Sociales y Salud e igualmente el Ciclo Largo de Ciencias Sociales. Por el contrario los graduados en las áreas de Técnicas o Ciencias muestran mayor tendencia a desarrollar el Pensamiento Metacognitivo durante sus estudios en la Universidad.

Respecto a las competencias de Comunicación los graduados en Humanidades, Educación, Ciencias Sociales y Salud desarrollan en mayor medida esta capacidad durante los estudios en la Universidad, al contrario de lo que ocurre con los graduados en Técnicas, Ciencias, Ciclos Largos de Salud y Economía y Empresa. 


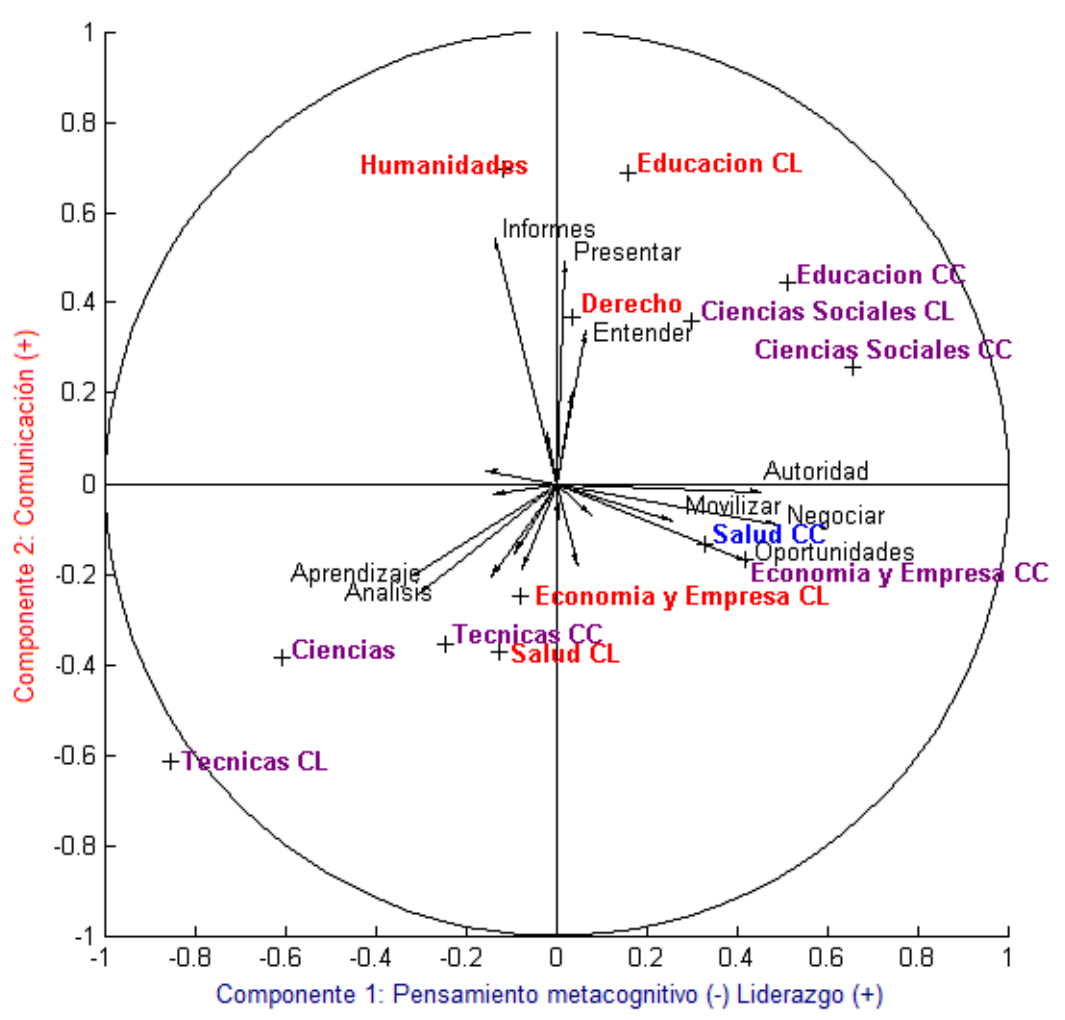

Figura 4.20: Representación simultánea sobre las componentes principales 1 y 2 de Competencias adquiridas en la Universidad. Área y Nivel de estudios

\section{Relaciones entre factores metodológicos y competenciales}

El objetivo de este apartado es analizar la relación entre las dimensiones identificadas entre las variables pertenecientes al contexto académico, y los factores de Competencias adquiridas en la Universidad. Ambos conjuntos de factores proceden de análisis de componentes principales realizados previamente. De esta forma, se obtendrá una perspectiva más sintética de la relación entre el empleo de distintas metodologías de enseñanza - aprendizaje y la adquisición de competencias en la Universidad. La diferencia entre ellos es que el conjunto de factores metodológicos se obtiene a partir de las variables clasificadas como Características de los estudios y Métodos de enseñanza - aprendizaje de los estudiantes mientras que los factores competenciales proceden de las valoraciones de los titulados a la contribución de la Universidad a cada una de las 19 competencias listadas en el cuestionario. 
Tanto en el caso de los factores metodológicos como en los factores competenciales, es posible calcular las puntuaciones factoriales correspondientes a cada encuestado en el nuevo plano factorial. Concretamente el programa estadístico SPSS (versión 16) ofrece tres posibilidades para la generación de dichas puntuaciones. En primer lugar, el Método por Regresión obtiene puntuaciones que pueden estar correlacionadas entre distintos factores, aun en el caso de asumir ortogonalidad. Del mismo modo tampoco la varianza de dichas puntuaciones alcanza el valor de la unidad. Por otro lado el Método de Anderson - Rubin aporta puntuaciones incorrelacionadas y con varianza igual a la unidad, mientras que el Método de Barlett aplica el método de máxima verosimilitud, partiendo del supuesto de que los factores comunes se distribuyen de forma normal. En principio, el método seleccionado para la generación de las puntuaciones factoriales debería ser el método de Anderson - Rubin, ya que aporta puntuaciones incorrelacionadas más acordes con la rotación ortogonal Varimax aplicada en la extracción previa de componentes principales. No obstante, precisamente gracias a que se ha empleado este método concreto para la extracción de factores, es posible generar estas puntuaciones de forma exacta, de forma que coinciden independientemente del método empleado para obtenerlas.

Una vez obtenidas las puntuaciones factoriales de un conjunto de factores, su proyección en el espacio dimensional determinado por el conjunto alternativo de factores se reduce a la proyección de las puntuaciones factoriales como variables continuas ilustrativas en el espacio definido por otro conjunto de variables activas.

En primer lugar, se proyectan como variables continuas ilustrativas las puntuaciones factoriales obtenidas en la extracción de las componentes principales relativas al contexto académico, en el espacio de 6 dimensiones definido por las componentes principales de las competencias adquiridas en la Universidad.

Para ello es necesario tipificar las variables continuas que van a proyectarse sobre el espacio de 6 dimensiones de competencias. Sin embargo, puesto que en este caso las variables continuas son puntuaciones factoriales procedentes de otro análisis de componentes principales no es necesario realizar esta transformación, ya que por su propia definición tienen media igual a cero y varianza igual a la unidad.

Por tanto, las coordenadas de las puntuaciones factoriales de los factores metodológicos sobre el espacio de los factores competenciales se calculan como: 


$$
\Psi_{M e t-C o m p ; 5 x 6}^{r o t}=\Psi_{M e t ; 5 x 5474}^{\prime} \cdot \Psi_{C o m p ; 5474 x 6}
$$

De este modo se obtienen las coordenadas de los factores metodológicos en cada uno de los factores competenciales.

\begin{tabular}{|l|cccccc|}
\hline & $\begin{array}{c}\text { Liderazgo y } \\
\text { Pensamiento } \\
\text { metacognitivo }\end{array}$ & \multicolumn{3}{c}{$\begin{array}{c}\text { Comunicación } \\
\text { Organización Cooperación } \\
\text { Conocimientos }\end{array}$} & $\begin{array}{c}\text { Innovación y Instrumental } \\
\text { Conocimientos }\end{array}$ \\
\hline Estudio y T. en grupo & 0.40 & 0.80 & 0.30 & 0.70 & 0.13 & -0.03 \\
Especialización & 0.31 & -0.25 & 0.06 & 0.41 & 0.46 & -0.69 \\
C. prác. y Prác. ext. & 0.11 & -0.16 & -0.06 & 0.01 & 0.37 & -0.33 \\
Esfuerzo y prestigio & -0.66 & -0.52 & 0.95 & -0.55 & 0.79 & -0.51 \\
C. Teóricas & -0.55 & -0.07 & 0.09 & -0.19 & 0.15 & 0.40 \\
\hline
\end{tabular}

Tabla 4.53: Proyección de las componentes de Características de la carrera y Métodos de enseñanza - aprendizaje sobre las componentes de Competencias adquiridas en la Universidad

A partir de dichas coordenadas, y aplicando la expresión para el cálculo de los valores - test empleada anteriormente se obtienen los valores - test correspondientes a las proyecciones de las puntuaciones factoriales de las dimensiones del contexto académico sobre el espacio de los factores competenciales.

\begin{tabular}{|l|cccccc|}
\hline & $\begin{array}{c}\text { Liderazgo y } \\
\text { Pensamiento } \\
\text { metacognitivo }\end{array}$ & \multicolumn{3}{c}{$\begin{array}{c}\text { Comunicación } \\
\text { Organización Cooperación } \\
\text { Conocimientos }\end{array}$} & & $\begin{array}{c}\text { Innovación y Instrumental } \\
\text { Conocimientos }\end{array}$ \\
\hline Estudio y T. en grupo & 29.08 & 58.43 & 22.02 & 50.98 & 9.45 & -2.26 \\
Especialización & 22.50 & -17.98 & 4.43 & 30.37 & 33.69 & -50.50 \\
C. prác. y Prác. ext. & 7.86 & -11.59 & -4.28 & 0.96 & 26.72 & -24.33 \\
Esfuerzo y prestigio & -48.14 & -38.38 & 69.33 & -40.57 & 57.62 & -37.08 \\
C.Teóricas & -40.50 & -5.17 & 6.47 & -14.22 & 10.62 & 29.14 \\
\hline
\end{tabular}

Tabla 4.54: Valores-test de las proyecciones de Características de la carrera y Métodos de enseñanza - aprendizaje sobre las componentes de Competencias adquiridas en la Universidad

$\mathrm{Al}$ igual que en el apartado anterior, se mostrarán únicamente los gráficos relativos a las dos primeras componentes principales, en este caso extraídas sobre las competencias. Así, se ha mantenido la leyenda de colores definida para el caso anterior, según la cual el morado señala las categorías en posiciones significativas respecto a ambos factores. En la Figura 4.21 se observa que todas las categorías están señaladas con este color y por tanto las posiciones que ocupan son significativas en todos los casos respecto a ambos factores. En este gráfico se observa que los métodos basados en el Estudio y trabajo en grupo se asocian en gran medida con la adquisición de competencias de Comunicación, pero ocurre exactamente lo contrario con los estudios en los 
que los alumnos perciben que Se requiere un gran esfuerzo para aprobar y que Su carrera tiene prestigio académico. Ambas características se vinculan con el desarrollo del Pensamiento metacognitivo, al igual que ocurre con las metodologías tradicionales basadas en Clases Teóricas. Por el contrario otras características como la especialización de los estudios así como su enfoque hacia el Estudio y trabajo en grupo en las metodologías docentes, muestran mayor relación con la adquisición de competencias de Liderazgo.

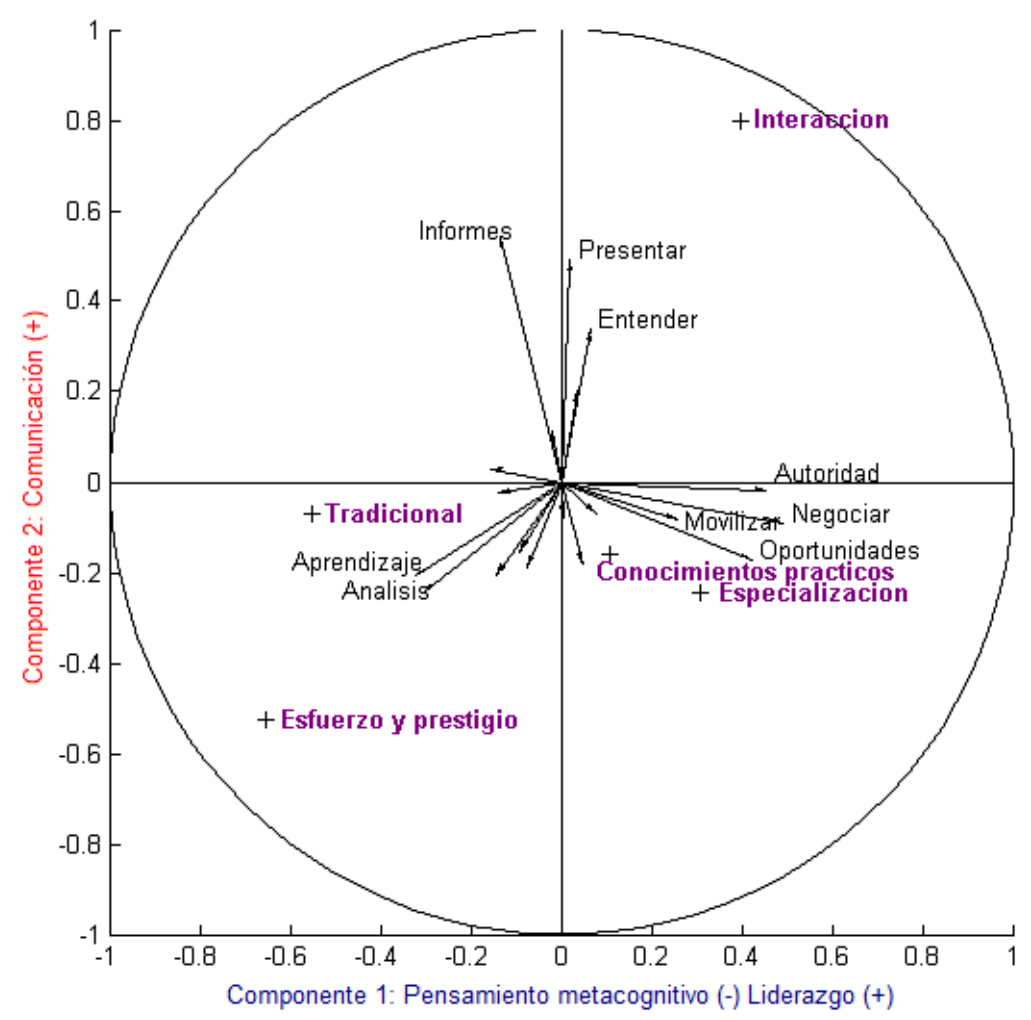

Figura 4.21: Representación simultánea de las componentes Características de la carrera y Métodos de enseñanza - aprendizaje sobre las componentes 1 y 2 de Competencias adquiridas en la Universidad

Las mismas conclusiones pueden extraerse desde otro punto de vista planteado a partir de las posiciones que ocupan los factores competenciales adquiridos en la Universidad sobre el plano factorial de las dimensiones del contexto académico. En este caso las variables proyectadas son las puntuaciones factoriales obtenidas mediante análisis de componentes principales en las variables de competencias. El espacio sobre el que se proyectan dichas 
variables son las componentes relativas a las Características de los estudios y los Métodos de enseñanza - aprendizaje empleados en la Universidad. El cálculo de las coordenadas aplicado a este nuevo conjunto de dimensiones proyectadas corresponde a la expresión:

$$
\Psi_{\text {Comp-Met } ; 6 x 5}^{r o t}=\Psi_{C o m p ; 6 x 5474}^{\prime} \cdot \Psi_{M e t ; 5474 x 5}
$$

De esta forma se obtienen las coordenadas de los factores competenciales proyectados en el espacio de 5 dimensiones referido al contexto académico del estudiante:

\begin{tabular}{|c|c|c|c|c|c|}
\hline & $\begin{array}{c}\text { Estudio y } \\
\text { trabajo } \\
\text { en grupo }\end{array}$ & Especialización & $\begin{array}{l}\text { Clases } \\
\text { prácticas }\end{array}$ & $\begin{array}{c}\text { Esfuerzo y } \\
\text { prestigio }\end{array}$ & $\begin{array}{l}\text { Clases } \\
\text { teóricas }\end{array}$ \\
\hline Liderazgo - Pensamiento metacognitivo & 0.25 & 0.35 & 0.30 & -0.48 & -1.36 \\
\hline Comunicación & 0.72 & -0.40 & -0.63 & -0.55 & -0.25 \\
\hline Organización personal -Conocimientos & 0.11 & 0.04 & -0.09 & 0.39 & 0.12 \\
\hline Cooperación & 0.69 & 0.75 & 0.06 & -0.64 & -0.76 \\
\hline Innovación - Conocimientos & 0.05 & 0.29 & 0.56 & 0.32 & 0.20 \\
\hline Instrumental & -0.01 & -0.45 & -0.53 & -0.21 & 0.56 \\
\hline
\end{tabular}

Tabla 4.55: Proyección de componentes del Competencias adquiridas en la Universidad sobre las componentes de Características de la carrera y Métodos de enseñanza - aprendizaje.

Nuevamente, y aplicando la expresión anterior para el cálculo de los valores - test, se han obtenido dichos valores con el fin de evaluar el nivel de significación de la posición que cada variable proyectada ocupa sobre los planos factoriales.

\begin{tabular}{|l|ccccc|}
\hline & \multicolumn{3}{|l}{$\begin{array}{l}\text { Estudio y Especialización } \\
\text { trabajo } \\
\text { en grupo }\end{array}$} & & Clases \\
prácticas & $\begin{array}{c}\text { Esfuerzo y } \\
\text { prestigio }\end{array}$ & Claseóricas \\
\hline Liderazgo - Pensamiento metacognitivo & 18.26 & 25.60 & 21.86 & -35.12 & -99.86 \\
Comunicación & 52.37 & -29.22 & -46.00 & -39.97 & -18.21 \\
Organización personal -Conocimientos & 7.77 & 2.83 & -6.69 & 28.41 & 8.96 \\
Cooperación & 50.56 & 54.59 & 4.22 & -46.74 & -55.37 \\
Innovación - Conocimientos & 3.30 & 21.28 & 41.24 & 23.33 & 14.53 \\
Instrumental & -0.81 & -32.81 & -38.62 & -15.44 & 41.02 \\
\hline
\end{tabular}

Tabla 4.56: Valores-test de la proyección de las componentes de Competencias adquiridas en la Universidad sobre las componentes de Características de la carrera y Métodos de enseñanza - aprendizaje

De forma coherente con los resultados anteriores es fácil observar en la Figura 4.22 que existe relación entre la Especialización de los conocimientos y la adquisición de competencias de Liderazgo. Asimismo, dicha especialización no produce el mismo efecto en cuanto a las competencias de Comunicación, lo que sí ocurre con el empleo de metodologías basadas en el Estudio y trabajo en grupo. Adicionalmente este gráfico permite identificar 
nuevas relaciones como las establecidas entre la Especialización de los conocimientos y las competencias de Cooperación, Innovación e Instrumentales. Estas últimas competencias se encuentran ubicadas en el sector inferior del gráfico pero se considera que están relacionadas con la Especialización de conocimientos al estar expresadas en términos negativos.

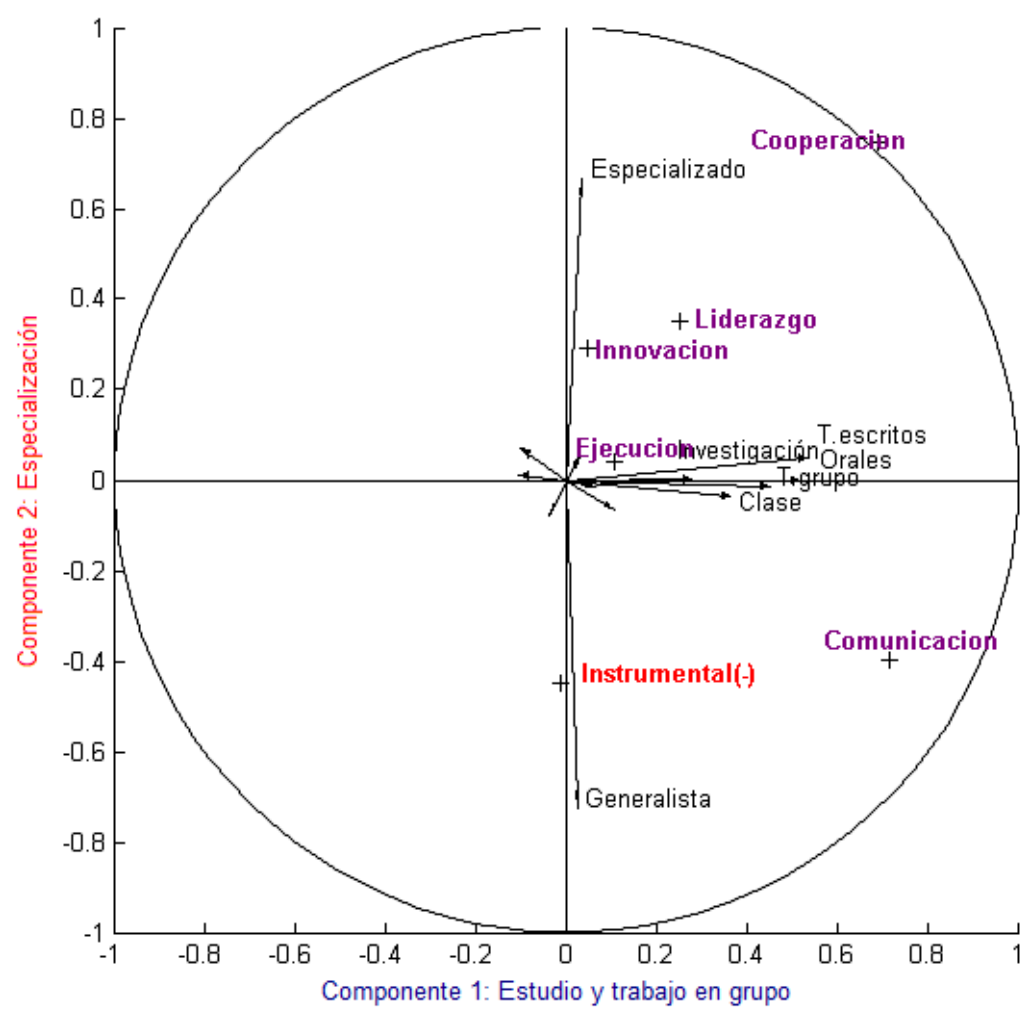

Figura 4.22: Representación simultánea de las componentes de Competencias adquiridas en la Universidad sobre las componentes 1 y 2 de Características de la carrera y Métodos de enseñanza - aprendizaje

\section{Relaciones entre variables dependientes y factores competenciales}

El objetivo de este apartado es analizar las relaciones que se establecen entre los factores de competencias adquiridos en la Universidad y dos variables pertenecientes al contexto académico, no independientes entre sí. Se trata por tanto de una aplicación del concepto del valor - test a la evaluación de relaciones de dependencia parcial respecto a los factores competenciales. 
Esta aplicación supone una ampliación de las posibilidades de análisis ofrecidas por el valor - test, ya que permite investigar la relación establecida entre los factores competenciales y un conjunto de variables no independientes entre sí.

No se trata en este punto de realizar un análisis exhaustivo de todas las posibles relaciones entre variables contextuales y factores competenciales, sino de verificar la adecuación de esta aplicación a los objetivos planteados. Por ello, y por razones de simplicidad, se muestran únicamente los resultados correspondientes a las relaciones entre factores competenciales y los siguientes pares de variables:

- Esfuerzo requerido para aprobar (C1) y Prestigio de los estudios (C6): Ambas variables ocupan posiciones significativas en el plano factorial y se encuentran vinculadas mediante una fuerte asociación positiva

- El profesor como principal fuente de información (V7) y Participación en proyectos de investigación (V3): Ambas variables ocupan posiciones significativas en el plano factorial y se encuentran vinculadas mediante una fuerte asociación negativa

Con el fin de analizar el comportamiento de ambos pares de variables, para cada uno de ellos se crea una nueva variable que contiene todas las combinaciones posibles en las opciones de respuesta:

Esfuerzo requerido para aprobar (C1) y Prestigio de los estudios (C6):

1. Esfuerzo requerido para aprobar (Sí) - Prestigio de los estudios (Sí)

2. Esfuerzo requerido para aprobar (Sí) - Prestigio de los estudios (No)

3. Esfuerzo requerido para aprobar (No) - Prestigio de los estudios (Sí)

4. Esfuerzo requerido para aprobar (No) - Prestigio de los estudios (No)

El profesor como principal fuente de información (V7) y Participación en proyectos de investigación (V3):

1. El profesor como principal fuente de información (Sí) - Participación en proyectos de investigación (Sí)

2. El profesor como principal fuente de información (Sí) - Participación en proyectos de investigación (No)

3. El profesor como principal fuente de información (No) - Participación en proyectos de investigación (Sí)

4. El profesor como principal fuente de información (No) - Participación en proyectos de investigación (No)

Seguidamente se desagrega la matriz de puntuaciones tipificada en 4 submatrices correspondientes a cada combinación de respuestas y se calculan las puntuaciones promedio de cada una de las 19 competencias listadas en 
el cuestionario. De esta forma se obtiene una matriz $\bar{\Psi}_{4 x 6}^{r o t}=\bar{\Psi}_{4 x 19} \cdot u_{j \alpha}$.

Las coordenadas obtenidas mediante esta expresión se muestran en las Tablas 4.57 y 4.58 , junto con las coordenadas de las categorías originales, antes de la combinación.

\begin{tabular}{|l|cccccc|}
\hline & F1 & F2 & F3 & F4 & F5 & F6 \\
\hline Sí Esfuerzo - Sí Prestigio & -0.285 & -0.296 & 0.092 & -0.343 & 0.040 & -0.074 \\
Sí Esfuerzo - No Prestigio & -0.034 & 0.058 & 0.083 & 0.058 & 0.023 & 0.042 \\
No Esfuerzo - Sí Prestigio & 0.125 & -0.079 & -0.120 & -0.048 & -0.001 & 0.045 \\
No Esfuerzo - No Prestigio & 0.222 & 0.195 & -0.112 & 0.225 & -0.047 & 0.017 \\
\hline Si Esfuerzo & -0.160 & -0.120 & 0.087 & -0.144 & 0.032 & -0.016 \\
No Esfuerzo & 0.208 & 0.155 & -0.113 & 0.186 & -0.041 & 0.021 \\
Si Prestigio & -0.211 & -0.257 & 0.054 & -0.290 & 0.032 & -0.053 \\
No Prestigio & 0.112 & 0.136 & -0.028 & 0.154 & -0.017 & 0.028 \\
\hline
\end{tabular}

Tabla 4.57: Coordenadas sobre las componentes principales de Competencias adquiridas en la Universidad. Asociación de En tu carrera había que trabajar mucho para aprobar y Tu carrera era una carrera con prestigio académico.

\begin{tabular}{|l|cccccc|}
\hline & F1 & F2 & F3 & F4 & F5 & F6 \\
\hline Sí Profesor - Sí Investigación & 0.056 & 0.276 & 0.044 & 0.256 & 0.182 & -0.079 \\
Sí Profesor - No Investigación & -0.087 & -0.105 & -0.009 & -0.055 & -0.006 & 0.028 \\
No Profesor - Sí Investigación & 0.224 & 0.391 & 0.104 & 0.281 & 0.169 & -0.249 \\
No Profesor - No Investigación & 0.026 & -0.003 & -0.003 & 0.025 & 0.021 & -0.051 \\
\hline Si Profesor & -0.038 & -0.054 & -0.007 & -0.033 & -0.017 & 0.021 \\
No Profesor & 0.071 & 0.099 & 0.013 & 0.061 & 0.030 & -0.039 \\
Si Investigación & 0.170 & 0.392 & 0.085 & 0.291 & 0.172 & -0.134 \\
No Investigación & -0.016 & -0.037 & -0.008 & -0.028 & -0.016 & 0.013 \\
\hline
\end{tabular}

Tabla 4.58: Coordenadas sobre las componentes principales de Competencias adquiridas en la Universidad. Asociación de El profesor era la principal fuente de información y Proyectos de investigación

El cálculo de los valores - test se realiza a partir de estos valores de coordenadas, considerando igualmente el número de encuestados total $n$ y el número de encuestados pertenecientes a cada combinación de categorías de respuesta. De este modo se obtienen las Tablas 4.59 y 4.60 que muestra los valores - test correspondientes a estas combinaciones de respuesta. Manteniendo la leyenda definida para el apartado anterior se han sombreado en gris claro los valores - test superiores a 1.96 y en gris oscuro los inferiores a -1.96. 


\begin{tabular}{|l|cccccc|}
\hline & F1 & F2 & F3 & F4 & F5 & F6 \\
\hline Sí Esfuerzo - Sí Prestigio & -8.23 & -9.33 & 3.38 & -13.31 & 1.67 & -3.15 \\
Sí Esfuerzo - No Prestigio & -0.98 & 1.85 & 3.08 & 2.27 & 0.98 & 1.80 \\
No Esfuerzo - Sí Prestigio & -3.44 & -3.90 & 1.41 & -5.56 & 0.70 & -1.31 \\
No Esfuerzo - No Prestigio & -1.19 & 2.23 & 3.72 & 2.74 & 1.19 & 2.17 \\
\hline Si Esfuerzo & -8.31 & -6.79 & 5.78 & -10.02 & 2.38 & -1.25 \\
No Esfuerzo & 8.41 & 6.87 & -5.85 & 10.11 & -2.40 & 1.25 \\
Si Prestigio & -7.06 & -9.37 & 2.28 & -13.02 & 1.57 & -2.58 \\
No Prestigio & 7.03 & 9.34 & -2.26 & 12.96 & -1.57 & 2.56 \\
\hline
\end{tabular}

Tabla 4.59: Valores-test sobre las componentes principales de Competencias adquiridas en la Universidad. Asociación de En tu carrera había que trabajar mucho para aprobar y Tu carrera era una carrera con prestigio académico

\begin{tabular}{|l|cccccc|}
\hline & F1 & F2 & F3 & F4 & F5 & F6 \\
\hline Sí Profesor - Sí Investigación & 0.55 & 2.97 & 0.55 & 3.39 & 2.60 & -1.14 \\
Sí Profesor - No Investigación & -4.72 & -6.24 & -0.59 & -4.00 & -0.46 & 2.23 \\
No Profesor - Sí Investigación & 0.55 & 2.99 & 0.56 & 3.41 & 2.62 & -1.15 \\
No Profesor - No Investigación & -2.79 & -3.70 & -0.35 & -2.37 & -0.27 & 1.32 \\
\hline Si Profesor & -2.25 & -3.43 & -0.53 & -2.61 & -1.40 & 1.83 \\
No Profesor & 2.55 & 3.88 & 0.59 & 2.95 & 1.59 & -2.07 \\
Si Investigación & 2.38 & 6.00 & 1.51 & 5.48 & 3.50 & -2.77 \\
No Investigación & -2.43 & -6.09 & -1.54 & -5.56 & -3.55 & 2.82 \\
\hline
\end{tabular}

Tabla 4.60: Valores-test sobre las componentes principales de Competencias adquiridas en la Universidad. Asociación de El profesor era la principal fuente de información y Proyectos de investigación

En las Figuras 4.23 y 4.24 se han representado las posiciones de las distintas combinaciones de respuestas para cada par de variables asociadas en los dos primeros factores. Por razones de simplicidad las representaciones gráficas del resto de factores se han incluido en el Anexo D. Se ha mantenido la leyenda definida en el apartado anterior, según la cual el color azul señala las categorías con posiciones significativas respecto al primer factor, e igualmente ocurre con el color rojo y el segundo factor. El color morado por tanto indica que la categoría se encuentra en una posición significativa considerando ambos factores.

Asimismo se han representado las categorías originales de respuesta antes de la combinación. Es fácil observar que en ambos casos la unión mediante líneas de todas las categorías, combinadas y no combinadas, forman un paralelepípedo en cuyos extremos se encuentran las categorías originales no combinadas. Por el contrario las categorías combinadas se sitúan en puntos a lo largo de las líneas que unen las categorías originales correspondientes.

El hecho de que en ambos gráficos la unión de las distintas categorías formen líneas paralelas indica que existe independencia entre cada uno de los factores competenciales, y cada uno de los pares de variables asociados, ya sea positiva o negativamente. 


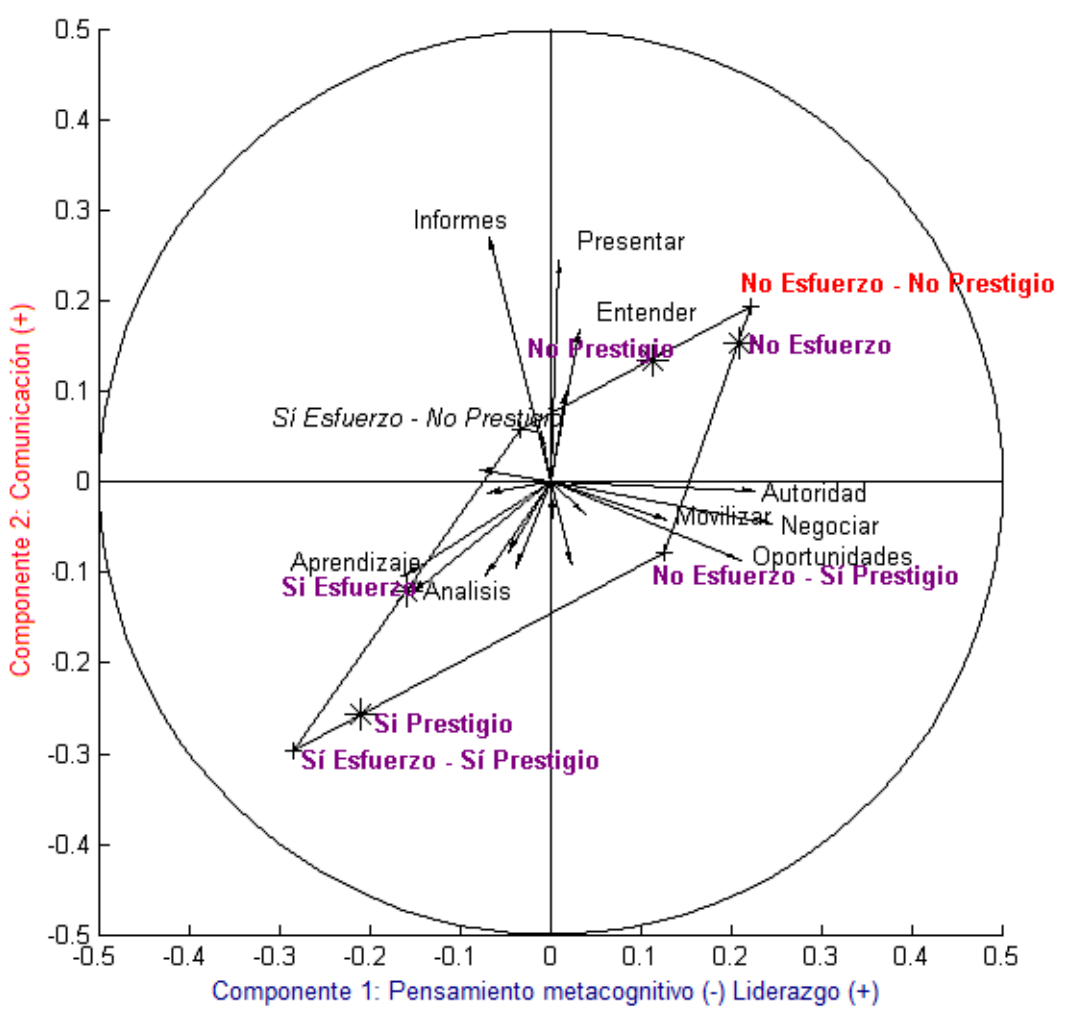

Figura 4.23: Representación simultánea sobre las componentes 1 y 2 de Competencias adquiridas en la Universidad. Asociación de En tu carrera había que trabajar mucho para aprobar y Tu carrera era una carrera con prestigio académico 


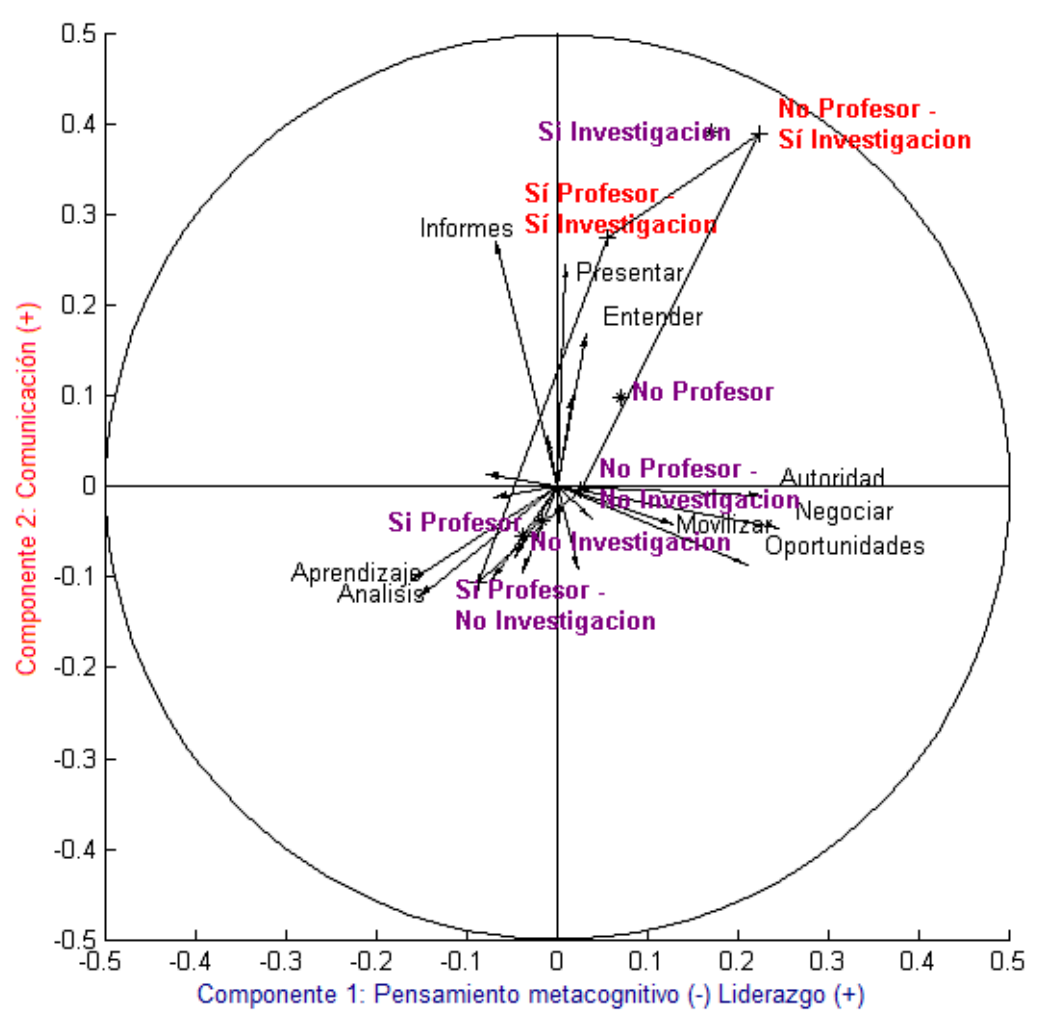

Figura 4.24: Representación simultánea sobre las componentes 1 y 2 de Competencias adquiridas en la Universidad. Asociación de El profesor era la principal fuente de información y Proyectos de investigación

Únicamente en el caso de las variables asociadas positivamente Esfuerzo para aprobar los estudios (C1) y Prestigio de los estudios (C6) se ha observado cierto nivel de dependencia respecto a los factores competenciales 5 y 6 - Conocimientos (-) - Innovación e Instrumental (-). En la Figura 4.25 se observa cómo la unión de las distintas categorías de respuesta combinadas condiciona el cruce de las líneas, indicando la presencia de relaciones de dependencia entre estos factores competenciales y las variables contextuales. No obstante, aunque se ha detectado la relación de dependencia parcial entre estas variables, hay que señalar que concretamente en este caso la magnitud de esta asociación es muy pequeña, como se observa en la Figura 4.25 cuya escala de los ejes se ha amplificado con el fin de observar con mayor detalle las direcciones de las líneas. En otras palabras, existen relaciones de dependencia parcial, en este caso con una intensidad pequeña por lo que las categorías tienden a ocupar posiciones cercanas al origen. De ahí la necesi- 
dad de amplificar la escala para apreciar el cruce de las líneas. De hecho, es fácil ver que por esta misma razón de cercanía al origen, ninguna de las categorías ocupa posiciones significativas respecto a ambos factores quinto y sexto, y de hecho, dos de las categorías combinadas ocupan posiciones no significativas respecto a ninguno de los factores.

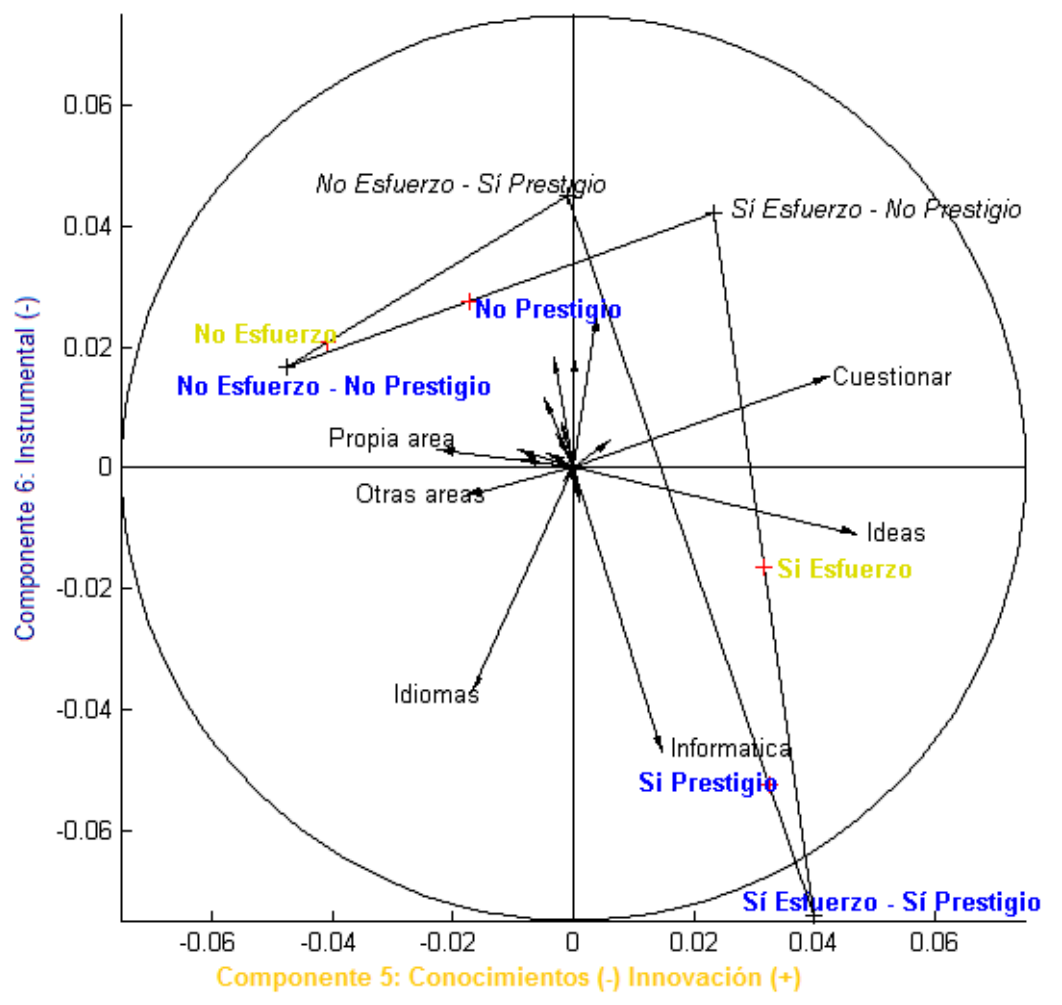

Figura 4.25: Representación simultánea sobre las componentes 5 y 6 de Competencias adquiridas en la Universidad. Asociación de En tu carrera había que trabajar mucho para aprobar y Tu carrera era una carrera con prestigio académico

Así, el empleo de valores - test ha permitido detectar la presencia de asociación entre variables de tipo dimensional y conjuntos de variables cualitativas relacionadas entre sí. Esta aplicación aporta un nuevo enfoque en el empleo de los valores - test como método de evaluación de la significación en las relaciones entre variables. 


\subsection{Resumen y conclusiones}

En este capítulo se ha estudiado cuál es la relación entre distintas variables pertenecientes a los contextos académico y personal de los estudiantes universitarios y las dimensiones principales de dos conjuntos de indicadores relativos a los Métodos de enseñanza - aprendizaje y las competencias adquiridas en la Universidad. Con este objetivo se han aplicado diversas técnicas de análisis de datos que aportan distintos enfoques al estudio de relaciones entre variables.

En primer lugar, la aplicación de análisis de componentes principales ha permitido reducir el conjunto inicial de once indicadores sobre Métodos de enseñanza - aprendizaje a cinco dimensiones: Estudio y trabajo en grupo, Especialización, Clases prácticas y prácticas externas, Esfuerzo y prestigio y Clases teóricas. Del mismo modo, previa transformación de las variables para la eliminación del factor tamaño, se han identificado seis dimensiones principales para el conjunto de diecinueve competencias adquiridas gracias a la contribución de los estudios universitarios. En este caso algunas de las dimensiones presentan un enfoque dual en el sentido de que permiten una doble interpretación debida a saturaciones de signo opuesto. Estas dimensiones se han denominado como Liderazgo - Pensamiento metacognitivo, Comunicación, Organización personal - Conocimientos, Cooperación, Innovación Conocimientos y Competencias Instrumentales (Conchado y Carot, 2011).

Por otro lado, los contrastes de independencia por pares revelan la presencia de numerosas relaciones de dependencia entre variables pertenecientes al mismo contexto, ya sea académico o personal. Por el contrario, se confirma la existencia de relaciones de independencia en la gran mayoría de los casos relativos a variables clasificadas en distintos contextos. En todos los casos el área de estudio actúa como variable mediadora de las relaciones entre variables, tanto a nivel global como a nivel parcial y condicional. En este aspecto, los contrastes condicionales sobre el riesgo ponderado y el cociente de ventajas común permiten describir con detalle la modificación del efecto introducida por esta variable. Los resultados de ambos contrastes muestran que en la mayoría de los casos la presencia del área de estudio implica una intensificación de la asociación entre variables, efecto que en principio no supone una distorsión substancial de las conclusiones pero que necesariamente debe ser tenido en consideración en el análisis de relaciones entre variables.

Respecto al contexto personal de los encuestados, la aplicación del análisis de correspondencias múltiples a las variables clasificadas en este grupo ha permitido identificar dos direcciones principales de inercia relativas al nivel socioeconómico familiar y el nivel de independencia económica y personal del encuestado. En este sentido cada una de las áreas de estudio se asocia 
con una determinada combinación de factores y circunstancias relativas a este entorno personal.

Por último, el empleo de los valores - test ha aportado una nueva perspectiva al estudio de las relaciones entre variables al introducir las dimensiones obtenidas mediante análisis de componentes principales como referencia para la proyección de variables en suplementario. De este modo, se han obtenido los valores - test correspondientes a las relaciones entre las dimensiones identificadas anteriormente y las variables pertenecientes a los contextos académico y personal de los encuestados. Adicionalmente se han ampliado las posibilidades de aplicación de esta técnica mediante el establecimiento de un paralelismo con los contrastes de independencia a nivel parcial y condicional. Esta aplicación de los valores - test para el análisis de la significatividad en las relaciones entre variables y factores latentes constituye una aportación muy interesante en el contexto de esta metodología como ampliación de sus posibilidades de empleo. 


\section{Capítulo 5}

\section{Modelización de los procesos de enseñanza - aprendizaje de competencias}

\subsection{Introducción}

El objetivo de este capítulo es modelizar mediante estructuras de covarianza los procesos de enseñanza - aprendizaje de competencias en Educación Superior. Estos procesos son evidentemente muy complejos puesto que engloban un gran y heterogéneo conjunto de circunstancias académicas y personales de los estudiantes. Por ello se ha segmentado en primera instancia el análisis de estos procesos mediante el estudio individual de los factores relativos a los Métodos de enseñanza - aprendizaje empleados en la Universidad, y por otro lado las Competencias adquiridas en la Universidad. Posteriormente se ha propuesto un modelo integrador de los efectos correspondientes a ambos subsistemas.

Asimismo el efecto del área de estudio se ha considerado mediante la segmentación de la muestra global en ocho submuestras correspondientes a cada una de las áreas de estudio definidas en el trabajo. Por otro lado se ha considerados que los factores relacionados con la Gestión del tiempo y las Características personales de los encuestados e encuentran vinculados a la elección del área de estudio, como se ha mostrado en el capítulo 4. Por ello, mediante la segmentación de la muestra en áreas de estudio, es posible analizar el efecto de dichos factores sobre los procesos de enseñanza - aprendizaje de forma indirecta.

Como resultado final de los análisis desarrollados en este capítulo se obtiene un modelo estadístico de estos procesos de enseñanza - aprendizaje en el ámbito universitario, aplicable a la práctica totalidad de las áreas de 
estudio y sin variaciones significativas en cuanto a las diferencias institucionales. No obstante, debe señalarse que en cualquier caso se trata de un modelo teórico cuya adecuación a la práctica debe tener en consideración los matices de la realidad de los contextos institucionales.

\subsection{Material y métodos}

El análisis con estructuras de covarianza, o ecuaciones estructurales, es una técnica multivariante que en la actualidad todavía se encuentra en investigación, sobre todo en lo referente a aplicaciones más avanzadas como son el análisis de datos longitudinales, el análisis de datos de muestras complejas y otros tipos de modelos. Sin embargo, las técnicas empleadas en este trabajo han sido estudiadas desde su inicio por un gran número de investigadores cuyas contribuciones han resultado muy signficativas para su avance.

En este trabajo se han empleado técnicas de Análisis Factorial Confirmatorio y Modelización causal. Asimismo se han estudiado los modelos validados mediante estos procedimientos desde el enfoque de la Invarianza Métrica y Estructural. Finalmente se han aplicado los fundamentos de los Modelos Multinivel con el fin de evaluar la adecuación de este tipo de modelos a los datos.

\subsubsection{Consideraciones generales}

\section{Tipologías de variables}

\section{Variables observables y factores latentes}

En los estudios del área de Ciencias Sociales, y concretamente en la gran mayoría de los desarrollados en Educación, es habitual plantear hipótesis acerca de determinados constructos teóricos que no pueden observarse directamente. Puesto que estas variables no pueden medirse, tampoco es posible observarlas de forma directa. Estas variables reciben el nombre de Variables o Factores Latentes. Habitualmente estas variables se miden a través de variables que sí son observables. Estas Variables Manifiestas tienen la función de actuar como indicadores del constructo subyacente a las variables. Así como las Variables Latentes también reciben el nombre de Variables No Observables y Variables No Medibles las variables manifiestas igualmente se denominan Variables Observables y Variables Medibles. 


\section{Variables exógenas y endógenas}

Se consideran Variables Exógenas aquellas variables que actúan como variables independientes del modelo. Su principal característica de dichas variables es que no es posible explicar su variabilidad a través del modelo, puesto que sus valores dependen de factores externos al mismo. Por el contrario, las Variables Endógenas cumplen la función de variables dependientes de modo que, al menos una parte de su variabilidad, puede ser explicada por medio del modelo.

\section{Tipologías de modelos}

\section{Modelo de medida y modelo estructural}

El Modelo de Medida describe las relaciones entre variables observables y las variables no observables y se centra en cuestiones como de qué forma y en qué medida las variables observables se vinculan a uno o varios factores latentes. Concretamente, se plantea cómo las variables observables pueden ser generadas a partir del constructo subyacente por lo que cobra gran importancia la magnitud de los coeficientes de regresión asociados a esta relación.

El Modelo Estructural describe las relaciones entre variables no observables y analiza el impacto de estas relaciones de forma directa e indirecta sobre los constructos latentes. Ambos modelos están relacionados y en la mayoría de los casos se recomienda validar en primer lugar el modelo de medida y a continuación el modelo estructural.

\section{Análisis factorial confirmatorio y Modelos causales}

El Análisis Factorial Confirmatorio constituye un caso particular de Análisis mediante Estructuras de Covarianzas. En el primer caso, el principal objetivo del análisis es contrastar un modelo de medida con los datos obtenidos en una muestra que, teóricamente, refleja fielmente las características de la población. Por ello, proporciona información sobre en qué medida los indicadores reflejan los factores latentes así como sobre la relación existente entre dichos factores. Asimismo analiza la magnitud de los errores de medida de los indicadores y aporta medidas de bondad de ajuste del modelo hipotetizado a los datos muestrales.

Las principales ventajas del análisis factorial confirmatorio son, por un lado la posibilidad de identificar los errores de medida de las variables y los errores asociados a los constructos latentes, y por otro lado la integración 
en un mismo procedimiento tanto la validación de las hipótesis teóricas subyacentes a los constructos latentes como la correspondiente a la medida de las variables observables.

Los Modelos Causales reciben otras denominaciones como Modelización con Estructuras de Covarianzas, Análisis de Variables Latentes y Análisis LISREL. En cualquier caso los modelos causales constituyen una metodología estadística, que incluye el Análisis Factorial Confirmatorio, basada en la aplicación de un enfoque confirmatorio al análisis de una teoría estructural sobre un determinado fenómeno (Byrne, 2006). Las relaciones propuestas en los modelos estructurales se basan en dos supuestos. En primer lugar la teoría es el punto de referencia a la hora de decidir la estructura del modelo o cualquier modificación en el mismo, como posibles exclusiones o inclusiones de variables. Asimismo en todos los casos se da por supuesto la naturaleza lineal de las relaciones, tanto en el modelo de medida como en el modelo estructural (Hair, Anderson, Tatham y Black, 2007).

Puesto que los modelos causales incluyen la metodología de análisis factorial confirmatorio, entre sus ventajas se encuentran las correspondientes a esta técnica estadística. Las ventajas específicamente características de los modelos causales incluyen el hecho de que se revelan relaciones causales y no únicamente medidas descriptivas de asociación o correlación entre las variables (Bollen, 1989). Asimismo permiten analizar este conjunto de relaciones causales de forma simultánea y exhaustiva entre las variables del modelo, incluyendo tanto variables observables como variables no observables. Por otro lado aporta un enfoque confirmatorio frente a los enfoques tradicionalmente exploratorios y descriptivos mediante los cuales no es posible la validación de hipótesis y aporta estimación específica de los errores de medida tanto de las variables observables como no observables.

\section{Modelos recursivos y no recursivos}

Los Modelos Recursivos incluyen relaciones de causalidad en una sola dirección mientras que los Modelos No Recursivos contienen relaciones de causalidad en ambas direcciones, al menos alguna de ellas relativa a la retroalimentación del proceso.

\section{Estrategias de modelización}

\section{Estrategia de modelización confirmatoria}

La Estrategia de Modelización Confirmatoria se fundamenta en la confirmación estricta de modelos basados en la teoría. Así, el primer paso que 
debe realizarse en cualquier procedimiento confirmatorio es proponer un modelo basado en la teoría y recoger adecuadamente los datos necesarios para la validación de dicha teoría. Tras la validación del modelo hipotetizado y el análisis de la bondad de ajuste se rechazará o aceptará el modelo inicial y no tendrán cabida nuevas modificaciones con el fin de mejorar el ajuste del modelo. Según Hair et al. (2007) esta estrategia posee cierto sesgo confirmatorio, es decir que existe una tendencia implícita hacia la confirmación del modelo. Sin embargo, el investigador debe ser consciente de que un buen ajuste del modelo no revela que sea la solución única, o incluso óptima, sino que se ha confirmado que es uno de los posibles modelos un ajuste aceptable.

\section{Estrategia de modelos rivales}

La Estrategia de Modelos Rivales se basa en la propuesta de diversos modelos alternativos al sugerido por la teoría. Cada uno de estos modelos alternativos se validan de forma individual y finalmente se escoge la alternativa que mejor represente los datos. Constituye la estrategia más rigurosa en el sentido de que identifica y constrasta diferentes modelos rivales que representan distintas relaciones estructurales hipotéticas procedentes de la teoría.

El concepto de Modelos Rivales engloba a los modelos equivalentes y los modelos anidados. Los Modelos Equivalentes poseen el mismo número de parámetros que el modelo original y el mismo nivel de ajuste pero distintas relaciones entre las variables. Estos modelos proporcionan un nuevo enfoque al desarrollo de modelos rivales y se ha demostrado que existen para cualquier modelo hipotetizado. Concretamente ningún modelo es único en el nivel de ajuste conseguido de forma que, para cualquier modelo, existe un número indeterminado de modelos alternativos con el mismo nivel de ajuste. En general, cuanto más complejo sea el modelo, mayor será el número de modelos equivalentes que podrán plantearse. El programa informático TETRAD examina las pautas de las relaciones entre variables, denominadas tetrads, y forma nuevos modelos rivales a partir de ellas. Por otro lado en la especificación de Modelos Anidados el número de constructos permanece constante pero es el número de relaciones estimadas el que varía.

\section{Estrategia de generación de nuevos modelos}

La Estrategia de Generación de Nuevos Modelos parte igualmente de un modelo basado la teoría, pero en caso de que dicho modelo hipotetizado sea rechazado, se proponen y contrastan las modificaciones necesarias para modificar y mejorar, si es posible, la bondad de ajuste del modelo. Así, posee un enfoque con carácter más exploratorio que las estrategias de modelización anteriores. Por tanto, aunque se base en un modelo especificado a partir de la teoría, el principal objetivo de esta estrategia es la mejora del modelo 
inicial mediante la introducción de nuevas modificaciones.

Según Jöreskog y Sörbom (1996), en caso de que el modelo inicialmente hipotetizado no obtenga un buen ajuste, no es habitual que la investigación concluya simplemente rechazando el modelo. Por esta razón es mucho más frecuente la estrategia de generación de nuevos modelos frente a la estrategia de modelización confirmatoria y de hecho la mayoría de las aplicaciones de esta metodología se basan en el este escenario. Sin embargo, aunque menos frecuentes, existen algunos trabajos realizados con la estrategia de modelos rivales y cada vez se están empleando más en la actualidad (MacCallum, Roznowsky y Necowitz, 1992; MacCallum, Wegener, Uchino y Fabrigar, 1993).

\section{Críticas a los modelos con estructuras de covarianza}

Respecto a las relaciones de causalidad formuladas en los modelos causales han surgido ciertas críticas escépticas. Concretamente se han planteado algunas dificultades filosóficas sobre la adecuación del término "causalidad" para definir las relaciones entre variables en estos modelos. Del mismo modo se ha aludido a la necesidad de diferenciar entre la consistencia del modelo con los datos y la consistencia del modelo con la realidad. Esta alusión ha sido bien recibida entre los investigadores en modelos causales y, de hecho, Bollen (1989) afirma que si un modelo es consistente con la realidad, los datos deberían ser consistentes con el modelo mientras que el hecho de los datos sean consistentes con la realidad no implica que el modelo se corresponda con la realidad.

\subsubsection{Formalización matemática}

Los modelos de ecuaciones estructurales, o modelos causales, se representan mediante ecuaciones de regresión que muestran la influencia de una o más variables sobre otra u otras variables. Gráficamente estas relaciones de influencia se representan mediante una flecha de sentido único que parte de la variable causal y apunta hacia la variable influenciada.

Existen dos posibles notaciones para la formalización matemática de los modelos causales. La notación LISREL procede del conocido programa estadístico LISREL desarrollado por Jöreskog y se basa en ocho matrices básicas (Jöreskog y Sörbom, 1984). De la misma forma la notación EQS procede del programa estadístico EQS y se conoce como la notación Bentler - Weeks (Bentler y Bonett, 1980). La diferencia entre ambas notaciones es que la notación LISREL aporta mayor información sobre el tipo de variables 
y las relaciones que se establecen entre ellas, mientras que la notación del programa EQS simplemente distingue entre variables dependientes e independientes. Por esta misma razón la notación LISREL puede resultar más compleja e incluso difícil de interpretar mientras que la notación EQS es más sencilla y comprensible.

\section{Notación LISREL}

La notación LISREL se fundamenta en en ocho matrices básicas. En primer lugar las matrices $B$ у $\Gamma$ definen las relaciones correspondientes a las ecuaciones estructurales entre constructos endógenos y entre constructos exógenos y endógenos, respectivamente. A continuación las matrices que definen la correspondencia entre indicadores y constructos son $\Lambda_{x}$ y $\Lambda_{y}$, , para los modelos de medida exógeno y endógeno, respectivamente. Las matrices $\Theta_{\delta}$ y $\Theta_{\epsilon}$ contienen las matrices de correlaciones entre los errores de medida de los indicadores exógenos y endógenos, respectivamente y finalmente $\Phi$ y $\Psi$ muestran las correlaciones entre constructos exógenos y endógenos, respectivamente. Los términos de error forman parte de estas ocho matrices. Específicamente los errores del modelo de medida $\delta_{p}$ y $\epsilon_{p}$ se encuentran en la diagonal de las matrices $\Theta_{\delta}$ y $\Theta_{\epsilon}$ mientras que los errores del modelo estructural $\zeta_{n}$ se ubican en la diagonal de la matriz $\Psi$ (Jöreskog y Sörbom, 1989).

Respecto a las variables $\xi$ y $\eta$ hacen referencia a las variables no observables (o constructos) exógenos y endógenos, respectivamente mientras que $X$ y $Y$ se refieren a los indicadores exógenos y endógenos. El número de constructos exógenos y endógenos se representan con las letras $m$ y $n$ mientras el número de indicadores exógenos y endógenos se representan con las letras $p$ y $q$. Esta nomenclatura se resume en las tablas siguientes (Hair et al., 2007):

\begin{tabular}{|l|c|l|c|}
\hline Modelo & Nombre & Descripción & Elemento \\
\hline \multirow{4}{*}{ Modelo de Medida } & $\Lambda_{x}$ & Ponderaciones de los indicadores exógenos & $\lambda_{p m}^{x}$ \\
& $\Lambda_{y}$ & Ponderaciones de los indicadores endógenos & $\lambda_{q n}^{y}$ \\
& $\Theta_{\delta}$ & Matriz de covarianzas entre los errores de medida de indicadores exógenos & $\theta_{p p}^{\delta}$ \\
& $\Theta_{\epsilon}$ & Matriz de covarianzas entre los errores de medida de indicadores endógenos & $\theta_{q q}^{\epsilon}$ \\
\hline \multirow{4}{*}{ Modelo Estructural } & $B$ & Relaciones de constructos endógenos a endógenos & $\beta_{n n}$ \\
& $\Gamma$ & Relaciones de constructos endógenos a exógenos & $\gamma_{n m}$ \\
& $\Phi$ & Correlaciones entre constructos exógenos & $\phi_{m m}$ \\
& $\Psi$ & Correlaciones entre constructos endógenos & $\psi_{n m}$ \\
& $\xi$ & Constructo exógeno & - \\
& $\eta$ & Constructo endógeno & - \\
& $X$ & Indicador exógeno & - \\
\hline
\end{tabular}

Tabla 5.1: Notación LISREL 
En la formulación del modelo de medida se asume siempre que el número de variables observables (indicadores de constructo) es superior al número de variables no observables (constructos), es decir $p>m$ para el modelo de medida exógeno y $q>n$ para el modelo de medida endógeno.

Por otro lado, se asumen que tanto las variables observables como las no observables se expresan como desviaciones respecto a su media, por lo que la esperanza de dichas variables y los errores de medida es nula. Concretamente en el modelo de medida exógeno se asumen las hipótesis $E(X)=0, E(\xi)=0$ y $E\left(\delta_{x}\right)=0$, mientras que en el modelo de medida endógeno estas hipótesis se formulan como $E(Y)=0, E(\eta)=0$ y $E\left(\delta_{y}\right)=0$. Dichas hipótesis justifican la ausencia de términos constantes en las ecuaciones de los modelos de medida. No obstante, cabe la posibilidad de incluir constantes con el fin de analizar las medias de las variables latentes. Dicho desplazamiento respecto al origen, no afecta a las covarianzas entre variables.

Asimismo se asume independencia entre las variables no observables y los errores de medida de las variables observables:

1. Modelo de medida exógeno: $\operatorname{cov}\left(\xi, \delta_{x}\right)$

2. Modelo de medida endógeno: $\operatorname{cov}\left(\eta, \delta_{y}\right)$

Finalmente los errores de medida de las variables observables pueden ser aleatorios o no aleatorios. En caso de que sean no aleatorios las matrices de covarianzas de los errores de medida, $\Theta_{\delta}$ y $\Theta_{\epsilon}$ para variables observables exógenas y endógenas respectivamente, pueden tener elementos no nulos fuera de sus diagonales, $\delta_{p}$ y $\epsilon_{p}$. Esta circunstancia no es habitual pero puede ser considerada en la especificación del modelo.

En cualquier caso, cualquiera de estos supuestos puede ser obviado por el investigador, siempre y cuando se justifique teóricamente en la fase de especificación.

\section{Formulación del modelo de medida}

Sea $\Sigma$ la matriz de varianzas - covarianzas entre las variables observadas $(X)$ una matriz $p x p$ simétrica. La hipótesis fundamental se basa en que esta matriz de varianzas - covarianzas poblacional $(\sigma)$ es función de un conjunto de parámetros $(\theta)$ de forma que, si el modelo hipotetizado es correcto y se conocen los parámetros adecuados, la matriz de varianzas - covarianzas estimada a partir de dichos parámetros $\sigma(\theta)$, reproducirá la matriz de de varianzas - covarianzas poblacional $(\Sigma)$.

$$
\Sigma=\Sigma(\theta)
$$


Según esta expresión es posible definir la matriz de varianzas - covarianzas de las variables observables como una función de los parámetros estructurales del modelo de medida:

$$
\Sigma=E\left(X X^{\prime}\right)=E\left[\left(\Lambda_{x} \xi+\delta\right)\left(\Lambda_{x} \xi+\delta^{\prime}\right)\right]=E\left[(\xi+\delta)\left(\xi^{\prime} \Lambda_{x}^{\prime}+\delta^{\prime}\right)\right]
$$

Considerando la propiedad distributiva y calculando la esperanza:

$\Sigma=E\left[\Lambda_{x} \xi \xi^{\prime} \Lambda_{x}^{\prime}+\Lambda_{x} \xi \delta^{\prime}+\delta \xi^{\prime} \Lambda_{x}^{\prime}+\delta \delta^{\prime}\right]=E\left[\Lambda_{x} \xi \xi^{\prime} \Lambda_{x}^{\prime}\right]+E\left[\Lambda_{x} \xi \delta^{\prime}\right]+E\left[\delta \xi^{\prime} \Lambda^{\prime}\right]+E\left[\delta \delta^{\prime}\right]$

La matriz $\Lambda_{x}$ no contiene variables aleatorias, al ser constantes los parámetros poblacionales:

$$
\Sigma=\Lambda_{x} E\left[\xi \xi^{\prime}\right] \Lambda_{x}^{\prime}+\Lambda_{x} E\left[\xi \delta^{\prime}\right]+E\left[\delta \xi^{\prime}\right]+E\left[\delta \delta^{\prime}\right]
$$

Si denominamos:

$$
\begin{gathered}
\Phi=E\left[\xi \xi^{\prime}\right] \\
\Theta_{\delta}=E\left[\delta \delta^{\prime}\right]
\end{gathered}
$$

Y asumimos que $\delta$ y $\xi$ están incorrelacionados:

$$
\begin{aligned}
& E\left[\xi \delta^{\prime}\right]=0 \\
& E\left[\delta \xi^{\prime}\right]=0
\end{aligned}
$$

Puede escribirse para el modelo de medida exógeno:

$$
\Sigma_{x x}=\Lambda_{x} \Phi \Lambda_{x}^{\prime}+\Theta_{\delta}
$$

Y de la misma forma puede escribirse para el modelo de medida endógeno:

$$
\Sigma_{y y}=\Lambda_{i} \eta \eta^{\prime} \Lambda_{y}^{\prime}+\Theta_{\epsilon}
$$

Aunque en este caso la expresión de $\eta \eta^{\prime}$ no puede simplificarse mediante una matriz, puesto que es función de otras matrices.

Esta expresión se conoce como ecuación de la covarianza y constituye la base de los modelos de medida, asumiendo los supuestos de independencia señalados anteriormente. Aplicando esta ecuación todos los términos de la matriz de covarianzas $\Sigma$, podrán expresarse en función de los parámetros del modelo. Sin embargo, no todos los parámetros estructurales son conocidos por lo que deberán estimarse. 
Esta ecuación de covarianza se aplica por igual a variables exógenas y endógenas, aunque la notación LISREL difiere según el carácter de las variables:

$$
\begin{aligned}
& x=\Lambda_{x} \xi+\delta \\
& y=\Lambda_{y} \eta+\epsilon
\end{aligned}
$$

\section{Formulación del modelo estructural}

La ecuación del modelo estructural se formula como:

$$
\eta=\beta \eta+\Gamma \xi+\delta
$$

En esta expresión $\beta$ es la matriz de coeficientes de regresión que relacionan las variables latentes endógenas $\eta, \Gamma$ es la matriz de coeficientes de regresión que relacionan las variables latentes exógenas $(\xi)$ las variables latentes endógenas $(\eta)$ y $\zeta$ es el vector de residuos de las variables latentes endógenas $(\eta)$. Si la matriz $\beta$ es o puede transformarse en una matriz triangular entonces el modelo es no recursivo.

Esta ecuación estructural puede escribirse como:

$$
(I-B) \eta=\Gamma \xi+\zeta
$$

Este formato de la ecuación del modelo estructural ha sido el empleado tradicionalmente en LISREL, aunque la matriz $(I-B)$ directamente se formulaba con la letra $\beta$. Sin embargo, en este caso era necesario invertir los signos de la matriz para su interpretación, lo cual introducía ciertas dificultades en la comprensión de los resultados.

El término $I-B$ se aplica para expresar la ecuación del modelo estructural en términos de covarianzas:

$$
\begin{gathered}
\eta=(I-B)^{-1} \Gamma \xi+(I-B)^{-1} \zeta \\
\eta \eta^{\prime}=(I-B)^{-1} \Gamma \xi \xi^{\prime} \Gamma^{\prime}(I-B)^{\prime-1}+(I-B)^{-1} \zeta \zeta^{\prime}(I-B)^{\prime-1} \\
\Sigma_{\eta \eta^{\prime}}=(I-B)^{-1} \Gamma \Phi \Gamma^{\prime}(I-B)^{\prime-1}-(I-B)^{-1} \Psi(I-B)^{\prime-1}
\end{gathered}
$$

En esta ecuación el primer término representa la varianza explicada del modelo estructural y el término situado a la derecha muestra la varianza no explicada del modelo. Esta expresión de la matriz de covarianzas de las variables latentes endógenas $\Sigma_{\eta \eta^{\prime}}$ muestra porqué la matriz $\eta \eta^{\prime}$ no puede 
simplificarse.

El proceso de estimación tiene como objetivo encontrar los valores de dichos parámetros, representados habitualmente mediante el vector $\theta$. La estimación de los parámetros da lugar al vector $\hat{\theta}$, mediante los cuales es posible generar la matriz de varianzas - covarianzas estimada $\Sigma(\hat{\theta})$ tan próxima a la matriz de varianzas - covarianzas muestral $\Sigma$ como sea posible. Así, de la diferencia entre ambas matrices $\Sigma-\Sigma(\hat{\theta})$ se obtiene una matriz de varianzas - covarianzas residual cuyos momentos son valores próximos a cero. Para determinar el grado de proximidad entre ambas matrices se define la función de ajuste entre ellas $F(\Sigma-\Sigma(\hat{\theta})) \simeq 0$.

A partir de esta función de ajuste se formula la hipótesis nula para el contraste de bondad de ajuste del modelo. Esta hipótesis nula plantea que el modelo hipotetizado sí es aplicable en la población de referencia:

$$
H_{0}: \Sigma=\Sigma(\hat{\theta})
$$

Al contrario de lo que ocurre en los contrastes de hipótesis tradicionales en este caso cabe esperar que no se rechace la hipótesis nula. De esta forma cuando la probabilidad asociada a este contraste sea superior a 0.05 , para o $-\alpha=0,95$, la probabilidad de encontrar un modelo que ajuste mejor a los datos es pequeña, y por tanto el modelo no deberá ser rechazado. Este umbral de 0.05 es el mínimo recomendado y debería superarse el nivel de 0.1 o 0.2 para confirmar la falta de significación.

Existen otros planteamientos alternativos a esta estrategia de validación de hipótesis. Por ejemplo MacCallum plantea que en muestras muy grandes es fácil obtener estimaciones muy precisas de los parámetros incluso si el modelo no ajusta por lo que propone invertir la hipótesis nula y utilizar intervalos de confianza en índices de ajuste, como los definidos sobre el Root Mean Square Error of Aproximation (Rigdon, 1996). En este caso un amplio intervalo de confianza del RMSEA, no permite determinar la precisión de la estimación de la discrepancia, y por tanto se concluye que no es posible determinar el grado de ajuste en la población de forma precisa. Por el contrario, un intervalo de confianza estrecho implica muy buena precisión en la estimación del RMSEA y por tanto del ajuste del modelo respecto a la población. No obstante estos intervalos de confianza sobre los índices de ajuste pueden verse seriamente influidos por el tamaño de muestra así como por la complejidad del modelo.

En modelos complejos, es necesario un gran tamaño de muestra para obtener intervalos de confianza suficientemente ajustados mientras que, si el número de parámetros a estimar es pequeño, entonces la probabilidad 
de obtener intervalos de confianza estrechos es alta incluso con muestras de tamaño moderado.

\section{Notación Bentler-Weeks}

La notación matemática empleada para representar los modelos causales procede del trabajo de Bentler y Weeks (Bentler y Weeks, 1979, 1980). La gran ventaja de la representación matemática de modelos en EQS es su simplicidad, puesto que todas las variables del modelo pueden clasificarse simplemente en variables dependientes o independientes. El criterio empleado para decidir si una variable se considera dependiente o independiente depende de si existe alguna flecha de relaciones apuntándole. Si ninguna flecha apunta a la variable, entonces se considera independiente, en caso contrario se considera dependiente. Este criterio es independiente de si la variable es observable o latente, o si se trata de algún término de error o perturbación.

Las variables observables se representan con la letra $V$ mientras que las variables latentes se representan con la letra $F$. La letra $E$ representa los términos de error de las variables observables (Rigdon, 1994) mientras que la letra $D$ representa los términos de error de las variables latentes, el error en la predicción del factor latente. En este caso no se utiliza el término residuo sino el término perturbación.

Una característica importante del modelo Bentler - Weeks es que las varianzas y covarianzas de las variables dependientes nunca pueden ser parámetros del modelo. Sin embargo, las varianzas y covarianzas de las variables independientes son importantes parámetros que deben ser estimados.

\subsubsection{Supuestos estadísticos y análisis previos}

\section{Normalidad}

La Normalidad de las variables implicadas en el análisis es un requerimiento para la correcta estimación de los parámetros del modelo así como para los contrastes de significación asociados a estos parámetros. Existen varios procedimientos disponibles para comprobar el supuesto de Normalidad Univariante. Los métodos gráficos aportan una solución inmediata. En primer lugar el Histograma permite observar si la distribución de los datos se asemeja a una distribución teórica normal. Asimismo el Gráfico de Probabilidad Normal, que representa ambas distribuciones de datos, muestra el grado de semejanza entre ambas mediante una línea recta a lo largo de la 
cual se alinean los datos. El principal inconveniente de estos métodos gráficos es su falta de precisión y el elevado componente subjetivo a cargo de la percepción del investigador. Por ello se han desarrollado diversas pruebas empíricas con el fin de comprobar de forma más precisa el cumplimiento de este supuesto.

El contraste empírico de uso más generalizado es el Contraste de Kolmogorov - Smirnov. Este contraste compara la distribución de los datos respecto a la distribución teórica normal. El contraste plantea como hipótesis nula que ambas distribuciones son semejantes. Esta hipótesis se rechaza cuando el valor del estadístico, que en este caso representa la máxima diferencia entre ambas distribuciones, es significativamente elevado.

Es bien sabido que existen otras muchas herramientas estadísticas para la verificación del cumplimiento de normalidad univariante, pero la descripción de todas y cada una de ellas escapa a los objetivos de este trabajo. Por otro lado, el hecho de que las variables se comporten de forma univariante no implica que conjuntamente sigan una distribución multivariante, aunque esta afirmación sí se cumple a la inversa. Es decir, un conjunto de variables que cumplan el supuesto de Normalidad Multivariante, también se comportarán individualmente como distribuciones normales univariantes. El supuesto de normalidad multivariante es posible comprobarlo mediante el test de Mardia (1970, 1974), incluido como opción de análisis por defecto en el programa EQS. En los casos en que el tamaño de muestra es suficientemente elevado y las variables implicadas en el análisis se distribuyen conjuntamente según una distribución normal multivariante, el Coeficiente Estandarizado Estimado de Mardia se distribuye igualmente según una distribución normal, aunque univariante. La presencia de elevados valores positivos en este coeficiente reflejan distribuciones significativamente leptocúrticas (curtosis positiva) mientras que los valores elevados negativos indican que la distribución es significativamente platicúrtica (curtosis negativa). Bentler (2006) sugiere que los valores estandarizados de este coeficiente superiores a 5 son indicadores de distribuciones de datos que no se comportan conjuntamente de forma normal.

\section{Linealidad}

El supuesto de Linealidad entre variables hace referencia al hecho de que estén relacionadas mediante relaciones lineales, y no cuadráticas o de orden superior. La herramienta más directa para comprobar este supuesto son los Gráficos de Dispersión, que representan los datos de una variable en relación con los de otra. Para analizar todos los posibles pares de variables es posible realizar este análisis mediante matrices que planteen los gráficos de todas 
las posibles combinaciones de variables dos a dos.

\section{Independencia}

El supuesto de Independencia entre variables requiere que ninguna de ellas pueda ser expresada como una combinación lineal de otras. En tal caso se consideraría que existen relaciones de colinealidad que podrían invalidar total o parcialmente los resultados. Para detectar si existen relaciones de colinealidad entre los datos es posible analizar los autovalores de la matriz de varianzas - covarianzas. En los casos en que uno o más autovalores tomaran valores próximos a cero se procedería a evaluar la suma de las componentes de sus autovectores asociados. Los sumatorios de dichas componentes con valores nulos o próximos a cero serían indicadores de relaciones de colinealidad entre variables, y por tanto se debería plantear la modificación o directamente la eliminación de las variables causantes de este efecto.

\section{Valores ausentes}

La presencia de datos ausentes es frecuente en cualquier investigación, y especialmente en las orientadas al ámbito de las ciencias sociales. No obstante, la gran mayoría de las técnicas estadísticas multivariantes requieren trabajar con conjuntos de datos completos y presentan ciertas limitaciones en los casos en que no se cumple este requerimiento. Concretamente en modelos de ecuaciones estructurales los valores perdidos son causantes de estimaciones sesgadas de los errores estándar de los parámetros así como de los estadísticos asociados a esta estimación.

Independientemente de los casos de no respuesta a la totalidad del cuestionario, que sin duda constituye un caso aparte en el tratamiento de valores ausentes, existen tres posibles situaciones en cuanto a la aleatoriedad de estos valores. En primer lugar se considera que los datos faltan completamente al azar (MCAR: Missing completely at random) si su ausencia o presencia es completamente independiente de las características de los individuos. Por el contrario los datos faltan no al azar cuando la ausencia o no de un dato depende del valor de la propia variable que falta, situación que también se denomina falta de datos no ignorable. El caso intermedio entre ambos corresponde a la situación en que los datos faltan al azar (MAR: Missing at random), que ocurre cuando la ausencia o no de un dato es independiente del valor de la propia variable donde falta, pero puede depender de las variables restantes (Coenders, Batista-Foguet y Saris, 2005). Específicamente se considera que existen una falta de datos ignorable si los datos faltan al azar y adicionalmente los parámetros que determinan la pérdida de datos son com- 
pletamente distintos de los parámetros del modelo a estimar (Allison, 2003). En la práctica ambos términos, falta de datos ignorable y falta de datos al azar, son equivalentes y se emplean indistintamente. Asimismo el hecho de que los datos falten completamente al azar implica el cumplimiento de la condición de falta de datos al azar, al ser la primera hipótesis más restrictiva que la segunda. Esta tipología de valores perdidos tiene una relevancia mucho mayor que la debida a las causas a las que se debe la presencia de los valores perdidos, en cuanto a su tratamiento para el análisis de los datos.

Para abordar el problema de los valores perdidos se han propuesto distintas metodologías basadas en la eliminación parcial de la información existente, la imputación de los valores perdidos y la estimación del modelo mediante máxima verosimilitud.

Entre las técnicas de eliminación de información la Eliminación completa del caso (Listwise) es la opción más directa, pero también la más ineficiente ya que elimina el caso completo en caso de haber al menos un valor ausente en alguna variable. En consecuencia, implica una disminución en la potencia de los contrastes y en la precisión de las estimaciones de los errores estándar. Este procedimiento es el más simple y viene implementado por defecto en la gran mayoría de los programas estadísticos como parte de las opciones de análisis ya que produce un nuevo conjunto de datos completo, aplicable a la totalidad de los métodos estadísticos. Este método tiene dos importantes propiedades estadísticas. En primer lugar si los datos faltan completamente al azar la submuestra resultante se extrae mediante muestreo aleatorio simple de la muestra original, y por tanto con la eliminación de esta información no se introduce sesgo alguno. Asimismo, las estimaciones de los errores estándar constituyen igualmente estimadores insesgados de los errores estándar reales. Sin embargo, en los casos en que los casos falten al azar, pero no completamente al azar, dichas estimaciones pueden presentar ciertos sesgos (Wothke, 2000). El gran inconveniente de este método es que conlleva la eliminación de una gran parte de la muestra original, sobre todo en los casos en que el número de variables es muy elevado. Por ello, a pesar de que los estimadores de los errores estándar sean insesgados, dichos errores pueden resultar mayores a los obtenidos con otras opciones de tratamiento de valores perdidos en los que se conserve la información. De hecho, las consecuencias directas del empleo de este método es el incremento del rango en los intervalos de confianza y la disminución de la potencia estadística de los contrastes asociados a la estimación.

La alternativa más inmediata a este método es la Eliminación por parejas (Pairwise), que emplea todos los individuos cuyas variables están disponibles a la hora de calcular las covarianzas. Esta técnica se basa en transformar el conjunto de datos original en una función de los momentos de primer o 
segundo orden, en los que se basan la gran mayoría de los modelos lineales. Concretamente en los modelos de estructuras de covarianza, se emplean para obtener la matriz de varianzas - covariazas. En los casos en que los datos faltan completamente al azar este método aporta estimaciones consistentes y por tanto insesgadas de los datos (Glasser, 1964). Sin embargo, presenta ciertos inconvenientes como la posibilidad de que la matriz de correlaciones resulte no definida positiva y por tanto no puedan estimarse ciertos parámetros del modelo. Asimismo, cabe la posibilidad de que se generen ciertas incoherencias en la matriz de varianzas - covarianzas, calculada en cada una de sus celdas sobre un conjunto de individuos diferente. Por último no se garantiza que los estimadores de los errores estándar sean consistentes e insesgados, afectando así a la validez de los intervalos de confianza y los estadísticos de los contrastes de hipótesis asociados a la estimación.

Una vez descartados estos métodos para el tratamiento de valores ausentes, se plantean los Métodos de imputación. La sustitución del valor ausente por el valor medio es el más simple pero no se considera una opción recomendable, ya que reduce la variabilidad de la varianble imputada sesgando las estimaciones de los parámetros del modelo (Little y Rubin, 1987). Asimismo se reducen las correlaciones entre dicha variable imputada y el resto de variables del modelo. También es posible realizar una regresión lineal entre la variable imputada como dependiente y las variables restantes como independientes. Este método tampoco solventa por completo la dificultad anterior y por ello se propone añadir un término aleatorio de modo que la regresión lineal sea adicionalmente estocástica. Otro método es la imputación mediante el algoritmo EM (Expectation - Maximization), traducido al español como algoritmo esperanza - maximización (Little y Rubin, 1987). Este método supone estimar la matriz de varianzas - covarianzas empleando este algoritmo, lo cual resulta especialmente eficiente en el caso de que los datos se distribuyan de forma normal. Aunque existen otros métodos de imputación en general estos métodos muestran cierta tendencia a estimar las varianzas y covarianzas de forma sesgada. Por ello, en términos generales, se recomienda trabajar con métodos de estimación que tomen en consideración la presencia de valores perdidos, en detrimento de la imputación de dichos valores. La razón para ello es que los métodos de estimación aplicados posteriormente a la imputación presuponen que la totalidad de los datos son reales, y por tanto dichos métodos son incapaces de gestionar las modificaciones en la variabilidad introducidas en la fase de imputación.

La última opción para el tratamiento de valores perdidos es considerarlos como parte de la matriz de datos originales, asunción que podrá hacerse siempre y cuando sea posible asumir que los datos faltan al azar. De hecho, la mayoría de los programas informáticos pueden asumir el cálculo de la matriz de varianzas - covarianzas en presencia de valores ausentes si cumplen 
esta condición y adicionalmente se distribuyen normalmente, como ocurre en MX, EQS 6.0, LISREL 8.5 y MPLUS para el método de estimación por máxima verosimlitud. El primero de los métodos para llevar a cabo este propósito es la estimación mediante el "Algoritmo E-M", que obtiene estimadores máxima verosimilitud de las medias y la matriz de varianzas covarianzas mediante un algoritmo en dos pasos que se repiten de forma iterativa: la estimación del valor esperado del estadístico log-verosimiltud y la posterior maximización de dicho valor para la nueva estimación de los parámetros del modelo. El inconveniente de este método de estimación es que los estimadores resultantes se han obtenido mediante máxima verosimilitud y por tanto son menos eficientes. Por otro lado la Estimación directa $M L$ o "Full information $M L$ " (FIML) soluciona este problema y en general es la opción más recomendada en la mayoría de las aplicaciones SEM. Sin embargo para aplicar este método es necesario asumir ciertas hipótesis, como que la falta de valores es ignorable y que se cumple el requerimiento de normalidad multivariante. En los casos en que el conjunto de datos se encuentra completo el incumplimiento de esta hipótesis no afecta a los estimadores de los parámetros, pero sí a las estimaciones de los errores estándar y el estadístico $\chi^{2}$ asociado. Estas circunstancias se aplican de forma equivalente a los conjuntos de datos con valores ausentes, de modo que en los casos que no se cumpla la hipótesis de normalidad multivariante se debe trabajar con los estadísticos paralelos desarrollados por Yuan y Bentler (2000). Así, los estadísticos Residual - based Test Statistic, Yuan - Bentler Residual - based test Statistic y Yuan - Bentler Residual -based F Statistic constituyen la alternativa al estadístico $\chi^{2}$ desarrollado por Satorra - Bentler en presencia de valores perdidos.

\subsubsection{Procedimiento para el análisis}

\section{Fase 1: Especificación del modelo}

El proceso de especificación tiene como objetivo establecer formalmente un modelo, ya sea un modelo de medida o un modelo estructural (Hoyle, 1995). Para ello es necesario tomar diversas decisiones sobre aspectos como el número de factores latentes, el número de variables observables, la relación entre los distintos factores latentes, la relación entre estos factores latentes y las variables observables y por último las relaciones entre factores únicos (Long, 1983).

\section{La teoría como elemento subyacente del modelo}

La teoría se define como un conjunto sistemático de relaciones que ofrecen una explicación exhaustiva y consistente de un fenómeno. Puede estar 
basada en la experiencia y la práctica por observación del comportamiento de la realidad, no necesariamente debe tratarse de conceptos abstractos no aplicables en la práctica.

Todos los modelos basados en estructuras de covarianza requieren una justificación teórica para la especificación de las relaciones de dependencia y la valoración de modificaciones a las relaciones propuestas. En este último caso la necesidad de un modelo teórico es especialmente crítica.

De hecho, existen cuatro criterios fundamentales a considerar a la hora de realizar afirmaciones sobre posibles relaciones causales: una base teórica subyacente al modelo, la existencia de asociaciones suficientes entre las variables, la existencia de antecedentes temporales de la causa frente al efecto y por último falta de alternativas a las variables causales (Hair et al., 2007).

\section{El Análisis Factorial Exploratorio}

Como se ha expuesto anteriormente, el Análisis Factorial Exploratorio es una técnica de reducción de datos que intenta describir, mediante un modelo lineal, un conjunto de variables observables a partir de un número menor de variables no observables (Levy y Varela, 2006). Por otro lado, el Análisis de Componentes Principales (ACP) es una técnica afín al Análisis Factorial Exploratorio, en el sentido de que ambas poseen el objetivo también de reducir la dimensionalidad de un conjunto de variables. No obstante existen diferencias entre ambas que desaconsejan su utilización indistintamente.

En primer lugar el Análisis de Componentes Principales analiza la varianza total del conjunto de variables observables y trata de maximizar la varianza explicada a través de las componentes extraídas. Por el contrario el Análisis Factorial Exploratorio analiza la varianza común entre las variables observables, denominada comunalidad, y busca un número reducido de variables no observables que expresen la parte común a las variables observables. Este hecho implica que la varianza de los errores sea nula en Análisis de Componentes Principales mientras que el Análisis Factorial Exploratorio sí considera la existencia de cierto error de medida de forma que:

$$
\sigma_{\text {total }}^{2}=\sigma_{\text {comun }}^{2}-\sigma_{\text {error }}^{2}
$$

En otras palabras, es posible expresar cada Componente Principal como una relación lineal de las variables observables sin necesidad de incluir ningún término de error, mientras que en Análisis Factorial Exploratorio son las variables observables las que se explican como la suma de las variables no observables correspondientes más un término de error. 
Una vez identificadas las diferencias entre Análisis Factorial Exploratorio y Análisis de Componentes Principales, cabe señalar igualmente las diferencias entre esta metodología y el Análisis Factorial Confirmatorio. Ambas se basan en la existencia de un conjunto reducido de variables no observables relativas a otro conjunto de variables observables. Sin embargo tanto el enfoque, como determinados detalles técnicos difieren notablemente. El Análisis Factorial Exploratorio se plantea cuando las relaciones entre variables observables y no observables no son conocidas o no están definidas con el fin de determinar cómo y en qué medida las variables observables se relacionan con las variables latentes subyacentes. En términos generales, el objetivo es identificar el mínimo número de factores que subyacen entre las variables observables. Por el contrario el Análisis Factorial Confirmatorio se utiliza cuando se poseen conocimientos previos sobre la estructura factorial, ya sea debido a conocimientos previos, a investigaciones empíricas anteriores o ambos, se parte de un conjunto conocido de relaciones entre las variables observables y las no observables.

Otra diferencia entre ambas metodologías es que el Análisis Factorial Exploratorio contempla las posibilidades de que todas las variables no observables estén correlacionadas (rotación oblicua), o que todas estas variables estén no correlacionadas (rotación ortogonal), mientras que en Análisis Factorial Confirmatorio es posible determinar a priori qué variables latentes estarán relacionadas. Del mismo modo, en Análisis Factorial Exploratorio todas las variables observables en principio saturan en todos los factores latentes mientras que en Análisis Factorial Confirmatorio es posible definir qué factores latentes afectan a determinadas variables observables.

Respecto a los términos de error, en Análisis Factorial Exploratorio no están correlacionados entre sí, pero sí pueden estarlo en Análisis Factorial Confirmatorio, aunque no es lo habitual. Al mismo tiempo en Análisis Factorial Exploratorio todas las variables observables están afectadas por un término de error no nulo, mientras que en Análisis Factorial Confirmatorio estas variables conllevan un error de medida, que puede fijarse a un valor nulo. Finalmente en Análisis Factorial Exploratorio todas las variables latentes están incorrelacionados con los términos de error de las variables observables mientras que sí existe esta posibilidad en Análisis Factorial Confirmatorio, aunque tampoco es habitual (Long, 1983).

\section{Definición del número de indicadores por constructo}

Las recomendaciones sobre el número de indicadores (variables observables) por factor (variables no observables) son vagas e imprecisas, aunque existen algunas indicaciones de carácter general. Sin ir más lejos, Bentler afir- 
ma que "escoger el número correcto de indicadores para cada variable latente es algo así como un arte" (Bentler y Bonett, 1980). En términos generales, se considera que un alto ratio de indicadores por constructo redundará en menos problemas de convergencia, mayor interpretabilidad, estimaciones más fiables y apropiadas y factores más fiables y se desaconseja utilizar pocos indicadores para muestras pequeñas (Marsh, Hau, Balla y Grayson, 1998). Concretamente si el tamaño de muestra es pequeño, se recomienda tener un amplio número de indicadores por factor.

A partir de los trabajos que analizan esta cuestión, parece haberse alcanzado un acuerdo en que se definan al menos dos indicadores por factor, aunque el mínimo deseable serían tres (Bollen, 1989). Estrictamente el número mínimo de indicadores por constructo es al menos uno, pero el uso de un solo indicador exige al investigador aportar rigurosas estimaciones de fiabilidad, que en la mayoría no alcanzan los límites exigidos. Así, aunque un constructo puede ser representado por dos indicadores, el número preferido es tres para constructos homogéneos, y un número comprendido entre 5 y 7 para constructos heterogéneos. No existe un límite superior para el número de indicadores por constructo, aunque debe considerarse que el número de variables observables que pueden incluirse es limitado. A medida que se añaden variables observables, y sobre todo cuando se superan las 20 variables, se incrementa en gran medida la dificultad de la interpretación de resultados y aumenta la significación estadística de los modelos, aún cuando sí son representativos de los datos (Hair et al., 2007). Este hecho no implica que se rechacen variables significativas en favor de la simplificación del modelo, sino que se valoren los modelos teóricos concisos y parsimoniosos. En cualquier caso esta cuestión adquiere mayor complejidad en modelos causales que en análisis factorial confirmatorio y en estos casos el criterio para escoger qué indicadores son más adecuados para cada constructo debe ser una combinación de la teoría y un número adecuado de indicadores por factor (Hagtvet y Nasser, 2004).

\section{Tamaño de muestra}

$\mathrm{Al}$ igual que ocurre con la ratio de indicadores por factor, las recomendaciones sobre el tamaño de muestra son igualmente vagas e imprecisas, aunque existen algunas indicaciones de carácter general. En Análisis Factorial se considera adecuado una ratio de al menos 10 veces el número de casos sobre el número de variables (Nunnally, 1978). Mientras que Bentler (2006) sugiere una ratio de 5:1 del tamaño muestral sobre los parámetros libres, siempre y cuando los datos sigan una distribución normal multivariante, Gorsuch sugiere un mínimo de 5 sujetos por variable en cualquier tipo de análisis factorial (Gorsuch, 1983). Anderson y Gerbin (1984) recomiendan el uso de una muestra con un tamaño cercano a 200 sujetos, cuando existen 
al menos tres indicadores por variable latente y una muestra de tamaño mayor cuando sólo se hayan definido dos indicadores por constructo. En este criterio coinciden con otros investigadores como Marsh, quien propone de forma general una muestra mínima de 200 observaciones (Marsh, Balla y McDonald, 1988; Marsh et al., 1998).

Sin embargo en la determinación del tamaño de la muestra deben considerarse otros factores potencialmente influyentes en el modelo (Raykov y Widaman, 1995). Primeramente la posible existencia de errores de especificación del modelo. La omisión de variables relevantes en la explicación del constructo o cualquier otro tipo de error de especificación debe implicar un incremento de las exigencias en el tamaño muestral. Asimismo debe considerarse el número de parámetros libres a estimar. A medida que la complejidad del modelo aumenta, también deben aumentar los requisitos del tamaño muestral. En este aspecto se han propuesto diversas indicaciones como un mínimo de 5-10 observaciones por parámetro a estimar o al menos un tamaño de muestra mínimo similar al número de covarianzas o correlaciones en la matriz de datos de entrada (Hair et al., 2007).

El incumplimiento del supuesto de normalidad multivariante conlleva igualmente el incremento del tamaño muestral requerido en la fase de estimación así como el empleo de algún método de estimación capaz de solventar esta circunstancia. En este caso el número de observaciones por parámetro debe aumentar al menos hasta 15. Finalmente el método que se vaya a emplear en la estimación también debe considerarse en la determinación del tamaño muestral. Aunque la estimación con máxima verosimilitud ofrece resultados válidos con sólo 50 observaciones, no es recomendable utilizar muestras tan pequeñas y se sugiere emplear tamaños de muestra entre $100 \mathrm{y}$ 200 observaciones. Sin embargo, aumentar el tamaño de muestra no siempre tiene un efecto positivo. Una vez superadas las 200 observaciones, la estimación por máxima verosimilitud se vuelve más sensible al tamaño muestral y pierde la capacidad de detectar diferencias significativas entre las matrices de varianzas - covarianzas. Por esta razón algunos investigadores han sugerido un número de observaciones igual a 200 como Tamaño de Muestra Crítico (Hoelter, 1983). Otros métodos de estimación, como Asymptotically Distribution Free que es resistente al incumplimiento del supuesto de normalidad multivariante, requieren un número muy amplio de observaciones.

En conclusión, el tamaño muestral recomendable debe rondar las 200 observaciones y este número debería aumentar en caso de posibles errores de especificación, falta de cumplimiento del supuesto de normalidad multivariante, elevada complejidad del modelo o determinados métodos de estimación. 


\section{Uso de la matriz de covarianzas o la matriz de correlaciones}

Una característica de los modelos de estructuras de covarianzas es que únicamente se formula a partir de la matriz de varianzas - covarianzas o la matriz de correlaciones, en ambos casos la matriz seleccionada recibe el nombre de Matriz de Información. Existe la posibilidad de introducir en los programas estadísticos las observaciones directas, pero estos programas los transforman internamente para obtener la matriz de información correspondiente. La razón para este emplear este procedimiento en el tratamiento de los datos es que el interés de estos modelos no estriba en las observaciones individuales, sino en los patrones de relaciones entre los encuestados. Inicialmente se formuló esta técnica para trabajar únicamente a partir de la matriz de varianzas - covarianzas, pero en los últimos años se han ampliado las posibilidades de los métodos de análisis para trabajar a partir de la matriz de correlaciones. Por ello, aunque se ha demostrado que existen ciertos problemas inherentes a la aplicación de estos modelos al análisis de matrices de correlación (Cudeck, 1989), resulta interesante considerar las aportaciones de ambas matrices de información a los análisis.

La Matriz de Varianzas - Covarianzas proporciona comparaciones válidas entre diferentes poblaciones o muestras aunque la interpretación de los resultados resulta bastante más compleja, debido al hecho de que los coeficientes de regresión entre variables observables y no observables se interpretan en términos de las unidades de medida de los constructos. La utilización de esta matriz resulta válida para contrastes de la teoría, en los que se considere la importancia tanto de las varianzas como de las covarianzas, y se cumplan los supuestos estadísticos.

Por el contrario la Matriz de Correlaciones no permite estas comparaciones entre diferentes poblaciones o muestras y por tratarse de una matriz de varianzas - covarianzas estandarizada sus coeficientes siempre están en el intervalo $[-1,1]$ y se expresan en unidades estandarizadas. De esta forma, es fácil la interpretación del patrón de relaciones entre constructos, pero no permite la explicación de la varianza total del constructo. Igualmente soluciona el problema de la comparabilidad entre distintas variables observables, puesto que no están afectadas por distintas unidades de medida no estandarizadas. Las estimaciones obtenidas a partir de la matriz de correlaciones son más conservadoras en cuanto a la significación de los parámetros, y no se encuentran sesgadas al alza como se pensaba anteriormente.

Existen varios tipos de correlación que pueden incluirse en esta matriz. La más habitual es la conocida Correlación de Pearson pero es posible utilizar otras medidas de correlación para la incorporación de medidas no métricas. Entre ellas la Correlación Policórica permite el análisis de varia- 
bles ordinales con tres o más de tres categorías y la Correlación Tetracórica se utiliza para el análisis de variables binarias. En medidas métricas las $C o$ rrelaciones Poliserial y Biserial se emplean respectivamente para variables relacionadas con medidas ordinales policotómicas y binarias.

\section{Fase 2: Identificación del modelo}

El problema de la identificación plantea si es posible estimar todos los parámetros estructurales, contenidos en el vector $\theta$, es decir si cada uno de los parámetros puede expresarse a partir de otro parámetro o la combinación lineal de otros parámetros procedentes de la matriz de varianzas - covarianzas poblacional $(\Sigma)$, o en su caso de la matriz muestral $S$. Este problema está directamente asociado con la posibilidad de transponer la matriz de varianzas - covarianzas de las variables observables estimada a partir de los parámetros estructurales del modelo (Byrne, 2006; Levy y Varela, 2006).

Para abordar esta cuestión surge el concepto de los Grados de Libertad, definidos como la diferencia entre el número de correlaciones o covarianzas en la matriz de información y el número de parámetros a estimar en el modelo hipotetizado. Según Hair et al. (2007), cuantos más grados de libertad tenga el modelo, mayor será su nivel de parsimonia. En este caso mejorarán tanto el ajuste que podrá conseguirse incluso con pocos coeficientes, como las propias medidas de bondad de ajuste del modelo y la confianza se podrá depositar en los resultados. Esta definición de los grados de libertad del modelo difiere de otras definiciones del mismo término en otros ámbitos de la estadística. Concretamente los grados de libertad en modelos con estructuras de covarianza compara el número de parámetros a estimar con el número de elementos en la matriz de información, pero no con el tamaño muestral como ocurre habitualmente.

Según el número de grados de libertad, caben tres posibles situaciones. En primer lugar puede ocurrir que el modelo esté Exactamente Identificado (just-identified). Este caso ocurre cuando existe una correspondencia unívoca entre el número de elementos de la matriz de información y el número de parámetros a estimar, de forma que sólo existe una solución única para la estimación de los parámetros. Aunque se considera que en estas circunstancias la estimación de los parámetros es posible y el modelo es estable, no resulta interesante desde el enfoque de la modelización estructural. Al existir una única solución para la estimación de los parámetros, el modelo no posee ningún grado de libertad y por tanto nunca podrá ser rechazado o en otras palabras, el modelo nunca podrá generalizarse.

Cuando el número de parámetros a estimar es inferior al número de ele- 
mentos de la matriz de información el modelo está Sobreidentificado (over identified) (Wheaton, 1987). Esta situación implica que el número de grados de libertad es positivo y por tanto es posible rechazar el modelo. Estas son las condiciones recomendables desde la perspectiva de los modelos estructurales ya que en este caso al menos un parámetro puede obtenerse a partir de dos o más ecuaciones diferentes.

El caso restante corresponde a la situación en que el número de grados de libertad sea negativo, es decir, que el número de parámetros a estimar sea superior al número de elementos de la matriz de información. El modelo con estas características recibe el nombre de Infraidentificado (underidentified) y no es posible encontrar una solución para la estimación de sus parámetros estructurales. En estas circunstancias se considera que los parámetros están sujetos a la arbitrariedad, lo que implica que múltiples parámetros pueden definir el mismo modelo. Asimismo, puesto que no es posible obtener estimaciones de todos los parámetros estructurales, el modelo no puede validarse empíricamente. La solución es definir algunos parámetros como fijos o imponerles determinadas restricciones.

Aunque la forma más efectiva de analizar la identificación de un modelo es demostrando algebraicamente que cada uno de los parámetros pueden expresarse en función de otro/s parámetros/s en la práctica esta tarea no es inmediata. Por esta razón se han propuesto diversas Condiciones Necesarias para comprobar la identificación de un modelo.

El cumplimiento de la Regla $t$ es una condición necesaria, pero no suficiente para la identificación del modelo (Bollen, 1989). También se considera que es una condición de orden puesto que hace referencia al número de grados de libertad. Según esta regla el número de parámetros estructurales a estimar, o parámetros libres, debe ser inferior o similar al número de varianzas y covarianzas de las variables observables (elementos de la matriz de información):

$$
t \leq \frac{1}{2} \cdot p \cdot(p+1)
$$

Donde $t$ es el número de parámetros libres y p es el número de variables observables.

Esta misma regla puede expresarse en referencia al número de grados de libertad necesarios para que el modelo esté identificado:

$$
g l=\frac{1}{2} \cdot p \cdot(p+1)-t
$$

De la misma forma existen otras recomendaciones que evitan tener que 
calcular algebraicamente si cada parámetros está específicamente identificado (Hatcher, 1994; Ullman, 1996). Una de ellas consiste en definir adecuadamente el número de ítems por factor. Independientemente de las recomendaciones generales sobre el número de indicadores por constructo, para asegurar la identificación del modelo se sugiere que haya al menos tres indicadores con cargas no nulas en caso de que haya un único constructo teórico. En el caso de hayan dos o más factores y que cada uno de ellos tenga tres o más indicadores, el modelo estará identificado si los errores de medida no están correlacionados entre sí. Por otro lado si hay dos o más factores, pero cada uno de ellos tiene dos indicadores, el modelo estará identificado no sólo si los errores de medida no están correlacionados entre sí, sino que también debe ocurrir que cada indicador cargue sobre un único factor y que ninguna de las covarianzas de los factores latentes sea nula (Long, 1983).

Otra condición necesaria para la identificación del modelo es haber definido una escala para las variables latentes. Este requerimiento se justifica por el hecho de que las variables latentes son no observables y por tanto, no tienen escala métrica definida. En caso de obviar este requerimiento puede darse el problema de la indeterminación entre la varianza y las cargas factoriales según el cual resulta imposible distinguir entre los casos en que un factor tiene varianza grande y cargas pequeñas o varianza pequeña y cargas grandes. Este requerimiento puede cumplirse a través de dos vías. En primer lugar es posible imponer la restricción de que el coeficiente de regresión entre la variable latente y alguna de sus variables observables asociadas tenga un valor distinto de cero (habitualmente 1.0). Esta restricción puede aplicarse tanto a variables observables exógenas como endógenas y desde este momento recibe el nombre de Variable de Referencia. También es posible especificar el valor del coeficiente de regresión como la raíz cuadrada de la fiabilidad deseada o estimada, o equivalentemente, especificando el término de error de esa variable como 1.0 menos el valor de fiabilidad deseado. Por otro lado también es posible fijar la varianza del factor latente a un valor distinto de cero (habitualmente 1.0). Esta opción resulta especialmente interesante cuando distintos indicadores de una misma variable latente estén medidos en distintas escalas métricas.

Una variante de la fijación de la fiabilidad de la escala completa es un enfoque propuesto por Hayduk que implica un proceso por pasos (Hayduk, 1996). En primer lugar, el investigador selecciona el indicador aislado que se cree es la mejor representación del constructo y se fija la fiabilidad de dicho indicador (coeficiente de regresión y término de error). A continuación, pueden añadirse indicadores adicionales, especificando de la misma forma sus coeficientes de regresión y términos de error. Así, se especifica significado deseado del constructo a través de este indicador clave, y a continuación se permite a otros indicadores añadir significados complementarios al concepto 
ya definido inicialmente.

No obstante, el cumplimiento de la Regla t y las anteriores condiciones necesarias, no aseguran la identificación del modelo. Para ello existen una serie de Condiciones Suficientes, aunque no necesarias, aplicables a los modelos de medida (Bollen, 1989; Long, 1983). El hecho de que haya al menos tres indicadores por variable latente, que cada fila de la matriz x tenga un solo elemento distinto de cero o que la matriz sea diagonal, constituyen el conjunto de condiciones suficientes para que el modelo de medida hipotetizado esté identificado.

Respecto a las Condiciones Necesarias y Suficientes no existe acuerdo sobre si realmente existen como tales en el ámbito de la identificación de modelos causales.

\section{Falta de identificación empírica}

A pesar de que se cumplan las condiciones anteriores, en ocasiones puede ocurrir que no puedan estimarse los parámetros estructurales del modelo. En tal caso el modelo se considera que está Empíricamente No Identificado (Kenny, 1979). Esta cuestión de falta de identificación empírica ha sido tratada no sólo por Kenny, sino también por numerosos investigadores en el área de los modelos estructurales (Bollen, 1989; Kline 2004; Maruyama, 1998; Wothke, 1993). Un modelo empíricamente no identificado implica que, pese al cumplimiento teórico de las reglas de identificación, la estimación del modelo proporciona coeficientes erróneos para alguno de sus parámetros (Levy y Varela, 2006; Uriel y Aldás, 2005). Estas estimaciones incorrectas reciben el nombre de Estimaciones Infractoras.

Un tipo de estimación infractora muy común son los parámetros que exceden los límites aceptables (Hair et al., 2007). Éste es el caso de las varianzas de error negativas, también denominados Casos Heywood, los coeficientes estandarizados muy próximos o superiores a la unidad y los errores estándar muy elevados asociados a cualquier parámetro estimado. En el caso de las varianzas de error negativas una posible solución es fijar las varianzas de los errores infractores a un error positivo muy pequeño (Bentler y Chou, 1987). Esta solución debe ser tenida en cuenta en la interpretación de los resultados, ya que constituye una solución para que sea posible la estimación del modelo pero no garantiza su validez. De la misma forma en el caso de los coeficientes estandarizados muy próximos o superiores a la unidad el investigador debe valorar la posibilidad de eliminar uno de los constructos asociados a dichos coeficientes y realizar las comprobaciones necesarias sobre la validez discriminante de los factores. 
Independientemente de los procedimientos propuestos para solucionar determinados tipos de estimaciones infractoras, existen ciertas recomendaciones generales para evitar la falta de identificación empírica. En términos generales se sugiriere aumentar el tamaño de la muestra, modificar el modelo o fijar a un valor los parámetros causantes de la falta de identificación (Dillon, Kumar y Mulani, 1987). Esta última opción se basa en el hecho de que al definir más restricciones al modelo, se eliminan parámetros a estimar. No obstante, no se trata de añadir restricciones sin más hasta que se solucione el problema, sino que se debe seguir un proceso estructurado en la adición gradual de restricciones hasta lograr que el modelo esté suficientemente sobreidentificado.

Concretamente Hayduk propone un procedimiento específico para solventar este tipo de situaciones. En primer lugar se construye el modelo teórico y en caso de falta de identificación empírica se fijan las varianzas del error de medida de los constructos. Si persiste el problema, entonces se fijarán los valores de determinados coeficientes estructurales, cuyo valor sea conocido de antemano con fiabilidad y si esta medida no tiene efecto sobre el problema entonces se eliminarán directamente las variables problemáticas o se reformulará completamente el modelo teórico (Hayduk, 1987).

Entre las posibles causas de la falta de identificación empírica es posible encontrar muy diversas razones. Es habitual que un parámetro del modelo sea linealmente dependiente de otros, provocando que la matriz de varianzas - covarianzas sea singular. También es posible que simplemente el modelo esté mal especificado, no se cumpla el supuesto de normalidad multivariante o la muestra sea demasiado pequeña. Asimismo puede ocurrir que algunos parámetros se encuentren demasiado próximos a los límites admisibles dando lugar a estimaciones difíciles o inestables. Incluso puede deberse a posibles efectos recíprocos entre variables o que los valores perdidos de algunas variables observadas hayan provocado que cada elemento de la matriz de covarianzas muestral esté calculado sobre una muestra diferente (Long, 1983).

\section{Fase 3: Estimación del modelo}

Según Sharma, el proceso de estimación puede sintetizarse en dos fases. En primer lugar estimar los parámetros estructurales a partir de la matriz de varianzas - covarianza, y a continuación determinar la bondad de ajuste del modelo hipotetizado. Es decir, en qué medida se ajusta la matriz de varianzas - covarianzas del modelo hipotetizado a la matriz de varianzas covarianzas muestral (Sharma, 1996). 
Existen varios métodos para realizar la estimación del modelo. Un estudio comparativo entre distintos métodos mediante simulación de Monte Carlo analizó cómo se comportaban ante diferentes tamaños muestrales y situaciones de falta de cumplimiento de los supuestos de normalidad multivariante e independencia entre los errores y las variables latentes $(\mathrm{Hu}$, Bentler y Kano, 1992).

Inicialmente se intentó aplicar la regresión por mínimos cuadrados ordinarios sobre los modelos estructurales (Cronbach, 1976), pero sus resultados fueron rápidamente superados por la estimación con Máxima Verosimili$t u d$. En términos generales se recomienda utilizar este tipo de estimación, así como la estimación por Mínimos Cuadrados Generalizados (GLS) con muestras pequeñas, siempre y cuando se cumplan los supuestos de normalidad multivariante e independencia. En caso de que no se cumplan, entonces se recomienda utilizar la Estimación Robusta o Escalada que ofrece EQS (Ullman, 1996).

EQS permite especificar varios métodos de estimación que se ejecuten de forma simultánea. El número máximo de métodos es dos, si no se incluye la Estimación Asintótica por Mínimos Cuadrados Generalizados (AGLS), en cuyo caso es posible incluir hasta tres métodos de estimación diferentes.

\section{Métodos de estimación}

\section{Estimación por Máxima Verosimilitud (ML)}

La función de ajuste se define como:

$$
F_{M L}\left(S ; \Sigma^{*}\right)=\operatorname{tr}\left(S \Sigma^{*-1}\right)+\log \left[\log \left|\Sigma^{*}\right|-\log |s|-p\right]
$$

Donde $p$ es el número de variables observables.

Este método de estimación proporciona estimaciones consistentes, eficientes y no sesgadas cuando se cumple el supuesto de normalidad multivariante. En este caso las estimaciones de los parámetros son más ajustadas en el sentido de que tienen errores estándar más pequeños cuando el tamaño de muestra es elevado (Bentler, 2006). Es el método utilizado por defecto por la mayoría de los investigadores.

Estimación por Mínimos Cuadrados No Ponderados (ULS)

La función de ajuste se define como:

$$
F_{U L S}\left(S ; \Sigma^{*}\right)=\frac{1}{2} \operatorname{tr}\left[\left(S-\Sigma^{*}\right)^{2}\right]
$$


Este método aporta ciertas ventajas respecto al resto (Long, 1983), como el hecho de que no es necesario asumir normalidad multivariante, ya que en caso de falta de normalidad es posible partir de la matriz de varianzas - covarianzas estandarizada. Igualmente puede proporcionar estimaciones más precisas de los parámetros cuando el tamaño de muestra es pequeño. Por el contrario existen ciertos inconvenientes como la inexistencia de contrastes estadísticos para este método de estimación o la dependencia de los estimadores de la escala de medida de las variables observables. En cualquier caso puede resultar especialmente útil cuando la estimación por máxima verosimilitud tiene tanta potencia que todas las discrepancias entre las matrices de covarianza poblacional y muestral son rechazadas, por pequeñas que sean.

Estimación por Mínimos Cuadrados Generalizados (GLS)

$$
F_{G L S}\left(S ; \Sigma^{*}\right)=\frac{1}{2} \operatorname{tr}\left[\left(S-\Sigma^{*}\right) S^{-1}\right]^{2}
$$

Se aplica, al igual que la estimación por máxima verosimilitud, cuando los datos siguen una distribución normal multivariante.

Estimación mediante Asymptotically Distribution Free (ADF)

La función de ajuste se define como:

$$
F_{A D F}\left(S ; \Sigma^{*}\right)=[s-\sigma(\Theta)]^{\prime} W_{-1}[s-\sigma(\Theta)]
$$

Donde $s$ es el vector de datos (la matriz de varianzas - covarianzas muestrales escritas en forma de un solo vector), $\sigma$ es la matriz de varianzas - covarianzas estimada y $\Theta$ indica que se deriva de los parámetros del modelo. $W$ es una matriz que pondera las diferencias cuadráticas entre las matrices de varianzas - covarianzas muestrales y estimadas. Este método de estimación fue desarrollado por Browne (1984).

Estimación por la teoría de la distribución elíptica

Según Sobel la función de ajuste se define como:

$$
\left.\left.F_{E D T}\left(S ; \Sigma^{*}\right)=\frac{1}{2}(K+1)^{-1} \operatorname{tr}\left[\left(S-\Sigma^{*}\right) W^{(}-1\right)\right]^{2}-\delta \operatorname{tr}\left[\left(S-\Sigma^{*}\right) W^{(}-1\right)\right]^{2}
$$

Siendo k y $\delta$ funciones de curtosis y $W$ cualquier estimador consistente de $\Sigma$, (Sobel y Bohrnstedt, 1985). 


\section{Otros métodos de estimación}

En esta línea de investigación se han definido otros muchos métodos para abordar un amplio espectro de circunstancias relativas a la distribución de los datos, el tamaño de muestra y otros aspectos. Aunque la descripción detallada de cada método escapa a los objetivos de este trabajo, se han incluido sus nombres y abreviaturas con el fin de proporcionar una perspectiva de la amplia variedad de métodos ofrecidos al investigador: Asymptotically Generalized Least Squares (AGLS), Eliptical Generalized Least Squares (EGLS), Eliptical Reweighted Least Squares (ERLS), Heterogeneus kurtosis Generalized Least Squares (HKGLS), Heterogeneus kurtosis Reweighted Least Squares (HKRLS) y Arbitrary Generalized Least Squares (AGLS).

\section{Estimación directa y múltiple}

Los métodos de estimación planteados hasta el momento corresponden a los procedimientos de Estimación Directa. Este tipo de estimación es el más habitual y se basa en un proceso iterativo en el que sucesivamente se iteran los parámetros, a partir de los cuales se calcula la matriz de covarianzas. De la función de minimización aplicada a esa matriz de covarianzas se obtiene un escalar con un intervalo de confianza y un error estándar asociado. Este proceso se repite hasta alcanzar el criterio de convergencia definido, según el cual la diferencia entre los escalares obtenidos en dos iteraciones sucesivas debe inferior a un valor de tolerancia especificado previamente (este valor se ha establecido en 0.001 por defecto en EQS). En este punto es posible que la función de minimización sea incapaz de alcanzar el mínimo final, es decir que no converja de forma que no se pueda realizar la estimación de los parámetros.

En contraposición a los métodos de estimación directa se han definido los procedimientos de Estimación Múltiple a partir de simulación y remuestreo. Los métodos de estimación múltiple más habituales son la estimación por Boostrapping, por Simulación y según el método Jacknife. La estimación por Boostrapping diseña en primer lugar la muestra original para que actúe como la población a efectos muestrales. Sobre esa muestra se remuestrea un número determinado de veces para generar muchas nuevas muestras, cada una de ellas definida como un subconjunto aleatorio de la muestra original. Para cada una estas nuevas muestras se realiza la estimación del modelo de forma que la estimación global se obtiene calculando el promedio de todas las estimaciones de cada parámetro. La estimación por Simulación es similar al Boostrapping pero es posible modificar determinadas características de la muestra para cumplir el objetivo concreto de investigación planteado. Por último la estimación por el método Jacknife es igualmente similar al Boostraping y la Simulación, aunque en este caso el número de nuevas 
muestras creadas es igual al tamaño muestral original, y no simplemente un número indefinido y elevado. En cada nueva muestra se omite una observación diferente, de forma que todas ellas tienen un tamaño muestral N-1, lo cual permite identificar con facilidad cualquier tipo de observación influyente mediante el análisis de los cambios en las distintas estimaciones de los parámetros.

\section{Fase 4: Validación e interpretación del modelo}

Si la función de ajuste alcanza el mínimo y se comprueba que no existen estimaciones infractores, entonces se procede al análisis de la bondad de ajuste del modelo (Levy y Varela, 2006).

\section{Estadístico $\chi^{2}$ para la valoración del ajuste global del modelo}

Inicialmente la evaluación de los modelos se realizaba exclusivamente en base a un contraste de hipótesis sobre una distribución $\chi^{2}$ (Jöreskog, 1969). Como se ha mostrado en la formalización matemática del modelo estructural, la formulación de la hipótesis nula asociada a este contraste de de bondad de ajuste del modelo es:

$$
H_{0}: \Sigma=\Sigma(\hat{\theta})
$$

El planteamiento de esta hipótesis nula propone que el modelo hipotetizado sí es aplicable en la población de referencia, o que la matriz de varianzas - covarianzas poblacional es similar a la estimada a partir de los parámetros estructurales. La hipótesis alternativa considera el caso en que la matriz de varianzas - covarianzas poblacional sea similar a cualquier matriz definida positiva, distinta a la matriz de varianzas - covarianzas estimada.

Para el contraste de estas hipótesis (Bentler y Bonett, 1980) proponen el estadístico $(N-1) F_{M L^{0}}$ donde $N$ es el número de datos y $F_{M L^{0}}$ es el valor de la función de ajuste en la estimación por Máxima Verosimilitud. Este estadístico se distribuye como una $\chi^{2}$ con $\frac{1}{2} p(p+1)-t$ grados de libertad, siendo $p$ el número de variables observables independientes y $t$ el número de parámetros libres. Este estadístico recibe el nombre de $\chi^{2}$ (Bollen, 1989).

El valor de significación asociado a este estadístico $\chi^{2}$ se interpreta como la probabilidad de obtener un ajuste mejor al obtenido mediante el modelo hipotetizado. Un elevado valor del estadístico $\chi^{2}$ indica que las matrices observadas y estimadas difieren notablemente mientras que los valores bajos, asociados a niveles de significación mayores de 0.05 o 0.01, indican que las 
diferencias entre las matrices de varianzas - covarianzas observadas y estimadas no son estadísticamente significativas (Hair et al., 2007). Evidentemente el interés del investigador es no rechazar esta hipótesis nula, ya que ello implicaría que el modelo hipotetizado no ajusta adecuadamente. Por ello, para un nivel de confianza del $95 \%$, una probabilidad asociada al estadístico $\chi^{2}$ superior a 0.05 indicará buen ajuste del modelo, o también, que la probabilidad de encontrar un modelo que ajuste mejor a los datos es pequeña, y por tanto que el modelo no deberá ser rechazado.

Sin embargo, la falta de significación estadística en las diferencias entre las matrices de varianzas - covarianzas estimada y muestral no garantiza haber identificado el modelo más correcto, sino simplemente que se ha encontrado un posible modelo que demostrado tener un buen ajuste empírico. Existen diversos factores que pueden afectar a este contraste sobre el estadístico $\chi^{2}$, como son la falta de cumplimiento del supuesto de normalidad multivariante, realizar la estimación de los parámetros a partir de la matriz de correlaciones y no partiendo de la matriz de varianzas - covarianzas o simplemente un tamaño de muestra demasiado elevado. Puesto que el valor de este estadístico está directamente relacionado con el tamaño de muestra $N$, en caso de que el tamaño de muestra sea demasiado elevado el estadístico $\chi^{2}$ tenderá a ser alto a pesar de que el modelo ajuste bien.

Concretamente en EQS existe la posibilidad de obtener los estadísticos asociados al contraste $\chi^{2}$ robustos a la falta de normalidad multivariante. Estos estadísticos robustos pueden calcularse con cualquier método de estimación, excepto mediante estimación asintótica por mínimos cuadrados generalizados (AGLS). El estadístico propuesto por Satorra y Bentler es un escalamiento del estadístico $\chi^{2}$ original y recibe el nombre de Satorra - Bentler $\chi^{2}$ (Satorra y Bentler, 1988, 1994). La principal función de este estadístico es corregir el estadístico original en casos de falta de normalidad multivariante, así como los errores estándar vinculados (Bentler y Dijkstra, 1985). En estas circunstancias se ha demostrado que el estadístico Satorra Bentler $\chi^{2}$ se aproxima adecuadamente al estadístico $\chi^{2}$ original y de hecho, se considera el contraste estadístico más fiable para la evaluación de modelos estructurales en conjuntos de datos con distintas distribuciones y tamaños de muestra (Bentler, 2006; Curran, West y Finch, 1996; Hu et al., 1992). El único inconveniente que pueden mostrar es que únicamente pueden ser calculados a partir de los datos originales y no directamente de la matriz de varianzas - covarianzas.

No obstante es necesario considerar un detalle en la comparación de modelos. Cuando la validación de los modelos se basa en el estadístico $\chi^{2}$ original, la la comparación entre modelos anidados simplemente es cuestión de calcular la diferencia entre los estadísticos $\chi^{2}$ y los grados de libertad 
asociados a cada modelo. Puesto que esta diferencia de valores se distribuye según una $\chi^{2}$ con tantos grados de libertad como la diferencia de grados de libertad entre ambos modelos, las conclusiones sobre la significatividad en la mejora del ajuste del modelo son inmediatas. Sin embargo, cuando los análisis se basan en la estimación robusta no es posible ejecutar este cálculo directo porque la diferencia de dos estadísticos Satorra - Bentler $\chi^{2}$ no se distribuye según una $\chi^{2}$. Por esta razón es necesario aplicar una corrección a la diferencia de estos estadísticos.

En primer lugar se calcula un Coeficiente de Escalamiento $\left(k_{0}\right.$ y $\left.k_{1}\right)$ para ambos modelos ( $M_{0}$ y $\left.M_{1}\right)$ según las expresiones:

$$
\begin{aligned}
& k_{0}=\frac{T_{0}}{\bar{T}_{0}}=\frac{M L \chi_{0}^{2}}{S-B \chi_{0}^{2}} \\
& k_{1}=\frac{T_{1}}{\bar{T}_{1}}=\frac{M L \chi_{1}^{2}}{S-B \chi_{1}^{2}}
\end{aligned}
$$

De la misma forma se calcula la diferencia entre los estadísticos Satorra - Bentler de ambos modelos e igualmente la diferencia de grados de libertad.

$$
\begin{gathered}
D=T_{0}-T_{1} \\
d=d_{0}-d_{1}
\end{gathered}
$$

Partiendo de estos resultados es posible obtener el Coeficiente de Escalamiento Global ( $k$ ) según la expresión:

$$
k=\frac{d_{0} k_{0}-d_{1} k_{1}}{d}
$$

Este coeficiente de escalamiento global es el que se utiliza posteriormente para corregir la diferencia entre los estadísticos Satorra - Bentler:

$$
\bar{D}=\frac{D}{k}
$$

Al mismo tiempo EQS proporciona otros tres estadísticos robustos complementarios a la Satorra - Bentler $\chi^{2}$, odos ellos basados en la distribución de los residuos. Entre ellos es posible encontrar el Residual - Based Statistic, desarrollado por Browne, cuya interpretación sólo es posible cuando el tamaño de muestra es muy elevado (Browne, 1984). Asimismo se presenta el Yuan - Bentler Residual - Based Statistic, propuesto por Yuan y Bentler como una extensión del Residual - Based Statistic, de forma que también puede utilizarse en muestras pequeñas, sin perder su utilidad en muestras de tamaño elevado (Bentler, 2006). Finalmente el Yuan-Bentler Residual Based F - Statistic, presentado por Yuan y Bentler (1998) se considera que 
es el mejor indicador puesto que considera el factor tamaño de muestra de la forma más precisa y adecuada.

En muchas ocasiones el valor del estadístico $\chi^{2}$, tanto en estimación robusta como en estimación estándar, se compara con el valor correspondiente al modelo de independencia (modelo nulo). Habitualmente este modelo representa una situación en que existe total independencia entre todas las variables, es decir, un modelo en el cual no existe relación entre sus distintas variables (Byrne, 2006). Aunque en realidad el modelo independencia debería ser simplemente un modelo realista cuyo ajuste cabe esperar que el resto de modelos sobrepasen, sobre todo aquellos basados en la teoría (Hair et al., 2007). Existe cierto desacuerdo sobre cómo especificar con exactitud el modelo de independencia más adecuado en cada situación y de hecho se han propuestos otros modelos de referencia alternativos (Sobel y Bohrnstedt, 1985). No obstante desde el primer trabajo de Tucker y Lewis, quienes propusieron por primera vez la comparación del estadístico $\chi^{2}$ del modelo al correspondiente al modelo de independencia, el modelo de independencia planteado anteriormente ha sido el más utilizado (Rigdon, 1996). La importancia de este modelo es que proporciona una referencia para el investigador a la hora de comparar y evaluar la mejora en el ajuste en muestras de tamaño elevado (Bentler y Bonett, 1980).

Como se ha podido observar, la dependencia del estadístico $\chi^{2}$ con el tamaño de la muestra es muy elevada (Bentler y Bonett, 1980; Bollen, 1990), llegando a ocurrir en muestras muy grandes que incluso pequeñas diferencias entre las matrices de varianza - covarianza sean evaluadas como significativas. Este problema resulta especialmente evidente en tamaños de muestra superiores a 200 observaciones. Sin embargo, mientras que la sensibilidad del estadístico $\chi^{2}$ limita el número de observaciones, el análisis de modelos estructurales sugiere trabajar con tamaños de muestra elevados con el fin de obtener estimaciones de los parámetros más precisas (Jöreskog y Sörbom, 1993). Al mismo tiempo el problema no se limita simplemente a la definición de un límite superior para el tamaño de la muestra. En los casos en que el tamaño muestral disminuye por debajo de 100 observaciones, aumenta la probabilidad de que las diferencias resulten no significativas, mostrando un ajuste aceptable incluso en los modelos que realmente no lo tienen. Así, la utilización del estadístico $\chi^{2}$ requiere la determinación del tamaño exacto de la muestra que no provoque distorsiones en la estimación de los parámetros o los estadísticos de bondad de ajuste.

Por otro lado este mismo estadístico $\chi^{2}$, unidas al creciente escepticismo respecto a los contrastes de significación estadística que se ha extendido en las últimas décadas entre la comunidad científica como exponen A. Fernández e I. Fernández (2009), han propiciado cierto recelo en la aplica- 
ción de este contraste. Según Cohen los contrastes de significación estadística tradicionales son una práctica de dudosa utilidad que todavía persiste en la actualidad (Cohen, 1994). Otros muchos investigadores han manifestado su adhesión a esta afirmación (Schmidt, 1996; Kirk, 1996; Thompson, 1996) y en la actualidad es muy probable que el uso de contrastes estadísticos de significación según la metodología tradicional esté en vías de extinción (Byrne, 2006).

Una sugerencia para solventar estas limitaciones fue el Estadístico $\chi^{2}$ Normado, propuesto por Jöreskog y Sörbom (1989). Se calcula como la ratio del estadístico $\chi^{2}$ y el número de grados de libertad. Este estadístico plantea dos formas de evaluar el ajuste del modelo. Los valores comprendidos en el intervalo [1,2] indican buen ajuste, mientras que los inferiores a 1 muestran modelos sobreajustados y los superiores a 2 son indicadores de modelos no representativos de los datos. Aunque su planteamiento es lógico se ha demostrado que es poco fiable como indicador de ajuste (Hayduk, 1987; Wheaton, 1987).

\section{Índices de bondad de ajuste}

Debido al elevado número de limitaciones y restricciones a considerar al aplicar el estadístico $\chi^{2}$ en la evaluación del ajuste, se han planteado infinidad de nuevos índices de ajuste aportando un enfoque más pragmático a esta cuestión. Asimismo surge el planteamiento paralelo de los intervalos de confianza de los distintos índices de bondad de ajuste. Esta línea de investigación surgió a partir del trabajo de Steiger quien plantea por primera vez la idea de proporcionar los intervalos de confianza de los índices de ajuste (Steiger y Lind, 1980; Steiger, 1990). Esta idea fue desarrollada más tarde por MacCallum (1996), especialmente en el caso del Root Mean Square Error of Aproximation (Rigdon, 1996). Finalmente el trabajo de Cliff pone de manifiesto la entonces reciente proliferación de nuevos índices de ajuste e intervalos de confianza, aparentemente carentes de coherencia, que inducían a incrementar el número de reespecificaciones de los modelos de forma innecesaria dando importancia a cambios aleatorios y efectos triviales (Cliff, 1983). El conjunto de todas estas investigaciones da lugar a la definición de determinados índices de evaluación (Browne y Cudeck, 1989; Cudeck y Browne, 1983) así como al desarrollo de procedimientos de validación cruzada (MacCallum et al., 1992). Sobre esta cuestión se han planteado numerosos trabajos que han abordado la obtención y las características de cada uno de los índices, así como las convenciones respecto a sus valores aceptables y los supuestos sobre los que se sustentan (Bollen, 1989; Hair et al., 2007; Hu y Bentler, 1995; Marsh et al., 1988; Tanaka, 1993).

Aunque en la mayoría de ocasiones los índices de ajuste varían en el 
mismo sentido y existe un alto grado de acuerdo entre ellos (Marsh et al., 1988), muchas otras veces aportan resultados dispares en la evaluación de un mismo modelo (La Du y Tanaka, 1989). Además, se ha demostrado que dada su disparidad, enfatizan en distintos aspectos del ajuste del modelo (Tanaka, 1993; West, Finch y Curran, 1995). Así, puede haber variaciones en las conclusiones extraídas a partir de cada uno de los índices en función del método de estimación empleado, el tamaño de la muestra y la distribución de los datos.

Existen tres tipologías de índices de bondad de ajuste por lo que se aconseja examinar varios índices pertenecientes a cada una de ellas, para comprobar la existencia de acuerdo generalizado entre ellos.

\section{Índices de ajuste absoluto}

También conocidas como Medidas Absolutas del Ajuste puesto que evalúan el ajuste global del modelo, tanto de los modelos de medida como de los modelos estructurales. Esta evaluación la realizan determinando el grado en que el modelo predice la matriz de covarianzas poblacional a partir de los parámetros estimados. La diferencia con otros índices es que no se basan en la comparación con un modelo de referencia, sino que únicamente dependen de si el modelo hipotetizado se ajusta a los datos.

\section{Goodness - of - Fit Index (GFI)}

Fue propuesto por Jöreskog y Sörbom (1984) y posteriormente reformulado por Cheung y Rensvold (2002). Es una medida de la proporción de varianza y covarianza explicada por la matriz de varianzas - covarianzas estimada, respecto a la matriz de varianzas - covarianzas muestral. Junto con el Adjusted Goodness - of - Fit Index (AGFI) son los dos únicos índices de proporción de varianza.

$$
G F I=\frac{\operatorname{tr}\left(\hat{\sigma}^{\prime} W \hat{\sigma}\right)}{\left.\operatorname{tr}\left(\hat{s}^{\prime} W \hat{s}\right)\right)}
$$

Donde el vector $\hat{\sigma}$ contiene las varianzas de la matriz de varianzas - covarianzas estimada, el vector $\hat{s}$ contiene las varianzas de la matriz de varianzas - covarianzas muestral y $W$ es una matriz de ponderación que varía en función del método de estimación seleccionado.

$\mathrm{Su}$ valor oscila en el intervalo $[0,1]$, siendo recomendables valores superiores a 0.90 para considerar el ajuste del modelo aceptable. 


\section{Adjusted Goodness - of - Fit Index (AGFI)}

Es una corrección del anterior índice GFI que considera tanto el número de parámetros libres $(k)$ como el número de datos disponibles en la matriz de información $(d)$.

$$
A G F I=1-\frac{1-G F I}{1-\frac{k}{d}}
$$

Hu y Bentler (1998) recomendaron no utilizar ambos índices de proporción de varianza (GFI y AGFI) por proporcionar información insuficiente y carente de coherencia en situaciones de mala especificación del modelo. Del mismo modo Marsh et al. (1988) demostraron que ambos índices eran muy sensibles al tamaño de muestra e incluso Jöreskog y Sörbom (1993) señalaron la posibilidad de que podría alcanzar valores negativos, situación que no debería ocurrir en la práctica ya que sería indicador de falta de ajuste absoluta.

Igualmente su valor oscila en el intervalo $[0,1]$, y se recomiendan valores superiores a 0.90 para considerar el ajuste del modelo aceptable.

\section{McDonald Fit Index (MFI)}

Propuesto por McDonald (1989) y McDonald y Marsh (1990) representa una medida normalizada de la centralidad del parámetro. En ocasiones su valor puede superar la unidad debido a errores muestrales (Hu y Bentler, $1995,1999)$ y se recomiendan valores superiores a 0.89 para considerar aceptable el ajuste del modelo.

$$
M F I=\exp ^{-\frac{1}{2} \frac{\chi_{\text {teorico }}^{2}-g l_{\text {teorico }}^{2}}{N}}
$$

Root Mean Square (RMS)

Tanto el índice Root Mean Square como los índices Root Mean Square Residual y Root Mean Square Error of Aproximation se consideran índices de ajuste absoluto, o más concretamente índices de falta de ajuste absolutos (Browne, MacCallum, Kim, Andersen y Glaser, 2002). Todos ellos están basado en los residuos entre las matrices de varianzas - covarianzas muestral y estimada que se derivan del modelo.

Específicamente el índice Root Mean Square representa el residuo medio derivado del ajuste entre las matrices de varianzas - covarianzas muestral y estimada. Sin embargo, puesto que estos residuos son relativos a los tamaños de las varianzas y covarianzas de las variables observables, en ocasiones pueden resultar difíciles de interpretar (Jöreskog y Sörbom, 1989). 


$$
R M R=\sqrt{\frac{\sum_{i=1}^{q} \sum_{j=1}^{i}\left(s_{i j}-\hat{\sigma}_{i j}\right)^{2}}{\frac{p(p+1)}{2}}}
$$

Donde $p$ es el número de variables observables.

Root Mean Square Residual (RMSR)

Se define como la raíz cuadrada de la media de los residuos entre las matrices de varianzas - covarianzas muestral y estimada, al cuadrado. Resulta más útil para residuos de matrices de correlaciones y no para residuos relativos a matrices de varianzas - covarianzas, ya que en primer caso todas las variables se expresan en la misma escala. Su valor está acotado en el intervalo $[0,1]$, siendo recomendables valores inferiores a 0.05 .

\section{$\underline{\text { Root Mean Square Error of Aproximation (RMSEA) }}$}

Este índice parte del trabajo de Steiger y Lind (1980) y plantea en qué medida ajustaría el modelo si fueran alcanzables y estuvieran disponibles los parámetros óptimos. A partir de este planteamiento logra expresar las discrepancias o residuos entre las matrices de varianzas - covarianzas muestral y estimada en términos de grados de libertad, introduciendo así el número de parámetros libres como factor a considerar. En este aspecto difiere del Root Mean Square Residual en que los residuos no se miden únicamente en términos de la muestra empleada (Steiger, 1990).

Respecto a los valores recomendables para considerar el ajuste del modelo aceptable no se ha alcanzado un consenso claro, aunque sí existen ciertas directrices. En términos generales se considera que valores inferiores a 0.05 indican buen ajuste, mientras que los valores comprendidos en el intervalo $[0.05,0.08]$ corresponden simplemente a un ajuste razonable. Al mismo tiempo los valores en el intervalo $[0.08,0.10]$ indican ajuste pobre y los superiores al valor 0.10 señalan falta de ajuste absoluto (Browne y Cudeck, 1993). MacCallum, Browne y Sugarawa (1996) matizan que los valores entre 0.08 y 0.10 indican ajuste mediocre mientras que los superiores a $0.10 \mathrm{sim}$ plemente indican ajuste muy pobre (MacCallum, 1996). Del mismo modo $\mathrm{Hu}$ y Bentler (1999) sugieren un valor de 0.06 para considerar buen ajuste y aconsejan precaución al aplicarlo a muestras pequeñas, ya que aumenta la probabilidad de rechazar el modelo aún cuando el ajuste sea bueno.

En cuanto a la conveniencia de su aplicación, Rigdon (1996) recomienda utilizarlo preferentemente en estrategias de modelización confirmatoria o estrategias de modelos rivales con tamaños de muestra elevados. Sin embargo, MacCallum et al. (1996) recomiendan utilizarlo de forma generalizada no 
sólo porque es adecuadamente sensible a la especificación del modelo, sino porque también aporta directrices para su interpretación a través de los intervalos de confianza. Estos intervalos permiten comparar tanto la bondad de ajuste de distintos modelos como la precisión con que se han realizado las estimaciones. En realidad la mayor parte de las investigaciones sobre intervalos de confianza en índices de ajuste se desarrolla sobre este índice de ajuste. Concretamente EQS proporciona los intervalos de confianza al $90 \%$ superior e inferior, que aportan información sobre la precisión de la estimación. MacCallum et al. (1996) afirman que los intervalos estrechos indican mayor precisión en la estimación mientras los intervalos anchos implican falta de precisión. En cualquier caso estos intervalos pueden estar influidos como el tamaño de muestra así como por la complejidad del modelo.

\section{Índices comparativos de ajuste}

Los índices comparativos de ajuste también se denominan Índices de Ajuste Incremental y comparan el modelo hipotetizado con otro modelo de referencia especificado por el investigador (Browne et al., 2002). Este modelo de referencia suele ser el modelo nulo o modelo de independencia en el cual no se especifica ninguna relación entre las variables. En general, suelen ser fáciles de interpretar ya que en todos los casos sus valores oscilan en el intervalo $[0,1]$ y se recomiendan valores superiores 0.90 para que el ajuste se considere aceptable.

\section{Normed Fit Index (NFI)}

Fue propuesto por Bentler y Bonett (1980) y durante varias décadas fue el principal índice de ajuste consultado de forma generalizada. No obstante se detectó cierta tendencia a infravalorar los modelos con pequeños tamaños de muestra y por esta razón se propone posteriormente el Comparative Fit Index (Rigdon, 1996).

Compara el valor del estadístico $\chi^{2}$ del modelo teórico con el del modelo independiente según la siguiente expresión:

$$
N F I=\frac{\chi_{i n d}^{2}-\chi_{t e o r}^{2}}{\chi_{\text {ind }}^{2}}
$$

Aunque se le aplica el criterio general para los índices comparativos de ajuste de valores que oscilan en el intervalo $[0,1]$ y valores superiores a 0.90 para considerar el ajuste aceptable (Bentler, 1992), posteriormente se ha propuesto un umbral de 0.95 para aceptar esta conclusión (Hu y Bentler, 1999). 
$\underline{\text { Nonnormed Fit Index (NNFI) }}$

Es una variación del Normed Fit Index (NFI), que incorpora los grados de libertad de los modelos teórico y de independencia. De este modo, se toma en consideración la complejidad del modelo y evita la subestimación del ajuste asociada al NFI. En casos extremos puede alcanzar valores fuera del intervalo $[0,1]$ y en muestras de tamaño muy pequeño puede indicar un ajuste excesivamente bajo al compararse con otros modelos (Anderson y Gerbing, 1984; Ullman, 1996).

$$
N N F I=\frac{\chi_{i n d}^{2}-\frac{g l_{\text {ind }}}{g l_{\text {teor }}} \chi_{\text {teor }}^{2}}{\chi_{\text {ind }}^{2}-g l_{\text {ind }}}
$$

$\underline{\text { Incremental Fit Index (IFI) }}$

El Índice Incremental de Ajuste fue propuesto por Bollen (1989) y pretende corregir la posibilidad de que el NNFI tome valores fuera del intervalo $[0,1]$. Asimismo se diseñó para tener en consideración factores como la complejidad del modelo y el tamaño de la muestra, condicionantes del NFI. En este caso se consideran adecuados valores superiores a 0.95 ( $\mathrm{Hu}$ y Bentler, 1999).

$$
I F I=\frac{\chi_{\text {ind }}^{2}-\chi_{\text {teor }}^{2}}{\chi_{\text {ind }}^{2}-g l_{\text {teor }}^{2}}
$$

$\underline{\text { Comparative Fit Index (CFI) }}$

Fue propuesto por Bentler (1990) y corrige los índices anteriores mediante el número de grados de libertad. Bentler lo propone como principal índice de referencia y, al igual que el Incremental Fit Index (IFI) se consideran adecuados valores superiores a 0.95 .

$$
C F I=\left|\frac{\left(\chi_{i n d}^{2}-g l_{i n d}\right)-\left(\chi_{i n d}^{2}-g l_{t e o r}\right)}{\chi_{i n d}^{2}}-g l_{i n d}\right|
$$

Índices de parsimonia

Los índices de parsimonia, también denominados Medidas de Ajuste de Parsimonia, ajustan las medidas de ajuste para ofrecer una comparación entre modelos con distinta complejidad. La interpretación de estos índices no se realiza en términos absolutos sino relativos, con el fin de determinar cuál de los modelos goza de mayor parsimonia. 


\section{Akaike Information Criterion (AIC)}

Fue propuesto por Aikaike y su interpretación se basa en que la parsimonia del modelo aumenta a medida que disminuye su valor (Aikaike, 1987).

$$
A I C=\chi_{\text {teor }}^{2}-2 \cdot g l_{\text {teor }}
$$

No existe un criterio para determinar cuándo el valor de este índice es suficientemente bajo. Según Ullman la evaluación del AIC sólo puede entenderse en términos comparativos con otros modelos teóricos (Ullman, 1996). Por ello siempre se presenta este resultado acompañado del correspondiente índice AIC del modelo independiente, considerado como el modelo base respecto al cual siempre debe haber una mejora. Así, cuanto mayor el decremento del índice en el modelo hipotetizado respecto al índice del modelo de independencia, mejor se considerará que ajusta el primero.

\section{Consistent Akaike Information Criterion (CAIC)}

El CAIC es una mejora del índice AIC anterior propuesta por Bozdogan (1987). Por consiguiente todas las consideraciones aplicables al AIC también lo son en este caso.

$$
C A I C=\chi_{\text {teor }}^{2}-(\ln N-1) g l_{\text {teor }}
$$

Ambos índices consideran tanto el ajuste del modelo como el número de parámetros libres a estimar. Sin embargo es fácil observa que, mientras que el AIC está relacionado con el número de grados de libertad, el CAIC está vinculado al tamaño de muestra.

\section{Parsimonius Goodness-of-Fit Index (PGFI)}

El índice PGFI se define como el Índice de Calidad de Ajuste De Parsimonia. Modifica el índice GFI, aunque de forma diferente al índice AGFI, ya que en este caso la modificación se plantea en base a la parsimonia del modelo. Así, un incremento en el valor del PGFI implica una mejora en la parsimonia del modelo.

$$
P G F I=\frac{g l_{\text {propuesto }}}{\frac{1}{2} N \cdot V_{\text {observables }} \cdot\left(N \cdot V_{\text {observables }}+1\right)} \cdot G F I
$$




\section{Parsimonius Normed Fit Index (PNFI)}

Se define como el índice de ajuste normalizdo de parsimonia y constituye una modificación del NFI para tener en cuenta el número de grados de libertad utilizados. Por ello se aplica en la comparación de modelos con diferentes grados de libertad. Al igual que en el caso anterior, un incremento en el valor del PNFI implica una mejora en el ajuste del modelo. Aunque no existen niveles recomendados de ajuste, para aceptar el modelo se sugiere que las diferencias oscilen en el intervalo [0.06, 0.09].

$$
\text { PNFI }=\frac{g l_{\text {propuesto }}}{g l_{\text {nulo }}} \cdot N F I
$$

Consideraciones respecto al uso de los índices de ajuste

A pesar de las limitaciones del estadístico $\chi^{2}$ en la evaluación de la bondad de ajuste del modelo, no resulta conveniente confiar únicamente en los índices de ajuste ya que no proporcionan una garantía de que el modelo sea útil. De hecho, cabe la posibilidad de que los índices señalen un buen ajuste del modelo, pero que esté mal especificado (Wheaton, 1987). Por consiguiente, los índices de ajuste aportan información sobre la posible falta de ajuste del modelo, pero no reflejan si el modelo es factible. La decisión final sobre la adecuación del modelo a los datos debe tomarla el investigador considerando tanto criterios estadísticos y prácticos como la base teórica subyacente al modelo (Sobel y Bohrnstedt, 1985).

\section{Valoración del ajuste del modelo de medida}

\section{Test de significación de parámetros}

A la hora de comprobar si la estimación de los parámetros es adecuada es necesario considerar su magnitud, su signo y su significación estadística son coherentes con la teoría (Byrne, 2006). En la estimación de los parámetros se proporcionan los coeficientes no estandarizados y los estandarizados, mostrados en la solución estandarizada del modelo. Para evaluar la significación estadística de las estimaciones de los parámetros no estandarizados se utiliza el Estadístico t, o Razón Critica, calculado a partir del cociente entre el valor del estimador y su error estándar. Este valor se comporta como un estadístico $Z$ en cuanto al constraste del estimador como valor distinto de cero. Entonces, para un nivel de significación de 0.05, un valor del estadístico t comprendido en el intervalo [-1.96, 1.96], indicará que el parámetro en cuestión no es significativo, en cuyo caso habrá que fijarlo a un valor o directamente eliminarlo. En caso de que el número de estimadores no estandarizados sea muy elevados el investigador debería plantearse si el 
tamaño de muestra es excesivamente bajo (Jöreskog y Sörbom, 1996).

Tradicionalmente se ha considerado el nivel de significación de 0.05 , pero en tamaños de muestra reducidos es recomendable reducirlo a niveles más pequeños como 0.025 o incluso 0.01 (Hair et al., 2007). Asimismo, si se supone una relación positiva o negativa, será posible emplear tests de significación unilaterales, y no bilaterales como es habitual. Todo ello repercutirá en los valores críticos del estadístico t utilizados para evaluar la significación.

Del mismo modo los errores estándar asociados a los estadísticos no estandarizados aportan información sobre la precisión en la estimación del parámetro. En este caso no se trata simplemente de valorar su magnitud. Mientras que los errores estándar de magnitud elevada que el parámetro no puede ser determinado, los errores próximos a cero señalan que la signficación estadística de la estimación del parámetro no puede ser calculada (Bentler, 2006). No obstante, puesto que los errores estándar están influidos por la escala de medida de las variables observables, no se ha definido ningún criterio sobre que lo constituye "pequeño" o "grande" (Jöreskog y Sörbom, 1989).

\section{Matriz residual de covarianzas}

La Matriz Residual de Covarianzas es una matriz formada por las diferencias entre las matrices de varianzas - covarianzas muestral y poblacional. Idealmente sus valores deberían tomar valores próximos a cero, de forma que el análisis de estos residuos permite identificar errores de predicción entre ambas matrices (Levy y Varela, 2006).

A simple vista es posible identificar en esta matriz determinados aspectos del ajuste del modelo. En primer lugar, la presencia de residuos de magnitud elevada asociados a un parámetro concreto, indica falta de especificación en este término que afecta al ajuste global del modelo. En este aspecto, al igual que en los análisis de regresión convencionales, no tendría sentido validar la significación estadística de los residuos de forma independiente. Únicamente resulta adecuado valorar en términos relativos la magnitud de los residuos para identificar posibles parámetros mal especificados como se ha indicado (Byrne, 2006). Del mismo modo, si en términos generales los valores de esta matriz son pequeños y se distribuyen adecuadamente la probabilidad de que el modelo ajuste bien globalmente aumenta.

Los residuos de esta matriz se expresan en las unidades de medida de la escala, y por tanto pueden ser difíciles de interpretar por lo que habitualmente se utilizan los residuos estandarizados. Estos últimos se calculan simplemente mediante el cociente entre los residuos no estandarizados y sus 
errores estándar correspondientes (Jöreskog y Sörbom, 1989). Por tanto los residuos estandarizados se comportan como las puntuaciones $\mathrm{Z}$ y resultan más fáciles de interpretar. Así, para un nivel de significación de 0.05, todo residuo estandarizado fuera del intervalo $[-2.58,+2.58]$ indicará que la matriz de covarianzas no se ha podido reproducir convenientemente a partir de la estimación de los parámetros estructurales, o en otras palabras, señalará un error de predicción para un determinado par de indicadores. Este criterio para evaluar el nivel de significación aporta información sobre la existencia de posibles diferencias significativas, pero no indica cómo eliminarlas. A partir de este resultado el investigador deberá decidir si las modifica o las añade a otras relaciones causales (Costner y Schoenberg, 1979; Glymour, 1988).

A partir de esta matriz EQS calcula dos promedios, el primero de ellos basado en todos los elementos de la matriz triangular inferior, incluyendo los elementos de la diagonal. El segundo promedio está basado en los elementos de la matriz triangular inferior, pero sin incluir estos elementos de la diagonal, ya que es este subconjunto de elementos el que tiene mayor influencia sobre la bondad de ajuste del modelo (Bentler, 2006).

\section{Índices de modificación}

Fueron propuestos por Jöreskog y Sörbom (1989) e informan del cambio esperado en el estadístico $\chi^{2}$ en caso de liberar el parámetro fijo considerado y reestimar el modelo manteniendo el resto de parámetros estables. Habitualmente se calculan como la diferencia entre los estadísticos $\chi^{2}$ del modelo con el parámetro fijo y el modelo con el parámetro libre. Este cálculo se aplica a todos los parámetros fijos del modelo y se muestran en un listado junto con los resultados de la estimación del modelo.

Puesto que el Índice de Modificación (IM) se calcula como la diferencia de dos estadísticos $\chi^{2}$, también se comporta como una distribución $\chi^{2}$ y su significación estadística puede evaluarse de forma tradicional. Así un valor del índice de modificación superior a 3.84, valor de la distribución $\chi^{2}$ con nivel de significación 0.05 y un grado de libertad, implica una reducción estadísticamente significativa en el estadístico $\chi^{2}$ original.

Este índice resulta muy útil para el análisis de posibles relaciones de multicolinealidad o saturaciones cruzadas entre indicadores. No obstante el investigador no debería hacer cambios basándose únicamente en los índices de modificación, ya que esta forma de proceder supone un enfoque ateórico completamente contrario a la metodología de los modelos estructurales.

Además del índice de modificación, LISREL ofrece un parámetro de cambio estimado, que hace referencia a la magnitud y dirección de cada paráme- 
tro fijo y EQS ofrece los contrastes de Lagrange y de Wald.

\section{Validez convergente y discriminante}

El análisis de la Validez Convergente y Discriminante fue propuesto por Campbell y Fiske (1959) y se basa en el análisis de las medidas correlacionales presentes en las Matrices Multirrasgo - Multimétodo. La evidencia de uno de los dos tipos de validez aportan información para tomar decisiones acerca de si agregar o desagregar factores, con el fin de que realmente midan conceptos distintos.

Los coeficientes de Validez Convergente hacen referencia a las correlaciones entre medidas de un mismo constructo usando diferentes indicadores. Así, las correlaciones altas muestran indicios de convergencia entre estos constructos similares que están medidos a través de indicadores diferentes. Por el contrario las correlaciones bajas indican ausencia de relación entre estos constructos. Se considera que existe validez convergente cuando la correlación entre factores es superior a 0.85 (Levy y Varela, 2006).

Los coeficientes de Validez Discriminante hacen referencia tanto a medidas de correlación entre diferentes constructos con el mismo método de medida (heterorasgo - monométodo) como a medidas de correlación entre diferentes constructos con distintos métodos de medida (heterorasgo - heterométodo). Cabe esperar que estas correlaciones sean bajas, puesto que reflejan el grado de diferenciación entre constructos. De este modo, a medida que decrece esta correlación, se evidencia que cada constructo es diferente al resto. Se considera que existe validez discriminante cuando la correlación entre factores es inferior a 0.50 .

\section{Fiabilidad y validez}

Levy y Varela (2006) definen la Validez como la adecuación de los indicadores a sus variables latentes correspondientes mientras que la Fiabilidad se basa en la obtención de resultados consistentes en mediciones sucesivas. Según Hair et al. (2007), la fiabilidad de una variable es el grado en que una variable independiente está "exenta de error", considerando que un coeficiente de regresión entre una variable dependiente y otra independiente está compuesto por un coeficiente estructural o "verdadero" y otra componente de error. Por tanto, a menos que la fiabilidad de un determinado indicador sea del $100 \%$, la correlación observada entre este indicador y el constructo al que se vincula, siempre será diferente de la relación estrictamente correcta.

La utilidad de los índices de fiabilidad y validez cobra mayor importancia 
en los casos en que el tamaño de muestra es elevado, ya que en este caso rara vez los estadísticos t resultarán no significativos. Esto ocurre así porque el error estándar disminuye a medida que se incrementa el tamaño muestral. En este caso el hecho de disponer de medidas alternativas sobre la representatividad de las variables respecto a los constructos constituye una herramienta de gran utilidad (Bollen, 1989).

\section{Coeficiente de fiabilidad Alfa de Cronbach}

Fue propuesto por Cronbach y desde entonces ha sido, sin duda, la medida de consistencia interna que más se ha utilizado en los últimos años (Cronbach, 1951). Sin embargo, su aplicación a la medida de fiabilidad de los modelos estructurales es bastante cuestionable, sobre todo en modelos multidimensionales. La razón es que se basa en un modelo muy restrictivo de un solo factor que requiere que todas las cargas factoriales y las varianzas de los errores sean iguales (Bentler, 2006). Este supuesto de unidimensionalidad subyace al cálculo de la fiabilidad y únicamente se cumple cuando los indicadores tienen un ajuste aceptable sobre un modelo de un único factor, o modelo unidimensional. Es decir, el coeficiente de fiabilidad Alfa de Cronbach supone que existe un modelo unidimensional y, puesto que es evidente que estas condiciones no se cumplen en la gran mayoría de los Análisis Factoriales Confirmatorios, este coeficiente no resulta un buen indicador de la consistencia interna de los constructos en estos casos. Adicionalmente Cronbach ha presentado recientemente un estudio sobre la no aplicabilidad de este contraste a determinados análisis (Cronbach y Shavelson, 2004).

\section{Coeficientes de fiabilidad de EQS}

Como contrapartida EQS proporciona otros coeficientes de fiabilidad que sí resultan buenos estimadores de la consistencia interna del modelo. Estos coeficientes no sólo no se ven influidos por la estructura del modelo sino que asumen que existe algún tipo de estructura factorial con un desconocido, pero elevado, número de factores latentes.

El primero de estos coeficientes es el Coeficiente Rho, que se basa en el modelo de variables latentes que se está ejecutando. A continuación los coeficientes Greatest Lower Bound Reliability, Bentler's Dimension - Free Lower Bound Reliability y Shapiro's Lower Bound Reliability se basan en una suma ponderada de las variables alcanzar una mayor fiabilidad. Concretamente el Shapiro's Lower Bound Reliability no se basa en ningún supuesto específico sobre el número de factores mientras que el Bentler's Dimension - Free Lower Bound Reliability se utiliza preferentemente en modelos unidimensionales y en los últimos años ha alcanzado bastante popularidad bajo el concepto de fiabilidad del constructo (Hancock y Mueller, 2006). 


\section{Coeficiente de determinación}

El Coeficiente de Determinación se estima para cada ecuación factorial, y para la ecuación $i$ se expresa según la nomenclatura $R_{x_{i}}^{2}$. Se calcula según la expresión:

$$
R_{x_{i}}^{2}=1-\frac{\operatorname{Var}\left(\delta_{i}\right)}{\hat{\sigma}_{i}^{2}}
$$

Donde $\operatorname{Var}\left(\delta_{i}\right)$ es la varianza estimada del error de medida de la ecuación y $\sigma_{i}^{2}$ es la varianza de la variable estimada.

Este coeficiente informa de la proporción de varianza de la variable observable que logra ser explicada por la/s variable/ s latente/s que lo afecta/n y según Bollen (1989), constituye una medida directa de fiabilidad sobre la adecuación de los indicadores en la medición de las variables latentes. Es decir, que aporta información sobre la parte de medida del indicador que está libre del error puramente aleatorio. En caso de que el indicador sólo dependa de una variable latente, el valor de este coeficiente coincide con el cuadrado de su saturación sobre dicha variable $\lambda_{i j}^{2}$.

Es recomendable obtener altos valores de este coeficiente (superiores a 0.50 ), ya que ello implica que los indicadores representan adecuadamente a las variables latentes. No obstante, los valores de $R_{x_{i}}^{2}$ pueden verse afectados por posibles relaciones de multicolinealidad entre variables. Por esta razón siempre que los valores de este coeficiente superen el umbral de 0.90 deberían examinarse los resultados por si realmente se deben a este efecto no deseado.

\section{Fiabilidad compuesta del constructo}

La mayoría de los programas estadísticos no proporcionan estos coeficientes, pero es fácil calcularlos a partir de la expresión:

$$
\rho_{x x}=\frac{\left(\sum \lambda_{j}^{s}\right)^{2}}{\left(\sum \lambda_{j}^{s}\right)^{2}+\sum \varepsilon_{j}}
$$

Donde $\lambda_{j}^{s}$ representa los coeficientes estandarizados de cada indicador del constructo y $\epsilon_{j}$ el error de medida asociado a los indicadores. Estos coeficientes estandarizados se obtienen directamente de la estimación del modelo y el error de medida correspondiente a cada indicador se calcula restando a la unidad el cuadrado del coeficiente estandarizado anterior.

El umbral recomendable para los coeficientes estandarizados es 0.7 , lo que corresponde a una fiabilidad compuesta de cada constructo similar a 
0.5. Este valor no es una referencia absoluta y se ha demostrado que son igualmente aceptables valores inferiores en investigaciones exploratorias.

\section{Varianza extraída por el constructo}

Este coeficiente aporta información sobre la cantidad total de la varianza de los indicadores considerada por el constructo latente de forma que se incrementa a medida que se aumenta la representatividad de los indicadores respecto al constructo. Es una medida complementaria a la fiabilidad compuesta del constructo y se calcula según la expresión:

$$
\operatorname{Var}=\frac{\sum\left(\lambda_{j}^{s}\right)^{2}}{\left(\lambda_{j}^{s}\right)^{2}+\sum \varepsilon_{j}}
$$

En este caso son recomendables valores iguales o superiores a 0.50.

\section{Especificación de la fiabilidad de los indicadores}

Así como es posible estimar la fiabilidad correspondiente a cada indicador, también es posible especificar a priori la fiabilidad de cada uno de ellos. Esta posibilidad parece no ser coherente con los procedimientos de estimación empírica de la fiabilidad descritos anteriormente. Sin embargo, en determinados casos está totalmente justificado e incluso es recomendable.

En primer lugar, puede ocurrir que no sea posible estimar la fiabilidad del constructo porque sólo se haya definido un indicador. En este caso se puede fijar la fiabilidad del indicador igual a la unidad, indicando que no existe error de medida. También es posible realizar alguna estimación de la fiabilidad y fijar ese valor estimado. Esta segunda opción resulta más correcta que la anterior, puesto que casi nunca las medidas están exentas de error, lo cual debería ser considerado en la especificación de la fiabilidad.

Asimismo cuando los indicadores se hayan utilizado previamente y se conozca su fiabilidad de antemano será conveniente utilizar esta fiabilidad. Esto se aplica fundamentalmente al uso de escalas validadas. Del mismo modo resulta aplicable en los enfoques de estimación en dos pasos, según los cuales se evalúa en primer lugar la fiabilidad de los indicadores para después ser seleccionados en la fase de especificación. Este método de estimación evita la interacción entre los modelos de medida y estructurales y resulta mejor aproximación cuando el modelo posee tanto fuerte lógica teórica como medidas altamente fiables. 


\section{Coeficientes de validez}

El Coeficiente de Validez No Estandarizado $\lambda_{i j}$ contiene la saturación de la variable latente $j$ en el indicador $i$. Resulta especialmente útil en la comparación de la validez de indicadores con igual escala de medida. Por otro lado el coeficiente de validez estandarizado $\lambda_{i j}^{s}$ se aplica en la comparación de indicadores con distintas escalas de medida.

\section{Validación cruzada}

El principal objetivo de los procedimientos de Validación Cruzada es comprobar en qué medida el modelo se ajustará también a otras posibles muestras de la misma población. Este procedimiento adaptado al análisis de estructuras de covarianza por Cudeck y Browne (1983) con el objetivo de posibilitar la comparación de modelos alternativos y seleccionar aquel que se aproximara mejor a la realidad. En términos generales se recomienda utilizar tamaños de muestra elevados (superiores a 800 observaciones) para obtener resultados fiables de validación cruzada (MacCallum et al., 1992).

Existen dos vías principales para evaluar los modelos mediante validación cruzada. El primer método consiste en dividir la muestra en dos submuestras de forma aleatoria: una germinal y otra de validación. A continuación se proponen distintos modelos alternativos y se realiza la estimación de sus parámetros sobre la muestra germinal. Se escoge aquel modelo que mejor ajuste haya obtenido y se valida sobre la muestra de validación. Finalmente se compara el ajuste obtenido por ese modelo en ambas muestras.

Del mismo modo es posible evaluar los valores de los llamados Índices de Validación Cruzada, que informan de la bondad de ajuste esperada por cada modelo en otra muestra del mismo tamaño. No existe un rango específico de valores aceptables, pero son preferibles los índices con menor valor absoluto.

El primero de ellos, el Índice de Validación Cruzada Esperada (ECVI) es una aproximación a la bondad de ajuste que conseguiría el modelo en otra misma muestra del mismo tamaño. No tiene un rango especificado de valores, pero se utiliza en la comparación de modelos alternativos.

$$
E C V I=\frac{\chi^{2}}{N-1}+\frac{2 \cdot N u m \text { parametros estimados }}{N-1}
$$

Por otro lado el Índice de Validación Cruzada (CVI) evalúa la calidad del ajuste cuando se ha llevado a cabo una validación cruzada y compara las matrices de varianzas - covarianzas sobre la muestra germinal y la muestra 
de validación.

\section{Soluciones estandarizadas}

La Solución Estandarizada muestra todas las ecuaciones del modelo de forma que todos los coeficientes tienen igual varianza y un valor máximo igual a la unidad. Por tanto los coeficientes cercanos a la unidad indican una relación fuerte mientras que los cercanos a cero señalan un efecto muy débil o prácticamente nulo. Son útiles en la determinación de la importancia relativa de cada coeficiente en cada muestra, pero no aportan información comparable entre distintas muestras. Para ello es necesario recurrir a los coeficientes no estandarizados, que sí se expresan en la escala de medida del constructo, y por tanto sí son comparables entre distintas muestras. Sin embargo, la interpretación de estos últimos coeficientes puede no ser inmediata, ya que la escala de medida puede variar para cada constructo.

\section{Fase 5: Reespecificación del modelo}

Si tanto el ajuste global del modelo como el correspondiente a cada parámetro se consideran aceptables, el modelo teórico será aceptado. En el caso de que no ocurra así, se revisarán tanto los tests de significación de los parámetros como la matriz de residuos normalizados y los índices de modificación. Entre las modificaciones que pueden realizarse es posible eliminar aquellos parámetros que no hayan resultado significativos, corregir la multicolinealidad o añadir parámetros que muestren un índice de modificación elevado.

Estas modificaciones deben introducirse de forma gradual y siempre apoyándose en una justificación teórica que sustente el cambio o corrección, ya que la modificación de un solo parámetro incide en la estimación de los demás. Por esta razón es necesario comprobar el ajuste del modelo cada vez que se introduce una nueva modificación. En esta línea Robles (1996) propone clasificar los parámetros en teóricos y empíricos y mantener intactas las relaciones esenciales subyacentes en la teoría. Así, se facilita la definición de un conjunto de modelos modelos rivales, o incluso anidados, con diferentes niveles de parsimonia para la misma teoría subyacente.

En la actualidad todavía no se ha alcanzado un consenso sobre la conveniencia o no conveniencia de los procedimientos de reespecificación. Algunos investigadores han criticado severamente esta práctica e incluso los más puristas han argumentado que, si se rechaza el modelo hipotetizado, no es correcto realizar modificaciones para subsanar este problema (Cliff, 1983; Cudeck y Browne, 1983). Por el contrario otros investigadores han señala- 
do que, sin olvidar el enfoque exploratorio del procedimiento, puede tener sentido considerar la significación estadística en la introducción de modificaciones al modelo (Byrne, Shavelson y Muthen, 1989). Acorde con esta opinión Jöreskog afirmó que, en caso de rechazar el modelo, es necesario determinar qué fallo ha habido en el modelo y cómo debería reespecificarse para que el ajuste mejore (Jöreskog y Sörbom, 1993).

\section{Introducción de modificaciones triviales}

En la introducción de modificaciones durante la fase de reespecificación es posible que el investigador no considere si éstas están soportadas por el marco teórico y simplemente las acepte porque mejoran empíricamente el ajuste del modelo. Esta forma de proceder es científicamente incorrecta, ya los cambios introducidos deben ser teóricamente interpretables y justificables en todos los casos (Pedhazur, 1982; Sörbom, 1989). De hecho, MacCallum et al. (1992) señalan que cuando un modelo ajusta bien inicialmente, probablemente no sea conveniente modificarlo para mejorar su ajuste, ya que las modificaciones introducidas pueden deberse simplemente a pequeñas características idiosincrásicas de la muestra. Asimismo Jöreskog y Sörbom (1993) comenta que no tendría sentido aceptar la primera modificación propuesta por el contraste de Lagrange, si no resulta coherente con el marco teórico. En este caso sería preferible considerar el parámetro situado en la segunda posición.

De acuerdo con esta afirmación, Hatcher (1994) y MacCallum et al. (1992) recomiendan realizar pocas modificaciones y aceptar únicamente los cambios que puedan ser interpretables y justificables según la teoría. Asimismo sugieren utilizar muestras de tamaño elevado (al menos superior a a 100 observaciones) y seguir determinados procedimientos, como trabajar con dos muestras independientes sobre las que se validen las modificaciones en paralelo, o comparar modelos alternativos y escoger el que muestre mejor ajuste. En cualquier caso en la redacción del trabajo se deberán describir detalladamente las limitaciones del estudio.

$\mathrm{Si}$, a pesar de estas consideraciones, el investigador decide continuar con la introducción de modificaciones desde un enfoque exploratorio debe ser consciente de ello. En este caso estos análisis post-hoc reciben el nombre de Búsquedas de Especificación (MacCallum, 1986). No obstante, como ya se ha comentado, esta práctica debe realizarse con precaución, ya que el hecho de sobreajustar un modelo conlleva el riesgo de añadir parámetros frágiles, en el sentido de que representan efectos muy débiles que son difícilmente replicables. Además estos parámetros suelen conllevar un incremento significativo del error estándar e influyen sobre los parámetros principales del modelo (Wheaton, 1987). 


\section{Contraste del multiplicador de Lagrange y Contraste de Wald}

El Contraste del Multiplicador de Lagrange permite evaluar la mejora que se obtendría al añadir una nueva relación causal o covarianza al modelo teórico. Por el contrario, el Contraste de Wald comprueba si determinados conjuntos de parámetros, especificados como libres en el modelo, pueden en realidad ponerse a cero sin pérdida sustantiva de significado en el ajuste del modelo. Es decir, comprueba si deberían suprimirse alguno de los parámetros existentes (Wald, 1943).

Tanto las mejoras por adición de parámetros, evaluadas mediante el contraste de Lagrange, como las mejoras por eliminación de parámetros, en este caso evaluadas mediante el contraste de Wald, conllevan un determinado nivel de significación que indican si la modificación introducida supondría una mejora significativa del ajuste del modelo. Las modificaciones propuestas por estos contrastes se basan únicamente en criterios estadísticos por lo que el investigador debe valorar si están sustentadas en la teoría (Byrne, 2006). Adicionalmente Ullman propone ser conservador con el nivel de significación considerado y reducirlo hasta $\alpha=0,01$ para el Contraste de Lagrange, ya que el error Tipo I suele incrementarse en los procedimientos "paso a paso" (Ullman, 1996). Asimismo se sugiere añadir primero los parámetros aceptados a partir del Contraste de Lagrange y seguidamente eliminar los seleccionados en el Contraste de Wald.

En el contraste de Lagrange no es necesario comprobar si el incremento en el estadístico $\chi^{2}$ es significativo ya que existe equivalencia entre este contraste y el correspondiente a las diferencias en el estadístico $\chi^{2}$. Es decir, que las modificaciones propuestas como significativas por el contraste de Lagrange siempre darán lugar a modelos con un ajuste significativamente mejor al anterior (Bentler, 2006; Yuan y Bentler, 2004b).

EQS muestra dos versiones de este contraste. La versión univariante presenta el incremento en el estadístico $\chi^{2}$ considerando de forma individual cada una de las relaciones, pero obviando las covarianzas entre los distintos estimadores de los parámetros. Por el contrario la versión multivariante, recomendada como referencia, identifica el parámetro que conduciría a un mayor incremento en el estadístico $\chi^{2}$ considerando todos los demás.

La forma de especificar en EQS qué parámetros deben ser considerados en cada caso se basa en las tres matrices fundamentales de este programa. Estas matrices son $\Phi(\mathrm{PHI})$ que contiene las correlaciones entre constructos exógenos, $\Gamma$ (GAMMA), que indica las relaciones de constructos endógenos a exógenos y $B$ (BETA), que muestra las relaciones de constructos endógenos 
a endógenos. Así, el comando SET del programa admite hasta tres letras de forma que la primera letra representa la matriz $(P, G$ o $B)$ y las otros dos representa una submatriz de las anteriores. Por ejemplo, $V F$ muestra la submatriz que contiene los coeficientes de regresión de las variables dependientes observables $V$ a las variables independientes latentes $F$ y $E E$ muestra la submatriz con las covarianzas entre los errores de medida de las variables independientes. De esta forma las combinaciones más frecuentes son $G V F$ y $P E E$, aunque es posible realizar otras muchas combinaciones. De hecho, las covarianzas entre los errores de medida de las variables observables suelen deberse a de medida aleatorios de las respuestas, y posiblemente de características específicas de las personas que responden a la encuesta por lo que Aish recomienda no considerarlos (Aish y Jöreskog, 1990).

\subsubsection{Modelos sobre múltiples muestras}

\section{Consideraciones generales}

La contrastación de la Invarianza Factorial analiza si existe equivalencia en la estructura factorial de varios grupos. Este análisis se sustenta en el supuesto de que un mismo instrumento opera de la misma manera para cada uno de los grupos considerados, suponiendo en todos los casos que los modelos de medida de los constructos tienen la misma estructura teórica. Sin embargo, rara vez se confirma esta suposición (Byrne, Stewart y Lee, 2004). Los análisis de invarianza no sólo se aplican para la contrastación de la invarianza de un instrumento de medida sino también en la generabilidad de un constructo teórico y las relaciones entre sus variables y la identificación de coeficientes de regresión estructurales comunes a distintos grupos (Byrne, 2006; Levy y Varela, 2006). En cualquier caso, aunque la mayoría de los trabajos de investigación plantean la equivalencia entre el número de factores y relaciones entre variables de distintos grupos, no se trata de una condición necesaria en la contrastación de la invarianza entre grupos.

\section{Procedimiento de análisis de modelos sobre múltiples muestras}

\section{Fase 1: Especificación del modelo con múltiples muestras}

El análisis de la equivalencia factorial entre grupos suele comenzar con un análisis preliminar en el que se examina la bondad de ajuste de un mismo modelo base sobre diferentes muestras por separado. El objetivo de este análisis es comprobar que el modelo base hipotetizado ajusta suficientemente bien en todas las muestras y que es interpretable en cada una de ellas (Levy y Varela, 2006). El hecho de que el modelo resulte aplicable en todos los grupos no implica que ya se haya alcanzado la invarianza, sino que es 
posible realizar la estimación de los parámetros en base a la misma estructura factorial. Así, al aplicar el mismo modelo base sobre diferentes muestras varía para cada muestra la matriz de varianzas - covarianzas y la correspondiente estimación de los parámetros. Este resultado permite conocer las diferencias entre distintas muestras y valorar la pertinencia de aplicar el examen de invarianza a los distintos grupos.

Incluso cabe la posibilidad de que, a partir de este análisis, el investigador decida trabajar con distintos modelos base en alguno de los grupos, con el fin de incrementar la bondad de ajuste del modelo global. En este caso, el test de invarianza se calcula de la misma forma, salvo por el hecho de que únicamente se aplica sobre los parámetros coincidentes en cada factor, mientras que los parámetros no coincidentes se estiman libremente (Byrne et al., 1989).

\section{Modelos anidados y definición de jerarquías de restricciones}

La contrastación de la invarianza factorial está ligada a la imposición de restricciones y su aceptación requerirá que algunos o todos los parámetros tomen el mismo valor en los diferentes grupos (Marsh et al., 1988). El procedimiento para imponer estas restricciones se fundamenta en una jerarquía de forma que el modelo con las restricciones que genere un mejor ajuste a los datos, será el que mejor defina el grado de invarianza entre los grupos.

El primer procedimiento parte de la definición de un modelo inicial sin ninguna restricción que se toma como referencia para el análisis de modelos más restrictivos, anidados a este modelo inicial. Esta forma de proceder resulta más adecuada en análisis exploratorios puesto que, partiendo de una hipótesis genérica, el objetivo es la identificación de los parámetros a restringir para lograr un mejor ajuste.

Por otro lado, Jöreskog (1971) propone definir el modelo inicial con todos los parámetros restringidos y liberarlos progresivamente de forma que se generen nuevos modelos comparables al modelo de base con todos los parámetros restringidos. Este procedimiento se aplica con mayor frecuencia en fases posteriores del análisis y sobre todo en los análisis de invarianza parcial (Byrne, 2006). En este caso el primer modelo totalmente restringido plantea la hipótesis nula:

$$
H_{0}: \Sigma_{1}=\Sigma_{2}=\ldots=\Sigma_{G}
$$

Donde G es el número de grupos.

Esta hipótesis propuesta por Jöreskog es la más restrictiva que puede 
plantearse e incluye todas las hipótesis menos restrictivas que progresivamente incorporan la liberación de determinados parámetros. Así, si esta hipótesis no puede rechazarse desde el punto de partida inicial, entonces puede considerarse directamente que todos los grupos tienen la misma estructura de covarianzas y por tanto no serán necesarios posteriores análisis de invarianza. Este procedimiento, que parece lógico y directo, puede llevar a conclusiones contradictorias, como por ejemplo rechazar la hipótesis nula global, aunque sea válida para los modelos de medida y estructural (Byrne, 1988).

Por ello se recomienda comenzar el análisis de invarianza según el primer procedimiento exploratorio, basado en añadir parámetros al modelo no restringido, y refinar los resultados obtenidos mediante el segundo procedimiento, consistente en la liberación progresiva de parámetros a partir de un modelo completamente restringido.

Entonces, en el caso del primer procedimiento es necesario aplicar una jerarquización de restricciones de forma exploratoria y adecuada a los objetivos de la investigación. Puesto que Jöreskog propone analizar en primer lugar el modelo de medida y a continuación el modelo estructural (Jöreskog, 1971) se muestra el orden habitual de restricciones, aunque no único, aplicado a los modelos de medida:

$H_{f}$ : La misma forma sin restricciones

$$
\begin{aligned}
& H_{\Lambda_{x}}: \Lambda_{x}^{(1)}=\Lambda_{x}^{(2)}=\ldots=\Lambda_{x}^{(g)} \\
& H_{\Lambda_{x} \Phi}: \Lambda_{x}^{(1)}=\Lambda_{x}^{(2)}=\ldots=\Lambda_{x}^{(g)} ; \Phi^{(1)}=\Phi^{(2)}=\ldots=\Phi^{(g)} \\
& H_{\Lambda_{x} \Phi \Theta_{\delta}}: \Lambda_{x}^{(1)}=\Lambda_{x}^{(2)}=\ldots=\Lambda_{x}^{(g)} ; \Phi^{(1)}=\Phi^{(2)}=\ldots=\Phi^{(g)} ; \Theta_{\delta}^{(1)}=\Theta_{\delta}^{(2)}=\ldots=\Theta_{\delta}^{(g)}
\end{aligned}
$$

\section{Fase 2: Identificación del modelo con múltiples muestras}

Todo lo expuesto anteriormente en cuanto a la identificación modelos sobre una única muestra se aplica igualmente a la identificación de modelos sobre varias muestras. La única diferencia es que el cálculo de los grados de libertad incorpora en este caso el número de grupos presentes en el análisis (Rigdon, 1994).

$$
g l=\frac{1}{2} \cdot G \cdot p \cdot(p+1)-t
$$


Donde $G$ es el número de grupos, $p$ es el número de variables observables y $t$ es el número de parámetros libres.

\section{Fase 3: Estimación del modelo con múltiples muestras}

Respecto a la estimación con múltiples muestras no varía la hipótesis nula planteada para los modelos simples, aunque en este caso cada grupo posee su propia matriz de varianzas - covarianzas muestral que se comparará con la correspondiente matriz estimada. Así, la estimación se considera un procedimiento agregado e iterativo de las estimaciones independientes para cada grupo y la función de ajuste que se minimiza en este caso está compuesta por una suma ponderada de las funciones correspondientes a cada grupo.

$$
F=\sum_{g=1}^{G}\left(\frac{n_{g}}{n}\right) F_{g}\left(S_{g}, \Sigma_{g}\left(\Theta_{g}\right)\right)
$$

En esta expresión se puede observar la necesidad de considerar el tamaño muestral de cada grupo, ya que los grupos con mayor tamaño muestral tendrán mayor peso en la minimización de la función de ajuste a través del término $\frac{n_{g}}{n}$. Asimismo, la definición una única función de minimización común a los grupos y la imposición de restricciones comunes sobre ellos implica que las estimación debe realizarse de forma simultánea (Bentler, 2006; Jöreskog y Sörbom, 1996).

\section{Fase 4: Validación e interpretación del modelo con múltiples muestras}

Se considera que existe invarianza entre los grupos si el ajuste del modelo con restricciones impuestas a todos los grupos resulta adecuado (Widaman y Reise, 1997) y además la diferencia de ajuste con el modelo hipotetizado es mínima. El grado de invarianza lo deteminará el nivel de restricciones impuestas al modelo que mejor ajuste ha obtenido.

Para la evaluación del ajuste el parámetro más utilizado es el estadístico $\chi^{2}$, cuya interpretación es la misma que en modelos sobre una única muestra, aunque en este caso aplicado a la estimación simultánea de parámetros en varias muestras. Este estadístico se calcula, como en el caso anterior, como el producto de la función de minimización por el término $(N-1)$, es decir $(N-1) F_{M L}^{0}$ la estimación de la función de minimización mediante máxima verosimilitud. Al igual que en modelos sobre una única muestra, este estadístico se distribuye según una $\chi^{2}$ e informa de probabilidad de aceptación de la hipótesis nula especificada (Browne, 1984).

En la práctica totalidad de las aplicaciones de los análisis de invarianza, 
el incremento en este estadístico $\chi^{2}$ resulta la principal evidencia utilizada para evaluar la bondad de ajuste (Yuan y Bentler, 2004b) y en la mayoría de los casos este cálculo se aplica sobre modelos anidados. Concretamente en este tipo de análisis un incremento significativo del estadístico $\chi^{2}$ implica que no es posible aceptar el siguiente nivel de restricción. Por el contrario un incremento no significativo indica que es posible aceptar el siguiente nivel de invarianza, ya que el modelo correspondiente a las restricciones del nivel superior en la jerarquía si ha obtenido un buen ajuste. Estos incrementos del estadístico $\chi^{2}$ entre modelos anidados se distribuyen como una nueva variable $\chi^{2}$ con tantos grados de libertad como la diferencia entre los grados de libertad de ambos modelo (Anderson y Gerbing, 1988). Esta estrategia de calcular el incremento del estadístico $\chi^{2}$ fue propuesta por Jöreskog (1971), aunque posteriormente se ha demostrado que no era fiable en modelos mal especificados y además es tan sensible al tamaño de muestra y la falta de cumplimiento del supuesto de normalidad multiviante como el propio estadístico $\chi^{2}$ (Little, 1997). Por tanto constituye un criterio poco realista y fiable en el que no deberían basarse exclusivamente conclusiones sobre el análisis de invarianza.

Por todo ello algunos investigadores ha propuesto trabajar con el incremento del CFI, en vez del incremento del estadístico $\chi^{2}$. En esta línea se han sugerido distintos límites en el valor del CFI para ser considerado significativo. Concrementamente Little sugiere un umbral de 0.05 en el incremento del CFI, mientras que Cheung y Rensvold (2002) recomendaron fijar el límite del incremento en 0.01 después de examinar las propiedades de diversos índices de ajuste.

\subsubsection{Análisis parcial de invarianza}

El Análisis Parcial de la Invarianza se caracteriza por contrastar la equivalencia de parámetros individuales en lugar de hacerlo a nivel matricial (Byrne et al., 1989). Este examen se aplica cuando ya se ha comprobado la invarianza a distintos niveles de la jerarquía de restricciones y se pretende identificar los parámetros que más contribuyen a la invarianza. Estos nuevos modelos se plantean anidados a los anteriores y por tanto la bondad de ajuste se evalúa de la misma forma (mediante el incremento en el estadístico $\chi^{2}$ o en el índice CFI). Así, el análisis parcial de invarianza debe realizarse después de analizar el modelo para los distintos niveles de la jerarquía de restricciones. Una vez alcanzado un determinado nivel en que el ajuste del modelo se considere aceptable, se refinará este modelo mediante la liberación progresiva de parámetros individuales. 


\subsubsection{Modelos multinivel}

Existen muchos métodos y procedimientos que de alguna forma abordan los análisis multinivel en distintas áreas de la estadística, como el Análisis de la Varianza (ANOVA), las Regresiones Multinivel en econometría e incluso algunos estimadores bayesianos de Modelos Lineales. Los modelos multinivel se aplican sobre una única muestra cuyos datos se estructuran jerárquicamente. Precisamente el término multinivel hace referencia al hecho de que los datos se estructuran según uno o más niveles de análisis, que pueden ser simplemente dos o incluso más. Estas estructuras de datos pueden estar asociadas al diseño muestral por conglomerados (Hox, 2002).

En los últimos años la aplicación práctica de los modelos multinivel está resultando mucho más lenta que los avances realizados en el ámbito de la teoría. De hecho, a pesar de la frecuente presencia de datos estructurados jerárquicamente en el área de las Ciencias Sociales, las investigaciones tradicionalmente desarrolladas en este campo de conocimiento a menudo no consideran esta circunstancia adecuadamente en los análisis (Bryk y Raudenbush, 1992). La razón para ello puede deberse a la falta de disponibilidad de programas estadísticos que incluyan esta opción de análisis y aborden correctamente las dificultades que implica.

\section{Simplificaciones en la estructura de los datos: Agregación y des- agregación de medidas}

El hecho de ignorar la estructuración de los datos en niveles jerárquicos puede llevar a deducir falsas relaciones entre las variables del modelo, así como a ignorar determinados aspectos del proceso social que se está analizando. En este aspecto es posible realizar dos simplificaciones distintas en la estructura de los datos: una desagregación o agregación de las medidas, cada una de ellas conlleva unas dificultades y limitaciones en el análisis de datos (Heck, 2001).

En el caso de realizar una desagregación de las medidas se parte del supuesto de que no existen características ni percepciones comunes entre las distintas medidas o respuestas de los individuos, lo cual es completamente irrealista. Así, cuanto más pronunciadas sean las características comunes entre medidas o individuos, más sesgadas resultarán las estimaciones de los parámetros, los errores estándar y por ende, las medidas de significación asociadas. Por tanto no se está cumpliendo el supuesto de independencia entre variables. Del mismo modo no se cumple el supuesto de que los errores aleatorios son independientes, se distribuyen según una normal multivariante y son homocedásticos. Al obviar las influencias que sistemáticamente proce- 
den del nivel superior de las variables, esta variabilidad es incluida en los errores de medida de dichas variables dando lugar al incumplimiento de los supuestos relativos a los errores aleatorios (Kreft y De Leeuw, 1998).

Respecto a la agregación de las medidas, habitualmente el nivel superior está compuesto por las organizaciones en las que se integran los individuos del nivel inferior (Heck, 2001). La principal dificultad de este análisis es que la variabilidad correspondiente a cada unidad del nivel superior se reduce a una única medida y por tanto cualquier tipo de diferencia de variabilidad dentro de estas unidades desaparece. De la misma manera, en aquellas unidades en que las medidas del nivel inferior varíen de forma especialmente característica, las estimaciones de los parámetros a nivel superior serán menos eficientes (Bryk y Raudenbush, 1992).

\section{Correlaciones intraclases}

Las Correlaciones Intraclases proporcionan información sobre la pertinencia de la aplicación del análisis multinivel a los datos. En ocasiones es posible que no exista ningún tipo de efecto debido a las diferencias entre grupos, es decir que no exista ninguna variabilidad de las medidas en los grupos. El análisis de estos coeficientes señalan la proporción de varianza explicada por la estructura de nivel superior respecto a la varianza total del modelo (Hox, 2002). Según Muthen (1997), dichos coeficientes suelen variar entre 0.0 y 0.5 . Considerando estos límites, realizar el análisis multinivel resulta adecuado en los casos en que estos coeficientes superen el umbral 0.1 y además los tamaños de muestra de cada grupo sean suficientemente elevados $N>15$.

$$
\text { Correlacion intraclases }=\frac{\sigma_{B}^{2}}{\sigma_{W}^{2}+\sigma_{B}^{2}}
$$

\section{Estimación en modelos multinivel}

\section{Descomposición de la matriz de covarianzas}

Los modelos multinivel descomponen la varianza total del modelo en dos componentes Intra-Grupos y Entre-Grupos y permiten especificar un modelo estructural diferente para cada uno de ellos. Así, la matriz de varianzas covarianzas $(\Sigma)$ se descompone en una Matriz de Varianzas - Covarianzas Intra-Grupos $\left(\Sigma_{W}\right)$ y otra Matriz de Varianzas - Covarianzas Entre-Grupos $\left(\Sigma_{B}\right)$.

$$
\Sigma=\Sigma_{W}+\Sigma_{B}
$$


La matriz de varianzas - covarianzas intra-grupos $\left(\Sigma_{W}\right)$ presenta la covarianza entre medidas individuales mientras que la matriz de varianzas covarianzas entre-grupos $\left(\Sigma_{B}\right)$ representa la covarianza entre las unidades del nivel superior. Esta descomposición se aplica a cada una de las medidas, y a partir de ellas se calculan cada una de las matrices (Heck, 2001; Hox, 2002). De hecho, McArdle y Hamagami (1996) proponen considerar los modelos multinivel como un análisis de invarianza estándar, en el que cada matriz de varianzas - covarianzas se aplique a ambos grupos, cada uno de ellos con un conjunto diferente de especificaciones.

\section{Métodos de estimación en modelos multinivel}

Existen tres procedimientos para la estimación de modelos multinivel. En primer lugar la estimación por Máxima Verosimilitud es el más utilizado (Hox, 2002), aunque la aplicación de este método puede dar lugar a estimaciones incorrectas del estadístico $\chi^{2}$ así como de los índices de ajuste y los errores estándar (Muthen, 1994). Además, este método requiere elevados tamaños de muestra, sobre todo en el nivel superior (Heck, 2001; Yuan y Bentler, 2004a) y de hecho Bentler sugiere que idealmente deberían haber cientos de unidades de nivel superior (Bentler, 2006). En la actualidad la última versión de EQS (6.1) permite realizar las estimaciones de los parámetros mediante este método, a pesar de que los grupos tengan distintos tamaños de muestra (Liang y Bentler, 2004).

Los otros dos métodos ofrecen una alternativa a estas limitaciones del método de estimación por máxima verosimilitud. El primer de ellos es la aproximación de Muthen (MUML) y el segundo el Modelado Lineal Jerárquico (HLM).

\subsubsection{Programas informáticos}

Existe una gran variedad de programas informáticos diseñados para el análisis de ecuaciones estructurales. Jöreskog y Sörbom (1996), desarrollaron el primer programa informático que abordaba este tipo de análisis, el programa LISREL, comercializado por la organización Scientific Software International (SSI). Dispone de dos módulos adicionales, PRELIS y SIMPLIS, que respectivamente ofrecen diversas opciones para el tratamiento de los datos y simplifican los resultados obtenidos en la estimación del modelo. Si bien la principal ventaja de este programa frente al resto es su flexibilidad y capacidad para ejecutar todo tipo de análisis, es más bien conocido por la dificultad para su aprendizaje, y en definitiva por su alto nivel de sofisticación. Se recomienda preferentemente a aquellos investigadores que ya posean conocimientos previos de ecuaciones estructurales y no para los 
recién iniciados. En este aspecto el software MX, en principio ofrece las mismas posibilidades que LISREL y su utilización es más intuitiva. Además, posee una ventaja innegable frente al resto y es que está clasificado como software libre.

Para estos casos resulta más conveniente utilizar el software AMOS (Arbuckle, 2009), perteneciente a IBM SPSS, cuya principal característica es un amigable entorno visual que facilita enormemente su aprendizaje. Sin embargo, se ha criticado su excesiva simplicidad por el hecho de que admite la validación de modelos cuya interpretación sencillamente no es posible y que ofrece opciones limitadas de análisis. Algo similar ocurre con el software MPLUS, desarrollado por Muthen y Muthen, que ofrece un entorno gráfico de trabajo y una sintaxis mejor estructurada que en otros programas pero parece ser que es más sensible a posibles errores de especificación del modelo.

Existen otros programas de uso menos generalizado como el procedimiento CALIS perteneciente a SAS, la librería SEM de R o el módulo RAMONA integrante del programa estadístico SYSTAT. Todos ellos ofrecen distintas posibilidades de análisis pero ninguno presenta tantas ventajas como el programa EQS (Bentler, 2006) distribuido por Multivariate Software, Inc. Este programa puede utilizarse como herramienta para el aprendizaje de la metodología de ecuaciones estructurales ya que no requiere el uso de matrices sino que admite la formulación directa de ecuaciones. Asimismo permite el análisis de los datos aunque no cumplan el supuesto de normalidad multivariante mediante el cálculo de los estadísticos robustos. Aun así precisa ciertas mejoras en su programación ya se han detectado ciertos defectos de software en la ejecución del programa. Sin embargo, por sus características es el programa más adecuado para la realización de los análisis en las condiciones de este trabajo y por ello ha sido el software seleccionado.

\subsection{Resultados y discusión}

\subsubsection{Supuestos estadísticos y análisis previos}

Los estadísticos descriptivos de los ítems que se van a incluir en los modelos de medida son muy heterogéneos. Entre los ítems de Métodos de enseñanza - aprendizaje los indicadores 3 y 4 - Proyectos de investigación y Prácticas en empresa - mostraron cierta asimetría positiva mientras que el coeficiente de asimetría de los ítem 6 y 7 - Teorías, conceptos y paradigmas y El profesor como fuente de información - toma un valor significativamente negativo. Prácticamente todos los ítems alcanzan valores significativos de curtosis positiva o negativa. 


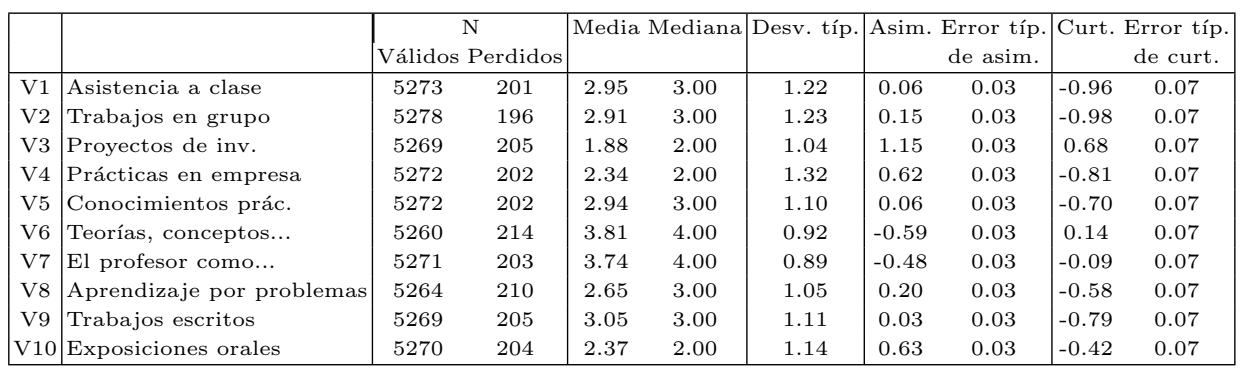

Tabla 5.2: Análisis descriptivo. Métodos de enseñanza - aprendizaje

Entre los ítems de Competencias adquiridas en la Universidad casi la mitad de los indicadores muestran indicios de asimetría positiva y todos ellos presentan coeficientes de curtosis negativos, con excepción del ítem 19 - Idiomas extranjeros - que alcanza un elevado valor de curtosis positiva.

\begin{tabular}{|c|c|c|c|c|c|c|c|c|c|c|}
\hline \multirow[b]{2}{*}{ V11 } & \multirow[b]{2}{*}{ Conocimientos del área } & \multicolumn{2}{|c|}{\begin{tabular}{|c|}
$\mathrm{N}$ \\
Válidos Perdidos \\
\end{tabular}} & \multicolumn{2}{|c|}{ Media Mediana } & \multirow{2}{*}{\begin{tabular}{|c|} 
Desv. típ. \\
1.73
\end{tabular}} & \multicolumn{2}{|c|}{$\begin{array}{l}\text { Asim. Error típ. } \\
\text { de asim. }\end{array}$} & \multicolumn{2}{|c|}{$\begin{array}{r}\text { Curt. Error típ. } \\
\text { de curt. }\end{array}$} \\
\hline & & 4802 & \begin{tabular}{l|l}
672 \\
\end{tabular} & 4.06 & 4.00 & & -0.06 & 0.04 & -0.85 & 0.07 \\
\hline $\mathrm{V} 12$ & Conocimientos de otras áreas & 4772 & 702 & 3.24 & 3.00 & 1.58 & 0.32 & 0.04 & -0.67 & 0.07 \\
\hline V13 & Pensamiento analítico & 4744 & 730 & 4.12 & 4.00 & 1.63 & -0.14 & 0.04 & -0.68 & 0.07 \\
\hline V14 & Aprender rápidamente & 4752 & 722 & 4.43 & 5.00 & 1.62 & -0.33 & 0.04 & -0.59 & 0.07 \\
\hline V15 & Negociar & 4735 & 739 & 2.79 & 3.00 & 1.57 & 0.67 & 0.04 & -0.28 & 0.07 \\
\hline V16 & Rendir bajo presión & 4767 & 707 & 3.61 & 4.00 & 1.81 & 0.19 & 0.04 & -0.99 & 0.07 \\
\hline V17 & Detectar oportunidades & 4720 & 754 & 3.00 & 3.00 & 1.54 & 0.44 & 0.04 & -0.52 & 0.07 \\
\hline V18 & Coordinar actividades & 4755 & 719 & 3.64 & 4.00 & 1.66 & 0.13 & 0.04 & -0.80 & 0.07 \\
\hline V19 & Uso eficaz del tiempo & 4770 & 704 & 3.89 & 4.00 & 1.70 & 0.00 & 0.04 & -0.85 & 0.07 \\
\hline V20 & Trabajar en equipo & 4765 & 709 & 4.26 & 4.00 & 1.78 & -0.20 & 0.04 & -0.93 & 0.07 \\
\hline V21 & Movilizar a otros & 4748 & 726 & 3.22 & 3.00 & 1.62 & 0.36 & 0.04 & -0.64 & 0.07 \\
\hline V22 & Hacerte entender & 4760 & 714 & 3.93 & 4.00 & 1.66 & 0.00 & 0.04 & -0.76 & 0.07 \\
\hline V23 & Hacer valer la autoridad & 4749 & 725 & 2.95 & 3.00 & 1.57 & 0.53 & 0.04 & -0.42 & 0.07 \\
\hline V24 & Herramientas informáticas & 4764 & 710 & 3.19 & 3.00 & 1.83 & 0.45 & 0.04 & -0.88 & 0.07 \\
\hline V25 & Ideas y soluciones & 4747 & 727 & 3.69 & 4.00 & 1.64 & 0.08 & 0.04 & -0.78 & 0.07 \\
\hline V26 & Cuestionar ideas & 4752 & 722 & 3.74 & 4.00 & 1.68 & 0.11 & 0.04 & -0.78 & 0.07 \\
\hline $\mathrm{V} 27$ & Presentar en público & 4756 & 718 & 3.61 & 4.00 & 1.75 & 0.19 & 0.04 & -0.94 & 0.07 \\
\hline V28 & Redactar documentos & 4761 & 713 & 4.09 & 4.00 & 1.74 & -0.12 & 0.04 & -0.90 & 0.07 \\
\hline V29 & Idiomas extranjeros & 4750 & 724 & 2.22 & 2.00 & 1.55 & 1.38 & 0.04 & 1.27 & 0.07 \\
\hline
\end{tabular}

Tabla 5.3: Análisis descriptivo. Competencias adquiridas en la Universidad

En ambas tablas se aprecia el elevado número de valores ausentes registrados en las valoraciones de los encuestados. Concretamente se observa que el número de valores ausentes respecto a la cuestión de las Competencias adquiridas en la Universidad es muy superior al correspondiente en la pregunta sobre los Métodos de enseñanza - aprendizaje. La justificación de este resultado se debe simplemente a que esta primera pregunta se encontraba situada en la parte final del cuestionario, mientras que en el segundo caso la cuestión se formuló al inicio del cuestionario. Debido a la longitud del mismo, las tasas de abandono en la cumplimentación de las respuestas, se incrementan progresivamente en cada sección a medida que aumenta el 
número de preguntas respondidas por el encuestado previamente.

En los casos en que el encuestado ha optado por no responder a la totalidad de las cuestiones relativas a los Métodos de enseñanza - aprendizaje y/o las Competencias adquiridas en la Universidad se ha aplicado directamente el método de eliminación del caso (Listwise) ya que ambos conjuntos de variables constituyen el núcleo central de todos los análisis posteriores. No obstante, en los casos en que se han detectado valores ausentes que faltan al azar, o incluso completamente al azar, se han imputado estos valores ausentes mediante el método de regresión. Al aplicar este método se ha seleccionado la opción de añadir un error aleatorio a los valores estimados con el fin de evitar un incremento en las correlaciones entre variables. Dada la falta de cumplimiento del supuesto de normalidad de las variables la estimación por máxima verosimilitud de la matriz de varianzas - covarianzas asumiendo que algunos valores falta al azar, no constituye una opción recomendable, ni tampoco la estimación mediante el algoritmo esperanza - maximización. Las Tablas 5.4 y 5.5 muestra los resultados de la prueba de Kolmogorov Smirnov, según la cual se rechaza en todos los todos los ítems la hipótesis nula de normalidad univariante:

\begin{tabular}{|l|l|ccc|cc|c|}
\hline & & \multicolumn{3}{|c|}{$\begin{array}{c}\text { Diferencias más extremas } \\
\text { Absoluta }\end{array}$} & $\begin{array}{c}\text { Z desitiva } \\
\text { Kolmogativa }\end{array}$ & Smirnov & Sig. \\
\hline V1 & Asistencia a clase & 0.17 & 0.17 & -0.15 & 12.14 & 0.000 \\
V2 & Trabajos en grupo & 0.19 & 0.19 & -0.15 & 13.70 & 0.000 \\
V3 & Proyectos de investigación & 0.27 & 0.27 & -0.20 & 19.32 & 0.000 \\
V4 & Prácticas en empresa & 0.21 & 0.21 & -0.15 & 14.97 & 0.000 \\
V5 & Conocimientos prácticos & 0.17 & 0.17 & -0.16 & 11.99 & 0.000 \\
V6 & Teorías, conceptos y paradigmas & 0.25 & 0.18 & -0.25 & 18.13 & 0.000 \\
V7 & El profesor como principal fuente de información & 0.26 & 0.19 & -0.26 & 19.17 & 0.000 \\
V8 & Aprendizaje basado en problemas & 0.19 & 0.19 & -0.17 & 14.00 & 0.000 \\
V9 & Trabajos escritos & 0.17 & 0.17 & -0.16 & 12.13 & 0.000 \\
V10 & Exposiciones orales & 0.25 & 0.25 & -0.13 & 18.12 & 0.000 \\
\hline
\end{tabular}

Tabla 5.4: Prueba de Kolmogorov-Smirnov. Métodos de enseñanza - aprendizaje

El hecho de que las variables no se comporten como distribuciones normales de forma independiente, condiciona la posibilidad de que sigan una distribución normal multivariante conjuntamente. Por tanto, no es necesario realizar el contraste de normalidad multivariante, ya que el resultado es ya conocido. Sin embargo, puesto que el programa EQS aporta los resultados de esta prueba como una opción por defecto en los análisis, se comentan a continuación los resultados obtenidos para los distintos conjuntos de variables.

El conjunto de indicadores referidos exclusivamente a los Métodos de enseñanza - aprendizaje obtiene un estimador normalizado del coeficiente 


\begin{tabular}{|l|l|ccc|c|c|}
\hline & & \multicolumn{2}{|c|}{$\begin{array}{l}\text { Diferencias más extremas } \\
\text { Absoluta }\end{array}$} & $\begin{array}{c}\text { Z de } \text { de Kolmogorov } \\
\text { Smirnov }\end{array}$ & Sig. \\
\hline V11 & Conocimientos del área & 0.12 & 0.10 & -0.12 & 8.05 & 0.000 \\
V12 & Conocimientos de otras áreas & 0.15 & 0.15 & -0.12 & 10.11 & 0.000 \\
V13 & Pensamiento analítico & 0.13 & 0.12 & -0.13 & 9.18 & 0.000 \\
V14 & Aprender rápidamente & 0.15 & 0.10 & -0.15 & 10.05 & 0.000 \\
V15 & Negociar & 0.18 & 0.18 & -0.13 & 12.62 & 0.000 \\
V16 & Rendir bajo presión & 0.13 & 0.13 & -0.10 & 9.13 & 0.000 \\
V17 & Detectar oportunidades & 0.16 & 0.16 & -0.12 & 10.88 & 0.000 \\
V18 & Coordinar actividades & 0.12 & 0.12 & -0.11 & 8.43 & 0.000 \\
V19 & Uso eficaz del tiempo & 0.12 & 0.11 & -0.12 & 8.06 & 0.000 \\
V20 & Trabajar en equipo & 0.13 & 0.09 & -0.13 & 9.07 & 0.000 \\
V21 & Movilizar a otros & 0.14 & 0.14 & -0.11 & 9.65 & 0.000 \\
V22 & Hacerte entender & 0.12 & 0.12 & -0.12 & 8.59 & 0.000 \\
V23 & Hacer valer la autoridad & 0.16 & 0.16 & -0.11 & 11.17 & 0.000 \\
V24 & Herramientas informáticas & 0.17 & 0.17 & -0.12 & 11.58 & 0.000 \\
V25 & Ideas y soluciones & 0.12 & 0.11 & -0.12 & 8.52 & 0.000 \\
V26 & Cuestionar ideas & 0.12 & 0.12 & -0.12 & 8.10 & 0.000 \\
V27 & Presentar en público & 0.13 & 0.13 & -0.11 & 9.17 & 0.000 \\
V28 & Redactar documentos & 0.13 & 0.10 & -0.13 & 8.88 & 0.000 \\
V29 & Idiomas extranjeros & 0.24 & 0.24 & -0.22 & 16.85 & 0.000 \\
\hline
\end{tabular}

Tabla 5.5: Prueba de Kolmogorov-Smirnov. Competencias adquiridas en la Universidad

de Mardia del 19.2. Este estimador alcanza el valor de 142.1 al incluir los ítems relativos a Competencias adquiridos en la Universidad. Puesto que se considera que los datos siguen una distribución normal multivariante cuando el valor de este estadístico no supera las 5 unidades, se concluye que los datos no se comportan conjuntamente según la distribución normal.

\subsubsection{Métodos de Enseñanza - Aprendizaje}

\section{Análisis Factorial Confirmatorio sobre la muestra general}

En primer lugar se ha realizado el análisis factorial confirmatorio sobre la tipología de métodos de enseñanza - aprendizaje propuesta por De Miguel (De Miguel, 2006). Tal como se ha expuesto anteriormente, De Miguel señala que existe una clara diferenciación entre los conceptos de Modalidades y Métodos en los procesos de enseñanza - aprendizaje. En cualquier caso, puesto es posible intuir cierta vinculación entre ambos conceptos, es posible proponer las siguientes estructuras factoriales a partir de la combinación de ambos elementos. De esta forma se plantea la validación de los ítems mostrados en el cuestionario como instrumento de medida de los siguientes factores latentes en cuanto a los métodos de enseñanza - aprendizaje en la Universidad. Estas estructuras factoriales definidas a priori, implican que los resultados del modelo tienen un carácter plenamente confirmatorio.

Cabe señalar que previamente se ha descartado uno de los indicadores incluidos en el apartado Métodos de enseñanza - aprendizaje por no considerarse una metodología sino más bien un procedimiento de evaluación. Este 
ítem, formulado en el cuestionario como Realización de pruebas tipo test, es denominado en investigaciones precedentes como Pruebas objetivas y se considera una técnica de evaluación válida para valorar el nivel de aprendizaje de los estudiantes a largo plazo (De Miguel, 2006).

\section{Modelo 1: AFC: Métodos de Enseñanza - Aprendizaje (4 Fac- tores)}

El modelo planteado está formado por 10 variables observables dependientes y 13 variables independientes, 3 de ellas son factores latentes y las 10 restantes son los errores de medida de las anteriores variables observables. El número de parámetros libres a estimar es 23 por lo que al aplicar la Regla t es fácil observar que el modelo está sobreidentificado:

$$
g l=\frac{1}{2} \cdot 10 \cdot 11-28=27
$$

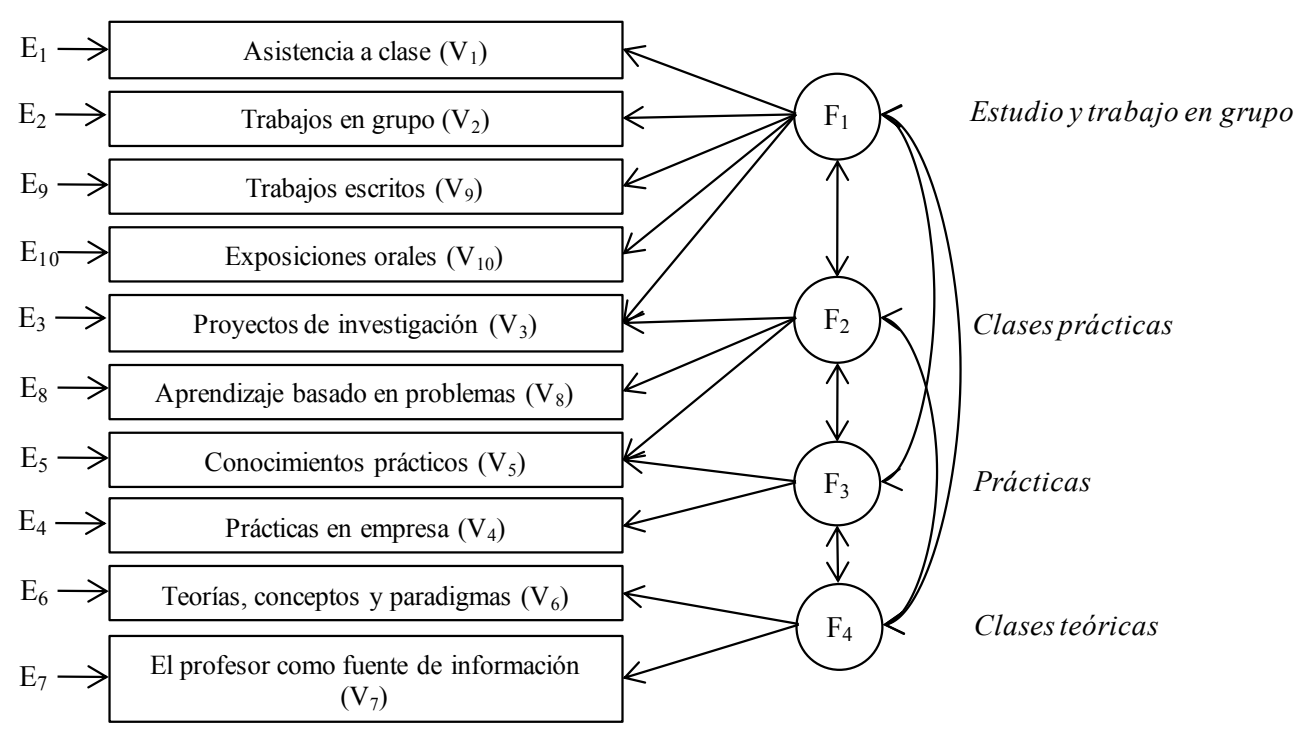

Figura 5.1: Modelo de medida inicial con 4 factores latentes. Métodos de enseñanza - aprendizaje

Tanto en esta estimación como en las siguientes, se ha empleado el método de estimación por Máxima Verosimilitud a través del análisis de los estadísticos robustos. Esta opción ha sido escogida dado el elevado tamaño de la muestra, que en estas circunstancias implica estimaciones más precisas de los parámetros, y al mismo tiempo que la probabilidad de rechazar 
el modelo siendo éste ajustado, es excesivamente elevada. Los estadísticos robustos, obtenidos mediante el programa EQS6.1, aportan la solución a la falta de cumplimiento de los datos del supuesto de normalidad multivariante.

Tras 15 iteraciones se obtienen las estimaciones de los parámetros con un ajuste muy bueno según los resultados aportados por los índices de bondad de ajuste. Según el contraste del estadístico $\chi^{2}$ el modelo no muestra un buen ajuste desde el enfoque exclusivamente estadístico. El hecho de que la probabilidad asociada a este contraste tome el valor nulo significa rechazar la hipótesis nula de que el modelo hipotetizado sí se ajusta bien a la población de referencia. Este valor es indicador de que las diferencias entre las matrices de varianzas - covarianzas observadas y estimadas son suficientemente grandes como para ser consideradas estadísticamente significativas. No obstante, este estadístico es muy influenciable por el tamaño de la muestra y en los casos en que el número de observaciones es superior a 200, como ocurre en este caso, cualquier pequeña diferencia entre ambas matrices puede ser evaluada como significativa.

Por el contrario, los índices de ajuste indican que la bondad de ajuste del modelo puede considerarse muy bueno. Concretamente los índices comparativos de ajuste alcanzan valores muy próximos a la unidad $(N F I=$ $0,953 ; N N F I=0,927 ; C F I=0,956 ; I F I=0,956)$ mientras que el índice de ajuste absoluto RMSEA toma un valor de 0.058 , con un intervalo de confianza $(90 \%)$ de $[0.053,0.062]$. Del mismo modo los índices de parsimonia AIC y CAIC en el modelo hipotetizado $(A I C=398,9 ; C A I C=197,5)$ presentan reducciones muy significativas respecto a los valores de dichos índices en el modelo de independencia $\left(A I C_{\text {Ind }}=9627,0 ; C A I C_{\text {Ind }}=9291,3\right)$.

El contraste $\chi^{2}$ del modelo de medida para Métodos de enseñanza aprendizaje con 4 factores latentes toma los valores:

$$
\begin{aligned}
& \chi_{\text {Ind }}^{2}=9717,0 \\
& g l_{\text {Ind }}=45 \\
& \chi^{2}=504,7 \\
& \chi_{S-B}^{2}=452,9 \\
& g l=27 \\
& \text { Prob }=0,000
\end{aligned}
$$

Por otro lado, la matriz residual de covarianzas arroja unos promedios muy pequeños, que evidencian la bondad de ajuste del modelo:

Average absolute residual $=0.0256$

Average off-diagonal absolute residual $=0.0313$ 
Average absolute standardized residual $=0.0214$

Average off-diagonal absolute standardized residual $=0.0261$

Por tanto, a pesar de que la probabilidad asociada al estadístico $\chi^{2}$ de Satorra - Bentler indique que debe rechazarse el modelo, el ajuste puede considerarse muy bueno, de acuerdo con los resultados de los índices de ajuste y también los promedios derivados de la matriz residual de covarianzas.

La fiabilidad de los factores de la escala se ha medido de forma global mediante el coeficiente Alfa de Cronbach y el coeficiente Rho. Este coeficiente Alfa de Cronbach no resulta aplicable en modelos multidimensionales como los planteados, sino que su aplicación se plantea fundamentalmente en contextos unidimensionales. Por ello su valor se incrementa en función del número de indicadores incluidos en su cálculo. En este caso únicamente se han considerado 10 indicadores, lo que puede considerarse un número relativamente bajo de variables. Por esta razón el valor alcanzado para el coeficiente Alfa de Cronbach (0.734) no resulta un buen indicador de la calidad del ajuste del modelo. Por el contrario, el coeficiente Rho alcanza un valor de 0.826 . No obstante, en este aspecto resulta determinante la evaluación de los porcentajes de fiabilidad compuesta de cada constructo y la varianza extraída. En la siguiente tabla se puede observar que únicamente el primer factor supera el umbral recomendado del $70 \%$ en cuanto a la fiabilidad compuesta mientras que el cuarto factor - Clases teóricas - es el único que alcanza el valor recomendado del $50 \%$ para la varianza extraída. Ambos factores obtienen asimismo valores aceptables de varianza extraída y fiabilidad compuesta respectivamente, mientras que el segundo - Clases prácticas- y el tercer factor - Prácticas - no superan estos umbrales. Concretamente el segundo factor obtiene los peores resultados de fiabilidad. Este hecho está relacionado con la limitación de únicamente se han definido dos indicadores para cada una de estas variables latentes, lo cual es muy poco aconsejable desde una perspectiva metodológica. En estas circunstancias, si alguno de los parámetros asociados a estos indicadores es restringido durante el proceso de estimación, la calidad de las estimaciones relacionadas disminuye inevitablemente, tal y como ha ocurrido con el error de medida del ítem 7 - El profesor como fuente de información.

El contraste de Lagrange aplicado a esta estimación propone la existencia de una relación fuerte entre dos ítems del primer factor - Estudio y trabajo en grupo. Estos ítems, el número 9 - Trabajos escritos - y el 10 Exposiciones orales - presentan un nivel de covarianza superior a la común al resto de indicadores asociados a este factor. Puesto que esta modificación resulta coherente con la teoría se acepta en la fase de reespecificación del modelo y se procede a una nueva estimación de los parámetros con el fin de evaluar nuevamente la bondad de ajuste del modelo. 


\begin{tabular}{|c|c|c|}
\hline & Fiabilidad compuesta & Varianza extraída \\
\hline$F_{1}$ & $76.5 \%$ & $41.2 \%$ \\
$F_{2}$ & $46.9 \%$ & $23.5 \%$ \\
$F_{3}$ & $52.4 \%$ & $38.1 \%$ \\
$F_{4}$ & $61.4 \%$ & $52.6 \%$ \\
\hline
\end{tabular}

Tabla 5.6: Fiabilidad compuesta y varianza extraída. Modelo de medida inicial para Métodos de enseñanza - aprendizaje con 4 factores latentes

Con la introducción de esta nueva relación el número de variables observables permanece constante pero se ha introducido un nuevo parámetro libre a estimar por lo que el número de grados de libertad se reduce en una unidad. En cualquier caso el modelo sigue estando sobreidentificado, según la aplicación de la Regla t:

$$
g l=\frac{1}{2} \cdot 10 \cdot 11-29=26
$$

A pesar de que esta modificación no evita que el error de medida del ítem 7 - El profesor como fuente de información - se fije nuevamente al valor nulo durante la estimación, sí favorece la disminución de los promedios de la matriz residual de covarianzas, que anteriormente tomaban los valores 0.0256 y 0.0313 para los promedios absolutos incluyendo y excluyendo los términos en la diagonal, y 0.0214 y 0.0261 para los mismos valores estandarizados:

Average absolute residual $=0.0194$

Average off-diagonal absolute residual $=0.0237$

Average absolute standardized residual $=0.0167$

Average off-diagonal absolute standardized residual $=0.0204$

Asimismo, la introducción de esta modificación supone un descenso en el estadístico $\chi^{2}$ de Satorra - Bentler de 452.9 a 285.7 , cuya diferencia corregida para la comparación del estadístico $\chi^{2}$ de Satorra - Bentler en modelos anidados es 134.0, así como un incremento de los índices de ajuste. En el caso de los índices comparativos de ajuste el incremento oscila entre 2 y 3 centésimas $(N F I=0,953 \rightarrow 0,971 ; N N F I=0,927 \rightarrow 0,954 ; C F I=$ $0,956 \rightarrow 0,973 ;$ IFI $=0,956 \rightarrow 0,973)$ mientras que el RMSEA disminuye de 0.058 a 0.046 , con un intervalo de confianza (90\% de [0.041, 0.051]. Los valores de los índices de parsimonia también disminuyen de forma significativa $(A I C=398,9 \rightarrow 233,7 ; C A I C=197,5 \rightarrow 39,8)$, permaneciendo invariantes los índices correspondientes al modelo de independencia, que evidentemente no se ve afectado por la introducción de esta modificación 
$\left(A I C_{\text {Ind }}=9627,0 ; C A I C_{\text {Ind }}=9291,3\right)$.

El contraste $\chi^{2}$ para este modelo de medida modificado para Métodos de enseñanza - aprendizaje con 4 factores se muestra a continuación:

$$
\begin{aligned}
& \chi_{\text {Ind }}^{2}=9717,0 \\
& g l_{\text {Ind }}=45 \\
& \chi^{2}=315,2 \\
& \chi_{S-B}^{2}=285,7 \\
& g l=26 \\
& \text { Prob }=0,000
\end{aligned}
$$

La disminución del estadístico $\chi^{2}$ de este segundo modelo respecto al primero resulta significativo como se muestra a continuación:

$$
\begin{aligned}
& k_{1}=\frac{\chi_{1}^{2}}{\chi_{S-B, 1}^{2}}=1,114 \\
& k_{2}=\frac{\chi_{2}^{2}}{\chi_{S-B, 2}^{2}}=1,103 \\
& D_{12}=\chi_{1}^{2}-\chi_{2}^{2}=189,5 \\
& d_{12}=1 \\
& k_{12}=\frac{d_{1} k_{1}-d_{2} k_{2}}{d_{12}}=1,410 \\
& \bar{D}_{12}=\frac{D_{12}}{k_{12}}=134,0>\chi_{\alpha=0,05 ; g l=1}^{2}=3,84
\end{aligned}
$$

Con esta modificación el valor del coeficiente Alfa de Cronbach permanece invariante, ya que no se ha modificado el número de ítems, pero el Coeficente Rho sí se modifica, disminuyendo desde el valor 0.826 hasta 0.814 . Igualmente se reduce la fiabilidad compuesta del primer factor - Estudio y trabajo en grupo - de $76.5 \%$ a $75.2 \%$, al tiempo que las fiabilidades del resto de factores permanecen aproximadamente similares.

\begin{tabular}{|c|c|c|}
\hline & Fiabilidad compuesta & Varianza extraída \\
\hline$F_{1}$ & $75.2 \%$ & $39.8 \%$ \\
$F_{2}$ & $47.6 \%$ & $24.0 \%$ \\
$F_{3}$ & $52.8 \%$ & $38.9 \%$ \\
$F_{4}$ & $61.4 \%$ & $52.6 \%$ \\
\hline
\end{tabular}

Tabla 5.7: Fiabilidad compuesta y varianza extraída. Modelo de medida modificado para Métodos de enseñanza - aprendizaje con 4 factores latentes

Una vez más el Contraste de Lagrange propone una nueva modificación que este caso hace referencia a los indicadores 9 y 2 - Trabajos escritos y 
Trabajos en grupo. Puesto que resulta coherente con la teoría asumir cierto grado de covariación entre estas variables, y cabe esperar que el descenso en el estadístico $\chi^{2}$ sea significativo, se opta por aceptar dicha modificación y reestimar nuevamente los parámetros del modelo.

Tal y como había sucedido anteriormente el número de variables observables no sufre ninguna alteración pero sí el número de parámetros libres a estimar. Con la introducción de esta nueva relación entre errores residuales el número de grados de libertad disminuye en una unidad de acuerdo con la Regla t:

$$
g l=\frac{1}{2} \cdot 10 \cdot 11-30=25
$$

En estas circunstancias se mantiene la imposición de fijar la varianza del error residual del ítem 7 - El profesor como fuente de información - y nuevamente se reducen los promedios de la matriz residual de varianzas covarianzas, que anteriormente tomaban los valores 0.0194 y 0.0237 para los promedios absolutos incluyendo y excluyendo los términos en la diagonal, y 0.0167 y 0.0204 para los mismos valores estandarizados:

Average absolute residual $=0.0158$

Average off-diagonal absolute residual $=0.0193$

Average absolute standardized residual $=0.0140$

Average off-diagonal absolute standardized residual $=0.0171$

Asimismo dicha modificación supone un descenso significativo del estadístico $\chi^{2}$ de Satorra - Bentler de 285.7 a 207.1, que corresponde a una diferencia corregida de 75.6, mientras que los índices de ajuste muestran en estas condiciones un ajuste excelente de los datos al modelo hipotetizado. Específicamente los índices comparativos de ajuste se incrementan aproximadamente en una centésima $(N F I=0,971 \rightarrow 0,979 ; N N F I=$ $0,954 \rightarrow 0,966 ; C F I=0,973 \rightarrow 0,981 ;$ IFI $=0,973 \rightarrow 0,981)$ mientras que el RMSEA disminuye de 0.046 a 0.039 , con intervalo de confianza $(90 \%)$ de $[0.034,0.044]$. Al mismo tiempo los índices de parsimonia se reducen notablemente $(A I C=233,7 \rightarrow 157,1 ; C A I C=39,8 \rightarrow-29,4)$, permaneciendo invariantes los respectivos índices del modelo de independencia $\left(A I C_{\text {Ind }}=9627\right) ; C A I C_{\text {Ind }}=9291,3$.

El contraste $\chi^{2}$ para el modelo de medida modificado nuevamente para Métodos de enseñanza - aprendizaje con 4 factores proporciona los siguientes resultados:

$\chi_{\text {Ind }}^{2}=9717,0$

$g l_{\text {Ind }}=45$ 
$\chi^{2}=228,0$

$\chi_{S-B}^{2}=207,1$

$g l=25$

Prob $=0,000$

La introducción de estas modificaciones genera nuevamente una disminución significativa del estadístico $\chi^{2}$ como se muestra a continuación:

$k_{3}=\frac{\chi_{3}^{2}}{\chi_{S-B, 3}^{2}}=1,101$
$k_{2}=\frac{\chi_{2}^{2}}{\chi_{S-B, 2}^{2}}=1,103$
$D_{23}=\chi_{2}^{2}-\chi_{3}^{2}=87,2$
$d_{23}=1$
$k_{23}=\frac{d_{2} k_{2}-d_{3} k_{3}}{d_{23}}=1,153$
$\bar{D}_{23}=\frac{D_{23}}{k_{23}}>\chi_{\alpha=0,05 ; g l=1}^{2}=75,43,84$

A pesar de que el coeficiente Alfa de Cronbach permanece imperturbable en el valor 0.784 el Coeficiente Rho disminuye desde 0.814 hasta alcanzar el valor 0.796 e igualmente disminuyen las fiabilidades de los dos primeros factores - Estudio y trabajo en grupo y Clases prácticas, en el primer caso de $75.2 \%$ a $73.7 \%$ y en el segundo de $47.6 \%$ a $45.8 \%$.

\begin{tabular}{|c|c|c|}
\hline & Fiabilidad compuesta & Varianza extraída \\
\hline$F_{1}$ & $73.7 \%$ & $37.5 \%$ \\
$F_{2}$ & $45.8 \%$ & $23.0 \%$ \\
$F_{3}$ & $53.3 \%$ & $38.7 \%$ \\
$F_{4}$ & $61.4 \%$ & $52.6 \%$ \\
\hline
\end{tabular}

Tabla 5.8: Modelo de medida final para Métodos de enseñanza - aprendizaje con 4 factores latentes. Fiabilidad compuesta y varianza extraída

De esta forma, aunque la modificación introducida mejora el ajuste del modelo, las nuevas relaciones actúan en detrimento de la fiabilidad de las variables latentes.

La siguiente modificación propuesta por el Contraste de Lagrange ya no puede sustentarse en conclusiones de investigaciones precedentes, puesto que hace referencia a la relación entre los ítems 6 y 4 - Teorías, conceptos y paradigmas y Prácticas en empresa. El descenso esperado en el valor del estadístico $\chi^{2}$ es muy inferior a los anteriores, a pesar de que los resultados de esta prueba indican que supondría una mejora significativa. Sin embargo, dado el elevado tamaño de la muestra, no es de extrañar la significación de 
este resultado. Por todo ello, y también con el fin de respetar el criterio de parsimonia, se descarta esta modificación, de forma que la estructura final de los métodos de enseñanza - aprendizaje con cuatro factores resulta tal y como se muestra en la siguiente figura:

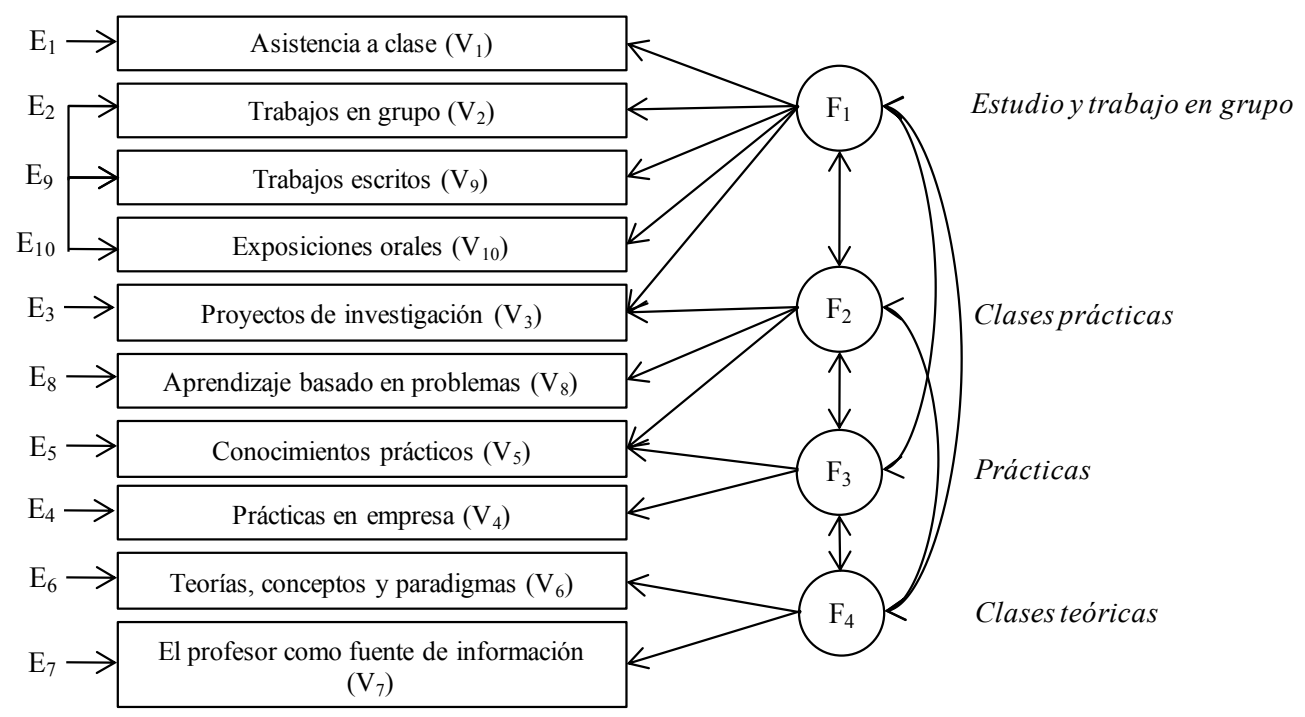

Figura 5.2: Modelo de medida final con 4 factores latentes. Métodos de enseñanza - aprendizaje

Las ecuaciones no estandarizadas correspondientes a este modelo muestran que el error de medida más elevado corresponde al ítem 1 - Asistencia a clase $\left(E_{1}=1,097^{*}\right)$ - mientras que el más reducido se encuentra en el ítem 2 - Trabajos en grupo $\left(E_{2}=0,405^{*}\right)$, ambos significativos como se indica mediante el símbolo *. El ítem 7 - El profesor como fuente de información - presenta un valor nulo en la estimación de este parámetro por imposición del programa EQS durante la ejecución del programa. Todas las saturaciones resultan estadísticamente significativas, pero dado el elevado tamaño de la muestra que incrementa la potencia de la prueba, este dato no resulta especialmente significativo. Por ello es más adecuado analizar las ecuaciones estandarizadas del modelo. 


$$
\begin{array}{ll}
V_{1}=1,000 \cdot F_{1}+1,000 \cdot E_{1} & E_{1}=1,097^{*} \\
V_{2}=1,671^{*} \cdot F_{1}+1,000 \cdot E_{2} & E_{2}=0,405^{*} \\
V_{3}=0,642^{*} \cdot F_{1}+1,000 \cdot F_{2}+1,000 \cdot E_{3} & E_{3}=0,659^{*} \\
V_{4}=1,000 \cdot F_{3}+1,000 \cdot E_{4} & E_{4}=0,666^{*} \\
V_{5}=1,455^{*} \cdot F_{2}+0,422^{*} \cdot F_{3}+1,000 \cdot E_{5} & E_{5}=0,577^{*} \\
V_{6}=1,000 \cdot F_{4}+1,000 \cdot E_{6} & E_{6}=0,797^{*} \\
V_{7}=4,246 \cdot F_{4}+1,000 \cdot E_{7} & E_{7}=0,000 \\
V_{8}=1,892^{*} \cdot F_{2}+1,000 \cdot E_{8} & E_{8}=0,677^{*} \\
V_{9}=0,994^{*} \cdot F_{1}+1,000 \cdot E_{9} & E_{9}=0,832^{*} \\
V_{10}=1,175^{*} \cdot F_{1}+1,000 \cdot E_{10} & E_{10}=0,747^{*}
\end{array}
$$

Tabla 5.9: Ecuaciones no estandarizadas. Modelo de medida final para Métodos de enseñanza - aprendizaje con 4 factores latentes

Las estimaciones de las matrices de varianzas - covarianzas y correlaciones muestran que los tres primeros factores latentes - Estudio y trabajo en grupo, Clases prácticas y Prácticas se encuentran fuertemente relacionados, como se observa en el nivel de significación de las covarianzas y las correlaciones superiores al valor 0.3. Sin embargo el cuarto factor - Clases teóricas - apenas sí cuenta con relaciones significativas con el resto de factores latentes. En las Tablas 5.10 y 5.11 se observa que en este caso los valores de covarianza y correlaciones son muy reducidos.

\begin{tabular}{|c|c|c|c|c|}
\hline & $F_{1}$ & $F_{2}$ & $F_{3}$ & $F_{4}$ \\
\hline$F_{1}$ & $0.389^{*}$ & & & \\
$F_{2}$ & 0.101 & $0.116^{*}$ & & \\
$F_{3}$ & $0.371^{*}$ & $0.156^{*}$ & $1.067^{*}$ & \\
$F_{4}$ & -0.015 & $-0.005^{*}$ & $-0.018^{*}$ & $0.043^{*}$ \\
\hline$E_{9}-E_{10}$ & \multicolumn{3}{|c|}{$0.162^{*}$} \\
$E_{9}-E_{2}$ & \multicolumn{3}{|c}{$0.241^{*}$} \\
\hline
\end{tabular}

Tabla 5.10: Matriz de varianzas - covarianzas. Modelo de medida final para Métodos de enseñanza - aprendizaje con 4 factores latentes

\begin{tabular}{|c|c|c|c|c|}
\hline & $F_{1}$ & $F_{2}$ & $F_{3}$ & $F_{4}$ \\
\hline$F_{1}$ & 1.000 & & & \\
$F_{2}$ & 0.4777 & 1.000 & & \\
$F_{3}$ & 0.576 & 0.442 & 1.000 & \\
$F_{4}$ & -0.115 & -0.076 & -0.082 & 1.000 \\
\hline$E_{9}-E_{10}$ & \multicolumn{3}{|c|}{0.278} \\
$E_{9}-E_{2}$ & \multicolumn{3}{|c}{0.306} \\
\hline
\end{tabular}

Tabla 5.11: Matriz de correlaciones. Modelo de medida final para Métodos de enseñanza - aprendizaje con 4 factores latentes 
De la misma forma es posible observar en el listado de ecuaciones estandarizadas que los ítems 1 y 6 - Asistencia a clase y Teorías, conceptos y paradigmas - obtienen los coeficientes de determinación más bajos $\left(R^{2}=0,262 ; R_{6}^{2}=0,052\right)$, indicando que dichos ítems no están siendo bien explicados por sus factores latentes correspondientes - Estudio y trabajo en grupo y Clases Teóricas, respectivamente. En estas ecuaciones se observa que todas las saturaciones correspondientes al primer factor - Estudio y trabajo en grupo - toman valores por encima de 0.3, con excepción del ítem 3 - Proyectos de investigación - siendo el ítem 2 - Trabajo en grupo - el que alcanza un valor más elevado de este coeficiente $\left(R^{2}=0,728\right)$. El segundo factor - Clases prácticas - presenta saturaciones entre 0.3 y 0.6 mientras que el tercer factor - Prácticas - está formado por dos ítems con coeficientes de determinación muy similares $\left(R^{2}=0,616 ; R^{2}=0,520\right)$, al contrario de lo que ocurre con el cuarto factor - Clases teóricas - formado por los ítems 6 y 7 con coeficientes de determinación muy distintos por imposición de EQS durante la estimación $\left(R^{2}=0,052 ; R^{2}=1,000\right)$.

$$
\begin{array}{lr}
V_{1}=0,512 \cdot F_{1}+0,859 \cdot E_{1} & R^{2}=0,262 \\
V_{2}=0,853 \cdot F_{1}+0,521 \cdot E_{2} & R^{2}=0,728 \\
V_{3}=0,388 \cdot F_{1}+0,329 \cdot F_{2}+0,787 \cdot E_{3} & R^{2}=0,381 \\
V_{4}=0,785 \cdot F_{3}+0,620 \cdot E_{4} & R^{2}=0,616 \\
V_{5}=0,451 \cdot F_{2}+0,397 \cdot F_{3}+0,693 \cdot E_{5} & R^{2}=0,520 \\
V_{6}=0,227 \cdot F_{4}+0,974 \cdot E_{6} & R^{2}=0,052 \\
V_{7}=1,000 \cdot F_{4}+0,000 \cdot E_{7} & R^{2}=1,000 \\
V_{8}=0,616 \cdot F_{2}+0,788 \cdot E_{8} & R^{2}=0,380 \\
V_{9}=0,562 \cdot F_{1}+0,827 \cdot E_{9} & R^{2}=0,316 \\
V_{10}=0,647 \cdot F_{1}+0,763 \cdot E_{10} & R^{2}=0,419
\end{array}
$$

Tabla 5.12: Ecuaciones estandarizadas. Modelo de medida final para Métodos de enseñanza - aprendizaje con 4 factores latentes

\section{Modelo 2: AFC: Métodos de Enseñanza - Aprendizaje (3 Fac- tores)}

Una vez evaluado el primer modelo basado en una estructura de cuatro factores latentes, y siguiendo la estrategia de evaluación de modelos rivales comentada anteriormente, se ha analizado un segundo modelo. Dicho modelo consta de tres factores latentes, formados a partir de la agrupación de los factores 2 y 3 - Clases prácticas y Prácticas - del modelo anterior. De esta forma este segundo modelo se fundamenta en los siguientes factores: Estudio y trabajo en grupo, Prácticas y Clases prácticas y Clases teóricas como se muestra en la siguiente figura:

Este modelo se encuentra sobreidentificado ya que el número de variables 


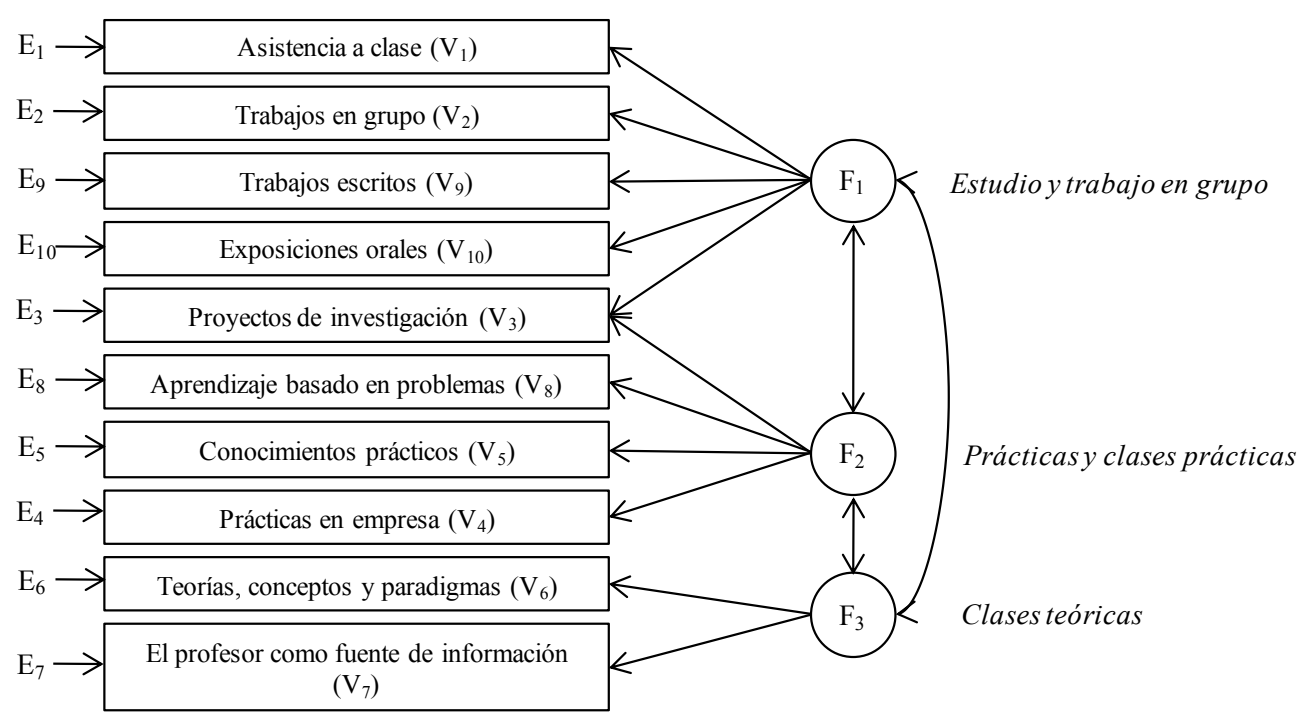

Figura 5.3: Modelo de medida inicial con 3 factores latentes. Métodos de enseñanza - aprendizaje

observables continúa siendo 10, como en el modelo anterior, pero al reducir el número de factores latentes disminuye en consonancia el número de parámetros libres a estimar. Así, aplicando la Regla t se obtiene el número de grados de libertad del modelo:

$$
g l=\frac{1}{2} \cdot 10 \cdot 11-23=32
$$

La imposición del error de medida del ítem 7 - El profesor como principal fuente de información - se mantiene en este modelo y los promedios de la matriz residual de covarianzas alcanzan el doble de su valor en el modelo de cuatro factores. Específicamente en el modelo final anterior con 4 factores latentes tomaban los valores 0.0158 y 0.0193 para los promedios absolutos incluyendo y excluyendo los términos en la diagonal, y 0.0140 y 0.0171 para los mismos valores estandarizados:

Average absolute residual $=0.0417$

Average off-diagonal absolute residual $=0.0468$

Average absolute standardized residual $=0.0332$

Average off-diagonal absolute standardized residual $=0.0376$

Evidentemente las estimaciones de los parámetros correspondientes al 
modelo de independencia no sufren ningún tipo de modificación, pero no ocurre lo mismo con los parámetros del modelo hipotetizado. Como cabía esperar, ocurre lo mismo con los índices AIC y CAIC de los modelos de independencia. Respecto al estadístico $\chi^{2}$ de Satorra - Bentler, ya desde un principio resulta muy superior al del modelo anterior, alcanzando un valor de 780.6 con 32 grados de libertad frente al obtenido en el modelo final con 4 factores de 207.1 con 25 grados de libertad. A pesar de que los resultados muestran que la bondad de ajuste de este modelo es ligeramente peor que el anterior, los índices de ajuste muestran que el ajuste del modelo con 3 factores es suficientemente bueno. Los índices comparativos de ajuste se aproximan suficientemente a la unidad $(N F I=0,920 ; N N F I=0,891 ; C F I=0,923 ; I F I=$ $0,923)$ mientras que el RMSEA toma el valor de 0.070 , con intervalo de confianza $(90 \%)$ de $[0.066,0.075]$ y los índices de parsimonia disminuyen hasta los valores $(A I C=716,6 ; C A I C=477,9)$ en relación a los mismos índices del modelo de independencia anterior $\left(A I C_{\text {Ind }}=9627 ; C A I C_{I n d}=9291,3\right)$.

El contraste $\chi^{2}$ del modelo de medida para Métodos de enseñanza aprendizaje con 3 factores latentes resulta:

$$
\begin{aligned}
& \chi_{\text {Ind }}^{2}=9717,0 \\
& g l_{\text {Ind }}=45 \\
& \chi^{2}=874,5 \\
& \chi_{S-B}^{2}=780,6 \\
& g l=32 \\
& \text { Prob }=0,000
\end{aligned}
$$

Puesto que no se han modificado las variables observables no se produce ninguna modificación en el valor del coeficiente Alfa de Cronbach, que se mantiene en 0.784 mientras que el Coeficiente Rho resulta algo inferior al obtenido anteriormente de 0.814 , limitándose a un valor de 0.802 . La fiabilidad compuesta de las variables latentes sólo alcanza el umbral del $70 \%$ en el primer factor - Estudio y trabajo en grupo - mientras que el límite inferior de varianza extraída del $50 \%$ únicamente lo supera el tercer factor - Clases teóricas. En este caso la unión de los anteriores factores 2 y 3 - Clases prácticas $(45.8 \%)$ y Prácticas $(53.3 \%)$ - ha supuesto un incremento de la fiabilidad compuesta del nuevo segundo factor - Clases prácticas y Prácticas $(65.3 \%)$.

En este caso el Contraste de Lagrange propone la misma modificación que se aceptó en el primer modelo relativa a la covarianza entre los errores residuales de los ítems 9 y 10 - Trabajos escritos y Exposiciones orales. Esta nueva relación resulta coherente con la teoría y por ello es igualmente aceptada en este modelo. Tal y como ocurría anteriormente, la introducción 


\begin{tabular}{|c|c|c|}
\hline & Fiabilidad compuesta & Varianza extraída \\
\hline$F_{1}$ & $72.7 \%$ & $39.0 \%$ \\
$F_{2}$ & $65.3 \%$ & $32.5 \%$ \\
$F_{3}$ & $61.4 \%$ & $52.6 \%$ \\
\hline
\end{tabular}

Tabla 5.13: Fiabilidad compuesta y varianza extraída. Modelo de medida inicial para Métodos de enseñanza - aprendizaje con 3 factores latentes

de esta nueva relación en el modelo supone un descenso de una unidad en el número de grados de libertad. Esto implica que este nuevo modelo posee ahora 31 grados de libertad por lo que sigue estando sobreidentificado.

$$
g l=\frac{1}{2} \cdot 10 \cdot 11-24=31
$$

Esta nueva relación tampoco evita la imposición de EQS de fijar a cero el error de medida del ítem 7 - El profesor como fuente de información. Sin embargo sí se reducen los promedios de la matriz residual de varianzas covarianzas, que anteriormente tomaban los valores 0.0417 y 0.0468 para los promedios absolutos incluyendo y excluyendo los términos en la diagonal, y 0.0332 y 0.0376 para los mismos valores estandarizados:

Average absolute residual $=0.0376$

Average off-diagonal absolute residual $=0.0418$

Average absolute standardized residual $=0.0299$

Average off-diagonal absolute standardized residual $=0.0337$

Asimismo se produce un descenso significativo del estadístico $\chi^{2}$ de Satorra - Bentler de 780.6 a 682.7, que corresponde a una diferencia corregida de 143.4, al tiempo que se incrementan los índices de bondad de ajuste, reflejando que la introducción de la nueva relación ha supuesto una mejora significativa del modelo hipotetizado. En esta ocasión los índices comparativos de ajuste se incrementan aproximadamente en dos centési$\operatorname{mas}(N F I=0,920 \rightarrow 0,937 ; N N F I=0,891 \rightarrow 0,913 ; C F I=0,923 \rightarrow$ $0,940 ;$ IFI $=0,924 \rightarrow 0,940)$ mientras que el RMSEA disminuye de 0.070 a 0.063 , con intervalo de confianza $(90 \%)$ de $[0.059,0.067]$. Al mismo tiempo los índices de parsimonia se reducen notablemente $(A I C=716,6 \rightarrow$ $551,6 ; C A I C=477,9 \rightarrow 320,4)$.Por tanto, en estas condiciones el ajuste del modelo puede considerarse muy bueno.

En esta ocasión el contraste $\chi^{2}$ del modelo de medida modificado para Métodos de enseñanza - aprendizaje con 3 factores latentes proporciona los siguientes resultados: 


$$
\begin{aligned}
& \chi_{\text {Ind }}^{2}=9717,0 \\
& g l_{\text {Ind }}=45 \\
& \chi^{2}=682,7 \\
& \chi_{S-B}^{2}=613,6 \\
& g l=31 \\
& \text { Prob }=0,000
\end{aligned}
$$

Esta disminución del estadístico $\chi^{2}$ es significativo como se muestra a continuación:

$$
\begin{aligned}
& k_{1}=\frac{\chi_{1}^{2}}{\chi_{S-B, 1}^{2}}=1,120 \\
& k_{2}=\frac{\chi_{2}^{2}}{\chi_{S-B, 2}^{2}}=1,113 \\
& D_{12}=\chi_{1}^{2}-\chi_{2}^{2}=191,8 \\
& d_{12}=1 \\
& k_{12}=\frac{d_{1} k_{1}-d_{2} k_{2}}{d_{12}}=1,337 \\
& \bar{D}_{12}=\frac{D_{12}}{k_{12}}=143,4>\chi_{\alpha=0,05 ; g l=1}^{2}=3,84
\end{aligned}
$$

No obstante, la introducción de esta relación de covarianza entre los errores residuales supone una reducción del Coeficiente Rho, hasta el valor 0.789. El coeficiente Alfa de Cronbach permanece inalterable ya que no se modifican las variables observables. Asimismo la fiabilidad del primer factor - Estudio y trabajo en grupo - disminuye de $72.7 \%$ a $71.1 \%$ mientras que el resto no sufren ninguna modificación.

\begin{tabular}{|c|c|c|}
\hline & Fiabilidad compuesta & Varianza extraída \\
\hline$F_{1}$ & $71.1 \%$ & $37.6 \%$ \\
$F_{2}$ & $65.5 \%$ & $32.7 \%$ \\
$F_{3}$ & $61.4 \%$ & $52.6 \%$ \\
\hline
\end{tabular}

Tabla 5.14: Fiabilidad compuesta y varianza extraída. Modelo de medida final para Métodos de enseñanza - aprendizaje con 3 factores latentes

En esta ocasión la modificación más significativa propuesta nuevamente por el Contraste de Lagrange hace referencia a la relación entre los errores de medida de los indicadores 4 y 8 - Prácticas en empresa y Aprendizaje basado en problemas. A pesar de ambos indicadores se asocian al mismo factor latente - Prácticas y clases prácticas - esta relación ya no resulta coherente con la teoría subyacente a la estructura factorial. Por esta razón, y también con el fin de no incrementar innecesariamente la complejidad del modelo, se descarta esta nueva relación. Así, el segundo modelo basado en una es- 
tructura de tres factores adquiere la siguiente forma después de la fase de reespecificación:

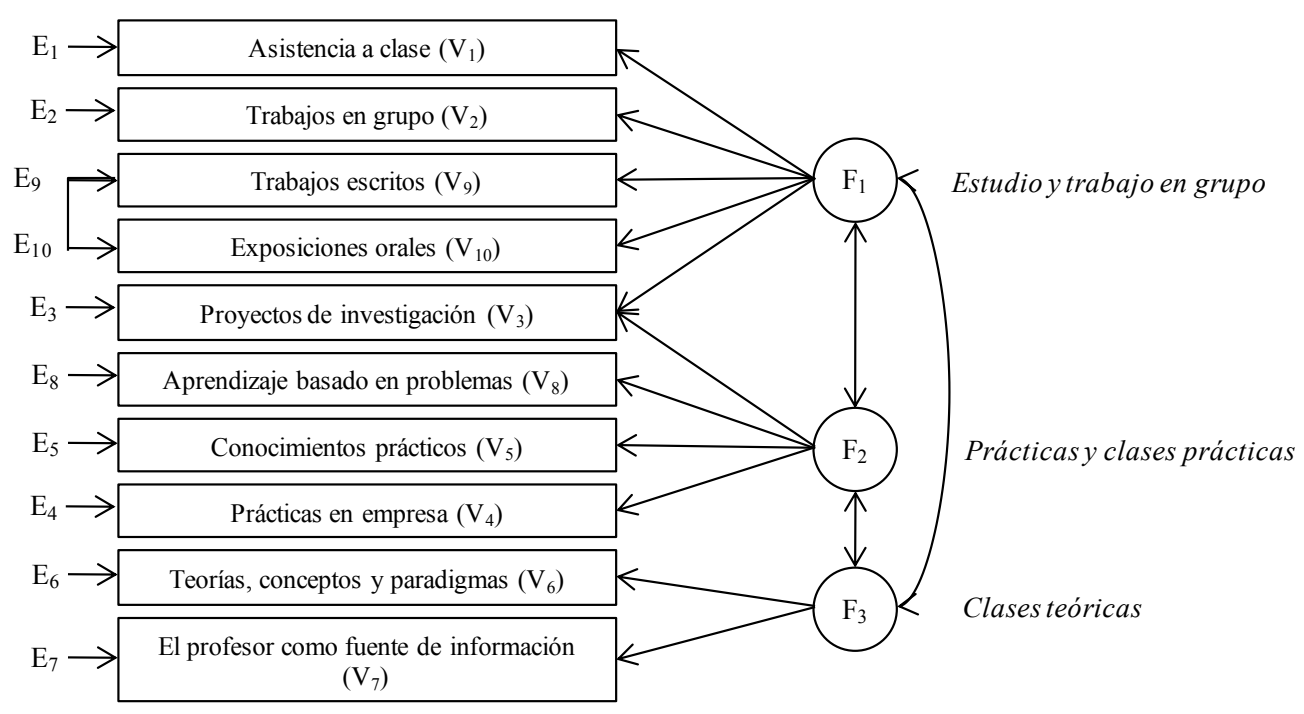

Figura 5.4: Modelo de medida final con 3 factores latentes. Métodos de enseñanza - aprendizaje

Las ecuaciones no estandarizadas del modelo con tres factores muestran bastantes similitudes con el modelo de cuatro factores, e incluso la estimación del coeficiente de regresión del ítem 7 - El profesor como fuente de información - sigue siendo no significativa. Evidentemente los ítems que mayor variación han experimentado en sus coeficientes de regresión son aquellos que en este modelo cargan sobre otros factores, como los indicadores 4 y 5 - Prácticas en empresa y Conocimientos prácticos.

Al igual que antes, en las correlaciones entre factores es fácil observar que el tercer factor - Clases teóricas - es prácticamente independiente y de hecho, todas las correlaciones entre dicho factor y el resto son prácticamente nulas al tiempo que las covarianzas son no significativas. 


$$
\begin{array}{lr}
V_{1}=1,000 \cdot F_{1}+1,000 \cdot E_{1} & E_{1}=1,122^{*} \\
V_{2}=1,830^{*} \cdot F_{1}+1,000 \cdot E_{2} & E_{2}=0,273^{*} \\
V_{3}=0,173^{*} \cdot F_{1}+1,000^{*} \cdot F_{2}+1,000 \cdot E_{3} & E_{3}=0,641^{*} \\
V_{4}=1,000 \cdot F_{2}+1,000 \cdot E_{4} & E_{4}=1,220^{*} \\
V_{5}=1,214^{*} \cdot F_{2}+1,000 \cdot E_{5} & E_{5}=0,627^{*} \\
V_{6}=1,000 \cdot F_{3}+1,000 \cdot E_{6} & E_{6}=0,797^{*} \\
V_{7}=4,246 \cdot F_{3}+1,000 \cdot E_{7} & E_{7}=0,000 \\
V_{8}=0,819^{*} \cdot F_{2}+1,000 \cdot E_{8} & E_{8}=0,829^{*} \\
V_{9}=1,194^{*} \cdot F_{1}+1,000 \cdot E_{9} & E_{9}=0,697^{*} \\
V_{10}=1,163^{*} \cdot F_{1}+1,000 \cdot E_{10} & E_{10}=0,792^{*}
\end{array}
$$

Tabla 5.15: Ecuaciones no estandarizadas. Modelo de medida final para Métodos de enseñanza - aprendizaje con 3 factores latentes

\begin{tabular}{|c|c|c|c|}
\hline & $F_{1}$ & $F_{2}$ & $F_{3}$ \\
\hline$F_{1}$ & $0.364^{*}$ & & \\
$F_{2}$ & $0.251^{*}$ & $0.391^{*}$ & \\
$F_{3}$ & -0.012 & -0.016 & 0.043 \\
\hline$E_{9}-E_{10}$ & \multicolumn{3}{|c|}{$0.189 *$} \\
\hline
\end{tabular}

Tabla 5.16: Matriz de varianzas - covarianzas. Modelo de medida final para Métodos de enseñanza - aprendizaje con 3 factores latentes

\begin{tabular}{|c|c|c|c|}
\hline & $F_{1}$ & $F_{2}$ & $F_{3}$ \\
\hline$F_{1}$ & 1.000 & & \\
$F_{2}$ & 0.666 & 1.000 & \\
$F_{3}$ & -0.097 & -0.019 & 1.000 \\
\hline$E_{9}-E_{10}$ & \multicolumn{3}{|c|}{0.255} \\
\hline
\end{tabular}

Tabla 5.17: Matriz de correlaciones. Modelo de medida final para Métodos de enseñanza - aprendizaje con 3 factores latentes

Las ecuaciones estandarizadas revelan que los coeficientes de determinación de los ítems 1, 4, 6 y 8 - Asistencia a clase, Prácticas en empresa, Teorías, conceptos y paradigmas y Aprendizaje basado en problemas - son especialmente reducidos, denotando cierta falta de calidad en la estimación de los parámetros estandarizados correspondientes. Al mismo tiempo los ítems que anteriormente pertenecían al tercer factor de Prácticas, ahora unido al relativo a las Clases prácticas, han experimentado notables modificaciones en sus valores. Estos indicadores son el 4 - Prácticas en empresa - y el 5 Conocimientos prácticos.

De esta forma se han evaluado dos modelos rivales para la estructura factorial de los Métodos de enseñanza - aprendizaje en Educación Supe- 


$\begin{array}{ll}V_{1}=0,495 \cdot F_{1}+0,869 \cdot E_{1} & R^{2}=0,245 \\ V_{2}=0,904 \cdot F_{1}+0,428 \cdot E_{2} & R^{2}=0,817 \\ V_{3}=0,098 \cdot F_{1}+0,588 \cdot F_{2}+0,753 \cdot E_{3} & R^{2}=0,433 \\ V_{4}=0,493 \cdot F_{2}+0,870 \cdot E_{4} & R^{2}=0,243 \\ V_{5}=0,692 \cdot F_{2}+0,722 \cdot E_{5} & R^{2}=0,479 \\ V_{6}=0,227 \cdot F_{3}+0,974 \cdot E_{6} & R^{2}=0,052 \\ V_{7}=1,000 \cdot F_{3}+0,000 \cdot E_{7} & R^{2}=1,000 \\ V_{8}=0,490 \cdot F_{2}+0,872 \cdot E_{8} & R^{2}=0,240 \\ V_{9}=0,653 \cdot F_{1}+0,757 \cdot E_{9} & R^{2}=0,427 \\ V_{10}=0,619 \cdot F_{1}+0,785 \cdot E_{10} & R^{2}=0,384\end{array}$

Tabla 5.18: Ecuaciones estandarizadas. Modelo de medida final para Métodos de enseñanza - aprendizaje con 3 factores latentes

rior. Ambos modelos hipotetizados han mostrado un ajuste excelente o muy bueno a los datos por lo que se consideran válidos para representar la teoría referente a esta área de investigación.

\section{Análisis Factorial Confirmatorio por áreas de estudio}

El análisis factorial confirmatorio sobre múltiples muestras recibe el nombre de Análisis de Invarianza. En los casos en que se aplica exclusivamente a los modelos de medida la invarianza se define como métrica, mientras que es calificada como estructural cuando se aplica a modelos causales o estructurales. Este análisis permite evaluar si un mismo modelo puede ser aplicado a distintas submuestras pertenecientes a la muestra global. En este caso estas submuestras las componen los graduados titulados en las distintas ocho de estudio consideradas a lo largo de este trabajo (Educación, Humanidades, Ciencias Sociales, Economía y empresa, Derecho, Técnicas, Salud y Ciencias).

En el apartado anterior se han evaluado dos modelos alternativos propuestos en base a la teoría sobre Métodos de enseñanza - aprendizaje en Educación Superior. Ambos modelos han aportado una representación muy ajustada del comportamiento de los datos. Puesto que hasta el momento no existe ninguna razón para descartar alguno de ellos se ha considerado apropiado evaluar ambos modelos desde el enfoque de la invarianza métrica.

En este punto del análisis se ha optado por evaluar únicamente la imposición de restricciones de invarianza métrica sobre las distintas estructuras factoriales. La evaluación de la invarianza estructural sobre estos modelos implicaría restringir parámetros como las varianzas y covarianzas de los factores. Si bien este tipo de análisis es posible en estas circuntancias, lo cierto es que no es habitual y, de hecho, resulta de poco interés y excesivamente 
restrictiva la evaluación del modelo en estas condiciones. Posteriormente, en la evaluación del modelo causal donde se definen relaciones estructurales entre variables latentes, se planteará este análisis con mayor fundamento metodológico.

Previamente a la evaluación de este análisis de invarianza métrica se recomienda validar el modelo en cada uno de los grupos con el fin de comprobar tanto si puede aplicarse el mismo modelo base a todos los grupos, como de obtener una perspectiva del orden de magnitud en el que variarán las estimaciones de los parámetros. La Tabla 5.19 muestra los estadísticos e índices de ajuste del modelo con cuatro factores en cada una de las áreas de estudio planteadas. En dicha tabla se observa que el modelo ajusta excelentemente en las áreas de Educación, Ciencias Sociales y Derecho, con valores del estadístico $\chi^{2}$ de Satorra - Bentler inferiores a 50. En las áreas de Técnicas, Salud y Economía y empresa el ajuste puede considerarse muy bueno, con valores del mismo estadístico entre 60 y 70, mientras que en las áreas de Ciencias y Humanidades el ajuste alcanzado por el modelo es simplemente bueno en relación a las anteriores, ya que los valores del estadístico $\chi^{2}$ de Satorra - Bentler son en este caso superiores a 80.

\begin{tabular}{|l|ccccccccc|}
\hline & \multicolumn{7}{|c|}{ Educación Humanidades Ciencias Economía Derecho Técnicas Salud Ciencias } \\
& \multicolumn{7}{|c}{ Sociales } & y & empresa \\
\hline$\chi_{\text {Ind }}^{2}$ & 883.9 & 814.2 & 1234.9 & 1728.8 & 489.3 & 1335.7 & 1102.6 & 998.3 \\
$g l_{\text {Ind }}$ & 45 & 45 & 45 & 45 & 45 & 45 & 45 & 45 \\
$\chi_{S-B}^{2}$ & 47.8 & 98.6 & 47.9 & 69.6 & 25.9 & 60.3 & 60.8 & 80.5 \\
$g l_{\text {Ind }}$ & 25 & 25 & 25 & 25 & 25 & 25 & 25 & 25 \\
\hline AIC $C_{\text {Ind }}$ & 793.9 & 724.2 & 1144.9 & 1638.8 & 399.3 & 1245.7 & 1012.6 & 908.3 \\
AIC & -2.2 & 48.6 & -2.1 & 19.6 & -24.1 & 10.3 & 10.8 & 30.5 \\
CAIC Ind & 555.8 & 494.5 & 905.0 & 1374.9 & 191.5 & 981.1 & 777.5 & 669.0 \\
CAIC & -134.5 & -79.1 & -135.4 & -127.0 & -139.5 & -136.7 & -119.8 & -102.4 \\
NFI & 0.946 & 0.879 & 0.961 & 0.960 & 0.947 & 0.955 & 0.945 & 0.919 \\
NNFI & 0.951 & 0.828 & 0.965 & 0.952 & 0.996 & 0.951 & 0.939 & 0.895 \\
CFI & 0.973 & 0.904 & 0.981 & 0.973 & 0.998 & 0.973 & 0.966 & 0.942 \\
IFI & 0.973 & 0.907 & 0.9810 & 0.974 & 0.998 & 0.973 & 0.967 & 0.943 \\
MFI & 0.979 & 0.921 & 0.980 & 0.977 & 0.998 & 0.982 & 0.965 & 0.951 \\
RMSEA & 0.041 & 0.081 & 0.040 & 0.043 & 0.012 & 0.038 & 0.053 & 0.063 \\
Lim Inf $90 \%$ & 0.023 & 0.064 & 0.022 & 0.031 & 0.000 & 0.026 & 0.036 & 0.048 \\
Lim Sup $90 \%$ & 0.059 & 0.098 & 0.057 & 0.055 & 0.050 & 0.051 & 0.070 & 0.079 \\
\hline
\end{tabular}

Tabla 5.19: Índices de ajuste y contraste $\chi^{2}$ por área de estudio. Invarianza métrica del modelo para Métodos de enseñanza - aprendizaje con 4 factores latentes

El hecho de que el modelo ajuste bien en cada una de las áreas resulta coherente con la calidad del ajuste a nivel global obtenido anteriormente. 
No obstante, los promedios de la matriz residual de varianzas - covarianzas resultan más elevados al segmentar la muestra global por áreas, y ni siquiera en las áreas de estudio con valores mínimos como Economía y empresa y Técnicas se obtienen valores similares a los globales correspondientes a los valores 0.0158 y 0.0193 para los promedios absolutos incluyendo y excluyendo los términos en la diagonal, y 0.0140 y 0.0171 para los mismos valores estandarizados.

\begin{tabular}{|l|cccccccc|}
\hline & \multicolumn{7}{|c|}{ Educación Humanidades Ciencias Economía Derecho Técnicas Salud Ciencias } \\
& \multicolumn{7}{|c}{ Sociales } & y empresa \\
\hline Avg. abs. residual & 0.0458 & 0.0572 & 0.0553 & 0.0246 & 0.0479 & 0.0273 & 0.0515 & 0.0416 \\
Avg. off-diag. abs. residual & 0.0472 & 0.0646 & 0.0559 & 0.0271 & 0.0482 & 0.0288 & 0.0568 & 0.0454 \\
Avg. abs. std. residual & 0.0425 & 0.0483 & 0.0462 & 0.0282 & 0.0537 & 0.0281 & 0.0452 & 0.0418 \\
Avg. off-diag. abs. std. residual & 0.0445 & 0.0548 & 0.0471 & 0.0315 & 0.0540 & 0.0295 & 0.0497 & 0.0459 \\
\hline
\end{tabular}

Tabla 5.20: Promedios de las matrices residuales de varianzas-covarianzas por área de estudio. Invarianza métrica del modelo para Métodos de enseñanza - aprendizaje con 4 factores latentes

El ajuste del modelo con las restricciones de invarianza métrica sobre las áreas de estudio sigue siendo muy bueno, a pesar de que ya no puede considerarse excelente como ocurría en la muestra global. Asimismo, el número de grados de libertad se ha incrementado notablemente ya que toma en consideración tanto el número de grupos analizado como las restricciones impuestas sobre el modelo. En este aspecto el estadístico $\chi^{2}$ de Satorra Bentler se incrementa respecto al modelo con 4 factores sin restricciones de invarianza donde tomaba un valor de 207.1 con 25 grados de libertad, hasta el valor de 696.9 con 256 grados de libertad en el modelo con restricciones de invarianza. Del mismo modo los índices comparativos de ajuste disminuyen aproximadamente en 4 centésimas $(N F I=0,979 \rightarrow 0,919 ; N N F I=$ $0,966 \rightarrow 0,924 ; C F I=0,981 \rightarrow 0,946 ;$ IFI $=0,981 \rightarrow 0,947)$ mientras que el RMSEA se incrementa de 0.039 a 0.054 , con intervalo de confianza (90\%) de $[0.049,0.058]$.

En este caso el contraste $\chi^{2}$ del modelo de medida final para Métodos de enseñanza - aprendizaje con 4 factores latentes y restricciones de invarianza métrica resulta:

$$
\begin{aligned}
& \chi_{\text {Ind }}^{2}=8560,2 \\
& g l_{\text {Ind }}=360 \\
& \chi_{S-B}^{2}=696,9 \\
& g l=256 \\
& \text { Prob }=0,000
\end{aligned}
$$


Por otro lado los coeficientes de fiabilidad muestran valores relativamente elevados, próximos a los obtenidos sobre el modelo con cuatro factores sin restricciones de invarianza, en el que los coeficientes Alfa de Cronbach y Rho tomaban valores de 0.784 y 0.796 , respectivamente.

\begin{tabular}{|l|cccccccc|}
\hline & \multicolumn{8}{|c|}{ Educación Humanidades Ciencias Economía Derecho Técnicas Salud Ciencias } \\
& 0.714 & 0.668 & 0.735 & 0.722 & 0.724 & 0.679 & 0.737 & 0.692 \\
\hline$\alpha$ Cronbach & 0.719 y empresa \\
Rho & 0.764 & 0.762 & 0.815 & 0.794 & 0.754 & 0.727 & 0.812 & 0.735 \\
\hline
\end{tabular}

Tabla 5.21: Índices de fiabilidad por área de estudio. Invarianza métrica del modelo para Métodos de enseñanza - aprendizaje con 4 factores latentes

Las ecuaciones no estandarizadas son comunes a todas las áreas de estudio analizadas por las propias restricciones de invarianza métrica. En dichas ecuaciones se observa que los coeficientes de regresión de los distintos ítems toman valores muy similares a los correspondientes a la muestra global. La única diferencia notable respecto a los anteriores es el coeficiente del ítem 7 - El profesor como principal fuente de información - cuya estimación no había resultado significativa hasta el momento y ahora sí lo es.

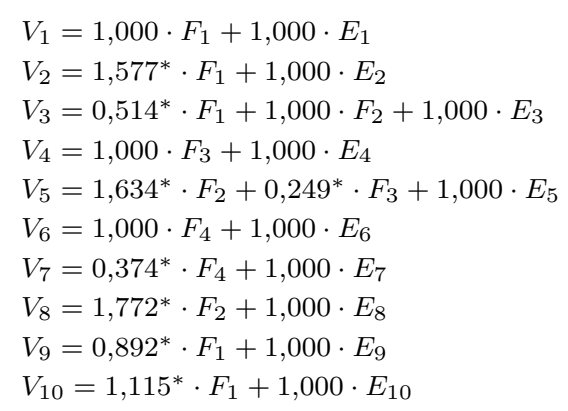

Tabla 5.22: Ecuaciones no estandarizadas. Invarianza métrica del modelo para Métodos de enseñanza - aprendizaje con 4 factores latentes

A pesar de que las ecuaciones no estandarizadas sí son comunes a todas las áreas de estudio no ocurre lo mismo con la estimación como los errores residuales del modelo de medida. En la imposición de restricciones cabía la posibilidad de haber definido una restricción adicional relativa a la invarianza de estos parámetros, pero en la práctica esta condición suele considerarse excesivamente restrictiva e igualmente así se ha valorado en este análisis. Por esta razón, se muestra en la Tabla 5.23 cómo varían las estimaciones de los errores de medida en cada área de estudio. Como venía sucediendo 
anteriormente los errores de medida más elevados corresponden al ítem 1 Asistencia a clase -, pero determinadas áreas de estudio, como Economía y empresa y Derecho logran reducir los valores estimados de todos los parámetros.

\begin{tabular}{|l|cccccccc|}
\hline & \multicolumn{7}{|c|}{ Educación Humanidades Ciencias Economía Derecho Técnicas Salud Ciencias } \\
& \multicolumn{7}{|c}{ Sociales y empresa } \\
\hline$E_{1}$ & $1.055^{*}$ & $1.231^{*}$ & $0.972^{*}$ & $1.053^{*}$ & $1.126^{*}$ & $1.140^{*}$ & $1.094^{*}$ & $1.061^{*}$ \\
$E_{2}$ & $0.259^{*}$ & $0.505^{*}$ & $0.342^{*}$ & $0.255^{*}$ & $0.274^{*}$ & $0.485^{*}$ & $0.491^{*}$ & $0.376^{*}$ \\
$E_{3}$ & $0.891^{*}$ & $0.832^{*}$ & $1.056^{*}$ & $0.399^{*}$ & $0.249^{*}$ & $0.591^{*}$ & $0.633^{*}$ & $0.716^{*}$ \\
$E_{4}$ & $0.412^{*}$ & 0.000 & 0.048 & $0.515^{*}$ & 0.010 & $0.694^{*}$ & 0.000 & $0.457^{*}$ \\
$E_{5}$ & $0.625^{*}$ & $0.616^{*}$ & $0.619^{*}$ & $0.506^{*}$ & $0.484^{*}$ & $0.527^{*}$ & $0.645^{*}$ & $0.613^{*}$ \\
$E_{6}$ & $0.314^{*}$ & $0.365^{*}$ & 0.223 & $0.310^{*}$ & $0.574^{*}$ & $0.351^{*}$ & $0.522^{*}$ & $0.329^{*}$ \\
$E_{7}$ & $0.751^{*}$ & $0.823^{*}$ & $0.761^{*}$ & $0.649^{*}$ & $0.725^{*}$ & $0.736^{*}$ & $0.623^{*}$ & $0.690^{*}$ \\
$E_{8}$ & $0.547^{*}$ & $0.570^{*}$ & $0.629^{*}$ & $0.524^{*}$ & $0.370^{*}$ & $0.702^{*}$ & $0.710^{*}$ & $0.670^{*}$ \\
$E_{9}$ & $0.622^{*}$ & $0.954^{*}$ & $0.635^{*}$ & $0.573^{*}$ & $0.737^{*}$ & $0.762^{*}$ & $0.765^{*}$ & $0.773^{*}$ \\
$E_{10}$ & $0.872^{*}$ & $1.075^{*}$ & $0.852^{*}$ & $0.471^{*}$ & $1.000^{*}$ & $0.553^{*}$ & $0.579^{*}$ & $0.578^{*}$ \\
\hline
\end{tabular}

Tabla 5.23: Errores de medida por área de estudio. Invarianza métrica del modelo para Métodos de enseñanza - aprendizaje con 4 factores latentes

Por otro lado, puesto que las restricciones de invarianza se han limitado al modelo de medida, las estimaciones de los parámetros estructurales asociados a este tipo de modelo, como varianzas y covarianzas en cada factor varían en cada área de estudio. En todas las áreas la varianza más elevada corresponde al tercer factor - Clases prácticas - como ocurría en la muestra global. Del mismo modo el cuarto factor - Clases teóricas - resulta prácticamente independiente del resto con correlaciones prácticamente nulas y covarianzas no significativas en la mayoría de los casos.

\begin{tabular}{|l|cccccccc|}
\hline & \multicolumn{7}{|c|}{ Educación Humanidades Ciencias Economía Derecho Técnicas Salud Ciencias } \\
& \multicolumn{7}{|c}{ Sociales y empresa } \\
\hline $\operatorname{Var}\left(F_{1}\right)$ & $0.283^{*}$ & $0.336^{*}$ & $0.369^{*}$ & $0.326^{*}$ & $0.222^{*}$ & $0.233^{*}$ & $0.335^{*}$ & $0.321^{*}$ \\
$\operatorname{Var}\left(F_{2}\right)$ & $0.113^{*}$ & $0.230^{*}$ & $0.122^{*}$ & $0.081^{*}$ & $0.095^{*}$ & $0.107^{*}$ & $0.095^{*}$ & $0.135^{*}$ \\
$\operatorname{Var}\left(F_{3}\right)$ & $1.016^{*}$ & $0.862^{*}$ & $1.772^{*}$ & $0.886^{*}$ & $0.654^{*}$ & $0.509^{*}$ & $2.369^{*}$ & $0.333^{*}$ \\
$\operatorname{Var}\left(F_{4}\right)$ & $0.434^{*}$ & $0.704^{*}$ & $0.547^{*}$ & $0.461^{*}$ & $0.491^{*}$ & $0.408^{*}$ & $0.475^{*}$ & $0.431^{*}$ \\
\hline
\end{tabular}

Tabla 5.24: Varianzas de los factores latentes por área de estudio. Invarianza métrica del modelo para Métodos de enseñanza - aprendizaje con 4 factores latentes 


\begin{tabular}{|l|ccccccccc|}
\hline & \multicolumn{7}{|c|}{ Educación Humanidades Ciencias Economía Derecho Técnicas } & Salud Ciencias \\
& \multicolumn{7}{|c}{ Sociales y empresa } \\
\hline $\operatorname{Cov}\left(F_{1}, F_{2}\right)$ & $0.112^{*}$ & $0.138^{*}$ & $0.154^{*}$ & $0.084^{*}$ & $0.114^{*}$ & $0.106^{*}$ & $0.141^{*}$ & $0.082^{*}$ \\
$\operatorname{Cov}\left(F_{1}, F_{3}\right)$ & $0.167^{*}$ & $0.150^{*}$ & $0.408^{*}$ & $0.273^{*}$ & $0.192^{*}$ & $0.191^{*}$ & $0.436^{*}$ & $0.193^{*}$ \\
$\operatorname{Cov}\left(F_{1}, F_{4}\right)$ & $0.043^{*}$ & -0.041 & -0.025 & 0.026 & -0.010 & -0.019 & -0.018 & $-0.048^{*}$ \\
$\operatorname{Cov}\left(F_{2}, F_{3}\right)$ & $0.159^{*}$ & $0.153^{*}$ & $0.232^{*}$ & $0.146^{*}$ & $0.124^{*}$ & $0.189^{*}$ & $0.247^{*}$ & $0.093^{*}$ \\
$\operatorname{Cov}\left(F_{2}, F_{4}\right)$ & $0.037^{*}$ & -0.026 & $-0.042^{*}$ & 0.015 & 0.021 & 0.016 & 0.038 & $0.077^{*}$ \\
$\operatorname{Cov}\left(F_{3}, F_{4}\right)$ & $0.046^{*}$ & -0.038 & $-0.284^{*}$ & -0.030 & -0.049 & -0.026 & $-0.140^{*}$ & -0.046 \\
$\operatorname{Cov}\left(E_{2}, E_{9}\right)$ & $0.068^{*}$ & 0.042 & 0.035 & $0.061^{*}$ & $0.058^{*}$ & $0.151^{*}$ & $0.161^{*}$ & $0.174^{*}$ \\
$\operatorname{Cov}\left(E_{2}, E_{10}\right)$ & $0.171^{*}$ & $0.314^{*}$ & $0.149^{*}$ & $0.111^{*}$ & $0.181^{*}$ & $0.121^{*}$ & $0.209^{*}$ & $0.213^{*}$ \\
\hline
\end{tabular}

Tabla 5.25: Covarianzas de los factores latentes por área de estudio. Invarianza métrica del modelo para Métodos de enseñanza - aprendizaje con 4 factores latentes

\begin{tabular}{|l|cccccccc|}
\hline & \multicolumn{7}{|c|}{ Educación Humanidades Ciencias Economía Derecho Técnicas Salud Ciencias } \\
& \multicolumn{7}{|c}{ Sociales y empresa } \\
\hline $\operatorname{Corr}\left(F_{1}, F_{2}\right)$ & 0.627 & 0.497 & 0.726 & 0.516 & 0.787 & 0.673 & 0.788 & 0.393 \\
$\operatorname{Corr}\left(F_{1}, F_{3}\right)$ & 0.311 & 0.278 & 0.504 & 0.509 & 0.503 & 0.555 & 0.489 & 0.591 \\
$\operatorname{Corr}\left(F_{1}, F_{4}\right)$ & 0.121 & -0.085 & -0.056 & 0.067 & -0.031 & -0.062 & -0.045 & -0.128 \\
$\operatorname{Corr}\left(F_{2}, F_{3}\right)$ & 0.470 & 0.343 & 0.498 & 0.545 & 0.499 & 0.811 & 0.521 & 0.439 \\
$\operatorname{Corr}\left(F_{2}, F_{4}\right)$ & 0.166 & -0.064 & -0.162 & 0.077 & 0.098 & 0.078 & 0.180 & 0.320 \\
$\operatorname{Corr}\left(F_{3}, F_{4}\right)$ & 0.070 & -0.049 & -0.289 & -0.047 & -0.087 & -0.057 & -0.132 & -0.121 \\
$\operatorname{Corr}\left(E_{2}, E_{9}\right)$ & 0.170 & 0.061 & 0.076 & 0.159 & 0.129 & 0.248 & 0.263 & 0.323 \\
$\operatorname{Corr}\left(E_{2}, E_{10}\right)$ & 0.232 & 0.310 & 0.202 & 0.214 & 0.211 & 0.187 & 0.314 & 0.319 \\
\hline
\end{tabular}

Tabla 5.26: Correlaciones de los factores latentes por área de estudio. Invarianza métrica del modelo para Métodos de enseñanza - aprendizaje con 4 factores latentes

Puesto que la principal utilidad de la estandarización de los coeficientes es proporcionar coeficientes comparables en distintos modelos aplicados a una misma muestra, cabe esperar que no serán de utilidad en la comparación de resultados de un mismo modelo sobre diferentes muestras. Atendiendo a este razonamiento, y con el fin de evitar redundancias que puedan llevar a confusión, se ha optado por no mostrar los resultados correspondientes a las ecuaciones estandarizadas del modelo de medida en cada área de estudio.

Respecto al modelo con estructura basada en tres factores, el análisis preliminar por áreas de estudio muestra que el ajuste es muy bueno en las mismas áreas donde el modelo anterior presentaba un ajuste excelente. Concretamente en las áreas de Educación, Derecho y Técnicas el estadístico $\chi^{2}$ de Satorra - Bentler toma valores inferiores a 80, mientras que en Humanidades, Ciencias Sociales y Ciencias oscila entre 100 y 115. En otras áreas como Economía y empresa y Salud la bondad de ajuste empeora tomando este estadístico valores superiores a 120 . 


\begin{tabular}{|l|ccccccccc|}
\hline & \multicolumn{7}{|c|}{ Educación Humanidades Ciencias Economía } & Derecho Técnicas Salud Ciencias \\
& \multicolumn{7}{c}{ Sociales } & y empresa \\
\hline$\chi_{\text {Ind }}^{2}$ & 883.9 & 814.2 & 1234.9 & 1728.8 & 489.3 & 1335.7 & 1102.6 & 998.3 \\
$g l_{\text {Ind }}$ & 45 & 45 & 45 & 45 & 45 & 45 & 45 & 45 \\
$\chi_{S-B}^{2}$ & 77.5 & 113.6 & 106.0 & 121.3 & 39.5 & 79.0 & 147.0 & 112.9 \\
$g l_{\text {Ind }}$ & 30 & 30 & 30 & 30 & 30 & 30 & 30 & 30 \\
\hline AIC Ind & 793.9 & 724.2 & 1144.9 & 1638.8 & 399.3 & 1245.7 & 1012.6 & 908.3 \\
AIC & 17.5 & 53.6 & 46.0 & 61.3 & -20.5 & 19.0 & 87.0 & 52.9 \\
CAIC & 555.8 & 494.5 & 905.0 & 1374.9 & 191.5 & 981.1 & 777.5 & 669.0 \\
CAIC & -141.2 & -99.6 & -114.0 & -114.7 & -159.0 & -157.4 & -69.7 & -106.6 \\
NFI & 0.912 & 0.861 & 0.914 & 0.930 & 0.919 & 0.941 & 0.867 & 0.887 \\
NNFI & 0.915 & 0.837 & 0.904 & 0.919 & 0.968 & 0.943 & 0.834 & 0.870 \\
CFI & 0.943 & 0.891 & 0.936 & 0.946 & 0.979 & 0.962 & 0.889 & 0.913 \\
IFI & 0.944 & 0.893 & 0.937 & 0.946 & 0.979 & 0.962 & 0.891 & 0.914 \\
MFI & 0.957 & 0.911 & 0.935 & 0.953 & 0.983 & 0.975 & 0.891 & 0.928 \\
RMSEA & 0.054 & 0.079 & 0.067 & 0.056 & 0.034 & 0.041 & 0.088 & 0.071 \\
Lim Inf $90 \%$ & 0.039 & 0.064 & 0.053 & 0.046 & 0.000 & 0.030 & 0.074 & 0.057 \\
Lim Sup $90 \%$ & 0.069 & 0.094 & 0.081 & 0.067 & 0.060 & 0.052 & 0.102 & 0.085 \\
\hline
\end{tabular}

Tabla 5.27: Índices de ajuste y contraste $\chi^{2}$ por área de estudio. Invarianza métrica del modelo para Métodos de enseñanza - aprendizaje con 3 factores latentes

Los promedios de la matriz residual de varianzas - covarianzas son superiores a los correspondientes al modelo anterior, y también al obtenido por el mismo modelo con tres factores pero sin restricciones de invarianza, que tomaba valores de 0.0417 y 0.0468 para los promedios absolutos incluyendo y excluyendo los términos en la diagonal, y 0.0332 y 0.0376 para los mismos valores estandarizados.

\begin{tabular}{|l|cccccccc|}
\hline & \multicolumn{7}{|l|}{ Educación Humanidades Ciencias Economía Derecho Técnicas Salud Ciencias } \\
& \multicolumn{7}{c}{ Sociales y empresa } \\
\hline Avg. abs. residual & 0.0508 & 0.0789 & 0.0682 & 0.0362 & 0.0502 & 0.0272 & 0.0751 & 0.0512 \\
Avg. off-diag. abs. residual & 0.0540 & 0.0820 & 0.0691 & 0.0388 & 0.0495 & 0.0292 & 0.0755 & 0.0554 \\
Avg. abs. std. residual & 0.0475 & 0.0668 & 0.0553 & 0.0388 & 0.0567 & 0.0279 & 0.0584 & 0.0514 \\
Avg. off-diag. abs. std. residual & 0.0513 & 0.0686 & 0.0571 & 0.0421 & 0.0557 & 0.0299 & 0.0597 & 0.0554 \\
\hline
\end{tabular}

Tabla 5.28: Promedios de las matrices residuales de varianzas-covarianzas por área de estudio. Invarianza métrica del modelo para Métodos de enseñanza - aprendizaje con 3 factores latentes

Del mismo modo, el ajuste del modelo con las restricciones de invarianza métrica no resulta tan bueno como el obtenido a partir del modelo anterior, pero sí puede considerarse suficientemente bueno como para ser considerado una alternativa. Concretamente el valor del estadístico $\chi^{2}$ 
de Satorra - Bentler se incrementa desde el valor correspondiente al modelo sin restricciones de 613.6 con 31 grados de libertad hasta 979.4 con 288 grados de libertad. En este sentido los índices comparativos de ajuste disminuyen entre 3 y 5 centésimas debido a las restricciones de invarianza $(N F I=0,937 \rightarrow 0,895 ; N N F I=0,913 \rightarrow 0,916 ; C F I=0,940 \rightarrow$ $0,931 ; I F I=0,940 \rightarrow 0,947)$ mientras que el RMSEA permanece invariante en el valor 0.063, con intervalo de confianza (90\%) en [0.059, 0.068].

En este caso el contraste $\chi^{2}$ del modelo de medida final para Métodos de enseñanza - aprendizaje con 3 factores latentes y restricciones de invarianza métrica aporta los siguientes resultados:

$$
\begin{aligned}
& \chi_{\text {Ind }}^{2}=8560,2 \\
& g l_{\text {Ind }}=360 \\
& \chi_{S-B}^{2}=979,4 \\
& g l=288 \\
& \text { Prob }=0,000
\end{aligned}
$$

Los coeficientes de fiabilidad se encuentran en el mismo orden de magnitud que los obtenidos en la validación del modelo anterior a las distintas áreas de estudio. De hecho los coeficientes Alfa de Cronbach son exactamente iguales ya que no se han modificado las variables observables o las distintas submuestras, y el Coeficiente Rho ha disminuido ligeramente en esta estimación respecto al valor en el modelo sin restricciones de 0.789.

\begin{tabular}{|l|cccccccc|}
\hline & \multicolumn{10}{|c|}{ Educación Humanidades Ciencias Economía Derecho Técnicas Salud Ciencias } \\
& Sociales & y empresa \\
\hline$\alpha$ Cronbach & 0.714 & 0.668 & 0.735 & 0.722 & 0.724 & 0.679 & 0.737 & 0.692 \\
Rho & 0.730 & 0.716 & 0.770 & 0.758 & 0.733 & 0.714 & 0.748 & 0.697 \\
\hline
\end{tabular}

Tabla 5.29: Índices de fiabilidad por área de estudio. Invarianza métrica del modelo para Métodos de enseñanza - aprendizaje con 3 factores latentes

Los valores de los coeficientes de regresión mostrados en las ecuaciones no estandarizadas son comunes a todas las áreas de estudio, tal y como se especifica en las restricciones de invarianza métrica. Respecto al modelo anterior evidentemente experimentan mayores variaciones aquellos ítems cuyas saturaciones se asocian ahora a factores diferentes, como el 4, el 5 y el 7 Prácticas en empresa, Conocimientos prácticos y El profesor como fuente de información. Concretamente este último ítem pasa a ser significativo, al igual que ocurría en el análisis de invarianza métrica sobre el modelo de 
cuatro factores.

$$
\begin{aligned}
& V_{1}=1,000 \cdot F_{1}+1,000 \cdot E_{1} \\
& V_{2}=1,586^{*} \cdot F_{1}+1,000 \cdot E_{2} \\
& V_{3}=0,470^{*} \cdot F_{1}+1,000 \cdot F_{2}+1,000 \cdot E_{3} \\
& V_{4}=1,818 \cdot F_{2}+1,000 \cdot E_{4} \\
& V_{5}=2,108^{*} \cdot F_{2}+1,000 \cdot E_{5} \\
& V_{6}=1,000 \cdot F_{3}+1,000 \cdot E_{6} \\
& V_{7}=1,871^{*} \cdot F_{3}+1,000 \cdot E_{7} \\
& V_{8}=1,548^{*} \cdot F_{2}+1,000 \cdot E_{8} \\
& V_{9}=0,897^{*} \cdot F_{1}+1,000 \cdot E_{9} \\
& V_{10}=1,120^{*} \cdot F_{1}+1,000 \cdot E_{10}
\end{aligned}
$$

Tabla 5.30: Ecuaciones no estandarizadas. Invarianza métrica del modelo para Métodos de enseñanza - aprendizaje con 3 factores latentes

En la Tabla 5.31 se muestran las estimaciones de los errores de medida, cuya invarianza no se ha restringido como se ha comentado anteriormente. $\mathrm{Al}$ igual que ocurría en el modelo con cuatro factores el primer indicador Asistencia a clase - obtiene las estimaciones más elevadas de este parámetro, con excepción de determinados errores en indicadores y áreas de estudio concretas. Las áreas con estimaciones de estos parámetros más pequeñas siguen siendo Economía y empresa y Derecho.

En cuanto a las variaciones de los parámetros estructurales, en ningún caso se alcanzan varianzas tan elevadas como las obtenidas en el tercer factor - Clases prácticas - del anterior modelo con cuatro factores. Sin embargo el factor Clases teóricas sigue obteniendo las varianzas más bajas e igualmente se comporta de forma prácticamente independiente respecto al resto de los factores, con la mayoría de covarianzas no significativas y correlaciones prácticamente nulas. 


\begin{tabular}{|l|llllllll|}
\hline & \multicolumn{7}{|c|}{ Educación Humanidades Ciencias Economía Derecho Técnicas Salud Ciencias } \\
Sociales y empresa \\
\hline$E_{1}$ & $1.058^{*}$ & $1.233^{*}$ & $0.973^{*}$ & $1.054^{*}$ & $1.124^{*}$ & $1.140^{*}$ & $1.094^{*}$ & $1.068^{*}$ \\
$E_{2}$ & $0.248^{*}$ & $0.496^{*}$ & $0.330^{*}$ & $0.255^{*}$ & $0.259^{*}$ & $0.480^{*}$ & $0.484^{*}$ & $0.386^{*}$ \\
$E_{3}$ & $0.922^{*}$ & $0.846^{*}$ & $1.070^{*}$ & $0.399^{*}$ & $0.257^{*}$ & $0.590^{*}$ & $0.659^{*}$ & $0.729^{*}$ \\
$E_{4}$ & $1.054^{*}$ & $0.597^{*}$ & $1.119^{*}$ & $0.995^{*}$ & $0.405^{*}$ & $0.828^{*}$ & $1.519^{*}$ & $0.577^{*}$ \\
$E_{5}$ & $0.571^{*}$ & $0.652^{*}$ & $0.557^{*}$ & $0.470^{*}$ & $0.460^{*}$ & $0.495^{*}$ & $0.604^{*}$ & $0.698^{*}$ \\
$E_{6}$ & $0.662^{*}$ & $0.923^{*}$ & $0.647^{*}$ & $0.682^{*}$ & $0.968^{*}$ & $0.675^{*}$ & $0.907^{*}$ & $0.672^{*}$ \\
$E_{7}$ & $0.524^{*}$ & $0.420^{*}$ & $0.481^{*}$ & $0.395^{*}$ & $0.460^{*}$ & $0.514^{*}$ & $0.339^{*}$ & $0.462^{*}$ \\
$E_{8}$ & $0.610^{*}$ & $0.838^{*}$ & $0.693^{*}$ & $0.568^{*}$ & $0.417^{*}$ & $0.728^{*}$ & $0.726^{*}$ & $0.853^{*}$ \\
$E_{9}$ & $0.618^{*}$ & $0.946^{*}$ & $0.638^{*}$ & $0.570^{*}$ & $0.730^{*}$ & $0.763^{*}$ & $0.767^{*}$ & $0.769^{*}$ \\
$E_{10}$ & $0.870^{*}$ & $1.075^{*}$ & $0.858^{*}$ & $0.471^{*}$ & $1.000^{*}$ & $0.552^{*}$ & $0.578^{*}$ & $0.569^{*}$ \\
\hline
\end{tabular}

Tabla 5.31: Errores de medida por área de estudio. Invarianza métrica del modelo para Métodos de enseñanza - aprendizaje con 3 factores latentes

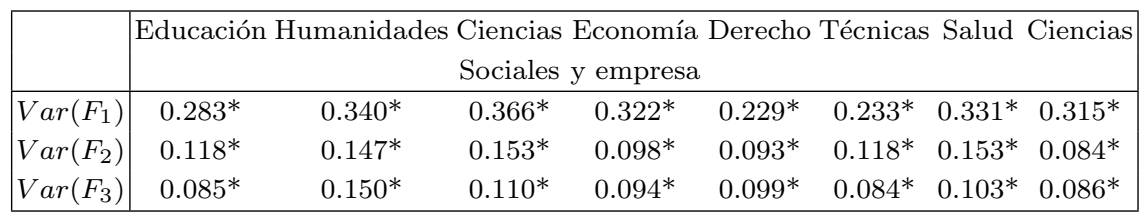

Tabla 5.32: Varianzas de los factores latentes por área de estudio. Invarianza métrica del modelo para Métodos de enseñanza - aprendizaje con 4 factores latentes

\begin{tabular}{|l|ccccccccc|}
\hline & \multicolumn{7}{|c|}{ Educación Humanidades Ciencias Economía Derecho Técnicas } & Salud Ciencias \\
& \multicolumn{7}{|c|}{ Sociales y empresa } \\
\hline $\operatorname{Cov}\left(F_{1}, F_{2}\right)$ & $0.109^{*}$ & $0.119^{*}$ & $0.172^{*}$ & $0.105^{*}$ & $0.116^{*}$ & $0.109^{*}$ & $0.171^{*}$ & $0.097^{*}$ \\
$\operatorname{Cov}\left(F_{1}, F_{3}\right)$ & 0.004 & $-0.030^{*}$ & $-0.027^{*}$ & -0.010 & -0.011 & -0.015 & $-0.036^{*}$ & $-0.046^{*}$ \\
$\operatorname{Cov}\left(F_{2}, F_{3}\right)$ & 0.008 & $-0.031^{*}$ & $-0.035^{*}$ & $-0.009^{*}$ & 0.001 & -0.002 & $-0.027^{*}$ & -0.001 \\
$\operatorname{Cov}\left(E_{2}, E_{9}\right)$ & 0.061 & $0.034^{*}$ & $0.035^{*}$ & $0.059^{*}$ & 0.048 & $0.151^{*}$ & $0.161^{*}$ & $0.174^{*}$ \\
$\operatorname{Cov}\left(E_{2}, E_{10}\right)$ & $0.169^{*}$ & $0.309^{*}$ & $0.153^{*}$ & $0.110^{*}$ & $0.178^{*}$ & $0.122^{*}$ & $0.210^{*}$ & $0.208^{*}$ \\
\hline
\end{tabular}

Tabla 5.33: Covarianzas de los factores latentes por área de estudio. Invarianza métrica del modelo para Métodos de enseñanza - aprendizaje con 4 factores latentes 


\begin{tabular}{|l|cccccccc|}
\hline & \multicolumn{7}{|c|}{ Educación Humanidades Ciencias Economía Derecho Técnicas Salud Ciencias } \\
& \multicolumn{7}{|c}{ Sociales y empresa } \\
\hline $\operatorname{Corr}\left(F_{1}, F_{2}\right)$ & 0.593 & 0.532 & 0.729 & 0.590 & 0.797 & 0.657 & 0.758 & 0.598 \\
$\operatorname{Corr}\left(F_{1}, F_{3}\right)$ & 0.026 & -0.132 & -0.135 & -0.057 & -0.070 & -0.105 & -0.196 & -0.281 \\
$\operatorname{Corr}\left(F_{2}, F_{3}\right)$ & 0.081 & -0.208 & -0.274 & -0.092 & 0.011 & -0.019 & -0.212 & -0.006 \\
$\operatorname{Corr}\left(E_{2}, E_{9}\right)$ & 0.157 & 0.050 & 0.076 & 0.155 & 0.111 & 0.249 & 0.265 & 0.319 \\
$\operatorname{Corr}\left(E_{2}, E_{10}\right)$ & 0.230 & 0.307 & 0.207 & 0.212 & 0.208 & 0.188 & 0.315 & 0.314 \\
\hline
\end{tabular}

Tabla 5.34: Correlaciones de los factores latentes por área de estudio. Invarianza métrica del modelo para Métodos de enseñanza - aprendizaje con 4 factores latentes

En conclusión, el análisis de invarianza métrica sobre ambos modelos de medida, con cuatro y tres factores respectivamente, muestra que ambos instrumentos pueden ser aplicados sobre las ocho áreas de estudio definidas para este análisis sin pérdida de generabilidad de los constructos teóricos ni fiabilidad en las medidas. El análisis de la bondad de ajuste muestra que el modelo de cuatro factores es ligeramente mejor que el modelo de tres factores tanto en la muestra global, como en cada una de las submuestras asociadas a las áreas de estudio. Por esta razón se ha seleccionado la estructura con cuatro factores como modelo de los constructos teóricos relativos a los Métodos de enseñanza - aprendizaje en Educación Superior. Dichos constructos conformarán posteriormente el conjunto de factores latentes exógenos en la modelización de los procesos de enseñanza - aprendizaje de competencias.

\subsubsection{Competencias adquiridas en la Universidad}

El análisis factorial confirmatorio sobre las distintos constructos asociados a las competencias adquiridas en la Universidad se ha planteado sobre dos modelos alternativos que reflejan los conceptos expuestos por De Miguel (2006). En su investigación De Miguel señala que las competencias se favorecen actuando tanto sobre aquellas características que constituyen la base de la personalidad de los estudiantes como sobre aquellas características más visibles de la competencia. En el primer grupo se engloban las características subyacentes a la competencia como la motivación del estudiante, los rasgos de su personalidad, el autoconcepto que tiene de sí mismo o sus actitudes o valores ante el estudio. Por el contrario las características visibles incluyen tanto los conocimientos como las habilidades. Resulta evidente que la medición de estas características es mucho más directa y fácil para el investigador que las anteriores. Por ello, y sin menospreciar en ningún momento la importancia de las características subyacentes de las competencias así como su vinculación intrínseca a las características visibles, el análisis de las competencias se focalizará en los conocimientos y habilidades de los graduados universitarios. 


\section{Análisis Factorial Confirmatorio sobre la muestra general}

Así, a partir de los fundamentos teóricos expuestos por De Miguel sobre Competencias adquiridas en Educación Superior se proponen dos modelos alternativos con cinco y seis factores de la siguiente forma:

1. Modelo 1: 5 Factores; Exclusión del ítem Hablar y escribir en idiomas extranjeros

2. Modelo 2: 6 Factores; Exclusión del ítem Hablar y escribir en idiomas extranjeros

3. Modelo 3: 5 Factores; Inclusión del ítem Hablar y escribir en idiomas extranjeros

4. Modelo 4: 6 Factores; Inclusión del ítem Hablar y escribir en idiomas extranjeros

En este punto se plantea la cuestión de si introducir el ítem Hablar y escribir en idiomas extranjeros incluido en el cuestionario. Dicho indicador no aparece reflejado en la teoría sobre competencias y de hecho, los resultados en análisis previos ya mostraron que su contribución a la definición de los constructos teóricos era prácticamente irrelevante. En cualquier caso se formularon dos nuevos modelos, a partir de los anteriores, que contemplan la introducción de este ítem como parte del Factor 3 - Comunicación. De esta forma el trabajo de selección del modelo con mejor ajuste supone el análisis de cuatro modelos rivales excluyendo e incluyendo este ítem e igualmente modificando el número de factores entre cinco y seis. A continuación se muestran las características diferenciadoras de cada uno de los modelos planteados.

En todos los casos se han numerado los factores a partir de 5, puesto que la numeración anterior se ha asignado ya a los factores sobre Métodos de enseñanza - aprendizaje. La selección del modelo que mejor ajusta a los datos se realiza en base a los estadísticos e índices de ajuste obtenidos a priori, sin realizar ninguna modificación de reespecificación. El objetivo es seleccionar cuál de los modelos planteados presenta un mejor ajuste sin necesidad de introducir nuevas relaciones que incrementen la complejidad del modelo. Ello no implica que sobre el modelo seleccionado deje de plantearse posteriormente la fase de reespecificación con el fin de mejorar el ajuste. 


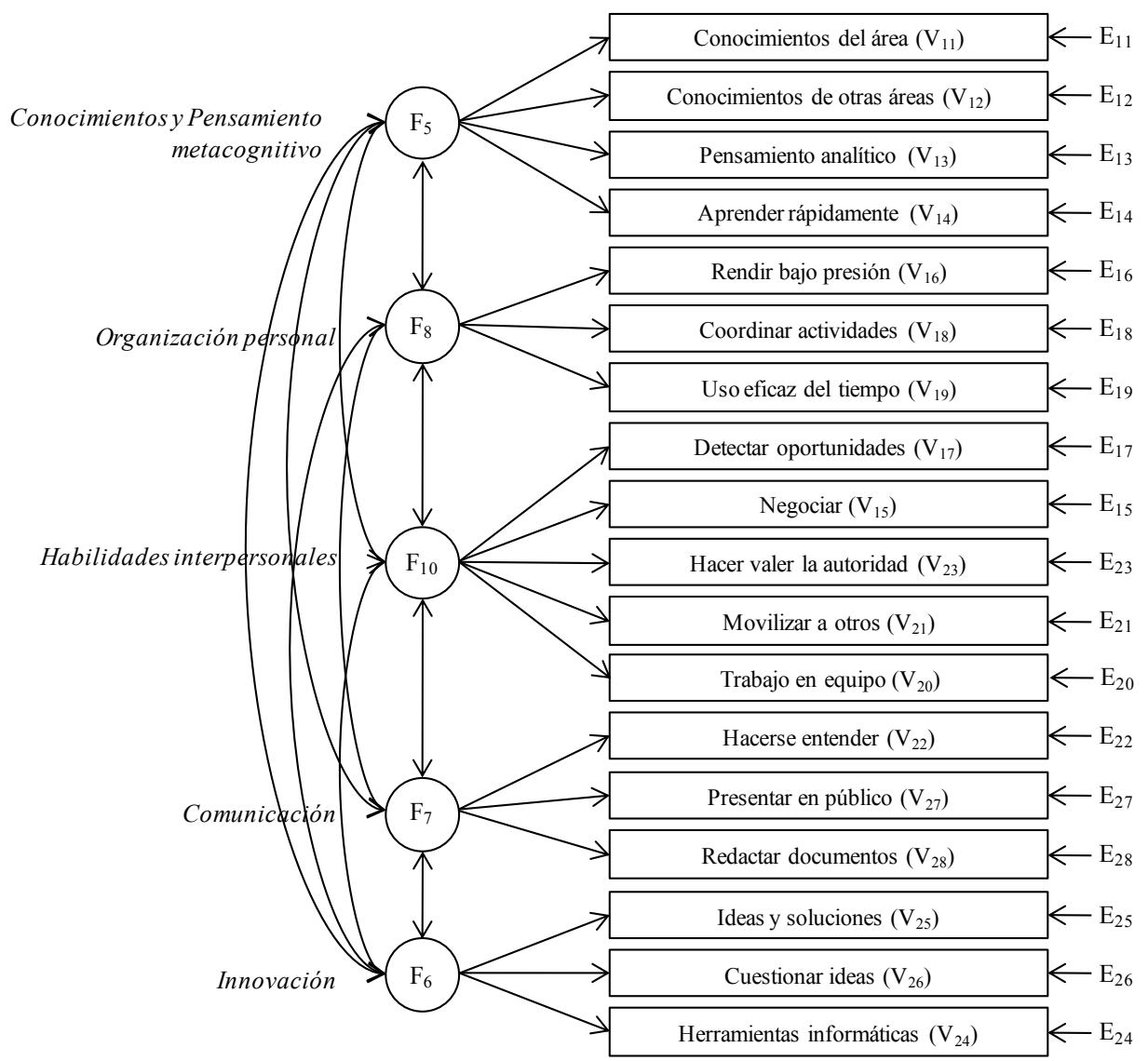

Figura 5.5: Modelo de medida 1, con 5 factores latentes y exclusión del ítem Hablar y escribir en idiomas extranjeros. Competencias adquiridas en la Universidad 


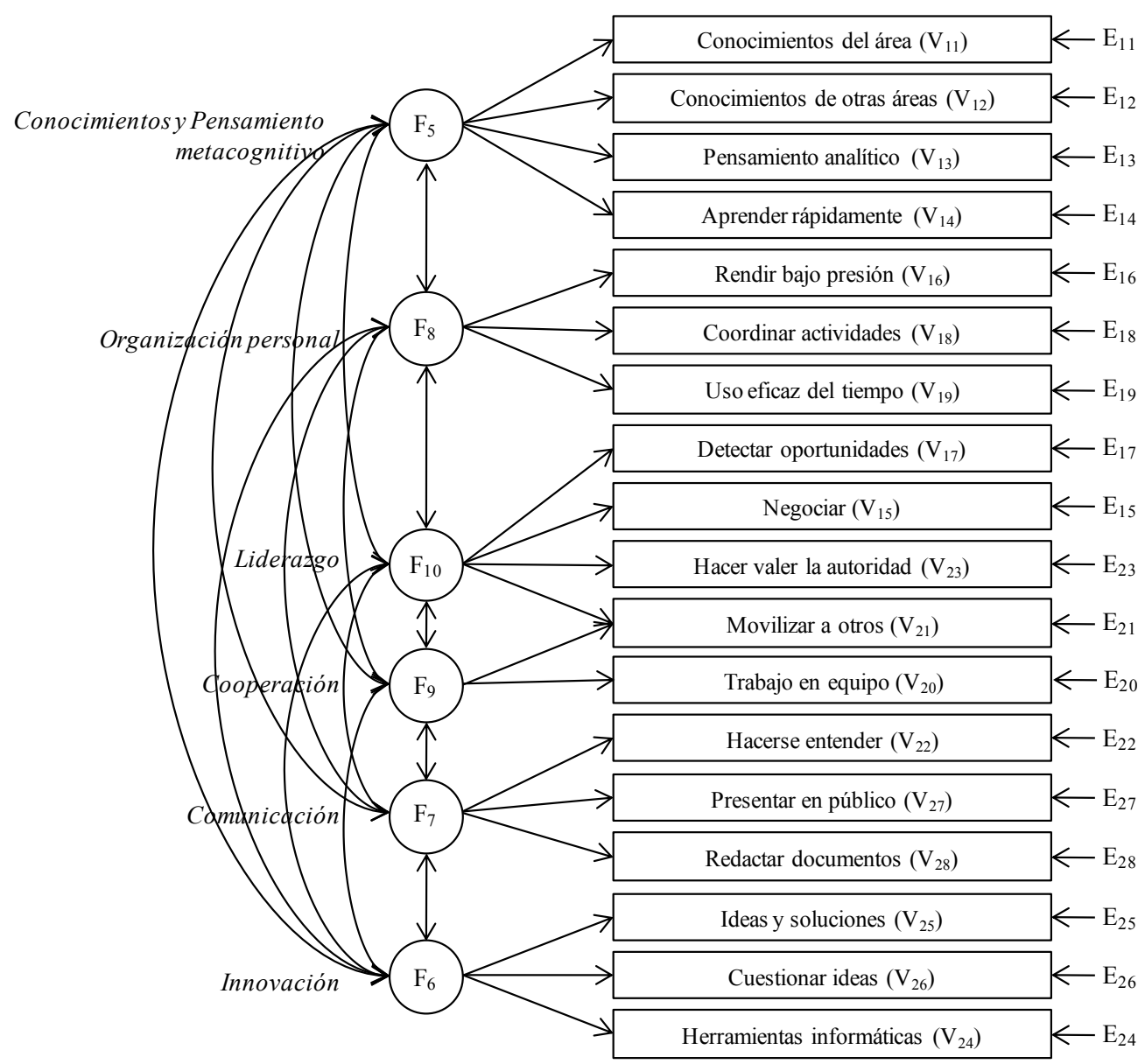

Figura 5.6: Modelo de medida 2, con 6 factores latentes y exclusión del ítem Hablar y escribir en idiomas extranjeros. Competencias adquiridas en la Universidad 


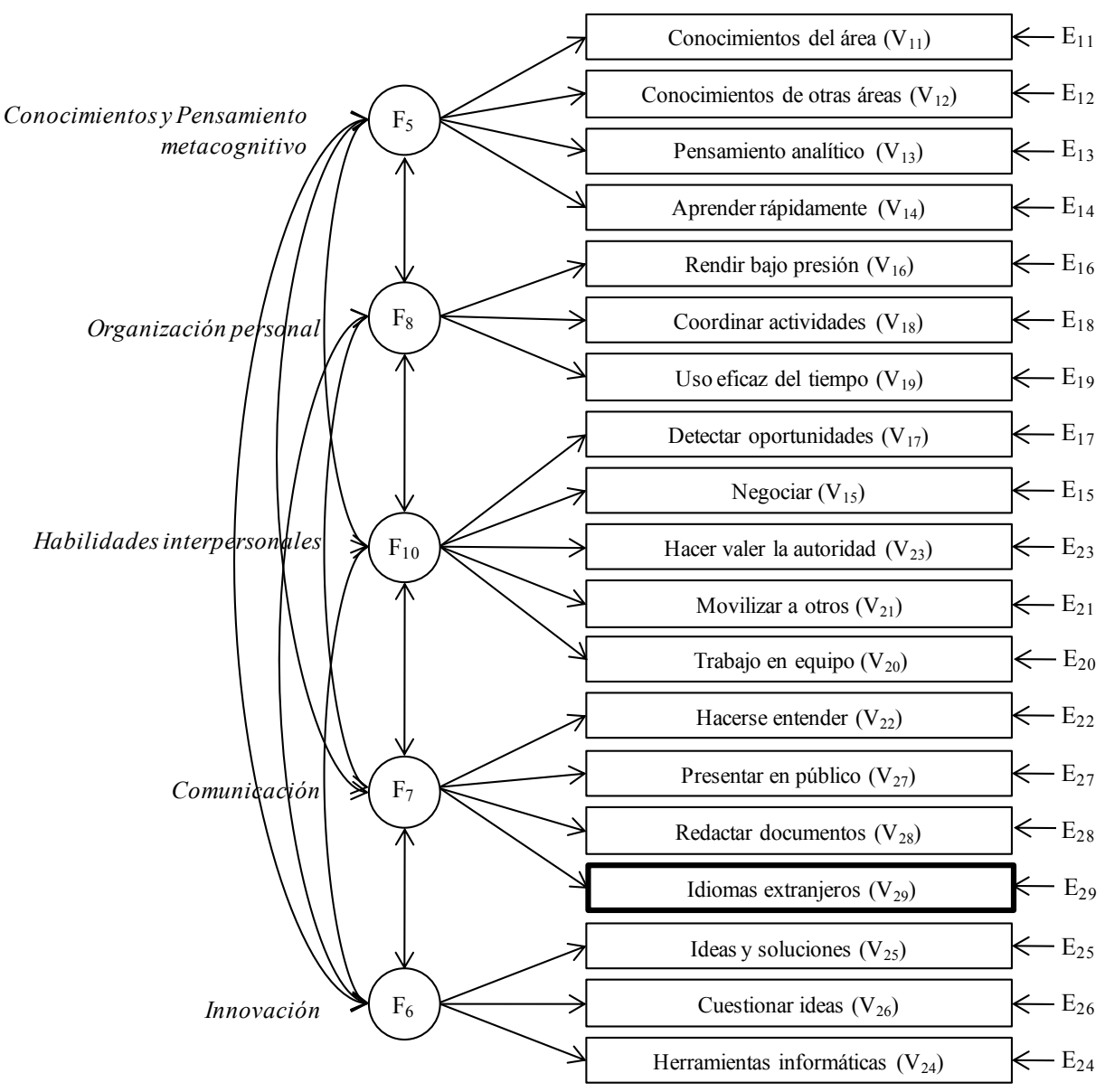

Figura 5.7: Modelo de medida 3, con 5 factores latentes e inclusión del ítem Hablar y escribir en idiomas extranjeros. Competencias adquiridas en la Universidad 


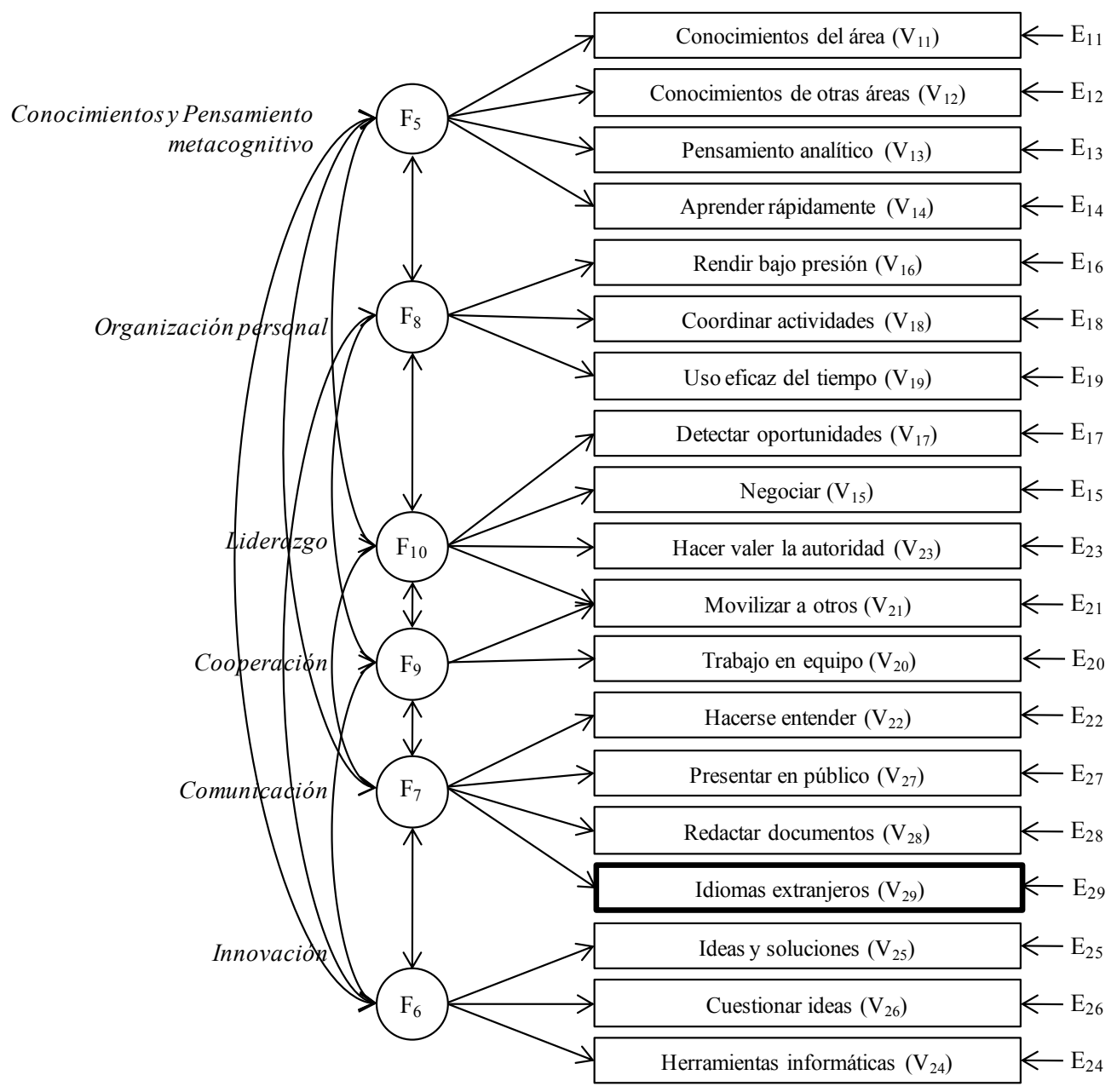

Figura 5.8: Modelo de medida 4, con 6 factores latentes e inclusión del ítem Hablar y escribir en idiomas extranjeros. Competencias adquiridas en la Universidad 


\begin{tabular}{|l|c|c|c|c|}
\hline & Modelo 1 & Modelo 2 & Modelo 3 & Modelo 4 \\
\hline$\chi_{\text {Ind }}^{2}$ & 38991.5 & 38991.5 & 38991.5 & 38991.5 \\
$g l_{\text {Ind }}$ & 153 & 153 & 153 & 153 \\
$\chi^{2}$ & 4507.4 & 3615.8 & 4801.3 & 3935.3 \\
$\chi_{S-B}^{2}$ & 3280.6 & 2628.2 & 3568.3 & 2923.6 \\
$g l$ & 125 & 119 & 142 & 136 \\
Prob. & 0.000 & 0.000 & 0.000 & 0.000 \\
\hline
\end{tabular}

Tabla 5.35: Contraste $\chi^{2}$ para los 4 modelos planteados inicialmente. Competencias adquiridas en la Universidad

La comparación de los estadísticos $\chi^{2}$ muestra que, entre los modelos que excluyen el ítem Hablar y escribir en idiomas extranjeros, el segundo modelo con seis factores latentes ajusta mejor que el primero, con sólo cinco factores latentes. Del mismo modo, entre los modelos que sí incluyen este ítem, el ajuste del cuarto modelo con seis factores latentes, es mejor que el del tercero. Las diferencias corregidas entre el valor del estadístico $\chi^{2}$ de Satorra - Bentler son significativas en todos los casos. Así, quedan descartados los modelos con cinco factores y se plantea ahora escoger el modelo de seis factores que mejor ajuste a los datos. Se trata entonces de comparar el segundo y cuarto modelo, con exclusión e inclusión del ítem sobre idiomas extranjeros. La diferencia corregida en el estadístico $\chi^{2}$ de Satorra - Bentler de ambos modelos muestra que existe una mejora significativa del segundo modelo respecto al cuarto. Por tanto se selecciona el segundo modelo con seis factores y exclusión del ítem Hablar y escribir en idiomas extranjeros.

La disminución del estadístico $\chi^{2}$ al pasar del Modelo 1 al 2 resulta significativa, es decir que el modelo 2 ajusta mejor que el modelo 1 :

$k_{1}=\frac{\chi_{1}^{2}}{\chi_{S-B, 1}^{2}}=1,374$

$k_{2}=\frac{\chi_{2}^{2}}{\chi_{S-B, 2}^{2}}=1,375$

$D_{12}=\chi_{1}^{2}-\chi_{2}^{2}=891,6$

$d_{12}=6$

$k_{12}=\frac{d_{1} k_{1}-d_{2} k_{2}}{d_{12}}=1,354$

$\bar{D}_{12}=\frac{D_{12}}{k_{12}}=658,5>\chi_{\alpha=0,05 ; g l=6}^{2}=12,59$

De la misma forma que resulta significativa la diferencia en el mismo estadístico al pasar del modelo 3 al 4 , por lo que el modelo 4 ajusta mejor que el modelo 3:

$k_{3}=\frac{\chi_{3}^{2}}{\chi_{S-B, 3}^{2}}=1,345$ 
$k_{4}=\frac{\chi_{4}^{2}}{\chi_{S-B, 4}^{2}}=1,346$

$D_{34}=\chi_{3}^{2}-\chi_{4}^{2}=866,0$

$d_{34}=6$

$k_{34}=\frac{d_{3} k_{3}-d_{4} k_{4}}{d_{34}}=0,597$

$\bar{D}_{34}=\frac{D_{34}}{k_{34}}=1450,6>\chi_{\alpha=0,05 ; g l=6}^{2}=12,59$

Entre los modelos 2 y 4 resulta significativa la diferencia en el estadístico $\chi^{2}$ al pasar del modelo 4 al 2. Por tanto el modelo que minimiza el valor del estadístico $\chi^{2}$ resulta ser el modelo 2 , que como se observa en la Tabla 5.36 también obtiene los índices de ajuste más elevados:

$D_{42}=\chi_{4}^{2}-\chi_{2}^{2}=319,5$

$d_{42}=17$

$k_{42}=\frac{d_{4} k_{4}-d_{2} k_{2}}{d_{42}}=1,143$

$\bar{D}_{42}=\frac{D_{42}}{k_{42}}=279,5>\chi_{\alpha=0,05 ; g l=17}^{2}=27,58$

Por tanto la comparación de los índices de ajuste correspondientes a los distintos modelos planteados muestra que el segundo modelo obtiene el mejor ajuste. Asimismo es fácil comprobar que son igualmente aplicables las conclusiones obtenidas mediante los estadísticos $\chi^{2}$. Los modelos dos y cuatro, ambos con seis factores latentes, ajustan mejor que los modelos con cinco factores y entre ellos, el segundo modelo que excluye el ítem Hablar y escribir en idiomas extranjeros, ajusta que mejor que el cuarto que sí lo incluye.

\begin{tabular}{|l|c|c|c|c|}
\hline & Modelo 1 & Modelo 2 & Modelo 3 & Modelo 4 \\
\hline AIC Ind & 38685.5 & 38685.6 & 39344.7 & 39344.7 \\
AIC & 3030.7 & 2390.2 & 3284.3 & 2651.6 \\
CAIC Ind & 37541.4 & 37541.3 & 38066.0 & 38066.0 \\
CAIC & 2095.9 & 1500.2 & 2222.5 & 1634.6 \\
NFI & 0.916 & 0.933 & 0.910 & 0.926 \\
NNFI & 0.901 & 0.917 & 0.896 & 0.911 \\
CFI & 0.919 & 0.935 & 0.913 & 0.929 \\
IFI & 0.919 & 0.935 & 0.913 & 0.930 \\
MFI & 0.720 & 0.770 & 0.700 & 0.748 \\
RMSEA & 0.072 & 0.066 & 0.071 & 0.065 \\
Lim Inf $90 \%$ & 0.070 & 0.064 & 0.069 & 0.063 \\
Lim Sup $90 \%$ & 0.075 & 0.068 & 0.073 & 0.067 \\
\hline
\end{tabular}

Tabla 5.36: Índices de ajuste para los cuatro modelos planteados inicialmente. Competencias adquiridas en la Universidad 
El número de grados de libertad de este modelo es el más pequeño de los cuatro modelos planteados ya que incluye una variable observable menos y adicionalmente la adición de un nuevo factor ha incrementado el número de parámetros libres a estimar. En cualquier caso al ser dicho número es positivo, el modelo se encuentra sobreidentificado.

$$
g l=\frac{1}{2} \cdot 18 \cdot 19-52=119
$$

Los promedios de la matriz residual de varianza resultan superiores a los obtenidos en el análisis de los Métodos de enseñanza - aprendizaje debido a la mayor complejidad del modelo. Concretamente resultan superiores al modelo con tres factores, que sin restricciones de invarianza alcanzaba los mayores promedios residuales, alcanzando valores de 0.0376 y 0.0418 para los promedios absolutos incluyendo y excluyendo los términos en la diagonal, y 0.0249 y 0.0376 para los mismos valores estandarizados.

Average absolute residual $=0.0904$

Average off-diagonal absolute residual $=0.1010$

Average absolute standardized residual $=0.0327$

Average off-diagonal absolute standardized residual $=0.0365$

Los coeficientes de fiabilidad de este modelo resultan muy elevados, ya que también lo es el número de variables observables contenidas en él. Así, el Coeficiente Alfa de Cronbach alcanza el valor 0.927 y el Coeficiente Rho se sitúa en 0.946. Asimismo los porcentajes de fiabilidad compuesta y porcentaje de varianza extraída por cada factor revelan que la mayoría de los factores alcanzan el umbral establecido para ambos parámetros. Únicamente en el caso del noveno factor - Cooperación - no se supera el límite mínimo recomendado para la fiabilidad compuesta del $70 \%$ (65.9\%) mientras que la recomendación de un obtener un porcentaje mínimo de varianza extraída del $50 \%$ no se cumple para el quinto factor - Conocimientos y Pensamiento metacognitivo (43.6\%).

La aplicación del Contraste de Lagrange a este modelo sugiere añadir una nueva relación entre el ítem 22 - Hacerse entender - y el décimo factor Liderazgo. Esta relación resulta coherente con la teoría y su introducción implicaría un descenso muy significativo en el estadístico $\chi^{2}$, mejorando así el ajuste del modelo a los datos. Puesto que no supone un incremento innecesario de la complejidad del modelo se opta por aceptar dicha modificación y volver a ejecutar la estimación de los parámetros del modelo tomando en cuenta esta consideración. En este caso el número de grados de libertad se reduce en una unidad al introducirse una nueva relación con un parámetro adicional a estimar. 


\begin{tabular}{|l|c|c|}
\hline & Fiabilidad compuesta & Varianza extraída \\
\hline$F_{5}$ & $74.8 \%$ & $43.6 \%$ \\
$F_{6}$ & $80.3 \%$ & $51.2 \%$ \\
$F_{7}$ & $80.4 \%$ & $57.8 \%$ \\
$F_{8}$ & $80.8 \%$ & $58.7 \%$ \\
$F_{9}$ & $65.9 \%$ & $55.0 \%$ \\
$F_{10}$ & $80.5 \%$ & $58.0 \%$ \\
\hline
\end{tabular}

Tabla 5.37: Fiabilidad compuesta y varianza extraída. Modelo de medida inicial para Competencias adquiridas en la Universidad con 6 factores latentes

$$
g l=\frac{1}{2} \cdot 18 \cdot 19-53=118
$$

Con esta modificación los promedios de la matriz residual de varianzas - covarianzas disminuyen respecto al modelo anterior, en el que tomaban valores de 0.0904 y 0.1010 para los promedios absolutos incluyendo y excluyendo los términos en la diagonal, y 0.0327 y 0.0365 para los mismos valores estandarizados.

Average absolute residual $=0.0840$

Average off-diagonal absolute residual $=0.0939$

Average absolute standardized residual $=0.0305$

Average off-diagonal absolute standardized residual $=0.0341$

E igualmente mejoran tanto los estadísticos $\chi^{2}$ como los índices de ajuste del modelo, indicando una mejora significativa de la bondad de ajuste del modelo gracias a la modificación introducida. El valor del estadístico $\chi^{2}$ disminuye de 2628.2 hasta 2345.5, que corresponde a una diferencia corregida de 374.5. Del mismo modo los índices comparativos de ajuste se incrementa aproximadamente en dos centésimas $(N F I=0,933 \rightarrow 0,940 ; N N F I=$ $0,917 \rightarrow 0,926 ; C F I=0,935 \rightarrow 0,943 ; I F I=0,935 \rightarrow 0,943)$ mientras que el RMSEA disminuye de 0.066 a 0.063 , con intervalo de confianza $(90 \%)$ entre los valores $[0.060,0.065]$. Los índices de parsimonia $(A I C=2390,2 \rightarrow$ $2109,5 ; C A I C=1500,2 \rightarrow 1227,0)$ igualmente disminuyen respecto a los obtenidos en el modelo de independencia $\left(A I C_{\text {Ind }}=38685,6 ; C A I C_{\text {Ind }}=\right.$ $37541,3)$.

El contraste $\chi^{2}$ del modelo modificado de medida para Competencias adquiridas en la Universidad con 6 factores latentes resulta: 
$\chi_{I n d}^{2}=38991,6$

$g l_{\text {Ind }}=153$

$\chi^{2}=3233,4$

$\chi_{S-B}^{2}=2345,5$

$g l=118$

Prob $=0,000$

La introducción de esta modificacion supone una disminución significativa del estadístico $\chi^{2}$ como se muestra a continuación del segundo modelo respecto al primer modelo anterior:

$$
\begin{aligned}
& k_{21}=\frac{\chi_{21}^{2}}{\chi_{S-B, 21}^{2}}=1,378 \\
& D_{2,21}=\chi_{2}^{2}-\chi_{21}^{2}=382,4 \\
& d_{2,21}=1 \\
& k_{12}=\frac{d_{2} k_{2}-d_{21} k_{21}}{d_{2,21}}=1,021 \\
& \bar{D}_{2,21}=\frac{D_{2,21}}{k_{2,21}}=374,5>\chi_{\alpha=0,05 ; g l=1}^{2} 3,84
\end{aligned}
$$

Con la introducción de esta nueva modificación los coeficientes de fiabilidad Alfa de Cronbach y Rho no sufren ninguna modificación mientras que los porcentajes de fiabilidad compuesta y variabilidad extraída por cada factor se reducen ligeramente en los factores 7, 9 y 10 - Comunicación, Cooperación y Liderazgo. Consecuentemente, si en la estimación del modelo sin reespecificar únicamente el quinto factor - Conocimientos y Pensamiento metacognitivo - no alcanzaba el umbral del $50 \%$ recomendado para el porcentaje de varianza extraída por cada factor limitándose al $43.6 \%$, en esta ocasión se encuentran también en esta situación el séptimo y el décimo factor - Comunicación (48.8\%) y Liderazgo (46.9\%).

\begin{tabular}{|l|c|c|}
\hline & Fiabilidad compuesta & Varianza extraída \\
\hline$F_{5}$ & $74.8 \%$ & $43.6 \%$ \\
$F_{6}$ & $80.6 \%$ & $51.6 \%$ \\
$F_{7}$ & $73.0 \%$ & $48.8 \%$ \\
$F_{8}$ & $80.9 \%$ & $58.7 \%$ \\
$F_{9}$ & $65.1 \%$ & $54.5 \%$ \\
$F_{10}$ & $76.9 \%$ & $46.9 \%$ \\
\hline
\end{tabular}

Tabla 5.38: Fiabilidad compuesta y varianza extraída. Modelo de medida final para Competencias adquiridas en la Universidad con 6 factores latentes

Las ecuaciones no estandarizadas muestran que los ítems 11, 12 y 24 Conocimientos del área, Conocimientos de otras áreas y Herramientas in- 
formáticas - alcanzan los valores máximos en las estimaciones del error de medida. El error de medida del ítem 20 - Trabajo en equipo -fue fijado a cero por imposición del programa EQS durante la estimación. Sin embargo eso no ha impedido obtener una estimación significativa del coeficiente de regresión que vincula dicho ítem con su factor correspondiente.

$$
\begin{array}{ll}
V_{11}=1,000 \cdot F_{5}+1,000 \cdot E_{11} & E_{11}=2,285^{*} \\
V_{12}=1,007^{*} \cdot F_{5}+1,000 \cdot E_{12} & E_{12}=1,777^{*} \\
V_{13}=1,500^{*} \cdot F_{5}+1,000 \cdot E_{13} & E_{13}=1,018^{*} \\
V_{14}=1,474^{*} \cdot F_{5}+1,000 \cdot E_{14} & E_{14}=1,041^{*} \\
V_{15}=0,963^{*} \cdot F_{10}+1,000 \cdot E_{15} & E_{15}=1,184^{*} \\
V_{16}=1,000 \cdot F_{8}+1,000 \cdot E_{16} & E_{16}=1,827^{*} \\
V_{17}=1,000 \cdot F_{10}+1,000 \cdot E_{17} & E_{17}=0,965^{*} \\
V_{18}=1,159^{*} \cdot F_{8}+1,000 \cdot E_{18} & E_{18}=0,841^{*} \\
V_{19}=1,128^{*} \cdot F_{8}+1,000 \cdot E_{19} & E_{19}=1,070^{*} \\
V_{20}=3,660^{*} \cdot F_{9}+1,000 \cdot E_{20} & E_{20}=0,000 \\
V_{21}=1,000 \cdot F_{9}+0,808 \cdot F_{10}+1,000 \cdot E_{21} & E_{21}=0,915^{*} \\
V_{22}=1,000 \cdot F_{7}+0,550 \cdot F_{10}+1,000 \cdot E_{22} & E_{22}=1,149^{*} \\
V_{23}=1,042^{*} \cdot F_{10}+1,000 \cdot E_{23} & E_{23}=0,957^{*} \\
V_{24}=0,763^{*} \cdot F_{6}+1,000 \cdot E_{24} & E_{24}=2,176^{*} \\
V_{25}=1,000 \cdot F_{6}+1,000 \cdot E_{25} & E_{25}=0,665^{*} \\
V_{26}=0,926^{*} \cdot F_{6}+1,000 \cdot E_{26} & E_{26}=1,077^{*} \\
V_{27}=1,958^{*} \cdot F_{7}+1,000 \cdot E_{27} & E_{27}=0,922^{*} \\
V_{28}=1,743^{*} \cdot F_{7}+1,000 \cdot E_{28} & E_{28}=1,317^{*}
\end{array}
$$

Tabla 5.39: Ecuaciones no estandarizadas. Modelo de medida final para Competencias adquiridas en la Universidad con 6 factores latentes

En las estimaciones de las varianzas y covarianzas de los factores latentes se observa que los valores máximos de varianza los obtienen los factores 6 y 8 - Innovación y Organización personal - mientras que el noveno factor Cooperación - presenta el mínimo valor de varianza alcanzado entre todos los factores. Respecto a las covarianzas y correlaciones entre factores parece existir relación entre todos los factores en mayor o menor medida. Sin embargo las correlaciones entre los factores 6, 8 y 10 - Innovación, Organización personal y Liderazgo - resultan más elevadas que las correspondientes al resto de factores. 


\begin{tabular}{|c|c|c|c|c|c|c|}
\hline & $F_{5}$ & $F_{6}$ & $F_{7}$ & $F_{8}$ & $F_{9}$ & $F_{10}$ \\
\hline$F_{5}$ & $0.718^{*}$ & & & & & \\
$F_{6}$ & $0.865^{*}$ & $2.009^{*}$ & & & & \\
$F_{7}$ & $0.325^{*}$ & $0.794^{*}$ & $0.559^{*}$ & & & \\
$F_{8}$ & $0.714^{*}$ & $1.284^{*}$ & $0.581 *$ & $1.424^{*}$ & & \\
$F_{9}$ & $0.174 *$ & $0.391 *$ & $0.232 *$ & $0.377^{*}$ & $0.235^{*}$ & \\
$F_{10}$ & $0.588^{*}$ & $1.212^{*}$ & $0.578 *$ & $1.202 *$ & $0.335^{*}$ & $1.391 *$ \\
\hline
\end{tabular}

Tabla 5.40: Matriz de varianzas-covarianzas. Modelo de medida final para Competencias adquiridas en la Universidad con 6 factores latentes

\begin{tabular}{|c|c|c|c|c|c|c|}
\hline & $F_{5}$ & $F_{6}$ & $F_{7}$ & $F_{8}$ & $F_{9}$ & $F_{10}$ \\
\hline$F_{5}$ & 1.000 & & & & & \\
$F_{6}$ & 0.720 & 1.000 & & & & \\
$F_{7}$ & 0.512 & 0.749 & 1.000 & & & \\
$F_{8}$ & 0.706 & 0.759 & 0.651 & 1.000 & & \\
$F_{9}$ & 0.422 & 0.569 & 0.641 & 0.653 & 1.000 & \\
$F_{10}$ & 0.588 & 0.725 & 0.655 & 0.854 & 0.586 & 1.000 \\
\hline
\end{tabular}

Tabla 5.41: Matriz de correlaciones. Modelo de medida final para Competencias adquiridas en la Universidad con 6 factores latentes

Congruentemente con la información aportada por las ecuaciones no estandarizadas, el conjunto de ecuaciones estandarizadas muestra que los ítems 11, 12 y 24 - Conocimientos del área, Conocimientos de otras áreas y $\mathrm{He}$ rramientas informáticas - presentan los coeficientes de determinación más bajos $\left(R^{2}=0,239 ; R^{2}=0,291 ; R^{2}=0,348\right)$, indicando que dichos ítems no se explican adecuadamente mediante sus factores latentes correspondientes. 


$$
\begin{array}{ll}
V_{11}=0,489 \cdot F_{5}+0,872 \cdot E_{11} & R^{2}=0,239 \\
V_{12}=0,539 \cdot F_{5}+0,842 \cdot E_{12} & R^{2}=0,291 \\
V_{13}=0,783 \cdot F_{5}+0,622 \cdot E_{13} & R^{2}=0,614 \\
V_{14}=0,775 \cdot F_{5}+0,632 \cdot E_{14} & R^{2}=0,600 \\
V_{15}=0,722 \cdot F_{10}+0,692 \cdot E_{15} & R^{2}=0,533 \\
V_{16}=0,662 \cdot F_{8}+0,750 \cdot E_{16} & R^{2}=0,438 \\
V_{17}=0,768 \cdot F_{10}+0,640 \cdot E_{17} & R^{2}=0,609 \\
V_{18}=0,833 \cdot F_{8}+0,553 \cdot E_{18} & R^{2}=0,697 \\
V_{19}=0,793 \cdot F_{8}+0,609 \cdot E_{19} & R^{2}=0,626 \\
V_{20}=1,000 \cdot F_{9}+0,000 \cdot E_{20} & R^{2}=1,000 \\
V_{21}=0,301 \cdot F_{9}+0,591 \cdot F_{6}+0,593 \cdot E_{21} & R^{2}=0,642 \\
V_{22}=0,450 \cdot F_{7}+0,390 \cdot F_{10}+0,645 \cdot E_{22} & R^{2}=0,607 \\
V_{23}=0,782 \cdot F_{10}+0,623 \cdot E_{23} & R^{2}=0,598 \\
V_{24}=0,591 \cdot F_{6}+0,807 \cdot E_{24} & R^{2}=0,348 \\
V_{25}=0,867 \cdot F_{6}+0,499 \cdot E_{25} & R^{2}=0,753 \\
V_{26}=0,784 \cdot F_{6}+0,620 \cdot E_{26} & R^{2}=0,615 \\
V_{27}=0,836 \cdot F_{7}+0,548 \cdot E_{27} & R^{2}=0,618 \\
V_{28}=0,750 \cdot F_{7}+0,661 \cdot E_{28} & R^{2}=0,508
\end{array}
$$

Tabla 5.42: Ecuaciones estandarizadas. Modelo de medida final para Competencias adquiridas en la Universidad con 6 factores latentes

La aplicación del Contraste de Lagrange a este modelo propone una nueva relación entre el ítem 17 - Detectar oportunidades - y el octavo factor Organización personal. Aunque cabría esperar que esta modificación produjera una disminución significativa en el valor del estadístico $\chi^{2}$, lo cierto es que esta relación ya no puede justificada en base a la teoría. Puesto que se considera que el ajuste del modelo ya es suficientemente bueno y que la adición de esta nueva relación incrementaría la complejidad del modelo de forma innecesaria, se ha optado por descartar esta propuesta. Así, la estructura factorial del modelo de Competencias adquiridas en la Universidad se representa mediante la siguiente figura: 


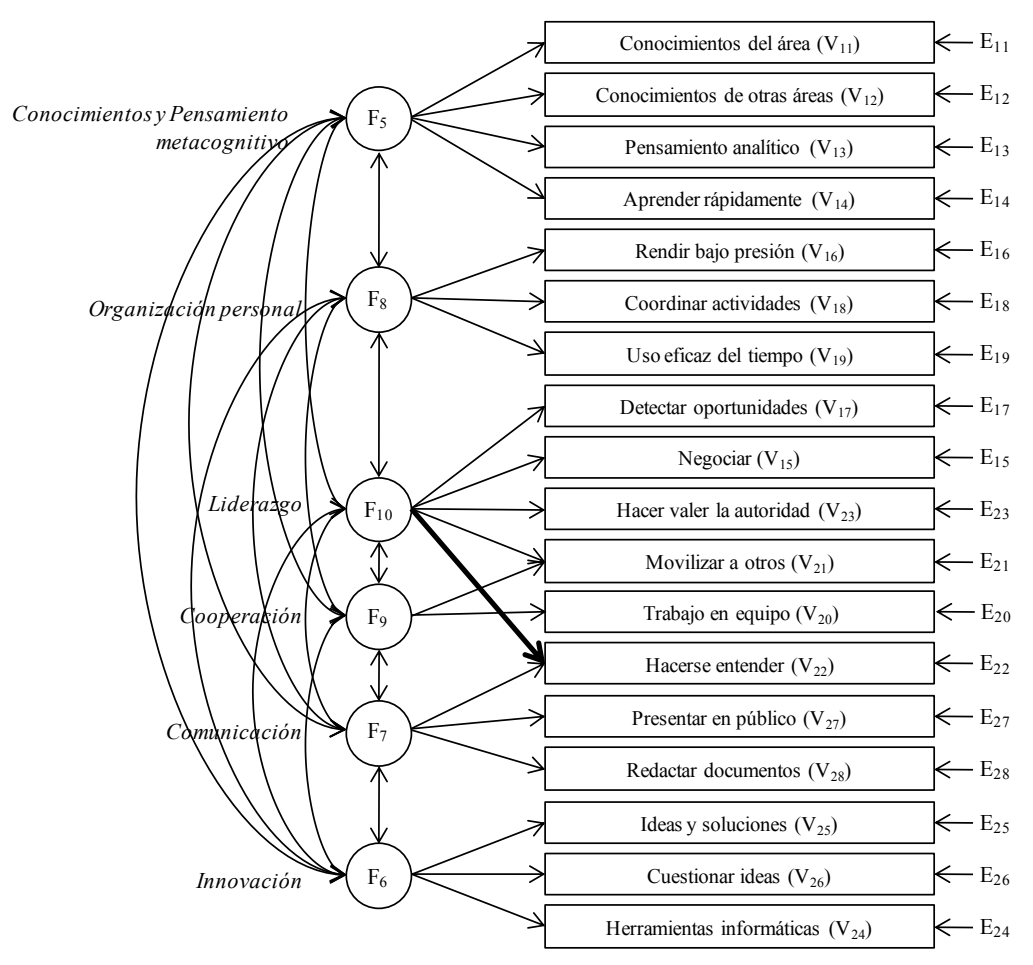

Figura 5.9: Modelo de medida final para Competencias adquiridas en la Universidad con 6 factores latentes

\section{Análisis Factorial Confirmatorio por áreas de estudio}

Una vez identificado el modelo definitivo para las Competencias adquiridas en la Universidad se comprueba su aplicabilidad a cada una de las áreas de estudio planteadas. Al igual que en el análisis aplicado a los Métodos de enseñanza - aprendizaje, las restricciones del análisis de invarianza se aplican exclusivamente sobre el modelo de medida. La restricción de los parámetros estructurales no resulta interesante en esta fase del análisis ya que únicamente afectaría a determinados parámetros de los factores latentes, como varianzas y covarianzas. Por la misma razón tampoco se impondrán restricciones sobre las covarianzas de los errores de medida.

El análisis previo de este modelo en cada una de las ocho áreas de estudio muestra un ajuste bastante bueno en todas ellas. Concretamente, las áreas de Derecho y Humanidades toman valores del estadístico $\chi^{2}$ de Satorra - Bentler entre 225 y 300, mientras que en las áreas de Educación, Salud, Ciencias Sociales y Ciencias toman valores comprendidos entre 300 y 400. Por último las áreas de Economía y empresa y Técnicas toman ya valores 
superiores a 400, siendo esta última la que obtiene el valor máximo para este estadístico. Sin embargo, con un índice de CFI en torno a 0.929 se puede considerar que el ajuste sigue siendo suficientemente bueno.

\begin{tabular}{|c|c|c|c|c|c|c|c|c|}
\hline \multirow{5}{*}{$\begin{array}{l}\chi_{\text {Ind }}^{2} \\
g l_{\text {Ind }} \\
\chi_{S-B}^{2} \\
g l_{\text {Ind }} \\
\end{array}$} & \multicolumn{8}{|c|}{$\begin{array}{l}\text { Educación Humanidades Ciencias Economía Derecho Técnicas Salud Ciencias } \\
\text { Sociales y empresa }\end{array}$} \\
\hline & 4722.7 & 4224.8 & 3963.8 & 6984.6 & 2272.4 & 6274.2 & 4262.0 & 4008.5 \\
\hline & 153 & 153 & 153 & 153 & 153 & 153 & 153 & 153 \\
\hline & 311.0 & 297.6 & 355.9 & 442.1 & 225.5 & 554.9 & 311.2 & 367.0 \\
\hline & 118 & 118 & 118 & 118 & 118 & 118 & 118 & 118 \\
\hline$A I C_{\text {Ind }}$ & 4416.7 & 3918.8 & 3657.8 & 6678.6 & 1966.4 & 5968.2 & 3956.0 & 3702.5 \\
\hline$A I C$ & 75.0 & 61.6 & 119.9 & 206.1 & -10.5 & 318.9 & 75.2 & 131.0 \\
\hline$C A I C_{\text {Ind }}$ & 3608.3 & 3140.2 & 2843.2 & 5783.9 & 1262.2 & 5070.2 & 3158.4 & 2889.8 \\
\hline$C A I C$ & -548.5 & -538.9 & -508.4 & -483.9 & -553.6 & -373.7 & -539.9 & -495.8 \\
\hline$N F I$ & 0.934 & 0.930 & 0.910 & 0.937 & 0.901 & 0.912 & 0.927 & 0.908 \\
\hline$N N F I$ & 0.945 & 0.943 & 0.919 & 0.938 & 0.934 & 0.907 & 0.939 & 0.916 \\
\hline$C F I$ & 0.958 & 0.956 & 0.938 & 0.953 & 0.949 & 0.929 & 0.953 & 0.935 \\
\hline$I F I$ & 0.958 & 0.956 & 0.938 & 0.953 & 0.950 & 0.929 & 0.953 & 0.936 \\
\hline$M F I$ & 0.835 & 0.816 & 0.808 & 0.842 & 0.820 & 0.797 & 0.824 & 0.798 \\
\hline$R M S E A$ & 0.055 & 0.059 & 0.060 & 0.054 & 0.058 & 0.062 & 0.057 & 0.062 \\
\hline Lim Inf $90 \%$ & 0.048 & 0.050 & 0.053 & 0.049 & 0.046 & 0.057 & 0.050 & 0.055 \\
\hline Lim Sup $90 \%$ & 0.063 & 0.067 & 0.067 & 0.059 & 0.069 & 0.067 & 0.065 & 0.069 \\
\hline
\end{tabular}

Tabla 5.43: Índices de ajuste y contraste $\chi^{2}$ por área de estudio. Invarianza métrica del modelo para Competencias adquiridas en la Universidad

Los promedios de la matriz residual de varianzas - covarianzas resultan aproximdamente similares a los obtenidos en el modelo equivalente sin restricciones de invarianza. En este modelo no restringido los promedios residuales tomaban valores de 0.0840 y 0.0939 para los promedios absolutos incluyendo y excluyendo los términos en la diagonal, y 0.0305 y 0.0341 para los mismos valores estandarizados.

\begin{tabular}{|c|c|c|c|c|c|c|c|c|}
\hline & Educación & umanidad & $\begin{array}{l}\text { Ciencias } \\
\text { Sociales }\end{array}$ & $\begin{array}{l}\text { Economía } \\
\text { y empresa }\end{array}$ & Derecho & Técnicas & Salud & Ciencias \\
\hline Avg. abs. residual & 0.0521 & 0.0565 & 0.0575 & 0.0416 & 0.0622 & 0.0531 & 0.0416 & 0.0661 \\
\hline Avg. off-diag. abs. residual & 0.0539 & 0.0588 & 0.0585 & 0.0439 & 0.0651 & 0.0570 & 0.0438 & 0.0674 \\
\hline Avg. absolute std. residual & 0.0371 & 0.0336 & 0.0431 & 0.0332 & 0.044 & 0.0416 & 0.0309 & 0.0471 \\
\hline Avg. off-diag. abs. std. residual & 0.0384 & 0.035 & 0.0439 & 0.0352 & 0.0461 & 0.0449 & 0.0325 & 0.048 \\
\hline
\end{tabular}

Tabla 5.44: Promedios de las matrices residuales de varianzas-covarianzas por área de estudio. Invarianza métrica del modelo para Competencias adquiridas en la Universidad

Al igual que ocurría en el análisis de invarianza métrica de los Métodos de enseñanza - aprendizaje el número de grados de libertad aumenta notablemente al considerar tanto las restricciones de invarianza métrica como el 
número de grupos en que se segmenta la muestra. Aún con estas restricciones el ajuste del modelo sigue siendo excelente por lo que puede concluirse que el modelo de Competencias adquiridas en la Universidad formulado es invariante ante modificaciones en el modelo de medida.

El contraste $\chi^{2}$ del modelo de medida para Competencias adquiridas en la Universidad, y sus correspondientes índices de ajuste resultan:

$$
\begin{aligned}
& \chi_{\text {Ind }}^{2}=37028,0 \\
& g l_{\text {Ind }}=1224 \\
& \chi_{S-B}^{2}=3071,1 \\
& g l=1042 \\
& \text { Prob }=0,000 \\
& A I C_{\text {Ind }}=34580,0 \\
& A I C=987,1 \\
& C A I C_{\text {Ind }}=25439,1 \\
& C A I C=-6794,5 \\
& N F I=0,917 \\
& N N F I=0,933 \\
& C F I=0,943 \\
& \text { IFI }=0,944 \\
& M F I=0,808 \\
& R M S E A=0,057 \\
& 90 \% \text { Interval }=[0,055,0,060]
\end{aligned}
$$

Respecto a la fiabilidad del modelo, el coeficiente Alfa de Cronbach sigue siendo muy elevado en todas las áreas de estudio y lo mismo ocurre con el Coeficiente Rho. Ambos coeficientes tomaban los valores 0.927 y 0.946 en el modelo equivalente no restringido.

\begin{tabular}{|l|cccccccc|}
\hline & \multicolumn{7}{|c|}{ Educación Humanidades Ciencias Economía Derecho Técnicas Salud Ciencias } \\
& \multicolumn{7}{|c}{ Sociales y empresa } \\
\hline$\alpha$ Cronbach & 0.938 & 0.933 & 0.918 & 0.93 & 0.929 & 0.912 & 0.935 & 0.908 \\
Rho & 0.952 & 0.951 & 0.937 & 0.946 & 0.947 & 0.934 & 0.951 & 0.934 \\
\hline
\end{tabular}

Tabla 5.45: Indices de fiabilidad por área de estudio. Invarianza métrica del modelo para Competencias adquiridas en la Universidad

La estimación de los coeficientes no estandarizados es común para todas las áreas de estudio. Dichos coeficientes son muy similares a los obtenidos para el modelo sin imposición de restricciones. 


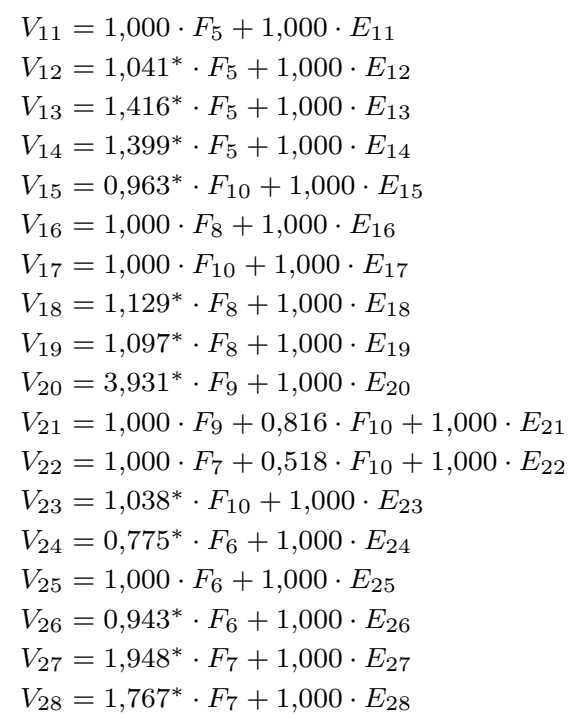

Tabla 5.46: Ecuaciones no estandarizadas. Invarianza métrica del modelo para Competencias adquiridas en la Universidad

No obstante, puesto que no se han restringido las covarianzas entre los errores residuales, la estimación de estos parámetros varía en función del área de estudio. En la Tabla 5.47 es posible observar ciertos patrones en las estimaciones de estos coeficientes como valores elevados en los ítems 11, 12, y 24 - Conocimientos del área, Conocimientos de otras áreas y Herramientas informáticas, en consonancia con los resultados obtenidos para el modelo sin restricciones de invarianza. Asimismo el error correspondiente al ítem 20 Trabajar en equipo -que durante la estimación del modelo sin restricciones se había fijado a cero, sigue siendo nulo en algunas áreas como Humanidades, Ciencias Sociales Derecho y Técnicas y muy pequeño en las áreas de estudio restantes.

Del mismo modo las estimaciones de las varianzas y covarianzas de los factores latentes muestran que el sexto factor - Innovación - presenta igualmente la varianza más elevada mientras que el noveno factor - Cooperación es el factor con la mínima varianza. Asimismo los factores 8 y 10 - Organización personal y Liderazgo - muestran varianzas considerablemente elevadas. Por el contrario el séptimo factor - Comunicación - presenta igualmente valores de varianza relativamente bajos. 


\begin{tabular}{|c|c|c|c|c|c|c|c|c|}
\hline & Educaci & anid & $\begin{array}{l}\text { Ciencias } \\
\text { Sociales }\end{array}$ & empres & Derecho & Técnicas & Salud & Ciencias \\
\hline$E_{11}$ & $1.078^{*}$ & $1.437^{*}$ & $1.127^{*}$ & $0.934^{*}$ & $1.174^{*}$ & $0.954^{*}$ & $0.953^{*}$ & $1.218^{*}$ \\
\hline$E_{12}$ & $0.866^{*}$ & $1.030^{*}$ & $0.958^{*}$ & $0.791^{*}$ & $0.868^{*}$ & $0.851^{*}$ & $0.905^{*}$ & $1.025^{*}$ \\
\hline$E_{13}$ & $0.501^{*}$ & $0.603^{*}$ & $0.620^{*}$ & $0.502^{*}$ & $0.614^{*}$ & $0.492^{*}$ & $0.501^{*}$ & $0.576^{*}$ \\
\hline$E_{14}$ & $0.465^{*}$ & $0.579 *$ & $0.603^{*}$ & $0.531^{*}$ & $0.654^{*}$ & $0.522^{*}$ & $0.545^{*}$ & $0.619 *$ \\
\hline$E_{15}$ & $0.667^{*}$ & $0.544^{*}$ & $0.738^{*}$ & $0.624^{*}$ & $0.542^{*}$ & $0.539^{*}$ & $0.632^{*}$ & $0.637^{*}$ \\
\hline$E_{16}$ & $0.835^{*}$ & $0.909 *$ & $0.802^{*}$ & $0.722^{*}$ & $0.814^{*}$ & $0.985^{*}$ & $0.928^{*}$ & 0.988* \\
\hline$E_{17}$ & $0.532^{*}$ & $0.632^{*}$ & $0.567^{*}$ & $0.478^{*}$ & $0.461^{*}$ & $0.498^{*}$ & $0.448^{*}$ & $0.584^{*}$ \\
\hline$E_{18}$ & $0.461^{*}$ & $0.401^{*}$ & $0.427^{*}$ & $0.478^{*}$ & $0.473^{*}$ & $0.456^{*}$ & $0.397^{*}$ & $0.618^{*}$ \\
\hline$E_{19}$ & $0.548^{*}$ & $0.596^{*}$ & $0.474^{*}$ & $0.565^{*}$ & $0.599 *$ & $0.546^{*}$ & $0.611^{*}$ & $0.573^{*}$ \\
\hline$E_{20}$ & 0.021 & 0.000 & 0.000 & 0.029 & 0.000 & 0.000 & 0.247 & 0.137 \\
\hline$E_{21}$ & $0.615^{*}$ & $0.524^{*}$ & $0.641^{*}$ & $0.385^{*}$ & $0.398^{*}$ & $0.470^{*}$ & $0.408^{*}$ & $0.449^{*}$ \\
\hline$E_{22}$ & $0.534^{*}$ & $0.723^{*}$ & $0.524^{*}$ & $0.530^{*}$ & $0.651^{*}$ & $0.576^{*}$ & $0.513^{*}$ & $0.742^{*}$ \\
\hline$E_{23}$ & $0.751^{*}$ & 0.599* & $0.607^{*}$ & $0.387^{*}$ & $0.531^{*}$ & $0.454^{*}$ & $0.455^{*}$ & $0.492^{*}$ \\
\hline$E_{24}$ & $0.850^{*}$ & $1.244^{*}$ & $1.152^{*}$ & $1.011^{*}$ & $0.918^{*}$ & $1.241^{*}$ & $0.866^{*}$ & $0.974^{*}$ \\
\hline$E_{25}$ & $0.326^{*}$ & $0.469^{*}$ & $0.411^{*}$ & $0.302^{*}$ & $0.529 *$ & $0.432^{*}$ & $0.288^{*}$ & $0.392^{*}$ \\
\hline$E_{26}$ & $0.432^{*}$ & $0.751^{*}$ & $0.624^{*}$ & $0.440^{*}$ & $0.592^{*}$ & $0.528^{*}$ & $0.435^{*}$ & $0.729^{*}$ \\
\hline$E_{27}$ & $0.418^{*}$ & $0.537^{*}$ & $0.523^{*}$ & $0.573^{*}$ & $0.458^{*}$ & $0.544^{*}$ & $0.479^{*}$ & $0.454^{*}$ \\
\hline$E_{28}$ & $0.612^{*}$ & $0.979 *$ & $0.581^{*}$ & $0.620^{*}$ & $0.594^{*}$ & $0.687^{*}$ & $0.572^{*}$ & $0.654^{*}$ \\
\hline
\end{tabular}

Tabla 5.47: Errores de medida por área de estudio. Invarianza métrica del modelo para Competencias adquiridas en la Universidad

\begin{tabular}{|l|cccccccc|}
\hline & \multicolumn{8}{|c|}{ Educación Humanidades Ciencias Economía Derecho Técnicas Salud Ciencias } \\
& \multicolumn{7}{|c}{ Sociales y empresa } \\
\hline $\operatorname{Var}\left(F_{5}\right)$ & $0.426^{*}$ & $0.506^{*}$ & $0.316^{*}$ & $0.334^{*}$ & $0.344^{*}$ & $0.326^{*}$ & $0.331^{*}$ & $0.346^{*}$ \\
$\operatorname{Var}\left(F_{6}\right)$ & $0.958^{*}$ & $1.158^{*}$ & $0.792^{*}$ & $0.832^{*}$ & $0.879^{*}$ & $0.812^{*}$ & $0.959^{*}$ & $1.156^{*}$ \\
$\operatorname{Var}\left(F_{7}\right)$ & $0.289^{*}$ & $0.340^{*}$ & $0.260^{*}$ & $0.235^{*}$ & $0.277^{*}$ & $0.198^{*}$ & $0.301^{*}$ & $0.238^{*}$ \\
$\operatorname{Var}\left(F_{8}\right)$ & $0.810^{*}$ & $0.981^{*}$ & $0.651^{*}$ & $0.603^{*}$ & $0.831^{*}$ & $0.642^{*}$ & $0.687^{*}$ & $0.728^{*}$ \\
$\operatorname{Var}\left(F_{9}\right)$ & $0.085^{*}$ & $0.114^{*}$ & $0.090^{*}$ & $0.084^{*}$ & $0.097^{*}$ & $0.088^{*}$ & $0.091^{*}$ & $0.086^{*}$ \\
$\operatorname{Var}\left(F_{10}\right)$ & $0.716^{*}$ & $0.830^{*}$ & $0.640^{*}$ & $0.695^{*}$ & $0.793^{*}$ & $0.677^{*}$ & $0.740^{*}$ & $0.693^{*}$ \\
\hline
\end{tabular}

Tabla 5.48: Varianza de los factores latentes por área de estudio. Invarianza métrica del modelo para Competencias adquiridas en la Universidad 


\begin{tabular}{|c|c|c|c|c|c|c|c|c|}
\hline \multirow[b]{2}{*}{$\operatorname{Cov}\left(F_{5}, F_{6}\right)$} & \multicolumn{8}{|c|}{$\begin{array}{c}\text { Educación Humanidades Ciencias Economía Derecho Técnicas Salud Ciencias } \\
\text { Sociales y empresa }\end{array}$} \\
\hline & $0.478^{*}$ & $0.565^{*}$ & $0.338^{*}$ & $0.364^{*}$ & $0.377^{*}$ & $0.347^{*}$ & $0.358^{*}$ & $0.499^{*}$ \\
\hline $\operatorname{Cov}\left(F_{5}, F_{7}\right)$ & $0.195^{*}$ & $0.247^{*}$ & $160^{*}$ & $0.172^{*}$ & $0.198^{*}$ & $0.127^{*}$ & $0.178^{*}$ & $0.123^{*}$ \\
\hline $\operatorname{Cov}\left(F_{5}, F_{8}\right)$ & $0.463^{*}$ & $0.509^{*}$ & $336^{*}$ & $0.322^{*}$ & $0.411^{*}$ & $0.280^{*}$ & $0.375^{*}$ & $0.318^{*}$ \\
\hline $\operatorname{Cov}\left(F_{5}, F_{9}\right)$ & 0.08 & $120^{*}$ & $75^{*}$ & $0.085^{*}$ & $0.095^{*}$ & $0.060 *$ & $0.103^{*}$ & $0.058^{*}$ \\
\hline $\operatorname{Cov}\left(F_{5}, F_{10}\right)$ & & 0.4 & & & $0.341^{*}$ & $0.203^{*}$ & $0.343^{*}$ & $0.199^{*}$ \\
\hline $\operatorname{Cov}\left(F_{6}, F_{7}\right)$ & 0.42 & 499 & & 0.36 & $0.393^{*}$ & $0.318^{*}$ & $0.450^{*}$ & $0.314^{*}$ \\
\hline $\operatorname{Cov}\left(F_{6}, F_{8}\right)$ & & $826^{*}$ & & & $0.659^{*}$ & $0.519^{*}$ & $0.633^{*}$ & $0.652^{*}$ \\
\hline $\operatorname{Cov}\left(F_{6}, F_{9}\right)$ & & & & & $0.1 \varepsilon$ & $0.145^{*}$ & $0.205^{*}$ & $0.137^{*}$ \\
\hline $\operatorname{Cov}\left(F_{6}, F_{10}\right)$ & 0.682 & & 0 & 0.614 & $0.715^{*}$ & $0.471^{*}$ & $0.679^{*}$ & $0.509^{*}$ \\
\hline $\operatorname{Cov}\left(F_{7}, F_{8}\right)$ & $0.342^{*}$ & $0.389^{*}$ & $0.258^{*}$ & $0.278^{*}$ & $0.296^{*}$ & $0.236^{*}$ & $0.308^{*}$ & $0.227^{*}$ \\
\hline $\operatorname{Cov}\left(F_{7}, F_{9}\right)$ & & $0.130^{*}$ & $0.097^{*}$ & $0.095^{*}$ & $0.074^{*}$ & $0.081^{*}$ & $0.119 *$ & $0.089 *$ \\
\hline $\operatorname{Cov}\left(F_{7}, F_{10}\right)$ & $0.303^{*}$ & $0.328^{*}$ & $0.256^{*}$ & $0.290^{*}$ & $0.318^{*}$ & $0.253^{*}$ & $0.308^{*}$ & $0.210^{*}$ \\
\hline $\operatorname{Cov}\left(F_{8}, F_{9}\right)$ & $0.182^{*}$ & $0.227^{*}$ & $0.143^{*}$ & $0.146^{*}$ & $0.171^{*}$ & $0.148^{*}$ & $0.192^{*}$ & $0.163^{*}$ \\
\hline $\operatorname{Cov}\left(F_{8}, F_{10}\right)$ & & $0.819^{*}$ & $0.591^{*}$ & $0.559^{*}$ & $0.635^{*}$ & $0.519 *$ & $0.662^{*}$ & $0.572^{*}$ \\
\hline $\operatorname{Cov}\left(F_{9}, F_{10}\right)$ & $0.127^{*}$ & $0.185^{*}$ & $0.131^{*}$ & $0.142^{*}$ & $0.157^{*}$ & $0.132^{*}$ & $0.182^{*}$ & $0.151^{*}$ \\
\hline
\end{tabular}

Tabla 5.49: Covarianzas entre factores latentes por área de estudio. Invarianza métrica del modelo para Competencias adquiridas en la Universidad

\begin{tabular}{|l|cccccccc|}
\hline & \multicolumn{7}{|c|}{ Educación Humanidades Ciencias Economía Derecho Técnicas Salud Ciencias } \\
& \multicolumn{7}{|c}{ Sociales } & empresa \\
\hline $\operatorname{Corr}\left(F_{5}, F_{6}\right)$ & 0.748 & 0.739 & 0.676 & 0.691 & 0.686 & 0.674 & 0.636 & 0.789 \\
$\operatorname{Corr}\left(F_{5}, F_{7}\right)$ & 0.557 & 0.596 & 0.557 & 0.613 & 0.643 & 0.501 & 0.564 & 0.431 \\
$\operatorname{Corr}\left(F_{5}, F_{8}\right)$ & 0.788 & 0.723 & 0.741 & 0.717 & 0.770 & 0.611 & 0.785 & 0.634 \\
$\operatorname{Corr}\left(F_{5}, F_{9}\right)$ & 0.455 & 0.499 & 0.448 & 0.507 & 0.520 & 0.352 & 0.592 & 0.334 \\
$\operatorname{Corr}\left(F_{5}, F_{10}\right)$ & 0.804 & 0.717 & 0.761 & 0.591 & 0.652 & 0.431 & 0.693 & 0.406 \\
$\operatorname{Corr}\left(F_{6}, F_{7}\right)$ & 0.815 & 0.795 & 0.800 & 0.827 & 0.795 & 0.793 & 0.838 & 0.599 \\
$\operatorname{Cor}\left(F_{6}, F_{8}\right)$ & 0.848 & 0.775 & 0.757 & 0.778 & 0.771 & 0.719 & 0.780 & 0.711 \\
$\operatorname{Corr}\left(F_{6}, F_{9}\right)$ & 0.627 & 0.621 & 0.556 & 0.617 & 0.622 & 0.541 & 0.693 & 0.434 \\
$\operatorname{Corr}\left(F_{6}, F_{10}\right)$ & 0.824 & 0.771 & 0.795 & 0.807 & 0.856 & 0.636 & 0.806 & 0.569 \\
$\operatorname{Corr}\left(F_{7}, F_{8}\right)$ & 0.707 & 0.674 & 0.628 & 0.738 & 0.615 & 0.664 & 0.677 & 0.545 \\
$\operatorname{Corr}\left(F_{7}, F_{9}\right)$ & 0.670 & 0.663 & 0.635 & 0.670 & 0.449 & 0.614 & 0.718 & 0.625 \\
$\operatorname{Corr}\left(F_{7}, F_{10}\right)$ & 0.667 & 0.617 & 0.628 & 0.717 & 0.678 & 0.692 & 0.653 & 0.518 \\
$\operatorname{Corr}\left(F_{8}, F_{9}\right)$ & 0.693 & 0.680 & 0.591 & 0.647 & 0.605 & 0.623 & 0.764 & 0.650 \\
$\operatorname{Corr}\left(F_{8}, F_{10}\right)$ & 0.919 & 0.908 & 0.916 & 0.864 & 0.782 & 0.787 & 0.929 & 0.806 \\
$\operatorname{Corr}\left(F_{9}, F_{10}\right)$ & 0.515 & 0.601 & 0.549 & 0.585 & 0.568 & 0.541 & 0.700 & 0.618 \\
\hline
\end{tabular}

Tabla 5.50: Correlaciones entre factores latentes por área de estudio. Invarianza métrica del modelo para Competencias adquiridas en la Universidad 


\subsubsection{Modelización causal de los Procesos de enseñanza - aprendizaje de competencias en la Universidad}

\section{Modelización causal sobre la muestra general}

Así, se han validado los modelos de medida propuestos para analizar tanto los Métodos de enseñanza - aprendizaje empleados en la Universidad como las Competencias adquiridas en la Universidad. Dichos modelos pueden ser ahora empleados en la formulación de modelos causales o estructurales que incluyan relaciones entre los factores latentes correspondientes a ambos modelos de medida. El planteamiento de estas relaciones se basa en la teoría precedente sobre Modelos de Enseñanza - Aprendizaje desarrollada a partir de la década de los ochenta. Concretamente el análisis específico de las relaciones entre los métodos de enseñanza y la adquisición de competencias fue estudiado por De Miguel (2005), a partir de las valoraciones de un grupo de expertos que asignaron puntuaciones a cada una de las posibles vinculaciones entre distintos elementos de ambos grupos. A partir de los resultados en esta investigación se plantean las hipótesis sobre las que se fundamentan modelos causales presentados en esta sección.

- $H_{1}$ : Las clases teóricas contribuyen al desarrollo de los conocimientos generales y académicos, así como a la adquisición de habilidades intelectuales

- $\mathrm{H}_{2}$ : Las clases prácticas contribuyen globalmente al desarrollo de competencias

- $H_{3}$ : Las prácticas externas contribuyen a la adquisición de habilidades de organización personal

- $H_{4}$ : El estudio y trabajo en grupo contribuye globalmente al desarrollo de competencias y específicamente a la adquisición de habilidades interpersonales y de comunicación

Según este modelo se considera que los métodos basados en el Estudio y trabajo en grupo se relacionan con la adquisición de competencias de $\mathrm{Li}$ derazgo, Cooperación y Comunicación, mientras que las Clases prácticas se asocian en mayor medida con la competencia de Innovación. Las Prácticas contribuyen al desarrollo de la capacidad de Organización personal mientras que las Clases teóricas se vinculan con la adquisición de Conocimientos y el desarrollo del Pensamiento metacognitivo.

Puesto que como resultado del análisis de los Métodos de enseñanza aprendizaje se formularon dos posibles estructuras factoriales, con cuatro y 
tres factores latentes, se han planteado los modelos causales correspondientes a ambos modelos de medida. Así, se plantea en primer lugar un primer modelo con cuatro factores exógenos de Métodos de enseñanza - aprendizaje y seis factores endógenos de Competencias adquiridas en la Universidad. Se ha seleccionado este modelo de medida en cuatro factores latentes para los Métodos de enseñanza - aprendizaje basado puesto que obtuvo el mejor ajuste entre los dos planteados en este apartado.

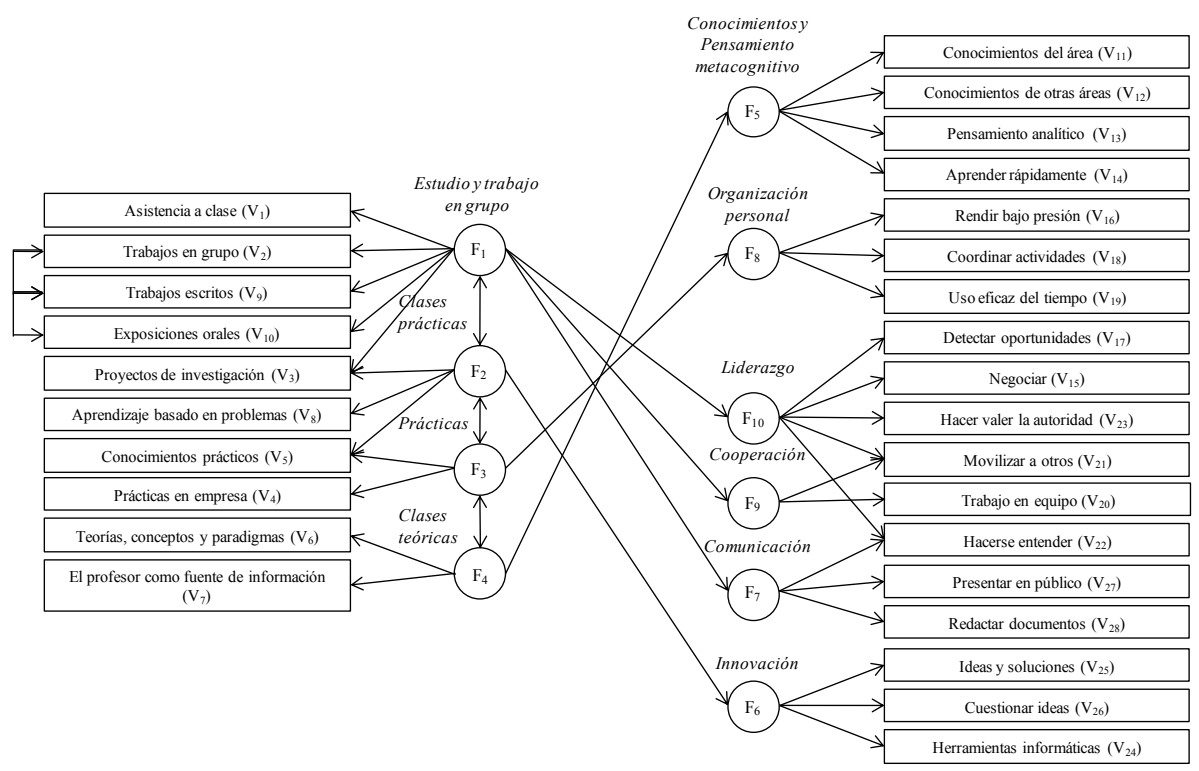

Figura 5.10: Modelo estructural inicial entre Métodos de enseñanza - aprendizaje y Competencias adquiridas en la Universidad con problemas de infraidentificación empírica

Asimismo la bondad de ajuste resultante para este modelo causal puede considerarse aceptable tanto en relación a los índices comparativos de ajuste $(N F I=0,808 ; N N F I=0,787 ; C F I=0,813 ; I F I=0,813)$ mientras que el RMSEA toma un valor de 0.076, con intervalo de confianza (90\%) en [0.075, 0.078]. Los índices de parsimonia igualmente toman valores muy reducidos $(A I C=8839,0 ; C A I C=6359,6)$ respecto a los obtenidos en el modelo de independencia $\left(A I C_{\text {Ind }}=48742,8 ; C A I C_{\text {Ind }}=45919,9\right)$.

En este caso el contraste $\chi^{2}$ para el primer modelo estructural entre Métodos de enseñanza - aprendizaje y Competencias adquiridas en la Universidad resulta: 
$\chi_{\text {Ind }}^{2}=49498,8$

$g l_{\text {Ind }}=378$

$\chi_{S-B}^{2}=9503,0$

$g l=332$

Prob $=0,000$

No obstante, en el examen de las estimaciones de los parámetros del modelo se detectan dos estimaciones infractoras, también denominadas estimaciones aberrantes, que invalidan todos los demás resultados correspondientes a este modelo. Concretamente la estimación de las correlaciones entre el segundo y el tercer factor - Clases Prácticas y Prácticas - resulta ser superior a la unidad.

\begin{tabular}{|c|c|c|c|c|}
\hline & $F_{1}$ & $F_{2}$ & $F_{3}$ & $F_{4}$ \\
\hline$F_{1}$ & $0.115^{*}$ & & & \\
$F_{2}$ & 0.001 & 0.000 & & \\
$F_{3}$ & $0.129^{*}$ & -0.001 & $0.100^{*}$ & \\
$F_{4}$ & -0.012 & -0.001 & 0.011 & 0.005 \\
\hline
\end{tabular}

Tabla 5.51: Matriz de varianzas-covarianzas entre factores latentes. Modelo estructural inicial entre Métodos de enseñanza - aprendizaje y Competencias adquiridas en la Universidad con problemas de infraidentificación empírica

\begin{tabular}{|c|c|c|c|c|}
\hline & $F_{1}$ & $F_{2}$ & $F_{3}$ & $F_{4}$ \\
\hline$F_{1}$ & 1.000 & & & \\
$F_{2}$ & 0.908 & 1.000 & & \\
$F_{3}$ & 0.873 & 1.176 & 1.000 & \\
$F_{4}$ & 0.040 & 0.065 & 0.010 & 1.000 \\
\hline
\end{tabular}

Tabla 5.52: Matriz de correlaciones entre factores latentes. Modelo estructural inicial entre Métodos de enseñanza - aprendizaje y Competencias adquiridas en la Universidad con problemas de infraidentificación empírica

Entre las estimaciones de los parámetros estructurales no se detecta ninguna varianza negativa que pueda corresponder a posibles casos Heywood. En todo caso, la varianza del segundo factor relativo a las Clases prácticas se estima con valor nulo. No obstante en la aplicación de este modelo a cada una de las áreas de estudio, como análisis previo a la realización del análisis de invarianza estructural, no solo aparecen de nuevo estas mismas estimaciones infractoras sino que surgen graves problemas de falta de identificación empírica. Este problema, que puede resultar potencialmente serio, 
no invalida la totalidad de la estimación como ocurre con las estimaciones infractoras, sino que en estas condiciones la estimación del modelo puede ser parcialmente informativa, si no afecta a un gran porcentaje de los parámetros libres. Sin embargo, en este caso el problema de la falta de identificación sí afecta considerablemente al resto de las estimaciones en cada una de las áreas de estudio. Con el fin de tratar de solventar esta dificultad se incrementa el valor de tolerancia en la determinación del nivel de significación pero sin ningún efecto significativo.

En las investigaciones sobre este problema se ha identificado mayor probabilidad de ocurrencia en condiciones de varios factores con tan sólo dos ítems, y que además se encuentren poco correlacionados con el resto de los factores. Asimismo se ha constatado que dicho problema puede quedar enmascarado en muestras de tamaño muy elevado de forma que, al segmentar la muestra global en submuestras de tamaño más reducido, se haya evidenciado el problema que antes no se detectaba.

En esta fase del análisis se emplea el método propuesto por Wothke (Wothke, 1993) para la identificación de colinearidades, basado en el análisis de la suma de los componentes de los autovectores de la matriz de varianzas - covarianzas asociados a autovalores con valores próximos a cero. En este caso los seis primeros autovalores de dicha matriz se encuentran próximos a cero, por lo que se calcula la suma de las componentes de sus autovectores asociados. Este cálculo igualmente resulta próximo a cero indicando colinealidad entre los seis primeros ítems del modelo.

Como solución a este problema se formula un nuevo modelo que elimina el problema de considerar varios factores con tan sólo dos ítems, y únicamente uno de ellos incorrelacionado con el resto. Este modelo se plantea a partir del segundo modelo de medida analizado para los Métodos de enseñanza aprendizaje basado en tres factores latentes. En este nuevo modelo ya sólo uno de los factores endógenos consta de dos ítems, y es precisamente el factor que se comporta de forma independiente respecto al resto. No obstante, esta modificación ya es suficiente para que desaparezcan los problemas de falta de identificación empírica y sobre todo las estimaciones infractoras en las correlaciones entre factores latentes. En este nuevo modelo se ha modificado la numeración de los factores endógenos de Competencias adquiridas en la Universidad con el fin de mantener la coherencia con la numeración de tres factores exógenos de Métodos de enseñanza - aprendizaje. 


\begin{tabular}{|c|c|ccccccc|}
\hline Autovalores & & 0.366 & 0.394 & 0.417 & 0.433 & 0.459 & 0.470 \\
\hline & 1 & 0.012 & 0.040 & 0.077 & -0.107 & -0.072 & -0.003 \\
& 2 & 0.049 & -0.251 & -0.419 & 0.370 & 0.188 & -0.029 \\
& 3 & -0.075 & 0.117 & 0.167 & -0.139 & -0.030 & -0.055 \\
4 & 0.027 & 0.019 & 0.095 & -0.046 & 0.015 & 0.017 \\
& 5 & -0.018 & 0.023 & -0.105 & -0.019 & -0.070 & -0.097 \\
& 6 & -0.002 & 0.024 & -0.017 & 0.034 & -0.005 & 0.088 \\
7 & -0.022 & -0.026 & 0.007 & 0.039 & -0.011 & -0.007 \\
& 8 & -0.039 & -0.073 & 0.014 & 0.022 & 0.063 & 0.116 \\
& 9 & 0.057 & 0.139 & 0.386 & -0.325 & 0.114 & -0.210 \\
10 & -0.070 & -0.007 & -0.137 & 0.165 & -0.207 & 0.231 \\
& 11 & -0.001 & -0.018 & -0.034 & -0.009 & 0.030 & 0.002 \\
& 12 & 0.027 & 0.033 & -0.087 & 0.024 & -0.002 & 0.091 \\
13 & -0.004 & -0.116 & 0.288 & 0.076 & -0.104 & 0.056 \\
14 & -0.048 & 0.116 & -0.278 & -0.126 & 0.182 & -0.346 \\
& 15 & 0.146 & 0.047 & 0.093 & -0.061 & 0.128 & 0.506 \\
16 & 0.043 & 0.047 & -0.049 & -0.060 & -0.017 & 0.088 \\
17 & -0.298 & -0.295 & 0.190 & 0.232 & -0.362 & -0.474 \\
& 18 & 0.098 & 0.293 & -0.396 & -0.369 & -0.182 & -0.042 \\
19 & 0.016 & -0.265 & 0.251 & 0.246 & 0.381 & 0.157 \\
& 20 & -0.387 & 0.167 & 0.197 & -0.058 & -0.141 & 0.243 \\
21 & 0.552 & -0.259 & -0.062 & -0.059 & 0.028 & -0.175 \\
22 & 0.093 & -0.178 & 0.021 & -0.075 & -0.456 & 0.002 \\
& 23 & -0.450 & 0.299 & -0.131 & 0.166 & 0.328 & -0.153 \\
24 & 0.011 & -0.154 & -0.012 & -0.160 & -0.051 & 0.059 \\
& 25 & 0.327 & 0.528 & 0.133 & 0.431 & -0.123 & -0.006 \\
26 & -0.240 & -0.315 & -0.135 & -0.353 & 0.155 & 0.168 \\
27 & 0.150 & 0.034 & 0.202 & -0.056 & 0.353 & -0.259 \\
28 & -0.060 & 0.017 & -0.173 & 0.187 & -0.142 & 0.098 \\
\hline & & -0.107 & -0.014 & 0.084 & -0.029 & -0.008 & 0.067 \\
\hline
\end{tabular}

Tabla 5.53: Autovalores y autovectores de la matriz de varianzas - covarianzas para la identificación de problemas de falta de identificación empírica

Por simplicidad en esta representación gráfica de este modelo ya no se han incluido los errores residuales de medida sobre los 28 ítems. Asimismo, tampoco se han señalado los términos de perturbaciones que ahora tienen efecto sobre los seis factores endógenos del modelo. Estas perturbaciones se suman al conjunto de variables independientes, hasta ahora compuesto únicamente por las variables latentes y los errores de medida. Así, según la Regla t el modelo consta en estas condiciones de 337 grados de libertad.

$$
g l=\frac{1}{2} \cdot 28 \cdot 29-69=337
$$

Durante la estimación de los parámetros el error de medida del ítem 20 - Trabajar en equipo - se fijó al valor nulo como venía ocurriendo en el análisis anterior del modelo de medida correspondiente y también se aplicó la 


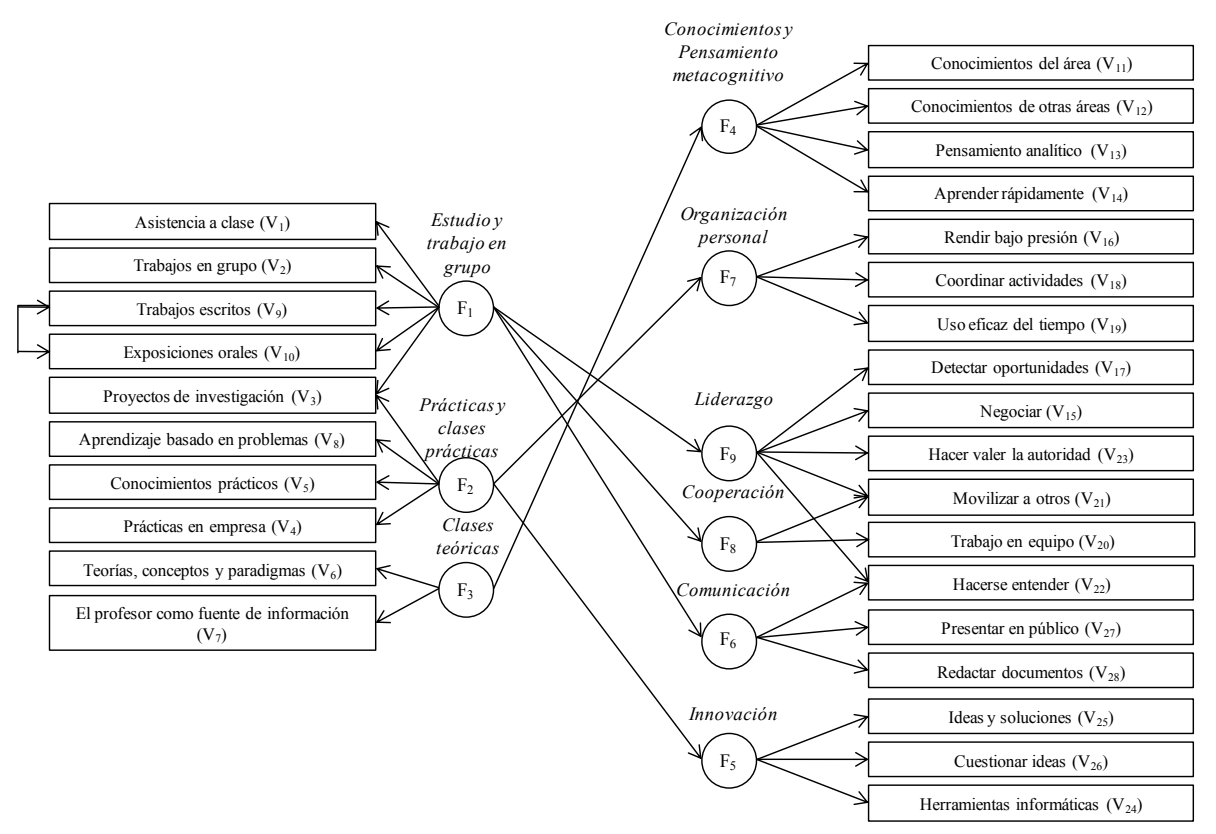

Figura 5.11: Modelo estructural inicial entre Métodos de enseñanza - aprendizaje y Competencias adquiridas en la Universidad

misma restricción sobre la perturbación del cuarto factor - Conocimientos y Pensamiento metacognitivo. Por otro lado se detectó cierta colinearidad entre los factores 2 y 5 - Prácticas y Clases prácticas e Innovación.

Average absolute residual $=0.0781$

Average off-diagonal absolute residual $=0.0838$

Average absolute standardized residual $=0.0586$

Average off-diagonal absolute standardized residual $=0.0630$

Respecto a la bondad de ajuste del modelo puede considerarse aceptable, aunque no alcance los valores tan elevados obtenidos en el análisis de los modelos de medida de forma independiente, debido al notable incremento de la complejidad del modelo. Concretamente, el estadístico $\chi^{2}$ de Satorra Bentler toma el valor de 10237.9 para 337 grados de libertad, mientras los índices comparativos de ajuste alcanzan valores considerados como aceptables $(N F I=0,793 ; N N F I=0,774 ; C F I=0,798 ; I F I=0,799)$ y el RMSEA disminuye hasta 0.079, con intervalo de confianza (90\%) en [0.077, 0.080]. Los índices de parsimonia toman valores muy reducidos $(A I C=$ $9563,9 ; C A I C=7047,2)$ respecto a los correspondientes al modelo de independencia $\left(A I C_{\text {Ind }}=48742,8 ; C A I C_{\text {Ind }}=45919,9\right)$ 
Indices de ajuste para el segundo modelo estructural entre Métodos de enseñanza - aprendizaje y Competencias adquiridas en la Universidad:

$$
\begin{aligned}
& \chi_{\text {Ind }}^{2}=49498,8 \\
& g l_{\text {Ind }}=378 \\
& \chi_{S-B}^{2}=10237,9 \\
& g l=337 \\
& \text { Prob }=0,000
\end{aligned}
$$

El coeficiente Alfa de Cronbach alcanza el valor de 0.904 mientras que el Coeficiente Rho se sitúa en 0.922. Los porcentajes de fiabilidad compuesta y varianza extraída de cada factor revelan que la fiabilidad de los factores de Métodos de enseñanza - aprendizaje es muy reducida respecto a los relativos a Competencias adquiridas en la Universidad. Ninguno de ellos supera los umbrales del $70 \%$ para fiabilidad compuesta y $50 \%$ para varianza extraída. Concretamente el tercer factor - Clases teóricas - que cuenta con sólo dos ítems presenta el valor más reducido $(1 \%)$ mientras que el primer factor Estudio y trabajo en grupo - obtiene los valores más elevados entre conjunto de factores exógenos (46.2\%). Entre los factores endógenos de Competencias adquiridas en la Universidad la fiabilidad es aceptable en la mayoría de los casos, y únicamente el octavo factor - Cooperación - presenta un valor inferior a umbral del $70 \%$ recomendado para el porcentaje de fiabilidad compuesta $(62.2 \%)$.

\begin{tabular}{|c|c|c|}
\hline & Fiabilidad compuesta & Varianza extraída \\
\hline$F_{1}$ & $46.2 \%$ & $15.0 \%$ \\
$F_{2}$ & $20.3 \%$ & $7.9 \%$ \\
$F_{3}$ & $1.0 \%$ & $0.8 \%$ \\
$F_{4}$ & $73.6 \%$ & $42.0 \%$ \\
$F_{5}$ & $78.5 \%$ & $55.5 \%$ \\
$F_{6}$ & $71.3 \%$ & $46.9 \%$ \\
$F_{7}$ & $79.6 \%$ & $56.8 \%$ \\
$F_{8}$ & $62.2 \%$ & $53.0 \%$ \\
$F_{9}$ & $79.3 \%$ & $44.5 \%$ \\
\hline
\end{tabular}

Tabla 5.54: Fiabilidad compuesta y varianza extraída. Modelo estructural final entre Métodos de enseñanza - aprendizaje y Competencias adquiridas en la Universidad 
Las ecuaciones no estandarizadas muestran tanto las estimaciones de los coeficientes para ambos modelos de medida, exógeno y endógeno, como las correspondientes al modelo estructural. En términos generales las estimaciones de los modelos de medida son aproximadamente similares a las obtenidas en los análisis anteriores. Sin embargo es posible detectar ciertas diferencias como las estimaciones en los ítems 4, 5 y 8 - Prácticas en empresa, Conocimientos prácticos y Aprendizaje basado en problemas - que anteriormente resultaban significativas y con valores en el rango habitual. Por otro lado las estimaciones de los coeficientes de Competencias son prácticamente iguales a los obtenidos en el análisis del modelo de medida, con pequeñas modificaciones apenas significativas. Incluso parecen que mejoran las estimaciones de determinados ítems, como el 11, 12 y 24 - Conocimientos del área, Conocimientos de otras áreas y Herramientas informáticas - que anteriormente obtenían estimaciones del error residual más elevadas. En esta ocasión, aunque sus estimaciones resultan más elevadas que el resto de ítems relativos a Competencias adquiridas en la Universidad, no resultan especialmente elevada en el conjunto de todos los ítems incluidos en el modelo.

Las estimaciones más interesantes en este análisis son las relativas al modelo estructural. A pesar de que se hayan obtenido dos coeficientes de regresión no significativos entre los factores 2 y 5 - Prácticas y Clases prácticas e Innovación - así como entre los factores 2 y 7 - Prácticas y Clases prácticas y Organización personal sí es posible observar que ambos valores toman valores positivos. De este modo aunque no es posible validar estadísticamente el cumplimiento de las hipótesis 2 y 3 , relativas a las Clases prácticas y Prácticas externas, tampoco existe evidencia de que deban ser rechazadas.

Por otro lado el resto de coeficientes de regresión resultan significativos. En primer lugar los coeficientes entre los factores 3 y 4 - Clases teóricas y Conocimientos y Pensamiento metacognitivo indican una fuerte relación entre estos constructos, validando la primera hipótesis de investigación planteada anteriormente. Asimismo es posible observar relaciones de carácter moderado entre el primer factor - Estudio y trabajo en grupo - y los factores 6, 8 y 9 - Comunicación, Cooperación y Liderazgo, validando de esta forma la cuarta hipótesis de investigación. 


$\begin{array}{ll}V_{1}=1,000 \cdot F_{1}+1,000 \cdot E_{1} & E_{1}=1,368^{*} \\ V_{2}=1,690^{*} \cdot F_{1}+1,000 \cdot E_{2} & E_{2}=1,151^{*} \\ V_{3}=1,165^{*} \cdot F_{1}+1,000 \cdot F_{2}+1,000 \cdot E_{3} & E_{3}=0,908^{*} \\ V_{4}=172,9 \cdot F_{2}+1,000 \cdot E_{4} & E_{4}=1,599^{*} \\ V_{5}=178,9 \cdot F_{2}+1,000 \cdot E_{5} & E_{5}=1,065^{*} \\ V_{6}=1,000 \cdot F_{3}+1,000 \cdot E_{6} & E_{6}=0,825^{*} \\ V_{7}=0,110 \cdot F_{3}+1,000 \cdot E_{7} & E_{7}=0,782^{*} \\ V_{8}=170,5 \cdot F_{2}+1,000 \cdot E_{8} & E_{8}=0,960^{*} \\ V_{9}=1,166^{*} \cdot F_{1}+1,000 \cdot E_{9} & E_{9}=1,055^{*} \\ V_{10}=1,297^{*} \cdot F_{1}+1,000 \cdot E_{10} & E_{10}=1,087^{*} \\ V_{11}=1,000 \cdot F_{4}+1,000 \cdot E_{11} & E_{11}=1,102^{*} \\ V_{12}=1,036^{*} \cdot F_{4}+1,000 \cdot E_{12} & E_{12}=0,922^{*} \\ V_{13}=1,499^{*} \cdot F_{4}+1,000 \cdot E_{13} & E_{13}=0,536^{*} \\ V_{14}=1,462^{*} \cdot F_{4}+1,000 \cdot E_{14} & E_{14}=0,562^{*} \\ V_{15}=1,012^{*} \cdot F_{9}+1,000 \cdot E_{15} & E_{15}=0,646^{*} \\ V_{16}=1,000 \cdot F_{7}+1,000 \cdot E_{16} & E_{16}=0,939^{*} \\ V_{17}=1,000 \cdot F_{9}+1,000 \cdot E_{17} & E_{17}=0,588^{*} \\ V_{18}=1,155^{*} \cdot F_{7}+1,000 \cdot E_{18} & E_{18}=0,462^{*} \\ V_{19}=1,127^{*} \cdot F_{7}+1,000 \cdot E_{19} & E_{19}=0,545^{*} \\ V_{20}=4,378^{*} \cdot F_{8}+1,000 \cdot E_{20} & E_{20}=0,000^{*} \\ V_{21}=1,000 \cdot F_{8}+0,920^{*} \cdot F_{9}+1,000 \cdot E_{21} & E_{21}=0,460^{*} \\ V_{22}=1,000 \cdot F_{6}+0,560^{*} \cdot F_{9}+1,000 \cdot E_{22} & E_{22}=0,603^{*} \\ V_{23}=1,117^{*} \cdot F_{9}+1,000 \cdot E_{23} & E_{23}=0,481^{*} \\ V_{24}=0,779^{*} \cdot F_{5}+1,000 \cdot E_{24} & E_{24}=1,133^{*} \\ V_{25}=1,000 \cdot F_{5}+1,000 \cdot E_{25} & E_{25}=0,345^{*} \\ V_{26}=0,892^{*} \cdot F_{5}+1,000 \cdot E_{26} & E_{26}=0,590^{*} \\ V_{27}=2,085^{*} \cdot F_{6}+1,000 \cdot E_{27} & E_{27}=0,464^{*} \\ V_{28}=1,787^{*} \cdot F_{6}+1,000 \cdot E_{28} & E_{28}=0,699^{*}\end{array}$

Tabla 5.55: Ecuaciones no estandarizadas del modelo de medida. Modelo estructural final entre Métodos de enseñanza - aprendizaje y Competencias adquiridas en la Universidad

Las estimaciones de los parámetros de varianzas y covarianzas permiten comprender porqué han resultado no significativos las relaciones estructurales asociadas al segundo factor relacionado con las Prácticas y clases prácticas. Como se puede comprobar la estimación de la varianza de este factor es nula. Este tipo de estimación se considera válida y no infractora, como ocurriría en caso de que fuera negativa. No obstante, condiciona las estimaciones de los parámetros relacionados como se observa en los coeficientes de regresión no estandarizados como se ha visto anteriormente.

Las estimaciones de las covarianzas y correlaciones entre factores confirman los resultados obtenidos anteriormente. Los factores 1 y 2 - Estudio y trabajo en grupo y Prácticas y clases prácticas - guardan cierta relación entre sí, y a su vez ambos mantienen relaciones muy débiles con el tercer factor - Clases teóricas. 


$$
\begin{aligned}
& F_{4}=5,103^{*} \cdot F_{3}+1,000 \cdot D_{4} D_{4}=0,000 \\
& F_{5}=396,4 \cdot F_{2}+1,000 \cdot D_{5} \quad D_{5}=0,296^{*} \\
& F_{6}=1,174^{*} \cdot F_{1}+1,000 \cdot D_{6} D_{6}=0,081^{*} \\
& F_{7}=340,2 \cdot F_{2}+1,000 \cdot D_{7} \quad D_{7}=0,171^{*} \\
& F_{8}=0,609^{*} \cdot F_{1}+1,000 \cdot D_{8} D_{8}=0,035^{*} \\
& F_{9}=1,896^{*} \cdot F_{1}+1,000 \cdot D_{9} D_{9}=0,225^{*}
\end{aligned}
$$

Tabla 5.56: Ecuaciones no estandarizadas del modelo estructural. Modelo estructural final entre Métodos de enseñanza - aprendizaje y Competencias adquiridas en la Universidad

\begin{tabular}{|c|c|c|c|}
\hline & $F_{1}$ & $F_{2}$ & $F_{3}$ \\
\hline$F_{1}$ & $0.119^{*}$ & & \\
$F_{2}$ & 0.265 & 0.000 & \\
$F_{3}$ & 0.022 & 0.015 & $0.013^{*}$ \\
\hline$E_{9}-E_{2}$ & \multicolumn{3}{|c|}{$0.513^{*}$} \\
\hline
\end{tabular}

Tabla 5.57: Matriz de varianzas-covarianzas. Modelo estructural final entre Métodos de enseñanza - aprendizaje y Competencias adquiridas en la Universidad

\begin{tabular}{|c|c|c|c|}
\hline & $F_{1}$ & $F_{2}$ & $F_{3}$ \\
\hline$F_{1}$ & 1.000 & & \\
$F_{2}$ & 0.658 & 1.000 & \\
$F_{3}$ & 0.135 & 0.112 & 1.000 \\
\hline$E_{9}-E_{2}$ & \multicolumn{3}{|c|}{0.479} \\
\hline
\end{tabular}

Tabla 5.58: Matriz de correlaciones. Modelo estructural final entre Métodos de enseñanza - aprendizaje y Competencias adquiridas en la Universidad

Las ecuaciones estandarizadas muestran resultados equivalentes a las no estandarizadas, aunque en este caso es posible apreciar que los coeficientes de determinación de las ecuaciones del modelo de medida de los Métodos de enseñanza- aprendizaje son prácticamente nulos, indicando la falta de capacidad de los factores latentes exógenos para explicar el comportamiento de dichos ítems. Por el contrario, los coeficientes de determinación del modelo de medida de las Competencias adquiridas en la Universidad resultan aceptables y únicamente resultan más pequeños en los ítems que hasta ahora han obtenido las peores estimaciones. Estos ítems son fundamentalmente el 11, 12 y 24 - Conocimientos del área, Conocimientos de otras áreas y Herramientas informáticas. 


$$
\begin{array}{ll}
V_{1}=0,283 \cdot F_{1}+0,959 \cdot E_{1} & R^{2}=0,080 \\
V_{2}=0,478 \cdot F_{1}+0,879 \cdot E_{2} & R^{2}=0,228 \\
V_{3}=0,389 \cdot F_{1}+0,002 \cdot F_{2}+0,923 \cdot E_{3} & R^{2}=0,153 \\
V_{4}=0,278 \cdot F_{2}+0,961 \cdot E_{4} & R^{2}=0,077 \\
V_{5}=0,344 \cdot F_{2}+0,939 \cdot E_{5} & R^{2}=0,119 \\
V_{6}=0,125 \cdot F_{3}+0,992 \cdot E_{6} & R^{2}=0,016 \\
V_{7}=0,014 \cdot F_{3}+1,000 \cdot E_{7} & R^{2}=0,000 \\
V_{8}=0,346 \cdot F_{2}+0,938 \cdot E_{8} & R^{2}=0,119 \\
V_{9}=0,365 \cdot F_{1}+0,931 \cdot E_{9} & R^{2}=0,133 \\
V_{10}=0,395 \cdot F_{1}+0,919 \cdot E_{10} & R^{2}=0,156 \\
V_{11}=0,487 \cdot F_{4}+0,873 \cdot E_{11} & R^{2}=0,238 \\
V_{12}=0,534 \cdot F_{4}+0,845 \cdot E_{12} & R^{2}=0,286 \\
V_{13}=0,768 \cdot F_{4}+0,640 \cdot E_{13} & R^{2}=0,590 \\
V_{14}=0,753 \cdot F_{4}+0,658 \cdot E_{14} & R^{2}=0,566 \\
V_{15}=0,713 \cdot F_{9}+0,701 \cdot E_{15} & R^{2}=0,508 \\
V_{16}=0,650 \cdot F_{7}+0,760 \cdot E_{16} & R^{2}=0,423 \\
V_{17}=0,726 \cdot F_{9}+0,688 \cdot E_{17} & R^{2}=0,526 \\
V_{18}=0,816 \cdot F_{7}+0,578 \cdot E_{18} & R^{2}=0,665 \\
V_{19}=0,785 \cdot F_{7}+0,619 \cdot E_{19} & R^{2}=0,616 \\
V_{20}=1,000 \cdot F_{8}+0,000 \cdot E_{20} & R^{2}=1,000 \\
V_{21}=0,243 \cdot F_{8}+0,641 \cdot F_{9}+0,585 \cdot E_{21} & R^{2}=0,658 \\
V_{22}=0,426 \cdot F_{6}+0,390 \cdot F_{9}+0,668 \cdot E_{22} & R^{2}=0,554 \\
V_{23}=0,793 \cdot F_{9}+0,609 \cdot E_{23} & R^{2}=0,629 \\
V_{24}=0,590 \cdot F_{5}+0,807 \cdot E_{24} & R^{2}=0,348 \\
V_{25}=0,862 \cdot F_{5}+0,507 \cdot E_{25} & R^{2}=0,743 \\
V_{26}=0,758 \cdot F_{5}+0,653 \cdot E_{26} & R^{2}=0,574 \\
V_{27}=0,835 \cdot F_{6}+0,551 \cdot E_{27} & R^{2}=0,697 \\
V_{28}=0,727 \cdot F_{6}+0,687 \cdot E_{28} & R^{2}=0,528
\end{array}
$$

Tabla 5.59: Ecuaciones estandarizadas del modelo de medida. Modelo estructural final entre Métodos de enseñanza - aprendizaje y Competencias adquiridas en la Universidad

En esta fase del análisis el Contraste de Lagrange se ejecuta evidentemente considerando las matrices relativas a los parámetros del modelo de medida, que incluyen los coeficientes de correlación entre ítems y factores y las covarianzas entre errores residuales, pero también las matrices referentes al modelo estructural, incluyendo los coeficientes de regresión entre factores exógenos y endógenos y las covarianzas entre perturbaciones. Como resultado de esta prueba se propone una nueva relación desde el séptimo factor Organización personal - al noveno - Liderazgo. Esta relación no es coherente con el planteamiento general del modelo, que plantea el efecto de un conjunto de factores exógenos sobre Métodos de enseñanza - aprendizaje sobre otro conjunto de factores endógenos sobre Competencias adquiridas en la Universidad. Asimismo tampoco es respaldada por ninguna de las investigaciones precedentes sobre modelos de enseñanza - aprendizaje. Las siguientes relaciones hicieron referencia a las covarianzas entre distintos errores de medida 


$$
\begin{aligned}
& F_{5}=0,839 \cdot F_{2}+0,544 \cdot D_{5} R^{2}=0,704 \\
& F_{6}=0,819 \cdot F_{1}+0,574 \cdot D_{6} R^{2}=0,671 \\
& F_{7}=0,867 \cdot F_{3}+0,498 \cdot D_{7} R^{2}=0,752 \\
& F_{8}=0,745 \cdot F_{1}+0,667 \cdot D_{8} R^{2}=0,555 \\
& F_{9}=0,810 \cdot F_{1}+0,587 \cdot D_{9} R^{2}=0,656
\end{aligned}
$$

Tabla 5.60: Ecuaciones no estandarizadas del modelo de medida. Modelo estructural final entre Métodos de enseñanza - aprendizaje y Competencias adquiridas en la Universidad

que, de haberse considerado, se hubieran incluido en las reespecificaciones de los anteriores modelos de medida. Por tanto, no se considera procedente aceptar la modificación propuesta y se acepta el modelo propuesto para los procesos de enseñanza - aprendizaje de competencias en la Universidad.

\section{Modelización causal por áreas de estudio}

Puesto que en este punto se parte de un modelo causal es posible plantear un análisis de invarianza estructural que considere no sólo las restricciones relativas a los modelos de medida sino también al modelo estructural. Este análisis se aplica, como es evidente, al modelo causal basado en tres factores de Métodos de enseñanza - aprendizaje que no ha mostrado evidencias de falta de identificación empírica ni estimaciones infractoras. La aplicación de este modelo a cada una de las áreas de estudio presenta un ajuste tan bueno como el obtenido en la muestra global, e incluso notablemente mejor en determinadas áreas como Educación, Economía y empresa y Salud. Por el contrario en el área de Ciencias no ha sido posible alcanzar la convergencia en dicho modelo. 


\begin{tabular}{|c|c|c|c|c|c|c|c|}
\hline \multirow{5}{*}{$\begin{array}{l}\chi_{\text {Ind }}^{2} \\
g l_{\text {Ind }} \\
\chi_{S-B}^{2} \\
g l\end{array}$} & \multicolumn{7}{|c|}{$\begin{array}{l}\text { Educación Humanidades Ciencias Economía Derecho Técnicas Salud } \\
\text { Sociales y empresa }\end{array}$} \\
\hline & 6173.7 & 5551.7 & 5763.2 & 9740.0 & 3230.1 & 8576.1 & 6071.9 \\
\hline & 378 & 378 & 378 & 378 & 378 & 378 & 378 \\
\hline & 1253.5 & 1352.1 & 1559.5 & 1976.5 & 896.5 & 1956.2 & 1298.6 \\
\hline & 337 & 337 & 337 & 337 & 337 & 337 & 337 \\
\hline$A I C_{\text {Ind }}$ & 5417.7 & 4795.7 & 5007.2 & 8984.0 & 2474.1 & 7820.1 & 5315.9 \\
\hline$A I C$ & 579.5 & 678.1 & 885.5 & 1302.5 & 222.5 & 1282.2 & 624.6 \\
\hline$C A I C_{\text {Ind }}$ & 3420.3 & 2872.0 & 2994.6 & 6773.4 & 734.5 & 5601.6 & 3345.6 \\
\hline$C A I C$ & -1201.2 & -1036.9 & -908.8 & -668.2 & -1328.4 & -695.7 & -1132.1 \\
\hline$N F I$ & 0.797 & 0.756 & 0.729 & 0.797 & 0.722 & 0.772 & 0.786 \\
\hline$N N F I$ & 0.823 & 0.780 & 0.745 & 0.804 & 0.780 & 0.778 & 0.811 \\
\hline$C F I$ & 0.842 & 0.804 & 0.773 & 0.825 & 0.804 & 0.802 & 0.831 \\
\hline$I F I$ & 0.843 & 0.805 & 0.775 & 0.826 & 0.807 & 0.803 & 0.832 \\
\hline$M F I$ & 0.425 & 0.316 & 0.334 & 0.419 & 0.356 & 0.431 & 0.382 \\
\hline$R M S E A$ & 0.071 & 0.083 & 0.081 & 0.072 & 0.078 & 0.071 & 0.076 \\
\hline Lim Inf $90 \%$ & 0.067 & 0.078 & 0.077 & 0.069 & 0.072 & 0.068 & 0.071 \\
\hline Lim Sup $90 \%$ & 0.075 & 0.087 & 0.085 & 0.075 & 0.084 & 0.074 & 0.080 \\
\hline
\end{tabular}

Tabla 5.61: Contraste $\chi^{2}$ e índices de ajuste por área de estudio. Invarianza estructural del modelo entre Métodos de enseñanza - aprendizaje y Competencias adquiridas en la Universidad

Por esta razón se plantean los análisis de invarianza métrica y estructural tanto para el conjunto de las ocho áreas de estudio consideradas incialmente como para un nuevo subconjunto de siete áreas que excluya al grupo de Ciencias. Como se observa en la Tabla 5.62 el análisis que mejores resultados obtuvo fue el análisis de invarianza métrica sobre el subconjunto de siete áreas de estudio excluyendo el grupo de Ciencias. No obstante, puesto que el análisis de invarianza estructural es más restrictivo que el de invarianza métrica, cabe esperar que en igualdad de condiciones, el ajuste obtenido mediante la imposición de restricciones sobre el modelo de medida sea mejor que sobre el modelo estructural. Por esa razón cabe destacar la mejora en el ajuste de los análisis de invarianza métrica y estructural sobre el subconjunto de áreas de estudio que excluye a las Ciencias, respecto al conjunto total de las ocho áreas de estudio. 


\begin{tabular}{|l|cc|cc|}
\hline & \multicolumn{2}{|c|}{8 Grupos } & \multicolumn{2}{c|}{7 Grupos (sin Ciencias) } \\
& Inv. Métrica & Inv. Estructural & Inv. Métrica & Inv. Estructural \\
\hline$\chi_{\text {Ind }}^{2}$ & 51603.7 & 58243.0 & 45809.9 & 45809.9 \\
$g l_{\text {Ind }}$ & 3024 & 3024 & 2646 & 2646 \\
$\chi^{2}$ & 13656.5 & 13971.5 & 11854.9 & 12078.4 \\
$\chi_{S-B}^{2}$ & 11946.3 & 12198.6 & 10332.4 & 10505.0 \\
$g l$ & 2850 & 2934 & 2491 & 2563 \\
Prob. & 0.000 & 0.000 & 0.000 & 0.000 \\
\hline
\end{tabular}

Tabla 5.62: Contraste $\chi^{2}$. Invarianza estructural del modelo entre Métodos de enseñanza - aprendizaje y Competencias adquiridas en la Universidad

La misma conclusión puede obtenerse mediante el análisis de los índices de ajuste. Los resultados obtenidos en el análisis de invarianza métrica excluyendo el área de Ciencias parecen ser los mejores, pero resultan muy próximos a los obtenidos en el análisis de invarianza estructural para el mismo subconjunto de áreas. En cualquier caso los resultados de ambos análisis son superiores a los obtenidos al aplicar los análisis de invarianza a las ocho áreas de estudio planteadas inicialmente. Así, los resultados sugieren descartar el área de Ciencias en los análisis de invarianza y contemplar los datos correspondientes a esta muestra de forma independiente.

De hecho, con el fin de encontrar más evidencias de la necesidad de descartar el área de Ciencias de los análisis de invarianza, se estudian las modificaciones propuestas por el Contraste de Lagrange en condiciones de invarianza parcial. Esta prueba de invarianza parcial se aplica sobre el análisis de invarianza estructural sobre las ocho áreas de estudio planteadas incialmente. Como se observa en la Tabla 5.64 la primer modificación propuesta hace referencia a la covarianza entre los factores 2 y 3 - Prácticas y clases prácticas y Clases teóricas - entre los grupos 1 y 8 - Educación y Ciencias. Asimismo la segunda modificación propuesta igualmente se refiere igualmente a los mismos grupos, pero en este caso sugiere liberar la varianza del segundo factor, que como se ha visto anteriormente es un parámetro problemático. En cualquier caso el hecho de que las dos primeras modificaciones del Contraste de Lagrange hagan referencia al grupo de Ciencias confirma que el modelo base aplicado a este grupo no puede ser el mismo que el aplicado al resto de las áreas. Por tanto, los análisis de invarianza realizados a partir de este punto se refieren exclusivamente a las áreas de Educación, Humanidades, Ciencias Sociales, Economía y empresa, Derecho, Técnicas y Salud. 


\begin{tabular}{|l|cc|cc|}
\hline & \multicolumn{2}{|c|}{ 8 Grupos } & \multicolumn{2}{c|}{7 Grupos (sin Ciencias) } \\
& Inv. Métrica Inv. Estructural & Inv. Métrica Inv. Estructural \\
\hline AIC Ind & 45555.7 & 45555.7 & 40517.9 & 40517.9 \\
AIC & 6246.3 & 6330.6 & 5350.4 & 5379.0 \\
C AIC Ind & 22986.5 & 22986.5 & 21097.0 & 21097.0 \\
CAIC & -15024.3 & -15566.9 & -12932.9 & -13432.8 \\
NFI & 0.768 & 0.764 & 0.774 & 0.771 \\
NNFI & 0.801 & 0.803 & 0.807 & 0.810 \\
CFI & 0.813 & 0.809 & 0.818 & 0.816 \\
IFI & 0.813 & 0.810 & 0.819 & 0.816 \\
MFI & 0.383 & 0.376 & 0.392 & 0.387 \\
RMSEA & 0.073 & 0.073 & 0.073 & 0.072 \\
Lim Inf $90 \%$ & 0.072 & 0.072 & 0.071 & 0.071 \\
Lim Sup $90 \%$ & 0.075 & 0.074 & 0.074 & 0.073 \\
\hline
\end{tabular}

Tabla 5.63: Indices de ajuste obtenidos sobre la totalidad de las áreas de estudio, y el subconjunto de 7 áreas excluyendo Ciencias. Invarianza métrica y estructural del modelo entre Métodos de enseñanza - aprendizaje y Competencias adquiridas en la Universidad

\begin{tabular}{|c|c|c|c|c|}
\hline Núm. & Restricciones & $\chi^{2}$ & g.l. & Prob. \\
\hline 1 & $C 285:\left(1, F_{2}, F_{3}\right)=\left(8, F_{2}, F_{3}\right)$ & 32.2 & 1 & 0.000 \\
2 & $C 257:\left(1, F_{2}, F_{2}\right)=\left(8, F_{2}, F_{2}\right)$ & 43.8 & 2 & 0.000 \\
3 & $C 269:\left(1, F_{1}, F_{2}\right)=\left(6, F_{1}, F_{2}\right)$ & 61.1 & 3 & 0.000 \\
4 & $C 283:\left(1, F_{2}, F_{3}\right)=\left(6, F_{2}, F_{3}\right)$ & 71.2 & 4 & 0.000 \\
5 & $C 267:\left(1, F_{1}, F_{2}\right)=\left(4, F_{1}, F_{2}\right)$ & 75.6 & 5 & 0.000 \\
\hline
\end{tabular}

Tabla 5.64: Imposición de restricciones de invarianza parcial. Invarianza métrica y estructural del modelo entre Métodos de enseñanza - aprendizaje y Competencias adquiridas en la Universidad 
Una vez realizada esta consideración, se observa que los promedios de la matriz residual de varianzas - covarianzas son relativamente pequeños en comparación con el obtenido sobre el mismo modelo causal sin restricciones de invarianza, en el que los promedios resduales tomaban valores de 0.0781 y 0.0838 para los promedios absolutos incluyendo y excluyendo los términos en la diagonal, y 0.0585 y 0.0630 para los mismos valores estandarizados.

\begin{tabular}{|c|c|c|c|c|c|c|c|}
\hline & Educación & Humanidades & $\begin{array}{l}\text { Ciencias } \\
\text { Sociales }\end{array}$ & $\begin{array}{l}\text { Economía } \\
\text { y empresa }\end{array}$ & Derecho & Técnicas & Salud \\
\hline Avg. abs. residual & 0.085 & 0.130 & 0.085 & 0.065 & 0.081 & 0.091 & 0.086 \\
\hline Avg. off-diagonal abs. residual & 0.087 & 0.132 & 0.087 & 0.067 & 0.084 & 0.092 & 0.088 \\
\hline Avg. abs. std. residual & 0.067 & 0.086 & 0.067 & 0.061 & 0.072 & 0.078 & 0.065 \\
\hline Avg. off-diagonal abs. std. residual & 0.069 & 0.088 & 0.069 & 0.062 & 0.074 & 0.079 & 0.067 \\
\hline
\end{tabular}

Tabla 5.65: Promedios de las matrices residuales de varianzas-covarianzas por área de estudio. Invarianza métrica y estructural del modelo entre Métodos de enseñanza - aprendizaje y Competencias adquiridas en la Universidad

Asimismo los coeficientes de fiabilidad Alfa de Cronbach y Rho son elevados y muy similares a los obtenidos en el modelo equivalente sin restricciones donde el coeficiente Alfa de Cronbach alcanza el valor de 0.904 y el coeficiente Rho toma el valor 0.922 .

\begin{tabular}{|l|ccccccc|}
\hline & \multicolumn{7}{|c|}{ Educación Humanidades Ciencias Economía Derecho Técnicas Salud } \\
& \multicolumn{7}{|c}{ Sociales y empresa } \\
\hline$\alpha$ Cronbach & 0.915 & 0.909 & 0.915 & 0.913 & 0.911 & 0.894 & 0.920 \\
Rho & 0.924 & 0.913 & 0.924 & 0.931 & 0.930 & 0.928 & 0.922 \\
\hline
\end{tabular}

Tabla 5.66: Indices de fiabilidad por área de estudio. Invarianza métrica y estructural del modelo entre Métodos de enseñanza - aprendizaje y Competencias adquiridas en la Universidad

Puesto que las restricciones de invarianza estructural son más restrictivas que las relativas a la invarianza métrica, la igualdad de las ecuaciones no estandarizadas en las distintas áreas de estudio se mantiene como ocurría en los análisis de invarianza anteriores. 


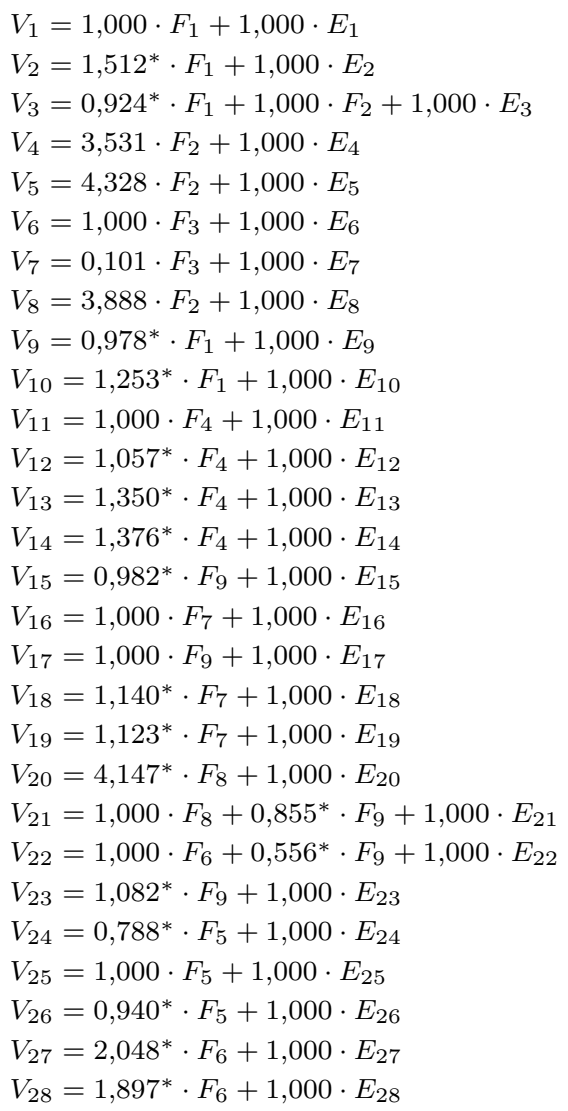

Tabla 5.67: Ecuaciones no estandarizadas del modelo de medida. Invarianza métrica y estructural del modelo entre Métodos de enseñanza - aprendizaje y Competencias adquiridas en la Universidad

Asimismo, las ecuaciones de regresión entre factores endógenos y exógenos son igualmente comunes a las siete áreas de estudio. Cabe destacar que las estimaciones de los parámetros asociados al segundo factor siguen sin ser significativas debido que la varianza de dicho factor sigue estimándose como nula. Al igual que ocurría en la validación del modelo sobre la muestra global, la relación de regresión significativa más fuerte se da entre los factores 3 y 4 - Clases teóricas y Conocimientos y Pensamiento metacognitivo. Al mismo tiempo es posible identificar relaciones de carácter moderado entre el primer factor - Estudio y trabajo en grupo - y los factores 6,8 y 9 - Comunicación, Cooperación y Liderazgo. 


$$
\begin{aligned}
& F_{4}=5,429^{*} \cdot F_{3}+1,000 \cdot D_{4} \\
& F_{5}=10,491 \cdot F_{2}+1,000 \cdot D_{5} \\
& F_{6}=1,577^{*} \cdot F_{1}+1,000 \cdot D_{6} \\
& F_{7}=9,405 \cdot F_{2}+1,000 \cdot D_{7} \\
& F_{8}=0,804^{*} \cdot F_{1}+1,000 \cdot D_{8} \\
& F_{9}=2,793^{*} \cdot F_{1}+1,000 \cdot D_{9}
\end{aligned}
$$

Tabla 5.68: Ecuaciones no estandarizadas del modelo estructural. Invarianza métrica y estructural del modelo entre Métodos de enseñanza - aprendizaje y Competencias adquiridas en la Universidad

Del mismo modo, al igual que la varianza del segundo factor - Prácticas y clases prácticas - sigue estimándose nula, las estimaciones correspondientes al resto de los parámetros son muy similares a las obtenidas en el modelo sin restringir. En este caso el tercer factor - Clases teóricas - sigue estando prácticamente incorrelacionado, al igual que ocurría en la validación del modelo.

\begin{tabular}{|c|c|c|c|}
\hline & $F_{1}$ & $F_{2}$ & $F_{3}$ \\
\hline$F_{1}$ & $0.112^{*}$ & & \\
$F_{2}$ & 0.226 & 0.000 & \\
$F_{3}$ & 0.017 & 0.009 & $0.005^{*}$ \\
\hline$E_{9}-E_{2}$ & \multicolumn{3}{|c|}{$0.476^{*}$} \\
\hline
\end{tabular}

Tabla 5.69: Matriz de varianzas-covarianzas. Invarianza métrica y estructural del modelo entre Métodos de enseñanza - aprendizaje y Competencias adquiridas en la Universidad

\begin{tabular}{|c|c|c|c|}
\hline & $F_{1}$ & $F_{2}$ & $F_{3}$ \\
\hline$F_{1}$ & 1.000 & & \\
$F_{2}$ & 0.532 & 1.000 & \\
$F_{3}$ & 0.110 & 0.096 & 1.000 \\
\hline$E_{9}-E_{2}$ & \multicolumn{3}{|c|}{0.367} \\
\hline
\end{tabular}

Tabla 5.70: Matriz de correlaciones. Invarianza métrica y estructural del modelo entre Métodos de enseñanza - aprendizaje y Competencias adquiridas en la Universidad

Sin embargo, los parámetros cuya variabilidad no se ha restringido toman valores distintos en cada una de las áreas de estudio. La Tabla 5.71 muestra las estimaciones de los errores de medida para los 28 ítems incluidos en el modelo en cada área de estudio. En términos generales los errores de los ítems relativos a los Métodos de enseñanza - aprendizaje son más 
elevados que los errores correspondientes a las Competencias adquiridas en la Universidad. Únicamente los ítems 11, 12 y 24 - Conocimientos del área, Conocimientos de otras áreas y Herramientas informáticas - que en todos los análisis precedentes presentaban estimaciones más elevadas de los errores, no cumplen en este caso este patrón. Por el contrario, la estimación del error residual del ítem 20 - Trabajar en equipo - que en los análisis precedentes tomaba el valor nulo por imposición de EQS, no llega a alcanzar valores no nulos aunque sí muy reducidos en todas las áreas de estudio, excepto Derecho y Técnicas.

\begin{tabular}{|c|c|c|c|c|c|c|c|}
\hline & Educació & 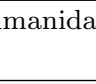 & Sociales & empres & & & \\
\hline$E_{1}$ & $1.277^{*}$ & $1.494^{*}$ & $1.277^{*}$ & $1.252^{*}$ & $1.377^{*}$ & $1.317^{*}$ & $1.375^{*}$ \\
\hline$E_{2}$ & $0.843^{*}$ & $1.273^{*}$ & $0.843^{*}$ & $0.901^{*}$ & $0.679 *$ & $0.931^{*}$ & $1.091^{*}$ \\
\hline$E_{3}$ & $1.159^{*}$ & $1.045^{*}$ & $1.159^{*}$ & $0.568^{*}$ & $0.396^{*}$ & $0.788^{*}$ & $0.807^{*}$ \\
\hline$E_{4}$ & $1.362^{*}$ & $0.871^{*}$ & $1.362^{*}$ & $1.272^{*}$ & $0.636^{*}$ & $1.138^{*}$ & $2.121^{*}$ \\
\hline$E_{5}$ & $0.919 *$ & $1.367^{*}$ & $0.919 *$ & $0.828^{*}$ & $0.830^{*}$ & $0.935^{*}$ & $1.090^{*}$ \\
\hline$E_{6}$ & $0.741^{*}$ & $1.015^{*}$ & $0.741^{*}$ & $0.768^{*}$ & $1.026^{*}$ & $0.754^{*}$ & $0.976^{*}$ \\
\hline$E_{7}$ & & $.920^{*}$ & $.808^{*}$ & $0.706^{*}$ & $0.793^{*}$ & $0.792^{*}$ & $0.682^{*}$ \\
\hline$E_{8}$ & & 1 & 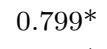 & $0.716^{*}$ & $0.583^{*}$ & $0.892^{*}$ & $0.901^{*}$ \\
\hline$E_{9}$ & $0.814^{*}$ & $.080^{*}$ & $0.814^{*}$ & $0.773^{*}$ & $0.907^{*}$ & $0.853^{*}$ & $0.945^{*}$ \\
\hline$E_{10}$ & $1.247^{*}$ & & $1.247^{*}$ & $0.791^{*}$ & $1.081^{*}$ & $0.732^{*}$ & $0.871^{*}$ \\
\hline$E_{11}$ & $1.073^{*}$ & $1.431^{*}$ & $.073^{*}$ & $0.924^{*}$ & $1.164^{*}$ & $0.943^{*}$ & $0.948^{*}$ \\
\hline$E_{12}$ & 0.863 & $1.027^{*}$ & $0.863^{*}$ & $0.777^{*}$ & $0.849^{*}$ & $0.833^{*}$ & $0.892^{*}$ \\
\hline$E_{13}$ & $0.509^{*}$ & $0.611^{*}$ & $0.509^{*}$ & $0.513^{*}$ & $0.599^{*}$ & $0.522^{*}$ & $0.505^{*}$ \\
\hline$E_{14}$ & $0.457^{*}$ & $0.580^{*}$ & $0.457^{*}$ & $0.542^{*}$ & $0.676^{*}$ & $0.526^{*}$ & $0.540^{*}$ \\
\hline$E_{15}$ & $0.701^{*}$ & $0.558^{*}$ & $0.701^{*}$ & $0.625^{*}$ & $0.530^{*}$ & $0.533^{*}$ & $0.641^{*}$ \\
\hline$E_{16}$ & $0.849 *$ & 0.899 & $0.849^{*}$ & $0.736^{*}$ & $0.819 *$ & $0.989 *$ & $0.963^{*}$ \\
\hline$E_{17}$ & $0.591^{*}$ & $0.685^{*}$ & $0.591^{*}$ & $0.501^{*}$ & $0.479^{*}$ & $0.515^{*}$ & $0.479^{*}$ \\
\hline$E_{18}$ & $0.453^{*}$ & $0.428^{*}$ & $0.453^{*}$ & $0.417^{*}$ & $0.489^{*}$ & $0.459 *$ & $0.388^{*}$ \\
\hline$E_{19}$ & $0.539 *$ & $0.569 *$ & $0.539^{*}$ & $0.555^{*}$ & $0.575^{*}$ & $0.541^{*}$ & $0.591^{*}$ \\
\hline$E_{20}$ & & & 0.183 & 0.073 & 0.000 & 0.000 & 0.273 \\
\hline$E_{21}$ & $0.582^{*}$ & $0.505^{*}$ & $0.582^{*}$ & $0.372^{*}$ & $0.392^{*}$ & $0.464^{*}$ & $0.399^{*}$ \\
\hline$E_{22}$ & $0.526^{*}$ & $0.729 *$ & $0.526^{*}$ & $0.531^{*}$ & $0.673^{*}$ & $0.585^{*}$ & $0.522^{*}$ \\
\hline$E_{23}$ & $0.703^{*}$ & $0.558^{*}$ & $0.703^{*}$ & $0.377^{*}$ & $0.529^{*}$ & $0.443^{*}$ & $0.433^{*}$ \\
\hline$E_{24}$ & $0.850^{*}$ & $1.213^{*}$ & $0.850^{*}$ & $0.995^{*}$ & $0.913^{*}$ & $1.226^{*}$ & $0.879^{*}$ \\
\hline$E_{25}$ & $0.313^{*}$ & $0.433^{*}$ & $0.313^{*}$ & $0.306^{*}$ & $0.511^{*}$ & $0.420^{*}$ & $0.275^{*}$ \\
\hline$E_{26}$ & $0.442^{*}$ & $0.804^{*}$ & $0.442^{*}$ & $0.445^{*}$ & $0.609^{*}$ & $0.544^{*}$ & $0.440^{*}$ \\
\hline$E_{27}$ & $0.427^{*}$ & $0.538^{*}$ & $0.427^{*}$ & $0.562^{*}$ & $0.474^{*}$ & $0.546^{*}$ & $0.449^{*}$ \\
\hline$E_{28}$ & $0.588^{*}$ & $0.978^{*}$ & $0.588^{*}$ & $0.617^{*}$ & $0.571^{*}$ & $0.670^{*}$ & $0.578^{*}$ \\
\hline
\end{tabular}

Tabla 5.71: Errores de medida por área de estudio. Invarianza métrica y estructural del modelo entre Métodos de enseñanza - aprendizaje y Competencias adquiridas en la Universidad

A continuación se muestran las estimaciones de las perturbaciones asociadas a los factores endógenos de Competencias adquiridas en la Universi- 
dad. Generalmente las perturbaciones de los factores 4,6 y 8 - Conocimientos y Pensamiento metacognitivo, Comunicación y Cooperación - presentan valores más reducidos de error mientras que las perturbaciones más elevadas se encuentran en el resto de factores. Concretamente en las áreas de $\mathrm{Hu}$ manidades, Técnicas y Derecho se localizan los valores máximos de estas perturbaciones. Igualmente se muestran las estimaciones de covarianza y correlación entre los errores residuales de los ítems 9 y 10 - Trabajos escritos y Exposiciones orales.

\begin{tabular}{|c|ccccccc|}
\hline \multicolumn{7}{|c|}{ Educación Humanidades Ciencias Economía Derecho Técnicas Salud } \\
Sociales y empresa \\
\hline$D_{4}$ & $0.018^{*}$ & 0.064 & 0.018 & 0.001 & 0.000 & 0.013 & 0.000 \\
$D_{5}$ & $0.187^{*}$ & $0.284^{*}$ & $0.187^{*}$ & $0.203^{*}$ & $0.184^{*}$ & $0.261^{*}$ & $0.259^{*}$ \\
$D_{6}$ & $0.083^{*}$ & $0.110^{*}$ & $0.083^{*}$ & $0.052^{*}$ & $0.108^{*}$ & $0.051^{*}$ & $0.096^{*}$ \\
$D_{7}$ & $0.127^{*}$ & $0.206^{*}$ & $0.127^{*}$ & $0.142^{*}$ & $0.254^{*}$ & $0.191^{*}$ & $0.108^{*}$ \\
$D_{8}$ & $0.030^{*}$ & $0.044^{*}$ & $0.030^{*}$ & $0.035^{*}$ & $0.046^{*}$ & $0.042^{*}$ & $0.027^{*}$ \\
$D_{9}$ & $0.147^{*}$ & $0.191^{*}$ & $0.147^{*}$ & $0.178^{*}$ & $0.238^{*}$ & $0.249^{*}$ & $0.136^{*}$ \\
\hline
\end{tabular}

Tabla 5.72: Perturbaciones por área de estudio. Invarianza métrica y estructural del modelo entre Métodos de enseñanza - aprendizaje y Competencias adquiridas en la Universidad

\begin{tabular}{|l|ccccccc|}
\hline & \multicolumn{7}{|c|}{ Educación Humanidades Ciencias Economía Derecho Técnicas Salud } \\
& \multicolumn{7}{|c|}{ Sociales y empresa } \\
\hline $\operatorname{Cov}\left(E_{9}, E_{10}\right)$ & $0.442^{*}$ & $0.511^{*}$ & $0.442^{*}$ & $0.363^{*}$ & $0.315^{*}$ & $0.252^{*}$ & $0.435^{*}$ \\
$\operatorname{Corr}\left(E_{9}, E_{10}\right)$ & 0.438 & 0.427 & 0.438 & 0.464 & 0.318 & 0.319 & 0.480 \\
\hline
\end{tabular}

Tabla 5.73: Covarianzas y correlaciones entre errores de medida por área de estudio. Invarianza métrica y estructural del modelo entre Métodos de enseñanza - aprendizaje y Competencias adquiridas en la Universidad

Los coeficientes estandarizados resultan muy similares en todos las áreas de estudio. En las ecuaciones relativas al modelo de medida los valores más bajos los alcanzan los ítems asociados a los factores sobre Métodos de enseñanza - aprendizaje. Entre los ítems relativos a Competencias adquiridas en la Universidad los ítems 11, 12 y 24 - Conocimientos del área, Conocimientos de otras áreas y Herramientas informáticas - obtienen coeficientes relativamente bajos, como había sucedido en modelos anteriores.

Como ya se ha comentado, la estandarización de los coeficientes resulta especialmente útil para comparar distintos modelos sobre una misma muestra, con lo cual no parece apropiado estimar estos coeficientes para valorar la aplicación de un mismo modelo sobre distintas muestras como ocurre en 
este caso. Sin embargo, puesto que las ecuaciones estandarizadas del modelo en cada una de las áreas de estudio constituye un resultado de la investigación relevante por sí mismo, se presentan a continuación dichas ecuaciones estandarizadas.

En todas las ecuaciones del modelo estructural es posible observar que la relación más fuerte se da entre el factor 3 - Clases teóricas - y el factor 4 Conocimientos y Pensamiento metacognitivo. Por el contrario las relaciones más débiles son las que vinculan al primer factor - Estudio y trabajo en grupo - con los factores 6 y 8 - Comunicación y Organización personal. El resto de relaciones estructurales se sitúan en un plano intermedio entre las anteriores.

$$
\begin{array}{ll}
V_{1}=0,219 \cdot F_{1}+0,976 \cdot E_{1} & R^{2}=0,048 \\
V_{2}=0,385 \cdot F_{1}+0,923 \cdot E_{2} & R^{2}=0,149 \\
V_{3}=0,209 \cdot F_{1}+0,070 \cdot F_{2}+0,960 \cdot E_{3} & R^{2}=0,079 \\
V_{4}=0,233 \cdot F_{2}+0,973 \cdot E_{4} & R^{2}=0,054 \\
V_{5}=0,336 \cdot F_{2}+0,942 \cdot E_{5} & R^{2}=0,113 \\
V_{6}=0,130 \cdot F_{3}+0,992 \cdot E_{6} & R^{2}=0,017 \\
V_{7}=0,013 \cdot F_{3}+1,000 \cdot E_{7} & R^{2}=0,000 \\
V_{8}=0,325 \cdot F_{2}+0,946 \cdot E_{8} & R^{2}=0,106 \\
V_{9}=0,265 \cdot F_{1}+0,964 \cdot E_{9} & R^{2}=0,070 \\
V_{10}=0,274 \cdot F_{1}+0,962 \cdot E_{10} & R^{2}=0,075 \\
V_{11}=0,518 \cdot F_{4}+0,856 \cdot E_{11} & R^{2}=0,268 \\
V_{12}=0,581 \cdot F_{4}+0,814 \cdot E_{12} & R^{2}=0,337 \\
V_{13}=0,765 \cdot F_{4}+0,645 \cdot E_{13} & R^{2}=0,585 \\
V_{14}=0,787 \cdot F_{4}+0,617 \cdot E_{14} & R^{2}=0,619 \\
V_{15}=0,687 \cdot F_{9}+0,727 \cdot E_{15} & R^{2}=0,472 \\
V_{16}=0,667 \cdot F_{7}+0,745 \cdot E_{16} & R^{2}=0,445 \\
V_{17}=0,723 \cdot F_{9}+0,690 \cdot E_{17} & R^{2}=0,523 \\
V_{18}=0,813 \cdot F_{7}+0,583 \cdot E_{18} & R^{2}=0,661 \\
V_{19}=0,784 \cdot F_{7}+0,621 \cdot E_{19} & R^{2}=0,614 \\
V_{20}=0,933 \cdot F_{8}+0,360 \cdot E_{20} & R^{2}=0,870 \\
V_{21}=0,227 \cdot F_{8}+0,588 \cdot F_{9}+0,651 \cdot E_{21} & R^{2}=0,577 \\
V_{22}=0,435 \cdot F_{6}+0,395 \cdot F_{9}+0,640 \cdot E_{22} & R^{2}=0,590 \\
V_{23}=0,721 \cdot F_{9}+0,693 \cdot E_{23} & R^{2}=0,519 \\
V_{24}=0,625 \cdot F_{5}+0,781 \cdot E_{24} & R^{2}=0,390 \\
V_{25}=0,858 \cdot F_{5}+0,513 \cdot E_{25} & R^{2}=0,736 \\
V_{26}=0,797 \cdot F_{5}+0,603 \cdot E_{26} & R^{2}=0,636 \\
V_{27}=0,839 \cdot F_{6}+0,543 \cdot E_{27} & R^{2}=0,705 \\
V_{28}=0,773 \cdot F_{6}+0,634 \cdot E_{28} & R^{2}=0,598
\end{array}
$$

Tabla 5.74: Ecuaciones estandarizadas del modelo de medida en Educación. Invarianza métrica y estructural del modelo entre Métodos de enseñanza aprendizaje y Competencias adquiridas en la Universidad 


$\begin{array}{ll}V_{1}=0,203 \cdot F_{1}+0,979 \cdot E_{1} & R^{2}=0,041 \\ V_{2}=0,322 \cdot F_{1}+0,947 \cdot E_{2} & R^{2}=0,104 \\ V_{3}=0,219 \cdot F_{1}+0,074 \cdot F_{2}+0,956 \cdot E_{3} & R^{2}=0,087 \\ V_{4}=0,286 \cdot F_{2}+0,958 \cdot E_{4} & R^{2}=0,082 \\ V_{5}=0,281 \cdot F_{2}+0,960 \cdot E_{5} & R^{2}=0,079 \\ V_{6}=0,111 \cdot F_{3}+0,994 \cdot E_{6} & R^{2}=0,012 \\ V_{7}=0,012 \cdot F_{3}+1,000 \cdot E_{7} & R^{2}=0,000 \\ V_{8}=0,272 \cdot F_{2}+0,962 \cdot E_{8} & R^{2}=0,074 \\ V_{9}=0,232 \cdot F_{1}+0,973 \cdot E_{9} & R^{2}=0,054 \\ V_{10}=0,266 \cdot F_{1}+0,964 \cdot E_{10} & R^{2}=0,071 \\ V_{11}=0,485 \cdot F_{4}+0,875 \cdot E_{11} & R^{2}=0,235 \\ V_{12}=0,569 \cdot F_{4}+0,823 \cdot E_{12} & R^{2}=0,323 \\ V_{13}=0,753 \cdot F_{4}+0,658 \cdot E_{13} & R^{2}=0,567 \\ V_{14}=0,768 \cdot F_{4}+0,641 \cdot E_{14} & R^{2}=0,589 \\ V_{15}=0,738 \cdot F_{9}+0,675 \cdot E_{15} & R^{2}=0,545 \\ V_{16}=0,676 \cdot F_{7}+0,737 \cdot E_{16} & R^{2}=0,458 \\ V_{17}=0,709 \cdot F_{9}+0,705 \cdot E_{17} & R^{2}=0,503 \\ V_{18}=0,835 \cdot F_{7}+0,550 \cdot E_{18} & R^{2}=0,697 \\ V_{19}=0,792 \cdot F_{7}+0,611 \cdot E_{19} & R^{2}=0,627 \\ V_{20}=0,978 \cdot F_{8}+0,209 \cdot E_{20} & R^{2}=0,956 \\ V_{21}=0,252 \cdot F_{8}+0,614 \cdot F_{9}+0,613 \cdot E_{21} & R^{2}=0,624 \\ V_{22}=0,420 \cdot F_{6}+0,374 \cdot F_{9}+0,691 \cdot E_{22} & R^{2}=0,523 \\ V_{23}=0,770 \cdot F_{9}+0,639 \cdot E_{23} & R^{2}=0,592 \\ V_{24}=0,576 \cdot F_{5}+0,817 \cdot E_{24} & R^{2}=0,332 \\ V_{25}=0,832 \cdot F_{5}+0,555 \cdot E_{25} & R^{2}=0,692 \\ V_{26}=0,718 \cdot F_{5}+0,696 \cdot E_{26} & R^{2}=0,516 \\ V_{27}=0,823 \cdot F_{6}+0,568 \cdot E_{27} & R^{2}=0,678 \\ V_{28}=0,706 \cdot F_{6}+0,708 \cdot E_{28} & R^{2}=0,498\end{array}$

Tabla 5.75: Ecuaciones estandarizadas del modelo de medida en Humanidades. Invarianza métrica y estructural del modelo entre Métodos de enseñanza - aprendizaje y Competencias adquiridas en la Universidad 


$\begin{array}{ll}V_{1}=0,219 \cdot F_{1}+0,976 \cdot E_{1} & R^{2}=0,048 \\ V_{2}=0,385 \cdot F_{1}+0,923 \cdot E_{2} & R^{2}=0,149 \\ V_{3}=0,209 \cdot F_{1}+0,070 \cdot F_{2}+0,960 \cdot E_{3} & R^{2}=0,079 \\ V_{4}=0,233 \cdot F_{2}+0,973 \cdot E_{4} & R^{2}=0,054 \\ V_{5}=0,336 \cdot F_{2}+0,942 \cdot E_{5} & R^{2}=0,113 \\ V_{6}=0,130 \cdot F_{3}+0,992 \cdot E_{6} & R^{2}=0,017 \\ V_{7}=0,013 \cdot F_{3}+1,000 \cdot E_{7} & R^{2}=0,000 \\ V_{8}=0,325 \cdot F_{2}+0,946 \cdot E_{8} & R^{2}=0,106 \\ V_{9}=0,265 \cdot F_{1}+0,964 \cdot E_{9} & R^{2}=0,070 \\ V_{10}=0,274 \cdot F_{1}+0,962 \cdot E_{10} & R^{2}=0,075 \\ V_{11}=0,518 \cdot F_{4}+0,856 \cdot E_{11} & R^{2}=0,268 \\ V_{12}=0,581 \cdot F_{4}+0,814 \cdot E_{12} & R^{2}=0,337 \\ V_{13}=0,765 \cdot F_{4}+0,645 \cdot E_{13} & R^{2}=0,585 \\ V_{14}=0,787 \cdot F_{4}+0,617 \cdot E_{14} & R^{2}=0,619 \\ V_{15}=0,687 \cdot F_{9}+0,727 \cdot E_{15} & R^{2}=0,472 \\ V_{16}=0,667 \cdot F_{7}+0,745 \cdot E_{16} & R^{2}=0,445 \\ V_{17}=0,723 \cdot F_{9}+0,690 \cdot E_{17} & R^{2}=0,523 \\ V_{18}=0,813 \cdot F_{7}+0,583 \cdot E_{18} & R^{2}=0,661 \\ V_{19}=0,784 \cdot F_{7}+0,621 \cdot E_{19} & R^{2}=0,614 \\ V_{20}=0,933 \cdot F_{8}+0,360 \cdot E_{20} & R^{2}=0,870 \\ V_{21}=0,227 \cdot F_{8}+0,588 \cdot F_{9}+0,651 \cdot E_{21} & R^{2}=0,577 \\ V_{22}=0,435 \cdot F_{6}+0,395 \cdot F_{9}+0,640 \cdot E_{22} & R^{2}=0,590 \\ V_{23}=0,721 \cdot F_{9}+0,693 \cdot E_{23} & R^{2}=0,519 \\ V_{24}=0,625 \cdot F_{5}+0,781 \cdot E_{24} & R^{2}=0,390 \\ V_{25}=0,858 \cdot F_{5}+0,513 \cdot E_{25} & R^{2}=0,736 \\ V_{26}=0,797 \cdot F_{5}+0,603 \cdot E_{26} & R^{2}=0,636 \\ V_{27}=0,839 \cdot F_{6}+0,543 \cdot E_{27} & R^{2}=0,705 \\ V_{28}=0,773 \cdot F_{6}+0,634 \cdot E_{28} & R^{2}=0,598\end{array}$

Tabla 5.76: Ecuaciones estandarizadas del modelo de medida en Ciencias Sociales. Invarianza métrica y estructural del modelo entre Métodos de enseñanza - aprendizaje y Competencias adquiridas en la Universidad 


$\begin{array}{ll}V_{1}=0,221 \cdot F_{1}+0,975 \cdot E_{1} & R^{2}=0,049 \\ V_{2}=0,375 \cdot F_{1}+0,927 \cdot E_{2} & R^{2}=0,140 \\ V_{3}=0,287 \cdot F_{1}+0,097 \cdot F_{2}+0,960 \cdot E_{3} & R^{2}=0,149 \\ V_{4}=0,240 \cdot F_{2}+0,971 \cdot E_{4} & R^{2}=0,058 \\ V_{5}=0,352 \cdot F_{2}+0,936 \cdot E_{5} & R^{2}=0,124 \\ V_{6}=0,128 \cdot F_{3}+0,992 \cdot E_{6} & R^{2}=0,016 \\ V_{7}=0,014 \cdot F_{3}+1,000 \cdot E_{7} & R^{2}=0,000 \\ V_{8}=0,341 \cdot F_{2}+0,940 \cdot E_{8} & R^{2}=0,116 \\ V_{9}=0,272 \cdot F_{1}+0,962 \cdot E_{9} & R^{2}=0,074 \\ V 10=0,337 \cdot F_{1}+0,942 \cdot E_{10} & R^{2}=0,113 \\ V_{11}=0,538 \cdot F_{4}+0,843 \cdot E_{11} & R^{2}=0,289 \\ V_{12}=0,592 \cdot F_{4}+0,806 \cdot E_{12} & R^{2}=0,351 \\ V_{13}=0,756 \cdot F_{4}+0,654 \cdot E_{13} & R^{2}=0,572 \\ V_{14}=0,754 \cdot F_{4}+0,657 \cdot E_{14} & R^{2}=0,568 \\ V_{15}=0,715 \cdot F_{9}+0,699 \cdot E_{15} & R^{2}=0,512 \\ V_{16}=0,697 \cdot F_{7}+0,717 \cdot E_{16} & R^{2}=0,485 \\ V_{17}=0,759 \cdot F_{9}+0,651 \cdot E_{17} & R^{2}=0,576 \\ V_{18}=0,827 \cdot F_{7}+0,563 \cdot E_{18} & R^{2}=0,683 \\ V_{19}=0,782 \cdot F_{7}+0,623 \cdot E_{19} & R^{2}=0,612 \\ V_{20}=0,973 \cdot F_{8}+0,230 \cdot E_{20} & R^{2}=0,947 \\ V_{21}=0,253 \cdot F_{8}+0,646 \cdot F_{9}+0,558 \cdot E_{21} & R^{2}=0,688 \\ V_{22}=0,409 \cdot F_{6}+0,407 \cdot F_{9}+0,647 \cdot E_{22} & R^{2}=0,581 \\ V_{23}=0,824 \cdot F_{9}+0,567 \cdot E_{23} & R^{2}=0,678 \\ V_{24}=0,598 \cdot F_{5}+0,802 \cdot E_{24} & R^{2}=0,357 \\ V_{25}=0,863 \cdot F_{5}+0,506 \cdot E_{25} & R^{2}=0,744 \\ V_{26}=0,799 \cdot F_{5}+0,601 \cdot E_{26} & R^{2}=0,638 \\ V_{27}=0,783 \cdot F_{6}+0,622 \cdot E_{27} & R^{2}=0,613 \\ V_{28}=0,744 \cdot F_{6}+0,669 \cdot E_{28} & R^{2}=0,553\end{array}$

Tabla 5.77: Ecuaciones estandarizadas del modelo de medida en Economía y empresa. Invarianza métrica y estructural del modelo entre Métodos de enseñanza - aprendizaje y Competencias adquiridas en la Universidad 


$\begin{array}{ll}V_{1}=0,211 \cdot F_{1}+0,977 \cdot E_{1} & R^{2}=0,045 \\ V_{2}=0,422 \cdot F_{1}+0,907 \cdot E_{2} & R^{2}=0,178 \\ V_{3}=0,333 \cdot F_{1}+0,112 \cdot F_{2}+0,894 \cdot E_{3} & R^{2}=0,201 \\ V_{4}=0,330 \cdot F_{2}+0,944 \cdot E_{4} & R^{2}=0,109 \\ V_{5}=0,351 \cdot F_{2}+0,936 \cdot E_{5} & R^{2}=0,124 \\ V_{6}=0,111 \cdot F_{3}+0,994 \cdot E_{6} & R^{2}=0,012 \\ V_{7}=0,013 \cdot F_{3}+1,000 \cdot E_{7} & R^{2}=0,000 \\ V_{8}=0,373 \cdot F_{2}+0,928 \cdot E_{8} & R^{2}=0,139 \\ V_{9}=0,252 \cdot F_{1}+0,968 \cdot E_{9} & R^{2}=0,064 \\ V_{10}=0,292 \cdot F_{1}+0,956 \cdot E_{10} & R^{2}=0,086 \\ V_{11}=0,494 \cdot F_{4}+0,870 \cdot E_{11} & R^{2}=0,244 \\ V_{12}=0,575 \cdot F_{4}+0,818 \cdot E_{12} & R^{2}=0,330 \\ V_{13}=0,730 \cdot F_{4}+0,683 \cdot E_{13} & R^{2}=0,533 \\ V_{14}=0,716 \cdot F_{4}+0,698 \cdot E_{14} & R^{2}=0,513 \\ V_{15}=0,758 \cdot F_{9}+0,653 \cdot E_{15} & R^{2}=0,574 \\ V_{16}=0,704 \cdot F_{7}+0,710 \cdot E_{16} & R^{2}=0,496 \\ V_{17}=0,779 \cdot F_{9}+0,627 \cdot E_{17} & R^{2}=0,607 \\ V_{18}=0,826 \cdot F_{7}+0,564 \cdot E_{18} & R^{2}=0,682 \\ V_{19}=0,799 \cdot F_{7}+0,601 \cdot E_{19} & R^{2}=0,639 \\ V_{20}=1,000 \cdot F_{8}+0,000 \cdot E_{20} & R^{2}=1,000 \\ V_{21}=0,263 \cdot F_{8}+0,653 \cdot F_{9}+0,556 \cdot E_{21} & R^{2}=0,691 \\ V_{22}=0,425 \cdot F_{6}+0,392 \cdot F_{9}+0,674 \cdot E_{22} & R^{2}=0,546 \\ V_{23}=0,788 \cdot F_{9}+0,616 \cdot E_{23} & R^{2}=0,621 \\ V_{24}=0,610 \cdot F_{5}+0,792 \cdot E_{24} & R^{2}=0,372 \\ V_{25}=0,794 \cdot F_{5}+0,608 \cdot E_{25} & R^{2}=0,630 \\ V_{26}=0,747 \cdot F_{5}+0,665 \cdot E_{26} & R^{2}=0,558 \\ V_{27}=0,838 \cdot F_{6}+0,545 \cdot E_{27} & R^{2}=0,703 \\ V_{28}=0,792 \cdot F_{6}+0,610 \cdot E_{28} & R^{2}=0,628\end{array}$

Tabla 5.78: Ecuaciones estandarizadas del modelo de medida en Derecho. Invarianza métrica y estructural del modelo entre Métodos de enseñanza aprendizaje y Competencias adquiridas en la Universidad 


$\begin{array}{ll}V_{1}=0,216 \cdot F_{1}+0,976 \cdot E_{1} & R^{2}=0,047 \\ V_{2}=0,369 \cdot F_{1}+0,929 \cdot E_{2} & R^{2}=0,136 \\ V_{3}=0,249 \cdot F_{1}+0,084 \cdot F_{2}+0,942 \cdot E_{3} & R^{2}=0,112 \\ V_{4}=0,253 \cdot F_{2}+0,967 \cdot E_{4} & R^{2}=0,064 \\ V_{5}=0,333 \cdot F_{2}+0,943 \cdot E_{5} & R^{2}=0,111 \\ V_{6}=0,129 \cdot F_{3}+0,992 \cdot E_{6} & R^{2}=0,017 \\ V_{7}=0,013 \cdot F_{3}+1,000 \cdot E_{7} & R^{2}=0,000 \\ V_{8}=0,309 \cdot F_{2}+0,951 \cdot E_{8} & R^{2}=0,096 \\ V_{9}=0,259 \cdot F_{1}+0,966 \cdot E_{9} & R^{2}=0,067 \\ V_{10}=0,348 \cdot F_{1}+0,937 \cdot E_{10} & R^{2}=0,121 \\ V_{11}=0,540 \cdot F_{4}+0,842 \cdot E_{11} & R^{2}=0,291 \\ V_{12}=0,585 \cdot F_{4}+0,811 \cdot E_{12} & R^{2}=0,342 \\ V_{13}=0,758 \cdot F_{4}+0,652 \cdot E_{13} & R^{2}=0,575 \\ V_{14}=0,763 \cdot F_{4}+0,646 \cdot E_{14} & R^{2}=0,583 \\ V_{15}=0,759 \cdot F_{9}+0,651 \cdot E_{15} & R^{2}=0,576 \\ V_{16}=0,655 \cdot F_{7}+0,756 \cdot E_{16} & R^{2}=0,429 \\ V_{17}=0,770 \cdot F_{9}+0,638 \cdot E_{17} & R^{2}=0,593 \\ V_{18}=0,823 \cdot F_{7}+0,568 \cdot E_{18} & R^{2}=0,678 \\ V_{19}=0,796 \cdot F_{7}+0,605 \cdot E_{19} & R^{2}=0,634 \\ V_{20}=1,000 \cdot F_{8}+0,000 \cdot E_{20} & R^{2}=1,000 \\ V_{21}=0,249 \cdot F_{8}+0,639 \cdot F_{9}+0,588 \cdot E_{21} & R^{2}=0,655 \\ V_{22}=0,397 \cdot F_{6}+0,415 \cdot F_{9}+0,660 \cdot E_{22} & R^{2}=0,564 \\ V_{23}=0,815 \cdot F_{9}+0,579 \cdot E_{23} & R^{2}=0,665 \\ V_{24}=0,570 \cdot F_{5}+0,822 \cdot E_{24} & R^{2}=0,325 \\ V_{25}=0,833 \cdot F_{5}+0,554 \cdot E_{25} & R^{2}=0,693 \\ V_{26}=0,779 \cdot F_{5}+0,628 \cdot E_{26} & R^{2}=0,606 \\ V_{27}=0,786 \cdot F_{6}+0,618 \cdot E_{27} & R^{2}=0,619 \\ V_{28}=0,729 \cdot F_{6}+0,684 \cdot E_{28} & R^{2}=0,532\end{array}$

Tabla 5.79: Ecuaciones estandarizadas del modelo de medida en Técnicas. Invarianza métrica y estructural del modelo entre Métodos de enseñanza aprendizaje y Competencias adquiridas en la Universidad 


$\begin{array}{ll}V_{1}=0,211 \cdot F_{1}+0,977 \cdot E_{1} & R^{2}=0,045 \\ V_{2}=0,345 \cdot F_{1}+0,939 \cdot E_{2} & R^{2}=0,119 \\ V_{3}=0,246 \cdot F_{1}+0,083 \cdot F_{2}+0,944 \cdot E_{3} & R^{2}=0,110 \\ V_{4}=0,188 \cdot F_{2}+0,982 \cdot E_{4} & R^{2}=0,035 \\ V_{5}=0,311 \cdot F_{2}+0,950 \cdot E_{5} & R^{2}=0,097 \\ V_{6}=0,113 \cdot F_{3}+0,994 \cdot E_{6} & R^{2}=0,013 \\ V_{7}=0,014 \cdot F_{3}+1,000 \cdot E_{7} & R^{2}=0,000 \\ V_{8}=0,308 \cdot F_{2}+0,951 \cdot E_{8} & R^{2}=0,095 \\ V_{9}=0,247 \cdot F_{1}+0,969 \cdot E_{9} & R^{2}=0,061 \\ V_{10}=0,322 \cdot F_{1}+0,947 \cdot E_{10} & R^{2}=0,104 \\ V_{11}=0,532 \cdot F_{4}+0,846 \cdot E_{11} & R^{2}=0,284 \\ V_{12}=0,565 \cdot F_{4}+0,825 \cdot E_{12} & R^{2}=0,320 \\ V_{13}=0,758 \cdot F_{4}+0,652 \cdot E_{13} & R^{2}=0,575 \\ V_{14}=0,754 \cdot F_{4}+0,657 \cdot E_{14} & R^{2}=0,568 \\ V_{15}=0,700 \cdot F_{9}+0,715 \cdot E_{15} & R^{2}=0,489 \\ V_{16}=0,638 \cdot F_{7}+0,770 \cdot E_{16} & R^{2}=0,407 \\ V_{17}=0,755 \cdot F_{9}+0,655 \cdot E_{17} & R^{2}=0,571 \\ V_{18}=0,830 \cdot F_{7}+0,558 \cdot E_{18} & R^{2}=0,689 \\ V_{19}=0,765 \cdot F_{7}+0,644 \cdot E_{19} & R^{2}=0,585 \\ V_{20}=0,901 \cdot F_{8}+0,435 \cdot E_{20} & R^{2}=0,811 \\ V_{21}=0,240 \cdot F_{8}+0,629 \cdot F_{9}+0,581 \cdot E_{21} & R^{2}=0,662 \\ V_{22}=0,446 \cdot F_{6}+0,390 \cdot F_{9}+0,636 \cdot E_{22} & R^{2}=0,595 \\ V_{23}=0,795 \cdot F_{9}+0,606 \cdot E_{23} & R^{2}=0,633 \\ V_{24}=0,633 \cdot F_{5}+0,774 \cdot E_{24} & R^{2}=0,401 \\ V_{25}=0,880 \cdot F_{5}+0,474 \cdot E_{25} & R^{2}=0,775 \\ V_{26}=0,810 \cdot F_{5}+0,587 \cdot E_{26} & R^{2}=0,655 \\ V_{27}=0,840 \cdot F_{6}+0,543 \cdot E_{27} & R^{2}=0,705 \\ V_{28}=0,784 \cdot F_{6}+0,621 \cdot E_{28} & R^{2}=0,615\end{array}$

Tabla 5.80: Ecuaciones estandarizadas del modelo de medida en Salud. Invarianza métrica y estructural del modelo entre Métodos de enseñanza aprendizaje y Competencias adquiridas en la Universidad 


$$
\begin{aligned}
& F_{4}=0,977 \cdot F_{3}+0,212 \cdot D_{4} R^{2}=0,955 \\
& F_{5}=0,886 \cdot F_{2}+0,463 \cdot D_{5} R^{2}=0,786 \\
& F_{6}=0,811 \cdot F_{1}+0,584 \cdot D_{6} R^{2}=0,658 \\
& F_{7}=0,902 \cdot F_{3}+0,433 \cdot D_{7} R^{2}=0,813 \\
& F_{8}=0,765 \cdot F_{1}+0,644 \cdot D_{8} R^{2}=0,585 \\
& F_{9}=0,879 \cdot F_{1}+0,476 \cdot D_{9} R^{2}=0,773
\end{aligned}
$$

Tabla 5.81: Ecuaciones estandarizadas del modelo estructural en Educación. Invarianza métrica y estructural del modelo entre Métodos de enseñanza aprendizaje y Competencias adquiridas en la Universidad

$$
\begin{aligned}
& F_{4}=0,924 \cdot F_{3}+0,382 \cdot D_{4} R^{2}=0,854 \\
& F_{5}=0,841 \cdot F_{2}+0,541 \cdot D_{5} R^{2}=0,708 \\
& F_{6}=0,770 \cdot F_{1}+0,638 \cdot D_{6} R^{2}=0,593 \\
& F_{7}=0,854 \cdot F_{3}+0,521 \cdot D_{7} R^{2}=0,729 \\
& F_{8}=0,699 \cdot F_{1}+0,715 \cdot D_{8} R^{2}=0,488 \\
& F_{9}=0,851 \cdot F_{1}+0,525 \cdot D_{9} R^{2}=0,725
\end{aligned}
$$

Tabla 5.82: Ecuaciones estandarizadas del modelo estructural en Humanidades. Invarianza métrica y estructural del modelo entre Métodos de enseñanza - aprendizaje y Competencias adquiridas en la Universidad

$$
\begin{aligned}
& F_{4}=0,977 \cdot F_{3}+0,212 \cdot D_{4} R^{2}=0,955 \\
& F_{5}=0,886 \cdot F_{2}+0,463 \cdot D_{5} R^{2}=0,786 \\
& F_{6}=0,811 \cdot F_{1}+0,584 \cdot D_{6} R^{2}=0,658 \\
& F_{7}=0,902 \cdot F_{3}+0,433 \cdot D_{7} R^{2}=0,813 \\
& F_{8}=0,765 \cdot F_{1}+0,644 \cdot D_{8} R^{2}=0,585 \\
& F_{9}=0,879 \cdot F_{1}+0,476 \cdot D_{9} R^{2}=0,773
\end{aligned}
$$

Tabla 5.83: Ecuaciones estandarizadas del modelo estructural en Ciencias Sociales. Invarianza métrica y estructural del modelo entre Métodos de enseñanza - aprendizaje y Competencias adquiridas en la Universidad 


$$
\begin{aligned}
& F_{4}=0,999 \cdot F_{3}+0,052 \cdot D_{4} R^{2}=0,997 \\
& F_{5}=0,879 \cdot F_{2}+0,477 \cdot D_{5} R^{2}=0,772 \\
& F_{6}=0,868 \cdot F_{1}+0,496 \cdot D_{6} R^{2}=0,754 \\
& F_{7}=0,892 \cdot F_{3}+0,452 \cdot D_{7} R^{2}=0,796 \\
& F_{8}=0,737 \cdot F_{1}+0,676 \cdot D_{8} R^{2}=0,544 \\
& F_{9}=0,859 \cdot F_{1}+0,512 \cdot D_{9} R^{2}=0,738
\end{aligned}
$$

Tabla 5.84: Ecuaciones estandarizadas del modelo estructural en Economía y empresa. Invarianza métrica y estructural del modelo entre Métodos de enseñanza - aprendizaje y Competencias adquiridas en la Universidad

$$
\begin{aligned}
& F_{4}=1,000 \cdot F_{3}+0,000 \cdot D_{4} R^{2}=1,000 \\
& F_{5}=0,888 \cdot F_{2}+0,460 \cdot D_{5} R^{2}=0,788 \\
& F_{6}=0,773 \cdot F_{1}+0,634 \cdot D_{6} R^{2}=0,598 \\
& F_{7}=0,828 \cdot F_{3}+0,561 \cdot D_{7} R^{2}=0,685 \\
& F_{8}=0,688 \cdot F_{1}+0,726 \cdot D_{8} R^{2}=0,474 \\
& F_{9}=0,824 \cdot F_{1}+0,567 \cdot D_{9} R^{2}=0,678
\end{aligned}
$$

Tabla 5.85: Ecuaciones estandarizadas del modelo estructural en Derecho. Invarianza métrica y estructural del modelo entre Métodos de enseñanza aprendizaje y Competencias adquiridas en la Universidad

$$
\begin{aligned}
& F_{4}=0,983 \cdot F_{3}+0,181 \cdot D_{4} R^{2}=0,967 \\
& F_{5}=0,851 \cdot F_{2}+0,525 \cdot D_{5} R^{2}=0,725 \\
& F_{6}=0,870 \cdot F_{1}+0,493 \cdot D_{6} R^{2}=0,757 \\
& F_{7}=0,862 \cdot F_{3}+0,507 \cdot D_{7} R^{2}=0,743 \\
& F_{8}=0,705 \cdot F_{1}+0,709 \cdot D_{8} R^{2}=0,497 \\
& F_{9}=0,818 \cdot F_{1}+0,576 \cdot D_{9} R^{2}=0,669
\end{aligned}
$$

Tabla 5.86: Ecuaciones estandarizadas del modelo estructural en Técnicas. Invarianza métrica y estructural del modelo entre Métodos de enseñanza aprendizaje y Competencias adquiridas en la Universidad 


$$
\begin{aligned}
& F_{4}=1,000 \cdot F_{3}+0,000 \cdot D_{4} R^{2}=1,000 \\
& F_{5}=0,852 \cdot F_{2}+0,523 \cdot D_{5} R^{2}=0,726 \\
& F_{6}=0,790 \cdot F_{1}+0,613 \cdot D_{6} R^{2}=0,625 \\
& F_{7}=0,914 \cdot F_{3}+0,405 \cdot D_{7} R^{2}=0,836 \\
& F_{8}=0,781 \cdot F_{1}+0,624 \cdot D_{8} R^{2}=0,611 \\
& F_{9}=0,887 \cdot F_{1}+0,461 \cdot D_{9} R^{2}=0,787
\end{aligned}
$$

Tabla 5.87: Ecuaciones estandarizadas del modelo estructural en Salud. Invarianza métrica y estructural del modelo entre Métodos de enseñanza aprendizaje y Competencias adquiridas en la Universidad

\section{Generación de un nuevo modelo causal para el área de Ciencias}

Los análisis precedentes han mostrado que el modelo propuesto de forma general para todas las áreas de estudio, no es aplicable al área de Ciencias. Hasta ahora los análisis se habían planteado con las estrategias de modelización confirmatoria o comparación de modelos rivales. Sin embargo, para abordar la problemática de la modelización causal en el área de ciencias, se utiliza la estrategia de generación de nuevos modelos. Esta estrategia tiene un carácter más exploratorio que confirmatorio, y su aplicación únicamente resulta adecuada si la investigación se orienta conscientemente hacia este enfoque. Así, se procederá a identificar en primer lugar las relaciones presentes en el modelo general que impiden la convergencia en la submuestra de Ciencias, y a continuación se determinarán las posibles modificaciones a dicho modelo de partida que mejoren el ajuste del modelo a los datos y resulten coherentes con la teoría.

Para identificar las relaciones responsables de la falta de convergencia del modelo en el área de ciencias, se han planteado seis nuevos modelos. En cada uno de ellos se ha eliminado una relación estructural entre factores exógenos y endógenos. Así, los tres primeros modelos consideran la eliminación de las tres relaciones estructurales entre el primer factor - Estudio y trabajo en grupo - y los factores 6, 8 y 9 - Comunicación, Cooperación y Liderazgo. Los modelos 4 y 5 excluyen las relaciones entre el segundo factor - Prácticas y clases prácticas - y los factores 5 y 7 - Innovación y Organización personal - y finalmente el sexto modelo descarta la relación entre los factores 3 y 4 Clases teóricas y Conocimientos y Pensamiento metacognitivo.

Entre todos los modelos planteados únicamente converge el quinto modelo que excluye la relación entre los factores 2 y 7 - Prácticas y clases prácticas y Organización personal. Sin embargo, el ajuste obtenido por este modelo se considera susceptible de ser mejorado. Las modificaciones propuestas por el Contraste de Lagrange resultan tener un efecto desfavorable ya que nuevamente provocan situaciones de falta de convergencia del mo- 
delo. Asimismo ninguna de las modificaciones propuestas por el Contraste de Wald causa mejora alguna en el ajuste. Por todo ello, se toma la determinación de analizar el efecto de introducir nuevas relaciones estructurales de forma exploratoria. Entre todas las relaciones planteadas, aquellas que resultan tener un efecto más significativo son las que asociaban el octavo factor - Organización personal - con los factores 1 y 3 - Estudio y trabajo en grupo y Clases teóricas. El hecho de que sean estas relaciones las que mayor significación hayan obtenido en la mejora del ajuste resulta coherente si se considera que la única relación estructural asociada a dicho factor había sido previamente eliminada, quedando aislado este factor del modelo causal.

De este modo, se plantean cuatro nuevos modelos, el primero de ellos simplemente excluye la relación mencionada anteriormente entre los factores 2 y 7 - Prácticas y clases prácticas y Organización personal. Los tres modelos restantes consideran la introducción de nuevas relaciones entre este factor de Organización personal y los factores 1 - Estudio y trabajo en grupo -, 3 - Clases teóricas - o ambos, respectivamente.

Como se observa en la Tabla 5.88 el valor más elevado del estadístico $\chi^{2}$ de Satorra - Bentler corresponde al primer modelo, en el que no se ha introducido ninguna relación estructural adicional. La mejora obtenida en los modelos 2 y 3 , en los que el factor de Organización personal se vincula al primer y tercer factor respectivamente, es muy similar entre ambos. En cualquier caso el mejor ajuste lo alcanza el cuarto modelo, que considera la introducción de ambas relaciones de forma simultánea. La diferencia corregida de los estadísticos $\chi^{2}$ de Satorra - Bentler entre el segundo modelo, cuyo ajuste resultó ser ligeramente mejor que el tercero, y el cuarto es significativa, y lo mismo ocurre con el tercer y cuarto modelo.

\begin{tabular}{|l|c|c|c|c|}
\hline & Modelo 1 & Modelo 2 & Modelo 3 & Modelo 4 \\
\hline$\chi_{\text {Ind }}^{2}$ & 5267.5 & 5267.5 & 5267.5 & 5267.5 \\
gl $l_{\text {Ind }}$ & 378 & 378 & 378 & 378 \\
$\chi^{2}$ & 1820.9 & 1539.0 & 1568.7 & 1493.8 \\
$\chi_{S-B}^{2}$ & 1644.5 & 1374.5 & 1401.4 & 1336.2 \\
$g l$ & 336 & 336 & 336 & 335 \\
Prob. & 0.000 & 0.000 & 0.000 & 0.000 \\
\hline
\end{tabular}

Tabla 5.88: Contraste $\chi^{2}$ para los 4 modelos planteados inicialmente. Modelos estructurales entre Métodos de enseñanza - aprendizaje y Competencias adquiridas en la Universidad para Ciencias

Los resultados de estos modelos indican que la disminución del estadísti- 
co $\chi^{2}$ es significativa al pasar del Modelo 2 al Modelo 4:

$k_{2}=\frac{\chi_{2}^{2}}{\chi_{S-B, 2}^{2}}=1,120$

$k_{4}=\frac{\chi_{4}^{2}}{\chi_{S-B, 4}^{2}}=1,118$

$D_{24}=\chi_{1}^{2}-\chi_{2}^{2}=45,2$

$d_{24}=1$

$k_{24}=\frac{d_{2} k_{2}-d_{4} k_{4}}{d_{24}}=1,79$

$\bar{D}_{24}=\frac{D_{24}}{k_{24}}=25,25>\chi_{\alpha=0,05 ; g l=1}^{2}=3,84$

Asimismo al pasar del Modelo 3 al 4 la disminución del estadístico $\chi^{2}$ también resulta significativa:

$k_{3}=\frac{\chi_{3}^{2}}{\chi_{S-B, 3}^{2}}=1,120$

$D_{34}=\chi_{3}^{2}-\chi_{4}^{2}=79,4$

$d_{34}=1$

$k_{34}=\frac{d_{3} k_{3}-d_{4} k_{4}}{d_{34}}=1,79$

$\bar{D}_{34}=\frac{D_{34}}{k_{34}}=41,89>\chi_{\alpha=0,05 ; g l=1}^{2}=3,84$

Las mismas conclusiones que se han extraído del análisis de los estadísti$\cos \chi^{2}$ pueden obtenerse mediante el estudio de los índices de ajuste. Mientras que al primer modelo le corresponden los índices más bajos, los resultados alcanzados por los modelos 2 y 3 son muy similares, y en ambos casos no superan los obtenidos por el cuarto modelo.

\begin{tabular}{|l|c|c|c|c|}
\hline & Modelo 1 & Modelo 2 & Modelo 3 & Modelo 4 \\
\hline AIC Ind & 4511.5 & 4511.5 & 4511.5 & 4511.5 \\
AIC & 972.5 & 702.5 & 729.4 & 666.2 \\
CAIC Ind & 2538.9 & 2538.9 & 2538.9 & 2538.9 \\
CAIC & -781.0 & -1050.9 & -1024.1 & -1082.0 \\
NFI & 0.688 & 0.739 & 0.734 & 0.746 \\
NNFI & 0.699 & 0.761 & 0.755 & 0.769 \\
CFI & 0.732 & 0.788 & 0.782 & 0.795 \\
IFI & 0.735 & 0.789 & 0.784 & 0.797 \\
MFI & 0.272 & 0.355 & 0.346 & 0.369 \\
RMSEA & 0.088 & 0.079 & 0.080 & 0.077 \\
Lim Inf 90\% & 0.084 & 0.074 & 0.075 & 0.073 \\
Lim Sup 90\% & 0.092 & 0.083 & 0.084 & 0.082 \\
\hline
\end{tabular}

Tabla 5.89: Indices de ajuste para los 4 modelos planteados inicialmente. Modelos estructurales entre Métodos de enseñanza - aprendizaje y Competencias adquiridas en la Universidad para Ciencias 
Nuevamente se analizaron las modificaciones propuestas por el Contraste de Lagrange y ninguna de ellas resultó tener un efecto significativo en la mejora del ajuste. Por tanto, el cuarto modelo es alcanza el mejor ajuste a los datos de la submuestra de Ciencias y se selecciona definitivamente para su aplicación en esta área.

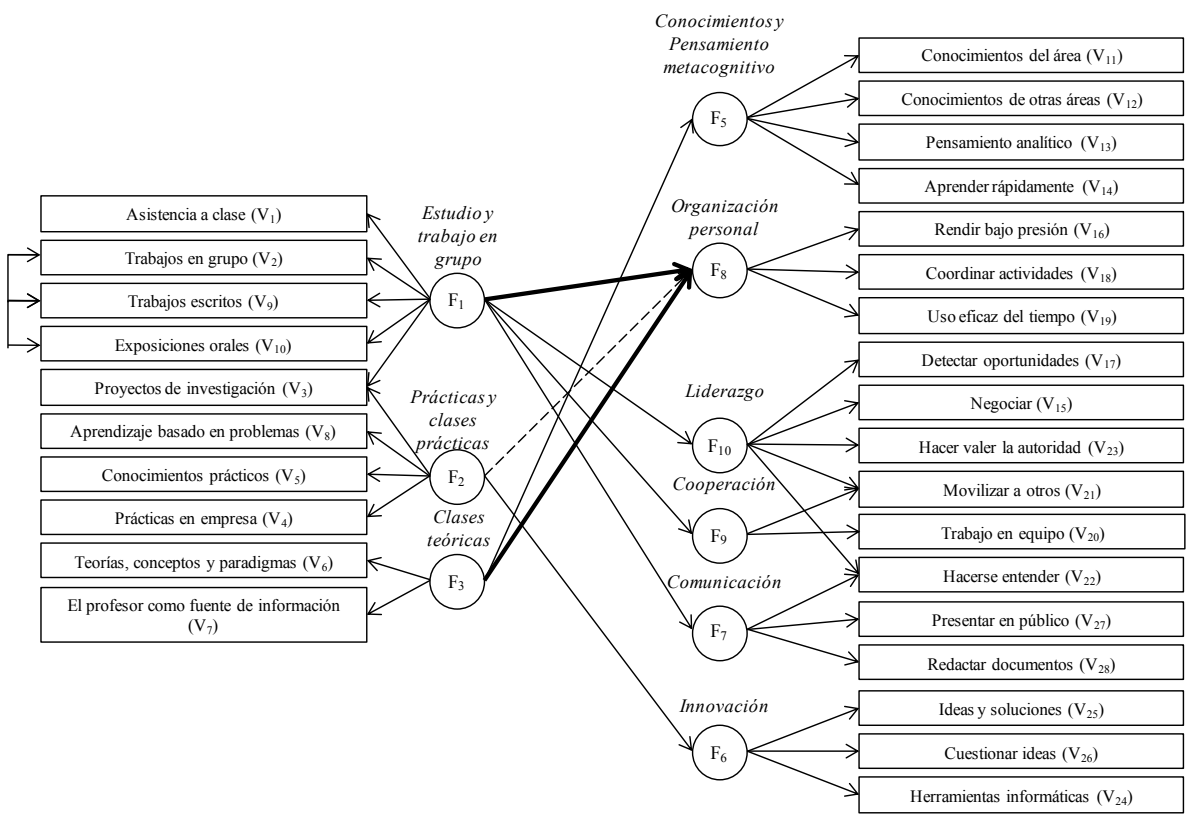

Figura 5.12: Adquisición de competencias mediante métodos de enseñanza - aprendizaje. Modelo estructural para el área de Ciencias

Este modelo específico para el área de ciencias consta de 335 grados de libertad según la aplicación de la Regla t:

$$
g l=\frac{1}{2} \cdot 28 \cdot 29-71=335
$$

Al igual que ocurría en la validación del modelo global, se fijó a cero el valor del error residual del ítem 20 - Trabajar en equipo - y se detectó cierta colinealidad entre los factores 2 y 5 - Prácticas y clases prácticas e Innovación. Como resultado de la estimación de este modelo se obtuvieron para los promedios de la matriz residual de varianzas - covarianzas no demasiado superiores a los obtenidos en el modelo causal para las áreas de estudio restantes, que tomaba valores de 0.0781 y 0.0838 para los promedios absolutos incluyendo y excluyendo los términos en la diagonal, y 0.0585 y 0.0630 para 
los mismos valores estandarizados.

Average absolute residual $=0.0846$

Average off-diagonal absolute residual $=0.0906$

Average absolute standardized residual $=0.0690$

Average off-diagonal absolute standardized residual $=0.0739$

Mientras que los coeficientes de fiabilidad Alfa de Cronbach y Rho resultaron aceptablemente elevados, alcanzando 0.890 en el primer caso y 0.909 en el segundo. Respecto a los porcentajes de fiabilidad compuesta y varianza extraída obtuvieron valores aceptables en los factores endógenos sobre Competencias adquiridas en la Universidad mientras que los relativos a Métodos de enseñanza - aprendizaje no alcanzaron los valores mínimos recomendables, al igual que en la aplicación del modelo general a las restantes áreas de estudio.

\begin{tabular}{|c|c|c|}
\hline & Fiabilidad compuesta & Varianza extraída \\
\hline$F_{1}$ & $43.9 \%$ & $14.3 \%$ \\
$F_{2}$ & $14.1 \%$ & $6.4 \%$ \\
$F_{3}$ & $2.2 \%$ & $2.1 \%$ \\
$F_{4}$ & $69.4 \%$ & $38.6 \%$ \\
$F_{5}$ & $81.2 \%$ & $59.4 \%$ \\
$F_{6}$ & $67.9 \%$ & $43.9 \%$ \\
$F_{7}$ & $77.7 \%$ & $54.0 \%$ \\
$F_{8}$ & $60.5 \%$ & $52.2 \%$ \\
$F_{9}$ & $79.2 \%$ & $44.3 \%$ \\
\hline
\end{tabular}

Tabla 5.90: Fiabilidad compuesta y varianza extraída. Modelo estructural entre Métodos de enseñanza - aprendizaje y Competencias adquiridas en la Universidad para Ciencias

De igual forma que las ecuaciones no estandarizadas del modelo general aplicado a las áreas de estudio restantes, los ítems con estimaciones más elevadas del error de medida resultan ser todos los correspondientes a los factores sobre Métodos de enseñanza - aprendizaje así como los ítems 11, 12 y 24 - Conocimientos del área, Conocimientos de otras áreas y Herramientas informáticas. Asimismo, los ítems 4, 5 y 8 - Prácticas en empresa, Conocimientos prácticos y Aprendizaje basado en problemas - que anteriormente obtenían estimaciones no significativas de los coeficientes de regresión, tampoco lo hacen en este modelo. En términos generales no se han producido grandes cambios en las estimaciones de los parámetros del modelo de medida, ya que éste no se ha modificado respecto al original. 


$\begin{array}{ll}V_{1}=1,000 \cdot F_{1}+1,000 \cdot E_{1} & E_{1}=1,337^{*} \\ V_{2}=1,975^{*} \cdot F_{1}+1,000 \cdot E_{2} & E_{2}=0,956^{*} \\ V_{3}=1,200^{*} \cdot F_{1}+1,000 \cdot F_{2}+1,000 \cdot E_{3} & E_{3}=0,993^{*} \\ V_{4}=40,4 \cdot F_{2}+1,000 \cdot E_{4} & E_{4}=0,770^{*} \\ V_{5}=162,5 \cdot F_{2}+1,000 \cdot E_{5} & E_{5}=1,001^{*} \\ V_{6}=1,000 \cdot F_{3}+1,000 \cdot E_{6} & E_{6}=0,712^{*} \\ V_{7}=0,110 \cdot F_{3}+1,000 \cdot E_{7} & E_{7}=0,759^{*} \\ V_{8}=211,6 \cdot F_{2}+1,000 \cdot E_{8} & E_{8}=0,870^{*} \\ V_{9}=1,782^{*} \cdot F_{1}+1,000 \cdot E_{9} & E_{9}=0,785^{*} \\ V_{10}=1,507^{*} \cdot F_{1}+1,000 \cdot E_{10} & E_{10}=0,750^{*} \\ V_{11}=1,000 \cdot F_{4}+1,000 \cdot E_{11} & E_{11}=1,241^{*} \\ V_{12}=0,868^{*} \cdot F_{4}+1,000 \cdot E_{12} & E_{12}=1,101 \\ V_{13}=1,916^{*} \cdot F_{4}+1,000 \cdot E_{13} & E_{13}=0,415^{*} \\ V_{14}=1,629^{*} \cdot F_{4}+1,000 \cdot E_{14} & E_{14}=0,617^{*} \\ V_{15}=0,899^{*} \cdot F_{9}+1,000 \cdot E_{15} & E_{15}=0,674^{*} \\ V_{16}=1,000 \cdot F_{7}+1,000 \cdot E_{16} & E_{16}=0,996^{*} \\ V_{17}=1,000 \cdot F_{9}+1,000 \cdot E_{17} & E_{17}=0,631^{*} \\ V_{18}=1,090^{*} \cdot F_{7}+1,000 \cdot E_{18} & E_{18}=0,665^{*} \\ V_{19}=1,221^{*} \cdot F_{7}+1,000 \cdot E_{19} & E_{19}=0,507^{*} \\ V_{20}=5,064^{*} \cdot F_{8}+1,000 \cdot E_{20} & E_{20}=0,000^{*} \\ V_{21}=1,000 \cdot F_{8}+0,990^{*} \cdot F_{9}+1,000 \cdot E_{21} & E_{21}=0,422^{*} \\ V_{22}=1,000 \cdot F_{6}+0,592^{*} \cdot F_{9}+1,000 \cdot E_{22} & E_{22}=0,776^{*} \\ V_{23}=1,165^{*} \cdot F_{9}+1,000 \cdot E_{23} & E_{23}=0,459^{*} \\ V_{24}=0,790^{*} \cdot F_{5}+1,000 \cdot E_{24} & E_{24}=0,964^{*} \\ V_{25}=1,000 \cdot F_{5}+1,000 \cdot E_{25} & E_{25}=0,310^{*} \\ V_{26}=0,820^{*} \cdot F_{5}+1,000 \cdot E_{26} & E_{26}=0,771^{*} \\ V_{27}=2,392^{*} \cdot F_{6}+1,000 \cdot E_{27} & E_{27}=0,447^{*} \\ V_{28}=2,126^{*} \cdot F_{6}+1,000 \cdot E_{28} & E_{28}=0,666^{*}\end{array}$

Tabla 5.91: Ecuaciones no estandarizadas del modelo de medida. Modelo estructural entre Métodos de enseñanza - aprendizaje y Competencias adquiridas en la Universidad para Ciencias

Por el contrario sí se observan modificaciones las ecuaciones referidas al modelo estructural. El coeficiente de regresión entre el segundo factor Prácticas y clases prácticas - y el quinto - Innovación - en este caso continua siendo no significativo. Sin embargo el anterior coeficiente entre este segundo factor y el relativo a la Organización personal, que anteriormente también era no significativo ahora se ha sustituido por dos nuevas relaciones con los factores 1 y 3 - Estudio y trabajo en grupo y Clases teóricas. Los coeficientes de regresión de dichas relaciones sí resultan significativos y con valores considerablemente elevados.

Las estimaciones de los parámetros estructurales son muy similares a los correspondientes al modelo general e incluso la varianza del factor 2 Prácticas y clases prácticas - continúa siendo nula. Al mismo tiempo el ter- 


$$
\begin{array}{lrl}
F_{4}=2,646^{*} \cdot F_{3}+1,000 \cdot D_{4} & D_{4}=0,000 \\
F_{5}=513,2 \cdot F_{2}+1,000 \cdot D_{5} & D_{5}=0,296^{*} \\
F_{6}=1,194^{*} \cdot F_{1}+1,000 \cdot D_{6} & D_{6}=0,081^{*} \\
F_{7}=2,100^{*} \cdot F_{1}+1,699^{*} \cdot F_{3}+1,000 \cdot D_{7} D_{7}=0,171^{*} \\
F_{8}=0,702^{*} \cdot F_{1}+1,000 \cdot D_{8} & D_{8}=0,035^{*} \\
F_{9}=2,321^{*} \cdot F_{1}+1,000 \cdot D_{9} & D_{9}=0,225^{*}
\end{array}
$$

Tabla 5.92: Ecuaciones no estandarizadas del modelo estructural. Modelo estructural entre Métodos de enseñanza - aprendizaje y Competencias adquiridas en la Universidad para Ciencias

cer factor relativo a las Clases teóricas es prácticamente independiente del resto.

\begin{tabular}{|c|c|c|c|}
\hline & $F_{1}$ & $F_{2}$ & $F_{3}$ \\
\hline$F_{1}$ & $0.107^{*}$ & & \\
$F_{2}$ & 0.202 & 0.000 & \\
$F_{3}$ & 0.011 & 0.004 & $0.009^{*}$ \\
\hline$E_{9}-E_{2}$ & \multicolumn{3}{|c|}{$0.412^{*}$} \\
\hline
\end{tabular}

Tabla 5.93: Matriz de varianzas-covarianzas entre factores latentes. Modelo estructural entre Métodos de enseñanza - aprendizaje y Competencias adquiridas en la Universidad para Ciencias

\begin{tabular}{|c|c|c|c|}
\hline & $F_{1}$ & $F_{2}$ & $F_{3}$ \\
\hline$F_{1}$ & 1.000 & & \\
$F_{2}$ & 0.560 & 1.000 & \\
$F_{3}$ & 0.104 & 0.105 & 1.000 \\
\hline$E_{9}-E_{2}$ & \multicolumn{3}{|c|}{0.395} \\
\hline
\end{tabular}

Tabla 5.94: Matriz de correlaciones entre factores latentes. Modelo estructural entre Métodos de enseñanza - aprendizaje y Competencias adquiridas en la Universidad para Ciencias

Las ecuaciones estandarizadas del modelo de medida aportan estimaciones de los coeficientes estandarizados. La relación entre sus valores es muy similar a los estimados para el resto de áreas de estudio.

Sin embargo los coeficientes de regresión estandarizados del modelo estructural permiten observar que la carga del primer factor - Estudio y trabajo en grupo - sobre el factor de Organización personal es superior a la correspondiente al tercer factor - Clases teóricas. Este resultado permite concluir que las clases teóricas tienen efecto fundamentalmente sobre los Conoci- 


$$
\begin{array}{ll}
V_{1}=0,216 \cdot F_{1}+0,976 \cdot E_{1} & R^{2}=0,047 \\
V_{2}=0,459 \cdot F_{1}+0,888 \cdot E_{2} & R^{2}=0,211 \\
V_{3}=0,294 \cdot F_{1}+0,002 \cdot F_{2}+0,955 \cdot E_{3} & R^{2}=0,087 \\
V_{4}=0,088 \cdot F_{2}+0,996 \cdot E_{4} & R^{2}=0,008 \\
V_{5}=0,297 \cdot F_{2}+0,955 \cdot E_{5} & R^{2}=0,088 \\
V_{6}=0,203 \cdot F_{3}+0,979 \cdot E_{6} & R^{2}=0,041 \\
V_{7}=0,009 \cdot F_{3}+1,000 \cdot E_{7} & R^{2}=0,000 \\
V_{8}=0,398 \cdot F_{2}+0,917 \cdot E_{8} & R^{2}=0,158 \\
V_{9}=0,457 \cdot F_{1}+0,889 \cdot E_{9} & R^{2}=0,209 \\
V_{10}=0,406 \cdot F_{1}+0,914 \cdot E_{10} & R^{2}=0,165 \\
V_{11}=0,415 \cdot F_{4}+0,910 \cdot E_{11} & R^{2}=0,172 \\
V_{12}=0,387 \cdot F_{4}+0,922 \cdot E_{12} & R^{2}=0,150 \\
V_{13}=0,834 \cdot F_{4}+0,552 \cdot E_{13} & R^{2}=0,695 \\
V_{14}=0,725 \cdot F_{4}+0,689 \cdot E_{14} & R^{2}=0,526 \\
V_{15}=0,656 \cdot F_{9}+0,755 \cdot E_{15} & R^{2}=0,430 \\
V_{16}=0,637 \cdot F_{7}+0,771 \cdot E_{16} & R^{2}=0,406 \\
V_{17}=0,707 \cdot F_{9}+0,708 \cdot E_{17} & R^{2}=0,499 \\
V_{18}=0,741 \cdot F_{7}+0,672 \cdot E_{18} & R^{2}=0,549 \\
V_{19}=0,816 \cdot F_{7}+0,577 \cdot E_{19} & R^{2}=0,667 \\
V_{20}=1,000 \cdot F_{8}+0,000 \cdot E_{20} & R^{2}=1,000 \\
V_{21}=0,209 \cdot F_{8}+0,687 \cdot F_{9}+0,568 \cdot E_{21} & R^{2}=0,677 \\
V_{22}=0,343 \cdot F_{6}+0,401 \cdot F_{9}+0,752 \cdot E_{22} & R^{2}=0,435 \\
V_{23}=0,806 \cdot F_{9}+0,592 \cdot E_{23} & R^{2}=0,650 \\
V_{24}=0,672 \cdot F_{5}+0,741 \cdot E_{24} & R^{2}=0,451 \\
V_{25}=0,897 \cdot F_{5}+0,443 \cdot E_{25} & R^{2}=0,804 \\
V_{26}=0,725 \cdot F_{5}+0,689 \cdot E_{26} & R^{2}=0,526 \\
V_{27}=0,821 \cdot F_{6}+0,570 \cdot E_{27} & R^{2}=0,675 \\
V_{28}=0,724 \cdot F_{6}+0,690 \cdot E_{28} & R^{2}=0,524
\end{array}
$$

Tabla 5.95: Ecuaciones estandarizadas del modelo de medida. Modelo estructural entre Métodos de enseñanza - aprendizaje y Competencias adquiridas en la Universidad para Ciencias

mientos y Pensamiento analítico, independientemente de que en el área de ciencias puedan tener un efecto débil sobre la Organización personal.

Una vez estimado el modelo se revisaron las modificaciones propuestas por el Contraste de Lagrange, cuya aplicación no se consideró adecuada. En primer lugar se propone introducir una nueva relación entre los factores 7 y 9 - Organización personal y Liderazgo -descartada por haberse planteado desde el inicio ambos factores como endógenos. Las siguientes modificaciones hacen referencia a las covarianzas entre distintos errores residuales de los modelos de medida. Puesto que la adición o eliminación de dichas relaciones se analiza y evalúa detalladamente en la validación de los modelos de medida no se considera pertinente introducir ninguna de las modificaciones propuestas por esta prueba, obteniendo así el modelo definitivo. 


$$
\begin{array}{ll}
F_{4}=0,913 \cdot F_{3}+0,408 \cdot D_{4} & R^{2}=0,833 \\
F_{5}=0,870 \cdot F_{2}+0,493 \cdot D_{5} & R^{2}=0,757 \\
F_{6}=0,759 \cdot F_{1}+0,652 \cdot D_{6} & R^{2}=0,575 \\
F_{7}=0,651 \cdot F_{1}+0,361 \cdot F_{3}+0,509 \cdot D_{7} R^{2}=0,741 \\
F_{8}=0,750 \cdot F_{1}+0,661 \cdot D_{8} & R^{2}=0,563 \\
F_{9}=0,748 \cdot F_{1}+0,664 \cdot D_{9} & R^{2}=0,560
\end{array}
$$

Tabla 5.96: Ecuaciones estandarizadas del modelo estructural. Modelo estructural entre Métodos de enseñanza - aprendizaje y Competencias adquiridas en la Universidad para Ciencias

\subsubsection{Modelización multinivel de los Procesos de enseñanza - aprendizaje de competencias en la Universidad}

En la última fase del análisis se plantea un modelo multinivel de los procesos de enseñanza - aprendizaje de competencias a partir del modelo planteado para todas las áreas de estudio, con excepción del área de Ciencias. Para la realización de este análisis se define un nivel superior como las universidades o Instituciones de Educación Superior donde los graduados cursaron sus estudios, mientras que el nivel inferior lo constituyen los graduados entrevistados. Al igual que se ha mantenido la confidencialidad de los encuestados, eliminando los campos de información identificativos, también se descartan los campos relativos a la identificación de la institución. Cabe señalar que este análisis se plantea con el fin de estudiar la posible variabilidad de la aplicación del modelo a distintas instituciones y en ningún momento se pretende utilizar esta herramienta con el fin de obtener posibles clasificaciones o rankings de universidades.

En cualquier caso este análisis no se lleva a cabo finalmente, ya que las estimaciones de las correlaciones intraclases no superan los umbrales recomendados para ello. Según Muthen, las estimaciones de dichos valores varían entre 0 y 0.5 , y sólo cuando son superiores a 0.1 y los tamaños de cluster exceden las 15 unidades del nivel inferior, será pertinente aplicar la modelización multinivel para analizar la variabilidad del modelo en distintos niveles.

En este caso el tamaño de los clusters está determinado por el número de graduados en cada universidad. Este dato es muy heterogéneo entre las 34 universidades participantes ya que en la fase de muestreo no se estableció un mínimo número de encuestados por institución para participar en la encuesta. Así, la institución que menos graduados logró encuestar obtuvo tan solo 2 respuestas mientras que el máximo fueron 848. Con el fin de obtener mayor representatividad se eliminaron aquellas instituciones cuyo número de encuestados fuera inferior a 15 de forma que la muestra inicial de 34 ins- 
tituciones se redujo a 28. Sin embargo, el tamaño de los clusters continúa siendo muy heterogéneo resultando el primer cuartil en 33 encuestados, la mediana en 60 y el tercero en 308 .

\begin{tabular}{|cc|cc|cc|cc|}
\hline $\begin{array}{r}\text { Ítem Correlación } \\
\text { intraclases }\end{array}$ & $\begin{array}{r}\text { Ítem Correlación } \\
\text { intraclases }\end{array}$ & $\begin{array}{r}\text { Ítem Correlación } \\
\text { intraclases }\end{array}$ & $\begin{array}{r}\text { Ítem Correlación } \\
\text { intraclases }\end{array}$ \\
\hline 1 & 0.0345 & 8 & 0.0676 & 15 & 0.0201 & 22 & 0.0268 \\
2 & 0.0698 & 9 & 0.0705 & 16 & 0.0167 & 23 & 0.0146 \\
3 & 0.0227 & 10 & 0.0488 & 17 & 0.0278 & 24 & 0.1189 \\
4 & 0.1094 & 11 & 0.0330 & 18 & 0.0289 & 25 & 0.0503 \\
5 & 0.0869 & 12 & 0.0244 & 19 & 0.0200 & 26 & 0.0084 \\
6 & 0.0652 & 13 & 0.0679 & 20 & 0.0428 & 27 & 0.0339 \\
7 & 0.0036 & 14 & 0.0551 & 21 & 0.0285 & 28 & 0.0363 \\
\hline
\end{tabular}

Tabla 5.97: Correlaciones intraclases. Modelo estructural entre Métodos de enseñanza - aprendizaje y Competencias adquiridas en la Universidad

Respecto a las correlaciones intraclases, el valor de 0.1 sólo fue superado por los ítems 4 y 24 - Prácticas en empresa y Herramientas informáticas - por lo que no se consideró pertinente aplicar el modelo multinivel. Sin embargo, este resultado es igualmente relevante ya que implica que la variabilidad asociada a la aplicación del modelo general en distintas instituciones no resulta significativa. En otras palabras, que el modelo es generalizable a todas las universidades que han participantes en el proyecto de investigación.

\subsection{Resumen y conclusiones}

En este capítulo se propone un modelo estructural en base a los procesos de enseñanza - aprendizaje de competencias en la Universidad. La gran mayoría de las investigaciones precedentes en este campo se han planteado desde un enfoque exclusivamente teórico y en la práctica apenas existen estudios que aborden esta cuestión de forma cuantitativa. Por tanto, el objetivo de este apartado es validar estas teorías en el ámbito de la educación universitaria en España.

En primer lugar se han validado dos modelos de medida relativos a los métodos de enseñanza - aprendizaje empleados en la Universidad. El primero de ellos consta de cuatro factores, denominados Estudio y trabajo en grupo, Clases prácticas, Prácticas externas y Clases teóricas mientras que el modelo alternativo se estructura únicamente en tres factores, mediante la unión de los factores anteriores Clases prácticas y Prácticas externas. 
Asimismo, tras la comparación del ajuste obtenido por cuatro modelos relativos a las competencias adquiridas en la Universidad, se ha seleccionado y validado un modelo estructurado en seis factores latentes: Conocimientos y pensamiento metacognitivo, Organización personal, Liderazgo, Cooperación, Comunicación e Innovación. Ambos modelos de medida obtuvieron un ajuste excelente tanto sobre el conjunto global de datos como tras la imposición de restricciones de invarianza métrica respecto al área de estudio.

A partir de estos modelos de medida se han propuesto dos modelos causales, basados en las investigaciones teóricas precedentes, en el cual se establecen diversas relaciones entre los métodos de enseñanza - aprendizaje y las competencias adquiridas en la Universidad. Ambos modelos plantean la existencia de relaciones causales entre el Estudio y trabajo en grupo y la adquisición de competencias de Liderazgo, Cooperación y Comunicación. Al mismo tiempo las Prácticas y clases prácticas se vinculan con la Organización personal y la Innovación y finalmente las Clases teóricas se asocian con la adquisición de Conocimientos y el desarrollo del Pensamiento metacognitivo. Una vez descartado por motivos de infraidentificación empírica el modelo causal construido a partir del modelo de medida relativo a los métodos de enseñanza - aprendizaje, y estructurado en cuatro factores, se ha validado el modelo alternativo obteniendo un ajuste aceptable. La bondad de ajuste del modelo no se vio modificada significativamente en los posteriores análisis de invarianza métrica y estructural en cuanto al área de estudio, con excepción del área de Ciencias. Ya en la ejecución de los análisis preliminares, consistentes en la validación del modelo causal para cada área de estudio, se verificó la falta de convergencia del modelo en esta área de estudio. Igualmente los análisis de invarianza parcial reflejaron la necesidad de considerar este grupo de forma independiente. Por esta razón, aplicando una estrategia de generación de modelos con fines exploratorios, se modificó el modelo convenientemente hasta alcanzar un ajuste aceptable en coherencia con el principio de parsimonia. Las modificaciones al modelo general incluyeron la eliminación de la relación entre las Prácticas y clases prácticas y la adquisición de competencias de Organización personal y la consiguiente introducción de dos nuevas relaciones entre esta competencia y los métodos basados en el Estudio y trabajo en grupo y las Clases teóricas.

Finalmente se planteó un análisis multinivel sobre el modelo estructural general, con el fin de estudiar la posible variabilidad en su aplicación a las 34 universidades que aportaron datos a la muestra global. No obstante, las estimaciones de las correlaciones intraclases desaconsejaron la realización de este análisis, lo cual constituye un resultado igualmente trascendente, indicando que la variabilidad de la aplicación del modelo causal en distintas universidades no resulta significativa. 


\section{Capítulo 6}

\section{Conclusiones y líneas futuras de investigación}

El empleo de metodologías cuantitativas en el área de la investigación educativa se ha incrementado notablemente en los últimos años gracias a los avances tecnológicos y el desarrollo de programas informáticos que han facilitado el acceso generalizado al empleo de técnicas de análisis de datos. Paralelamente se ha desarrollado una línea de investigación en torno a la cuestión específica de cómo se producen los procesos de enseñanza - aprendizaje en el contexto universitario. Sobre este problema de investigación se han aportado numerosas contribuciones científicas, así como una gran profusión de modelos relativos a la estructura, componentes y relaciones que describen dichos procesos. No obstante, a pesar del elevado número de aportaciones y del énfasis puesto en la importancia de las metodologías cuantitativas en el área de la investigación educativa, la gran mayoría de estos trabajos carecen de apoyo empírico sobre el que sustentarse.

En esta Tesis Doctoral se ha abordado el estudio de los procesos de enseñanza - aprendizaje de competencias en la Universidad desde un enfoque cuantitativo, aunque siempre tomando como referencia los resultados obtenidos en investigaciones precedentes, con independencia de su orientación metodológica. Con este objetivo se presenta una extensa revisión bibliográfica sobre el concepto de competencia, los enfoques de aprendizaje adoptados por los estudiantes universitarios, la gestión de su tiempo durante los estudios, los métodos de enseñanza - aprendizaje empleados por el profesorado universitario y por supuesto los modelos propuestos para la descripción de estos procesos en el ámbito universitario.

Con el fin de reflejar adecuadamente la complejidad de dichos procesos se han considerado desde un principio muy diversos factores pertenecientes al contexto de los estudiantes y potencialmente influyentes en la adquisición de 
competencias. Estos factores se han clasificado en dos categorías principales correspondientes tanto al contexto académico de los encuestados en su etapa como estudiantes como al contexto personal. Así, los factores relativos a las características de los estudios y los métodos de enseñanza - aprendizaje se han clasificado como parte del contexto académico universitario, mientras que los factores sobre la gestión del tiempo y las características personales a nivel individual se han incluido como componentes del contexto personal.

En este sentido, las competencias adquiridas en la Universidad constituyen el eje central de la investigación en torno al cual se articula el presente trabajo. Sobre este aspecto, los resultados de los análisis de componentes principales y análisis factorial confirmatorio sobre las valoraciones los titulados universitarios españoles muestran que es posible distinguir seis dimensiones distintas, que en este trabajo se han denominado como Conocimientos y pensamiento metacognitivo, Comunicación, Organización personal, Cooperación, Innovación y Liderazgo, agrupación que resulta acorde con los resultados obtenidos en investigaciones previas en este campo, así como con otras clasificaciones posteriores basadas en un mayor número de categorías. El ajuste de esta estructura factorial ha resultado ser excelente, incluso tras la imposición de restricciones relativas a la invarianza métrica respecto al área de estudio. Del mismo modo, el cálculo de valores - test ha permitido identificar relaciones significativas entre dichas componentes y cada una de las variables relativas al contexto de los encuestados.

Específicamente el estudio del contexto académico y personal cobra gran importancia en los procesos de enseñanza - aprendizaje. Los resultados obtenidos mediante la aplicación de contrastes de independencia y medidas de asociación revelan la existencia de numerosas relaciones de dependencia entre estas variables contextuales. Por la propia naturaleza de las variables, el análisis de ambos contextos se ha abordado mediante el empleo de distintas técnicas multivariantes. Concretamente, el contexto personal de los estudiantes se ha descrito mediante análisis de correspondencias múltiples, gracias al cual se han identificado dos dimensiones principales de inercia relativas al nivel socioeconómico familiar y el nivel de independencia económica y personal de los encuestados. Por otro lado, la aplicación de análisis de componentes principales y análisis factorial confirmatorio, de forma similar al análisis de las competencias adquiridas en la Universidad, ha permitido reducir la información relativa a metodologías a tres métodos de enseñanza - aprendizaje principales, denominados en este trabajo como Estudio y trabajo en grupo, Clases prácticas y prácticas externas y Clases teóricas. La estructura de este modelo de medida ha resultado de igual modo invariante respecto al área de estudio.

El área de estudio constituye en este trabajo un elemento de gran rele- 
vancia en sí mismo, puesto que introduce una modificación del efecto muy significativa en la relación entre el contexto del encuestado y su percepción sobre la adquisición de competencias. La práctica totalidad de los análisis realizados en este trabajo confirma el condicionamiento de los resultados finales al área de estudio. Por esta razón se ha seleccionado como variable de segmentación de la muestra global en los análisis de invarianza métrica anteriores, así como en el análisis de invarianza estructural aplicado sobre el modelo causal formulado en la sección final del trabajo.

El modelo estructural propuesto en este trabajo plantea la existencia de relaciones causales entre el empleo de determinados métodos de enseñanza aprendizaje y la adquisición de unas u otras competencias. Para la formulación de estas relaciones se toman como referencia las aportaciones realizadas en investigaciones precedentes según las cuales las metodologías basadas en el Estudio y trabajo en grupo se vinculan en gran medida con la adquisición de competencias de Liderazgo, Cooperación y Comunicación. Por otro lado, las Clases prácticas y prácticas externas se asocian con competencias relativas a la Organización personal y la Innovación mientras que las Clases teóricas se relacionan con la adquisición de Conocimientos y el desarrollo del Pensamiento metacognitivo. Este modelo obtiene un ajuste aceptable y cumple las restricciones de invarianza tanto a nivel métrico como estructural, con excepción del área de Ciencias, donde se demuestra que la aplicación de este modelo no resulta adecuada, para lo cual se proponen determinadas modificaciones, ya no desde una perspectiva confirmatoria sino exploratoria. Estas modificaciones consisten fundamentalmente en la sustitución de la relación entre Clases prácticas y prácticas externas y la adquisición de competencias de Organización personal, por dos nuevas relaciones entre esta competencia y las metodologías basadas en el Estudio y trabajo en grupo y las Clases teóricas. Esta alteración constituye la única excepción que afecta a la generabilidad del modelo y, de hecho, el planteamiento del análisis de este mismo modelo desde una perspectiva multinivel en función de las diferencias entre universidades no se consideró apropiado, puesto que la variabilidad debida a las instituciones no alcanzó valores suficientemente significativos.

Sobre estas conclusiones deben matizarse algunos aspectos relativos a las limitaciones del estudio. En primer lugar la investigación se basa en un estudio de corte transversal en el cual se aplica una sola herramienta de investigación, el cuestionario. No se dispone de otras fuentes de información complementarias que completen o maticen las valoraciones de los encuestados. Por otro lado se plantea a los graduados que evalúen de forma subjetiva la contribución de sus estudios al desarrollo de competencias. Ambas limitaciones pueden ser aceptadas, dado que el objetivo del estudio es el análisis de las competencias de los titulados universitarios, que en gran medida se trata de una cuestión intrínsecamente personal. En cualquier caso, se ha de tener 
siempre presente que los resultados proceden de valoraciones personales de los encuestados. Asimismo cabe la posibilidad de que en la selección de las competencias se haya omitido algún ítem relevante para los encuestados. En cualquier caso, considerando el enfoque internacional con que se ha planteado la investigación, las limitaciones en este sentido resultan relativamente inevitables.

A raíz de los resultados obtenidos en este trabajo se propone como línea de investigación futura profundizar en el estudio de los procesos de enseñanza - aprendizaje de competencias en el ámbito de cada una de las áreas de estudio planteadas en este trabajo, e incluso hasta alcanzar un nivel de detalle equivalente al de titulación universitaria. De este modo se pretende minimizar la heterogeneidad de la muestra y lograr estimaciones más precisas de los parámetros que conforman los modelos causales. Asimismo, se plantea la posibilidad de enriquecer la información contenida en el contexto de aprendizaje de los estudiantes universitarios, incluyendo las variables relativas a los valores y expectativas de los estudiantes respecto al trabajo, cuya relación con los procesos de adquisición de competencias puede resultar relevante, a pesar de que anteriormente no se habían considerado en el análisis en favor de la parsimonia del modelo. Por último, se sugiere aplicar este procedimiento metodológico de investigación al nivel de competencias adquirido en el momento de la entrevista, que corresponde un período de tiempo cinco años posterior a la graduación. En este nuevo planteamiento se tendrán en consideración las variables relativas a aquellos factores, académicos, personales e incluso laborales, que potencialmente hayan tenido algún tipo de influencia o relación con el aprendizaje de nuevas competencias durante el período de transición al mercado laboral. 


\section{Capítulo 7}

\section{Referencias}

Abalde, E., Muñoz, M., Buendía, L., Olmedo, E. M., Berrocal, E. y Cajide, J. (2001). Los enfoques de aprendizaje en estudiantes universitarios españoles. Revista de Investigación Educativa, 19(2):465-489.

Acemoglu, D. y Pischke, J. S. (1998). Why do firms train? Theory and evidence. The Quarterly Journal of Economics, 113(1):79-118.

Adam, S. (2008). Learning outcomes current developments in Europe: Update on the issues and applications of learning outcomes associated with the Bologna Process. Edinburgh: The Scottish Governement. Bologna Seminar. Learning Outcomes based higher education: the Scottish experience.

Agresti, A. (1996). An introduction to categorical data analysis. New York: John Wiley and Sons.

Agresti, A. (2002). Categorical data analysis. New York: John Wiley and Sons.

Aguilera, A. M. (2001). Tablas de contingencia bidimensionales. Madrid: Hespérides. La Muralla.

Aguilera, A. M. (2006). Modelización de tablas de contingencia multidimensionales. Madrid: Hespérides. La Muralla.

Ahrens, W. y Pigeot, I. (2001). Handbook of Epidemiology. New York: Springer.

Aikaike, H. (1987). Factor Analysis and AIC. Psychometrika, 52(3):317332 . 
Aish, A. M. y Jöreskog, K. G. (1990). A panel model for political efficacy and responsiveness: An application of LISREL 7 with weighted least squares. Quality and Quantity, 24(4):405-426.

Alevizos, P. y Morineau, A. (1992). Tests et valeurs-test: application à l'étude de mastics utilisés dans la fabrication des vitraux. Revue de statistique appliquée, 40(4):27-43.

Aliaga, F. (1999). Análisis de Correspondencias. Estudio Bibliométrico sobre su uso en la Investigación Educativa. Relieve: Revista Electrónica de Investigación y Evaluación Educativa, 5(1). Consultado en http://www.uv.es/ RELIEVE [Consulta: 14/11/2010].

Allen, J., Arnesen, C. Å., Calmand, J., Frontini, M., Paul, J.-J., Rostan, M., Schomburg, H., Støren, L. A., Teichler, U. y Van der Velden, R., (2007). The Flexible Professional in the Knowledge Society: General Results of the REFLEX Project. Maastricht: Open Access publications from Maastricht University.

Allen, J. y De Weert, E. (2007). What Do Educational Mismatches Tell Us About Skill Mismatches? A Cross-country Analysis. European Journal of Education, 42(1):59-73.

Allen, J. y Van der Velden, R. (2001). Educational Mismatches versus Skill Mismatches: Effects on Wages, Job Satisfaction and On-the-job Search. Oxford Economic Papers, 53(3):434-452.

Allen, J., y Van der Velden, R. (Eds.). (2009). Competencies and early labour market careers of higher education graduates. Report on the large-scale graduate survey. Consultado en: http://www.hegesco.org/content/view/36/108/ [Consulta: 15/01/2010].

Allen, J. y Van der Velden, R. (Eds.) (2011). The flexible professional in the knowledge society: New challenges for Higher Education. Dordrecht: Springer.

Allison, P. D. (2003). Missing data techniques for Structural Equation Modeling. Journal of Abnormal Psychology, 112(4):545-557.

Aluja, T. y Morineau, A. (1999). Aprender de los datos: El Análisis de Componentes Principales. Barcelona: Promociones y Publicaciones Universitarias.

Anderson, J. C. y Gerbing, D. W. (1984). The effect of sampling error on 
convergence, improper solutions, and goodness of fit indices for maximum likelihood confirmatory factor analysis. Psychometrika, 49(2):155-173.

Anderson, J. C. y Gerbing, D. W. (1988). Structural Equation modeling in practice: A review and recommended two-step approach. Psychological Bulletin, 103(3):411-423.

Arbuckle, J. (2009). Amos User's Guide. Version 18. Chicago: SPSS Inc.

Badillo - Amador, L., García-Sánchez, A. y Vila, L. (2005). Mismatches in the Spanish Labor Market: Education vs. Competence Match. International Advances in Economic Research, 11(1):93-109.

Barlett, M. S. (1935). Contingency table interactions. Journal of the Royal Statistical Society - Supplement, 2(2), 248-252.

Bécue, M., Lebart, L. y Rajadell, N. (1992). El análisis estadístico de datos textuales. La lectura según los escolares de enseñanza primaria. Anuario de Psicología, 55, 7-22.

Becker, G. S. (1964). Human Capital: A Theoretical and Empirical Analysis, with Special Reference to Education. Chicago: University of Chicago.

Belfield, C. R., Bullock, A. D. y Fielding, A. (1999). Graduates'views on the contribution of their higher education to their general development: A Retrospective Evaluation for United Kingdom. Research in Higher Education, 40(4):409-438.

Beltrán, J. (1993). Procesos, estrategias y técnicas de aprendizaje. Madrid: Síntesis.

Bentler, P. M. (1990). Comparative fit indexes in structural models. Psychological Bulletin, 107(2):238-246.

Bentler, P. M. (1992). On the fit of models to covariances and methodology to the Bulletin. Psychological Bulletin, 112(3):400-404.

Bentler, P. M. (2006). EQS 6 Structural Equations Program Manual. Encino, C.A.: Multivariate Software, Inc.

Bentler, P. M. y Bonett, D. G. (1980). Significance tests and goodness of fit in the analysis of covariance structures. Psychological Bulletin, 88(3):588606. 
Bentler, P. M. y Chou, C. P. (1987). Practical issues in structural modelling. Sociological Methods and Research, 16(1):78-117.

Bentler, P. M. y Dijkstra, T. (1985). Efficient estimation via linearization in structural models. En P. R. Krishnaiah (Ed.) Multivariate analysis (pp.9-42). Amsterdam: North-Holland.

Bentler, P. M. y Weeks, D. G. (1979). Interrelations among models for the analysis of moment structures. Multivariate Behavioral Research, 14(2):169185.

Bentler, P. M. y Weeks, D. G. (1980). Linear structural equations with latent variables. Psychometrika, 45(3):289-308.

Benzécri, J. P. (1979). Sur le calcul des taux d'inertie dans l'analyse dún questionnaire. Les Cahiers de l'Analyse des Données, 3, 377-378.

Benzécri, J. P. (1980). L'analyse des données. Tome II. L'analyse des correspondances. Paris: Dunod.

Benzécri, J. P. (1992). Correspondence analysis handbook. Paris: CRC Press.

Biggs, J. (1976). Dimensions of study behaviour: Another look at ATI. British Journal of Educational Psychology, 46(1):68-80.

Biggs, J. (1978). Individual and group differences in study processes. British Journal of Educational Psychology, 48(3):266-279.

Biggs, J. (1982). Student motivation and study strategies in University and CAE populations. Higher Education Research and Development, $1(1): 33-55$.

Biggs, J. (1984). Learning strategies, student motivation patterns and subjectively perceived success. En J. R. Kirby (Ed.), Cognitive strategies and educational performance (pp.111-134). New York: Academic Press.

Biggs, J. (1987). Study Process Questionnaire (SPQ). Manual. Melbourne: Australian Council for Educational Research.

Biggs, J. (1988). The role of metacognition in enhacing learning. Australian Journal of Education, 32(2):127-138. 
Biggs, J. (1985). The role of metalearning in study processes. British Journal of Educational Psychology, 55(3):185-212.

Biggs, J. (1989). Approaches to the enhancement of tertiary teaching. Higher Education Research and Development, 8(1):7-25.

Biggs, J. (1993). What do investories of students'learning processes really measure? A theoretical review and clarification. British Journal of Educational Psychology, 63(1):3-19.

Biggs, J. (2003). Teaching for quality learning at University: What the student does. Buckingham: Open University Press.

Biggs, J., Kember, D. y Leung, D. (2001). The revised two-factor Study Process Questionnaire: R-SPQ-2F. British Journal of Educational Psychology, 71(1):133-149.

Biggs, J. y Kirby, J. R. (1984). Differentiation of learning processes within ability groups. Educational Psychology, 4(1):21-39.

Bishop, J. H. (1995). Vocational Education and At-Risk Youth in the United States. (CAHRS Working Paper, 95-19). Ithaca, NY: Cornell University, School of Industrial and Labor Relations, Center for Advanced Human Resource Studies.

Bishop, Y. M., Fienberg, S. E., Holland, P. W., Light, R. J. y Mosteller, F. (2007). Discrete Multivariate Analysis. Theory and Practice. United States of America: Springer.

Bollen, K. A. (1989). Structural equations with latent variables. New York: Wiley.

Bollen, K. A. (1990). Overall fit in covariance structure models: Two types of sample size effects. Psychological Bulletin, 107(2):256-259.

Boshuizen, H. P. A. (2004). Does Practice Make Perfect? A slow and discontinuous process. En H. P. A. Boshuizen, R. Bromme y H. Gruber (Eds.) Professional Learning: Gaps and Transitions on the way from novice to expert Vol. 2, Part 3, (pp.75-95). Dordrecht: Kluwer Academic Publishers.

Bou - Llusar, J. C., Camisón - Zornoza, C. y Escrig - Tena, A. B. (2001). Measuring the relationship between firm perceived quality and customer satisfaction and its influence on purchase intentions. Total Quality Management, 12(6): 719-734. 
Bou - Llusar, J. C., Escrig - Tena, A. B., Roca - Puig, V. y Beltrán Martin, I. (2009). An empirical assessment of the EFQM Excellence Model: Evaluation as a TQM framework relative to the MBNQA Model. Journal of Operations Management, 27: 1-22.

Bowen, H. R. (1977). Investment in Learning. San Francisco: JosseyBass.

Bozdogan, H. (1987). Model selection and Akaike's information criteria (AIC): The general theory and its analytical extensions. Psychometrika, 52(3):345-370.

Brennan, J., Patel, K. y Tang, W. (2009). Diversity in the student learning experience and time devoted to study: a comparative analysis of the $U K$ and European evidence. London: The Open University. Centre for Higher Education Research and Information.

Breslow, N. y Day, N. E. (1975). Indirect standarization and multivariate models for rates, with reference to the age adjustment of cancer incidence and relative frequency data. Journal of Chronic Diseases, 28(5-6):289-303.

Breslow, N. y Day, N. E. (1980). Statistical methods in cancer research: The analysis of case-control studies. Lyon: International Agency for Research on Cancer (IARC).

Browne, M. W. (1984). Asymptotically distribution-free methods for the analysis of covariance structures. British Journal of Mathematical and Statistical Psychology, 37(1):62-83.

Browne, M. W. y Cudeck, R. (1989). Single sample cross-validation indices for covariance structures. Multivariate Behavioral Research, 24(4):445455.

Browne, M. W. y Cudeck, R. (1993). Alternative ways of assesing model fit. En K. A. Bollen y J. S. Long (Eds.) Testing structural equation models (pp.256-293). Newbury Park, C.A.: Sage.

Browne, M. W., MacCallum, R. C., Kim, C. T., Andersen, B. L. y Glaser, R. (2002). When fit indices and residuals are incompatible. Psychological Methods, 7(4):403-421.

Bryk, A. S. y Raudenbush, S. W. (1992). Hierarchical linear models: Applications and data analysis methods. Newbury Park, CA: Sage. 
Buendía, L. y Olmedo, E. A. (2002). El género, ¿constructo mediador en los enfoques de aprendizaje universitario? Revista de Investigación Educativa, 20(2):371-386.

Bullón, L. (2002). La estadística en la investigación epidemiológica: El estudio de casos y controles. Anales de la Facultad de Medicina, 63(2):46-56.

Bunk, G. P. (1994). La transmisión de las competencias en la formación y perfeccionamiento profesionales en la RFA. Revista Europea de Formación Profesional, 1:8-14.

Busato, V. V., Prins, F. J., Elshout, J. J. y Hamaker, C. (2000). Intellectual ability, learning style, personality, achievement motivation and academic success of psychology students in higher education. Personality and Individual Differences, 29(6):1057-1068.

Byrne, B. M. (1988). The self description questionnaire III: Testing for equivalent factorial validity across ability. Educational and Psychological Measurement, 48(2):397-406.

Byrne, B. M. (2006). Structural Equation Modeling with EQS. New York: Taylor and Francis Group.

Byrne, B. M., Shavelson, R. J. y Muthen, B. (1989). Testing for the equivalence of factor covariance and mean structures: The issue of partial measurement invariance. Psychological Bulletin, 105(3):456-466.

Byrne, B. M., Stewart, S. M. y Lee, P. W. H. (2004). Validating the beck depression inventory-II for Hong Kong Community Adolescents. International Journal of Testing, 4(3):199-216.

Campbell, D. T. y Fiske, D. W. (1959). Convergent and discriminant validation by the multitrait-multimethod matrix. Psychological Bulletin, 56(2):81105 .

Cano, F. (2000). Diferencias de género en estrategias y estilos de aprendizaje. Psicothema, 12(3):360-367.

Cazes, P. (1982). Note sur les éléments supplementaires en analyse des correspondances. Pratique et utilisation. Les Cahiers de l'Analyse des Données, $7(1): 9-23$. 
Cheung, G. W. y Rensvold, R. B. (2002). Evaluating goodness-of-fit indexes for testing measurement invariance. Structural Equation Modeling: A Multidisciplinary Journal, 9(2):233-255.

Clarke, R. M. (1986). Students' approaches to learning in an innovative medial school: a cross-sectional study. British Journal of Educational Psychology, 56(3):309-321.

Clausen, S. E. (1988). Applied correspondence analysis: An introduction. Thousand Oaks: Sage.

Cliff, N. (1983). Some cautions concerning the application of causal modeling methods. Multivariate Behavioral Research, 18(1):115-126.

Coenders, G., Batista - Foguet, J. M. y Saris, W. E. (2005). Temas avanzados en modelos de ecuaciones estructurales. Madrid: La Muralla.

Cohen, J. (1994). The earth is round $(p<0,05)$. American Psychologist, 49(12):997-1993.

Comunicado de Bergen (2005). The European Higher Education Area. Achieving the Goals. Consultado en http://www.eua.be/eua-work-and-policyarea/building-the-european-higher-education-area/bologna-basics.aspx [Consulta: $19 / 07 / 2008]$.

Comunicado de Londres (2007). Towards the European Higher Education Area: responding to challenges in a globalised world. Consultado en http://www.eua.be/eua-work-and-policy-area/building- the-european-highereducation-area/bologna-basics.aspx [Consulta: 18/07/2008].

Comunicado de Praga (2001). Towards the European Higher Education Area. Consultado en http://www.eua.be/eua-work-and-policy-area/buildingthe-european-higher-education-area/bologna-basics.aspx [Consulta: 18/07/ 2008].

Conchado, A. y Carot, J. M. (2009) El papel de los estudios de seguimiento de egresados como fuente de información estratégica para las universidades. En M. Parellada (Ed.) Informe CyD 2009. La contribución de las universidades al desarrollo (pp. 163-165). Madrid: Fundación Conocimiento y Desarrollo.

Conchado, A. y Carot, J. M. (2011). La enseñanza y el aprendizaje de competencias en la Universidad. En J. G. Mora (Ed.) Formando en competencias: ¿un nuevo paradigma? (pp. 119-125). Madrid: Fundación Conoci- 
miento y Desarrollo.

Conchado, A., Cortés, A., Mora, J.-G. y Carot, J. M. (2009). Los valores laborales de los graduados en educación en España. Revista de Educación. En prensa. (DOI: 10-4438/1988-592X-RE-2010-359-095).

Costner, H. L. y Schoenberg, R. (1979). Diagnosing indicator ills in multiple indicator models. En A. Goldberger y O. Duncan (Eds.) Structural equation models in the Social sciences (pp.167-199). New York: Seminar Press.

Cox, D. R. (1984). Interaction. International Statistical Review, 52(1):131 .

Cox, D. R. y Snell, E. J. (1990). Analysis of binary data. London: Chapman and Hall.

Cramer, H. (1946). Mathematical methods of statistics. Princeton, N.J.: Princeton University Press.

Cronbach, L. J. (1951). Coefficient alpha and the internal structure of tests. Psychometrika, 16(3):297-334.

Cronbach, L. J. (1967). How can instruction be adapted to individual differences? En R. M. Gagné (Ed.) Learning and individual differences (pp.2339). Ohio: Merrill.

Cronbach, L. J. (1976). Research on Classrooms and Schools: Formulation of Questions, Design and Analysis. Stanford Univ., CA: Stanford Evaluation Consortium.

Cronbach, L. J. y Shavelson, R. J. (2004). My current thoughts on coeffcient alpha and successor procedures. Los Angeles, CA: Center for the Study of Evaluation (CSE). National Center for Research on Evaluation, Standards, and Student Testing (CRESST).

Cuadras, C. M. (1991). Métodos de Análisis Multivariante. Barcelona: Promociones y Publicaciones Universitarias.

Cuadras, C. M. (2006). Modelos Estadísticos Multivariantes. Barcelona: Promociones y Publicaciones Universitarias.

Cudeck, R. (1989). Analysis of correlation matrices using covariance structure models. Psychological Bulletin, 105(2):317-327. 
Cudeck, R. y Browne, M. W. (1983). Cross-validation of covariance structures. Multivariate Behavioral Research, 18(2):147-167.

Curran, P. J., West, S. G. y Finch, J. F. (1996). The robustness of test statistics to nonnormality and specification error in confirmatory factor analysis. Psychological Methods, 1(1):16-29.

De Irala, J. y Fernández-Crehuet, R. (1999). Apuntes de epidemiología general: conceptos teóricos y ejercicios prácticos. Pamplona: Newbook Ediciones.

De Irala, J., Martínez-González, M. A. y Guillén, F. (2001a) ¿Qué es una variable de confusión? Medicina Clínica, 117(10):377-385.

De Irala, J., Martínez-González, M. A. y Guillén, F. (2001b) ¿Qué es una variable modificadora del efecto? Medicina Clínica, 117(10):297-302.

De la Fuente, A. y Ciccone, A. (2002). Human capital in a global and knowledge-based economy. European Commission: DG for Employment and Social Affairs. UFAE and IAE Working Papers.

De Miguel, M. (2006). Metodologías de enseñanza y aprendizaje para el desarrollo de competencias. Orientaciones para el profesorado universitario ante el Espacio Europeo de Educación Superior. Madrid: Alianza Editorial.

De Miguel, M., Alfaro, I., Apodaca, P., Arias, J. M., García, E., Lobato, C. y Pérez, A. (2005). Modalidades de enseñanza centradas en el desarrollo de competencias. Orientaciones para promover el cambio metodológico en el Espacio Europeo de Educación Superior. Madrid: Programa de Estudios y Análisis. Dirección General de Universidades. Ministerio de Educación y Ciencia.

Dekker, R., De Grip, A. y Heijke, H. (1980). The effects of training and overeducation on career mobility in a segmented labour market. International Journal of Manpower, 23(2):106-125.

Dewey, J. (1916). Democracy and education. New York: Dover Publications, Inc.

Dillon, W. A., Kumar, A. y Mulani, N. (1987). Offending estimates in covariance structure analysis. Comments on the causes and solutions to Heywood cases. Psychological Bulletin, 101(1):126-135. 
Ducoing, A. y Lecumberri, J. (1998). El control de factores de confusión en estudios epidemiológicos. Ciencia Veterinaria, 8:29-50.

Dunkin, M. J. y Biddle (1974). The study of teaching. Oxford: Rinehart and Winston.

Entwistle, N. (1987). A model of the teachign-learning process derived from research on student learning. En J. Richardson, M. Eysenck y D. Warren-Piper (Eds.) Student learning: research in education and congnitive psychology (pp.13-28). London: Open University Press.

Entwistle, N., Hanley, M. y Hounsell, D. (1979). Identifying distinctive approaches to studying. Higher Education, 8(4):365-380.

Entwistle, N. y Ramsden, P. (1982). Understanding student learning. London: Croom Helm.

Entwistle, N. y Tait, H. (1990). Approaches to evaluation, evaluation of teaching and preferent for contrating academia environment. Higher Education, 19(2):169-194.

Erkel-Rousse, H. (1995). Détection de la multicolinéarité dans un modèle linéaire ordinaire: quelques éléments pour un usage averti des indicateurs de Belsley, Kuh et Welsh. Revue de statistique appliquée, 43(4):19-42.

Escofier, B. y Pagès, J. (1992). Análisis factoriales simples y múltiples. Objetivos, métodos e interpretación. Bilbao: Servicio Editorial. Universidad del País Vasco.

Eurostat. (2009). The Bologna Process in Higher Education in Europe. Key indicators on the social dimension and mobility, http://www.eurostudent.eu/, [Consulta: 13/19/2010].

Everitt, B. (1977). The analysis of contingency tables. London: Chapman and Hall.

Everwijn, S. E. M., Bomers, G. B. J. y Knubben, J. A. (1993). Ability or competence-based education: bridging the gap between knowledge acquisition and ability to apply. Higher Education, 25(4):425-438.

Falconer, S. y Pettigrew, M. (2000). Developing added value skills within an academic program through work based learning. En L. Borghans (Ed.) Educational innovation in Economics and Business IV: Learning in a changing environment (pp.127-141). Dordrecht / Boston / London: Kluwer 
Academic Publishers.

Fernández, A. y Fernández, I. (2009). Crítica y alternativas a la significación estadística en el contraste de hipótesis. Madrid: Hespérides. La Muralla.

Fienberg, S. E. (2007). The analysis of cross-classified categorical data. Cambridge: Springer.

Fisher, R. A. (1922). On the interpretation of $\chi^{2}$ from contingency tables, and the calculation of $p$. Journal of the Royal Statistical Society, 85(1):87-94.

Fisher, R. A. (1924). The conditions under which chi-square measures the discrepancy between observation and hypothesis. Journal of the Royal Statistical Society, 87:442-450.

Fisher, R. A. (1925). Statistical methods for research workers. Edinburgh: Oliver and Boyd.

García-Aracil, A. y Van der Velden, R. (2008). Competencies for young European higher education graduates: labor market mismatches and their payoffs. Higher Education, 55(2):219-239.

Gart, J. J. y Zweiful, J. R. (1967). On the bias of various estimators of the logit and its variance with applications to quantal bioassay. Biometrika, 54(1-2):181-187.

Gerritsen, R. (1999). Do students study more throughly within a problembased learning environments course. En J. Hommes (Ed.) Educational innovation in Economics and Business IV: Learning in a changing environment (pp.127-141). Dordrecth / Boston / London: Kluwer Academic Publishers.

Gini, C. (1912). Variabilità e mutabilità, contributo allo studio delle distribuzioni: relazione statische. Cagliari: Giuridici della R. Università di Cagliari. Studi Economico.

Glasser, M. (1964). Linear regression analysis with missing observations among the independent variables. Journal of the American Statistical Association, 59(307):834-844.

Glymour, C. (1988). Discovering causal structure. Orlando, Fla: Academic Press.

Goldberger, S. A. y Duncan, O. D. (1973). Structural equation models in the Social Sciences. New York: Seminar Press. 
Gómez-Restrepo, C. y Okuda, M. (2006). Metodología y lectura crítica de estudios. Factores de confusión. Revista Colombiana de Psiquiatría, $35(2): 242-248$.

González, J. y Wagenaar, R. (2005). Tuning Educational Structures in Europe. Universities'contribution to the Bologna Process. Bilbao: Universidad de Deusto.

Goodman, L. A. y Kruskal, W. H. (1954). Measures of association for cross classifications, Part I. Journal of the American Statistical Association, 49(268):732-764.

Goodman, L. A. y Kruskal, W. H. (1959). Measures of association for cross classifications, Part II. Journal of the American Statistical Association, 54(285):123-163.

Goodman, L. A. y Kruskal, W. H. (1963). Measures of association for cross classifications, Part III, Approximate sampling theory. Journal of the American Statistical Association, 58(302):310-364.

Goodman, L. A. y Kruskal, W. H. (1972). Measures of association for cross classifications, Part IV, Simplification of asymptotic variances. Journal of the American Statistical Association, 67(338):415-421.

Gorsuch, R. L. (1983). Factor Analysis. Hillsdale, N.J.: Lawrence Erlbaum and Associates.

Greenacre, M. (2008). La práctica del análisis de correspondencias. Bilbao: Fundación BBVA.

Greenland, S. y Robins, P. J. (1999). Causal diagrams for epidemiologic research. Epidemiology, 10(1):37-48.

Guérin-Pace, F. (1997). La statistique textuelle. Un outil exploratoire en sciences sociales. Population, 52(4):865-887.

Haberman, S. J. (1978). Analysis of qualitative data. San Diego: Academic Press.

Hagtvet, K. A. y Nasser, F. M. (2004). How well do item parcels represent conceptually defined latent constructs? A two-facet approach. Structural Equation Modeling: A Multidisciplinary Journal, 11(2):168-193. 
Hair, J. F., Anderson, R., Tatham, R. y Black, W. (2007). Análisis Multivariante. Madrid: Prentice Hall.

Haldane, J. B. (1955). The estimation and significance of the logarithm of a ratio of frequencies. Annals of Human Genetics, 20(4):309-311.

Hancock, G. R. y Mueller, R. (2006). Structural equation modeling: A second course. Greenwich, Conneticut: Information Age Publishing.

Harman, H. H. (1980). Análisis factorial moderno. Madrid: Saltés.

Hartog, J. (1980). Earnings and Capability Requirements. The Review of Economics and Statistics, 62(2):230-240.

Hartog, J. (1988). An ordered response model for allocation and earnings. International Review for Social Sciences (Kylos), 41(1):113-141.

Hartog, J. (1992). Capabilities, Allocation and Earnings. Boston: Kluwer.

Hartog, J. (2000). Human Capital as a Instrument of Analysis for the Economics of the Education. European Journal of Education, 35(1):7-20.

Hatcher, L. (1994). A step-by-step approach to using the SAS system for factor analysis and structural equation modelling. Cary, NC: Sas Institute Inc.

Hayduk, L. A. (1987). Structural equation modelling with LISREL: Essentials and Advances. Baltimore: Johns Hopkins University Press.

Hayduk, L. A. (1996). LISREL Issues, Debates and Strategies. Baltimore: Johns Hopkins University Press.

Heck, R. H. (2001). Multilevel modeling with SEM. En G. A. Marcoulides y R. Schumacker (Eds.) New developments and techniques in structural equation modeling (pp.89-127). Mahwah, NJ: Lawrence Erlbaum and Associates.

Heijke, H. y Meng, C. (2006). The effects of Higher Education programme characteristics on allocation and performance of the graduates: A European view. (ROA-RM-2006/4E). Maastricht: Maastricht University. Faculty of Economics and Business Administration. Research Centre for Education and the Labour Market. 
Heijke, H., Meng, C. y Ramaekers, G. (2003). An investigation into the role of human capital competences and their pay-off. International Journal of Manpower, 24(7):750-773.

Heijke, H., Meng, C. y Ramaekers, G. (2010). Activating learning environments, student time use and competence acquisition. A study among European Economic and Business Administration students. En R. Jürgen y D. Eerma (Eds.) Economics of Education: Issues of Transition and Transformation (pp.75-111). Berlin: LitVerlag.

Heijke, H.; Meng, C. y Ris, C. (2003). Fitting to the job: the role of generic and vocational competencies in adjustement and performance. Labour Economics, 10(2):215-229.

Hernández, F. (1999). Los enfoques de aprendizaje en el contexto de la evaluación de la calidad de las universidades. Madrid: Ministerio de Educación y Ciencia. Programa sectorial de promoción general del conocimiento. Convocatoria 1995.

Hernández, F., García, P. y Maquillón, J. (2005). Análisis del cuestionario de procesos de estudio - 2 factores de Biggs en estudiantes universitarios españoles. Revista Fuentes, 6.

Hernández, F., García, P., Martínez, R. y Maquillón, J. (2002). Consistencia entre motivos y estrategias de aprendizaje en estudiantes universitarios. Revista de Investigación Educativa, 20(2):487-510.

Hernández, F. y Hervás, R. M. (2005). Enfoques y estilos de aprendizaje en Educación Superior. Revista española de orientación y psicopedagogía, 16(2):283-299.

Hernández, F. y Maquillón, J. (2000). Enfoques de aprendizaje en alumnos de COU y Reforma que pretenden acceder a la Universidad y alumnos de primer curso de carrera. En Congrés d'Orientació Universitaria (pp.107125). Barcelona, Edicions Universitat de Barcelona.

Hoelter, J. W. (1983). The analysis of covariance structures: Goodnessof-Fit Indices. Sociological Methods and Research, 11(3):325-344.

Hotelling, H. (1933) Analysis of a complex of statistical variables into principal components. Journal of Educational Psychology, 24(7):417-441.

Hox, J. (2002). Multilevel analysis: Techniques and applications. Mahwah, NJ: Lawrence Erlbaum and Associates. 
Hoyle, R. H. (1995). Structural equation modeling: Concepts, issues and applications. Thousand Oaks: Sage Publications.

Hu, L. y Bentler, P. M. (1995). Evaluating model fit. En R. H. Hoyle (Ed.) Structural equation modeling: Concepts, issues and applications (pp.76-99). Thousand Oaks: Sage.

Hu, L. y Bentler, P. M. (1998). Fit indices in covariance structure modeling: Sensitivity to underparameterized model misspecification. Psychological Methods, 3(4):424-453.

Hu, L. y Bentler, P. M. (1999). Cutoff criteria for fit indexes in covariance structure analysis: Conventional criteria versus new alternatives. Structural Equation Modeling: A Multidisciplinary Journal, 6(1):1-55.

Hu, L., Bentler, P. M. y Kano, Y. (1992). Can test statistics in covariance structure analysis be trusted? Psychological Bulletin, 112(2):351-362.

Ishikawa, M. y Ryan, D. (2002). Schooling, basic skills and economic outcomes. Economics of Education Review, 21(3):231-243.

Joaristi, L. y Lizasoain, L. (2000). Análisis de correspondencias. Madrid: La Muralla.

Johnson, D. W., Johnson, R. y Smith, K. (1991). Active learning: Cooperation in the college classroom. Edina, MN: Interaction Book Company.

Jöreskog, K. G. (1969). A general approach to confirmatory factor analysis. Psychometrika, 34(2):183-202.

Jöreskog, K. G. (1971). Statistical analysis of sets of congeneric tests. Psychometrika, 36(2):409-426.

Jöreskog, K. G. y Sörbom, D. (1984). LISREL 6: User's guide. Mooresville: Scientific Software Inc.

Jöreskog, K. G. y Sörbom, D. (1989). LISREL 7: A guide to the program applications. Chicago: SPSS Inc.

Jöreskog, K. G. y Sörbom, D. (1993). LISREL 8: Structural equation modeling with the SIMPLIS command languaje. Chicago: Scientific Software International. 
Jöreskog, K. G. y Sörbom, D. (1996). LISREL 8: User's reference guide. Chicago: Scientific Software International.

Kaiser, H. F. (1958). The varimax criterion for analytic rotation in factor analysis. Psychometrika, 23(3):187-200.

Keeley, B. (2007). Human capital. How what you know shapes your life. Paris: OECD Publishing.

Kellerman, P. (2003). Las competencias y los requisitos de los empleos de los titulados: La empleabilidad como ideología de la Educación Superior en Europa. En J. D. Jiménez, J. Sánchez y R. Montero (Eds.) Educación Superior y empleo: La situación de los jóvenes titulados en Europa. La encuesta CHEERS (pp.91-112). Granada: Editorial Universidad de Granada.

Kellerman, P. (2007). Acquired and Required Competencies Of Graduates. En U. Teichler (Ed.) Careers of University Graduates: Views and Experiences in Comparative Perspectives (pp.115-131). Dordrecht: Springer.

Kember, D. (2000). Misconceptions about the learning approaches, motivation and study practices of Asian students. Higher Education, 40(1):99121.

Kember, D. y Wong, A. (2000). Implications for evaluation from a study of students'perceptions of good and poor teaching. Higher Education, 40(1):6997.

Kennedy, D., Hyland, A. y Ryan, N. (2006). Writing and using learning outcomes: a practical guide. En J. Raabe (Ed.) EUA Bologna Handbook. Making Bologna Work (pp.1-30). Berlín: Raabe Verlag.

Kenny, D. A. (1979). Correlation and causality. New York: Wiley.

Kirk, R. E. (1996). Practical significance: A concept whose time has come. Educational and Psychological Measurement, 56(5):746-759.

Kish, L. (1978). Chance, statistics and statisticians. Journal of the American Statistical Association, 73(361):1-6.

Kleinbaum, D. G. y Klein, M. (2010). Logistic regression. A self-learning text. New York: Springer.

Kline, R. B. (2004). Principles and practice of structural equation modeling. New York: Guilford Press. 
Kreft, I. y De Leeuw, J. (1998). Introducing multilevel modeling. Newbury Park, CA: Sage.

La Du, T. J. y Tanaka, J. S. (1989). Influence of sample size, estimation method, and model specification on goodness-of-fit assessments in structural equation modeling. Journal of Applied Psychology, 74(4):625-636.

Lancaster, H. O. (1949). The derivation and partition of $\chi^{2}$ in certain discrete distributions. Biometrika, 36(1-2):117-129.

Last, J. M. (2001). A dictionary of epidemiology. London: Oxford University Press.

Laurillard, D. (1979). The processes of student learning. Higher Education, 8(4):395-409.

Lebart, L., Morineau, A. y Piron, M. (1995). Statistique Exploratoire Multidimensionnelle. Paris: Dunod.

Lebart, L., Morineau, A. y Warwick, K. M. (1984).Multivariate descriptive analysis: Correspondence analysis and related techniques for large matrices. New York: John Wiley and Sons, Inc.

Lebart, L., Salem, A. y Bécue, M. (2000). Análisis estadístico de textos. Lleida: Milenio.

Lebart, L., Salem, A. y Berry, L. (1998). Exploring textual data. Dordrecht: Kluwer Academic.

Leckey, J. F. y McGuigan, M. A. (1997). Right Tracks - Wrong Trails. The development of generic skills in Higher Education. Research in Higher Education, 38(3):365-378.

Levy, J. P. y Varela, J. (2006). Modelización con estructuras de covarianzas en Ciencias Sociales. Madrid: NetBiblo.

Liang, J. J. y Bentler, P. M. (2004). An EM algorithm for fitting twolevel structural equation models. Psychometrika, 69(1):101-122.

Light, R. J. y Margolin, B. H. (1971). An analysis of variance for categorical data. Journal of the American Statistical Association, 66(335):534-544. 
Little, R. J. A y Rubin, D. B. (1987). Statistical analysis with missing data. New York: Wiley.

Little, T. D. (1997). Mean and covariance structures (MACS) analyses of cross-cultural data: Practical and theoretical issues. Multivariate Behavioral Research, 32(1):53-76.

Long, J. S. (1983). Confirmatory factor analysis. Beverly Hills, CA: Sage Publications.

MacCallum, R. C. (1986). Specification searches in covariance structure modeling. Psychological Bulletin, 100(1):107-120.

MacCallum, R. C., Browne, M. W. y Sugawara, H. M. (1996). Power analysis and determination of sample size for covariance structure modeling. Psychological Methods, 1(2):130-149.

MacCallum, R. C., Roznowsky, M. y Necowitz, L. B. (1992). Model modifications in covariance structure analysis: The problem of capitalization on chance. Psychological Bulletin, 111(3):490-504.

MacCallum, R. C., Wegener, D. T., Uchino, B. N. y Fabrigar, L. R. (1993). The problem of equivalent models in applications o covariance structure analysis. Psychological Bulletin, 114(1):185-199.

Maldonado, G. y Greenland, S. (1993). Simulation study of confounder - selection strategies. American Journal of Epidemiology, 138(11):923-936.

Mantel, N. (2011). Chi-square tests with one degree of freedom; Extensions of the Mantel-Haenszel procedure. Journal of the American Statician Association, 58(303):690-700.

Mantel, N. y Haënszel, W. (1959). Statistical aspects of the analysis of data from retrospective studies of disease. Journal of the National Cancer Institute, 22, 719-727.

Maquillón, J. (2003) Diseño y evaluación del diseño de un programa de intervención para la mejora de las habilidades de aprendizaje de los estudiantes universitarios (Tesis Doctoral). Murcia: Universidad de Murcia. Facultad de Educación. Departamento de Métodos de Investigación y Diagnóstico en Educación.

Mardia, K. V. (1970). Measures of multivariate skewness and kurtosis with applications. Biometrika, 57(3):519-530. 
Mardia, K. V. (1974). Applications of some measures of multivariate skewness and kurtosis in testing normality and robustness studies. The Indian Journal of Statistics. 36(2):115-128.

Marton, F. y Säljö, R. (1976a). On qualitative differences in learning: I Outcome and process. British Journal of Educational Psychology, 46(1):3-11.

Marton, F. y Säljö, R. (1976b). On qualitative differences in learning: II Outcome as a function of the learner's conception of the task. British Journal of Educational Psychology, 46(2):115-127.

Marton, F., Hounsell, D. y Entwistle, N. (1984). The experience of learning. Edinburgh: Scottish Academia Press.

Marton, F. y Svensson, L. (1979). Conceptions of research in student learning. Higher Education, 8(4):471-486.

Marsh, H. W., Balla, J. R. y McDonald, R. P. (1988). Goodness of fit indexes in confirmatory factor analysis: The effect of sample size. Psychological Bulletin, 103(3):391-410.

Marsh, H. W., Hau, K., Balla, J. R. y Grayson, D. (1998). Is more ever too much? The number of indicators per factor in confirmatory factor analysis. Multivariate Behavioral Research, 33(2):181-220.

Martínez-González, M. A., De Irala, J. y Guillén-Grima, F. (1999) ¿Qué es una odds-ratio? Medicina Clínica, 112(1):416-422.

Maruyama, G. (1998). Basics of Structural Equation Modeling. Thousand Oaks: Sage Publications.

McArdle, J. J. y Hamagami, F. (1996). Multilevel models from a multiple group structural equation perspective. En G. A. Marcoulides y R. Schumacker (Eds.) Advanced structural equation modeling (pp.89-124). Mahwah, NJ: Lawrence Erlbaum and Associates.

McDonald, R. P. (1989). An index of goodness-of-fit based on noncentrality. Journal of Classification, 6(1):97-103.

McDonald, R. P. y Marsh, H. W. (1990). Choosing a multivariate model: noncentrality and goodness of fit. Psychological Bulletin, 107(2):247-255. 
Meng, C. y Heijke, H. (2005). Student Time Allocation, the Learning Environment and the Acquisition of Competencies (ROA-RM-2005/1E). Maastricht: Maastricht University. Faculty of Economics and Business Administration. Research Centre for Education and the Labour Market.

Miettinen O. (1974). Confounding and effect modification. American Journal of Epidemiology, 100(5):350-353.

Mora, J.-G, Badillo-Amador, L., Carot, J. M., Conchado, A, Nyssen, J. M. y Vila, L. E. (2008). El "éxito laboral" de los jóvenes graduados universitarios europeos. Revista de Educación, No Extraordinario, 41-58.

Mora, J.-G, Badillo-Amador, L., Conchado, A., Carot, J. M., Martínez, M., Martínez, D., Nyssen, J. M. y Vila, L. E. (2007a). El mercado de trabajo y las competencias profesionales de los jóvenes graduados: resultados del proyecto REFLEX. En M. Parellada (Ed.) Informe CyD 200\%. La contribución de las universidades al desarrollo (pp. 387-396). Madrid: Fundación Conocimiento y Desarrollo.

Mora, J.-G, Badillo-Amador, L., Conchado, A., Carot, J. M., Martínez, M., Martínez, D., Nyssen, J. M. y Vila, L. E. (2007b). Las competencias de los graduados universitarios europeos. Panorama Social, 6, 10-21.

Mora, J.-G., Carot, J. M. y Conchado, A. (2010). PROFLEX. El Profesional Flexible en la Sociedad del Conocimiento. Valencia: Autores.

Morineau, A. (1984). Note sur la caractérisation statistique dúne classe et les valeurs-tests, Bulletin Technique du Centre de Statistique et dÍnformatique Appliquées, 2(1-2), 20-27

Muthen, B. O. (1994). Multilevel covariance structure analysis. Sociological Methods and Research, 22(3):376-398.

Muthen, B. O. (1997). Latent variable modeling of longitudinal and multilevel data. En A. E. Raftery (Ed.) Sociological Methodology (pp.453-480). Washington, DC: American Sociological Association.

Nordaug, O. (1993). Human capital in organizations, competence, training and learning. Oslo: Scandinavian University Press.

Nunnally, J. C. (1978). Psychometric theory. New York: Mc. Graw Hill.

Organización para la Cooperación y el Desarrollo Económico. (2005). La definición y selección de competencias clave. Proyecto DeSeCo. Resumen 
ejecutivo.

Pavlin, S. (Ed.) (2009). Report on the qualitative analysis of Higher Education institutions and employers in five countries: Development of competencies in the world of work and education. Slovenia: University of Ljubljana, Faculty of Social Sciences.

Pearson, K. (1900). On a criterion that a given system of deviations from the probable in the case of a correlated system of variables is such it can be reasonably supposed to have arisen from random sampling. Philosophical Magazine, 5:157-175.

Pearson, K. (1901). On lines and planes of closest fit to systems of points in space. Philosophical Magazine 2:559-572.

Pearson, K. (1904). On the theory of contingency and its relation to association and normal correlation. Biometric Series 1. London: Drapers'Company Research Memoirs

Pedhazur, E. (1982). Multiple regression in behavioral research. New York: Holt.

Peña, D. (2002). Análisis de datos multivariantes. Madrid: McGraw Hill.

Pérez, M. L. (2001). El aprendizaje escolar desde el punto de vista del alumno: los enfoques del aprendizaje. En C. Coll, J. Palacios y A. Marchesi (Eds.) Desarrollo psicológico y educación. Vol. 2: Psicología de la educación escolar (pp.285-307). Madrid: Alianza Editorial.

Porto, A. M. (1994). Las aproximaciones al proceso de aprendizaje en estudiantes universitarios. Santiago de Compostela: Universidad de Santiago.

Ramsden, P. (1988). Context and strategy: Situational influences on learning. En R. R. Schmeck (Ed.) Learning strategies and learning styles (pp. 150-184). New York: Plenum Press.

Ramsden, P. (2003). Learning to teach in Higher Education. London: RoutledgeFalmer.

Raykov, T. y Widaman, K. F. (1995). Issues in structural equation modeling research. Structural Equation Modeling: A Multidisciplinary Journal, 2(4):289-318. 
Reichter, S. y Tauch, C. (2003). Tendencias 2003. Progreso hacia el Espacio Europeo de Educación Superior. Cuatro años después de Bolonia: Pasos hacia una Reforma Sostenible de la Educación Superior en Europa.

Richardson, J. y King, S. (1991). Gender differences in the experience of Higher Education: Quantitative and qualitative approaches. Educational Psychology, 11(3):363-382.

Rigdon, E. E. (1994). Demonstrating the Effects of Unmodeled Random Measurement Error. Structural Equation Modeling: A Multidisciplinary Journal, 1(4):375-380.

Rigdon, E. E. (1996). CFI versus RMSEA: A comparison of two fit indexes for structural equation modeling. Structural Equation Modeling: A Multidisciplinary Journal, 3(4):369-379.

Robles, J. (1996). Confirmation bias in structural equation modeling. Structural Equation Modeling: A Multidisciplinary Journal. 3(1):307-322.

Rodríguez, J. y Agulló, E. (1999). Estilo de vida, cultura, ocio y tiempo libre de los estudiantes universitarios. Psicothema, 11(2):247-259.

Rodríguez, A. y Vieira, M. J. (2009). La formación en competencias en la Universidad: Un estudio empírico sobre su tipología. Revista de Investigación Educativa, 27(1):27-47.

Rosario, P. L. S. (1997). Abordagens à aprendizagem dos alunos universitarios: Resultados no questionário SPQ de Biggs. En M. Gonçalves, S. Ribeiro, C. Araujo, L. Machado, L. Almeida y M. Simoes (Eds.) Actas da V Conferência Internacional sobre Avaliação Psicológica: Formas e Contextos (pp.101-117). Braga.

Rothman, K. J. y Greenland, S. (1998). Modern epidemiology. Washington: Lippincott - Williams and Wilkins.

Ruiz, E., Hernández, F. y Ureña, F. (2008). Evaluación y calidad del aprendizaje en Educación Superior. Murcia: Diego Marín Librero Editor.

Ruiz-Maya, L., Martín-Pliego, J., López, J., Montero, J. M. y Uriz, P. (1990). Metodología estadística para el análisis de datos cualitativos. Madrid: Centro de Investigaciones Sociológicas (CIS).

Ruiz-Maya, L., Martín Pliego, F. J., Montero, J. M. y Uriz Tome, P. (1995). Análisis estadístico de encuestas: datos cualitativos. Madrid: Edito- 
rial $\mathrm{AC}$.

Salanova, M., Martínez, I., Bresó, E., Llorens, S. y Grau, R. (2005). Bienestar psicológico en estudiantes universitarios: facilitadores y obstaculizadores del desempeño académico. Anales de Psicología, 21(1):170-180.

Salas, R., Santos, M. A. y Parra, S. (2004). Enfoques del aprendizaje y dominancias cerebrales en estudiantes universitarios. Aula abierta, 84:3-22.

Säljö, R. (1979). Learning about learning. Higher Education, 8(4):443451.

Satorra, A. y Bentler, P. M. (1988). Scaling corrections for chi square statistics in covariance structure analysis. American Statistical Association. Proceedings of the business and economic sections. Alexandria, VA: American Statistical Association.

Satorra, A. y Bentler, P. M. (1994). Corrections to test statistics and standard errors in covariance structure analysis. En A. Von Eye y C. C. Clogg (Eds.) Latent variables analysis: Applications for developmental research (pp.399-419). Thousand Oaks, CA: Sage.

Schmid, G. (2001). The dynamics of full employment. Social integration through transitional labour markets. Cheltenham: Edward Elgar.

Schmidt, F. L. (1996). Statistical significance testing and cumulative knowledge in psychology: Implications for training of researchers. Psychological Methods, 1(2):115-129.

Schomburg, H. y Teichler, U. (2006). Higher education and graduate employment in Europe: Results from graduate surveys from twelve countries. Dordrecht: Springer.

Schön, D. (1987). Educating the reflective practitioner: towards a new design for teaching and learning in the professions. San Francisco: Jossey Bass Publishers.

Semejin, J. H.; Van der Velden, R.,Heijke, H., Van der Vleuten, C. y Boshuizen, H. P. A. (2005). Competence indicators in academic education and early labour market success of graduates in health sciences (ROA-RM2005/2E). Maastricht: Maastricht University. Faculty of Economics and Business Administration. Research Centre for Education and the Labour Market. 
Sharma, S. (1996). Applied Multivariate Techniques. New York: John Wiley and Sons, Inc.

Simpson, E. (1951). The interpretation of interaction in contingency tables. Journal of the Royal Statistical Society, 13(2):238-241.

Sleiman, H., Bernier, C. y Roy, V. (2001). Gestion de projets ERP: étude exploratoire du profil managérial des chefs de projet (Cahier du GReSI, 01-04). Montreal: École des Hautes Études Commerciales de Montréal.

Sobel, M. E. y Bohrnstedt, G. W. (1985). Use of null models in evaluating the fit of covariance structure models. En N. B. Tuma (Ed.) Sociological Methodology (pp.152-178). San Francisco: Jossey-Bass.

Sonis, J. (1998). A closer look at confounding. Family Medicine Journal, 30(8):584-588.

Sörbom, D. (1989). Model modification. Psychometrika, 54(3):371-384.

Stasz, C. (1998). Generic Skills at Work: Implications for OccupationallyOriented Education. En W. J. Nijhof y J. N. Streumer (Eds.) Key Qualifications in Work and Education (pp.187-206). Dordrecht: Kluwer Academic Publishers.

Steiger, J. y Lind, J. C. (1980). Statistically based tests for the number of common factors. Psychometric Society Anual Meeting. Iowa City, IA.

Steiger, J. (1990). Structural Model Evaluation and Modification: An Interval Estimation Approach. Multivariate Behavioral Research, 25(2):173180 .

Stenberg, J. R. (2003). What is an expert student. Educational Researcher, 32(8):5-9.

Tait, H., Entwistle, N. y McCune, V. (1998). ASSIST: A reconceptualization of the approaches to Studying Inventory. En C. Rust (Ed.) Improving Student Learning: Improving students as learners (pp.262-270).Oxford: Oxford Brookes University. The Oxford Centre for Staff and Learning Development.

Tanaka, J. S. (1987). "How big is big enough?": Sample size and goodness of fit in structural equation models with latent variables. Child Development, 58(1):134-146. 
Tanaka, J. S. (1993). Multifaceted conceptions of fit in structural equation models. En K. A. Bollen y J. S. Long (Eds.) Testing structural equation models (pp.136-162). Newbury Park, C.A.: Sage.

Tejada, C. M., Tobón, S., Martínez, J. A., Mendo, C., Moreiro, J. A. y Ramos, L. F. (2006). El diseño del plan docente en Información y Documentación acorde con el Espacio Europeo de Educación Superior: un enfoque por competencias. Facultad de Ciencias de la Documentación. Universidad Complutense de Madrid.

Theil, H. (1970). On estimation of relationships involving qualitative variables. American Journal of Sociology, 76(1):103-154.

Thompson, B. (1996). Research news and Comment: AERA Editorial Policies Regarding Statistical Significance Testing: Three Suggested Reforms. Educational Researcher, 25(2):26-30.

Thrustone, L. L. (1931). The measurement of social attitudes. The Journal of Abnormal and Social Psychology, 26(3):249-269.

Thrustone, L. L. (1945). The vectors of mind. Chicago: University of Chicago Press.

Torrens-Ibern, J. (1972). Modèles et méthodes de l'analyse factorielle. Paris: Dunod.

Ullman, J. D. (1996). Structural Equation Modelling. En B. G. Tabachnick y L. S. Fidell (Eds). Using multivariate statistics (pp.653-771). Needham Heights, MA: Allyn and Bacon.

Uriel, E. y Aldás, J. (2005). Análisis Multivariante Aplicado. Aplicaciones al Marketing, Investigación de Mercados, Economía, Dirección de Empresas y Turismo. Madrid: Thomson.

Vaatstra, R. y De Vries, R. (2007). The effect of the learning environments on competences and training for the workplace according to graduates. Higher Education, 53(3):335-357.

Valle, A., González, R., Núñez, J. C., Suárez, J. M., Piñeiro, I. y Rodríguez, I. (2000). Enfoques de aprendizaje en estudiantes universitarios. Psicothema, 12(3):368-375.

Van der Velden, R. y Wolbers, M. (2004). How much does education matter and why? The Effects of Education on Socio-economic Outcomes among 
School-leavers in the Netherlands. European Sociological Review, 23(1):6580 .

Vermunt, J. D. (1996). Metacognitive, cognitive and affective aspects of learning styles and strategies: A phenomenographic analysis. Higher Education, 31(1):25-50.

Vila, L. (2000). The non-monetary Benefits of Education. European Journal of Education, 35(1):21-32.

Vila, L. (2005). Capital Humano Heterogéneo y Productividad de los Graduados de Educación Superior. Revista Económica de Castilla-La Mancha, 7:61-82.

Wald, A. (1943). Tests of statistical hypotheses concerning several parameters when the number of observations is large. Transactions of the American Mathematical Society, 54:426-482.

Watkins, D. y Hattie, J. (1981). The learning processes of australian university students: investigations of contextual and personal factors. British Journal of Educational Psychology, 51(3):384-393.

West, S. G., Finch, J. F. y Curran, P. J. (1995). Structural equation models with nonnormal variables: Problems and remedies. En R. H. Hoyle (Ed.) Structural equation modeling: Concepts, issues, and applications (pp.56-75). Thousand, Oaks, CA: Sage.

Wheaton, B. (1987). Assessment of fit in overidentified models with latent variables. Sociological Methods and Research, 16(1):118-154.

Widaman, K. F. y Reise, S. P. (1997). Exploring the measurement invariance of psychological instruments: Applications in the substance use domain. En K. J. Bryant, M. Windle y S. G. West (Eds.) The science of prevention: Methodological advances from alcohol and substance abuse research (pp.281-324). Washington, DC: American Psychological Association.

Wilcox, R. R. (2010). Fundamentals of modern statistical methods. Substantially improving power and accuracy. New York: Springer.

Woolf, B. (1955). On estimating the relation between blood group and disease. Annals of Human Genetics, 19:251-253.

Wothke, W. (1993). Nonpositive definite matrices in structural modeling. En K. A. Bollen y J. S. Long (Eds.) Testing structural equation models 
(pp.205-234). Newbury Park, C.A.: Sage.

Wothke, W. (2000). Longitudinal and multi-group modeling with missing data. En T. D. Little, K. U. Schnabel, y J. Baumert (Eds.) Modeling longitudinal and multiple group data: Practical issues, applied approaches and specific examples (pp.1-24). Mahwah, NJ: Erlbaum.

Yates, F. (1934). Contingency tables involving small numbers and $\chi^{2}$ test. Journal of the Royal Statistical Society - Supplement, 1(2):217-235.

Yuan, K. H. y Bentler, P. M. (1998). Normal theory based test statistics in structural equation modeling. British Journal of Mathematical and Statistical Psychology, 51(2):289-309.

Yuan, K. H. y Bentler, P. M. (2000). Three likelihood-based methods for mean and covariance structure analysis with nonnormal missing data. Sociological Methodology, 30(1):165-200.

Yuan, K. H. y Bentler, P. M. (2004a). On the asymptotic distributions of two statistics for two-level covariance structure models within the class of elliptical distributions. Psychometrika, 69(3):437-457.

Yuan, K. H. y Bentler, P. M. (2004b). On chi square difference and z-tests in mean and covariance structure analysis when the base model is misspecified. Educational and Psychological Measurement, 64(5):737-757.

Yule, G. U. (1900). On the association of attributes in statistics: with illustratrations from the material of the childhood society. Philosophical Transactions of the Royal Society of London, 194:257-319.

Yule, G. U. (1903). Notes on the theory of association of attributes in statistics. Biometrika, 2:121-134.

Yule, G. U. (1912). On the methods of measuring asoociation between two attributes (with discussion). Journal of the Royal Statistical Society, 75(6):579-652.

Yule, G. U. y Kendall, M. G. (1969). An introduction to the theory of statistics. New York: Hafner.

Zabalza, M. (2010). Competencias docentes del profesorado universitario. Calidad y desarrollo profesional. Madrid: Narcea.

Zeegers, P. (2001). Approaches to learning in science: a longitudinal 
study. British Journal of Educational Psychology, 71(1):115-132. 


\section{Capítulo 8}

\section{Apéndices}

\subsection{Anexo A: Preguntas seleccionadas del cues- tionario REFLEX}

\section{CARACTERÍSTICAS DE LOS ESTUDIOS}

En tu carrera...

- había que trabajar mucho para aprobar $(1=N a d a, 5=$ Mucho $)$

- su contenido era conocido por los empleadores $(1=N a d a, 5=$ Mucho $)$

- podía elegir las asignaturas que quería cursar (1=Nada, 5=Mucho)

- el enfoque era generalista $(1=N a d a, 5=M u c h o)$

- el enfoque era especializado (1=Nada, $5=$ Mucho)

- era una carrera con prestigio académico $(1=N a d a, 5=$ Mucho $)$

\section{MÉTODOS DE ENSEÑANZA - APRENDIZAJE}

¿En qué medida se hacía énfasis en tu carrera en los siguientes métodos de enseñanza y aprendizaje?

- Asistencia a clase $(1=N a d a, 5=$ Mucho $)$

- Trabajos en grupo ( $1=N a d a, 5=$ Mucho $)$ 
- Participación en proyectos de investigación (1=Nada, 5=Mucho)

- Prácticas en empresas, instituciones o similares ( $1=$ Nada, $5=$ Mucho)

- Conocimientos prácticos y metodológicos (1=Nada, 5=Mucho)

- Teorías, conceptos y paradigmas ( $1=N a d a, 5=$ Mucho $)$

- El profesor era la principal fuente de información $(1=N a d a, 5=M u c h o)$

- Aprendizaje basado en proyectos o problemas ( $1=N a d a, 5=$ Mucho)

- Trabajos escritos $(1=N a d a, 5=$ Mucho $)$

- Exposiciones orales $(1=N a d a, 5=$ Mucho $)$

- Realización de pruebas tipo test $(1=N a d a, 5=$ Mucho $)$

\section{GESTIÓN DEL TIEMPO}

¿Qué nota media obtuviste en la carrera?

- Aprobado

- Notable

- Sobresaliente

- Matrícula de Honor

¿Cuál era tu situación durante los dos últimos años de carrera?

- Los estudios eran mi actividad principal

- Los estudios no eran mi actividad principal

¿Realizaste una o más prácticas en empresas, instituciones o similares como parte de tu carrera?

- Sí

- No

Aproximadamente, cuántas horas destinabas a tus estudios (asistencia a clase, estudio personal... ): (Haz referencia a una semana lectiva normal durante los dos últimos años de carrera)

¿Has residido en el extranjero durante la carrera por motivos de estudio o trabajo? 
- Sí, para estudiar

- Sí, por cuestiones de trabajo

- No

¿Has residido en el extranjero después de la carrera por motivos de estudio o trabajo?

- Sí, para estudiar

- Sí, por cuestiones de trabajo

- No

\section{CARACTERÍSTICAS PERSONALES}

Sexo

- Hombre

- Mujer

Año de nacimiento

¿Cómo vives actualmente?

- Solo (incl. familia monoparental)

- Con tu pareja (con o sin hijos)

- Con tus padres

- Otros

¿Tienes hijos?

- Sí, 1 hijo

- Sí, 2 hijos

- Sí, 3 hijos o más

- No

¿Cuál es / era el nivel de estudios de tus padres y, si corresponde, de tu pareja actual? 
- Sin estudios

- Primaria

- Secundaria

- Terciaria

- No aplicable

\section{COMPETENCIAS}

¿En qué medida ha contribuido la carrera en que te graduaste en el año 1999/2000 al desarrollo de estas competencias?

En todos los casos: (1=Muy bajo, $7=$ Muy alto $)$

- a. Dominio de tu área o disciplina

- b. Conocimientos de otras áreas o disciplinas

- c. Pensamiento analítico

- d. Capacidad para adquirir con rapidez nuevos conocimientos

- e. Capacidad para negociar de forma eficaz

- f. Capacidad para rendir bajo presión

- g. Capacidad para detectar nuevas oportunidades

- h. Capacidad para coordinar actividades

- i. Capacidad para usar el tiempo de forma efectiva

- j. Capacidad para trabajar en equipo

- k. Capacidad para movilizar las capacidades de otros

- 1. Capacidad para hacerte entender

- m. Capacidad para hacer valer tu autoridad

- n. Capacidad para utilizar herramientas informáticas

- o. Capacidad para encontrar nuevas ideas y soluciones

- p. Predisposición para cuestionar ideas propias o ajenas

- q. Capacidad para presentar en público productos, ideas o informes

- r. Capacidad para redactar informes o documentos

- s. Capacidad para escribir y hablar en idiomas extranjeros 


\subsection{Anexo B: Agrupación de titulaciones univer- sitarias en áreas de estudio}

\section{Educación}

Ciclo Largo: Licenciatura en Pedagogía, Licenciatura en Educación Física y Licenciatura en Ciencias de la Actividad Física y el Deporte

Ciclo Corto: Diplomatura en Profesorado de E G B, Maestro de Audición y Lenguaje, Maestro de Educación Especial, Maestro de Educación Física, Maestro de Educación Infantil, Maestro de Educación Musical, Maestro de Educación Primaria y Maestro de Lengua Extranjera

\section{Humanidades}

Ciclo Largo: Diplomatura en Biblioteconomía y Documentación, Licenciatura en Antropología Social y Cultural, Licenciatura en Bellas Artes, Licenciatura en Documentación, Licenciatura en Filología, Licenciatura en Filología Alemana, Licenciatura en Filología Arabe, Licenciatura en Filología Clásica, Licenciatura en Filología Eslava, Licenciatura en Filología Francesa, Licenciatura en Filología Gallega, Licenciatura en Filología Hispánica, Licenciatura en Filología Inglesa, Licenciatura en Filología Italiana, Licenciatura en Filología Portuguesa, Licenciatura en Filología Románica, Licenciatura en Filología Vasca, Licenciatura en Filosofía y Letras Sección Geografía, Licenciatura en Filosofía, Licenciatura en Filosofía y Ciencias de la Educación, Licenciatura en Filosofía y Ciencias de la Educación, Licenciatura en Filosofía y Letras, Licenciatura en Geografía, Licenciatura e, Geografía e Historia, Licenciatura en Historia, Licenciatura en Historia del Arte, Licenciatura en Historia y Ciencias de de la Música, Licenciatura en Humanidades, Licenciatura en Lingüística y Licenciatura en Traducción e Interpretación

\section{Ciencias Sociales}

Ciclo Largo: Licenciatura en Ciencias de la Información, Licenciatura en Ciencias Políticas y Sociología Sección Políticas, Licenciatura e, Ciencias de Políticas y Administración, Licenciatura en Ciencias de Políticas y Sociología, Licenciatura en Comunicación Audiovisual, Licenciatura en Periodismo., Licenciatura en Psicología, Licenciatura en Psicopedagogía, Licenciatura en Publicidad , Relaciones Públicas y Licenciatura en Sociología 
Ciclo Corto: Diplomatura en Educación Social, Diplomatura en Trabajo Social, Diplomatura en Turismo y Graduado Social

\section{Economía y empresa}

Ciclo Largo: Licenciatura en Administración y Dirección de Empresas, Licenciatura en Ciencias de Actuariales y Financieras, Licenciatura e, Ciencias de Economía y Empresa Sección Empresariales, Licenciatura en Ciencias de Económicas, Licenciatura en Ciencias d, Económicas y Empresarial, Licenciatura en Ciencias de Empresariales, Licenciatura en Economía y Licenciatura en Investigación y Técnicas Mercado

Ciclo Corto: Diplomatura en Ciencias Empresariales, Diplomatura en Estudios Empresariales, Diplomatura en Gestión y Administración Pública y Diplomatura en Relaciones Laborales

\section{Derecho}

Ciclo Largo: Licenciatura en Derecho

\section{Técnicas}

Ciclo Largo: Arquitectura, Ingeniería Aeronáutica, Ingeniería Agrónoma, Ingeniería en Automática y Electrónica Industrial, Ingeniería d, Caminos, Canales y Puertos, Ingeniería Industrial, Ingeniería Informática, Ingeniería de Minas, Ingeniería de Montes, Ingenierí, Química, Ingeniería Técnica de Telecomunicación, Licenciatura en Informática y Licenciatura en Marina Civil

Ciclo Corto: Arquitectura Técnica, Diplomatura en de la Marina Civil, Diplomatura en Informática, Ingeniería Técnic, Aeronáutico, Ingeniería Técnica Agrícola, Ingeniería Técnica Diseño Industrial, Ingeniería Técnica Forestal, Ingeniería Técnic, Industrial, Ingeniería Técnica Informática de Gestión, Ingeniería Técnica Informática de Sistemas, Ingeniería Técnica Minas, Ingeniería Técnica de Obras Públicas, Ingeniería Técnica de Telecomunicaciones e Ingeniería Técnica de Topografía

Salud

Ciclo Largo: Licenciatura en Farmacia, Licenciatura en Medicina, Licenciatura en Medicina y Cirugía, Licenciatura en Odontología y Licenciatura en Veterinaria 
Ciclo Corto: Diplomatura en Enfermería, Diplomatura en Fisioterapia, Diplomatura en Logopedia, Diplomatura en Nutrición, Humana y Dietética, Diplomatura en Podología y Diplomatura en Terapia Ocupacional

\section{Ciencias}

Ciclo Largo: Diplomatura en Estadística, Diplomatura en Óptica y Optometría, Licenciatura en Biología, Licenciatura en Bioquímica, Licenciatura en Ciencias de Ambientales, Licenciatura en Ciencias de Biológicas, Licenciatura en Ciencias de del Mar, Licenciatur, en Ciencias de Físicas, Licenciatura en Ciencias de Matemáticas, Licenciatura en Ciencias de Químicas, Licenciatura en Ciencias de Tecnología de Alimentos, Licenciatura en Ciencias, , Licenciatura en Física, Licenciatura en Geología, Licenciatura en Matemáticas y Licenciatura en Química 


\subsection{Anexo C: Medidas de asociación}

\begin{tabular}{|c|c|c|c|c|c|c|c|c|c|c|c|c|c|c|c|c|c|}
\hline & $\mathrm{C} 1$ & $\mathrm{C} 2$ & C3 & $\mathrm{C} 4$ & C5 & C6 & V1 & $\mathrm{V} 2$ & V3 & V4 & V5 & V6 & V7 & V8 & V9 & V10 & VT \\
\hline $\mathrm{C} 1$ & 1 & & & & & & & & & & & & & & & & \\
\hline $\mathrm{C} 2$ & 0.151 & 1 & & & & & & & & & & & & & & & \\
\hline C3 & -0.112 & 0.061 & 1 & & & & & & & & & & & & & & \\
\hline $\mathrm{C} 4$ & & -0.087 & -0.037 & 1 & & & & & & & & & & & & & \\
\hline C5 & 0.105 & 0.223 & 0.156 & -0.654 & 1 & & & & & & & & & & & & \\
\hline C6 & 0.483 & 0.228 & -0.108 & -0.031 & 0.148 & 1 & & & & & & & & & & & \\
\hline V1 & & 0.056 & 0.056 & -0.044 & 0.125 & -0.032 & 1 & & & & & & & & & & \\
\hline $\mathrm{V} 2$ & -0.120 & 0.055 & 0.214 & -0.096 & 0.186 & -0.183 & 0.437 & 1 & & & & & & & & & \\
\hline V3 & & 0.092 & 0.162 & -0.135 & 0.227 & & 0.230 & 0.471 & 1 & & & & & & & & \\
\hline V4 & -0.068 & 0.150 & 0.121 & -0.094 & 0.184 & & 0.231 & 0.394 & 0.320 & 1 & & & & & & & \\
\hline V5 & 0.027 & 0.191 & 0.137 & -0.200 & 0.320 & 0.072 & 0.255 & 0.400 & 0.391 & 0.476 & 1 & & & & & & \\
\hline V6 & 0.158 & & & 0.140 & -0.058 & 0.119 & & -0.034 & & -0.080 & & 1 & & & & & \\
\hline V7 & 0.040 & 0.034 & & 0.078 & & 0.043 & & -0.086 & -0.130 & -0.078 & -0.079 & 0.223 & 1 & & & & \\
\hline V8 & 0.181 & 0.139 & 0.075 & -0.151 & 0.256 & 0.209 & 0.150 & 0.269 & 0.324 & 0.225 & 0.375 & & -0.027 & 1 & & & \\
\hline V9 & -0.131 & & 0.219 & -0.076 & 0.139 & -0.226 & 0.290 & 0.592 & 0.326 & 0.211 & 0.242 & 0.041 & & 0.166 & 1 & & \\
\hline V10 & -0.121 & 0.072 & 0.142 & -0.068 & 0.144 & -0.167 & 0.317 & 0.548 & 0.374 & 0.284 & 0.286 & & -0.090 & 0.137 & 0.552 & 1 & \\
\hline VT & & 0.069 & & 0.046 & -0.017 & 0.058 & & 0.083 & 0.065 & 0.249 & 0.122 & 0.073 & 0.039 & & & 0.066 & 1 \\
\hline
\end{tabular}

Tabla 8.1: Coeficiente de correlación Rho de Spearman. Características de la carrera y Métodos de enseñanza - aprendizaje

\begin{tabular}{|c|c|c|c|c|c|c|c|c|c|c|c|c|c|c|c|c|}
\hline & $\mathrm{C} 1$ & $\mathrm{C} 2$ & C3 & $\mathrm{C} 4$ & C5 & C6 & V1 & V2 & V3 & $\mathrm{V} 4$ & V5 & V6 & V7 & V8 & V9 & $\begin{array}{lll}\text { V10 } & \text { VT }\end{array}$ \\
\hline C1 & & & & & & & & & & & & & & & & \\
\hline $\mathrm{C} 2$ & 0.117 & & & & & & & & & & & & & & & \\
\hline C3 & 0.048 & 0.103 & & & & & & & & & & & & & & \\
\hline $\mathrm{C} 4$ & & 0.065 & & & & & & & & & & & & & & \\
\hline C5 & 0.081 & 0.121 & 0.089 & 0.362 & & & & & & & & & & & & \\
\hline C6 & 0.345 & 0.141 & 0.032 & & 0.090 & & & & & & & & & & & \\
\hline V1 & 0.030 & 0.061 & 0.030 & 0.044 & 0.094 & & & & & & & & & & & \\
\hline $\mathrm{V} 2$ & 0.091 & 0.054 & 0.118 & 0.064 & 0.142 & 0.133 & 0.326 & & & & & & & & & \\
\hline V3 & & 0.055 & 0.088 & 0.054 & 0.132 & & 0.121 & 0.279 & & & & & & & & \\
\hline $\mathrm{V} 4$ & & 0.115 & 0.086 & 0.061 & 0.118 & & 0.174 & 0.277 & 0.210 & & & & & & & \\
\hline V5 & 0.046 & 0.151 & 0.114 & 0.141 & 0.241 & 0.055 & 0.191 & 0.265 & 0.235 & 0.365 & & & & & & \\
\hline V6 & 0.112 & 0.086 & 0.038 & 0.087 & & 0.099 & 0.055 & 0.027 & & 0.037 & 0.083 & & & & & \\
\hline V7 & 0.030 & 0.075 & 0.037 & 0.055 & & & 0.044 & 0.028 & 0.077 & 0.052 & 0.026 & 0.207 & & & & \\
\hline V8 & 0.158 & 0.091 & 0.073 & 0.075 & 0.165 & 0.134 & 0.093 & 0.143 & 0.200 & 0.150 & 0.267 & 0.033 & & & & \\
\hline V9 & 0.080 & 0.034 & 0.146 & 0.079 & 0.105 & 0.162 & 0.221 & 0.455 & 0.212 & 0.176 & 0.193 & 0.079 & & 0.107 & & \\
\hline V10 & 0.058 & 0.057 & 0.094 & 0.053 & 0.111 & 0.109 & 0.241 & 0.424 & 0.270 & 0.211 & 0.197 & & 0.061 & 0.111 & 0.411 & \\
\hline VT & & 0.053 & 0.056 & 0.032 & & 0.029 & 0.043 & 0.053 & & 0.136 & 0.096 & 0.108 & 0.067 & & & 0.028 \\
\hline
\end{tabular}

Tabla 8.2: Coeficiente de contingencia. Características de la carrera y Métodos de enseñanza - aprendizaje 


\begin{tabular}{|c|c|c|c|c|c|c|c|c|c|c|c|c|c|c|c|c|c|}
\hline & C1 & $\mathrm{C} 2$ & C3 & $\mathrm{C} 4$ & C5 & C6 & V1 & V2 & V3 & V4 & V5 & V6 & V7 & V8 & V9 & V10 & VT \\
\hline G1 & 0.041 & 0.037 & & & 0.028 & & & 0.048 & & 0.060 & 0.047 & 0.027 & 0.034 & & 0.041 & 0.038 & 0.048 \\
\hline G2 & 0.097 & 0.029 & 0.038 & 0.036 & 0.056 & 0.072 & 0.114 & 0.255 & 0.074 & 0.364 & 0.163 & & & 0.043 & 0.131 & 0.146 & 0.161 \\
\hline G3 & 0.070 & & & 0.035 & & 0.081 & 0.115 & 0.173 & 0.094 & 0.156 & 0.124 & & & & 0.156 & 0.166 & \\
\hline G4 & 0.233 & & & 0.048 & 0.050 & 0.145 & 0.064 & 0.056 & & 0.039 & 0.052 & & & 0.089 & 0.058 & 0.042 & \\
\hline G5 & & & & 0.034 & & 0.035 & & 0.046 & 0.031 & 0.054 & 0.032 & 0.038 & 0.029 & & & & 0.052 \\
\hline $\mathrm{P} 1$ & 0.045 & & & 0.028 & & 0.143 & 0.099 & 0.149 & 0.043 & 0.117 & 0.089 & & 0.027 & 0.029 & 0.135 & 0.112 & 0.070 \\
\hline $\mathrm{P} 2$ & 0.123 & 0.050 & 0.050 & 0.038 & & 0.127 & 0.045 & 0.105 & & 0.105 & 0.047 & 0.057 & 0.053 & 0.034 & 0.093 & 0.063 & 0.040 \\
\hline P3 & 0.066 & & & 0.044 & & 0.086 & & & & 0.049 & 0.030 & 0.023 & & 0.043 & 0.043 & 0.041 & \\
\hline P4 & & & & & & 0.049 & & & & & & & 0.030 & & & & \\
\hline P5 & & & 0.029 & & & 0.085 & & 0.030 & & 0.029 & & & 0.039 & & 0.029 & & 0.027 \\
\hline P6 & & & & & & 0.067 & & 0.036 & 0.000 & 0.034 & & 0.029 & 0.032 & & 0.029 & & \\
\hline AREA & 0.378 & 0.092 & 0.168 & 0.169 & 0.169 & 0.394 & 0.205 & 0.411 & 0.198 & 0.348 & 0.257 & 0.094 & 0.102 & 0.260 & 0.397 & 0.345 & 0.371 \\
\hline CICLO & 0.061 & & & 0.096 & 0.083 & 0.116 & 0.089 & 0.137 & 0.029 & 0.236 & 0.131 & 0.137 & 0.038 & 0.112 & 0.053 & 0.078 & \\
\hline
\end{tabular}

Tabla 8.3: Coeficiente de contingencia. Características de la carrera y Métodos de enseñanza - aprendizaje frente a Gestión del tiempo y Características personales

\begin{tabular}{|c|c|c|c|c|c|c|}
\hline & G2 & G3 $\quad$ G4 & G5 & P2 & P3 & P5 \\
\hline G1 & & & & & & \\
\hline G2 & 0.076 & & & & & \\
\hline G3 & 0.1230 .152 & & & & & \\
\hline G4 & 0.145 & 0.145 & & & & \\
\hline G5 & 0.035 & 50.094 & & & & \\
\hline P1 & $\begin{array}{lll}0.066 & 0.124\end{array}$ & 40.054 & & & & \\
\hline P2 & $\begin{array}{lll}0.374 & 0.154\end{array}$ & $\begin{array}{lll}40.213 & 0.088\end{array}$ & 0.040 & 0.114 & & \\
\hline P3 & $\begin{array}{lll}0.145 & 0.042\end{array}$ & & 0.085 & $0.086 \quad 0.209$ & & \\
\hline P4 & 0.209 & 0.0440 .046 & 0.038 & 0.328 & 0.331 & \\
\hline P5 & 0.044 & 0.082 & 0.164 & 0.053 & $\begin{array}{lll}0.069 & 0.025\end{array}$ & \\
\hline P6 & 0.044 & 0.081 & 0.151 & 0.043 & 0.044 & 0.575 \\
\hline AREA & $\begin{array}{lll}0.150 & 0.437\end{array}$ & $\begin{array}{llll}7 & 0.251 & 0.241\end{array}$ & 0.180 & $0.289 \quad 0.200$ & 0.136 & 0.1350 .109 \\
\hline CICLO & 0.0460 .194 & & 0.162 & 0.078 & 0.049 & $\begin{array}{lll}0.143 & 0.117\end{array}$ \\
\hline
\end{tabular}

Tabla 8.4: Coeficiente de contingencia. Gestión del tiempo y Características personales del individuo

\begin{tabular}{|c|c|c|c|c|c|c|c|c|c|c|c|c|c|c|c|}
\hline & $\mathrm{C} 1$ & $\mathrm{C} 2$ & $\mathrm{C} 3$ & $\mathrm{C} 4$ & $\mathrm{C} 5$ & C6 & V1 & V2 & V3 & V4 & V5 & V6 & V7 & V9 & V10 VT \\
\hline C1 & & & & & & & & & & & & & & & \\
\hline $\mathrm{C} 2$ & 0.118 & & & & & & & & & & & & & & \\
\hline C3 & 0.048 & 0.103 & & & & & & & & & & & & & \\
\hline C4 & & 0.065 & & & & & & & & & & & & & \\
\hline C5 & 0.081 & 0.122 & 0.089 & 0.388 & & & & & & & & & & & \\
\hline C6 & 0.368 & 0.143 & 0.032 & & 0.091 & & & & & & & & & & \\
\hline V1 & 0.030 & 0.061 & 0.030 & 0.044 & 0.094 & & & & & & & & & & \\
\hline $\mathrm{V} 2$ & 0.091 & 0.054 & 0.119 & 0.064 & 0.143 & 0.135 & 0.344 & & & & & & & & \\
\hline V3 & & 0.055 & 0.089 & 0.054 & 0.134 & & 0.122 & 0.291 & & & & & & & \\
\hline $\mathrm{V} 4$ & & 0.116 & 0.087 & 0.061 & 0.119 & & 0.177 & 0.288 & 0.215 & & & & & & \\
\hline V5 & 0.046 & 0.153 & 0.115 & 0.142 & 0.248 & 0.056 & 0.194 & 0.275 & 0.242 & 20.392 & & & & & \\
\hline V6 & 0.112 & 0.086 & 0.038 & 0.087 & & 0.099 & 0.055 & 0.027 & & 0.037 & 0.083 & & & & \\
\hline V7 & 0.030 & 0.075 & 0.037 & 0.055 & & & 0.044 & 0.028 & 0.078 & 0.052 & & 0.211 & & & \\
\hline V8 & 0.160 & 0.091 & 0.073 & 0.075 & 0.167 & 0.135 & 0.093 & 0.144 & 0.204 & 40.152 & 0.277 & 0.033 & & & \\
\hline V9 & 0.080 & 0.034 & 0.148 & 0.079 & 0.106 & 0.164 & 0.227 & 0.511 & 0.217 & 70.179 & 0.197 & 0.079 & & 0.108 & \\
\hline V10 & 0.058 & 0.057 & 0.095 & 0.053 & 0.112 & 0.110 & 0.248 & 0.468 & 0.280 & 0.216 & 0.200 & & 0.062 & 0.1120 .451 & \\
\hline VT & & 0.053 & 0.056 & 0.032 & & 0.029 & 0.043 & 0.053 & & 0.138 & 0.096 & 0.108 & 0.067 & & 0.028 \\
\hline
\end{tabular}

Tabla 8.5: V de Cramer. Características de la carrera y Métodos de enseñan$z a-$ aprendizaje 


\begin{tabular}{|c|c|c|c|c|c|c|c|c|c|c|c|c|c|c|c|c|c|}
\hline & C1 & C2 & C3 & $\mathrm{C} 4$ & C5 & C6 & V1 & V2 & V3 & V4 & V5 & V6 & V7 & V8 & V9 & V10 & VT \\
\hline G1 & 0.041 & 0.037 & & & 0.028 & & & 0.048 & & 0.060 & 0.047 & 0.027 & 70.034 & & 0.041 & 0.038 & 0.048 \\
\hline G2 & 0.098 & 0.029 & 0.038 & 0.036 & 0.056 & 0.073 & 0.115 & 0.264 & 0.074 & 0.390 & 0.165 & & & 0.043 & 0.133 & 0.148 & 0.164 \\
\hline G3 & 0.070 & & & 0.036 & & 0.082 & 0.115 & 0.176 & 0.094 & 0.158 & 0.125 & & & 0.028 & 0.158 & 0.168 & \\
\hline G4 & 0.239 & & & 0.048 & 0.050 & 0.147 & 0.064 & 0.056 & & 0.039 & 0.052 & & & 0.089 & 0.058 & 0.042 & \\
\hline G5 & & & & 0.034 & & 0.035 & & 0.046 & 0.031 & 0.054 & 0.032 & 0.038 & 30.029 & & & & 0.052 \\
\hline P1 & 0.045 & & & 0.028 & & 0.144 & 0.100 & 0.151 & 0.044 & 0.117 & 0.089 & & 0.027 & 0.029 & 0.137 & 0.113 & 0.070 \\
\hline P2 & 0.124 & 0.050 & 0.050 & 0.038 & & 0.128 & 0.045 & 0.105 & & 0.105 & 0.048 & 0.058 & 0.053 & & 0.093 & 0.063 & 0.040 \\
\hline P3 & 0.066 & & & 0.044 & & 0.086 & & & & 0.049 & & & & 0.043 & 0.043 & 0.041 & \\
\hline $\mathrm{P} 4$ & & & & & & 0.049 & & & & & & & 0.030 & & & & \\
\hline P5 & & & 0.029 & & & 0.085 & & 0.030 & & 0.029 & & & 0.039 & & 0.029 & & 0.027 \\
\hline P6 & & & & & & 0.067 & & 0.036 & & 0.034 & & 0.029 & 0.032 & & 0.029 & & \\
\hline AREA & 0.408 & 0.092 & 0.171 & 0.172 & 0.171 & 10.429 & 0.210 & 0.450 & 0.202 & 0.371 & 0.266 & 0.095 & 0.102 & 0.269 & 0.432 & 0.367 & 0.400 \\
\hline CICLO & 0.061 & & & 0.096 & 0.083 & 30.116 & 0.089 & 0.138 & 0.029 & 0.243 & 0.132 & 0.139 & 0.038 & 0.113 & 0.053 & 0.078 & \\
\hline
\end{tabular}

Tabla 8.6: V de Cramer. Características de la carrera y Métodos de enseñanza-aprendizaje frente a Gestión del tiempo y Características personales

\begin{tabular}{|c|c|c|c|c|c|c|c|c|c|}
\hline & G2 & G4 & G5 & P1 & $\mathrm{P} 2$ & P3 & P4 & P5 & $\mathrm{P} 6$ \\
\hline G1 & & & & & & & & & \\
\hline G2 & 0.077 & & & & & & & & \\
\hline G3 & 0.1240 .154 & & & & & & & & \\
\hline G4 & 0.146 & 0.104 & & & & & & & \\
\hline G5 & 0.035 & 50.094 & & & & & & & \\
\hline $\mathrm{P} 1$ & $\begin{array}{lll}0.066 & 0.125\end{array}$ & 50.055 & & & & & & & \\
\hline P2 & $\begin{array}{lll}0.403 & 0.156\end{array}$ & 0.1550 .062 & 0.040 & 0.115 & & & & & \\
\hline P3 & 0.1460 .042 & & 0.086 & 0.086 & 0.151 & & & & \\
\hline $\mathrm{P} 4$ & 0.213 & 0.0440 .046 & 0.038 & & 0.347 & 0.351 & & & \\
\hline P5 & 0.044 & 0.082 & 0.167 & & 0.053 & 0.069 & & & \\
\hline P6 & 0.044 & 0.081 & 0.153 & & 0.043 & 0.044 & & 0.702 & \\
\hline AREA & $\begin{array}{lll}0.152 & 0.486\end{array}$ & 0.1830 .143 & 0.183 & 0.302 & 0.144 & 0.079 & & 0.136 & 0.110 \\
\hline CICLO & $\begin{array}{lll}0.046 & 0.197\end{array}$ & & 0.164 & & 0.079 & 0.049 & & 0.144 & 0.118 \\
\hline
\end{tabular}

Tabla 8.7: V de Cramer. Gestión del tiempo y Características personales

\begin{tabular}{|c|c|c|c|c|c|c|c|c|c|c|c|c|c|c|c|c|}
\hline & $\mathrm{C} 1$ & $\mathrm{C} 2$ & C3 & $\mathrm{C} 4$ & C5 & C6 & V1 & V2 & V3 & V4 & V5 & V6 & V7 & V8 & V9 & V10 VT \\
\hline C1 & & & & & & & & & & & & & & & & \\
\hline $\mathrm{C} 2$ & 0.000 & & & & & & & & & & & & & & & \\
\hline C3 & 0.000 & 0.000 & & & & & & & & & & & & & & \\
\hline $\mathrm{C} 4$ & & 0.027 & & & & & & & & & & & & & & \\
\hline C5 & 0.000 & 0.000 & 0.000 & 0.220 & & & & & & & & & & & & \\
\hline $\mathrm{C} 6$ & 0.123 & 0.000 & 0.000 & & 0.000 & & & & & & & & & & & \\
\hline V1 & 0.000 & 0.000 & 0.000 & 0.017 & 0.000 & & & & & & & & & & & \\
\hline $\mathrm{V} 2$ & 0.004 & 0.000 & 0.000 & 0.028 & 0.000 & 0.000 & 0.120 & & & & & & & & & \\
\hline V3 & & 0.000 & 0.000 & 0.023 & 0.000 & & 0.008 & 0.113 & & & & & & & & \\
\hline V4 & & 0.000 & 0.000 & 0.029 & 0.000 & & 0.000 & 0.069 & 0.000 & & & & & & & \\
\hline V5 & 0.000 & 0.000 & 0.000 & 0.075 & 0.000 & 0.000 & 0.000 & 0.0260 & 0.066 & 0.112 & & & & & & \\
\hline V6 & 0.012 & 0.000 & 0.000 & 0.040 & & 0.000 & 0.000 & 0.000 & & 0.000 & 0.000 & & & & & \\
\hline V7 & 0.000 & 0.000 & 0.000 & 0.021 & & & 0.000 & 0.000 & 0.001 & 0.000 & 0.000 & 0.016 & & & & \\
\hline V8 & 0.000 & 0.000 & 0.000 & 0.037 & 0.000 & 0.000 & 0.000 & 0.000 & 0.000 & 0.000 & 0.032 & 0.000 & & & & \\
\hline V9 & 0.000 & 0.000 & 0.000 & 0.036 & 0.000 & 0.000 & 0.007 & 0.352 & 0.075 & 0.015 & 0.001 & 0.000 & & 0.000 & & \\
\hline V10 & 0.000 & 0.000 & 0.000 & 0.025 & 0.000 & 0.000 & 0.056 & 0.208 & 0.024 & 0.000 & 0.000 & & 0.000 & 0.000 & 0.211 & \\
\hline VT & & 0.000 & 0.000 & 0.000 & & 0.000 & 0.000 & 0.000 & & 0.000 & 0.000 & 0.000 & 0.000 & & & 0.000 \\
\hline
\end{tabular}

Tabla 8.8: Medida Lambda de Goodman y Kruskal simétrica. Características de la carrera y Métodos de enseñanza - aprendizaje 


\begin{tabular}{|c|c|c|c|c|c|c|c|c|c|c|c|c|c|c|c|c|c|}
\hline & C1 & $\mathrm{C} 2$ & $\mathrm{C} 3$ & C4 & C5 & C6 & V1 & $\mathrm{V} 2$ & V3 & V4 & V5 & V6 & V7 & V8 & V9 & V10 & VT \\
\hline G1 & 0.000 & 0.000 & & & 0.000 & & & 0.000 & & 0.000 & 0.000 & 0.000 & 0.000 & & 0.000 & 0.000 & 0.000 \\
\hline G2 & 0.000 & 0.000 & 0.000 & 0.005 & 0.000 & 0.000 & 0.000 & 0.054 & 0.000 & 0.096 & 0.000 & & & 0.000 & 0.000 & 0.000 & 0.000 \\
\hline G3 & 0.023 & & & 0.012 & & 0.031 & 0.043 & 0.078 & 0.011 & 0.062 & 0.043 & & & & 0.067 & 0.064 & \\
\hline G4 & 0.039 & & & 0.012 & 0.000 & 0.009 & 0.000 & 0.000 & & 0.000 & 0.000 & & & 0.000 & 0.000 & 0.000 & \\
\hline G5 & & & & 0.000 & & 0.000 & & 0.000 & 0.000 & 0.000 & 0.000 & 0.000 & 0.000 & & & & 0.000 \\
\hline P1 & 0.000 & & & 0.000 & & 0.000 & 0.000 & 0.000 & 0.000 & 0.000 & 0.000 & & 0.000 & 0.000 & 0.000 & 0.000 & 0.000 \\
\hline $\mathrm{P} 2$ & 0.000 & 0.000 & 0.000 & 0.005 & & 0.000 & 0.000 & 0.000 & & 0.000 & 0.000 & 0.000 & 0.000 & 0.000 & 0.000 & 0.000 & 0.000 \\
\hline P3 & 0.000 & & & 0.007 & & 0.000 & & & & 0.000 & 0.000 & 0.000 & & 0.000 & 0.000 & 0.000 & \\
\hline $\mathrm{P} 4$ & & & & & & 0.000 & & & & & & & 0.000 & & & & \\
\hline P5 & & & 0.000 & & & 0.000 & & 0.000 & & 0.000 & & & 0.000 & & 0.000 & & 0.000 \\
\hline P6 & & & & & & 0.000 & & 0.000 & 0.000 & 0.000 & & 0.000 & 0.000 & & 0.000 & & \\
\hline AREA & 0.153 & 0.000 & 0.014 & 0.057 & 0.022 & 0.113 & 0.018 & 0.129 & 0.018 & 0.029 & 0.029 & 0.005 & 0.004 & 0.05 & 0.122 & 0.04 & 0.080 \\
\hline CICLO & 0.000 & & & 0.042 & 0.002 & 0.000 & 0.000 & 0.023 & 0.000 & 0.106 & 0.025 & 0.017 & 0.000 & 0.026 & 0.000 & 0.006 & \\
\hline
\end{tabular}

Tabla 8.9: Medida Lambda de Goodman y Kruskal simétrica. Características de la carrera y Métodos de enseñanza - aprendizaje frente a Gestión del tiempo y Características personales

\begin{tabular}{|c|c|c|c|c|c|c|}
\hline & G2 & G4 & G5 & P2 & P3 & P5 \\
\hline G1 & & & & & & \\
\hline G2 & 0.012 & & & & & \\
\hline G3 & 0.0540 .081 & & & & & \\
\hline G4 & 0.000 & 0.037 & & & & \\
\hline G5 & 0.000 & 0.006 & & & & \\
\hline P1 & 0.0000 .021 & 1 0.021 & & & & \\
\hline P2 & $\begin{array}{lll}0.118 & 0.029\end{array}$ & 0.0940 .000 & 0.000 & 0.000 & & \\
\hline P3 & 0.0000 .000 & & 0.000 & $0.000 \quad 0.054$ & & \\
\hline P4 & 0.000 & 0.0180 .000 & 0.000 & 0.054 & 0.047 & \\
\hline P5 & 0.000 & 0.000 & 0.000 & 0.000 & 0.0000 .000 & \\
\hline P6 & 0.000 & 0.000 & 0.000 & 0.000 & 0.000 & 0.452 \\
\hline AREA & $0.008 \quad 0.140$ & 0.0810 .053 & 0.011 & 0.0720 .027 & 0.027 & 0.0150 .005 \\
\hline CICLO & $0.000 \quad 0.050$ & & 0.000 & 0.000 & 0.000 & $0.000 \quad 0.000$ \\
\hline
\end{tabular}

Tabla 8.10: Medida Lambda de Goodman y Kruskal simétrica. Gestión del tiempo y Características personales

\begin{tabular}{|c|c|c|c|c|c|c|c|c|c|c|c|c|c|c|c|c|}
\hline & $\mathrm{C} 1$ & $\mathrm{C} 2$ & C3 & $\mathrm{C} 4$ & $\mathrm{C} 5$ & $\mathrm{C} 6$ & V1 & V2 & V3 & V4 & V5 & V6 & V7 & V8 & V9 & $\mathrm{V} 10 \mathrm{VT}$ \\
\hline C1 & & & & & & & & & & & & & & & & \\
\hline $\mathrm{C} 2$ & 0.010 & & & & & & & & & & & & & & & \\
\hline C3 & 0.002 & 0.008 & & & & & & & & & & & & & & \\
\hline C4 & & 0.003 & & & & & & & & & & & & & & \\
\hline C5 & 0.005 & 0.012 & 0.007 & 0.130 & & & & & & & & & & & & \\
\hline C6 & 0.108 & 0.015 & 0.001 & & 0.007 & & & & & & & & & & & \\
\hline V1 & 0.001 & 0.003 & 0.001 & 0.001 & 0.007 & & & & & & & & & & & \\
\hline $\mathrm{V} 2$ & 0.006 & 0.002 & 0.011 & 0.003 & 0.017 & 0.015 & 0.091 & & & & & & & & & \\
\hline V3 & & 0.003 & 0.008 & 0.003 & 0.019 & & 0.015 & 0.085 & & & & & & & & \\
\hline V4 & & 0.011 & 0.007 & 0.003 & 0.013 & & 0.026 & 0.069 & 0.048 & & & & & & & \\
\hline V5 & 0.002 & 0.018 & 0.011 & 0.016 & 0.051 & 0.002 & 0.030 & 0.059 & 0.058 & 0.127 & & & & & & \\
\hline V6 & 0.009 & 0.006 & 0.001 & 0.006 & & 0.008 & 0.002 & 0.001 & & 0.001 & 0.006 & & & & & \\
\hline V7 & 0.001 & 0.004 & 0.001 & 0.002 & & & 0.002 & 0.001 & 0.006 & 50.002 & 0.001 & 0.033 & & & & \\
\hline V8 & 0.022 & 0.007 & 0.005 & 0.005 & 0.025 & 0.015 & 0.007 & 0.018 & 0.044 & 40.021 & 0.065 & 0.001 & & & & \\
\hline V9 & 0.005 & 0.001 & 0.017 & 0.005 & 0.009 & 0.021 & 0.039 & 0.204 & 0.047 & 70.027 & 0.030 & 0.005 & & 0.010 & & \\
\hline V10 & 0.003 & 0.003 & 0.008 & 0.002 & 0.011 & 0.011 & 0.052 & 0.188 & 0.079 & 9.042 & 0.035 & & 0.003 & 0.012 & 0.177 & \\
\hline VT & & 0.002 & 0.003 & 0.001 & & 0.001 & 0.002 & 0.002 & & 0.016 & 0.008 & 0.010 & 0.004 & & & 0.001 \\
\hline
\end{tabular}

Tabla 8.11: Coeficiente de incertidumbre simétrico. Características de la carrera y Métodos de enseñanza - aprendizaje 


\begin{tabular}{|c|c|c|c|c|c|c|c|c|c|c|c|c|c|c|c|c|c|}
\hline & $\mathrm{C} 1$ & $\mathrm{C} 2$ & C3 & $\mathrm{C} 4$ & C5 & C6 & V1 & V2 & V3 & V4 & V5 & V6 & V7 & V8 & V9 & V10 & VT \\
\hline G1 & 0.001 & 0.001 & & & 0.001 & & & 0.002 & & 0.004 & 0.002 & 0.001 & 10.001 & & 0.002 & 0.002 & 0.002 \\
\hline G2 & 0.007 & 0.001 & 0.001 & 0.001 & 0.003 & 0.004 & 0.010 & 0.055 & 0.006 & 0.152 & 0.022 & & & 0.002 & 0.013 & 0.020 & 0.021 \\
\hline G3 & 0.003 & & & 0.001 & & 0.004 & 0.009 & 0.021 & 0.008 & 0.019 & 0.011 & & & & 0.017 & 0.022 & \\
\hline G4 & 0.029 & & & 0.001 & 0.001 & 0.011 & 0.002 & 0.002 & & 0.001 & 0.001 & & & 0.004 & 0.002 & 0.001 & \\
\hline G5 & & & & 0.001 & & 0.001 & & 0.002 & 0.002 & 0.004 & 0.001 & 0.001 & 10.001 & & & & 0.003 \\
\hline $\mathrm{P} 1$ & 0.002 & & & 0.001 & & 0.016 & 0.008 & 0.019 & 0.002 & 0.013 & 0.007 & & 0.001 & 0.001 & 0.015 & 0.012 & 0.004 \\
\hline P2 & 0.010 & 0.002 & 0.002 & 0.001 & & 0.011 & 0.001 & 0.008 & & 0.009 & 0.002 & 0.002 & 20.002 & 0.001 & 0.006 & 0.003 & 0.001 \\
\hline P3 & 0.002 & & & 0.001 & & 0.004 & & & & 0.001 & 0.001 & 0.000 & & 0.001 & 0.001 & 0.001 & \\
\hline P4 & & & & & & 0.002 & & & & & & & 0.001 & & & & \\
\hline P5 & & & 0.001 & & & 0.006 & & 0.001 & & 0.001 & & & 0.001 & & 0.001 & & 0.001 \\
\hline P6 & & & & & & 0.004 & & 0.001 & 0.000 & 0.001 & & 0.001 & 0.001 & & 0.001 & & \\
\hline AREA & 0.066 & 0.003 & 0.012 & 0.011 & 0.012 & 0.074 & 0.017 & 0.077 & 0.018 & 30.056 & 0.028 & 0.003 & 30.004 & 0.029 & 0.071 & 0.051 & 0.062 \\
\hline CICLO & 0.003 & & & 0.007 & 0.006 & 0.010 & 0.006 & 0.014 & 0.001 & 0.049 & 0.014 & 0.014 & 40.001 & 0.011 & 0.002 & 0.005 & \\
\hline
\end{tabular}

Tabla 8.12: Coeficiente de incertidumbre simétrico. Características de la carrera y Métodos de enseñanza - aprendizaje frente a Gestión del tiempo y Características personales

\begin{tabular}{|c|c|c|c|c|c|c|c|c|}
\hline & G2 & G4 & G5 & P2 & P3 & $\mathrm{P} 4$ & P5 & P6 \\
\hline G1 & & & & & & & & \\
\hline G2 & 0.005 & & & & & & & \\
\hline G3 & $\begin{array}{lll}0.012 & 0.015\end{array}$ & & & & & & & \\
\hline G4 & 0.012 & 0.010 & & & & & & \\
\hline G5 & 0.001 & 0.007 & & & & & & \\
\hline P1 & $\begin{array}{lll}0.004 & 0.012\end{array}$ & 0.002 & & & & & & \\
\hline P2 & 0.1090 .016 & 0.0290 .003 & 0.001 & 0.009 & & & & \\
\hline P3 & 0.0140 .001 & & 0.005 & 0.0040 .024 & & & & \\
\hline P4 & 0.047 & 0.0020 .001 & 0.002 & 0.076 & 0.094 & & & \\
\hline P5 & 0.002 & 0.004 & 0.027 & 0.002 & 0.003 & 0.001 & & \\
\hline P6 & 0.002 & 0.004 & 0.025 & 0.002 & 0.001 & & 0.485 & \\
\hline AREA & $\begin{array}{lll}0.010 & 0.098\end{array}$ & 0.0230 .018 & 0.013 & $\begin{array}{lll}0.035 & 0.015\end{array}$ & 0.006 & & 0.007 & 0.005 \\
\hline CICLO & $\begin{array}{lll}0.002 & 0.029\end{array}$ & & 0.027 & 0.004 & 0.001 & & 0.017 & 0.013 \\
\hline
\end{tabular}

Tabla 8.13: Coeficiente de incertidumbre simétrico. Gestión del tiempo y Características personales 


\subsection{Anexo D: Relaciones entre variables contex- tuales y factores competenciales. Figuras}

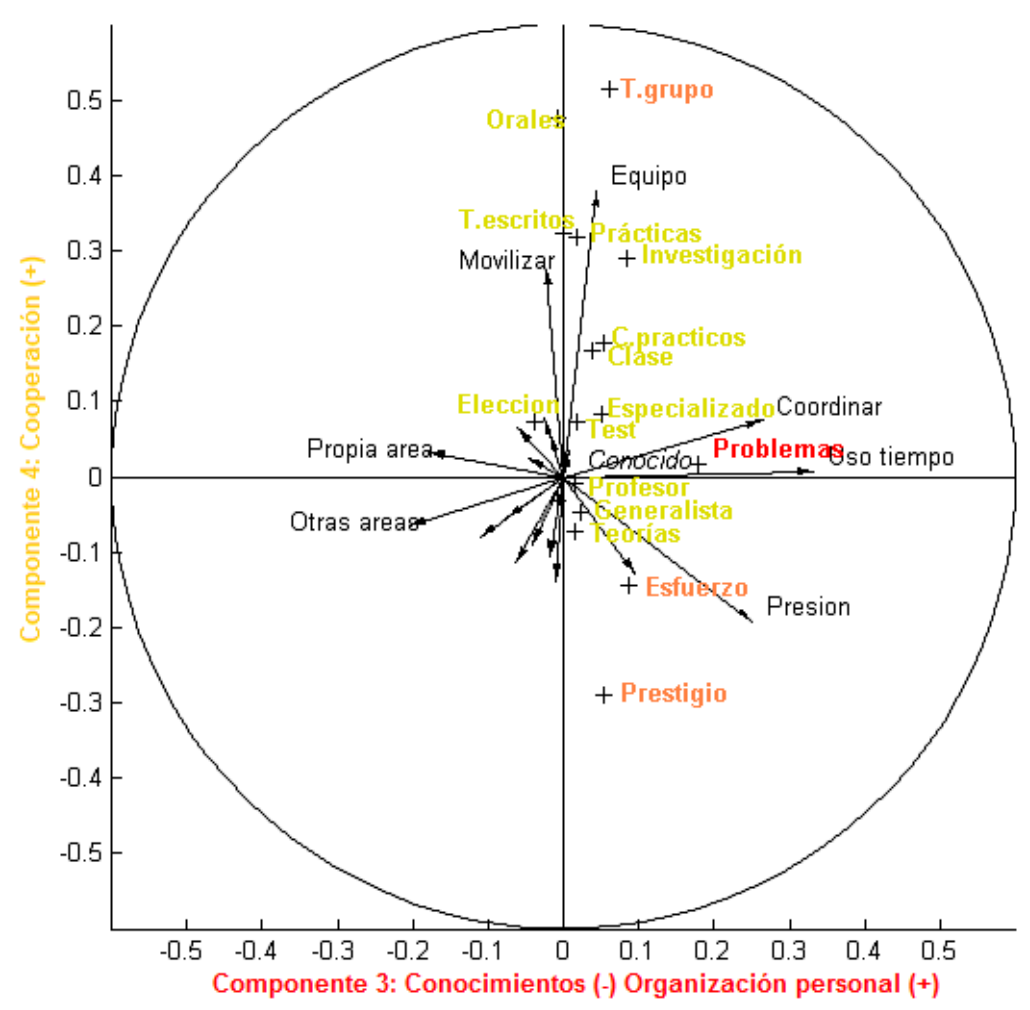

Figura 8.1: Representación simultánea sobre las componentes principales 3 y 4 de Competencias adquiridas en la Universidad. Características de los estudios y Métodos de enseñanza - aprendizaje 


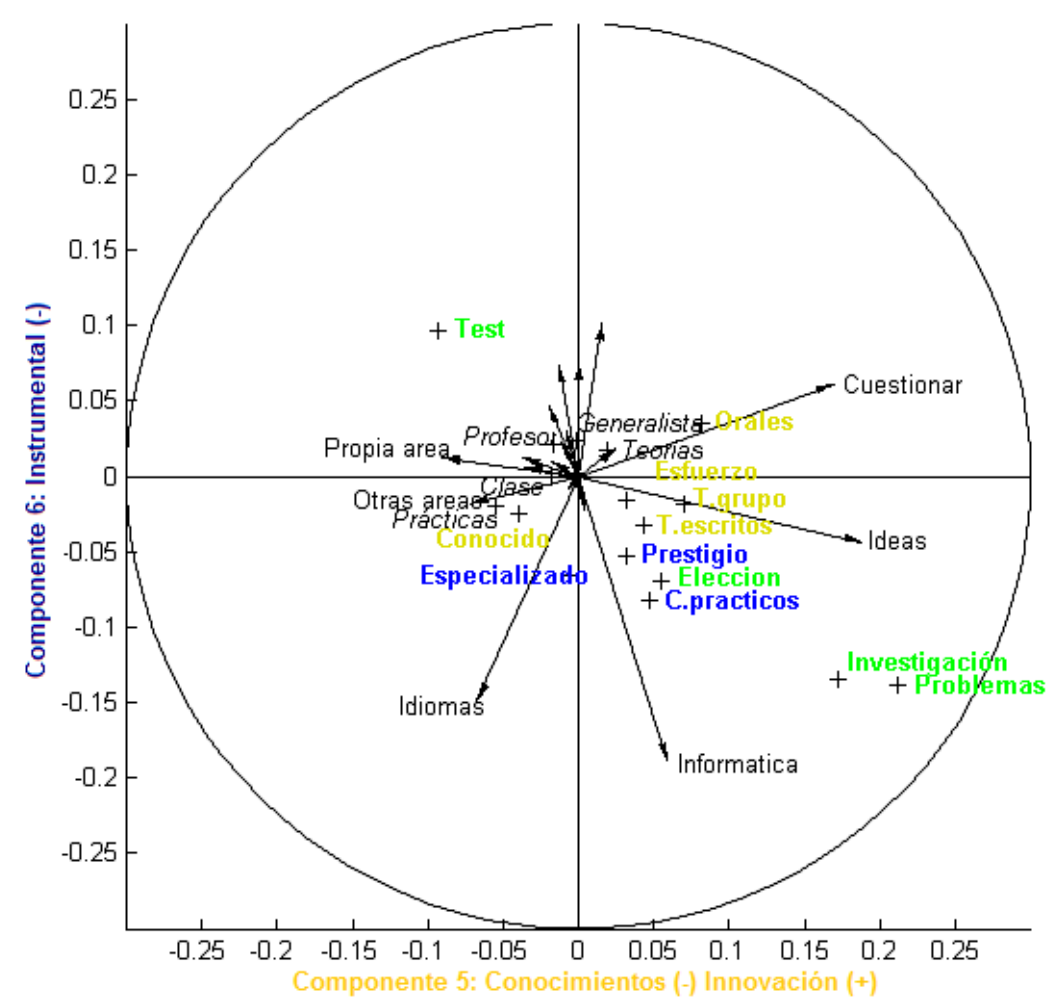

Figura 8.2: Representación simultánea sobre las componentes principales 5 y 6 de Competencias adquiridas en la Universidad. Características de los estudios y Métodos de enseñanza - aprendizaje 


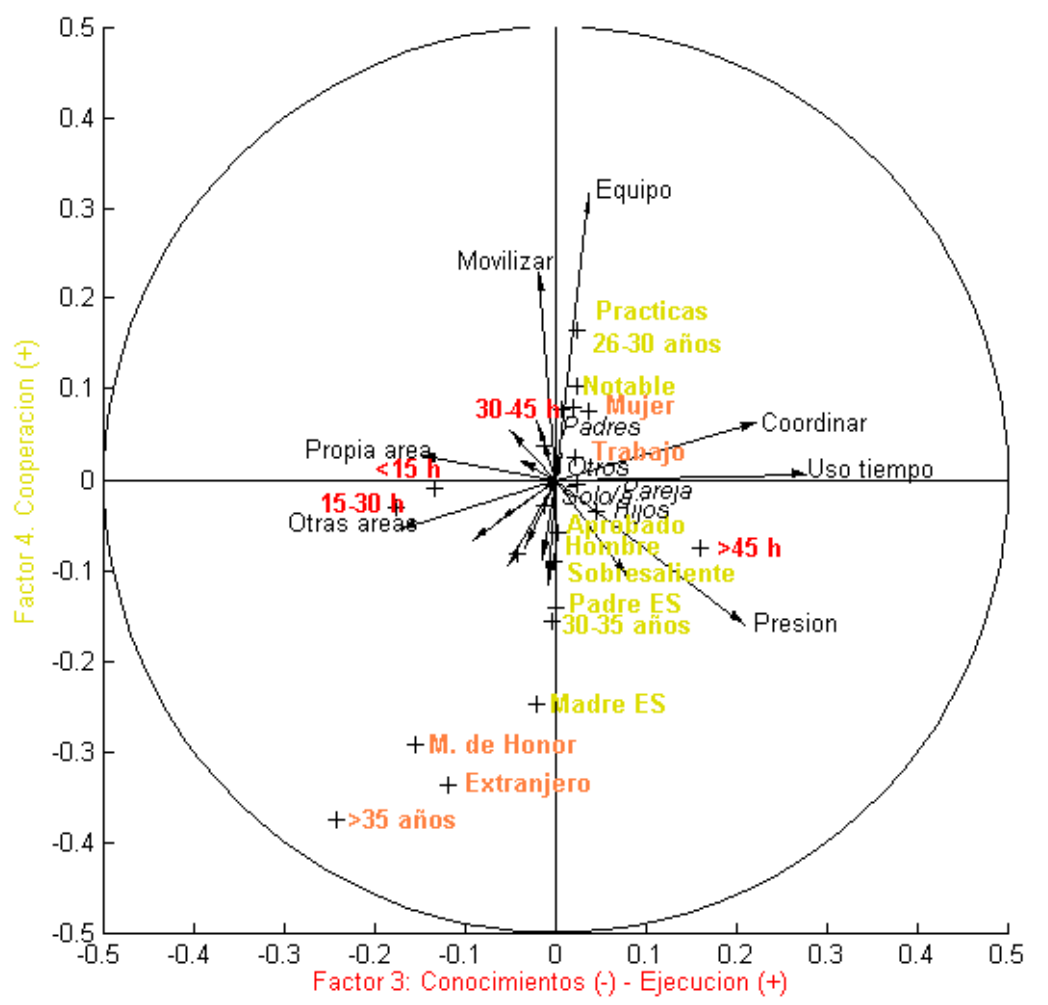

Figura 8.3: Representación simultánea sobre las componentes principales 3 y 4 de Competencias adquiridas en la Universidad. Gestión del tiempo y Características personales 


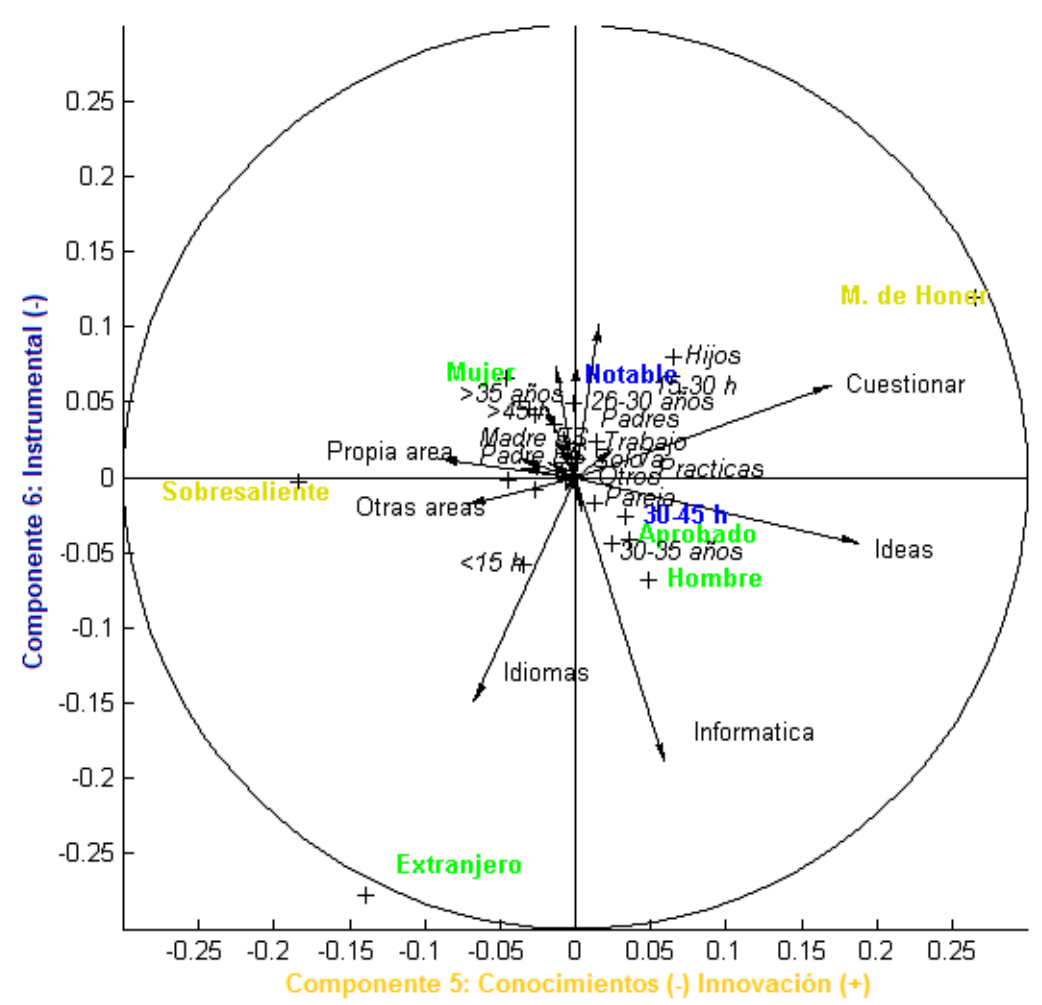

Figura 8.4: Representación simultánea sobre las componentes principales 5 y 6 de Competencias adquiridas en la Universidad. Gestión del tiempo y Características personales 


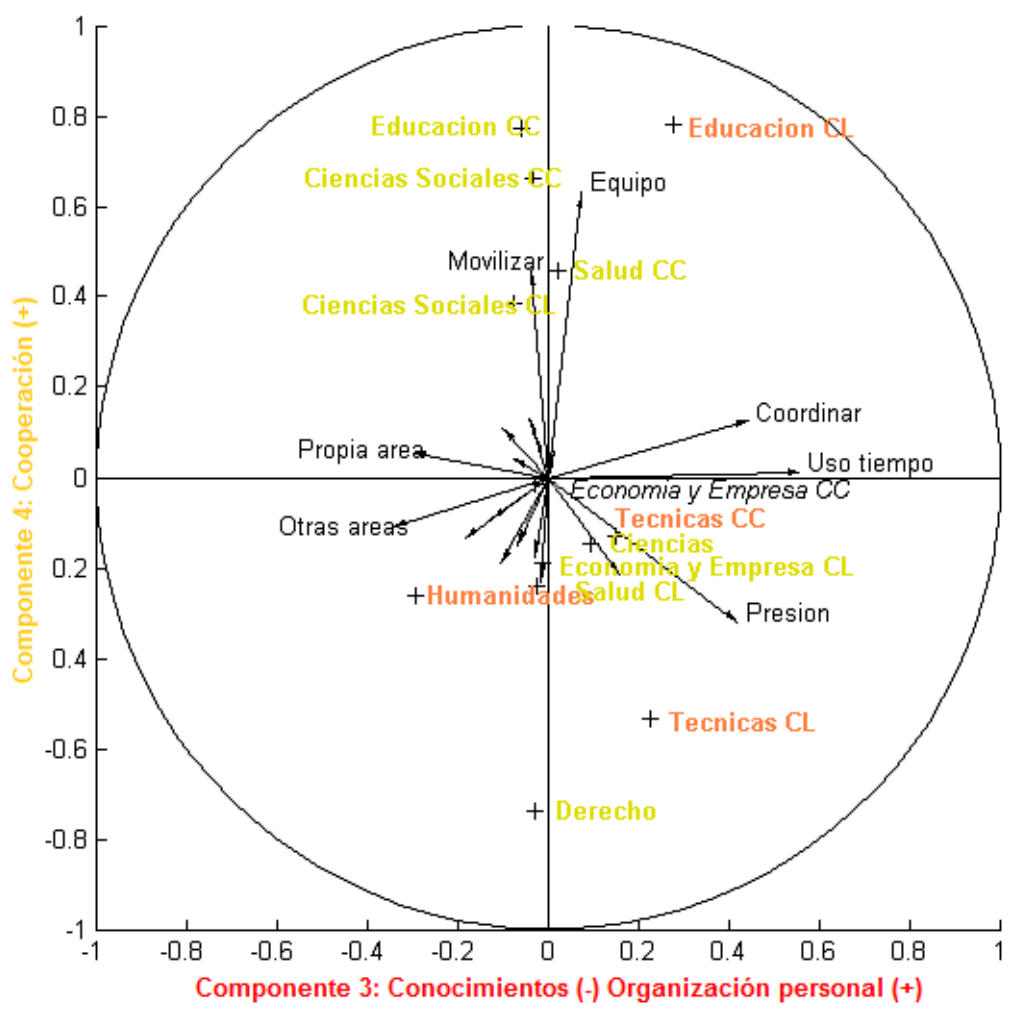

Figura 8.5: Representación simultánea sobre las componentes principales 3 y 4 de Competencias adquiridas en la Universidad. Área y nivel de estudios 


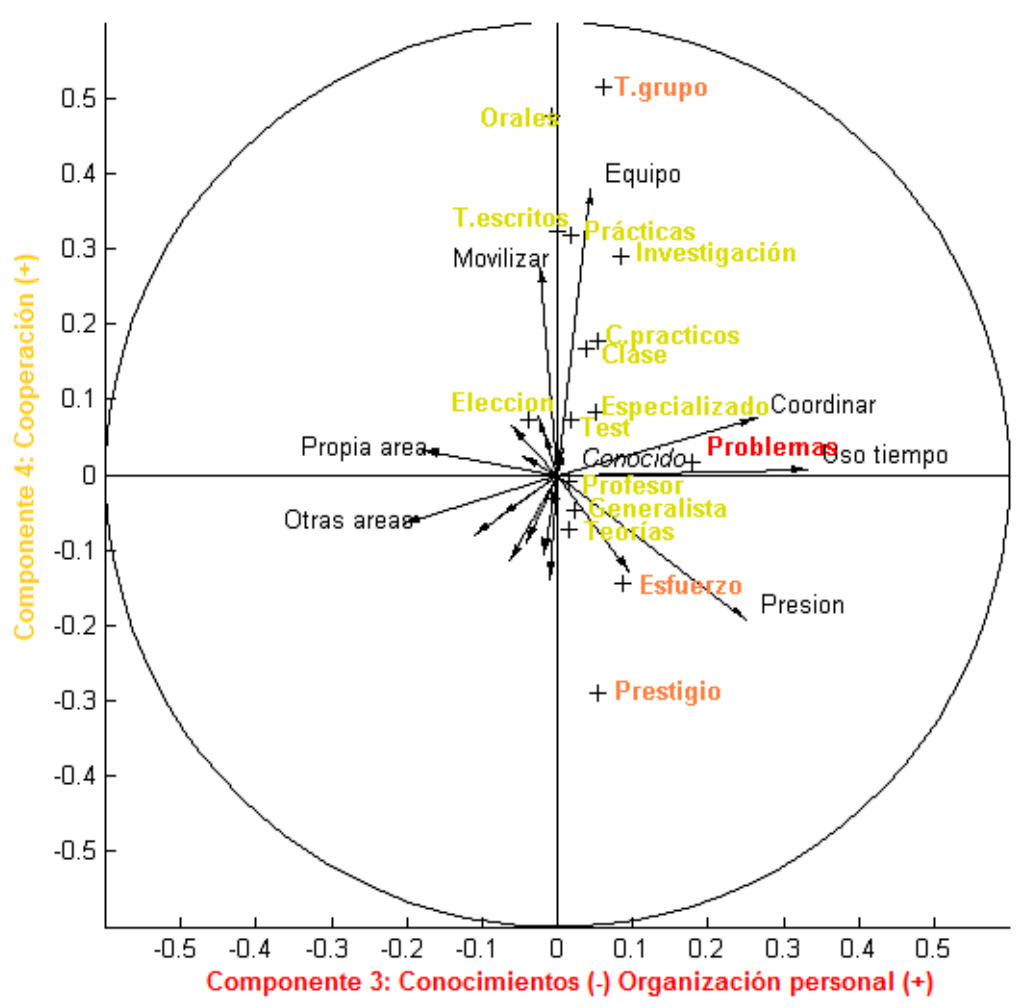

Figura 8.6: Representación simultánea sobre las componentes principales 5 y 6 de Competencias adquiridas en la Universidad. Área y nivel de estudios 


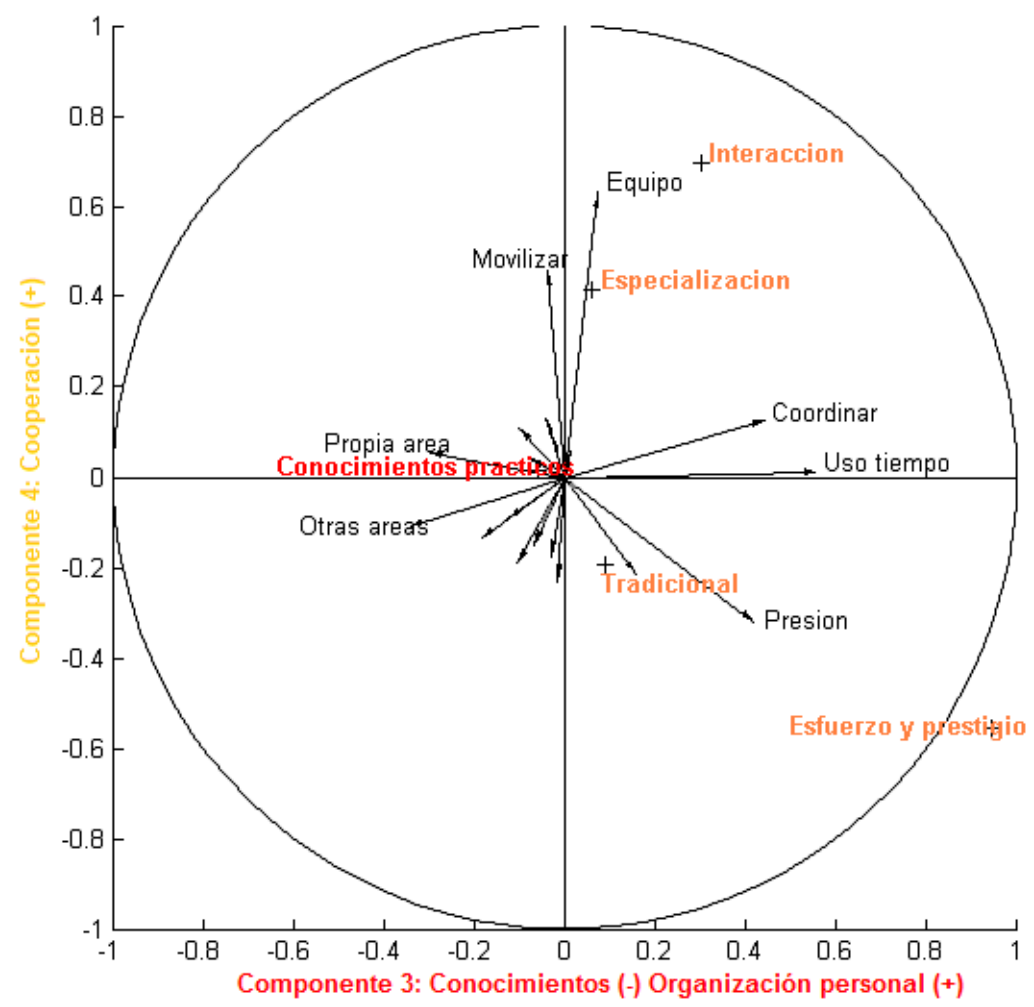

Figura 8.7: Representación simultánea sobre las componentes principales 3 y 4 de Competencias adquiridas en la Universidad. Características de la carrera y Métodos de enseñanza - aprendizaje 


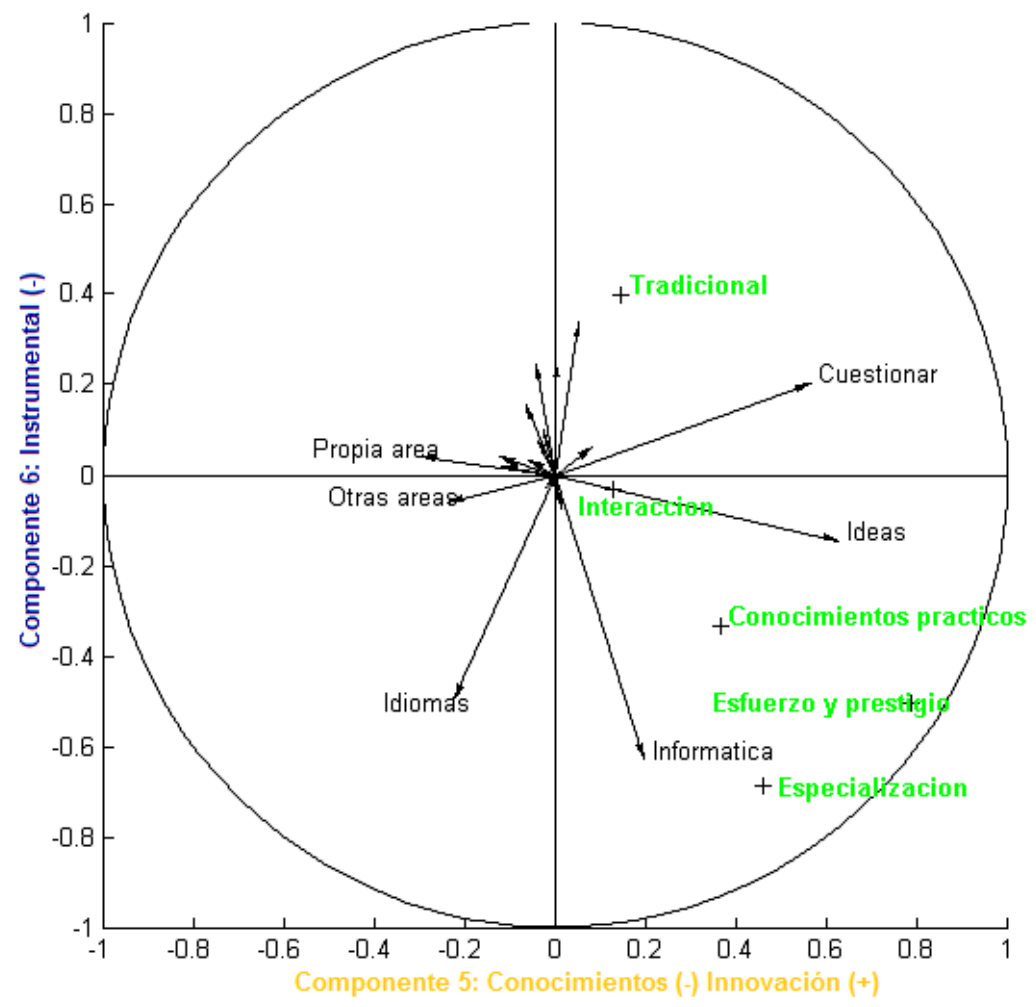

Figura 8.8: Representación simultánea sobre las componentes principales 5 y 6 de Competencias adquiridas en la Universidad. Características de la carrera y Métodos de enseñanza - aprendizaje 


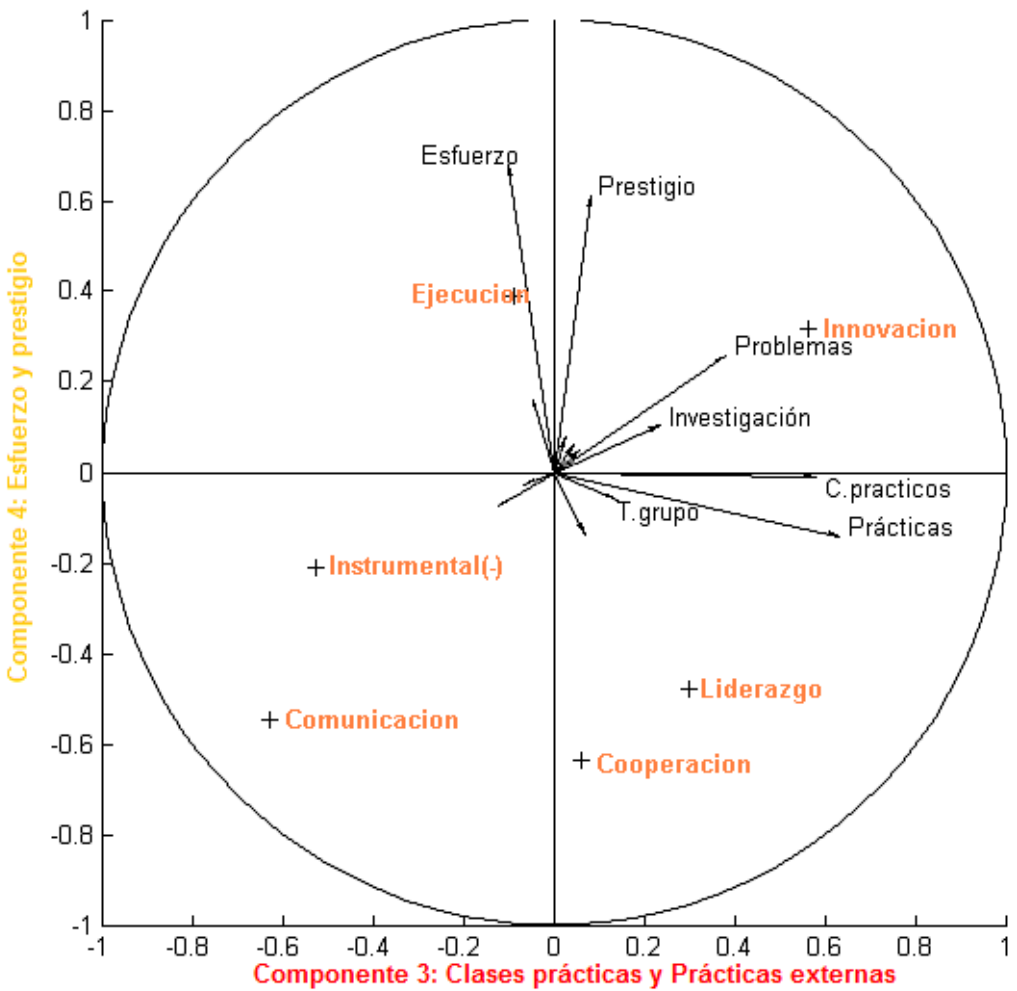

Figura 8.9: Representación simultánea sobre las componentes principales 3 y 4 de Competencias adquiridas en la Universidad. Características de la carrera y Métodos de enseñanza - aprendizaje 


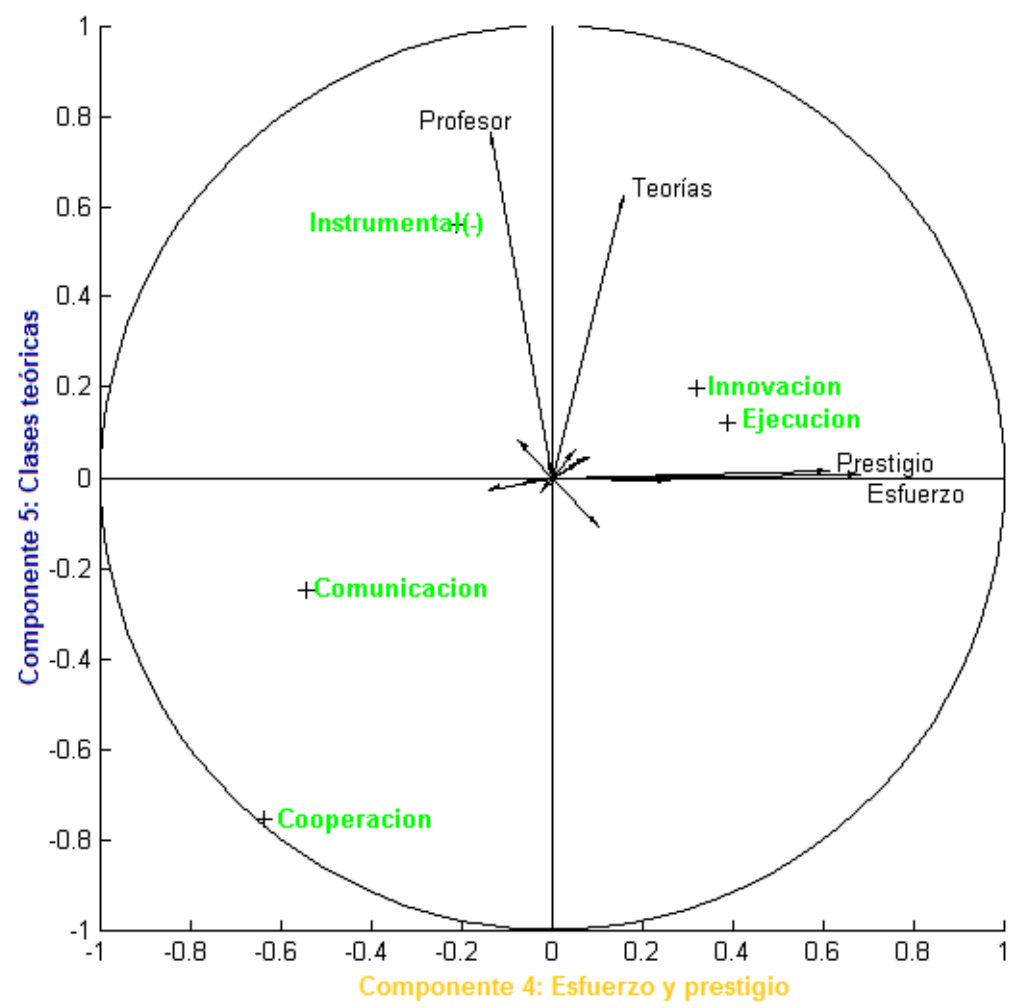

Figura 8.10: Representación simultánea sobre las componentes principales 5 y 6 de Competencias adquiridas en la Universidad. Características de la carrera y Métodos de enseñanza - aprendizaje 


\subsection{Anexo E: Abreviaturas y siglas}

\begin{tabular}{|c|c|}
\hline \multicolumn{2}{|r|}{ Características de los estudios } \\
\hline $\mathrm{C} 1$ & En tu carrera había que trabajar mucho para aprobar \\
\hline $\mathrm{C} 2$ & En tu carrera el contenido era conocido por los empleadores \\
\hline $\mathrm{C} 3$ & En tu carrera podías elegir las asignaturas que querías cursar \\
\hline $\mathrm{C} 4$ & En tu carrera el enfoque era generalista \\
\hline C5 & En tu carrera el enfoque era especializado \\
\hline C6 6 & Tu carrera era una carrera con prestigio académico \\
\hline \multicolumn{2}{|r|}{ Métodos de enseñanza - aprendizaje } \\
\hline V1 & Asistencia a clase \\
\hline $\mathrm{V} 2$ & Trabajos en grupo \\
\hline V3 & Participación en proyectos de investigación \\
\hline $\mathrm{V} 4$ & Prácticas en empresas, instituciones o similares \\
\hline V5 & Conocimientos prácticos y metodológicos \\
\hline V6 & Teorías, conceptos y paradigmas \\
\hline V7 & El profesor era la principal fuente de información \\
\hline V8 & Aprendizaje basado en proyectos o problemas \\
\hline V9 & Trabajos escritos \\
\hline V10 & Exposiciones orales \\
\hline VT & Realización de pruebas tipo test \\
\hline \multicolumn{2}{|r|}{ Gestión del tiempo } \\
\hline G1 & Dedicación exclusiva a los estudio \\
\hline G2 & Prácticas en empresa \\
\hline G3 & Nota obtenida en los estudios \\
\hline G4 & Dedicación de tiempo semanal a los estudios \\
\hline G5 & Estancia en el extranjero \\
\hline \multicolumn{2}{|r|}{ Características personales } \\
\hline P1 & Sexo \\
\hline $\mathrm{P} 2$ & Edad \\
\hline P3 & Situación de convivencia \\
\hline $\mathrm{P} 4$ & Tiene hijos \\
\hline P5 & Nivel de estudios del padre \\
\hline P6 & Nivel de estudios de la madre \\
\hline \multicolumn{2}{|r|}{ Área y nivel de estudios } \\
\hline A & Área de estudios \\
\hline $\mathrm{C}$ & Nivel de estudios \\
\hline \multicolumn{2}{|r|}{ Competencias adquiridas en la Universidad } \\
\hline V11 & Dominio de tu área o disciplina \\
\hline V12 & Conocimientos de otras áreas o disciplinas \\
\hline V13 & Pensamiento analítico \\
\hline V14 & Capacidad para adquirir con rapidez nuevos conocimientos \\
\hline V15 & Capacidad para negociar de forma eficaz \\
\hline V16 & Capacidad para rendir bajo presión \\
\hline V17 & Capacidad para detectar nuevas oportunidades \\
\hline V18 & Capacidad para coordinar actividades \\
\hline V19 & Capacidad para usar el tiempo de forma efectiva \\
\hline $\mathrm{V} 20$ & Capacidad para trabajar en equipo \\
\hline V21 & Capacidad para movilizar las capacidades de otros \\
\hline $\mathrm{V} 22$ & Capacidad para hacerte entender \\
\hline V23 & Capacidad para hacer valer tu autoridad \\
\hline $\mathrm{V} 24$ & Capacidad para utilizar herramientas informáticas \\
\hline $\mathrm{V} 25$ & Capacidad para encontrar nuevas ideas y soluciones \\
\hline V26 & Predisposición para cuestionar ideas propias o ajenas \\
\hline V27 & Capacidad para presentar en público productos, ideas o informes \\
\hline V28 & Capacidad para redactar informes o documentos \\
\hline V29 & Capacidad para escribir y hablar en idiomas extranjeros \\
\hline
\end{tabular}

Tabla 8.14: Variables contextuales del entorno académico y personal de los encuestados y Competencias adquiridas en la Universidad. Abreviaturas 